\title{
One-pot Parallel Synthesis of 5-(Dialkylamino)tetrazoles
}

Olena Savych, ${ }^{\text {a,b }}$ Yuliya O. Kuchkovska, ${ }^{\text {a,c }}$ Andrey V. Bogolyubsky, ${ }^{\text {a }}$ Anzhelika I. Konovets, ${ }^{\text {a }}$ Kateryna E. Gubina, ${ }^{\mathrm{c}}$ Sergey E. Pipko, ${ }^{\mathrm{d}}$ Anton V. Zhemera, ${ }^{\mathrm{a}}$ Alexander V. Grishchenko, ${ }^{\mathrm{a}}$ Dmytro N. Khomenko, ${ }^{\mathrm{c}}$ Volodymyr S. Brovarets, ${ }^{b}$ Roman Doroschuk, ${ }^{c}$ Yurii S. Moroz, ${ }^{\mathrm{c}, \mathrm{d}}$ Oleksandr O. Grygorenko*,a,c

${ }^{a}$ Enamine Ltd. (www.enamine.net) Chervonotkatska Street 78, Kyiv 02094, Ukraine

${ }^{b}$ V. P. Kukhar Institute of Bioorganic Chemistry and Petrochemistry, National Academy of Sciences of Ukraine, Kyiv, 02094, Ukraine

${ }^{c}$ Taras Shevchenko National University of Kyiv, Volodymyrska Street 60, Kyiv 01601, Ukraine

${ }^{d}$ Chemspace, Ilukstes iela 38-5, Riga, LV-1082, Latvia

*Corresponding author. E-mail: gregor@univ.kiev.ua.

Table of contents.

$\begin{array}{ll}\text { 1. Library characterization data } & \text { S2 }\end{array}$

2. Figure S1. Structures of the reagents $\mathbf{1 0}\{1-58\} \quad$ S21

$\begin{array}{lr}\text { 3. Figure S2. Structures of the reagents } 11\{1-511\} & \text { S24 }\end{array}$

4. Figure S3. Structures of the reagents $\mathbf{1 5}\{1-378\} \quad$ S56

5. Table S1. SMILES strings for the regents $\mathbf{1 0} \quad$ S80

6. Table S2. SMILES strings for the regents $\mathbf{1 1 .} \quad$ S83

$\begin{array}{ll}\text { 7. Table S3. SMILES strings for the regents } \mathbf{1 5 . .} & \text { S102 }\end{array}$

8. Table S4. Parallel synthesis of 5-aminotetrazoles $8 \quad$ S116

9. Table S5. Parallel synthesis of 5-aminotetrazoles $9 \quad$ S137

$\begin{array}{lr}\text { 10. Copies of NMR spectra } & \text { S180 }\end{array}$ 


\section{Library characterization data}

General Methods. The solvents were purified according to the standard procedures. All starting materials were taken at Enamine Ltd. Reverse phase column chromatography was performed using $\mathrm{C}_{18^{-}}$ modified silica gel as a stationary phase. ${ }^{1} \mathrm{H},{ }^{19} \mathrm{~F}$, and ${ }^{13} \mathrm{C}$ NMR spectra were recorded at $500 \mathrm{MHz}, 470$, and $126 \mathrm{MHz}$, respectively. Chemical shifts are reported in ppm downfield from TMS as internal standards. Mass spectra were recorded on an LC-MS instrument with chemical ionization (CI). LC-MS data were acquired on an Agilent 1200 HPLC system equipped with DAD/ELSD/LC-MS-6120 diodematrix and mass-selective detector. Melting points were measured on a MPA100 OptiMelt automated melting point system. Elemental analyses were performed at the Laboratory of Organic Analysis, Department of Chemistry, Taras Shevchenko National University of Kyiv.

\section{Products obtained by isothiocyanate-based method.}

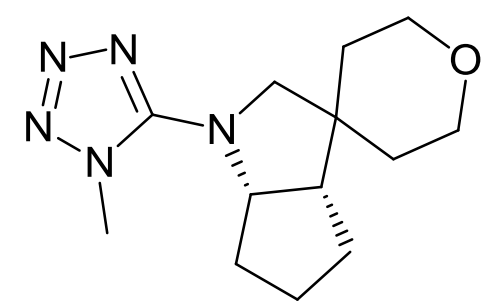

rac-(3aR,6aR)-1-(1-Methyl-1H-tetrazol-5-yl)decahydro-1 $H$-spiro[cyclopenta[b]pyrrole-3,4'-pyran] $(\mathbf{8}\{2,8\})$. Beige solid, $\mathrm{mp}=137-139{ }^{\circ} \mathrm{C} .{ }^{1} \mathrm{H}$ NMR $\left(500 \mathrm{MHz}\right.$, DMSO- $\left.d_{6}\right): \delta 4.46(\mathrm{td}, J=6.7,3.1 \mathrm{~Hz}$, $1 \mathrm{H}), 3.92(\mathrm{~s}, 3 \mathrm{H}), 3.67-3.54(\mathrm{~m}, 4 \mathrm{H}), 3.51-3.44(\mathrm{~m}, 1 \mathrm{H}), 3.36(\mathrm{~d}, J=9.9 \mathrm{~Hz}, 1 \mathrm{H}), 2.48-2.41(\mathrm{~m}$, $1 \mathrm{H}), 1.98-1.91(\mathrm{~m}, 1 \mathrm{H}), 1.66-1.60(\mathrm{~m}, 2 \mathrm{H}), 1.60-1.51(\mathrm{~m}, 2 \mathrm{H}), 1.49-1.33(\mathrm{~m}, 5 \mathrm{H}) \mathrm{ppm} .{ }^{13} \mathrm{C}\left\{{ }^{1} \mathrm{H}\right\}$ NMR (126 MHz, DMSO- $\left.d_{6}\right): \delta$ 155.6, 64.6, 63.9, 63.6, 56.8, 51.8, 41.2, 36.1, 33.6, 33.1, 31.4, 26.1, 25.5 ppm. LC-MS $(m / z): 264\left(\mathrm{M}+\mathrm{H}^{+}\right)$. Anal. Calcd. for $\mathrm{C}_{13} \mathrm{H}_{21} \mathrm{~N}_{5} \mathrm{O}: \mathrm{C}, 59.29 ; \mathrm{H}, 8.04 ; \mathrm{N}, 26.59$. Found: $\mathrm{C}$, 59.48; H, 8.19; N, 26.67.

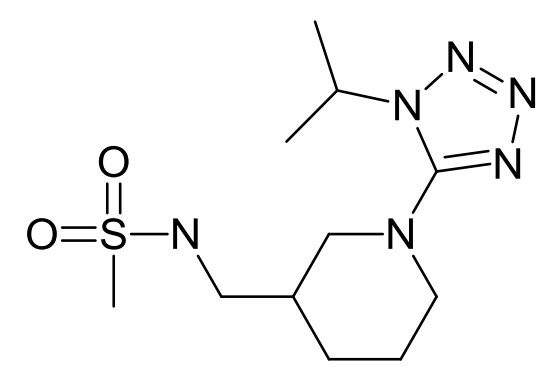

$\boldsymbol{N}$-((1-(1-Isopropyl-1H-tetrazol-5-yl)piperidin-3-yl)methyl)methanesulfonamide $(8\{10,50\})$. White solid, mp $=114-116^{\circ} \mathrm{C} .{ }^{1} \mathrm{H}$ NMR $\left(500 \mathrm{MHz}\right.$, DMSO- $\left.d_{6}\right): \delta 7.08(\mathrm{~s}, 1 \mathrm{H}), 4.56$ (hept, $\left.J=6.5 \mathrm{~Hz}, 1 \mathrm{H}\right)$, $3.38(\mathrm{dd}, J=12.3,3.5 \mathrm{~Hz}, 1 \mathrm{H}), 3.28(\mathrm{dt}, J=13.4,3.9 \mathrm{~Hz}, 1 \mathrm{H}), 2.94-2.90(\mathrm{~m}, 3 \mathrm{H}), 2.89(\mathrm{~s}, 3 \mathrm{H}), 2.74$ $(\mathrm{dd}, J=12.2,9.4 \mathrm{~Hz}, 1 \mathrm{H}), 1.94-1.80(\mathrm{~m}, 1 \mathrm{H}), 1.80-1.70(\mathrm{~m}, 2 \mathrm{H}), 1.68-1.56(\mathrm{~m}, 1 \mathrm{H}), 1.47(\mathrm{~d}, J=$ $6.4 \mathrm{~Hz}, 3 \mathrm{H}), 1.45(\mathrm{~d}, J=6.4 \mathrm{~Hz}, 3 \mathrm{H}), 1.26-1.17(\mathrm{~m}, 1 \mathrm{H}) \mathrm{ppm} .{ }^{13} \mathrm{C}\left\{{ }^{1} \mathrm{H}\right\}$ NMR $\left(126 \mathrm{MHz}\right.$, DMSO- $\left.d_{6}\right): \delta$ 158.5, 53.7, 50.9, 49.5, 45.2, 35.3, 27.1, 23.5, 22.22, $22.16 \mathrm{ppm}$. LC-MS $(\mathrm{m} / \mathrm{z}): 303\left(\mathrm{M}+\mathrm{H}^{+}\right)$. Anal. Calcd. for $\mathrm{C}_{11} \mathrm{H}_{22} \mathrm{~N}_{6} \mathrm{O}_{2} \mathrm{~S}: \mathrm{C}, 43.69 ; \mathrm{H}, 7.33 ; \mathrm{N}, 27.79 ; \mathrm{S}, 10.60$. Found: $\mathrm{C}, 43.29 ; \mathrm{H}, 7.54 ; \mathrm{N}, 27.85 ; \mathrm{S}$, 10.82 . 


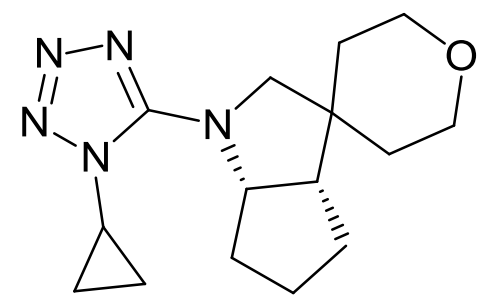

rac-(3a $R, 6 \mathrm{a} R)$-1-(1-Cyclopropyl-1H-tetrazol-5-yl)decahydro-1H-spiro[cyclopenta[b]pyrrole-3,4' pyran] $(8\{15,8)\})$. Yellow solid, $\mathrm{mp}=92-95{ }^{\circ} \mathrm{C} .{ }^{1} \mathrm{H}$ NMR $\left(500 \mathrm{MHz}, \mathrm{DMSO}-d_{6}\right): \delta 4.53(\mathrm{td}, J=6.8$, $2.9 \mathrm{~Hz}, 1 \mathrm{H}), 3.75(\mathrm{~d}, J=10.1 \mathrm{~Hz}, 1 \mathrm{H}), 3.70(\mathrm{tt}, J=7.1,3.7 \mathrm{~Hz}, 1 \mathrm{H}), 3.66-3.52(\mathrm{~m}, 3 \mathrm{H}), 3.51-3.44$ $(\mathrm{m}, 1 \mathrm{H}), 3.40(\mathrm{~d}, J=10.1 \mathrm{~Hz}, 1 \mathrm{H}), 1.98(\mathrm{dt}, J=11.4,6.5 \mathrm{~Hz}, 1 \mathrm{H}), 1.69-1.55(\mathrm{~m}, 4 \mathrm{H}), 1.54-1.40(\mathrm{~m}$, $5 \mathrm{H}), 1.39-1.29(\mathrm{~m}, 1 \mathrm{H}), 1.25-1.06(\mathrm{~m}, 3 \mathrm{H}), 1.05-0.96(\mathrm{~m}, 1 \mathrm{H}) \mathrm{ppm} .{ }^{13} \mathrm{C}\left\{{ }^{1} \mathrm{H}\right\} \mathrm{NMR}(126 \mathrm{MHz}$, DMSO- $\left.d_{6}\right): \delta 156.2,64.6,64.2,63.7,56.6,51.5,41.2,36.1,33.2,31.4,27.6,26.0,25.5,7.9,7.4$ ppm. LC-MS $(\mathrm{m} / z)$ : $290\left(\mathrm{M}+\mathrm{H}^{+}\right)$. Anal. Calcd. for $\mathrm{C}_{15} \mathrm{H}_{23} \mathrm{~N}_{5} \mathrm{O}: \mathrm{C}, 62.26 ; \mathrm{H}, 8.01 ; \mathrm{N}, 24.20$. Found: C, 62.12; $\mathrm{H}, 7.70 ; \mathrm{N}, 23.94$.<smiles>CCn1cc(CN(C)c2nnnn2C2CC2)cn1</smiles>

1-Cyclopropyl- $\boldsymbol{N}$-((1-ethyl-1H-pyrazol-4-yl)methyl)- $\boldsymbol{N}$-methyl-1 $\boldsymbol{H}$-tetrazol-5-amine $\quad(8\{15,71\})$. Yellow oil. ${ }^{1} \mathrm{H}$ NMR (500 MHz, DMSO-d $): \delta 7.72(\mathrm{~s}, 1 \mathrm{H}), 7.40(\mathrm{~s}, 1 \mathrm{H}), 4.46(\mathrm{~s}, 2 \mathrm{H}), 4.08(\mathrm{q}, J=7.2$ $\mathrm{Hz}, 2 \mathrm{H}), 3.73-3.67(\mathrm{~m}, 1 \mathrm{H}), 2.99(\mathrm{~s}, 3 \mathrm{H}), 1.33(\mathrm{t}, J=7.2 \mathrm{~Hz}, 3 \mathrm{H}), 1.21-1.12(\mathrm{~m}, 4 \mathrm{H}) \mathrm{ppm} .{ }^{13} \mathrm{C}\left\{{ }^{1} \mathrm{H}\right\}$ NMR $\left(126 \mathrm{MHz}, \mathrm{DMSO}-d_{6}\right): \delta 158.6,138.5,128.8,115.3,46.5,46.1,37.1,28.5,15.5,7.7$ ppm. LC-MS $(m / z):=248\left(\mathrm{M}+\mathrm{H}^{+}\right)$. Anal. Calcd. for $\mathrm{C}_{11} \mathrm{H}_{17} \mathrm{~N}_{7}: \mathrm{C}, 53.42 ; \mathrm{H}, 6.93 ; \mathrm{N}, 39.65$. Found: $\mathrm{C}, 53.28 ; \mathrm{H}, 7.11$; $\mathrm{N}, 39.43$.<smiles>CCN(Cc1ccoc1)c1nnnn1C1CC1</smiles>

1-Cyclopropyl- $\boldsymbol{N}$-ethyl- $\boldsymbol{N}$-(furan-3-ylmethyl)-1H-tetrazol-5-amine $(\mathbf{8}\{15,72)\})$. Beige solid, $\mathrm{mp}=$ 60-62 ${ }^{\circ} \mathrm{C} .{ }^{1} \mathrm{H}$ NMR (500 MHz, DMSO-d $): \delta 7.67$ (s, 1H), 7.62 (s, 1H), $6.44(\mathrm{~s}, 1 \mathrm{H}), 4.47$ (s, 2H), 3.64 (quint, $J=5.3 \mathrm{~Hz}, 1 \mathrm{H}), 3.43(\mathrm{q}, J=7.1 \mathrm{~Hz}, 2 \mathrm{H}), 1.15(\mathrm{~d}, J=5.3 \mathrm{~Hz}, 4 \mathrm{H}), 1.10(\mathrm{t}, J=7.1 \mathrm{~Hz}, 3 \mathrm{H}) \mathrm{ppm}$. ${ }^{13} \mathrm{C}\left\{{ }^{1} \mathrm{H}\right\}$ NMR $\left(126 \mathrm{MHz}, \mathrm{DMSO}-d_{6}\right): \delta 158.2,143.7,141.2,121.0,110.7,44.0,43.9,28.4,12.4,7.4$ ppm. LC-MS $(\mathrm{m} / \mathrm{z}): 234\left(\mathrm{M}+\mathrm{H}^{+}\right)$. Anal. Calcd. for $\mathrm{C}_{11} \mathrm{H}_{15} \mathrm{~N}_{5} \mathrm{O}: \mathrm{C}, 56.64 ; \mathrm{H}, 6.48 ; \mathrm{N}, 30.02$. Found: C, $56.98 ; \mathrm{H}, 6.76 ; \mathrm{N}, 29.86$. 


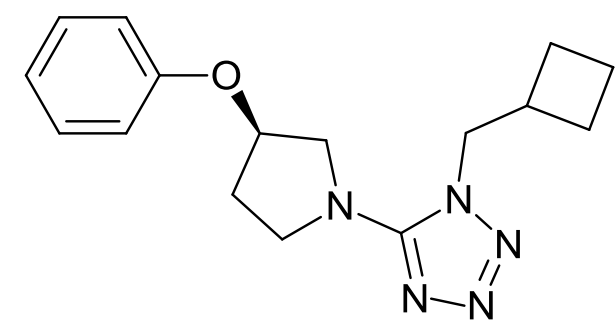

(R)-1-(Cyclobutylmethyl)-5-(3-phenoxypyrrolidin-1-yl)-1H -tetrazole $(\mathbf{8}\{36,164\})$. White solid, $\mathrm{mp}=$ 52-54 ${ }^{\circ} \mathrm{C}$. ${ }^{1} \mathrm{H}$ NMR $\left(500 \mathrm{MHz}, \mathrm{DMSO}-d_{6}\right): \delta 7.30(\mathrm{t}, J=7.8 \mathrm{~Hz}, 2 \mathrm{H}), 6.98-6.94(\mathrm{~m}, 3 \mathrm{H}), 5.16-5.12$ $(\mathrm{m}, 1 \mathrm{H}), 4.33(\mathrm{~d}, J=2.3 \mathrm{~Hz}, 1 \mathrm{H}), 4.32(\mathrm{~d}, J=2.2 \mathrm{~Hz}, 1 \mathrm{H}), 3.88(\mathrm{dd}, J=11.2,4.6 \mathrm{~Hz}, 1 \mathrm{H}), 3.70-3.62$ (m, 3H), 2.74 (hept, $J=7.6 \mathrm{~Hz}, 1 \mathrm{H}), 2.33-2.23(\mathrm{~m}, 1 \mathrm{H}), 2.20-2.12(\mathrm{~m}, 1 \mathrm{H}), 1.98-1.91(\mathrm{~m}, 2 \mathrm{H}), 1.86$ $-1.73(\mathrm{~m}, 4 \mathrm{H}) \mathrm{ppm} .{ }^{13} \mathrm{C}\left\{{ }^{1} \mathrm{H}\right\}$ NMR $\left(126 \mathrm{MHz}, \mathrm{DMSO}-d_{6}\right): \delta 156.8,155.7,129.6,121.0,115.5,75.7$, 54.7, 51.1, 47.4, 34.5, 30.9, 25.15, 25.12, $17.6 \mathrm{ppm}$. LC-MS $(\mathrm{m} / \mathrm{z}): 300\left(\mathrm{M}+\mathrm{H}^{+}\right)$. Anal. Calcd. for $\mathrm{C}_{16} \mathrm{H}_{21} \mathrm{~N}_{5} \mathrm{O}$ : C, 64.19; H, 7.07; N, 23.39. Found: C, 64.52; H, 6.87; N, 23.42.

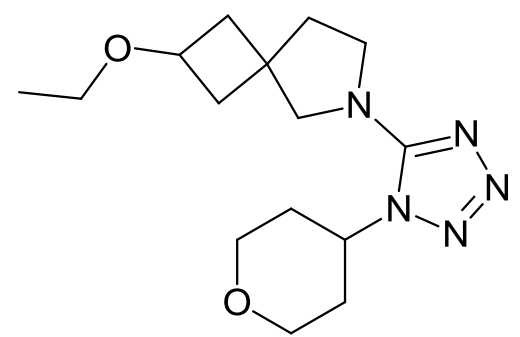

1-(1-(1-Methylcyclopropyl)-1H-tetrazol-5-yl)-4-((methylsulfonyl)methyl)piperidine $\quad(\mathbf{8}\{40,188\})$. Beige solid, $\mathrm{mp}=66-69{ }^{\circ} \mathrm{C}$. The compound existed as a ca. 3:2 mixture of rotamers. ${ }^{1} \mathrm{H}$ NMR (500 $\left.\mathrm{MHz}, \mathrm{DMSO}-d_{6}\right): \delta 4.68-4.56(\mathrm{~m}, 1 \mathrm{H}), 4.02-3.91(\mathrm{~m}, 3 \mathrm{H}), 3.57-3.50(\mathrm{~m}, 4 \mathrm{H}), 3.49(\mathrm{~s}, 2 \mathrm{H}), 3.35-$ $3.30(\mathrm{~m}, 2 \mathrm{H}), 2.29$ (ddd, $J=9.7,7.0,2.8 \mathrm{~Hz}, 0.8 \mathrm{H}), 2.23$ (ddd, $J=9.6,6.9,2.9 \mathrm{~Hz}, 1.2 \mathrm{H}), 2.03-1.85$ $(\mathrm{m}, 8 \mathrm{H}), 1.09(\mathrm{t}, J=7.0 \mathrm{~Hz}, 3 \mathrm{H}) \mathrm{ppm} .{ }^{13} \mathrm{C}\left\{{ }^{1} \mathrm{H}\right\}$ NMR $\left(126 \mathrm{MHz}, \mathrm{DMSO}-d_{6}\right): \delta 155.8$ and $155.5,68.1$ and 67.9, 65.73 and 65.70, 62.4, 60.5 and 59.9, 53.3, 48.5 and 47.9, 38.1, 37.4, 36.8 and 36.4, 32.85 and 32.76, 15.2 ppm. LC-MS $(\mathrm{m} / z): 308\left(\mathrm{M}+\mathrm{H}^{+}\right)$. Anal. Calcd. for $\mathrm{C}_{15} \mathrm{H}_{25} \mathrm{~N}_{5} \mathrm{O}_{2}: \mathrm{C}, 58.61 ; \mathrm{H}, 8.20 ; \mathrm{N}$, 22.78. Found: C, 58.70; H, 8.00; N, 22.60.<smiles>Cc1cccc(CN(C)c2nnnn2C2CCOCC2)n1</smiles>

$\boldsymbol{N}$-Methyl- $\boldsymbol{N}$-((6-methylpyridin-2-yl)methyl)-1-(tetrahydro-2H-pyran-4-yl)-1H-tetrazol-5-amine (8\{40,192\}). Brown solid, mp $=71-74{ }^{\circ} \mathrm{C} .{ }^{1} \mathrm{H}$ NMR $\left(500 \mathrm{MHz}, \mathrm{DMSO}-d_{6}\right): \delta 7.68(\mathrm{t}, J=7.7 \mathrm{~Hz}, 1 \mathrm{H})$, $7.20(\mathrm{~d}, J=7.7 \mathrm{~Hz}, 1 \mathrm{H}), 7.18(\mathrm{~d}, J=7.7 \mathrm{~Hz}, 1 \mathrm{H}), 4.82(\mathrm{tt}, J=10.4,5.3 \mathrm{~Hz}, 1 \mathrm{H}), 4.48(\mathrm{~s}, 2 \mathrm{H}), 3.96(\mathrm{~d}, J$ $=11.3 \mathrm{~Hz}, 2 \mathrm{H}), 3.42(\mathrm{td}, J=11.4,3.5 \mathrm{~Hz}, 2 \mathrm{H}), 3.00(\mathrm{~s}, 3 \mathrm{H}), 2.43(\mathrm{~s}, 3 \mathrm{H}), 2.05-1.96(\mathrm{~m}, 4 \mathrm{H}) \mathrm{ppm}$. ${ }^{13} \mathrm{C}\left\{{ }^{1} \mathrm{H}\right\}$ NMR $\left(126 \mathrm{MHz}, \mathrm{DMSO}-d_{6}\right): \delta 158.1,157.7,155.8,137.2,122.0,119.2,65.9,58.9,53.6,39.6$, 32.5, 24.0 ppm. LC-MS $(m / z): 289\left(\mathrm{M}+\mathrm{H}^{+}\right)$. Anal. Calcd. for $\mathrm{C}_{14} \mathrm{H}_{20} \mathrm{~N}_{6} \mathrm{O}: \mathrm{C}, 58.31 ; \mathrm{H}, 6.99 ; \mathrm{N}, 29.15$. Found: C, 58.48; H, 7.31; N, 29.38. 
<smiles>Clc1cnn(C2CCN(c3nnnn3CCc3cccs3)C2)c1</smiles>

5-(3-(4-Chloro-1H-pyrazol-1-yl)pyrrolidin-1-yl)-1-(2-(thiophen-2-yl)ethyl)-1 $H$-tetrazole $(8\{41,147\})$. Beige solid, mp $=92-94{ }^{\circ} \mathrm{C} .{ }^{1} \mathrm{H}$ NMR $\left(500 \mathrm{MHz}\right.$, DMSO- $\left.d_{6}\right): \delta 8.08(\mathrm{~s}, 1 \mathrm{H}), 7.58(\mathrm{~s}, 1 \mathrm{H})$, $7.33(\mathrm{~d}, J=5.1 \mathrm{~Hz}, 1 \mathrm{H}), 6.93(\mathrm{dd}, J=5.1,3.4 \mathrm{~Hz}, 1 \mathrm{H}), 6.81(\mathrm{~d}, J=3.4 \mathrm{~Hz}, 1 \mathrm{H}), 5.09-5.01(\mathrm{~m}, 1 \mathrm{H})$, $4.54(\mathrm{t}, J=7.0 \mathrm{~Hz}, 2 \mathrm{H}), 3.91(\mathrm{dd}, J=10.4,6.3 \mathrm{~Hz}, 1 \mathrm{H}), 3.77(\mathrm{dd}, J=10.4,3.9 \mathrm{~Hz}, 1 \mathrm{H}), 3.67(\mathrm{q}, J=7.9$ $\mathrm{Hz}, 1 \mathrm{H}), 3.60(\mathrm{dt}, J=9.0,4.4 \mathrm{~Hz}, 1 \mathrm{H}), 3.35-3.29(\mathrm{~m}, 2 \mathrm{H}), 2.44-2.32(\mathrm{~m}, 2 \mathrm{H}) \mathrm{ppm} .{ }^{13} \mathrm{C}\left\{{ }^{1} \mathrm{H}\right\} \mathrm{NMR}$ $\left(126 \mathrm{MHz}, \mathrm{DMSO}-d_{6}\right): \delta 155.6,138.8,137.2,127.3,127.1,126.1,124.8,108.3,60.3,54.1,47.8,47.6$, 31.0, 29.0 ppm. LC-MS $(\mathrm{m} / z):=350\left(\mathrm{M}+\mathrm{H}^{+}\right)$. Anal. Calcd. for $\mathrm{C}_{14} \mathrm{H}_{16} \mathrm{ClN}_{7} \mathrm{~S}: \mathrm{C}, 48.07 ; \mathrm{H}, 4.61 ; \mathrm{N}$, 28.03; S, 9.16; Cl, 10.13. Found: C, 47.96; H, 4.57; N, 28.14; S, 8.93; Cl, 9.87.<smiles>Cc1csc(C2CCN(c3nnnn3CCc3cccs3)C2)n1</smiles>

4-Methyl-2-(1-(1-(2-(thiophen-2-yl)ethyl)-1 $\boldsymbol{H}$-tetrazol-5-yl)pyrrolidin-3-yl)thiazole $\quad(8\{41,148\})$. Brown oil. ${ }^{1} \mathrm{H}$ NMR (500 MHz, DMSO- $\left.d_{6}\right): \delta 7.33(\mathrm{~d}, J=5.1 \mathrm{~Hz}, 1 \mathrm{H}), 7.17(\mathrm{~s}, 1 \mathrm{H}), 6.92(\mathrm{t}, J=5.1,3.4$ $\mathrm{Hz}, 1 \mathrm{H}), 6.81(\mathrm{~d}, J=3.4 \mathrm{~Hz}, 1 \mathrm{H}), 4.54(\mathrm{t}, J=7.0 \mathrm{~Hz}, 2 \mathrm{H}), 3.90-3.82(\mathrm{~m}, 2 \mathrm{H}), 3.69-3.52(\mathrm{~m}, 3 \mathrm{H})$, $3.33(\mathrm{t}, J=6.9 \mathrm{~Hz}, 2 \mathrm{H}), 2.43-2.36(\mathrm{~m}, 1 \mathrm{H}), 2.32(\mathrm{~s}, 3 \mathrm{H}), 2.19-2.11(\mathrm{~m}, 1 \mathrm{H}) \mathrm{ppm} .{ }^{13} \mathrm{C}\left\{{ }^{1} \mathrm{H}\right\} \mathrm{NMR}(126$ MHz, DMSO- $\left.d_{6}\right): \delta 169.5,155.7,151.6,138.8,127.1,126.2,124.9,113.7,54.5,48.5,47.8,41.6,32.1$, 29.0, 16.8 ppm. LC-MS $(m / z):=347\left(\mathrm{M}+\mathrm{H}^{+}\right)$. Anal. Calcd. for $\mathrm{C}_{15} \mathrm{H}_{18} \mathrm{~N}_{6} \mathrm{~S}_{2}: \mathrm{C}, 52.00 ; \mathrm{H}, 5.24 ; \mathrm{N}$, 24.26; S, 18.51. Found: C, 52.17; H, 5.34; N, 24.21; S, 18.37.<smiles>Oc1cccc2c1CCN(c1nnnn1CCc1cccs1)C2</smiles> 

$\mathrm{Hz}, 1 \mathrm{H}), 6.98(\mathrm{t}, J=7.8 \mathrm{~Hz}, 1 \mathrm{H}), 6.91(\mathrm{t}, J=5.1,3.4 \mathrm{~Hz}, 1 \mathrm{H}), 6.81(\mathrm{~d}, J=3.4 \mathrm{~Hz}, 1 \mathrm{H}), 6.67(\mathrm{~d}, J=7.9$ $\mathrm{Hz}, 1 \mathrm{H}), 6.58(\mathrm{~d}, J=7.6 \mathrm{~Hz}, 1 \mathrm{H}), 4.53(\mathrm{t}, J=6.8 \mathrm{~Hz}, 2 \mathrm{H}), 4.30(\mathrm{~s}, 2 \mathrm{H}), 3.44-3.39(\mathrm{~m}, 4 \mathrm{H}), 2.72(\mathrm{t}, J=$ $6.0 \mathrm{~Hz}, 2 \mathrm{H}) \mathrm{ppm} .{ }^{13} \mathrm{C}\left\{{ }^{1} \mathrm{H}\right\}$ NMR $\left(126 \mathrm{MHz}, \mathrm{DMSO}-d_{6}\right): \delta 158.7,154.8,138.9,134.0,127.1,126.4$, 126.1, 124.9, 120.4, 116.8, 112.4, 50.8, 48.2, 47.1, 27.8, 22.5 ppm. LC-MS $(\mathrm{m} / \mathrm{z}): 328\left(\mathrm{M}+\mathrm{H}^{+}\right)$. Anal. Calcd. for $\mathrm{C}_{16} \mathrm{H}_{17} \mathrm{~N}_{5} \mathrm{OS}$ : C, 58.70; H, 5.23; N, 21.39; S, 9.79. Found: C, 58.98; H, 5.44; N, 21.04; S, 9.56 .

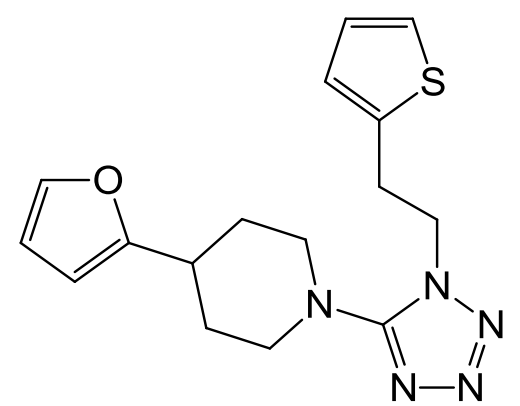

4-(Furan-2-yl)-1-(1-(2-(thiophen-2-yl)ethyl)-1 $\boldsymbol{H}$-tetrazol-5-yl)piperidine $(\mathbf{8}\{41,211\})$. Yellow oil. ${ }^{1} \mathrm{H}$ NMR $\left(500 \mathrm{MHz}\right.$, DMSO- $\left.d_{6}\right): \delta 7.53(\mathrm{~s}, 1 \mathrm{H}), 7.32(\mathrm{~d}, J=5.2 \mathrm{~Hz}, 1 \mathrm{H}), 6.93-6.88(\mathrm{~m}, 1 \mathrm{H}), 6.78(\mathrm{~d}, J=$ $3.4 \mathrm{~Hz}, 1 \mathrm{H}), 6.37(\mathrm{~s}, 1 \mathrm{H}), 6.12(\mathrm{~d}, J=3.1 \mathrm{~Hz}, 1 \mathrm{H}), 4.46(\mathrm{t}, J=6.8 \mathrm{~Hz}, 2 \mathrm{H}), 3.42-3.36(\mathrm{~m}, 4 \mathrm{H}), 3.00(\mathrm{t}$, $J=12.2 \mathrm{~Hz}, 2 \mathrm{H}), 2.89-2.82(\mathrm{~m}, 1 \mathrm{H}), 2.02-1.90(\mathrm{~m}, 2 \mathrm{H}), 1.72-1.62(\mathrm{~m}, 2 \mathrm{H}) \mathrm{ppm} .{ }^{13} \mathrm{C}\left\{{ }^{1} \mathrm{H}\right\} \mathrm{NMR}$ $\left(126 \mathrm{MHz}, \mathrm{DMSO}-d_{6}\right): \delta 159.0,158.2,141.3,138.9,127.0,126.1,124.8,110.2,103.7,49.2,48.1,33.8$, 29.6, 27.8 ppm. LC-MS $(m / z): 330\left(\mathrm{M}+\mathrm{H}^{+}\right)$. Anal. Calcd. for $\mathrm{C}_{16} \mathrm{H}_{19} \mathrm{~N}_{5} \mathrm{OS}: \mathrm{C}, 58.34 ; \mathrm{H}, 5.81 ; \mathrm{N}, 21.26$; S, 9.73. Found: C, 58.07; H, 5.83; N, 21.40; S, 9.64.

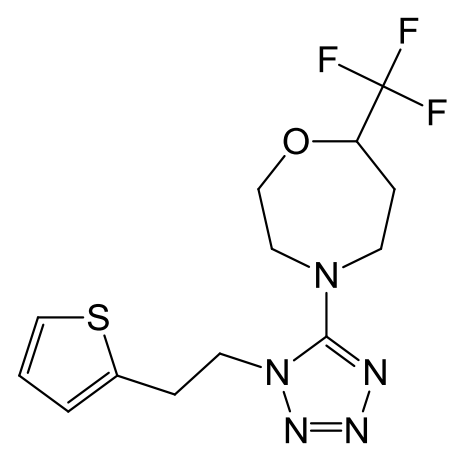

4-(1-(2-(Thiophen-2-yl)ethyl)-1H-tetrazol-5-yl)-7-(trifluoromethyl)-1,4-oxazepane $\quad(8\{41,212\})$. Yellow oil. ${ }^{1} \mathrm{H}$ NMR $\left(500 \mathrm{MHz}, \mathrm{DMSO}-d_{6}\right): \delta 7.34(\mathrm{~d}, J=5.1 \mathrm{~Hz}, 1 \mathrm{H}), 6.94-6.90(\mathrm{~m}, 1 \mathrm{H}), 6.83-6.77$ $(\mathrm{m}, 1 \mathrm{H}), 4.54-4.43(\mathrm{~m}, 2 \mathrm{H}), 4.31-4.24(\mathrm{~m}, 1 \mathrm{H}), 4.02(\mathrm{~d}, J=12.9 \mathrm{~Hz}, 1 \mathrm{H}), 3.78-3.71(\mathrm{~m}, 1 \mathrm{H}), 3.58-$ $3.46(\mathrm{~m}, 4 \mathrm{H}), 3.37(\mathrm{t}, J=6.9 \mathrm{~Hz}, 2 \mathrm{H}), 2.13-2.05(\mathrm{~m}, 1 \mathrm{H}), 2.01-1.94(\mathrm{~m}, 1 \mathrm{H}) \mathrm{ppm} .{ }^{13} \mathrm{C}\left\{{ }^{1} \mathrm{H}\right\} \mathrm{NMR}$ $\left(126 \mathrm{MHz}, \mathrm{DMSO}-d_{6}\right): \delta 157.9,138.9,127.1,126.2,124.9,124.8(\mathrm{q}, J=281.1 \mathrm{~Hz}), 75.4(\mathrm{q}, J=29.8$ $\mathrm{Hz}), 68.8,53.1,48.4,48.3,28.3,28.1 \mathrm{ppm} .{ }^{19} \mathrm{~F}\left\{{ }^{1} \mathrm{H}\right\}$ NMR $\left(376 \mathrm{MHz}\right.$, DMSO- $\left.d_{6}\right): \delta-77.6 \mathrm{ppm}$. LC-MS $(\mathrm{m} / \mathrm{z}): 348\left(\mathrm{M}+\mathrm{H}^{+}\right)$. Anal. Calcd. for $\mathrm{C}_{13} \mathrm{H}_{16} \mathrm{~F}_{3} \mathrm{~N}_{5} \mathrm{OS}$ : C, 44.95; H, 4.64; N, 20.16; S, 9.23. Found: C, 44.92; H, 4.83; N, 19.96; S, 9.00. 


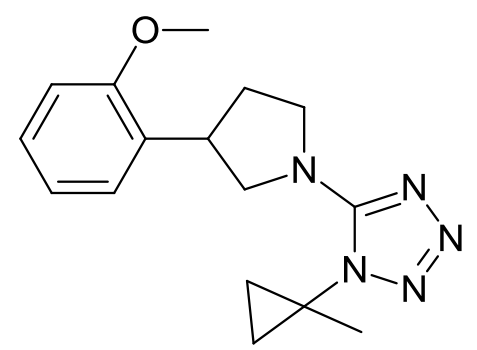

5-(3-(2-Methoxyphenyl)pyrrolidin-1-yl)-1-(1-methylcyclopropyl)-1H-tetrazole $(8\{42,193)\})$. White solid, $\mathrm{mp}=106-108{ }^{\circ} \mathrm{C} .{ }^{1} \mathrm{H}$ NMR $\left(500 \mathrm{MHz}, \mathrm{DMSO}-d_{6}\right): \delta 7.29-7.21(\mathrm{~m}, 2 \mathrm{H}), 7.01(\mathrm{~d}, J=8.1 \mathrm{~Hz}$, $1 \mathrm{H}), 6.93(\mathrm{t}, J=7.5 \mathrm{~Hz}, 1 \mathrm{H}), 4.00(\mathrm{dd}, J=9.5,7.3 \mathrm{~Hz}, 1 \mathrm{H}), 3.82(\mathrm{~s}, 3 \mathrm{H}), 3.78-3.65(\mathrm{~m}, 3 \mathrm{H}), 3.54-$ $3.49(\mathrm{~m}, 1 \mathrm{H}), 2.33-2.24(\mathrm{~m}, 1 \mathrm{H}), 2.11(\mathrm{dq}, J=12.0,8.6 \mathrm{~Hz}, 1 \mathrm{H}), 1.56(\mathrm{~s}, 3 \mathrm{H}), 1.35-1.26(\mathrm{~m}, 2 \mathrm{H})$, $1.13-1.06(\mathrm{~m}, 2 \mathrm{H}) \mathrm{ppm} .{ }^{13} \mathrm{C}\left\{{ }^{1} \mathrm{H}\right\}$ NMR $\left(126 \mathrm{MHz}, \mathrm{DMSO}-d_{6}\right): \delta 157.1,156.0,129.0,127.9,126.7$, 120.5, 110.9, 55.4, 54.5, 49.2, 37.3, 34.2, 30.7, 23.9, 15.1, 14.9 ppm. LC-MS $(\mathrm{m} / \mathrm{z}): 300\left(\mathrm{M}+\mathrm{H}^{+}\right)$. Anal. Calcd. for $\mathrm{C}_{16} \mathrm{H}_{21} \mathrm{~N}_{5} \mathrm{O}$ : C, 64.19; H, 7.07; N, 23.39. Found: C, 64.57; H, 7.44; N, 23.30 .

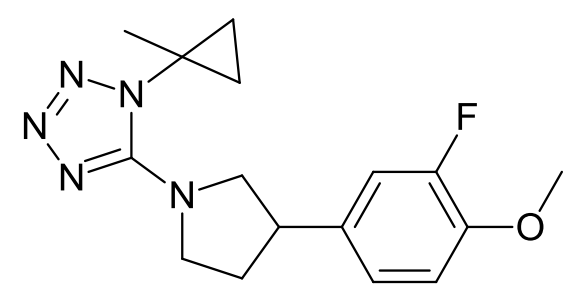

\section{5-(3-(3-Fluoro-4-methoxyphenyl)pyrrolidin-1-yl)-1-(1-methylcyclopropyl)-1H-tetrazole}

(8 $\{42,227\})$. White solid, $\mathrm{mp}=95-97{ }^{\circ} \mathrm{C} .{ }^{1} \mathrm{H}$ NMR $\left(500 \mathrm{MHz}\right.$, DMSO- $\left.d_{6}\right): \delta 7.24(\mathrm{~d}, J=13.1 \mathrm{~Hz}, 1 \mathrm{H})$, $7.13-7.10(\mathrm{~m}, 2 \mathrm{H}), 4.03-3.94(\mathrm{~m}, 1 \mathrm{H}), 3.82(\mathrm{~s}, 3 \mathrm{H}), 3.80-3.76(\mathrm{~m}, 1 \mathrm{H}), 3.68(\mathrm{td}, J=9.4,6.9 \mathrm{~Hz}$, $1 \mathrm{H}), 3.53-3.44(\mathrm{~m}, 2 \mathrm{H}), 2.37-2.29(\mathrm{~m}, 1 \mathrm{H}), 2.10-2.01(\mathrm{~m}, 1 \mathrm{H}), 1.56(\mathrm{~s}, 3 \mathrm{H}), 1.37-1.24(\mathrm{~m}, 2 \mathrm{H})$, $1.14-1.08(\mathrm{~m}, 2 \mathrm{H}) \mathrm{ppm} .{ }^{13} \mathrm{C}\left\{{ }^{1} \mathrm{H}\right\}$ NMR $\left(126 \mathrm{MHz}, \mathrm{DMSO}-d_{6}\right): \delta 155.9,151.4(\mathrm{~d}, J=243.4 \mathrm{~Hz}), 145.8$ $(\mathrm{d}, J=10.5 \mathrm{~Hz}), 134.3(\mathrm{~d}, J=6.0 \mathrm{~Hz}), 123.3(\mathrm{~d}, J=3.2 \mathrm{~Hz}), 114.8(\mathrm{~d}, J=18.0 \mathrm{~Hz}), 113.9(\mathrm{~d}, J=2.1$ $\mathrm{Hz}), 56.0,55.8,49.6,42.4,34.2,32.4,23.9,15.1,14.8 \mathrm{ppm} .{ }^{19} \mathrm{~F}$ NMR (470 MHz, DMSO- $\left.d_{6}\right): \delta-135.2-$ $-135.3(\mathrm{~m}) \mathrm{ppm}$. LC-MS $(\mathrm{m} / \mathrm{z}): 318\left(\mathrm{M}+\mathrm{H}^{+}\right)$. Anal. Calcd. for $\mathrm{C}_{16} \mathrm{H}_{20} \mathrm{FN}_{5} \mathrm{O}: \mathrm{C}, 60.55 ; \mathrm{H}, 6.35 ; \mathrm{N}$, 22.07. Found: C, 60.54; H, 6.62; N, 21.69.<smiles>CN1CCN(c2nnnn2CC2CCOC2)Cc2cccnc21</smiles>

1-Methyl-4-(1-((tetrahydrofuran-3-yl)methyl)-1H-tetrazol-5-yl)-2,3,4,5-tetrahydro-1 $H$-pyrido[2,3e][1,4]diazepine (8 $\{51,251\})$. Brownish oil. ${ }^{1} \mathrm{H}$ NMR $\left(500 \mathrm{MHz}, \mathrm{DMSO}-d_{6}\right): \delta 8.05$ (dd, $J=4.8,1.8$ $\mathrm{Hz}, 1 \mathrm{H}), 7.50(\mathrm{dd}, J=7.3,1.8 \mathrm{~Hz}, 1 \mathrm{H}), 6.71(\mathrm{dd}, J=7.3,4.8 \mathrm{~Hz}, 1 \mathrm{H}), 4.53(\mathrm{~s}, 2 \mathrm{H}), 4.22(\mathrm{~d}, J=7.6 \mathrm{~Hz}$, $2 \mathrm{H}), 3.73-3.69(\mathrm{~m}, 3 \mathrm{H}), 3.61(\mathrm{td}, J=9.1,7.1 \mathrm{~Hz}, 2 \mathrm{H}), 3.55-3.51(\mathrm{~m}, 2 \mathrm{H}), 3.37(\mathrm{dd}, J=8.8,5.4 \mathrm{~Hz}$, 1H), $2.99(\mathrm{~s}, 3 \mathrm{H}), 2.77-2.65(\mathrm{~m}, 1 \mathrm{H}), 1.94-1.83(\mathrm{~m}, 1 \mathrm{H}), 1.57-1.46(\mathrm{~m}, 1 \mathrm{H}) \mathrm{ppm} .{ }^{13} \mathrm{C}\left\{{ }^{1} \mathrm{H}\right\} \mathrm{NMR}$ (126 MHz, DMSO- $\left.d_{6}\right): \delta 160.2,157.9,146.2,137.8,119.9,114.1,70.0,66.7,52.5,52.1,51.4,49.6$, 
39.6, 37.9, 29.2 ppm. LC-MS $(m / z): 316\left(\mathrm{M}+\mathrm{H}^{+}\right)$. Anal. Calcd. for $\mathrm{C}_{15} \mathrm{H}_{21} \mathrm{~N}_{7} \mathrm{O}: \mathrm{C}, 57.13 ; \mathrm{H}, 6.71 ; \mathrm{N}$, 31.09. Found: C, 57.39; H, 7.08; N, 31.47.<smiles>Oc1ccc2c(c1)CN(c1nnnn1CCc1ccc(F)cc1)CC2</smiles>

2-(1-(4-Fluorophenethyl)-1H-tetrazol-5-yl)-1,2,3,4-tetrahydroisoquinolin-7-ol (8\{53,236\}). Yellow oil. ${ }^{1} \mathrm{H}$ NMR $\left(500 \mathrm{MHz}, \mathrm{DMSO}-d_{6}\right): \delta 9.23(\mathrm{~s}, 1 \mathrm{H}), 7.19-7.15(\mathrm{~m}, 2 \mathrm{H}), 7.08-7.03(\mathrm{~m}, 2 \mathrm{H}), 6.95(\mathrm{~d}, J$ $=8.3 \mathrm{~Hz}, 1 \mathrm{H}), 6.61(\mathrm{~d}, J=8.3 \mathrm{~Hz}, 1 \mathrm{H}), 6.53(\mathrm{~s}, 1 \mathrm{H}), 4.52(\mathrm{t}, J=7.1 \mathrm{~Hz}, 2 \mathrm{H}), 4.28(\mathrm{~s}, 2 \mathrm{H}), 3.40(\mathrm{t}, J=$ $6.0 \mathrm{~Hz}, 2 \mathrm{H}), 3.16(\mathrm{t}, J=6.9 \mathrm{~Hz}, 2 \mathrm{H}), 2.77(\mathrm{t}, J=6.0 \mathrm{~Hz}, 2 \mathrm{H}) \mathrm{ppm} .{ }^{13} \mathrm{C}\left\{{ }^{1} \mathrm{H}\right\}$ NMR (126 MHz, DMSO$\left.d_{6}\right): \delta 161.1(\mathrm{~d}, J=242.4 \mathrm{~Hz}), 158.5,155.4,133.7,133.4(\mathrm{~d}, J=2.9 \mathrm{~Hz}), 130.6(\mathrm{~d}, J=8.0 \mathrm{~Hz}), 129.5$, 123.5, $115.1(\mathrm{~d}, J=21.1 \mathrm{~Hz}), 114.1,112.4,50.6,48.1,47.4,33.0,26.8 \mathrm{ppm} .{ }^{19} \mathrm{~F}\left\{{ }^{1} \mathrm{H}\right\}$ NMR $(376 \mathrm{MHz}$, DMSO- $\left.d_{6}\right): \delta-116.7 \mathrm{ppm}$. LC-MS $(\mathrm{m} / z): 340\left(\mathrm{M}+\mathrm{H}^{+}\right)$. Anal. Calcd. for $\mathrm{C}_{18} \mathrm{H}_{18} \mathrm{FN}_{5} \mathrm{O}: \mathrm{C}, 63.70 ; \mathrm{H}$, 5.35; N, 20.64. Found: C, 64.08; H, 4.98; N, 20.68.<smiles>O=C1CCCN1C1CCN(c2nnnn2Cc2ccon2)CC1</smiles>

1-(1-(1-(Isoxazol-3-ylmethyl)-1 $\boldsymbol{H}$-tetrazol-5-yl)piperidin-4-yl)pyrrolidin-2-one $(\mathbf{8}\{56,108\})$. Brown solid, $\mathrm{mp}=88-92{ }^{\circ} \mathrm{C} .{ }^{1} \mathrm{H}$ NMR $\left(500 \mathrm{MHz}, \mathrm{DMSO}-d_{6}\right): \delta 8.98(\mathrm{~d}, J=1.7 \mathrm{~Hz}, 1 \mathrm{H}), 6.59(\mathrm{~d}, J=1.7 \mathrm{~Hz}$, $1 \mathrm{H}), 5.68(\mathrm{~s}, 2 \mathrm{H}), 3.96(\mathrm{tt}, J=12.1,4.1 \mathrm{~Hz}, 1 \mathrm{H}), 3.64(\mathrm{~d}, J=12.8 \mathrm{~Hz}, 2 \mathrm{H}), 3.27(\mathrm{t}, J=7.0 \mathrm{~Hz}, 2 \mathrm{H}), 3.07$ $(\mathrm{td}, J=12.8,2.4 \mathrm{~Hz}, 2 \mathrm{H}), 2.22(\mathrm{t}, J=8.1 \mathrm{~Hz}, 2 \mathrm{H}), 1.90$ (quint, $J=7.5 \mathrm{~Hz}, 2 \mathrm{H}), 1.78-1.66(\mathrm{~m}, 2 \mathrm{H}$ ), $1.61-1.53(\mathrm{~m}, 2 \mathrm{H}) \mathrm{ppm} .{ }^{13} \mathrm{C}\left\{{ }^{1} \mathrm{H}\right\}$ NMR $\left(126 \mathrm{MHz}, \mathrm{DMSO}-d_{6}\right): \delta 173.4,161.1,158.2,157.6,104.3$, 48.8, 47.7, 42.4, 42.4, 30.9, 28.0, 17.7 ppm. LC-MS $(m / z): 318\left(\mathrm{M}+\mathrm{H}^{+}\right)$. Anal. Calcd. for $\mathrm{C}_{14} \mathrm{H}_{19} \mathrm{~N}_{7} \mathrm{O}_{2}$ : C, 52.99; H, 6.03; N, 30.90. Found: C, 52.85; H, 6.07; N, 30.72. 


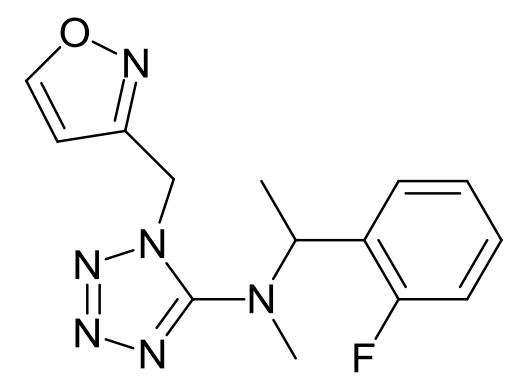

$N$-(1-(2-Fluorophenyl)ethyl)-1-(isoxazol-3-ylmethyl)- $N$-methyl-1H-tetrazol-5-amine $\quad(8\{56,271\})$. Brown oil. ${ }^{1} \mathrm{H}$ NMR $\left(500 \mathrm{MHz}, \mathrm{DMSO}-d_{6}\right): \delta 8.96(\mathrm{~s}, 1 \mathrm{H}), 7.47$ (t, $\left.J=7.5 \mathrm{~Hz}, 1 \mathrm{H}\right), 7.35(\mathrm{q}, J=6.5 \mathrm{~Hz}$, $1 \mathrm{H}), 7.20(\mathrm{t}, J=7.5 \mathrm{~Hz}, 1 \mathrm{H}), 7.13(\mathrm{dd}, J=10.8,8.2 \mathrm{~Hz}, 1 \mathrm{H}), 6.52(\mathrm{~s}, 1 \mathrm{H}), 5.76(\mathrm{~d}, J=16.8 \mathrm{~Hz}, 1 \mathrm{H})$, $5.72(\mathrm{~d}, J=16.8 \mathrm{~Hz}, 1 \mathrm{H}), 5.23(\mathrm{q}, J=6.9 \mathrm{~Hz}, 1 \mathrm{H}), 2.71(\mathrm{~s}, 3 \mathrm{H}), 1.52(\mathrm{~d}, J=7.0 \mathrm{~Hz}, 3 \mathrm{H}) \mathrm{ppm} .{ }^{13} \mathrm{C}\left\{{ }^{1} \mathrm{H}\right\}$ NMR (126 MHz, DMSO- $\left.d_{6}\right): \delta 161.1,160.2(\mathrm{~d}, J=246.0 \mathrm{~Hz}), 158.2,157.9,129.8(\mathrm{~d}, J=8.6 \mathrm{~Hz}), 128.7$ $(\mathrm{d}, J=4.0 \mathrm{~Hz}), 126.5(\mathrm{~d}, J=13.7 \mathrm{~Hz}), 124.3(\mathrm{~d}, J=3.3 \mathrm{~Hz}), 115.4(\mathrm{~d}, J=22.1 \mathrm{~Hz}), 104.0,53.1,42.5$, 32.8, 15.8 ppm. ${ }^{19} \mathrm{~F}$ NMR $\left(470 \mathrm{MHz}, \mathrm{DMSO}-d_{6}\right): \delta-117.0(\mathrm{dt}, J=11.8,6.4 \mathrm{~Hz}) \mathrm{ppm}$. LC-MS $(\mathrm{m} / \mathrm{z})$ : $303\left(\mathrm{M}+\mathrm{H}^{+}\right)$. Anal. Calcd. for $\mathrm{C}_{14} \mathrm{H}_{15} \mathrm{FN}_{6} \mathrm{O}: \mathrm{C}, 55.62 ; \mathrm{H}, 5.00 ; \mathrm{N}, 27.80$. Found: $\mathrm{C}, 55.77 ; \mathrm{H}, 4.61 ; \mathrm{N}$, 27.73 .<smiles>CC1(n2nnnc2N2CCCN(c3ncccc3F)CC2)CCC1</smiles>

1-(3-Fluoropyridin-2-yl)-4-(1-(1-methylcyclobutyl)-1 $H$-tetrazol-5-yl)-1,4-diazepane $\quad(8\{58,280\})$. Brownish oil. ${ }^{1} \mathrm{H}$ NMR $\left(500 \mathrm{MHz}, \mathrm{DMSO}-d_{6}\right): \delta 7.90-7.87(\mathrm{~m}, 1 \mathrm{H}), 7.41(\mathrm{dd}, J=14.6,7.8 \mathrm{~Hz}, 1 \mathrm{H})$, $6.66(\mathrm{ddd}, J=7.8,4.7,2.9 \mathrm{~Hz}, 1 \mathrm{H}), 3.85(\mathrm{t}, J=5.2 \mathrm{~Hz}, 2 \mathrm{H}), 3.68(\mathrm{t}, J=5.3 \mathrm{~Hz}, 2 \mathrm{H}), 3.51(\mathrm{t}, J=5.3 \mathrm{~Hz}$, $2 \mathrm{H}), 3.36-3.33(\mathrm{~m}, 2 \mathrm{H}), 2.71(\mathrm{qd}, J=10.0,2.7 \mathrm{~Hz}, 2 \mathrm{H}), 2.25(\mathrm{tt}, J=8.8,2.6 \mathrm{~Hz}, 2 \mathrm{H}), 2.01-1.89(\mathrm{~m}$, $3 \mathrm{H}), 1.82-1.75(\mathrm{~m}, 1 \mathrm{H}), 1.64(\mathrm{~s}, 3 \mathrm{H}) \mathrm{ppm} .{ }^{13} \mathrm{C}\left\{{ }^{1} \mathrm{H}\right\}$ NMR $\left(126 \mathrm{MHz}, \mathrm{DMSO}-d_{6}\right): \delta 157.1,148.0(\mathrm{~d}, J=$ $126.3 \mathrm{~Hz}), 147.0(\mathrm{~d}, J=132.7 \mathrm{~Hz}), 142.9(\mathrm{~d}, J=5.0 \mathrm{~Hz}), 123.5(\mathrm{~d}, J=19.5 \mathrm{~Hz}), 113.5(\mathrm{~d}, J=2.1 \mathrm{~Hz})$, $59.9,52.7,50.9,49.4(\mathrm{~d}, J=4.7 \mathrm{~Hz}), 48.4(\mathrm{~d}, J=7.1 \mathrm{~Hz}), 33.9,27.4,24.9,14.1 \mathrm{ppm} .{ }^{19} \mathrm{~F}$ NMR $(470$ MHz, DMSO- $\left.d_{6}\right): \delta-131.8(\mathrm{~d}, J=14.6 \mathrm{~Hz}) \mathrm{ppm}$. LC-MS $(\mathrm{m} / \mathrm{z}): 332\left(\mathrm{M}+\mathrm{H}^{+}\right)$. Anal. Calcd. for $\mathrm{C}_{16} \mathrm{H}_{22} \mathrm{FN}_{7}$ : C, 57.99; H, 6.69; N, 29.59. Found: C, 57.72; H, 6.69; N, 29.19.

\section{Products obtained by BTTC-based method.}

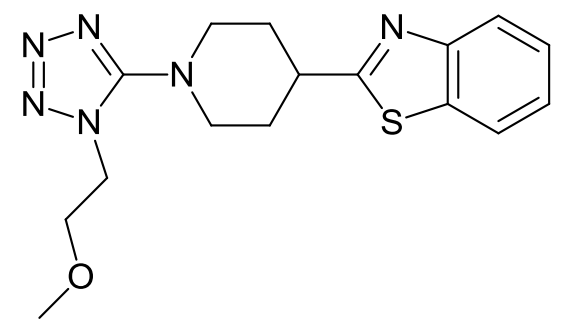

2-(1-(1-(2-Methoxyethyl)-1H-tetrazol-5-yl)piperidin-4-yl)benzo[d]thiazole $(\mathbf{9}\{4,289\})$. Beige solid, $\mathrm{mp}=90-93{ }^{\circ} \mathrm{C} .{ }^{1} \mathrm{H}$ NMR $\left(500 \mathrm{MHz}, \mathrm{DMSO}-d_{6}\right): \delta 8.08(\mathrm{~d}, J=8.0 \mathrm{~Hz}, 1 \mathrm{H}), 7.96(\mathrm{~d}, J=8.1 \mathrm{~Hz}, 1 \mathrm{H})$, 
$7.50(\mathrm{t}, J=7.6 \mathrm{~Hz}, 1 \mathrm{H}), 7.42(\mathrm{t}, J=7.5 \mathrm{~Hz}, 1 \mathrm{H}), 4.41(\mathrm{t}, J=5.2 \mathrm{~Hz}, 2 \mathrm{H}), 3.82(\mathrm{t}, J=5.2 \mathrm{~Hz}, 2 \mathrm{H}), 3.71-$ $3.61(\mathrm{~m}, 2 \mathrm{H}), 3.47-3.39(\mathrm{~m}, 1 \mathrm{H}), 3.23(\mathrm{~s}, 3 \mathrm{H}), 3.17(\mathrm{t}, J=11.7 \mathrm{~Hz}, 2 \mathrm{H}), 2.23-2.17(\mathrm{~m}, 2 \mathrm{H}), 2.00(\mathrm{qd}$, $J=12.1,3.8 \mathrm{~Hz}, 2 \mathrm{H}) \mathrm{ppm} .{ }^{13} \mathrm{C}\left\{{ }^{1} \mathrm{H}\right\} \mathrm{NMR}\left(126 \mathrm{MHz}, \mathrm{DMSO}-d_{6}\right): \delta 174.8,159.1,152.6,134.1,126.1$, 124.9, 122.4, 122.2, 68.6, 58.1, 49.4, 46.6, 39.5, 31.0 ppm. LC-MS (m/z): $345\left(\mathrm{M}+\mathrm{H}^{+}\right)$. Anal. Calcd. for $\mathrm{C}_{16} \mathrm{H}_{20} \mathrm{~N}_{6} \mathrm{OS}$ : C, 55.79; H, 5.85; N, 24.40; S, 9.31. Found: C, 55.42; H, 5.56; N, 24.75; S, 9.48.<smiles>COCCn1nnnc1N1CCN(C(=O)c2ccc(F)cc2)CC1</smiles>

(4-Fluorophenyl)(4-(1-(2-methoxyethyl)-1H-tetrazol-5-yl)piperazin-1-yl)methanone (9\{4,291\}). Brown oil. ${ }^{1} \mathrm{H}$ NMR $\left(500 \mathrm{MHz}, \mathrm{DMSO}-d_{6}\right): \delta 7.52(\mathrm{dd}, J=8.5,5.6 \mathrm{~Hz}, 2 \mathrm{H}), 7.29(\mathrm{t}, J=8.8 \mathrm{~Hz}, 2 \mathrm{H})$, $4.41(\mathrm{t}, J=5.2 \mathrm{~Hz}, 2 \mathrm{H}), 3.79(\mathrm{t}, J=5.2 \mathrm{~Hz}, 2 \mathrm{H}), 3.74-3.40(\mathrm{~m}, 4 \mathrm{H}), 3.33-3.24(\mathrm{~m}, 4 \mathrm{H}), 3.21(\mathrm{~s}, 3 \mathrm{H})$ ppm. ${ }^{13} \mathrm{C}\left\{{ }^{1} \mathrm{H}\right\}$ NMR $\left(126 \mathrm{MHz}, \mathrm{DMSO}-d_{6}\right): \delta 168.3,162.6(\mathrm{~d}, J=246.7 \mathrm{~Hz}), 158.6,132.0(\mathrm{~d}, J=3.3$ $\mathrm{Hz}), 129.7$ (d, $J=8.7 \mathrm{~Hz}), 115.4(\mathrm{~d}, J=21.6 \mathrm{~Hz}), 68.7,58.1,49.3(2 \mathrm{C}), 46.6 \mathrm{ppm} .{ }^{19} \mathrm{~F} \mathrm{NMR}(470 \mathrm{MHz}$, DMSO- $\left.d_{6}\right): \delta-111.0(\mathrm{tt}, J=9.3,5.5 \mathrm{~Hz}) \mathrm{ppm}$. LC-MS $(\mathrm{m} / \mathrm{z}): 335\left(\mathrm{M}+\mathrm{H}^{+}\right)$. Anal. Calcd. for $\mathrm{C}_{15} \mathrm{H}_{19} \mathrm{FN}_{6} \mathrm{O}_{2}$ : C, 53.88; H, 5.73; N, 25.14. Found: C, 53.61; H, 5.83; N, 24.93.

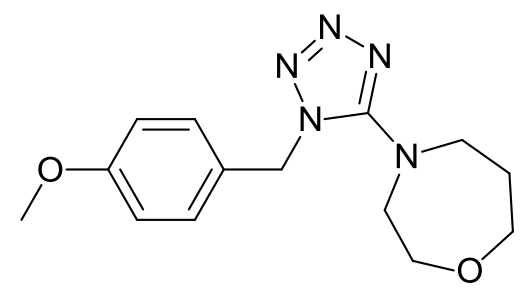

4-(1-(4-Methoxybenzyl)-1H-tetrazol-5-yl)-1,4-oxazepane (9\{18,300\}). Yellow oil. ${ }^{1} \mathrm{H}$ NMR (500 $\left.\mathrm{MHz}, \mathrm{DMSO}-d_{6}\right): \delta 7.10(\mathrm{~d}, J=8.6 \mathrm{~Hz}, 2 \mathrm{H}), 6.93(\mathrm{~d}, J=8.6 \mathrm{~Hz}, 2 \mathrm{H}), 5.49(\mathrm{~s}, 2 \mathrm{H}), 3.73(\mathrm{~s}, 3 \mathrm{H}), 3.69-$ $3.64(\mathrm{~m}, 2 \mathrm{H}), 3.60(\mathrm{t}, J=5.6 \mathrm{~Hz}, 2 \mathrm{H}), 3.57-3.50(\mathrm{~m}, 4 \mathrm{H}), 1.81$ (quint, $J=5.7 \mathrm{~Hz}, 2 \mathrm{H}) \mathrm{ppm} .{ }^{13} \mathrm{C}\left\{{ }^{1} \mathrm{H}\right\}$ NMR (126 MHz, DMSO- $\left.d_{6}\right): \delta$ 158.9, 157.2, 128.1, 127.2, 114.3, 68.8, 68.8, 55.1, 52.8, 49.8, 49.3, 29.8 ppm. LC-MS $(m / z):=290\left(\mathrm{M}+\mathrm{H}^{+}\right)$. Anal. Calcd. for $\mathrm{C}_{14} \mathrm{H}_{19} \mathrm{~N}_{5} \mathrm{O}_{2}: \mathrm{C}, 58.12 ; \mathrm{H}, 6.62 ; \mathrm{N}, 24.21$. Found: C, 58.43; H, 6.59; N, 24.32.<smiles>Clc1cnn(C2CCN(c3nnnn3CCC3=CCCCC3)C2)c1</smiles> 
5-(3-(4-Chloro-1H-pyrazol-1-yl)pyrrolidin-1-yl)-1-(2-(cyclohex-1-en-1-yl)ethyl)-1H-tetrazole (9 $\{25,147\})$. Brown oil. ${ }^{1} \mathrm{H}$ NMR $\left(500 \mathrm{MHz}, \mathrm{DMSO}-d_{6}\right): \delta 8.13(\mathrm{~s}, 1 \mathrm{H}), 7.58(\mathrm{~s}, 1 \mathrm{H}), 5.29-5.26(\mathrm{~m}$, $1 \mathrm{H}), 5.09(\mathrm{tt}, J=6.3,3.9 \mathrm{~Hz}, 1 \mathrm{H}), 4.35(\mathrm{t}, J=7.3 \mathrm{~Hz}, 2 \mathrm{H}), 3.96(\mathrm{dd}, J=10.5,6.3 \mathrm{~Hz}, 1 \mathrm{H}), 3.83(\mathrm{dd}, J=$ $10.4,3.7 \mathrm{~Hz}, 1 \mathrm{H}), 3.74(\mathrm{q}, J=8.0 \mathrm{~Hz}, 1 \mathrm{H}), 3.66(\mathrm{td}, J=8.6,5.1 \mathrm{~Hz}, 1 \mathrm{H}), 2.47-2.39(\mathrm{~m}, 2 \mathrm{H}), 2.34(\mathrm{t}, J$ $=7.3 \mathrm{~Hz}, 2 \mathrm{H}), 1.91-1.86(\mathrm{~m}, 2 \mathrm{H}), 1.85-1.80(\mathrm{~m}, 2 \mathrm{H}), 1.54-1.48(\mathrm{~m}, 2 \mathrm{H}), 1.45-1.40(\mathrm{~m}, 2 \mathrm{H}) \mathrm{ppm}$.

${ }^{13} \mathrm{C}\left\{{ }^{1} \mathrm{H}\right\}$ NMR $\left(126 \mathrm{MHz}, \mathrm{DMSO}-d_{6}\right): \delta 155.7,137.2,133.1,127.4,123.6,108.3,60.3,54.3,47.7,45.3$, 37.2 , 30.9, 27.4, 24.6, 22.2, 21.7 ppm. LC-MS $(\mathrm{m} / \mathrm{z}): 348\left(\mathrm{M}+\mathrm{H}^{+}\right)$. Anal. Calcd. for $\mathrm{C}_{16} \mathrm{H}_{22} \mathrm{ClN}_{7}: \mathrm{C}_{\text {, }}$ 55.25; H, 6.38; N, 28.19; Cl, 10.19. Found: C, 54.89; H, 6.05; N, 28.50; Cl, 9.87.

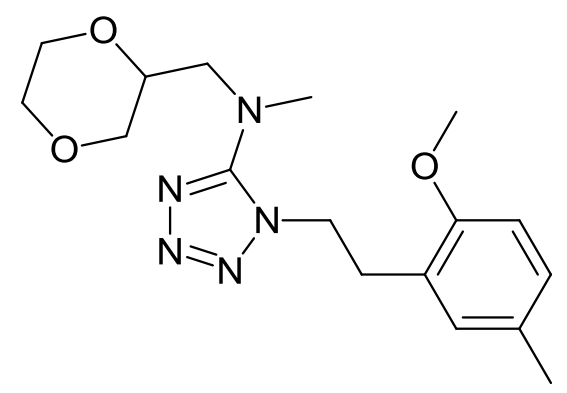

$N$-((1,4-Dioxan-2-yl)methyl)-1-(2-methoxy-5-methylphenethyl)- $N$-methyl-1H-tetrazol-5-amine (9 $\{52,319\})$. Brown oil. ${ }^{1} \mathrm{H}$ NMR $\left(500 \mathrm{MHz}, \mathrm{DMSO}-d_{6}\right): \delta 7.02(\mathrm{~d}, J=8.2 \mathrm{~Hz}, 1 \mathrm{H}), 6.88-6.83(\mathrm{~m}$, $2 \mathrm{H}), 4.42(\mathrm{t}, J=7.4 \mathrm{~Hz}, 2 \mathrm{H}), 3.79-3.74(\mathrm{~m}, 1 \mathrm{H}), 3.73(\mathrm{~s}, 3 \mathrm{H}), 3.67(\mathrm{~d}, J=11.5 \mathrm{~Hz}, 2 \mathrm{H}), 3.62(\mathrm{~d}, J=$ $11.3 \mathrm{~Hz}, 1 \mathrm{H}), 3.53(\mathrm{td}, J=11.3,2.5 \mathrm{~Hz}, 1 \mathrm{H}), 3.42(\mathrm{td}, J=11.3,2.5 \mathrm{~Hz}, 1 \mathrm{H}), 3.38-3.33(\mathrm{~m}, 1 \mathrm{H}), 3.28-$ $3.22(\mathrm{~m}, 1 \mathrm{H}), 3.23-3.12(\mathrm{~m}, 1 \mathrm{H}), 3.03-3.00(\mathrm{~m}, 2 \mathrm{H}), 2.99(\mathrm{~s}, 3 \mathrm{H}), 2.18(\mathrm{~s}, 3 \mathrm{H}) \mathrm{ppm} .{ }^{13} \mathrm{C}\left\{{ }^{1} \mathrm{H}\right\} \mathrm{NMR}$ (126 MHz, DMSO- $\left.d_{6}\right): \delta 158.1,155.2,131.0,128.9,128.5,124.5,110.6,72.7,68.1,65.9,65.7,55.3$, 53.9, 46.7, 39.2, 30.0, 20.0 ppm. LC-MS $(\mathrm{m} / z):=348\left(\mathrm{M}+\mathrm{H}^{+}\right)$. Anal. Calcd. for $\mathrm{C}_{17} \mathrm{H}_{25} \mathrm{~N}_{5} \mathrm{O}_{3}:$ C, 58.77; H, 7.25; N, 20.16. Found: C, 58.97; H, 7.31; N, 20.05.<smiles>CN(CC1CCOC1)c1nnnn1Cc1ccc(C(F)(F)F)cc1</smiles>

$\boldsymbol{N}$-Methyl- $\boldsymbol{N}$-((tetrahydrofuran-3-yl)methyl)-1-(4-(trifluoromethyl)benzyl)-1H-tetrazol-5-amine (9 $\{55,337\})$. Colorless oil. ${ }^{1} \mathrm{H}$ NMR $\left(500 \mathrm{MHz}, \mathrm{DMSO}-d_{6}\right): \delta 7.76(\mathrm{~d}, J=8.0 \mathrm{~Hz}, 2 \mathrm{H}), 7.35(\mathrm{~d}, J=8.0$ $\mathrm{Hz}, 2 \mathrm{H}), 5.71(\mathrm{~s}, 2 \mathrm{H}), 3.60(\mathrm{td}, J=8.1,5.3 \mathrm{~Hz}, 1 \mathrm{H}), 3.55-3.43(\mathrm{~m}, 2 \mathrm{H}), 3.24(\mathrm{qd}, J=13.6,7.7 \mathrm{~Hz}, 2 \mathrm{H})$, $3.17(\mathrm{dd}, J=8.5,5.7 \mathrm{~Hz}, 1 \mathrm{H}), 2.92(\mathrm{~s}, 3 \mathrm{H}), 2.41(\mathrm{dq}, J=14.1,7.0 \mathrm{~Hz}, 1 \mathrm{H}), 1.78-1.69(\mathrm{~m}, 1 \mathrm{H}), 1.34-$ $1.25(\mathrm{~m}, 1 \mathrm{H}) \mathrm{ppm} .{ }^{13} \mathrm{C}\left\{{ }^{1} \mathrm{H}\right\}$ NMR $\left(126 \mathrm{MHz}, \mathrm{DMSO}-d_{6}\right): \delta 158.3,140.1,128.6(\mathrm{q}, J=31.9 \mathrm{~Hz}), 127.6$, $125.8(\mathrm{q}, J=3.8 \mathrm{~Hz}), 124.1(\mathrm{q}, J=272.1 \mathrm{~Hz}), 70.0,66.6,55.6,49.6,38.7,36.9,29.1 \mathrm{ppm} .{ }^{19} \mathrm{~F}$ NMR $\left(470 \mathrm{MHz}, \mathrm{DMSO}-d_{6}\right): \delta-61.1 \mathrm{ppm}$. LC-MS $(\mathrm{m} / z):=342\left(\mathrm{M}+\mathrm{H}^{+}\right)$. Anal. Calcd. for $\mathrm{C}_{15} \mathrm{H}_{18} \mathrm{~F}_{3} \mathrm{~N}_{5} \mathrm{O}: \mathrm{C}$, 52.78; H, 5.32; N, 20.52. Found: C, 52.49; H, 5.39; N, 20.39. 


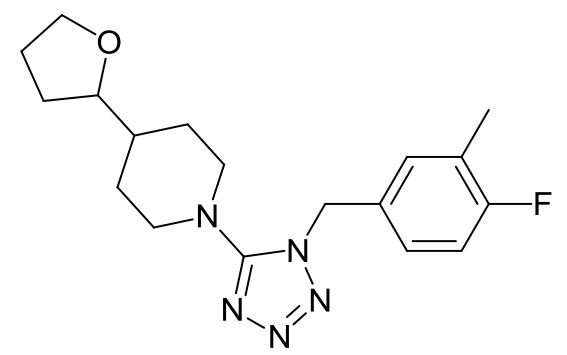

1-(1-(4-Fluoro-3-methylbenzyl)-1H-tetrazol-5-yl)-4-(tetrahydrofuran-2-yl)piperidine (9\{57,261\}). Beige solid, $\mathrm{mp}=79-81^{\circ} \mathrm{C}$. ${ }^{1} \mathrm{H}$ NMR $\left(500 \mathrm{MHz}, \mathrm{DMSO}-d_{6}\right): \delta 7.18-7.12(\mathrm{~m}, 2 \mathrm{H}), 7.08-7.03(\mathrm{~m}$, $1 \mathrm{H}), 5.45$ (s, 2H), 3.69 (q, $J=7.1 \mathrm{~Hz}, 1 \mathrm{H}), 3.58(\mathrm{q}, J=7.2 \mathrm{~Hz}, 1 \mathrm{H}), 3.51(\mathrm{~d}, J=12.5 \mathrm{~Hz}, 2 \mathrm{H}), 3.44(\mathrm{q}, J$ $=7.2 \mathrm{~Hz}, 1 \mathrm{H}), 2.87(\mathrm{dt}, J=12.8,10.1 \mathrm{~Hz}, 2 \mathrm{H}), 2.21(\mathrm{~s}, 3 \mathrm{H}), 1.90-1.82(\mathrm{~m}, 1 \mathrm{H}), 1.81-1.74(\mathrm{~m}, 3 \mathrm{H})$, $1.54(\mathrm{~d}, J=13.5 \mathrm{~Hz}, 1 \mathrm{H}), 1.50-1.40(\mathrm{~m}, 2 \mathrm{H}), 1.31-1.17(\mathrm{~m}, 2 \mathrm{H}) \mathrm{ppm} .{ }^{13} \mathrm{C}\left\{{ }^{1} \mathrm{H}\right\} \mathrm{NMR}(126 \mathrm{MHz}$, DMSO- $\left.d_{6}\right): \delta 161.2,159.3,158.4,130.6(\mathrm{t}, J=4.7 \mathrm{~Hz}), 126.8(\mathrm{~d}, J=8.4 \mathrm{~Hz}), 124.7(\mathrm{~d}, J=17.7 \mathrm{~Hz})$, $115.3(\mathrm{~d}, J=22.5 \mathrm{~Hz}), 82.0,67.0,49.37,49.36,49.28,28.4,27.8,27.2,25.3(2 \mathrm{C}), 14.1(\mathrm{~d}, J=3.3 \mathrm{~Hz})$ ppm. ${ }^{19} \mathrm{~F}\left\{{ }^{1} \mathrm{H}\right\}$ NMR (470 MHz, DMSO-d $): \delta-118.6$ ppm. LC-MS $(\mathrm{m} / z): 346\left(\mathrm{M}+\mathrm{H}^{+}\right)$. Anal. Calcd. for $\mathrm{C}_{18} \mathrm{H}_{24} \mathrm{FN}_{5} \mathrm{O}$ : C, 62.59; H, 7.00; N, 20.28. Found: C, 62.45; H, 6.88; N, 20.02.

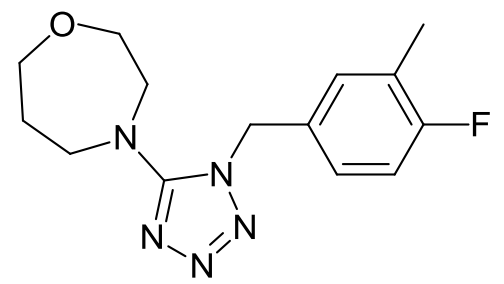

4-(1-(4-Fluoro-3-methylbenzyl)-1H-tetrazol-5-yl)-1,4-oxazepane (9\{57,300\}). White solid, $\mathrm{mp}=$ 107-109 ${ }^{\circ} \mathrm{C} .{ }^{1} \mathrm{H}$ NMR $\left(500 \mathrm{MHz}, \mathrm{DMSO}-d_{6}\right): \delta 7.14(\mathrm{t}, J=9.1 \mathrm{~Hz}, 1 \mathrm{H}), 7.10(\mathrm{~d}, J=6.5 \mathrm{~Hz}, 1 \mathrm{H}), 7.02-$ $6.95(\mathrm{~m}, 1 \mathrm{H}), 5.52(\mathrm{~s}, 2 \mathrm{H}), 3.66(\mathrm{t}, J=4.9 \mathrm{~Hz}, 2 \mathrm{H}), 3.60(\mathrm{t}, J=5.5 \mathrm{~Hz}, 2 \mathrm{H}), 3.58-3.52(\mathrm{~m}, 4 \mathrm{H}), 2.20(\mathrm{~s}$, $3 \mathrm{H}), 1.85-1.78(\mathrm{~m}, 2 \mathrm{H}) \mathrm{ppm} .{ }^{13} \mathrm{C}\left\{{ }^{1} \mathrm{H}\right\}$ NMR (126 MHz, DMSO-d $): \delta 160.2(\mathrm{~d}, J=243.4 \mathrm{~Hz}), 157.3$, $131.2(\mathrm{~d}, J=3.4 \mathrm{~Hz}), 130.0(\mathrm{~d}, J=5.3 \mathrm{~Hz}), 126.1(\mathrm{~d}, J=8.4 \mathrm{~Hz}), 124.8(\mathrm{~d}, J=17.7 \mathrm{~Hz}), 115.4(\mathrm{~d}, J=$ $22.6 \mathrm{~Hz}), 68.83,68.77,52.9,49.6,49.3,29.8,14.1(\mathrm{~d}, J=3.3 \mathrm{~Hz}) \mathrm{ppm} .{ }^{19} \mathrm{~F}\left\{{ }^{1} \mathrm{H}\right\} \mathrm{NMR}(470 \mathrm{MHz}$, DMSO-d $)_{6}: \delta-118.63$ ppm. LC-MS $(\mathrm{m} / z):=292\left(\mathrm{M}+\mathrm{H}^{+}\right)$. Anal. Calcd. for $\mathrm{C}_{14} \mathrm{H}_{18} \mathrm{FN}_{5} \mathrm{O}: \mathrm{C}, 57.72 ; \mathrm{H}$, $6.23 ; \mathrm{N}, 24.04$. Found: C, 57.59; H, 6.20; N, 24.18.

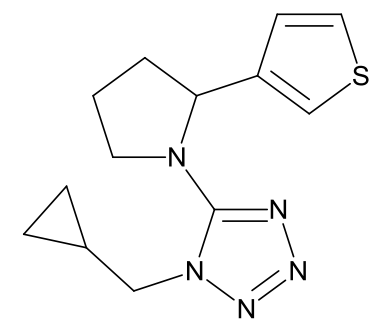

1-(Cyclopropylmethyl)-5-(2-(thiophen-3-yl)pyrrolidin-1-yl)-1H-tetrazole $(9\{63,327\})$. Beige solid, $\mathrm{mp}=65-67{ }^{\circ} \mathrm{C} .{ }^{1} \mathrm{H}$ NMR $\left(500 \mathrm{MHz}, \mathrm{DMSO}-d_{6}\right): \delta 7.46(\mathrm{dd}, J=4.9,2.9 \mathrm{~Hz}, 1 \mathrm{H}), 7.27-7.25(\mathrm{~m}, 1 \mathrm{H})$, $7.03(\mathrm{~d}, J=4.9 \mathrm{~Hz}, 1 \mathrm{H}), 5.21(\mathrm{dd}, J=7.5,4.9 \mathrm{~Hz}, 1 \mathrm{H}), 4.07(\mathrm{~d}, J=7.1 \mathrm{~Hz}, 2 \mathrm{H}), 3.92(\mathrm{dt}, J=9.4,6.4$ $\mathrm{Hz}, 1 \mathrm{H}), 3.54(\mathrm{dt}, J=9.6,7.2 \mathrm{~Hz}, 1 \mathrm{H}), 2.37-2.26(\mathrm{~m}, 1 \mathrm{H}), 1.98$ (quint, $J=6.7 \mathrm{~Hz}, 2 \mathrm{H}), 1.90-1.80(\mathrm{~m}$, 
$1 \mathrm{H}), 1.24-1.15(\mathrm{~m}, 1 \mathrm{H}), 0.56-0.42(\mathrm{~m}, 2 \mathrm{H}), 0.37-0.29(\mathrm{~m}, 1 \mathrm{H}), 0.30-0.21(\mathrm{~m}, 1 \mathrm{H}) \mathrm{ppm} .{ }^{13} \mathrm{C}\left\{{ }^{1} \mathrm{H}\right\}$ NMR (126 MHz, DMSO- $\left.d_{6}\right): \delta 155.8,144.5,126.6,126.1,120.9,60.2,50.8,50.4,34.4,23.9,10.7,3.9$, 3.6 ppm. LC-MS $(\mathrm{m} / \mathrm{z})$ : $276\left(\mathrm{M}+\mathrm{H}^{+}\right)$. Anal. Calcd. for $\mathrm{C}_{13} \mathrm{H}_{17} \mathrm{~N}_{5} \mathrm{~S}$ : C, 56.70; H, 6.22; N, 25.43; $\mathrm{S}$, 11.64. Found: C, 56.81; H, 6.48; N, 25.60; S, 11.47.<smiles>COc1ccc(OC)c(Cn2nnnc2N2CCCOCC2)c1</smiles>

4-(1-(2,5-Dimethoxybenzyl)-1H-tetrazol-5-yl)-1,4-oxazepane (9\{68,300\}). Yellow solid, $\mathrm{mp}=78-81$ ${ }^{\circ} \mathrm{C} .{ }^{1} \mathrm{H}$ NMR $\left(500 \mathrm{MHz}, \mathrm{DMSO}-d_{6}\right): \delta 7.00(\mathrm{~d}, J=8.9 \mathrm{~Hz}, 1 \mathrm{H}), 6.90(\mathrm{dd}, J=8.9,3.0 \mathrm{~Hz}, 1 \mathrm{H}), 6.40(\mathrm{~d}, J$ $=3.0 \mathrm{~Hz}, 1 \mathrm{H}), 5.41(\mathrm{~s}, 2 \mathrm{H}), 3.74(\mathrm{~s}, 3 \mathrm{H}), 3.70-3.67(\mathrm{~m}, 2 \mathrm{H}), 3.65(\mathrm{~s}, 3 \mathrm{H}), 3.62(\mathrm{t}, J=5.7 \mathrm{~Hz}, 2 \mathrm{H}), 3.57$ $-3.53(\mathrm{~m}, 4 \mathrm{H}), 1.84$ (quint, $J=5.7 \mathrm{~Hz}, 2 \mathrm{H}) \mathrm{ppm} .{ }^{13} \mathrm{C}\left\{{ }^{1} \mathrm{H}\right\}$ NMR $\left(126 \mathrm{MHz}\right.$, DMSO- $\left.d_{6}\right): \delta 157.6,153.1$, 150.2, 124.0, 114.2, 113.4, 112.3, 68.9, 68.9, 56.0, 55.4, 52.9, 49.2, 45.8, 29.9 ppm. LC-MS $(\mathrm{m} / \mathrm{z}):=$ $320\left(\mathrm{M}+\mathrm{H}^{+}\right)$. Anal. Calcd. for $\mathrm{C}_{15} \mathrm{H}_{21} \mathrm{~N}_{5} \mathrm{O}_{3}$ : C, 56.41; H, 6.63; N, 21.93. Found: C, 56.34; H, 6.61; N, 22.03 .

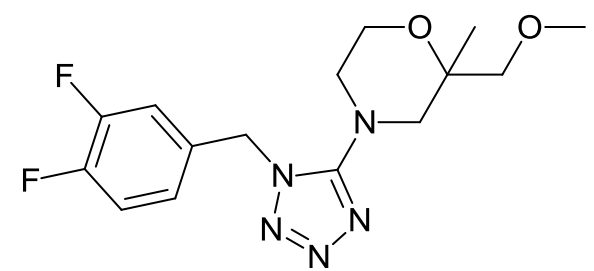

4-(1-(3,4-Difluorobenzyl)-1H-tetrazol-5-yl)-2-(methoxymethyl)-2-methylmorpholine (9\{77,363\}). Beige solid, $\mathrm{mp}=89-91{ }^{\circ} \mathrm{C} .{ }^{1} \mathrm{H}$ NMR $\left(500 \mathrm{MHz}\right.$, DMSO- $\left.d_{6}\right): \delta 7.48(\mathrm{dt}, J=10.7,8.5 \mathrm{~Hz}, 1 \mathrm{H}), 7.34$ (ddd, $J=10.8,7.8,2.1 \mathrm{~Hz}, 1 \mathrm{H}), 7.08-7.01(\mathrm{~m}, 1 \mathrm{H}), 5.57(\mathrm{~d}, J=16.4 \mathrm{~Hz}, 1 \mathrm{H}), 5.52(\mathrm{~d}, J=16.4 \mathrm{~Hz}$, $1 \mathrm{H}), 3.70(\mathrm{t}, J=5.0 \mathrm{~Hz}, 2 \mathrm{H}), 3.35(\mathrm{~d}, J=9.3 \mathrm{~Hz}, 1 \mathrm{H}), 3.21(\mathrm{~s}, 3 \mathrm{H}), 3.20-3.16(\mathrm{~m}, 3 \mathrm{H}), 3.15(\mathrm{~d}, J=$ $12.4 \mathrm{~Hz}, 1 \mathrm{H}), 2.96(\mathrm{~d}, J=12.4 \mathrm{~Hz}, 1 \mathrm{H}), 1.07(\mathrm{~s}, 3 \mathrm{H}) \mathrm{ppm} .{ }^{13} \mathrm{C}\left\{{ }^{1} \mathrm{H}\right\}$ NMR $\left(126 \mathrm{MHz}, \mathrm{DMSO}-d_{6}\right): \delta$ $158.3,149.4(\mathrm{dd}, J=246.7,12.8 \mathrm{~Hz}), 149.2(\mathrm{dd}, J=246.3,12.4 \mathrm{~Hz}), 132.2(\mathrm{~d}, J=5.4 \mathrm{~Hz}), 124.4(\mathrm{dd}, J$ $=6.8,3.5 \mathrm{~Hz}), 118.0(\mathrm{~d}, J=17.4 \mathrm{~Hz}), 116.8(\mathrm{~d}, J=18.1 \mathrm{~Hz}), 74.6,72.1,59.4,58.8,54.3,48.8(2 \mathrm{C})$, $20.3 \mathrm{ppm} .{ }^{19} \mathrm{~F}$ NMR $\left(470 \mathrm{MHz}\right.$, DMSO- $\left.d_{6}\right): \delta-137.8--137.9(\mathrm{~m}, 1 \mathrm{~F}),-139.3--139.4(\mathrm{~m}, 1 \mathrm{~F}) \mathrm{ppm}$. LC-MS $(\mathrm{m} / \mathrm{z}): 340\left(\mathrm{M}+\mathrm{H}^{+}\right)$. Anal. Calcd. for $\mathrm{C}_{15} \mathrm{H}_{19} \mathrm{~F}_{2} \mathrm{~N}_{5} \mathrm{O}_{2}: \mathrm{C}, 53.09 ; \mathrm{H}, 5.64 ; \mathrm{N}, 20.64$. Found: $\mathrm{C}$, 53.44; H, 5.96; N, 20.87.<smiles>CC(C)Oc1cccc(Cn2nnnc2N(C)CC2COCCO2)c1</smiles>

$N$-((1,4-Dioxan-2-yl)methyl)-1-(3-isopropoxybenzyl)- $N$-methyl-1 $H$-tetrazol-5-amine $(9\{84,319\})$. Yellow oil. ${ }^{1} \mathrm{H}$ NMR $\left(500 \mathrm{MHz}, \mathrm{DMSO}-d_{6}\right): \delta 7.27(\mathrm{t}, J=7.8 \mathrm{~Hz}, 1 \mathrm{H}), 6.87(\mathrm{~d}, J=8.0 \mathrm{~Hz}, 1 \mathrm{H}), 6.66(\mathrm{~s}$, 
2H), $5.56(\mathrm{~s}, 2 \mathrm{H}), 4.55(\mathrm{sept}, J=6.0 \mathrm{~Hz}, 1 \mathrm{H}), 3.73-3.67(\mathrm{~m}, 1 \mathrm{H}), 3.65(\mathrm{~d}, J=12.3 \mathrm{~Hz}, 1 \mathrm{H}), 3.58(\mathrm{~d}, J=$ $11.4 \mathrm{~Hz}, 1 \mathrm{H}), 3.55-3.44(\mathrm{~m}, 2 \mathrm{H}), 3.40-3.35(\mathrm{~m}, 1 \mathrm{H}), 3.31-3.20(\mathrm{~m}, 2 \mathrm{H}), 3.06-3.01(\mathrm{~m}, 1 \mathrm{H}), 2.99$ $(\mathrm{s}, 3 \mathrm{H}), 1.23(\mathrm{~d}, J=6.0 \mathrm{~Hz}, 6 \mathrm{H}) \mathrm{ppm} .{ }^{13} \mathrm{C}$ NMR $\left\{{ }^{1} \mathrm{H}\right\}\left(126 \mathrm{MHz}, \mathrm{DMSO}-d_{6}\right): \delta 158.0,157.7,137.2$, $130.0,118.4,114.8,114.0,72.6,69.2,67.9,65.9,65.6,53.7,49.9,39.2,21.7 \mathrm{ppm}$. LC-MS $(\mathrm{m} / \mathrm{z}):=348$ $\left(\mathrm{M}+\mathrm{H}^{+}\right)$. Anal. Calcd. for $\mathrm{C}_{17} \mathrm{H}_{25} \mathrm{~N}_{5} \mathrm{O}_{3}: \mathrm{C}, 58.77 ; \mathrm{H}, 7.25 ; \mathrm{N}, 20.16$. Found: C, 58.76; H, 7.17; N, 20.10 .<smiles>CC(C)OCCn1nnnc1N1CCOC2(CCc3ccccc32)C1</smiles>

4'-(1-(2-Isopropoxyethyl)-1H-tetrazol-5-yl)-2,3-dihydrospiro[indene-1,2'-morpholine] $(9\{90,371\})$. Beige solid, $\mathrm{mp}=104-106{ }^{\circ} \mathrm{C} .{ }^{1} \mathrm{H}$ NMR $\left(500 \mathrm{MHz}, \mathrm{DMSO}-d_{6}\right): \delta 7.48(\mathrm{~d}, J=7.5 \mathrm{~Hz}, 1 \mathrm{H}), 7.30-7.27$ $(\mathrm{m}, 2 \mathrm{H}), 7.24-7.19(\mathrm{~m}, 1 \mathrm{H}), 4.42-4.30(\mathrm{~m}, 2 \mathrm{H}), 3.90-3.86(\mathrm{~m}, 2 \mathrm{H}), 3.85-3.77(\mathrm{~m}, 2 \mathrm{H}), 3.48-3.39$ (m, 3H), $3.32-3.26(\mathrm{~m}, 1 \mathrm{H}), 3.17(\mathrm{~d}, J=12.2 \mathrm{~Hz}, 1 \mathrm{H}), 3.02-2.94(\mathrm{~m}, 1 \mathrm{H}), 2.90-2.82(\mathrm{~m}, 1 \mathrm{H}), 2.56-$ $2.52(\mathrm{~m}, 1 \mathrm{H}), 2.12(\mathrm{dt}, J=14.3,7.6 \mathrm{~Hz}, 1 \mathrm{H}), 0.88(\mathrm{~d}, J=6.1 \mathrm{~Hz}, 3 \mathrm{H}), 0.85(\mathrm{~d}, J=6.1 \mathrm{~Hz}, 3 \mathrm{H}) \mathrm{ppm}$.

${ }^{13} \mathrm{C}\left\{{ }^{1} \mathrm{H}\right\}$ NMR $\left(126 \mathrm{MHz}\right.$, DMSO- $\left.d_{6}\right): \delta 159.2,143.7,143.3,128.7,126.2,124.8,124.1,83.3,71.1$, 64.8, 61.1, 56.4, 49.3, 47.3, 34.4, 28.8, $21.5 \mathrm{ppm}$. LC-MS $(\mathrm{m} / \mathrm{z}): 344\left(\mathrm{M}+\mathrm{H}^{+}\right)$. Anal. Calcd. for $\mathrm{C}_{18} \mathrm{H}_{25} \mathrm{~N}_{5} \mathrm{O}_{2}$ : C, 62.95; H, 7.34; N, 20.39. Found: C, 63.11; H, 6.95; N, 20.38.<smiles>CN(CC1CCC1)c1nnnn1Cc1ccsc1</smiles>

$\boldsymbol{N}$-(Cyclobutylmethyl)- $\boldsymbol{N}$-methyl-1-(thiophen-3-ylmethyl)-1H-tetrazol-5-amine $\quad(\mathbf{9}\{102,356\})$. Yellow oil. ${ }^{1} \mathrm{H}$ NMR $\left(500 \mathrm{MHz}\right.$, DMSO- $\left.d_{6}\right): \delta 7.61-7.54(\mathrm{~m}, 1 \mathrm{H}), 7.37(\mathrm{~s}, 1 \mathrm{H}), 6.95(\mathrm{~d}, J=5.1 \mathrm{~Hz}$, $1 \mathrm{H}), 5.52(\mathrm{~s}, 2 \mathrm{H}), 3.29(\mathrm{~d}, J=7.2 \mathrm{~Hz}, 2 \mathrm{H}), 2.92(\mathrm{~s}, 3 \mathrm{H}), 2.50-2.44(\mathrm{~m}, 1 \mathrm{H}), 1.88-1.80(\mathrm{~m}, 2 \mathrm{H}), 1.80-$ $1.72(\mathrm{~m}, 1 \mathrm{H}), 1.71-1.62(\mathrm{~m}, 1 \mathrm{H}), 1.58-1.47(\mathrm{~m}, 2 \mathrm{H}) \mathrm{ppm} .{ }^{13} \mathrm{C}\left\{{ }^{1} \mathrm{H}\right\}$ NMR $(126 \mathrm{MHz}$, DMSO $\delta 158.0$, 135.9, 127.5, 126.5, 123.3, 58.1, 46.1, 38.6, 33.3, 25.8, $18.1 \mathrm{ppm}$. LC-MS $(\mathrm{m} / \mathrm{z}):=264\left(\mathrm{M}+\mathrm{H}^{+}\right)$. Anal. Calcd. for $\mathrm{C}_{12} \mathrm{H}_{17} \mathrm{~N}_{5} \mathrm{~S}$ : C, 54.73; H, 6.51; N, 26.59; S, 12.17. Found: C, 54.63; H, 6.26; N, 26.55; S, 12.15 .<smiles>CN(Cc1ccc(F)cc1)c1nnnn1CC1CCOC1</smiles> 
$\boldsymbol{N}$-(4-Fluorobenzyl)- $\boldsymbol{N}$-methyl-1-((tetrahydrofuran-3-yl)methyl)-1H-tetrazol-5-amine $(9\{103,386\})$. Brownish oil. ${ }^{1} \mathrm{H}$ NMR $\left(500 \mathrm{MHz}, \mathrm{DMSO}-d_{6}\right): \delta 7.36(\mathrm{t}, J=8.5,5.7 \mathrm{~Hz}, 2 \mathrm{H}), 7.18(\mathrm{t}, J=8.9 \mathrm{~Hz}, 2 \mathrm{H})$, $4.47(\mathrm{~s}, 2 \mathrm{H}), 4.25(\mathrm{~d}, J=7.8 \mathrm{~Hz}, 2 \mathrm{H}), 3.76(\mathrm{td}, J=8.2,5.5 \mathrm{~Hz}, 1 \mathrm{H}), 3.69-3.59(\mathrm{~m}, 2 \mathrm{H}), 3.42(\mathrm{dd}, J=$ $8.9,5.4 \mathrm{~Hz}, 1 \mathrm{H}), 2.91(\mathrm{~s}, 3 \mathrm{H}), 2.81-2.73(\mathrm{~m}, 1 \mathrm{H}), 1.98-1.88(\mathrm{~m}, 1 \mathrm{H}), 1.59-1.51(\mathrm{~m}, 1 \mathrm{H}) \mathrm{ppm}$.

${ }^{13} \mathrm{C}\left\{{ }^{1} \mathrm{H}\right\}$ NMR $\left(126 \mathrm{MHz}, \mathrm{DMSO}-d_{6}\right): \delta 161.5(\mathrm{~d}, J=243.2 \mathrm{~Hz}), 158.6,132.9(\mathrm{~d}, J=3.0 \mathrm{~Hz}), 130.0(\mathrm{~d}$, $J=8.2 \mathrm{~Hz}), 115.3(\mathrm{~d}, J=21.3 \mathrm{~Hz}), 70.0,66.7,56.0,49.3,38.5,38.3,29.2 \mathrm{ppm} .{ }^{19} \mathrm{~F} \mathrm{NMR}(470 \mathrm{MHz}$, DMSO- $\left.d_{6}\right): \delta-115.1(\mathrm{tt}, J=9.6,5.7 \mathrm{~Hz}) \mathrm{ppm}$. LC-MS $(\mathrm{m} / \mathrm{z}): 292\left(\mathrm{M}+\mathrm{H}^{+}\right)$. Anal. Calcd. for $\mathrm{C}_{14} \mathrm{H}_{18} \mathrm{FN}_{5} \mathrm{O}: \mathrm{C}, 57.72 ; \mathrm{H}, 6.23 ; \mathrm{N}, 24.04$. Found: C, 57.45; H, 5.90; N, 24.30.

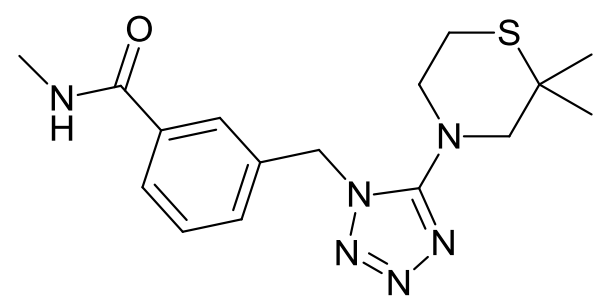

3-((5-(2,2-Dimethylthiomorpholino)-1H-tetrazol-1-yl)methyl)- $N$-methylbenzamide $\quad(9\{110,333\})$. Yellow solid, $\mathrm{mp}=72-75{ }^{\circ} \mathrm{C} .{ }^{1} \mathrm{H}$ NMR $\left(500 \mathrm{MHz}, \mathrm{DMSO}-d_{6}\right): \delta 8.51-8.42(\mathrm{~m}, 1 \mathrm{H}), 7.80(\mathrm{~d}, J=7.7$ $\mathrm{Hz}, 1 \mathrm{H}), 7.69(\mathrm{~s}, 1 \mathrm{H}), 7.48(\mathrm{t}, J=7.7 \mathrm{~Hz}, 1 \mathrm{H}), 7.35(\mathrm{~d}, J=7.7 \mathrm{~Hz}, 1 \mathrm{H}), 5.60(\mathrm{~s}, 2 \mathrm{H}), 3.42-3.36(\mathrm{~m}$, $2 \mathrm{H}), 3.23(\mathrm{~s}, 2 \mathrm{H}), 2.76(\mathrm{~d}, J=4.5 \mathrm{~Hz}, 3 \mathrm{H}), 2.73-2.69(\mathrm{~m}, 2 \mathrm{H}), 1.12(\mathrm{~s}, 6 \mathrm{H}) \mathrm{ppm} .{ }^{13} \mathrm{C}\left\{{ }^{1} \mathrm{H}\right\}$ NMR $(126$ $\left.\mathrm{MHz}, \mathrm{DMSO}-d_{6}\right): \delta 166.0,158.7,135.0,134.8,129.9,128.9,126.6,126.3,62.4,51.0,49.9,38.9,26.6$, 26.2, 24.0 ppm. LC-MS $(m / z):=347\left(\mathrm{M}+\mathrm{H}^{+}\right)$. Anal. Calcd. for $\mathrm{C}_{16} \mathrm{H}_{22} \mathrm{~N}_{6} \mathrm{OS}: \mathrm{C}, 55.47 ; \mathrm{H}, 6.40 ; \mathrm{N}$, 24.26; S, 9.25. Found: C, 55.29; H, 6.21; N, 24.59; S, 9.65.<smiles>CN(CCOc1ccc(F)cc1)c1nnnn1CC1CCOCC1</smiles>

$\boldsymbol{N}$-(2-(4-Fluorophenoxy)ethyl)- $\boldsymbol{N}$-methyl-1-((tetrahydro-2H-pyran-4-yl)methyl)-1H-tetrazol-5amine (9\{121,302\}). Yellow oil. ${ }^{1} \mathrm{H}$ NMR (500 MHz, DMSO- $\left.d_{6}\right): \delta 7.10(\mathrm{t}, J=8.8 \mathrm{~Hz}, 2 \mathrm{H}), 6.87(\mathrm{dd}, J$ $=9.1,4.2 \mathrm{~Hz}, 2 \mathrm{H}), 4.19(\mathrm{~d}, J=7.3 \mathrm{~Hz}, 2 \mathrm{H}), 4.15(\mathrm{t}, J=5.1 \mathrm{~Hz}, 2 \mathrm{H}), 3.76(\mathrm{dd}, J=11.3,3.1 \mathrm{~Hz}, 2 \mathrm{H})$, $3.69(\mathrm{t}, J=5.1 \mathrm{~Hz}, 2 \mathrm{H}), 3.16(\mathrm{td}, J=11.8,2.2 \mathrm{~Hz}, 2 \mathrm{H}), 3.07(\mathrm{~s}, 3 \mathrm{H}), 2.14-2.01(\mathrm{~m}, 1 \mathrm{H}), 1.41-1.31$ $(\mathrm{m}, 2 \mathrm{H}), 1.29-1.17(\mathrm{~m}, 2 \mathrm{H}) \mathrm{ppm} .{ }^{13} \mathrm{C}\left\{{ }^{1} \mathrm{H}\right\}$ NMR $\left(126 \mathrm{MHz}, \mathrm{DMSO}-d_{6}\right): \delta 158.5,156.6(\mathrm{~d}, J=236.0$ Hz), 154.5 (d, $J=1.7 \mathrm{~Hz}), 115.9$ (d, $J=23.0 \mathrm{~Hz}), 115.6$ (d, $J=8.1 \mathrm{~Hz}), 66.3,65.4,52.4,52.0,39.1$, 34.4, 29.7 ppm. ${ }^{19} \mathrm{~F}$ NMR (470 MHz, DMSO- $\left.d_{6}\right): \delta-123.7(\mathrm{tt}, J=8.8,4.5 \mathrm{~Hz}) \mathrm{ppm}$. LC-MS $(\mathrm{m} / \mathrm{z}):=$ $336\left(\mathrm{M}+\mathrm{H}^{+}\right)$. Anal. Calcd. for $\mathrm{C}_{16} \mathrm{H}_{22} \mathrm{FN}_{5} \mathrm{O}_{2}: \mathrm{C}, 57.30 ; \mathrm{H}, 6.61 ; \mathrm{N}, 20.88$. Found: $\mathrm{C}, 57.12 ; \mathrm{H}, 6.84 ; \mathrm{N}$, 20.61. 
<smiles>Cc1cccc(C(=O)NCCn2nnnc2N(C)C2CC2)c1</smiles>

$\boldsymbol{N}$-(2-(5-(Cyclopropyl(methyl)amino)-1H-tetrazol-1-yl)ethyl)-3-methylbenzamide $\quad(\mathbf{9}\{146,322\})$. White solid, $\mathrm{mp}=75-77^{\circ} \mathrm{C} .{ }^{1} \mathrm{H}$ NMR $\left(500 \mathrm{MHz}, \mathrm{DMSO}-d_{6}\right): \delta 8.60(\mathrm{t}, J=5.8 \mathrm{~Hz}, 1 \mathrm{H}), 7.60(\mathrm{~s}, 1 \mathrm{H})$, $7.56(\mathrm{t}, J=5.0 \mathrm{~Hz}, 1 \mathrm{H}), 7.34(\mathrm{~d}, J=4.6 \mathrm{~Hz}, 2 \mathrm{H}), 4.54(\mathrm{t}, J=6.1 \mathrm{~Hz}, 2 \mathrm{H}), 3.63(\mathrm{dt}, J=5.8,6.1 \mathrm{~Hz}, 2 \mathrm{H})$, $2.93(\mathrm{~s}, 3 \mathrm{H}), 2.84(\mathrm{tt}, J=6.9,3.6 \mathrm{~Hz}, 1 \mathrm{H}), 2.35(\mathrm{~s}, 3 \mathrm{H}), 0.75-0.70(\mathrm{~m}, 2 \mathrm{H}), 0.54-0.50(\mathrm{~m}, 2 \mathrm{H}) \mathrm{ppm}$. ${ }^{13} \mathrm{C}\left\{{ }^{1} \mathrm{H}\right\}$ NMR $\left(126 \mathrm{MHz}, \mathrm{DMSO}-d_{6}\right): \delta 166.7,158.6,137.5,134.0,131.9,128.2,127.6,124.2,46.1$, 39.4, 38.8, 33.4, 20.9, 8.2 ppm. LC-MS $(m / z):=301\left(\mathrm{M}+\mathrm{H}^{+}\right)$. Anal. Calcd. for $\mathrm{C}_{15} \mathrm{H}_{20} \mathrm{~N}_{6} \mathrm{O}: \mathrm{C}, 59.98$; H, 6.71; N, 27.98. Found: C, 60.24; H, 6.96; N, 28.09.<smiles>CN(CC1COCCO1)c1nnnn1CCc1ccc(F)cc1F</smiles>

$N$-((1,4-Dioxan-2-yl)methyl)-1-(2,4-difluorophenethyl)- $N$-methyl-1H-tetrazol-5-amine

(9 $\{151,319\})$. Beige solid, $\mathrm{mp}=41-44{ }^{\circ} \mathrm{C} .{ }^{1} \mathrm{H}$ NMR $\left(500 \mathrm{MHz}, \mathrm{DMSO}-d_{6}\right): \delta 7.26(\mathrm{q}, J=8.5 \mathrm{~Hz}, 1 \mathrm{H})$, $7.19(\mathrm{td}, J=9.9,2.6 \mathrm{~Hz}, 1 \mathrm{H}), 7.01(\mathrm{td}, J=8.6,2.6 \mathrm{~Hz}, 1 \mathrm{H}), 4.52(\mathrm{t}, J=7.1 \mathrm{~Hz}, 2 \mathrm{H}), 3.79-3.72(\mathrm{~m}$, $1 \mathrm{H}), 3.67(\mathrm{~d}, J=11.4 \mathrm{~Hz}, 2 \mathrm{H}), 3.62(\mathrm{~d}, J=11.2 \mathrm{~Hz}, 1 \mathrm{H}), 3.53(\mathrm{td}, J=11.3,2.5 \mathrm{~Hz}, 1 \mathrm{H}), 3.42(\mathrm{td}, J=$ 11.2, $2.7 \mathrm{~Hz}, 1 \mathrm{H}), 3.31-3.21(\mathrm{~m}, 2 \mathrm{H}), 3.18(\mathrm{dd}, J=11.4,9.9 \mathrm{~Hz}, 1 \mathrm{H}), 3.12(\mathrm{t}, J=7.0 \mathrm{~Hz}, 2 \mathrm{H}), 2.98(\mathrm{~s}$, $3 \mathrm{H}) \mathrm{ppm} .{ }^{13} \mathrm{C}\left\{{ }^{1} \mathrm{H}\right\}$ NMR $\left(126 \mathrm{MHz}, \mathrm{DMSO}-d_{6}\right): \delta 161.3(\mathrm{dd}, J=245.3,12.2 \mathrm{~Hz}), 160.6(\mathrm{dd}, J=246.6$, $12.2 \mathrm{~Hz}), 158.3,132.3(\mathrm{dd}, J=9.7,6.2 \mathrm{~Hz}), 120.3(\mathrm{dd}, J=15.9,3.7 \mathrm{~Hz}), 111.5(\mathrm{dd}, J=21.0,3.6 \mathrm{~Hz})$, $103.7(\mathrm{t}, J=26.1 \mathrm{~Hz}), 72.6,68.1,65.9,65.7,54.1,46.7,39.3,27.5 \mathrm{ppm} .{ }^{19} \mathrm{~F}$ NMR (470 MHz, DMSO$\left.d_{6}\right): \delta-111.9$ (quint, $\left.J=7.9 \mathrm{~Hz}\right),-114.4(\mathrm{q}, J=8.9 \mathrm{~Hz}) \mathrm{ppm}$. LC-MS $(m / z):=340\left(\mathrm{M}+\mathrm{H}^{+}\right)$. Anal. Calcd. for $\mathrm{C}_{15} \mathrm{H}_{19} \mathrm{~F}_{2} \mathrm{~N}_{5} \mathrm{O}_{2}$ : C, 53.09; H, 5.64; N, 20.64. Found: C, 53.37; H, 5.60; N, 20.31 .<smiles>C=C(Br)Cn1nnnc1N1CCC(CO)(OC)CC1</smiles>

(1-(1-(2-Bromoallyl)-1H-tetrazol-5-yl)-4-methoxypiperidin-4-yl)methanol $(9\{165,299\})$. Brown oil. ${ }^{1} \mathrm{H}$ NMR $\left(500 \mathrm{MHz}, \mathrm{DMSO}-d_{6}\right): \delta 6.05(\mathrm{~d}, J=2.6 \mathrm{~Hz}, 1 \mathrm{H}), 5.82(\mathrm{~d}, J=2.6 \mathrm{~Hz}, 1 \mathrm{H}), 5.25(\mathrm{~s}, 2 \mathrm{H}), 3.36$ $(\mathrm{s}, 2 \mathrm{H}), 3.35-3.31(\mathrm{~m}, 2 \mathrm{H}), 3.17-3.12(\mathrm{~m}, 5 \mathrm{H}), 1.75-1.66(\mathrm{~m}, 2 \mathrm{H}), 1.63-1.55(\mathrm{~m}, 2 \mathrm{H}) \mathrm{ppm}$. 
${ }^{13} \mathrm{C}\left\{{ }^{1} \mathrm{H}\right\}$ NMR $\left(126 \mathrm{MHz}, \mathrm{DMSO}-d_{6}\right): \delta 158.4,124.8,121.9,72.9,64.0,53.8,48.6,45.1,29.2 \mathrm{ppm} . \mathrm{LC}-$ MS $(m / z):=332 / 334\left(\mathrm{M}+\mathrm{H}^{+}\right)$. Anal. Calcd. for $\mathrm{C}_{11} \mathrm{H}_{18} \mathrm{BrN}_{5} \mathrm{O}_{2}: \mathrm{C}, 39.77 ; \mathrm{H}, 5.46 ; \mathrm{N}, 21.08 ; \mathrm{Br}, 24.05$. Found: C, 39.41; H, 5.73; N, 20.96; Br, 24.22.<smiles>CCC(Cn1nnnc1N1CCC(CO)(OC)CC1)c1ccccc1</smiles>

(4-Methoxy-1-(1-(2-phenylbutyl)-1H-tetrazol-5-yl)piperidin-4-yl)methanol (9\{175,299\}). Yellow oil. ${ }^{1} \mathrm{H}$ NMR $\left(500 \mathrm{MHz}, \mathrm{DMSO}-d_{6}\right): \delta 7.23(\mathrm{t}, J=7.5 \mathrm{~Hz}, 2 \mathrm{H}), 7.17(\mathrm{t}, J=7.2 \mathrm{~Hz}, 1 \mathrm{H}), 7.11(\mathrm{~d}, J=7.0$ $\mathrm{Hz}, 2 \mathrm{H}), 4.63(\mathrm{t}, J=5.6 \mathrm{~Hz}, 1 \mathrm{H}), 4.49(\mathrm{dd}, J=14.2,6.0 \mathrm{~Hz}, 1 \mathrm{H}), 4.36(\mathrm{dd}, J=14.2,9.2 \mathrm{~Hz}, 1 \mathrm{H}), 3.37$ $(\mathrm{s}, 1 \mathrm{H}), 3.36(\mathrm{~s}, 1 \mathrm{H}), 3.14(\mathrm{~s}, 3 \mathrm{H}), 3.12-3.06(\mathrm{~m}, 1 \mathrm{H}), 3.04-2.91(\mathrm{~m}, 3 \mathrm{H}), 1.75-1.62(\mathrm{~m}, 4 \mathrm{H}), 1.61-$ $1.48(\mathrm{~m}, 2 \mathrm{H}), 0.75(\mathrm{t}, J=7.3 \mathrm{~Hz}, 3 \mathrm{H}) \mathrm{ppm} .{ }^{13} \mathrm{C}\left\{{ }^{1} \mathrm{H}\right\}$ NMR $\left(126 \mathrm{MHz}, \mathrm{DMSO}-d_{6}\right): \delta 158.9,140.7,128.3$, $127.7,126.8,72.8,64.1,51.8,48.6,45.9,45.4,45.1,29.34,29.31,25.6,11.7$ ppm. LC-MS $(\mathrm{m} / \mathrm{z}):=346$ $\left(\mathrm{M}+\mathrm{H}^{+}\right)$. Anal. Calcd. for $\mathrm{C}_{18} \mathrm{H}_{27} \mathrm{~N}_{5} \mathrm{O}_{2}: \mathrm{C}, 62.58 ; \mathrm{H}, 7.88 ; \mathrm{N}, 20.27$. Found: $\mathrm{C}, 62.77 ; \mathrm{H}, 7.62 ; \mathrm{N}, 20.08$.

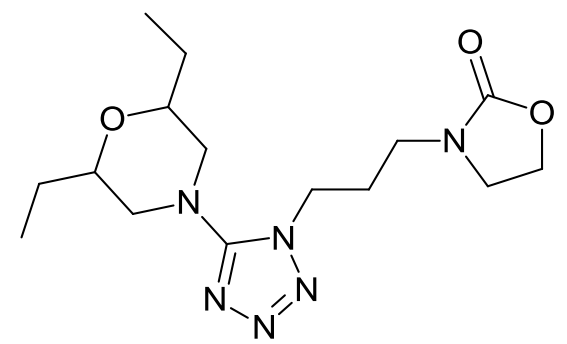

3-(3-(5-(2,6-Diethylmorpholino)-1H-tetrazol-1-yl)propyl)oxazolidin-2-one $\quad(\mathbf{9}\{188,338\})$. White solid, mp $=79-81{ }^{\circ} \mathrm{C} .{ }^{1} \mathrm{H}$ NMR $\left(500 \mathrm{MHz}, \mathrm{DMSO}-d_{6}\right): \delta 4.25(\mathrm{~d}, J=6.9 \mathrm{~Hz}, 2 \mathrm{H}), 4.23(\mathrm{t}, J=7.9 \mathrm{~Hz}$, $2 \mathrm{H}), 3.53-3.48(\mathrm{~m}, 4 \mathrm{H}), 3.37(\mathrm{~d}, J=12.1 \mathrm{~Hz}, 2 \mathrm{H}), 3.17(\mathrm{t}, J=6.9 \mathrm{~Hz}, 2 \mathrm{H}), 2.65(\mathrm{t}, J=12.1,10.5 \mathrm{~Hz}$, 2H), 2.09 (quint, $J=6.9 \mathrm{~Hz}, 2 \mathrm{H}), 1.53-1.37(\mathrm{~m}, 4 \mathrm{H}), 0.92(\mathrm{t}, J=7.4 \mathrm{~Hz}, 6 \mathrm{H}) \mathrm{ppm} .{ }^{13} \mathrm{C}\left\{{ }^{1} \mathrm{H}\right\} \mathrm{NMR}$ (126 MHz, DMSO- $d_{6}$ ): $\delta$ 158.7, 158.4, 76.1, 62.2, 53.7, 44.8, 44.5, 41.3, 26.2, 26.1, 10.0 ppm. LC-MS $(m / z):=339\left(\mathrm{M}+\mathrm{H}^{+}\right)$. Anal. Calcd. for $\mathrm{C}_{15} \mathrm{H}_{26} \mathrm{~N}_{6} \mathrm{O}_{3}: \mathrm{C}, 53.24 ; \mathrm{H}, 7.74 ; \mathrm{N}, 24.83$. Found: $\mathrm{C}, 53.56 ; \mathrm{H}$, 8.04; N, 24.88 .<smiles>COc1ccc(CCn2nnnc2N2CCCC2)cc1F</smiles>

1-(3-Fluoro-4-methoxyphenethyl)-5-(pyrrolidin-1-yl)-1H-tetrazole (9\{190,341\}). Whire solid, $\mathrm{mp}=$ 70-72 ${ }^{\circ} \mathrm{C}$. ${ }^{1} \mathrm{H}$ NMR $\left(500 \mathrm{MHz}, \mathrm{DMSO}-d_{6}\right): \delta 7.07-7.01(\mathrm{~m}, 2 \mathrm{H}), 6.87(\mathrm{~d}, J=8.5 \mathrm{~Hz}, 1 \mathrm{H}), 4.48(\mathrm{t}, J=$ $7.2 \mathrm{~Hz}, 2 \mathrm{H}), 3.78(\mathrm{~s}, 3 \mathrm{H}), 3.43-3.38(\mathrm{~m}, 4 \mathrm{H}), 3.01(\mathrm{t}, J=7.2 \mathrm{~Hz}, 2 \mathrm{H}), 1.90-1.83(\mathrm{~m}, 4 \mathrm{H}) \mathrm{ppm}$. 
${ }^{13} \mathrm{C}\left\{{ }^{1} \mathrm{H}\right\}$ NMR $\left(126 \mathrm{MHz}\right.$, DMSO- $\left.d_{6}\right): \delta 155.9,151.2(\mathrm{~d}, J=243.5 \mathrm{~Hz}), 145.8(\mathrm{~d}, J=10.4 \mathrm{~Hz}), 130.2(\mathrm{~d}$, $J=6.2 \mathrm{~Hz}), 125.0(\mathrm{~d}, J=3.3 \mathrm{~Hz}), 116.3(\mathrm{~d}, J=17.7 \mathrm{~Hz}), 113.7,55.9,49.0,47.5,34.1,25.1 \mathrm{ppm} .{ }^{19} \mathrm{~F}$ NMR $\left(470 \mathrm{MHz}\right.$, DMSO- $\left.d_{6}\right): \delta-135.6(\mathrm{dd}, J=12.5,9.2 \mathrm{~Hz}) \mathrm{ppm}$. LC-MS $(m / z):=292\left(\mathrm{M}+\mathrm{H}^{+}\right)$. Anal. Calcd. for $\mathrm{C}_{14} \mathrm{H}_{18} \mathrm{FN}_{5} \mathrm{O}$ : C, 57.72; H, 6.23; N, 24.04. Found: C, 58.04; H, 6.03; N, 23.88.

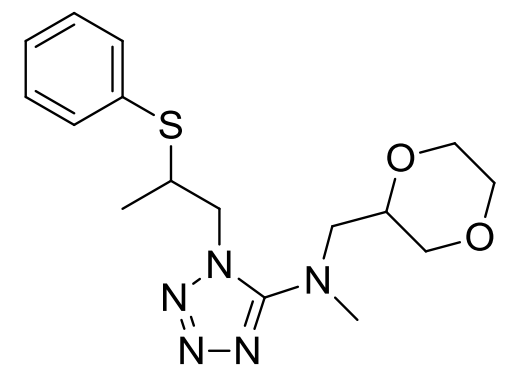

$N$-((1,4-Dioxan-2-yl)methyl)- $N$-methyl-1-(2-(phenylthio)propyl)-1H-tetrazol-5-amine (9\{262,319\}). Yellow oil. The compound existed as $c a$. 1:1 mixture of diastereomers. ${ }^{1} \mathrm{H}$ NMR (500 MHz, DMSO- $\left.d_{6}\right)$ : $\delta 7.43(\mathrm{t}, J=8.3 \mathrm{~Hz}, 2 \mathrm{H}), 7.40-7.29(\mathrm{~m}, 3 \mathrm{H}), 4.44-4.32(\mathrm{~m}, 2 \mathrm{H}), 3.79-3.69(\mathrm{~m}, 2 \mathrm{H}), 3.67-3.59(\mathrm{~m}$, $3 \mathrm{H}), 3.50(\mathrm{t}, J=11.4 \mathrm{~Hz}, 1 \mathrm{H}), 3.44-3.36(\mathrm{~m}, 1 \mathrm{H}), 3.28-3.08(\mathrm{~m}, 3 \mathrm{H}), 2.92(\mathrm{~s}, 1.5 \mathrm{H})$ and $2.91(\mathrm{~s}$, $1.5 \mathrm{H}), 1.23(\mathrm{~d}, J=6.8 \mathrm{~Hz}, 1.5 \mathrm{H})$ and $1.19(\mathrm{~d}, J=6.8 \mathrm{~Hz}, 1.5 \mathrm{H}) \mathrm{ppm} .{ }^{13} \mathrm{C}\left\{{ }^{1} \mathrm{H}\right\}$ NMR $(126 \mathrm{MHz}$, DMSO$\left.d_{6}\right)$ : $\delta 158.5$ and $158.4,132.57$ and $132.55,132.4$ and $132.3,129.3$ and $129.2,127.8$ and 127.7, 72.7, 72.5, 68.1 and 68.0, 65.9, 65.7, 54.02 and 53.95, 51.9 and 51.8, 41.64 and 41.56, 18.4 and $18.3 \mathrm{ppm}$. LC-MS $(m / z):=350\left(\mathrm{M}+\mathrm{H}^{+}\right)$. Anal. Calcd. for $\mathrm{C}_{16} \mathrm{H}_{23} \mathrm{~N}_{5} \mathrm{O}_{2} \mathrm{~S}: \mathrm{C}, 54.99 ; \mathrm{H}, 6.63 ; \mathrm{N}, 20.04 ; \mathrm{S}, 9.17$. Found: C, 54.87; H, 6.82; N, 20.09; S, 8.90.<smiles>CC(Cn1nnnc1N1CCCC1)Oc1ccccc1F</smiles>

1-(2-(2-Fluorophenoxy)propyl)-5-(pyrrolidin-1-yl)-1H -tetrazole $(9\{263,341\})$. Beige solid, $\mathrm{mp}=78-$ $80{ }^{\circ} \mathrm{C} .{ }^{1} \mathrm{H}$ NMR $\left(500 \mathrm{MHz}, \mathrm{DMSO}-d_{6}\right): \delta 7.15(\mathrm{dd}, J=11.7,8.1 \mathrm{~Hz}, 1 \mathrm{H}), 7.06-7.02(\mathrm{~m}, 2 \mathrm{H}), 6.94-$ $6.87(\mathrm{~m}, 1 \mathrm{H}), 4.85(\mathrm{sext}, J=6.2 \mathrm{~Hz}, 1 \mathrm{H}), 4.62-4.56(\mathrm{~m}, 2 \mathrm{H}), 3.63-3.55(\mathrm{~m}, 2 \mathrm{H}), 3.51-3.43(\mathrm{~m}, 2 \mathrm{H})$, $1.94-1.88(\mathrm{~m}, 4 \mathrm{H}), 1.34(\mathrm{~d}, J=6.2 \mathrm{~Hz}, 3 \mathrm{H}) \mathrm{ppm} .{ }^{13} \mathrm{C}\left\{{ }^{1} \mathrm{H}\right\}$ NMR $\left(126 \mathrm{MHz}\right.$, DMSO- $\left.d_{6}\right): \delta 155.9,152.3$ $(\mathrm{d}, J=243.6 \mathrm{~Hz}), 144.7(\mathrm{~d}, J=10.4 \mathrm{~Hz}), 124.7(\mathrm{~d}, J=3.7 \mathrm{~Hz}), 121.8(\mathrm{~d}, J=7.0 \mathrm{~Hz}), 116.4,116.2(\mathrm{~d}, J$ $=18.2 \mathrm{~Hz}), 73.8,51.0,48.9,25.2,17.0 \mathrm{ppm} .{ }^{19} \mathrm{~F}$ NMR $\left(470 \mathrm{MHz}, \mathrm{DMSO}-d_{6}\right): \delta-134.51--134.66(\mathrm{~m})$ ppm. LC-MS $(\mathrm{m} / z):=292\left(\mathrm{M}+\mathrm{H}^{+}\right)$. Anal. Calcd. for $\mathrm{C}_{14} \mathrm{H}_{18} \mathrm{FN}_{5} \mathrm{O}: \mathrm{C}, 57.72 ; \mathrm{H}, 6.23 ; \mathrm{N}, 24.04$. Found: C, 57.44; H, 6.51; N, 24.32. 


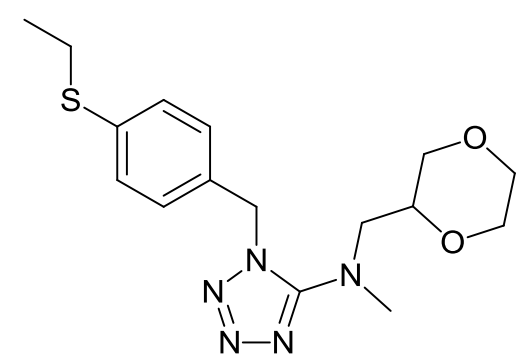

$\boldsymbol{N}$-((1,4-Dioxan-2-yl)methyl)-1-(4-(ethylthio)benzyl)- $\boldsymbol{N}$-methyl-1H-tetrazol-5-amine $\quad(\mathbf{9}\{272,319\})$. Yellow oil. ${ }^{1} \mathrm{H}$ NMR $\left(500 \mathrm{MHz}, \mathrm{DMSO}-d_{6}\right): \delta 7.31(\mathrm{~d}, J=8.3 \mathrm{~Hz}, 2 \mathrm{H}), 7.10(\mathrm{~d}, J=8.3 \mathrm{~Hz}, 2 \mathrm{H}), 5.56(\mathrm{~s}$, 2H), $3.72-3.67(\mathrm{~m}, 1 \mathrm{H}), 3.65(\mathrm{~d}, J=11.3 \mathrm{~Hz}, 1 \mathrm{H}), 3.58(\mathrm{~d}, J=11.1 \mathrm{~Hz}, 1 \mathrm{H}), 3.53(\mathrm{dd}, J=11.4,2.6$ $\mathrm{Hz}, 1 \mathrm{H}), 3.48(\mathrm{td}, J=11.3,2.6 \mathrm{~Hz}, 1 \mathrm{H}), 3.36(\mathrm{td}, J=11.3,2.7 \mathrm{~Hz}, 1 \mathrm{H}), 3.32-3.21(\mathrm{~m}, 2 \mathrm{H}), 3.03(\mathrm{dd}, J$ $=11.4,9.9 \mathrm{~Hz}, 1 \mathrm{H}), 2.98(\mathrm{~s}, 3 \mathrm{H}), 2.96(\mathrm{q}, J=7.3 \mathrm{~Hz}, 2 \mathrm{H}), 1.21(\mathrm{t}, J=7.3 \mathrm{~Hz}, 3 \mathrm{H}) \mathrm{ppm} .{ }^{13} \mathrm{C}\left\{{ }^{1} \mathrm{H}\right\} \mathrm{NMR}$ (126 MHz, DMSO- $\left.d_{6}\right): \delta 158.0,136.3,132.7,128.0,127.5,72.5,67.9,65.8,65.6,53.7,49.6,39.3,25.9$, 14.1 ppm. LC-MS $(\mathrm{m} / z):=350\left(\mathrm{M}+\mathrm{H}^{+}\right)$. Anal. Calcd. for $\mathrm{C}_{16} \mathrm{H}_{23} \mathrm{~N}_{5} \mathrm{O}_{2} \mathrm{~S}: \mathrm{C}, 54.99 ; \mathrm{H}, 6.63 ; \mathrm{N}, 20.04 ; \mathrm{S}$, 9.17. Found: C, 54.79; H, 6.72; N, 19.85; S, 9.54.

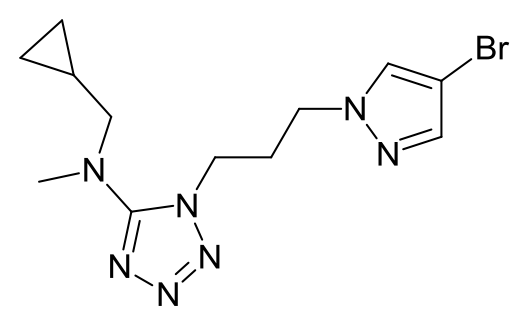

1-(3-(4-Bromo-1H-pyrazol-1-yl)propyl)- $N$-(cyclopropylmethyl)- $\boldsymbol{N}$-methyl- $1 H$-tetrazol-5-amine (9 $\{280,391\})$. Yellow oil. ${ }^{1} \mathrm{H}$ NMR $\left(500 \mathrm{MHz}, \mathrm{DMSO}-d_{6}\right): \delta 7.98(\mathrm{~s}, 1 \mathrm{H}), 7.56(\mathrm{~s}, 1 \mathrm{H}), 4.17(\mathrm{q}, J=6.8$ $\mathrm{Hz}, 4 \mathrm{H}), 3.05(\mathrm{~d}, J=6.8 \mathrm{~Hz}, 2 \mathrm{H}), 2.95(\mathrm{~s}, 3 \mathrm{H}), 2.31$ (quint, $J=6.8 \mathrm{~Hz}, 2 \mathrm{H}), 0.98-0.90(\mathrm{~m}, 1 \mathrm{H}), 0.48-$ $0.39(\mathrm{~m}, 2 \mathrm{H}), 0.15(\mathrm{dt}, J=5.8,4.3 \mathrm{~Hz}, 2 \mathrm{H}) \mathrm{ppm} .{ }^{13} \mathrm{C}\left\{{ }^{1} \mathrm{H}\right\}$ NMR $\left(126 \mathrm{MHz}, \mathrm{DMSO}-d_{6}\right): \delta 158.9,139.7$, 130.9, 92.0, 57.9, 49.3, 44.7, 38.8, 29.3, 9.0, 3.5 ppm. LC-MS $(m / z):=340 / 342\left(\mathrm{M}+\mathrm{H}^{+}\right)$. Anal. Calcd. for $\mathrm{C}_{12} \mathrm{H}_{18} \mathrm{BrN}_{7}$ : C, 42.36; H, 5.33; N, 28.82; Br, 23.49. Found: C, 42.26; H, 5.31; N, 28.73; Br, 23.52.

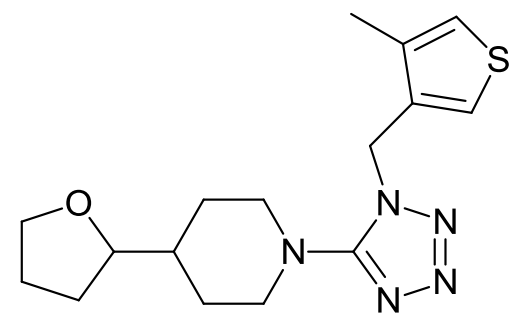

1-(1-((4-Methylthiophen-3-yl)methyl)-1H-tetrazol-5-yl)-4-(tetrahydrofuran-2-yl)piperidine (9 $\{306,261\})$. Brown solid, $\mathrm{mp}=64-66{ }^{\circ} \mathrm{C} .{ }^{1} \mathrm{H}$ NMR $\left(500 \mathrm{MHz}, \mathrm{DMSO}-d_{6}\right): \delta 7.24(\mathrm{~d}, J=3.1 \mathrm{~Hz}, 1 \mathrm{H})$, $7.19(\mathrm{~d}, J=3.1 \mathrm{~Hz}, 1 \mathrm{H}), 5.39(\mathrm{~s}, 2 \mathrm{H}), 3.70(\mathrm{dt}, J=8.1,6.7 \mathrm{~Hz}, 1 \mathrm{H}), 3.59(\mathrm{q}, J=7.3 \mathrm{~Hz}, 1 \mathrm{H}), 3.54-3.49$ $(\mathrm{m}, 2 \mathrm{H}), 3.45(\mathrm{q}, J=7.3 \mathrm{~Hz}, 1 \mathrm{H}), 2.94-2.83(\mathrm{~m}, 2 \mathrm{H}), 2.04(\mathrm{~s}, 3 \mathrm{H}), 1.88-1.83(\mathrm{~m}, 1 \mathrm{H}), 1.82-1.75(\mathrm{~m}$, $3 \mathrm{H}), 1.58-1.53(\mathrm{~m}, 1 \mathrm{H}), 1.51-1.43(\mathrm{~m}, 2 \mathrm{H}), 1.33-1.20(\mathrm{~m}, 2 \mathrm{H}) \mathrm{ppm} .{ }^{13} \mathrm{C}\left\{{ }^{1} \mathrm{H}\right\} \mathrm{NMR}(126 \mathrm{MHz}$, DMSO- $\left.d_{6}\right): \delta 158.5,135.8,134.2,124.6,122.8,82.1,66.9,49.5,49.4,45.3,39.9,28.5,27.9,27.3,25.3$, 13.7 ppm. LC-MS $(\mathrm{m} / z): 334\left(\mathrm{M}+\mathrm{H}^{+}\right)$. Anal. Calcd. for $\mathrm{C}_{16} \mathrm{H}_{23} \mathrm{~N}_{5} \mathrm{OS}: \mathrm{C}, 57.63 ; \mathrm{H}, 6.95 ; \mathrm{N}, 21.00 ; \mathrm{S}$, 9.61. Found: C, 57.86; H, 6.58; N, 20.84; S, 9.37. 
<smiles>CCN(C)c1nnnn1Cc1nc(C(F)(F)F)n2c1CCCC2</smiles>

$N$-Ethyl- $N$-methyl-1-((3-(trifluoromethyl)-5,6,7,8-tetrahydroimidazo[1,5-a]pyridin-1-yl)methyl)1H-tetrazol-5-amine $(9\{341,346\})$. Beige solid, $\mathrm{mp}=60-62{ }^{\circ} \mathrm{C} .{ }^{1} \mathrm{H}$ NMR $\left(500 \mathrm{MHz}, \mathrm{DMSO}-d_{6}\right): \delta$ $5.35(\mathrm{~s}, 2 \mathrm{H}), 4.08(\mathrm{t}, J=6.1 \mathrm{~Hz}, 2 \mathrm{H}), 3.41(\mathrm{q}, J=7.1 \mathrm{~Hz}, 2 \mathrm{H}), 3.02(\mathrm{~s}, 3 \mathrm{H}), 2.75(\mathrm{t}, J=6.5 \mathrm{~Hz}, 2 \mathrm{H}), 1.94$ $-1.88(\mathrm{~m}, 2 \mathrm{H}), 1.80-1.74(\mathrm{~m}, 2 \mathrm{H}), 1.07(\mathrm{t}, J=7.1 \mathrm{~Hz}, 3 \mathrm{H}) \mathrm{ppm} .{ }^{13} \mathrm{C}\left\{{ }^{1} \mathrm{H}\right\}$ NMR $(126 \mathrm{MHz}$, DMSO$\left.d_{6}\right): \delta 157.8,131.7(\mathrm{q}, J=38.4 \mathrm{~Hz}), 131.2,130.0,119.0(\mathrm{q}, J=268.8 \mathrm{~Hz}), 47.9,43.6,43.5,37.8,21.7$, 20.0, 18.6, 11.7 ppm. ${ }^{19} \mathrm{~F}$ NMR $\left(470 \mathrm{MHz}, \mathrm{DMSO}-d_{6}\right): \delta-60.9 \mathrm{ppm}$. LC-MS $(\mathrm{m} / \mathrm{z}):=330\left(\mathrm{M}+\mathrm{H}^{+}\right)$. Anal. Calcd. for $\mathrm{C}_{13} \mathrm{H}_{18} \mathrm{~F}_{3} \mathrm{~N}_{7}$ : C, 47.41; H, 5.51; N, 29.77. Found: C, 47.53; H, 5.82; N, 29.58 .

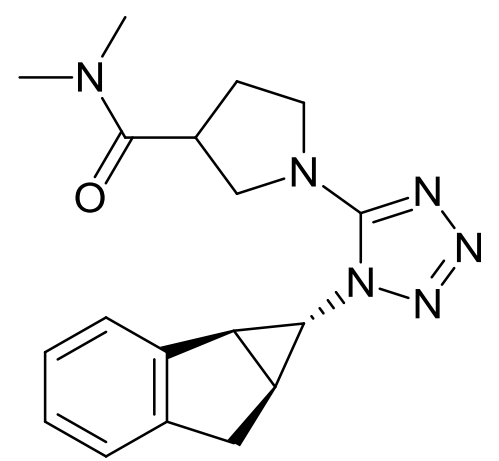

$r a c-N, N$-dimethyl-1-(1-((1R,1aS,6aS)-1,1a,6,6a-tetrahydrocyclopropa[a]inden-1-yl)-1H-tetrazol-5yl)pyrrolidine-3-carboxamide $(9\{375,511\})$. Brown solid, $\mathrm{mp}=60-63{ }^{\circ} \mathrm{C}$. The compound existed as ca. 1:1 mixture of diastereomers. ${ }^{1} \mathrm{H}$ NMR $\left(500 \mathrm{MHz}, \mathrm{DMSO}-d_{6}\right): \delta 7.49-7.41(\mathrm{~m}, 1 \mathrm{H}), 7.29-7.24$ $(\mathrm{m}, 1 \mathrm{H}), 7.21-7.17(\mathrm{~m}, 2 \mathrm{H}), 3.88(\mathrm{dd}, J=9.5,7.8 \mathrm{~Hz}, 0.5 \mathrm{H}), 3.81(\mathrm{~d}, J=7.5 \mathrm{~Hz}, 1 \mathrm{H}), 3.79-3.72(\mathrm{~m}$, $0.5 \mathrm{H}), 3.71-3.57(\mathrm{~m}, 2 \mathrm{H}), 3.56-3.47(\mathrm{~m}, 1 \mathrm{H}), 3.30(\mathrm{dd}, J=7.0,3.8 \mathrm{~Hz}, 0.5 \mathrm{H}), 3.22-3.12(\mathrm{~m}, 3.5 \mathrm{H})$, $3.06(\mathrm{~s}, 1.5 \mathrm{H})$ and $3.03(\mathrm{~s}, 1.5 \mathrm{H}), 2.87(\mathrm{~s}, 1.5 \mathrm{H})$ and $2.85(\mathrm{~s}, 1.5 \mathrm{H}), 2.70(\mathrm{t}, J=6.9 \mathrm{~Hz}, 0.5 \mathrm{H})$ and $2.66(\mathrm{t}$, $J=5.9 \mathrm{~Hz}, 0.5 \mathrm{H}), 2.25-2.14(\mathrm{~m}, 1 \mathrm{H}), 2.10-1.97(\mathrm{~m}, 1 \mathrm{H}) \mathrm{ppm} .{ }^{13} \mathrm{C}\left\{{ }^{1} \mathrm{H}\right\} \mathrm{NMR}\left(126 \mathrm{MHz}, \mathrm{DMSO}-d_{6}\right)$ : $\delta 171.4,156.32$ and 156.27, 142.1 and 142.0, 141.4 and 141.3, 126.8, 126.52 and 126.49, 125.25 and 125.23, 124.0, 51.5 and 51.4, 48.9 and 48.8, 40.4, 39.8, 36.72 and 36.70, 35.2 and 35.1, 34.1 and 34.0, 33.0, 28.73 and 28.70, 24.81 and $24.77 \mathrm{ppm}$. LC-MS $(\mathrm{m} / \mathrm{z}):=339\left(\mathrm{M}+\mathrm{H}^{+}\right)$. Anal. Calcd. for $\mathrm{C}_{18} \mathrm{H}_{22} \mathrm{~N}_{6} \mathrm{O}$ : C, 63.89; H, 6.55; N, 24.83. Found: C, 63.66; H, 6.18; N, 24.49. 
Figure S1. Structures of the reagents $\mathbf{1 0}\{1-58\}$

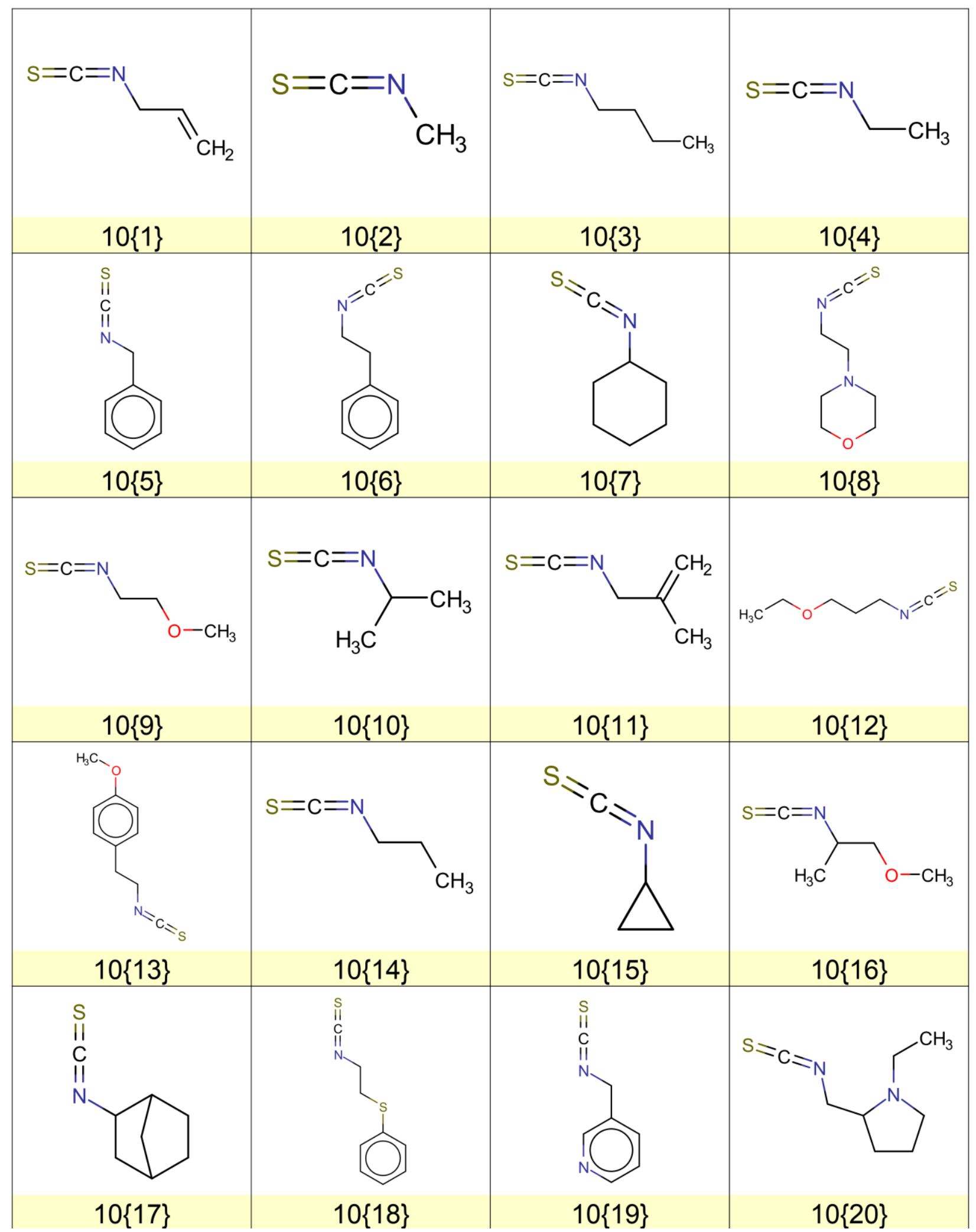




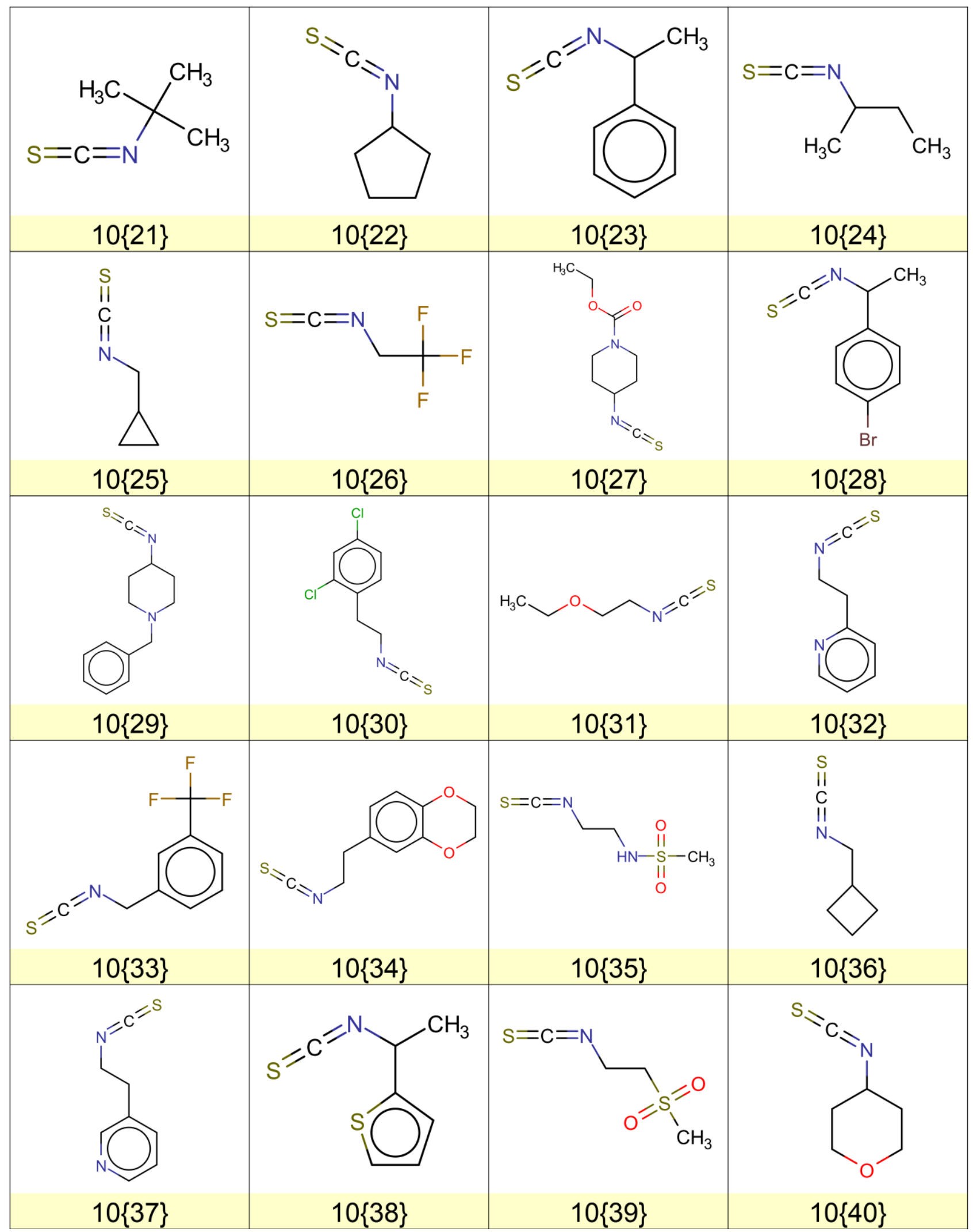




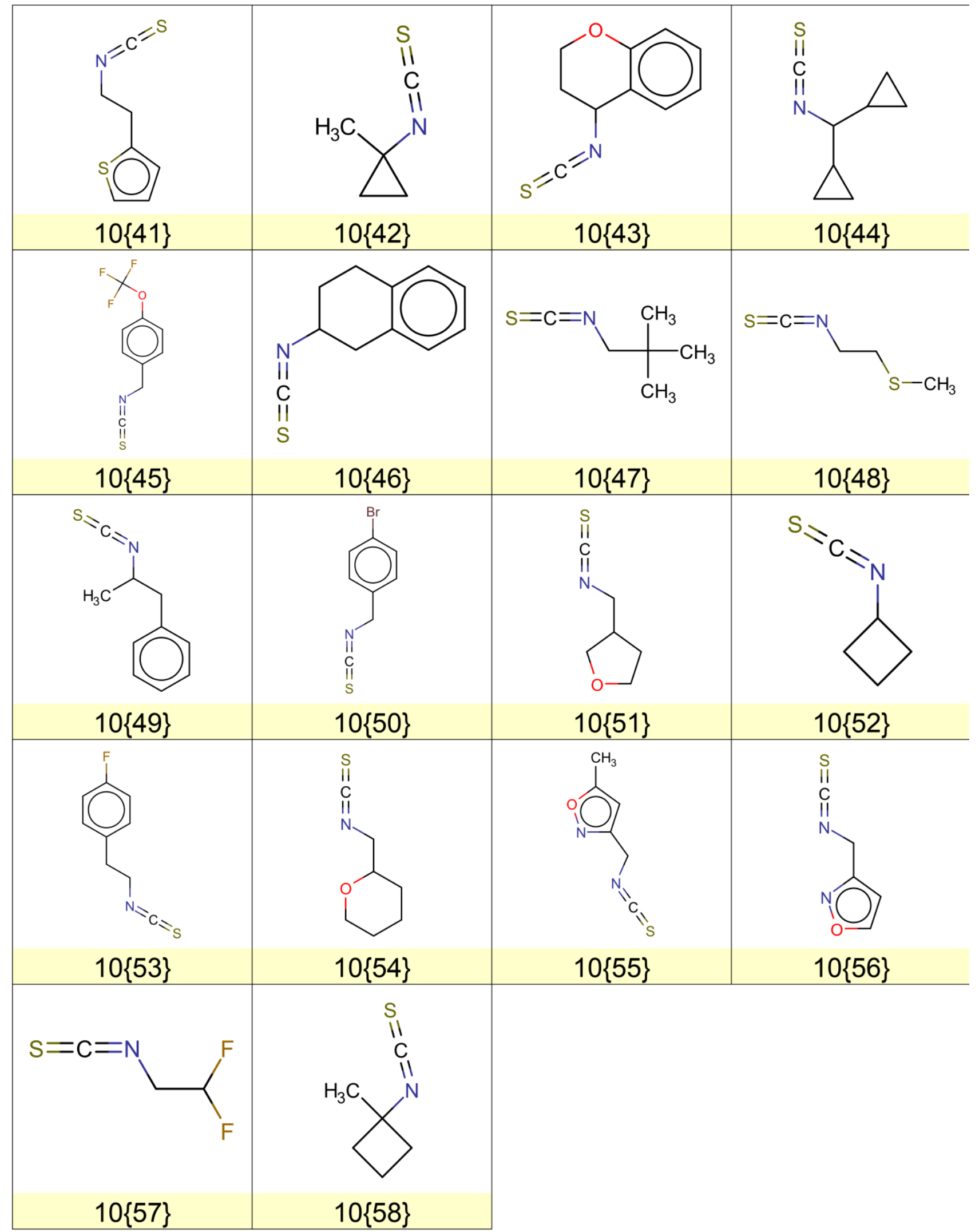


Figure S2. Structures of the reagents $11\{1-511\}$

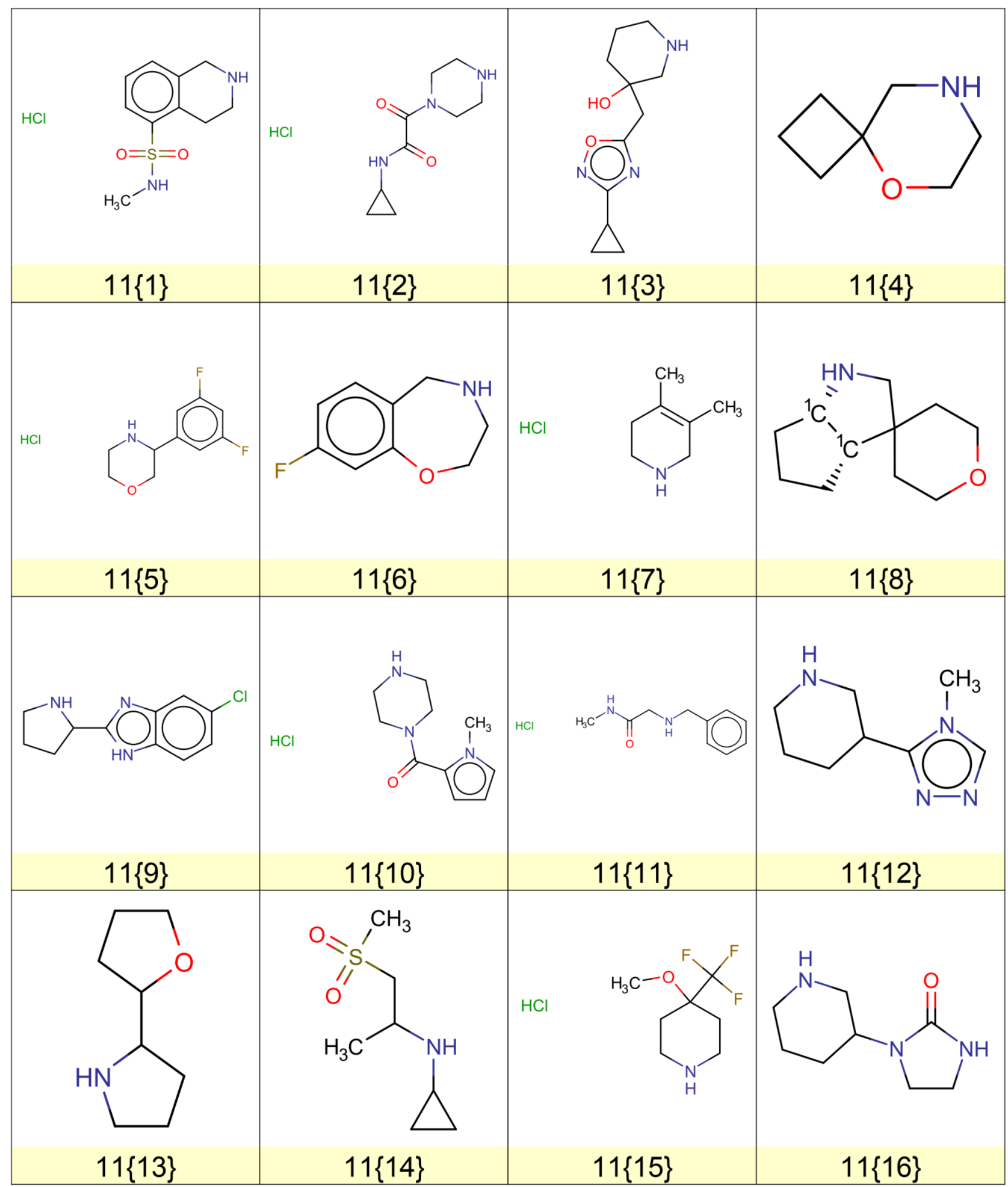




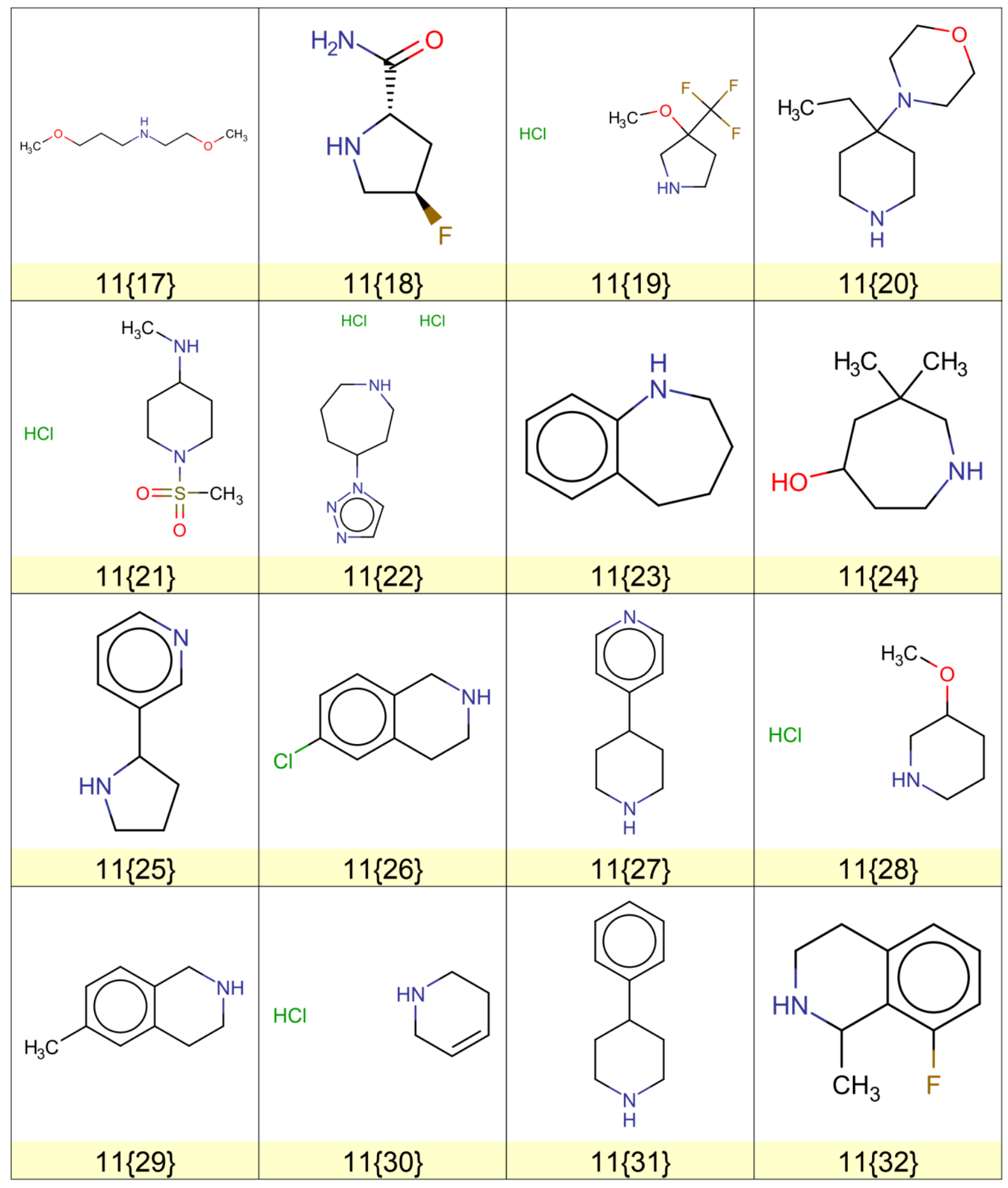




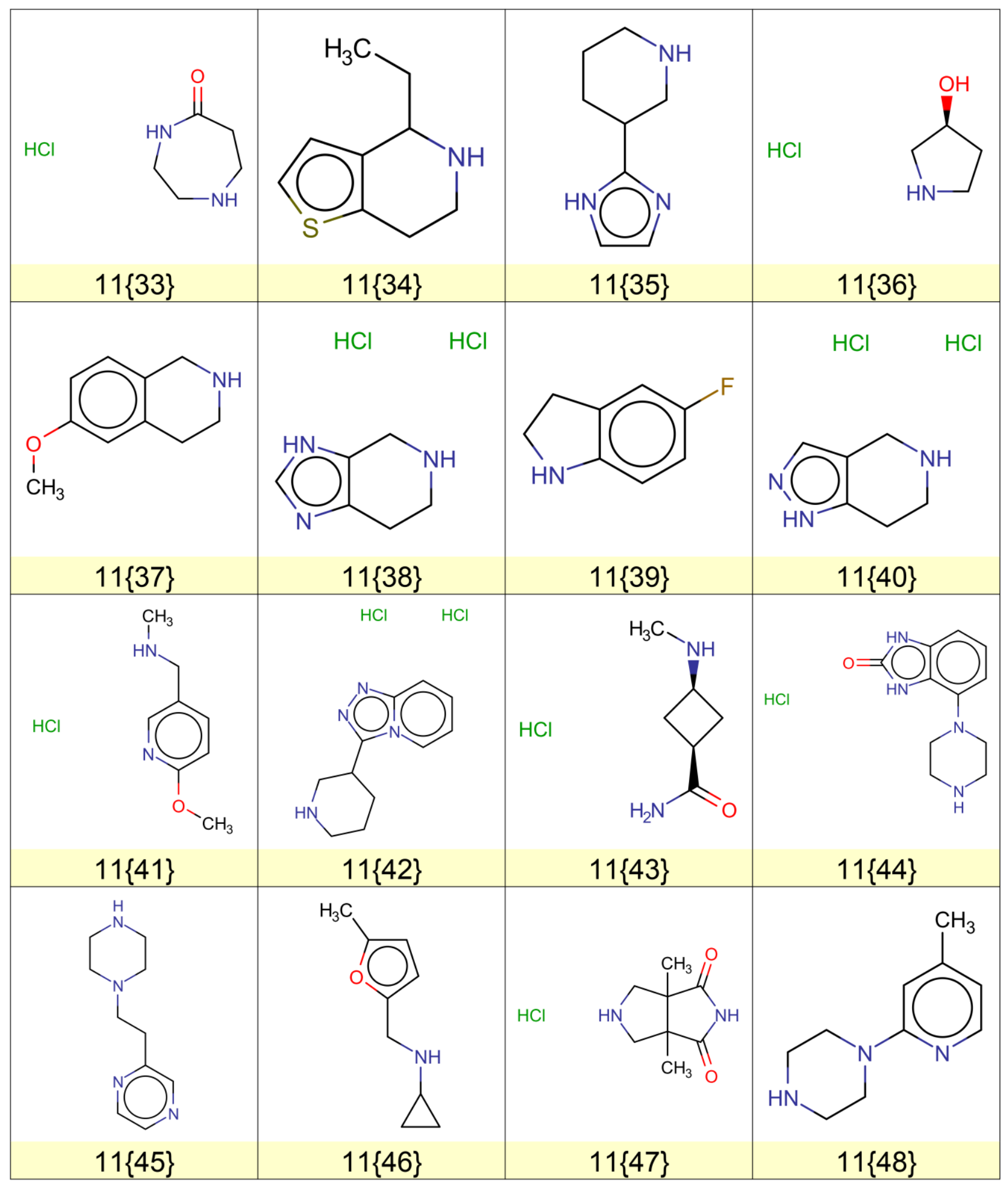




(1)




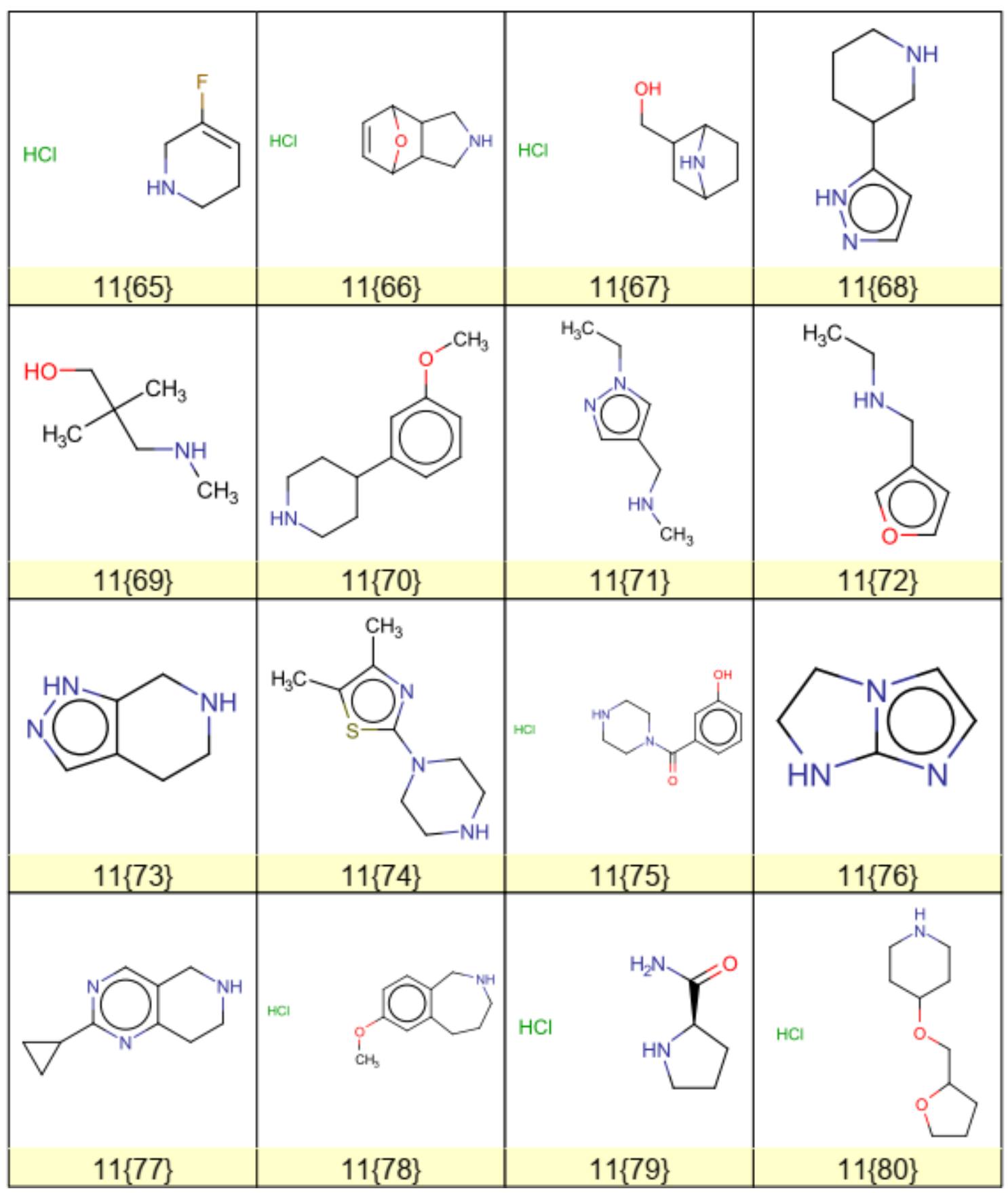




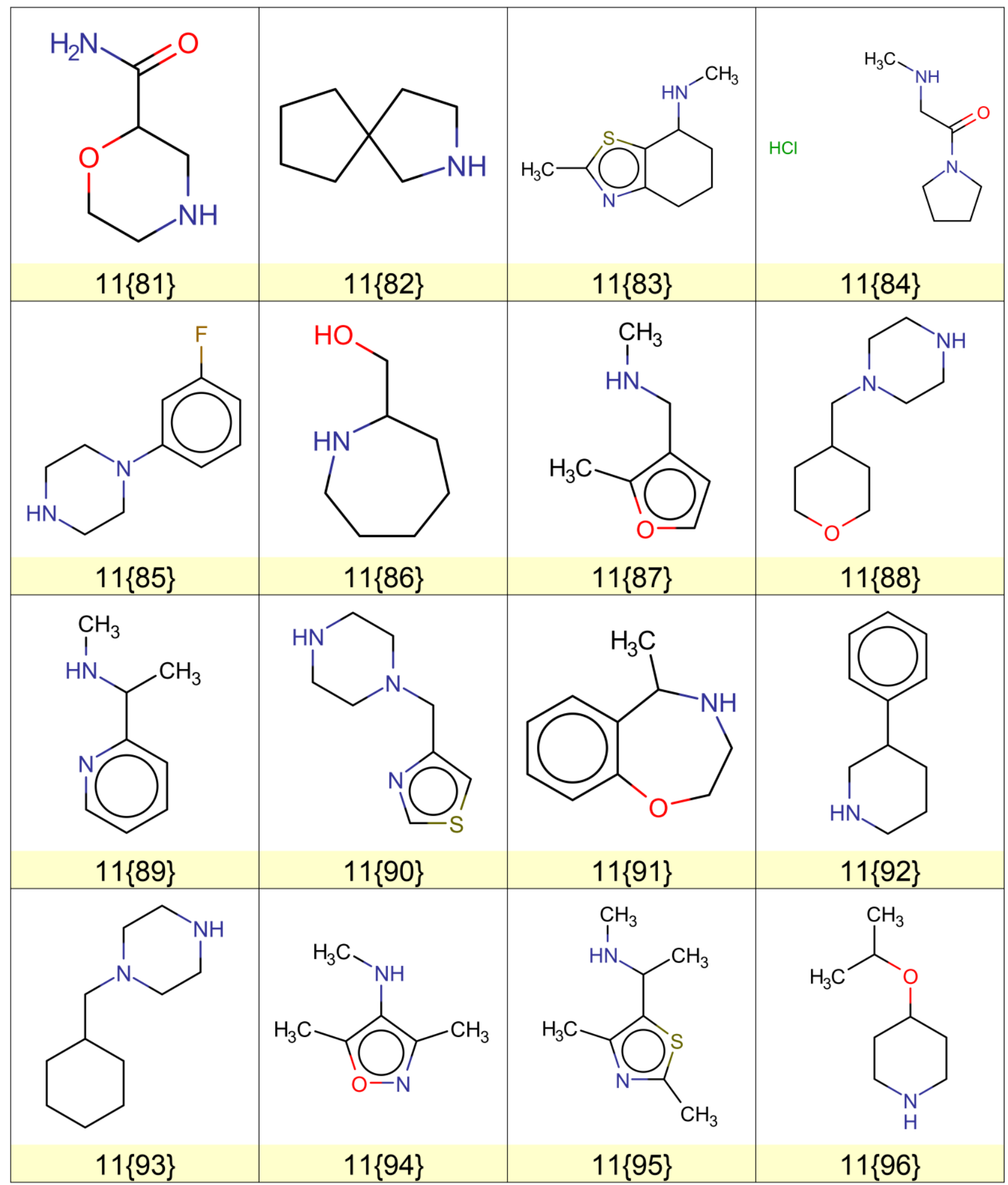




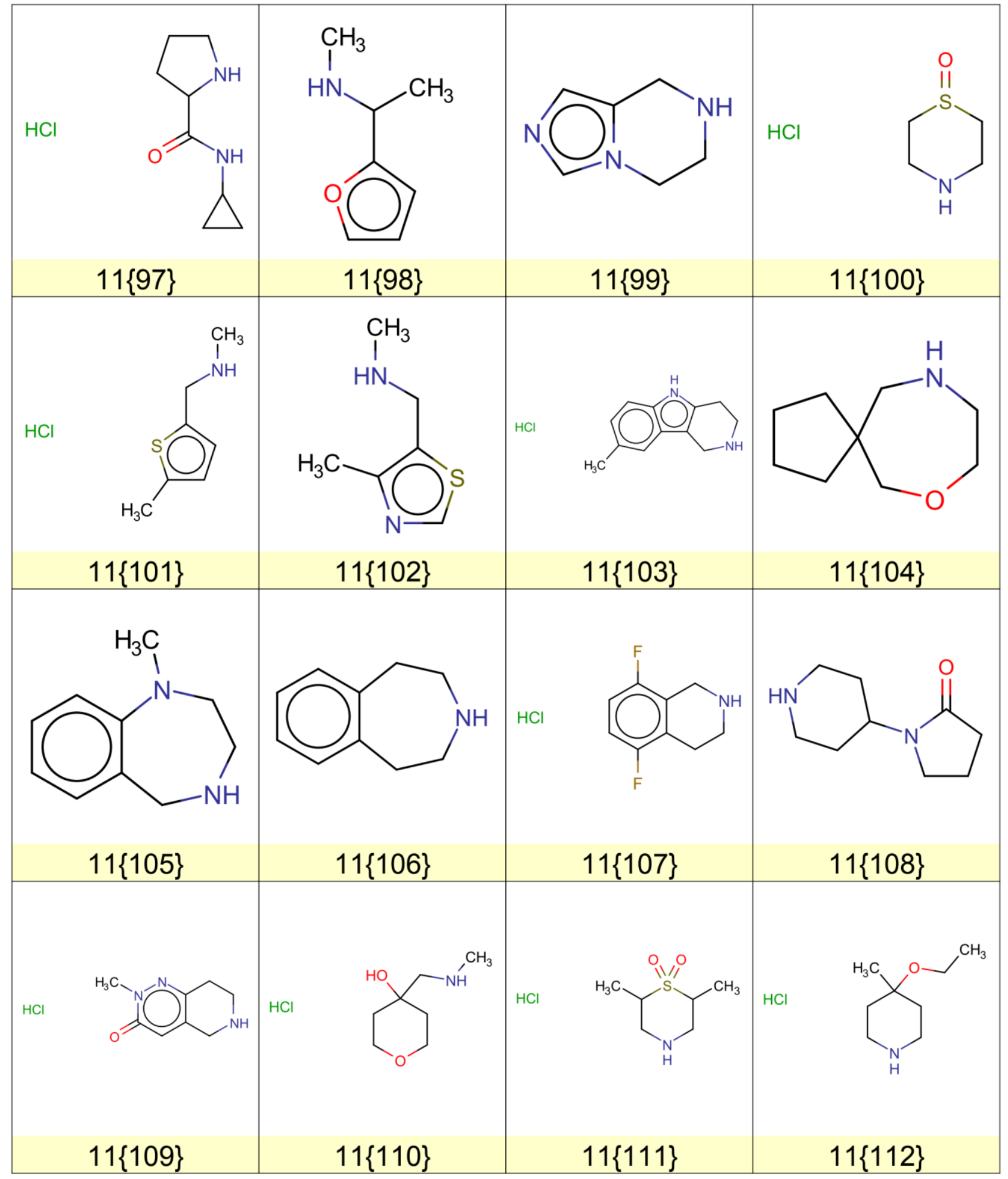




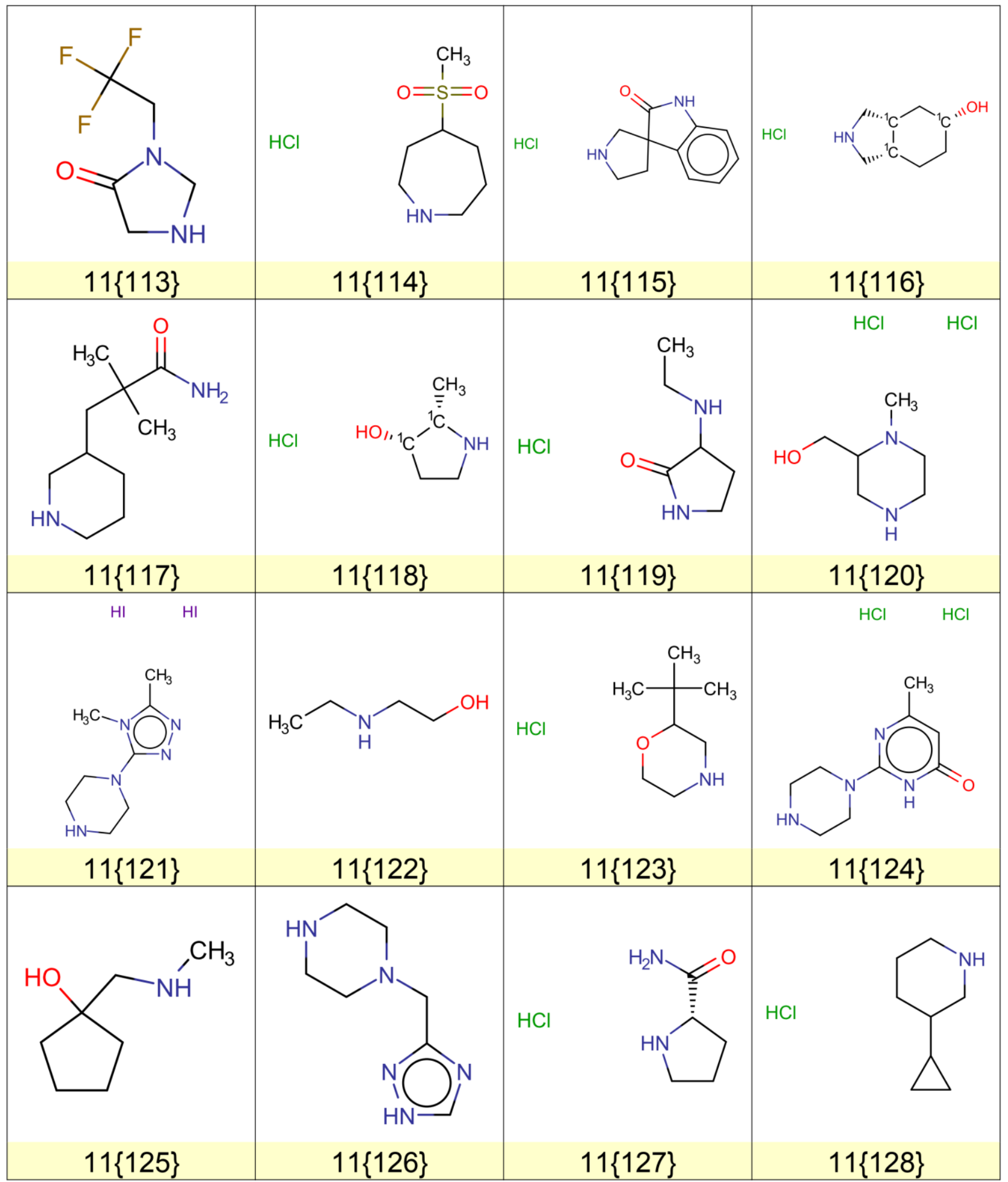




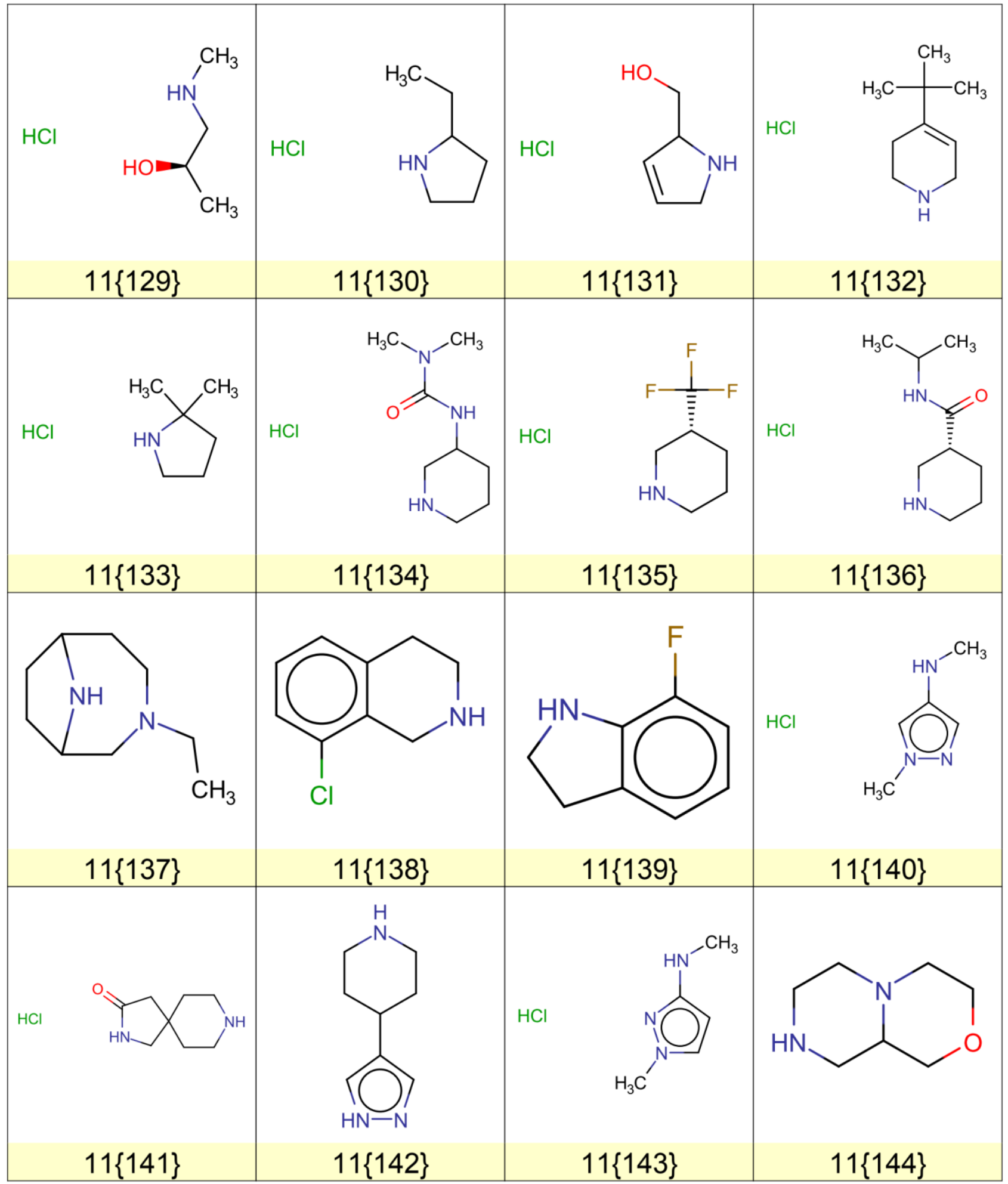




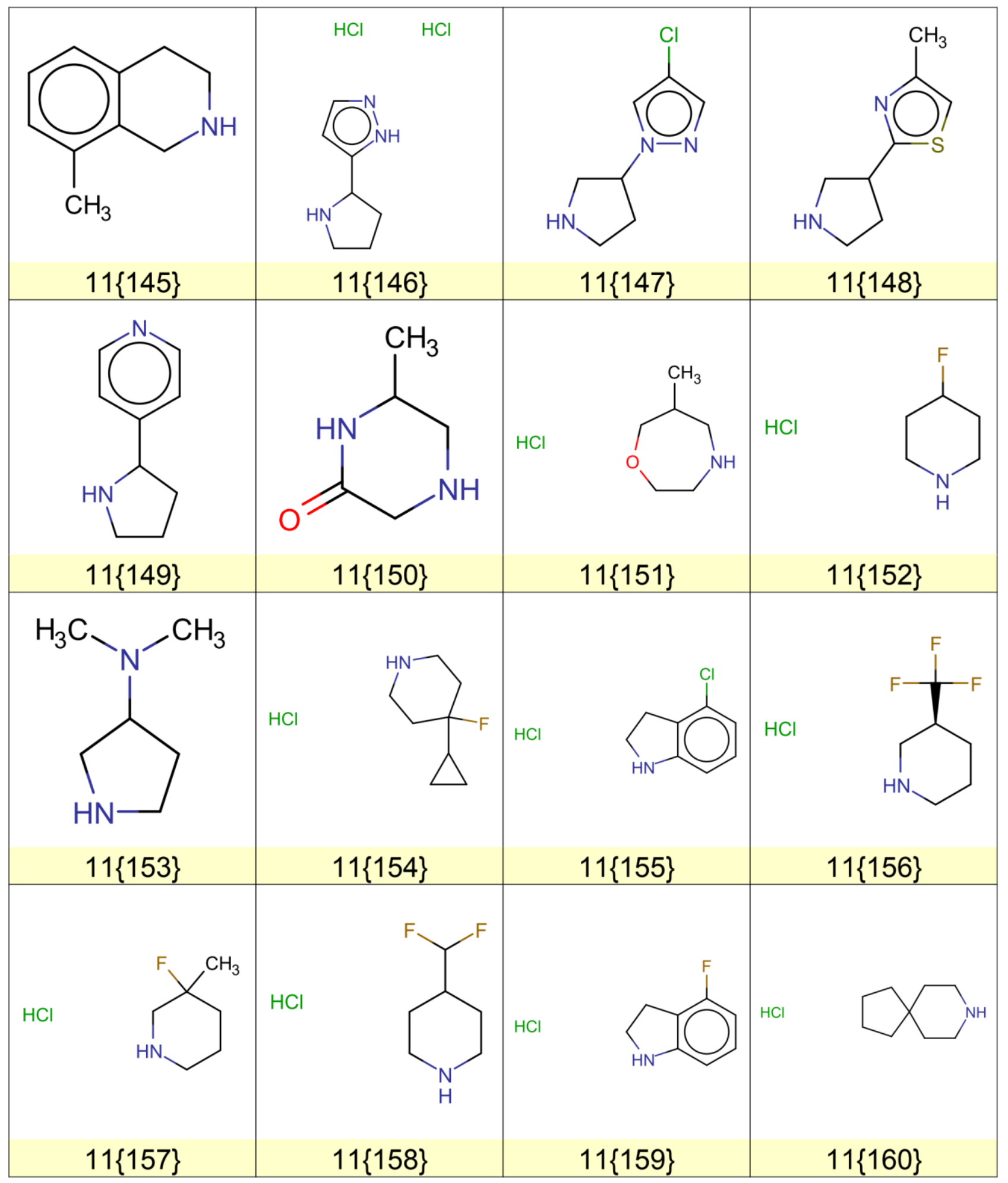




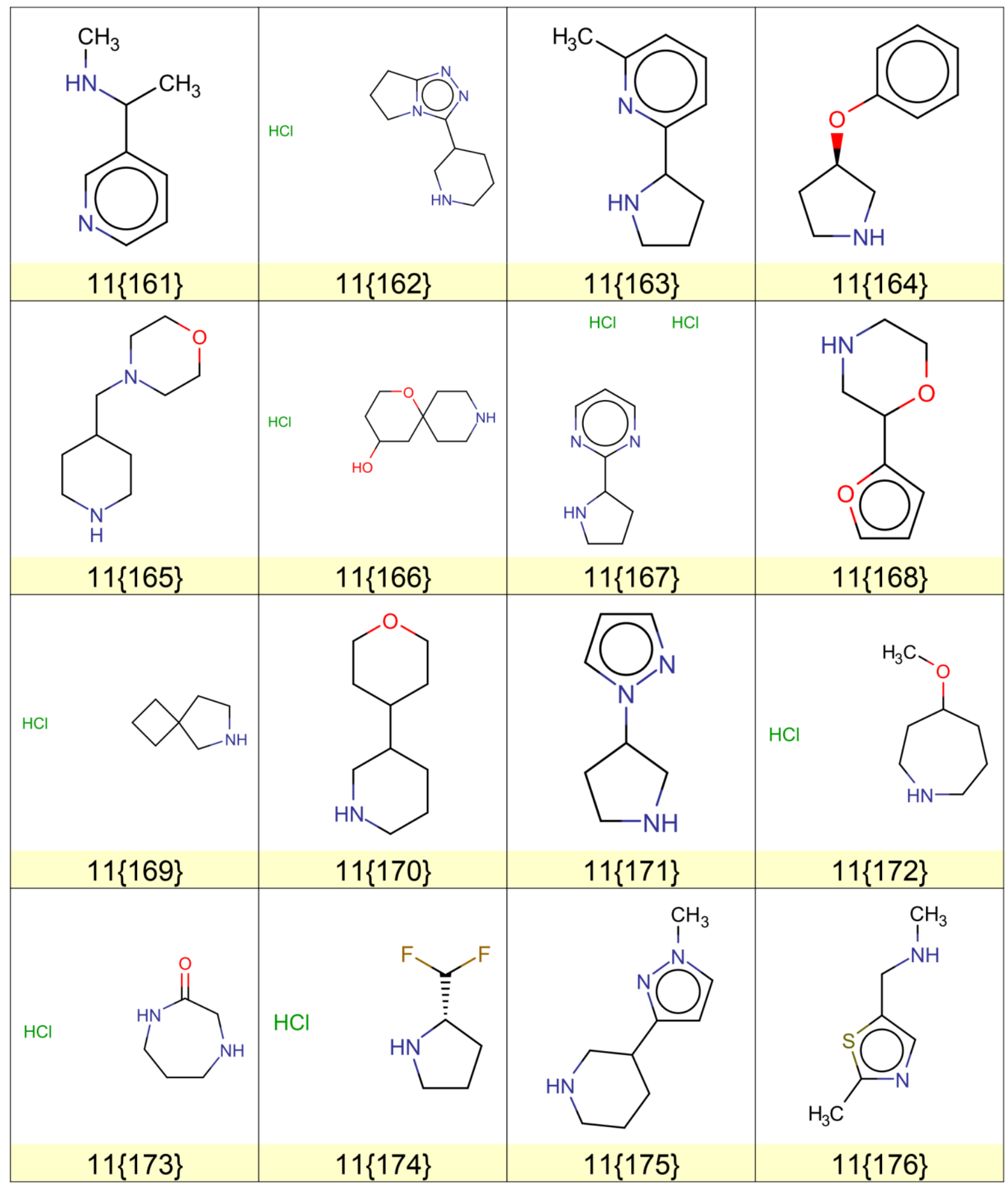




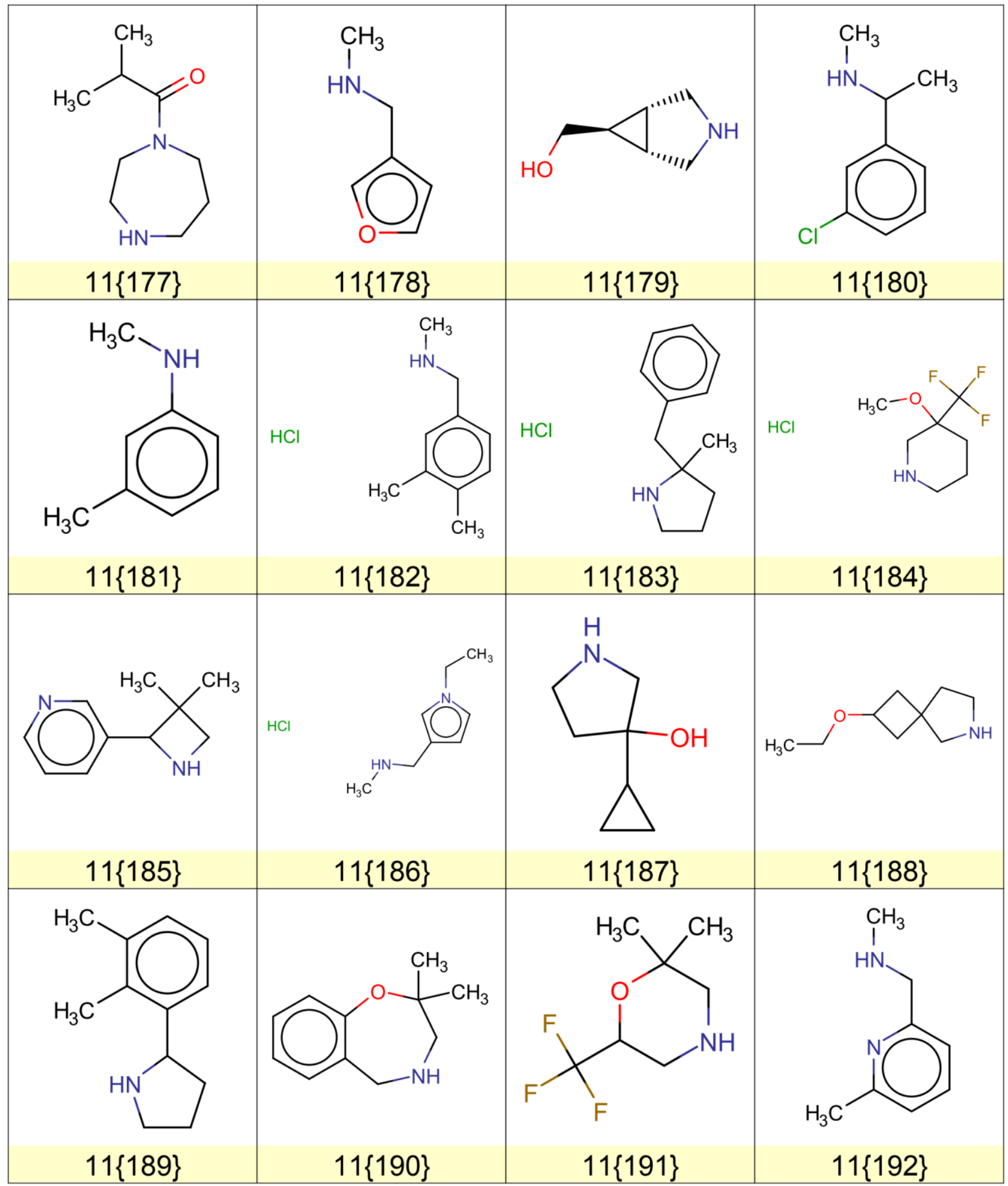




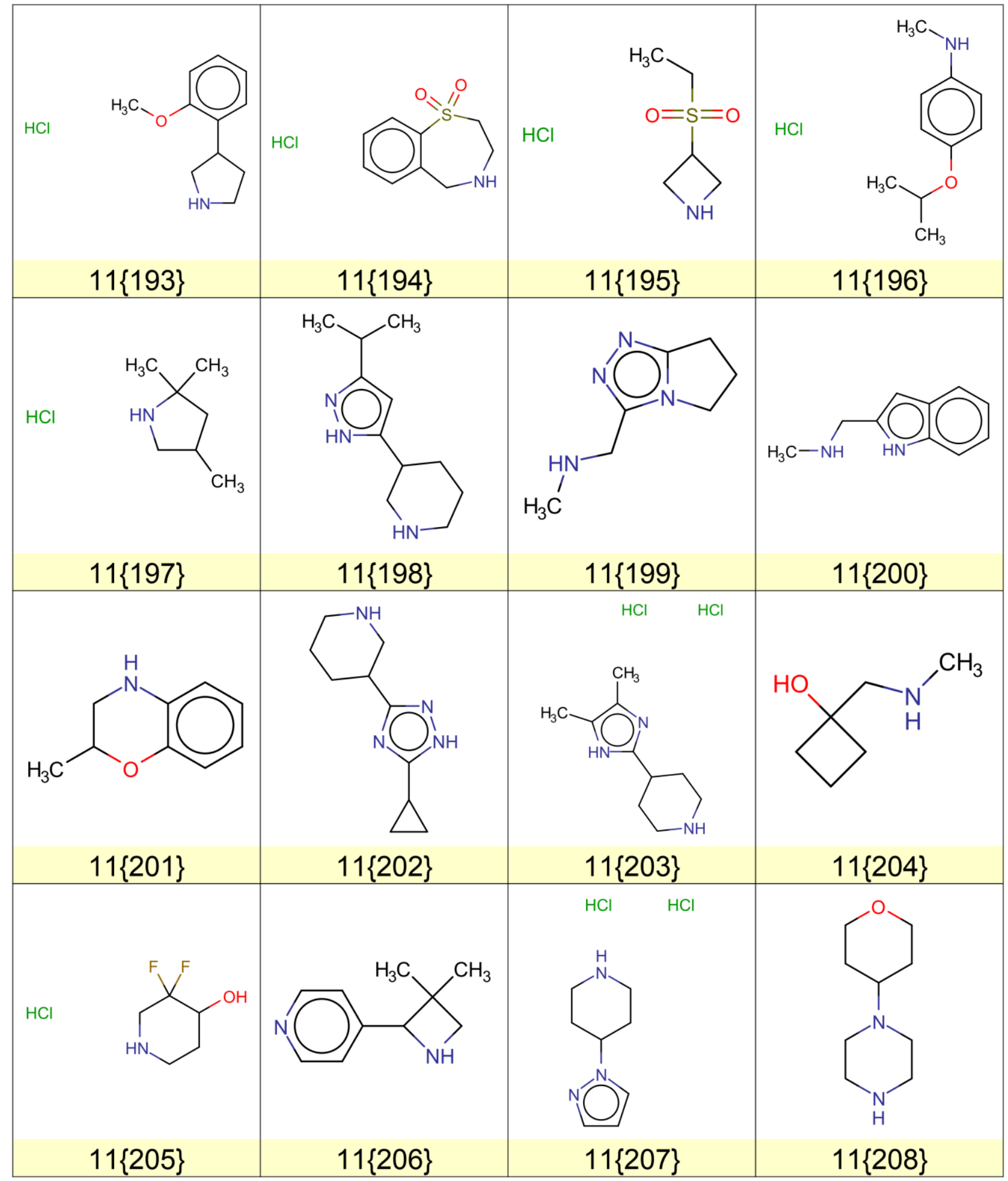




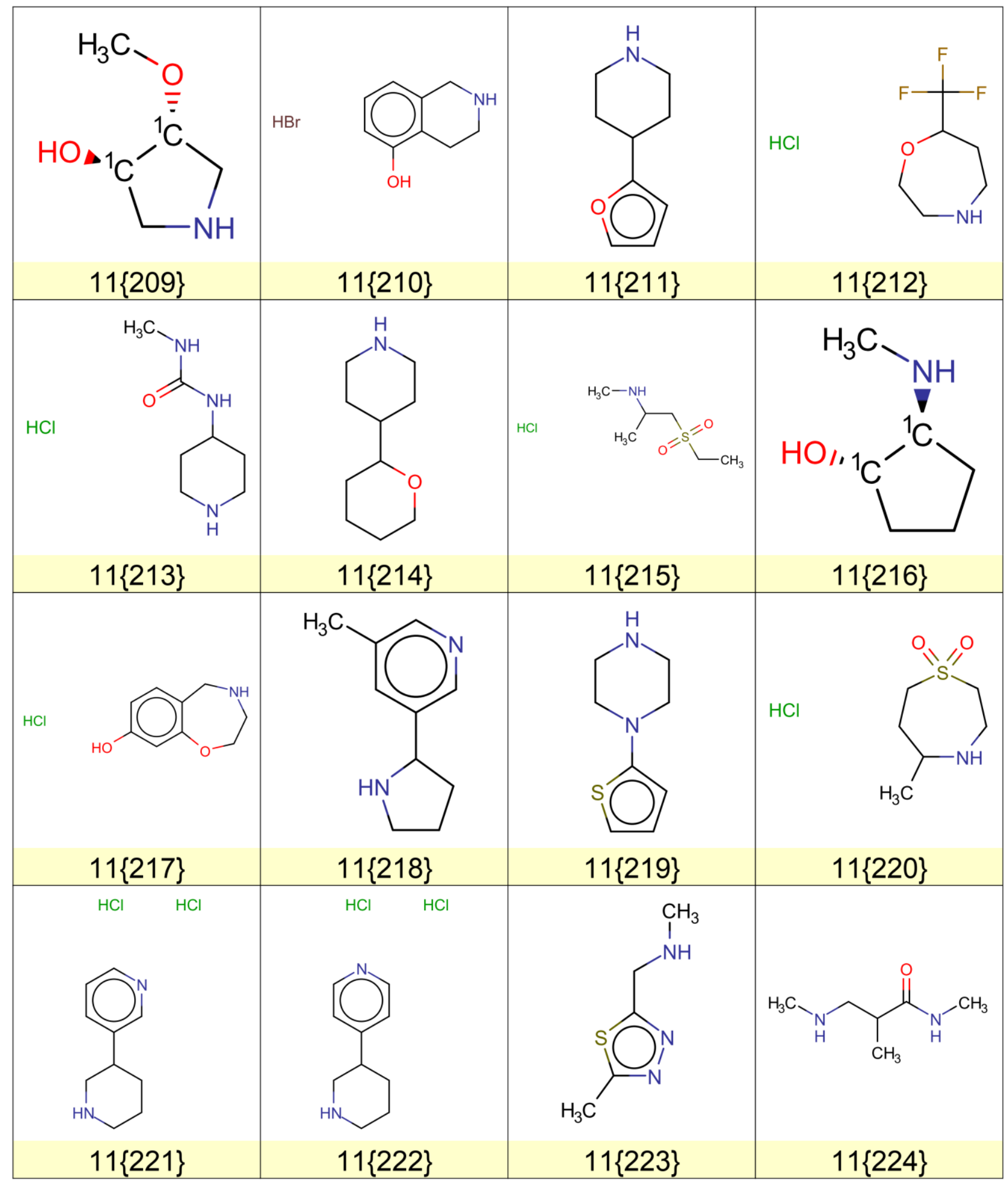




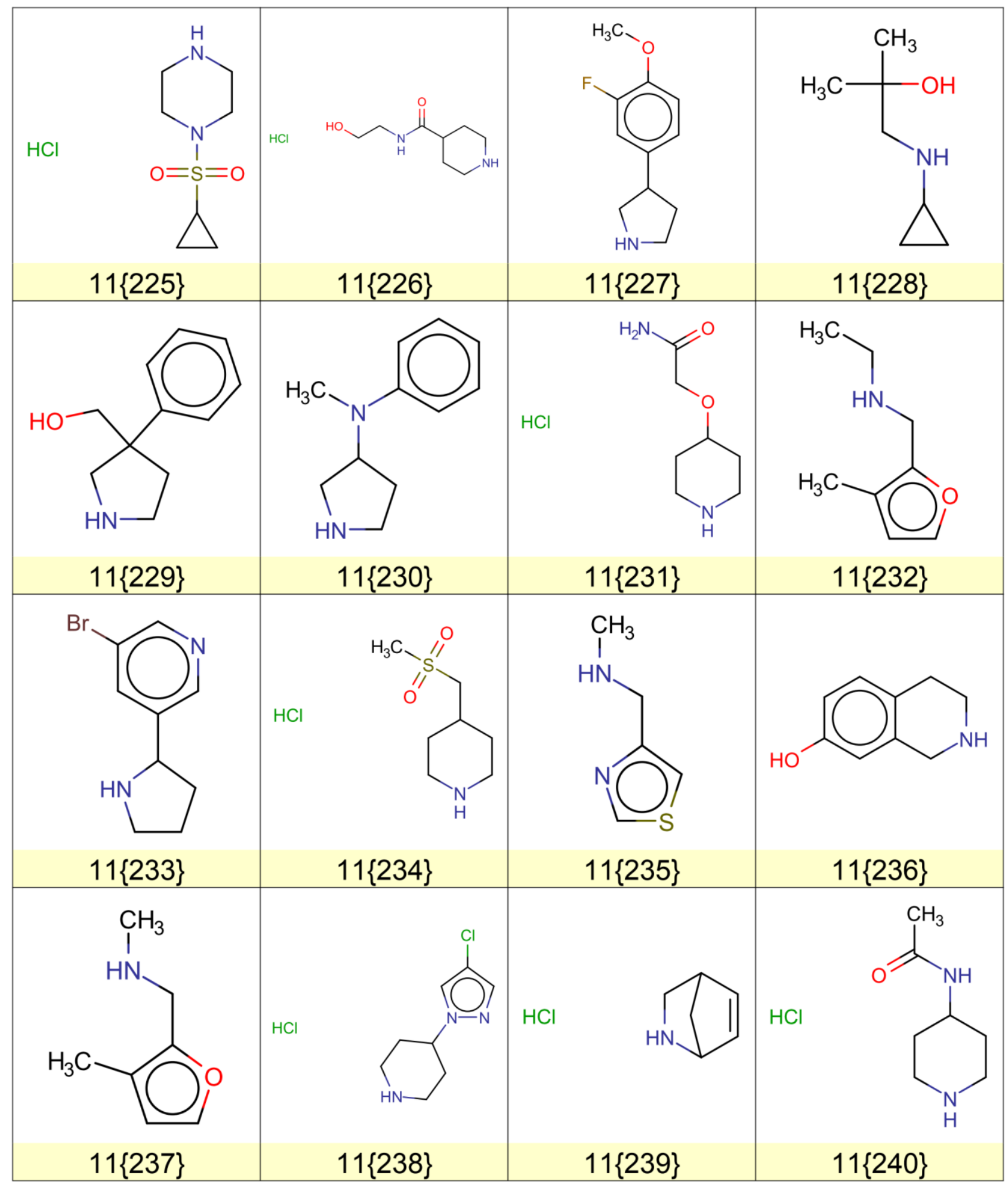




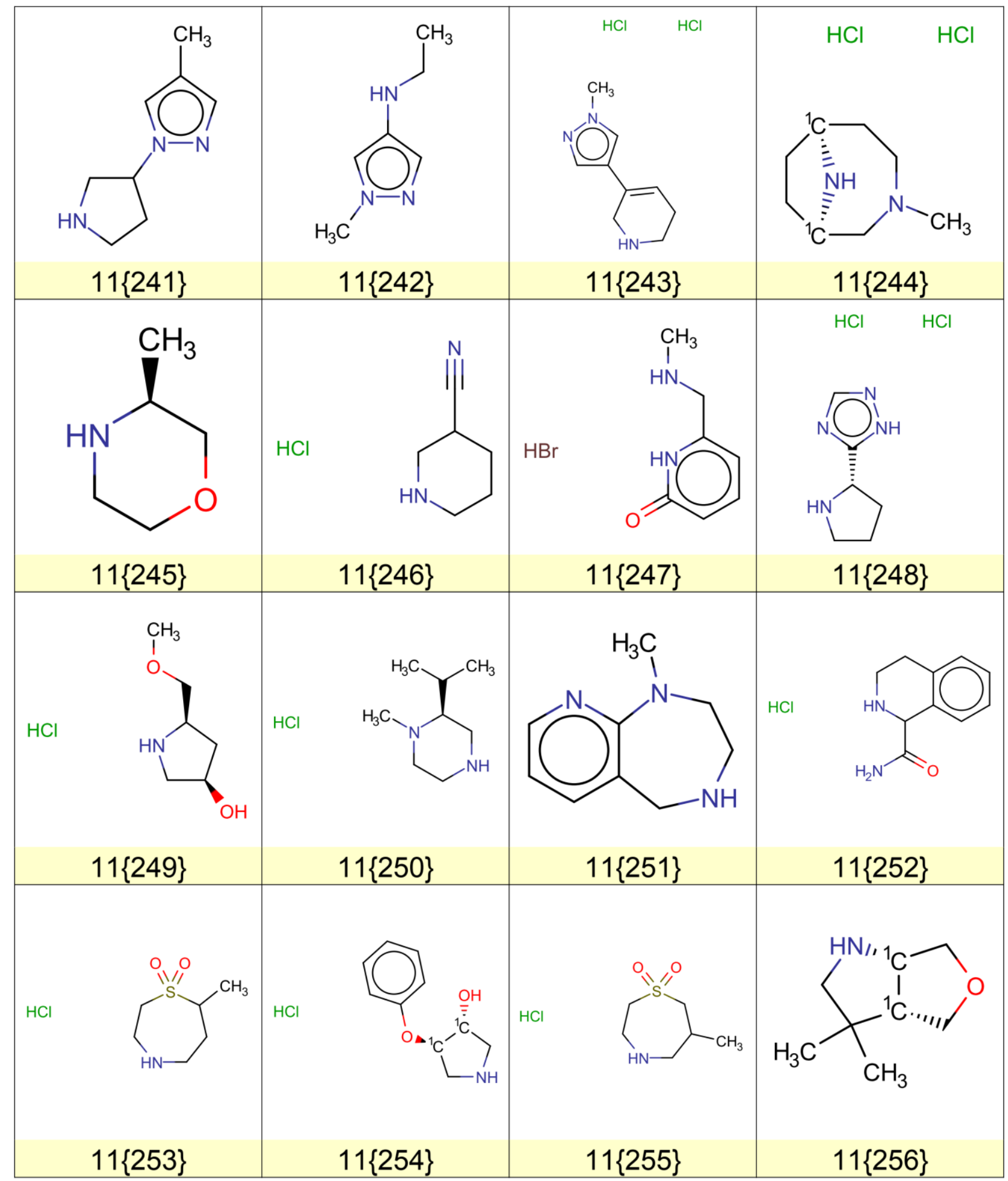




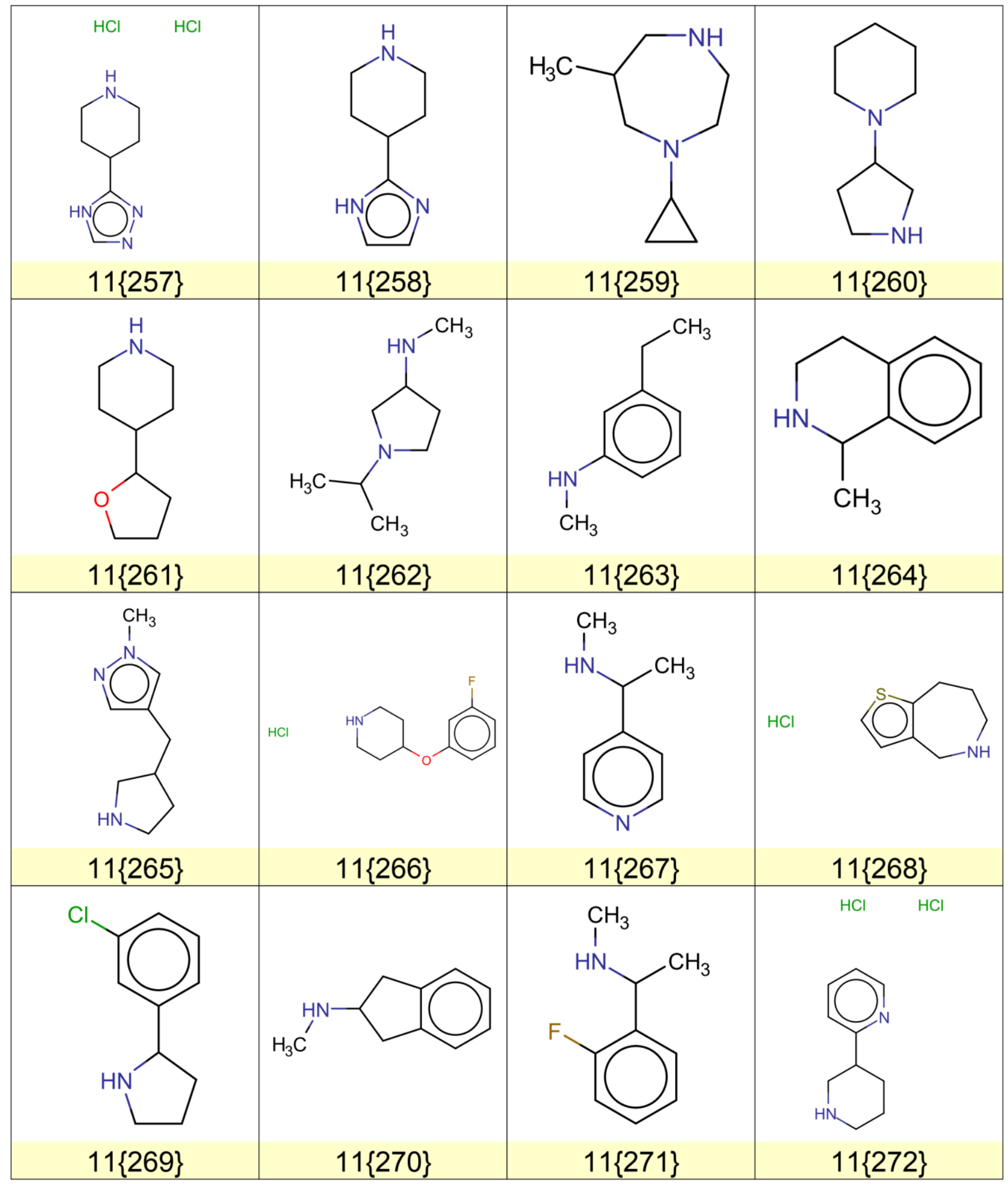




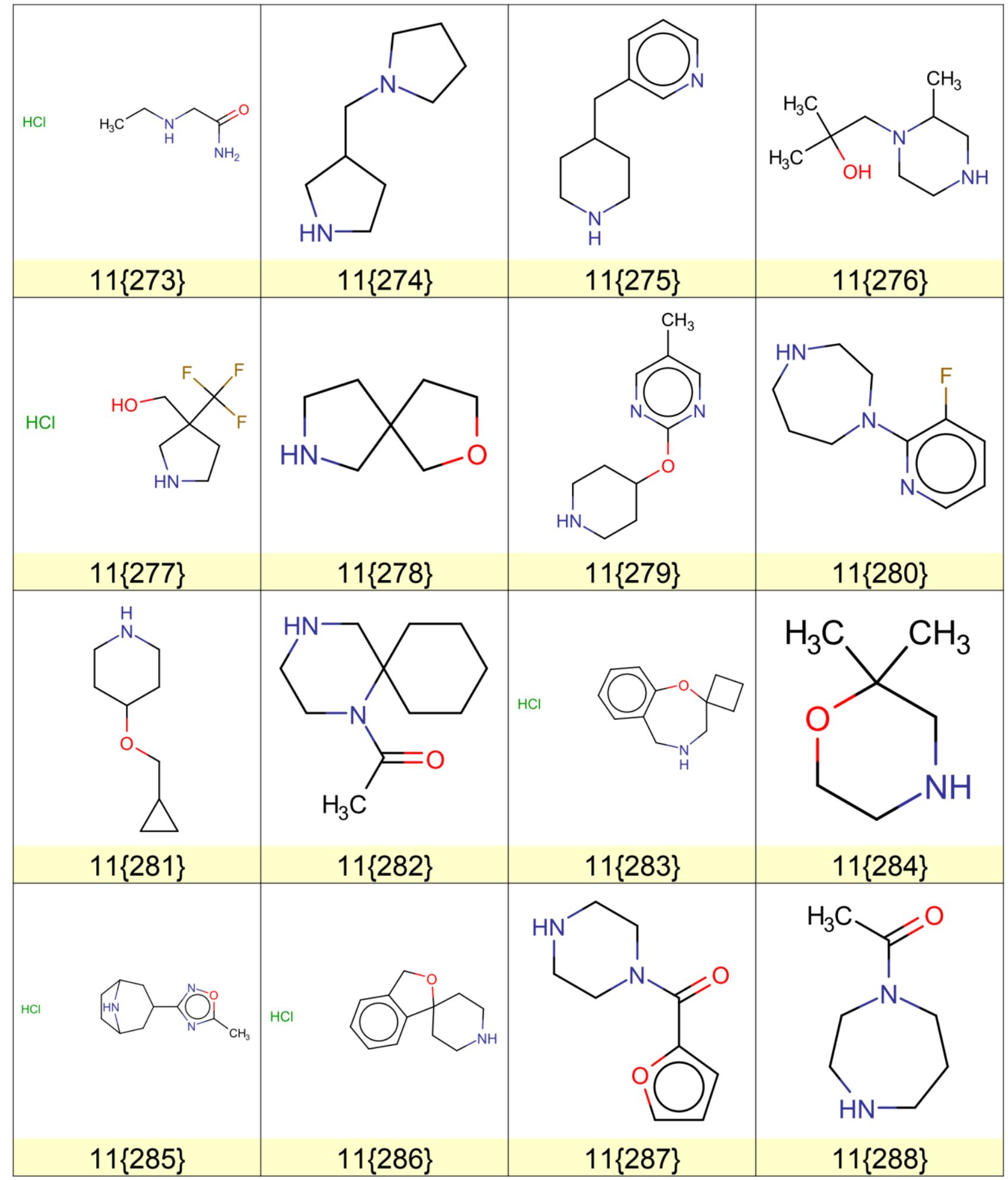




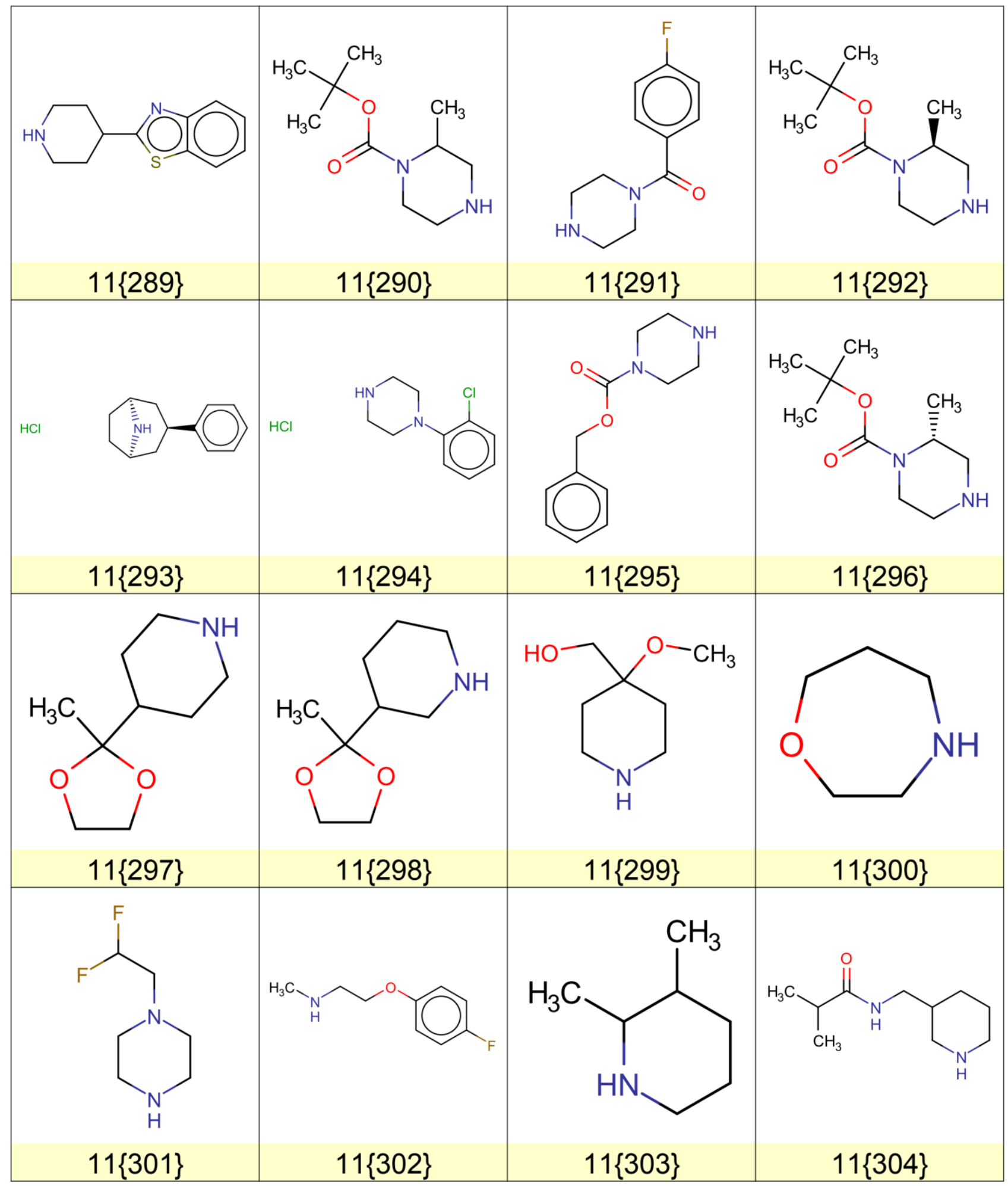




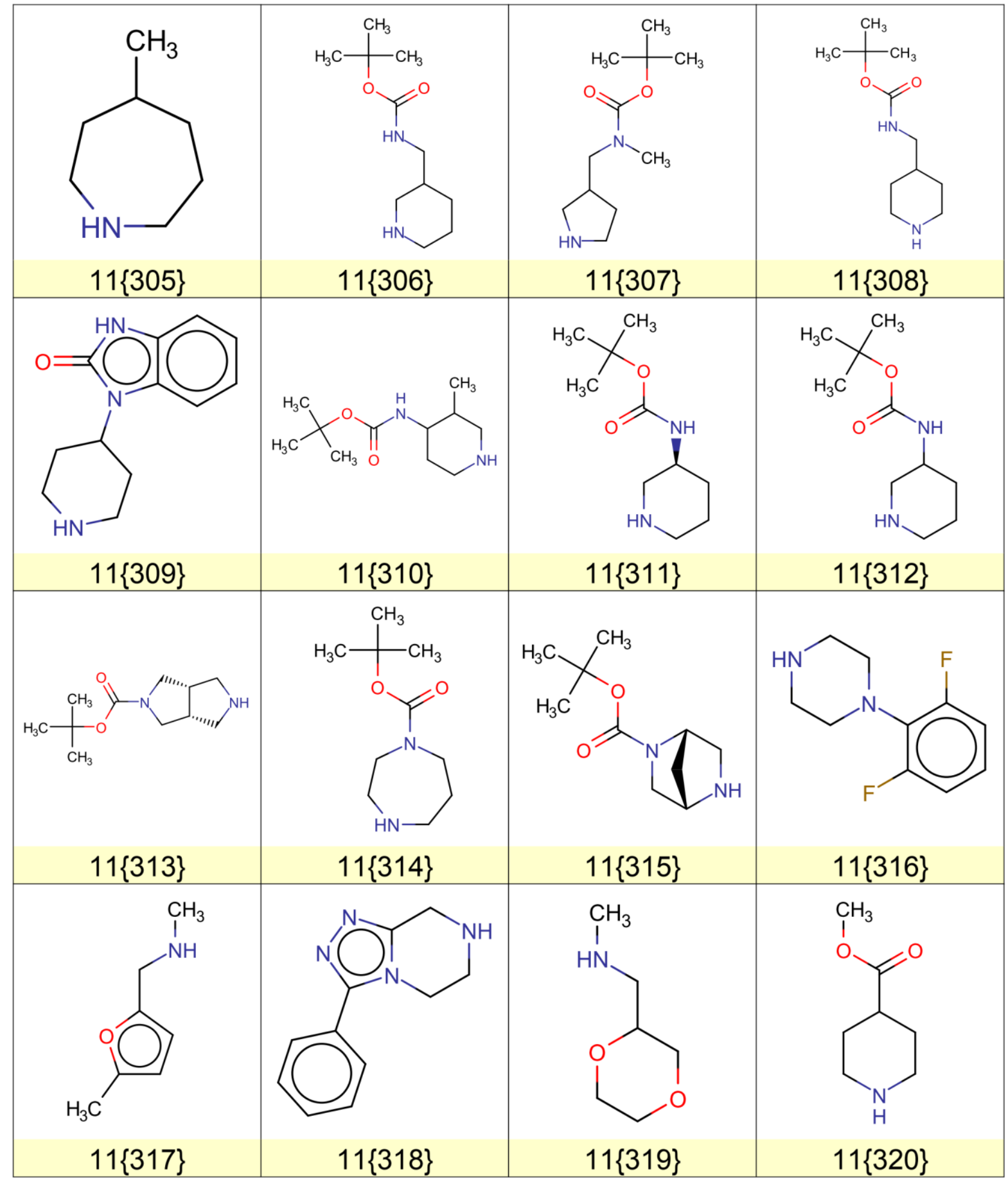




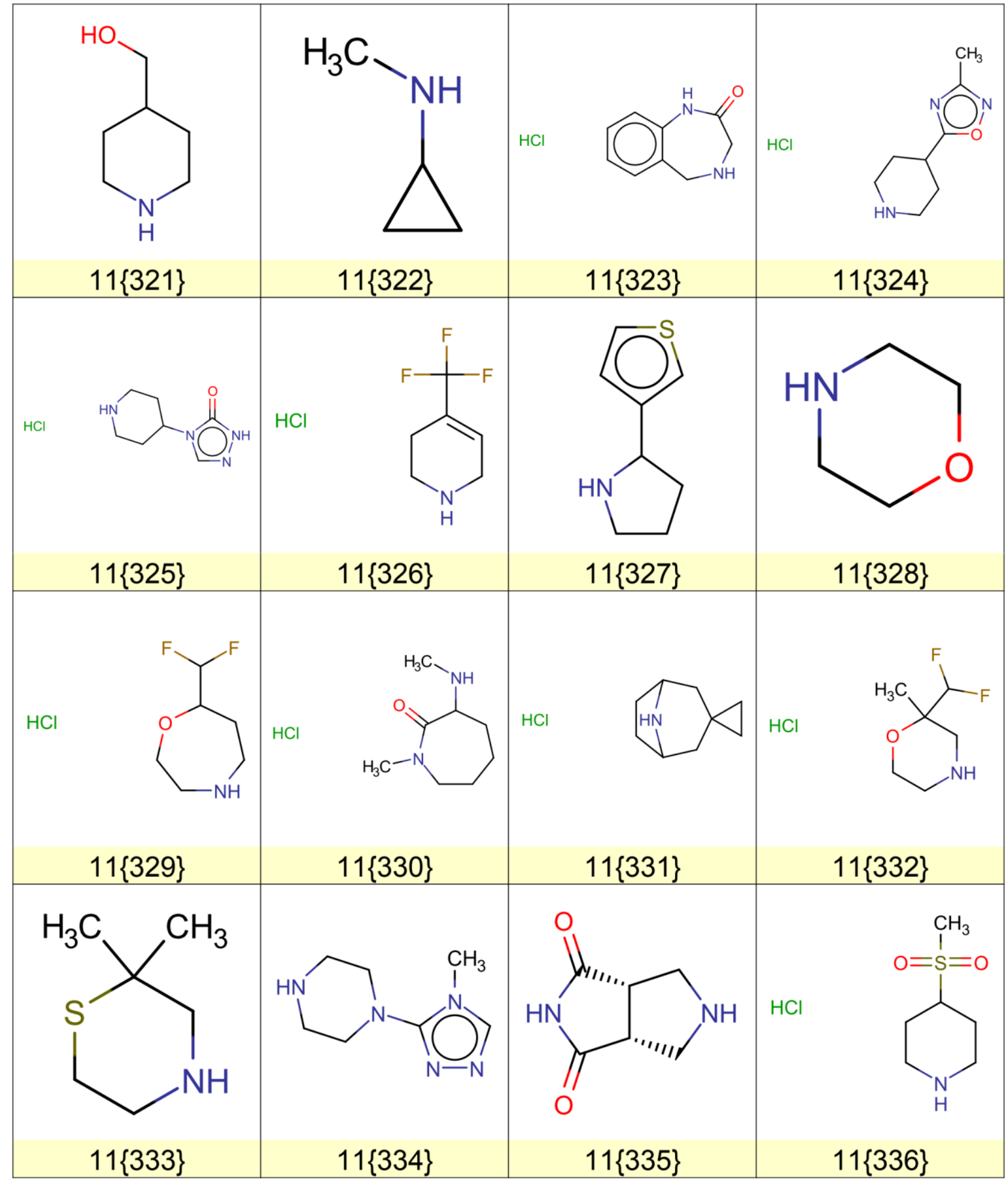




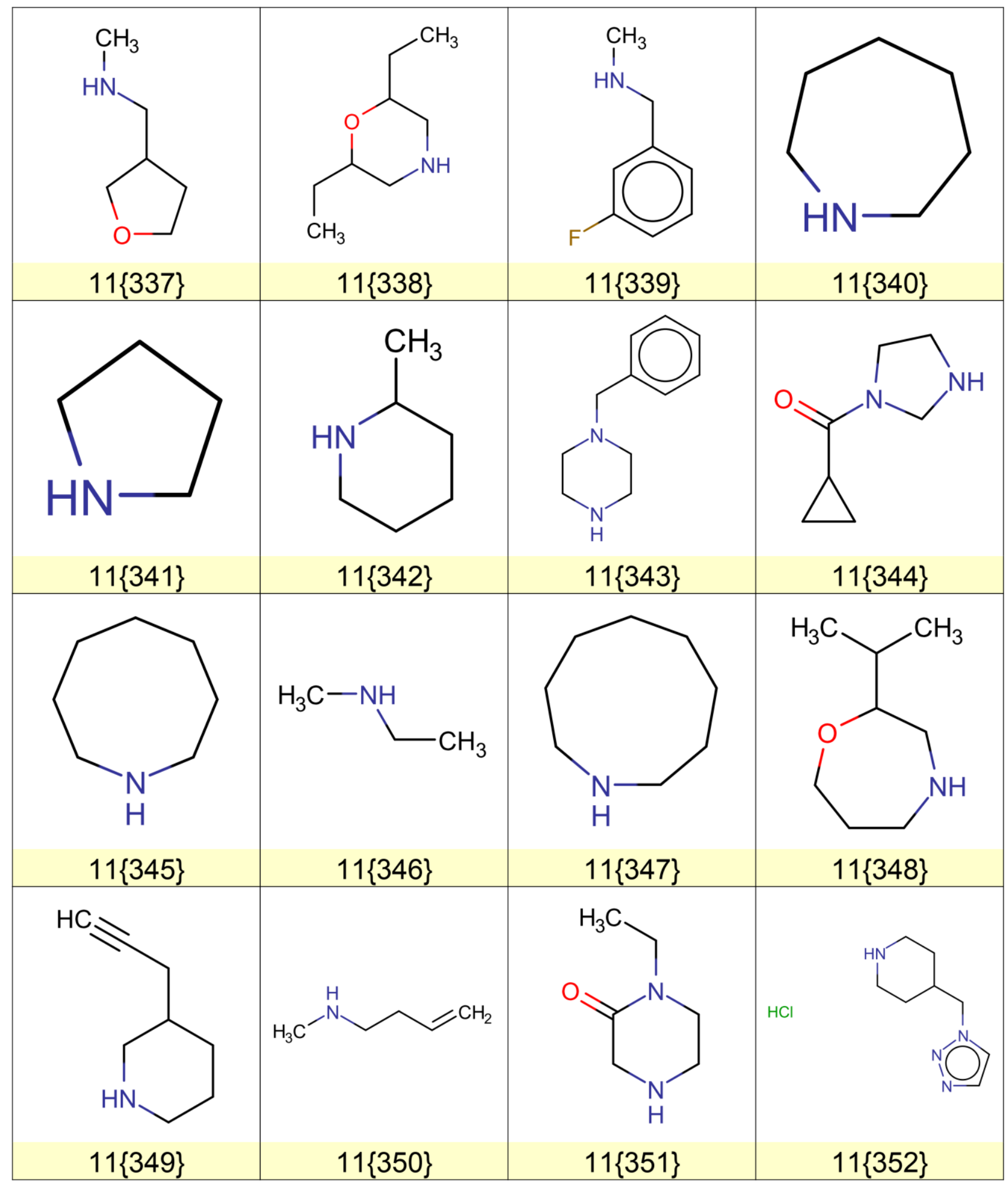




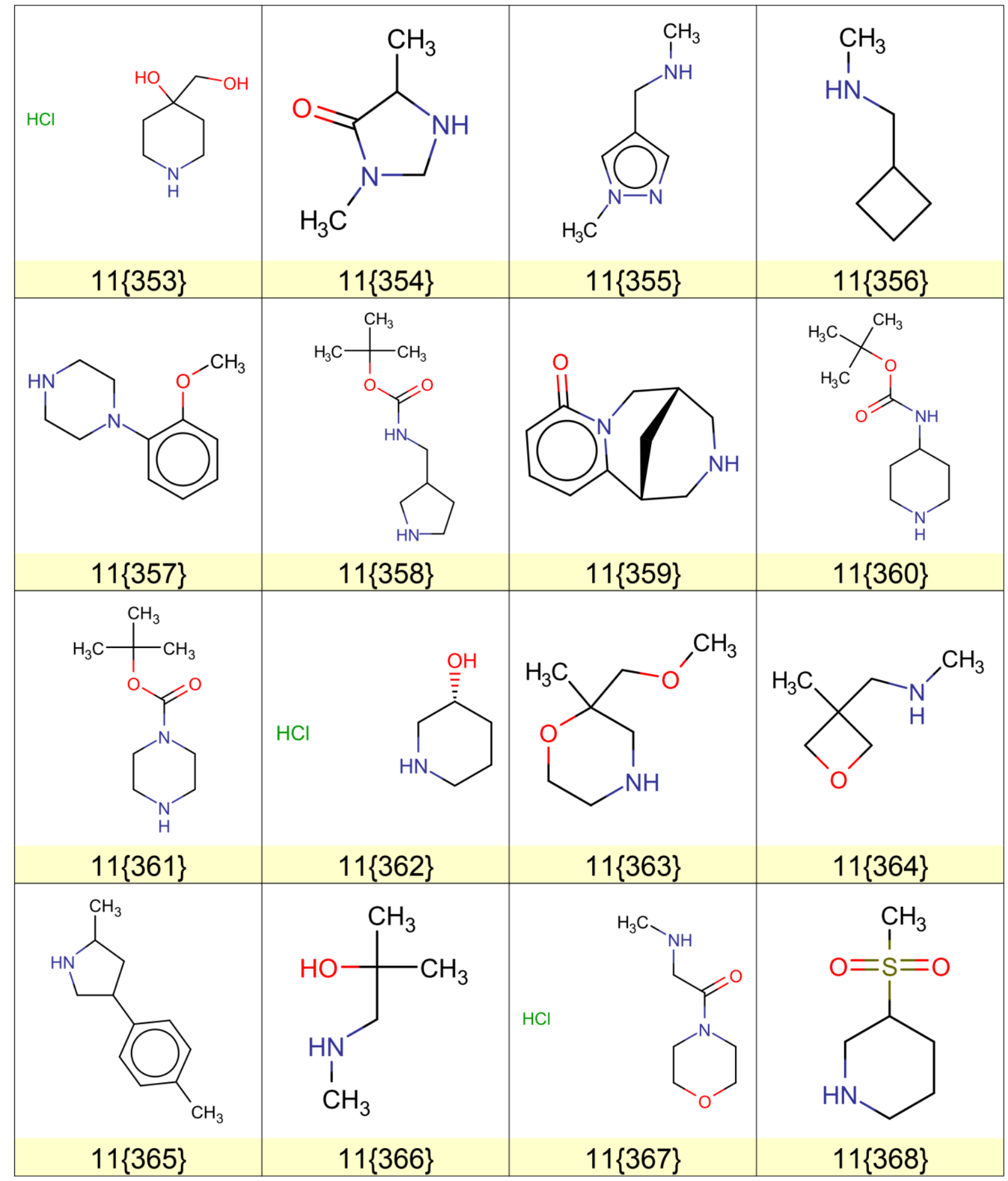




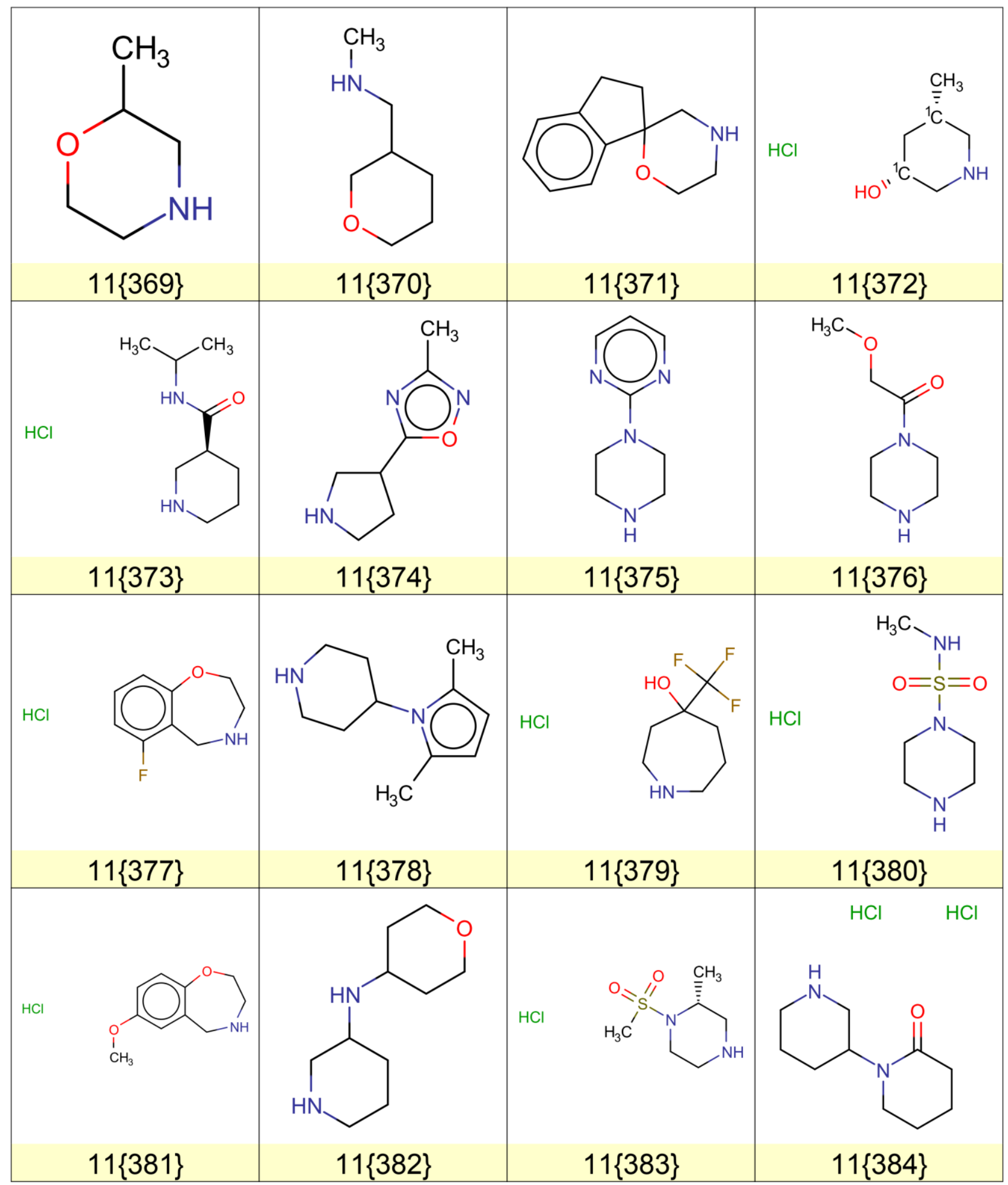




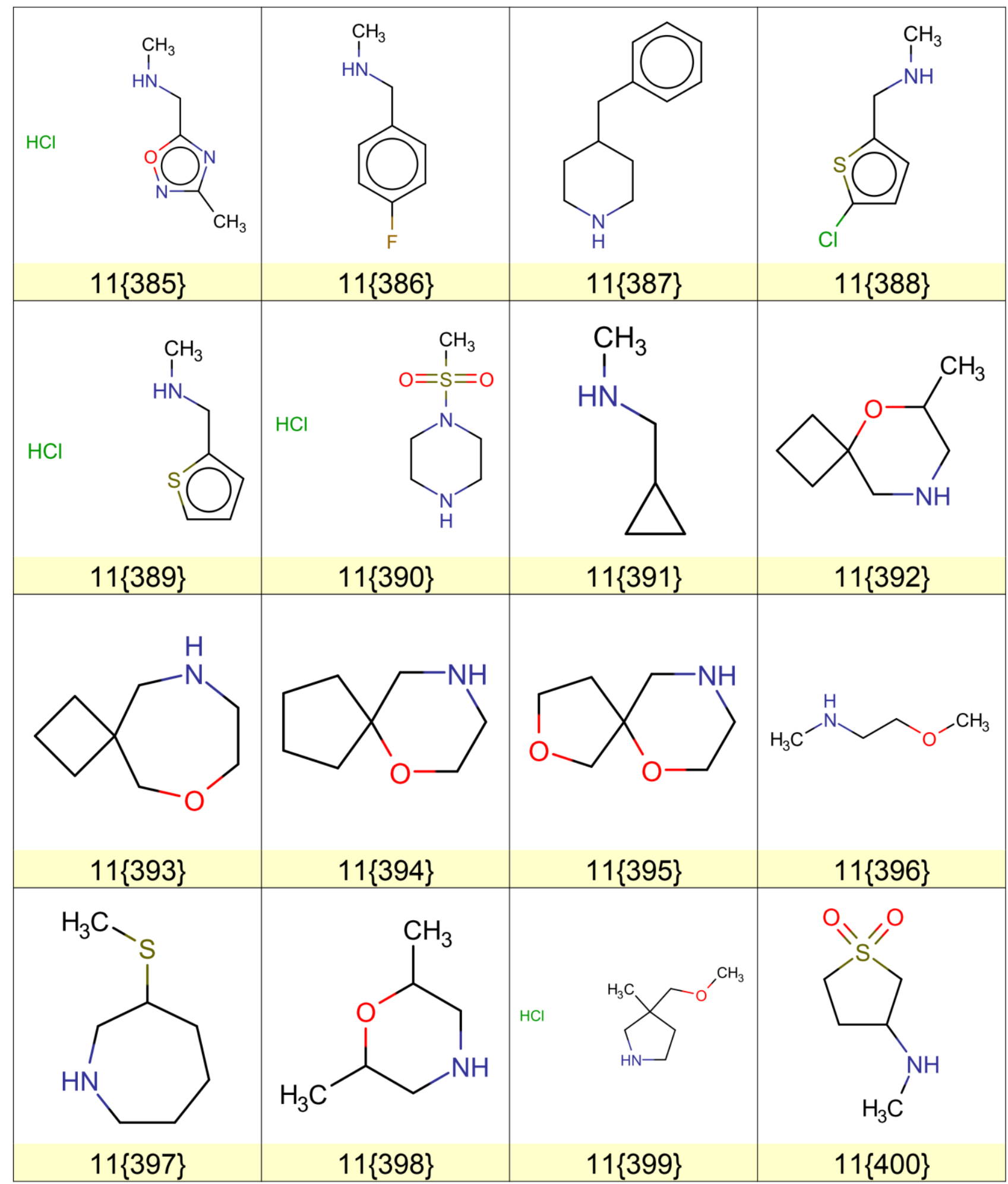




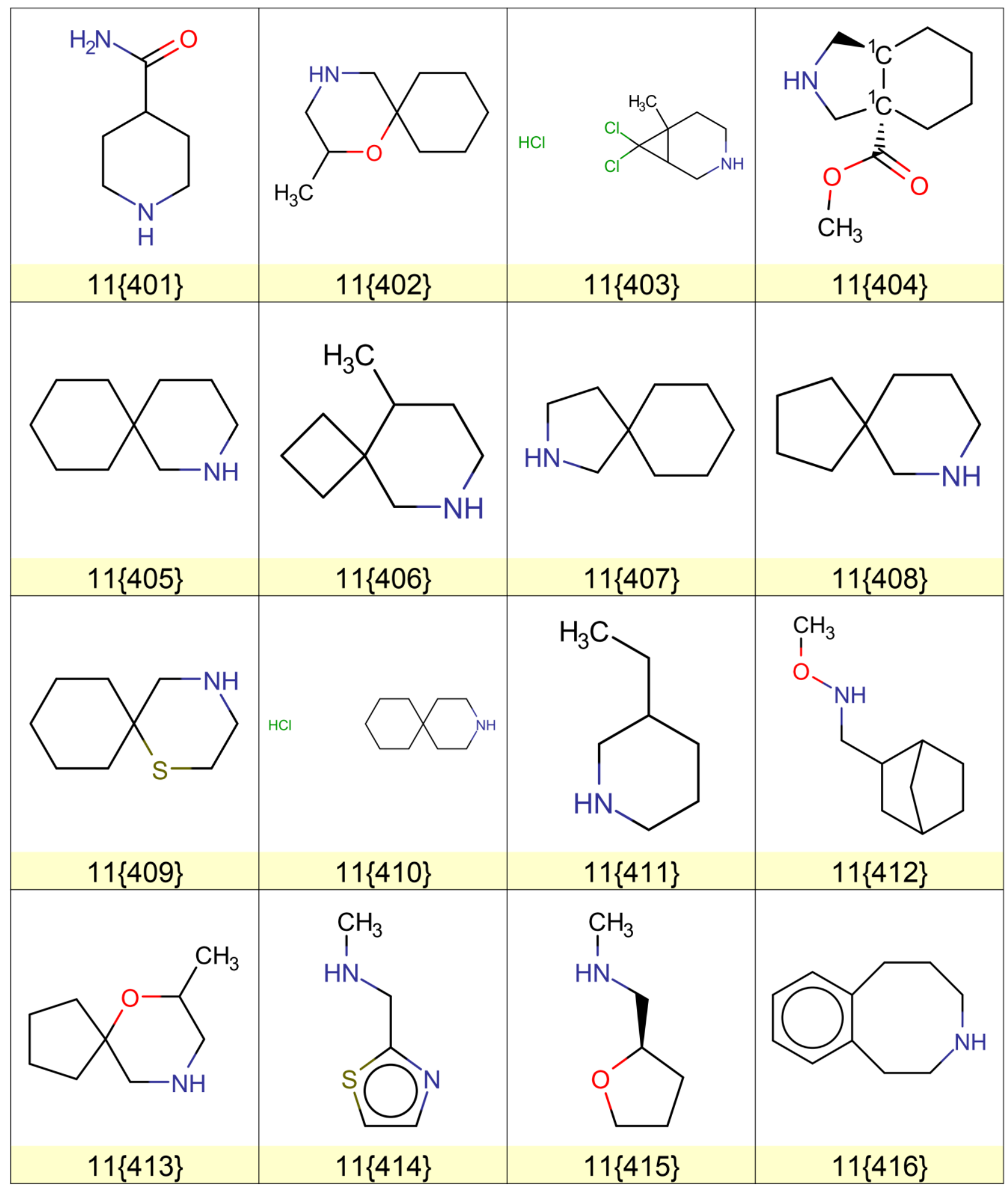




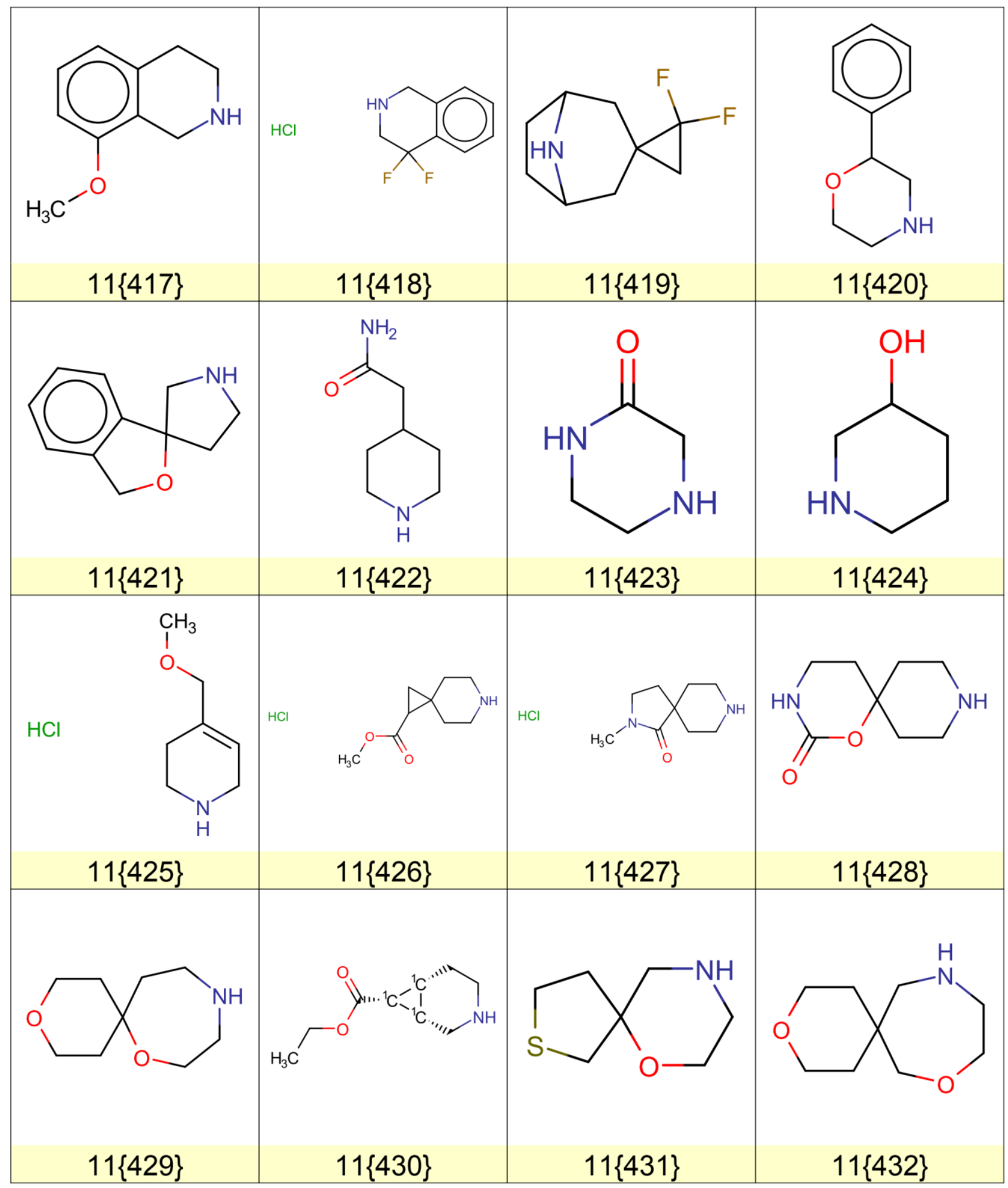




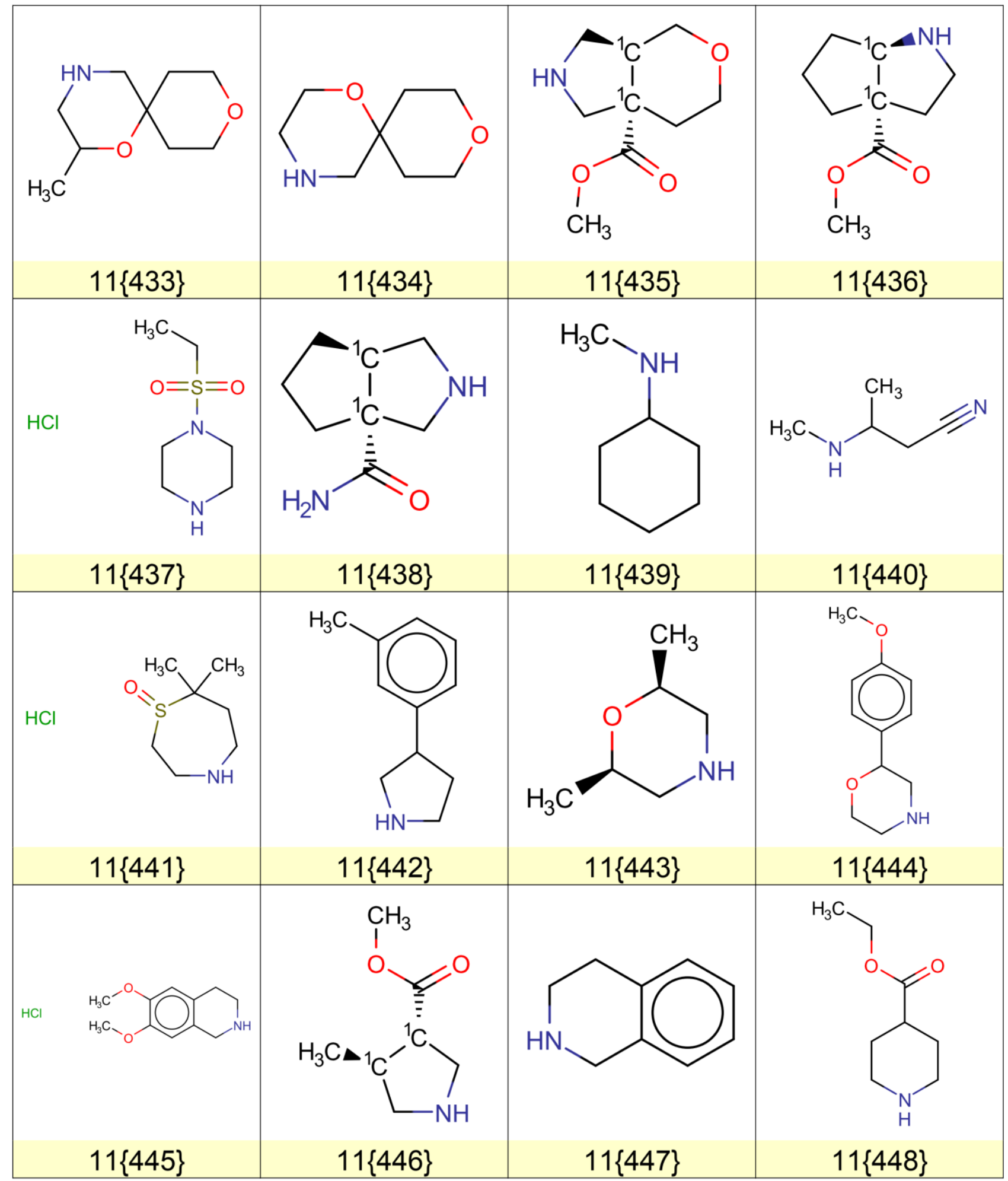




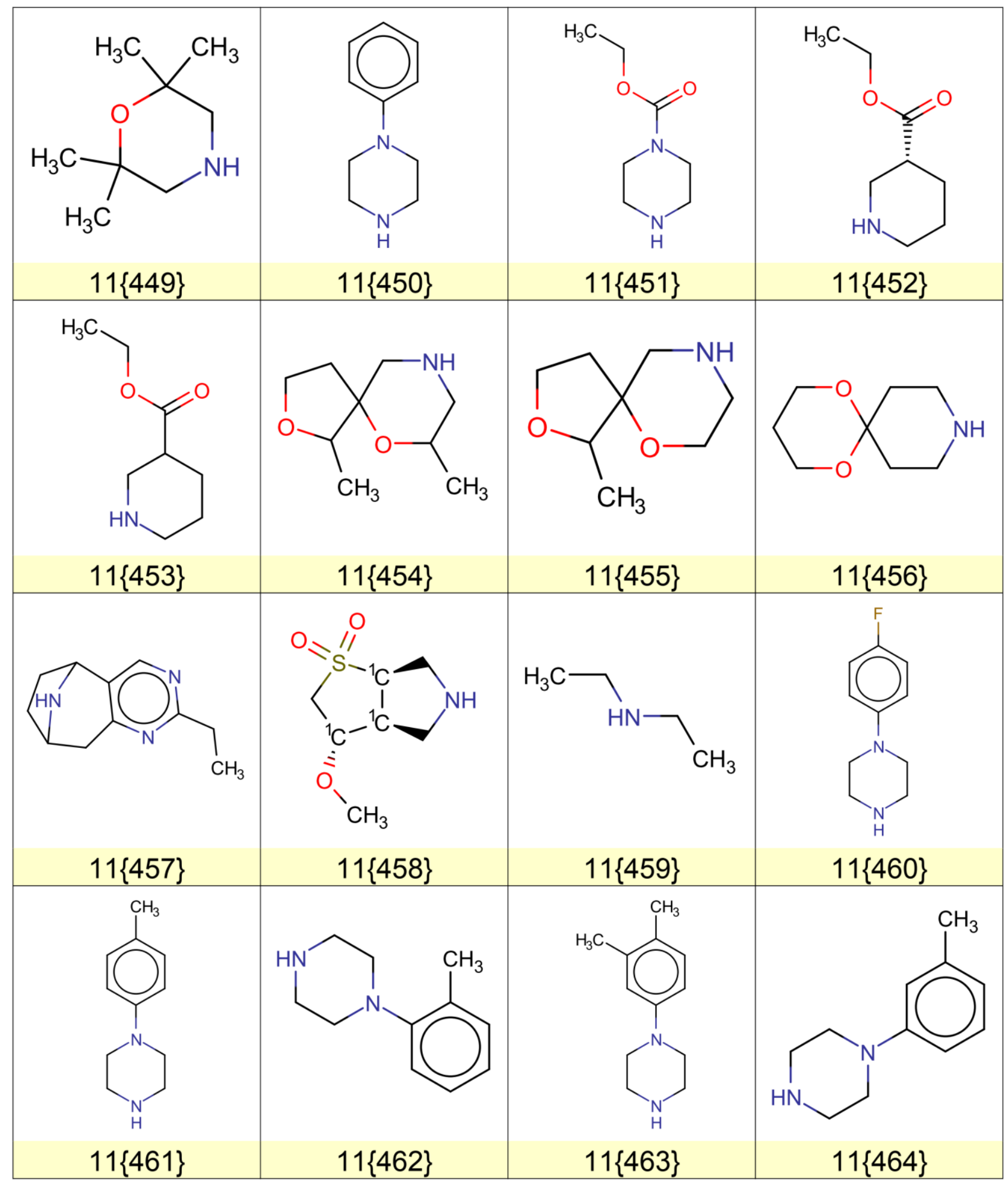




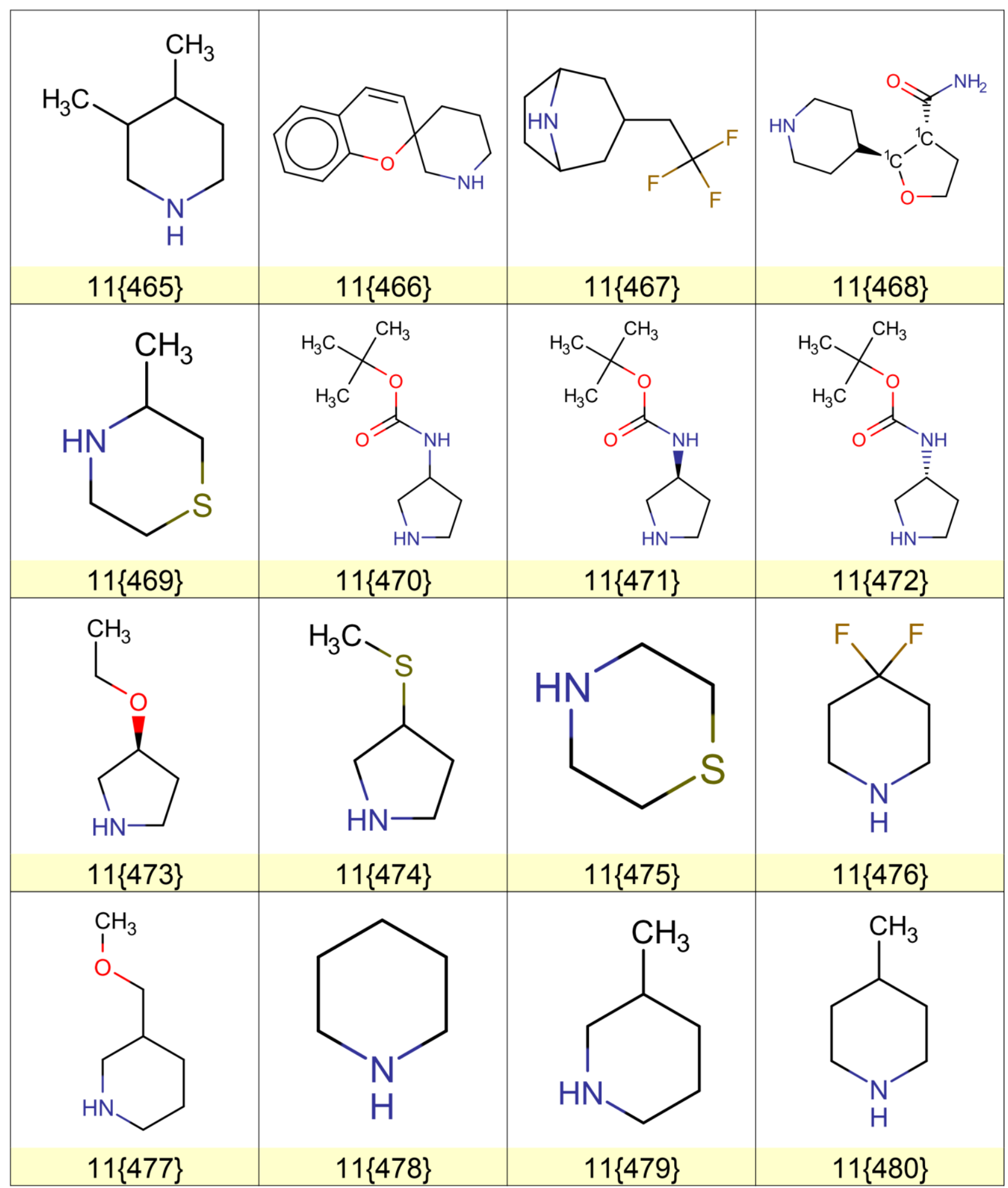




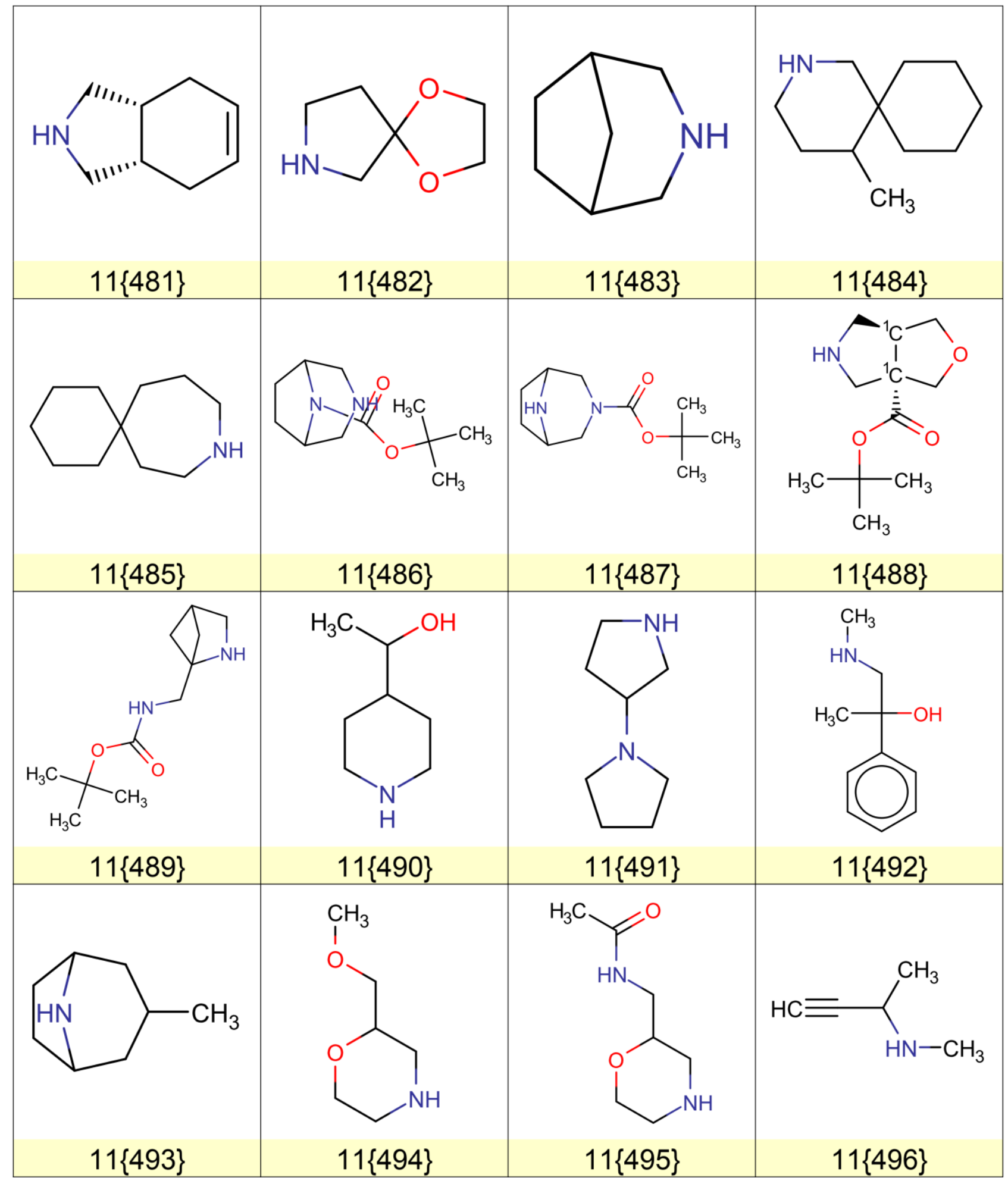




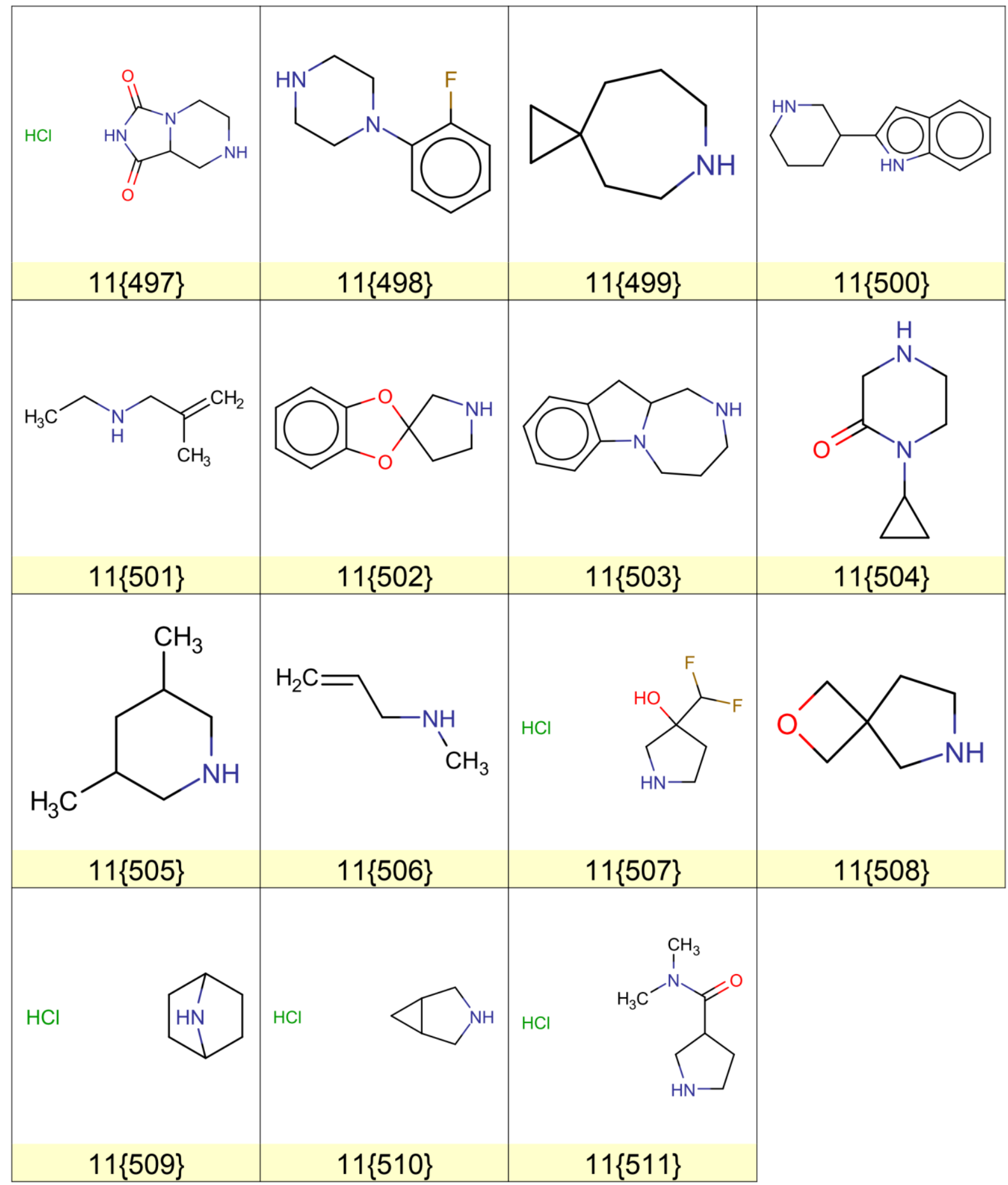


Figure S3. Structures of the reagents $15\{1-378\}$

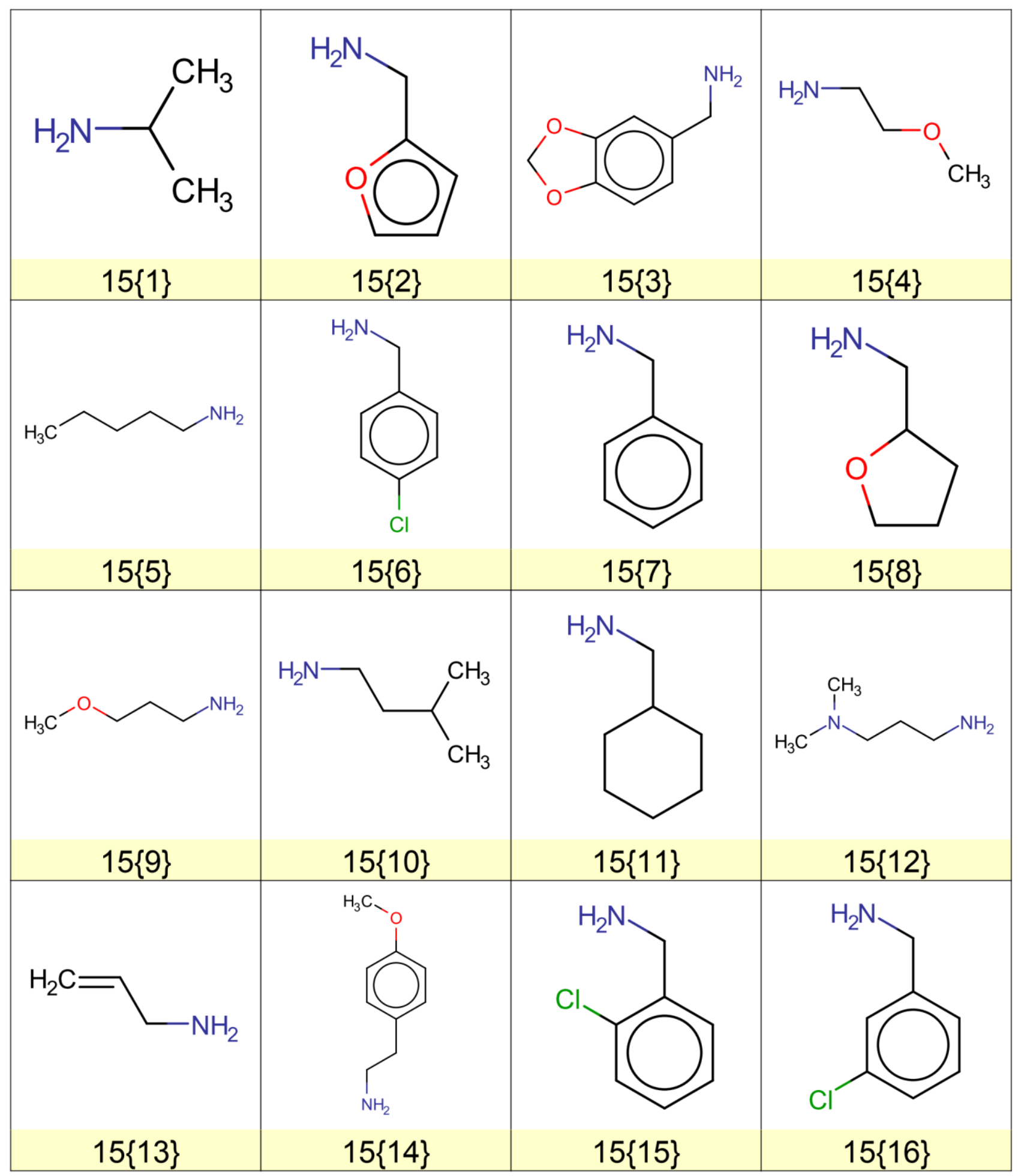




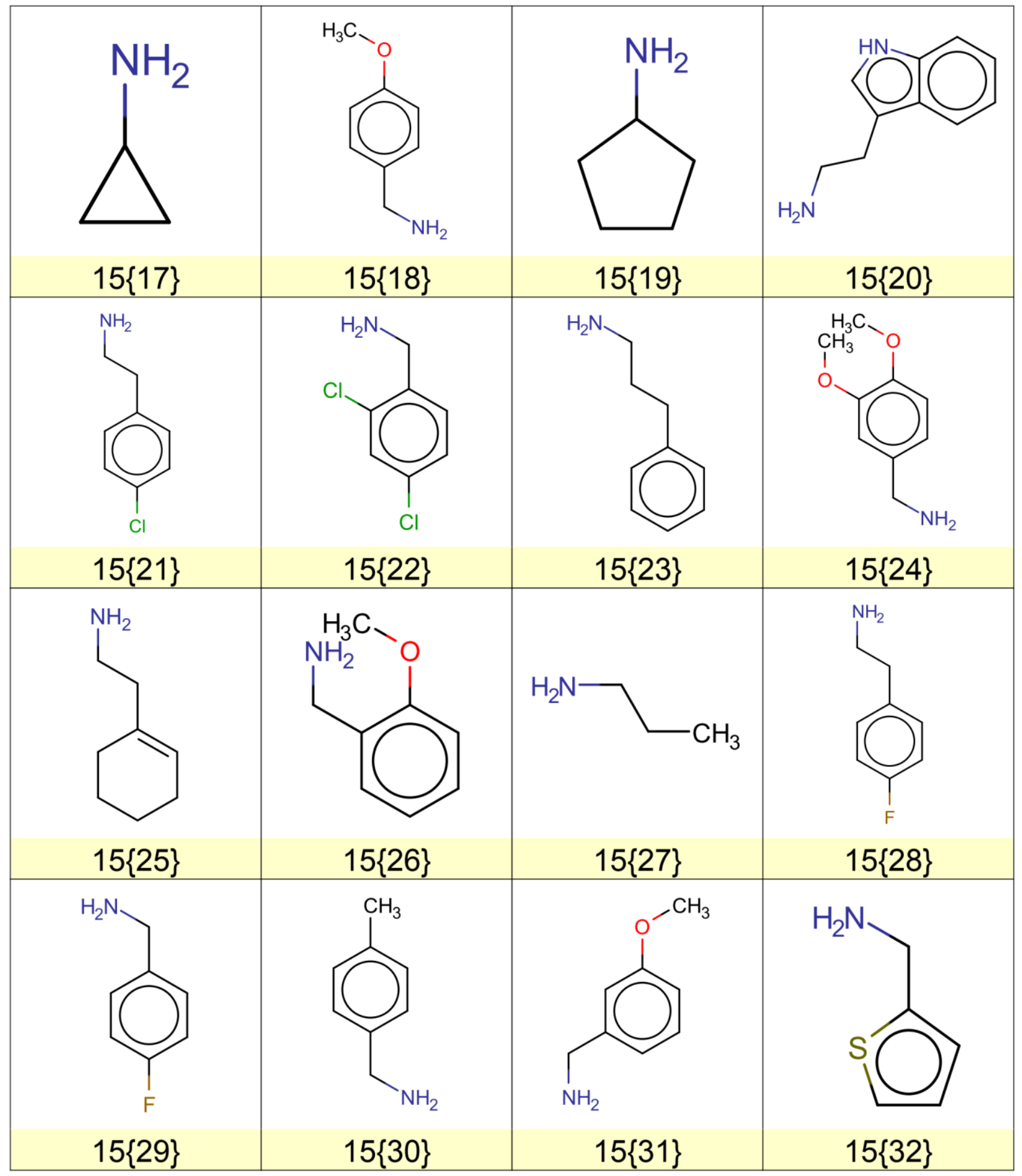




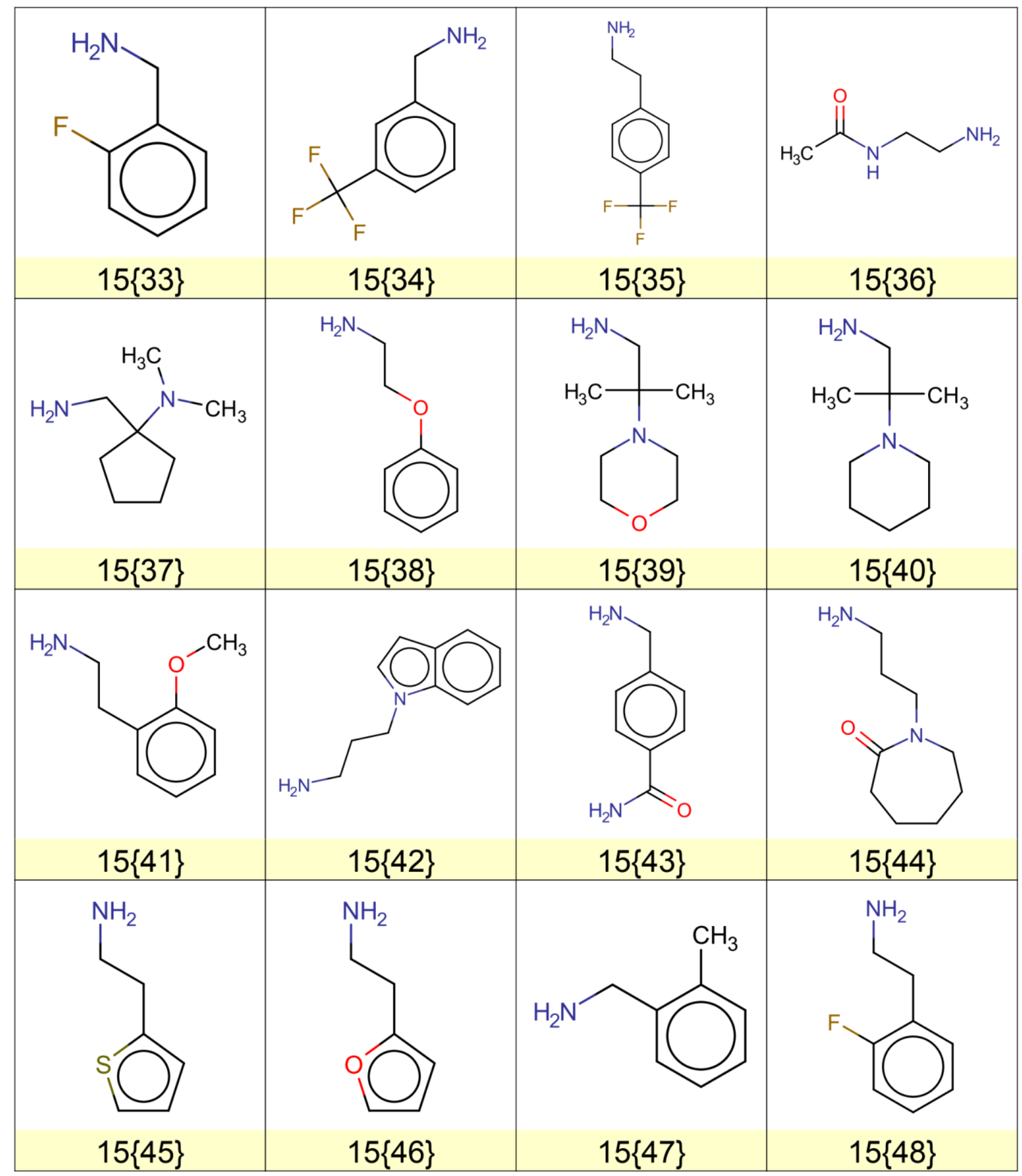




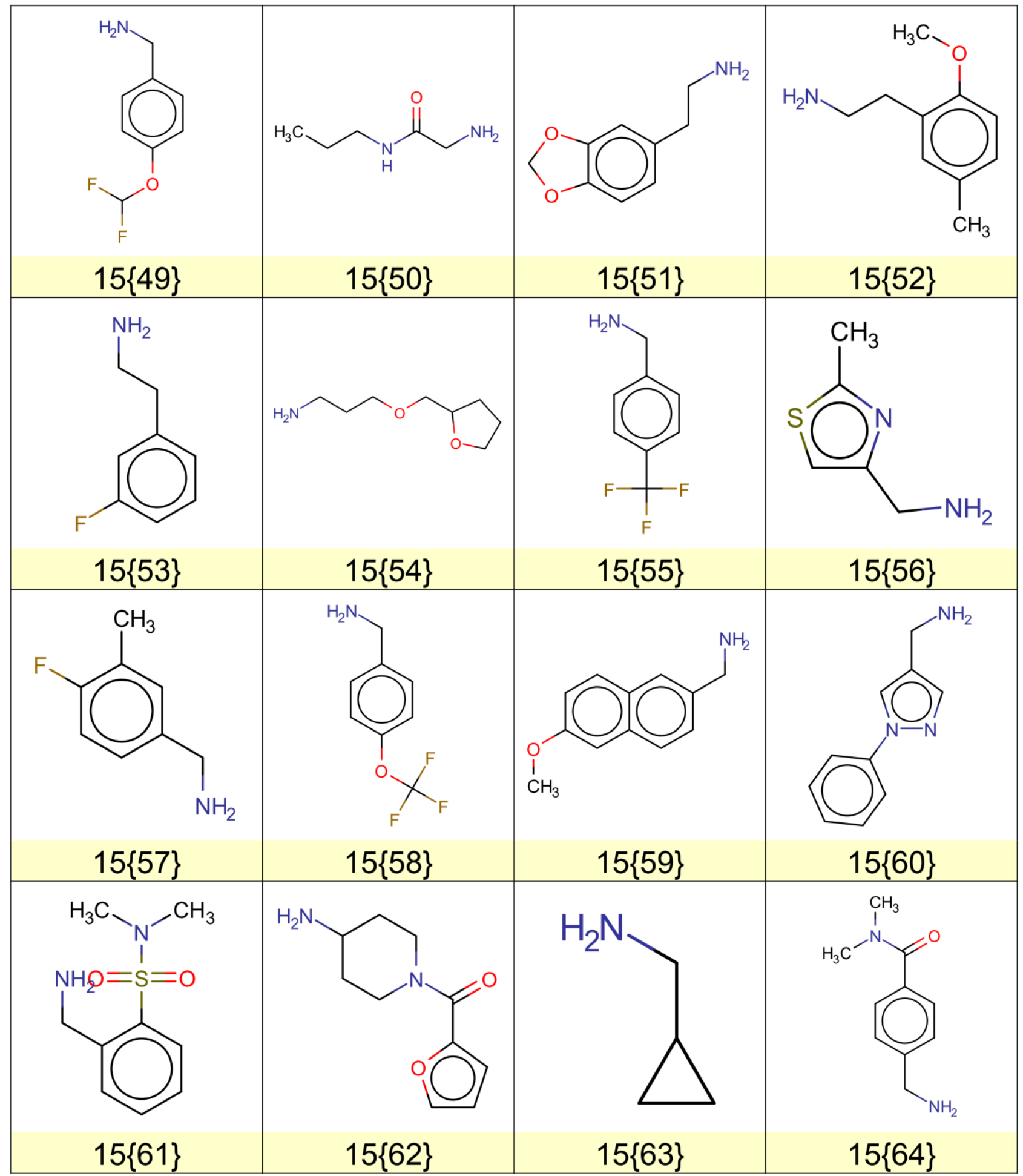




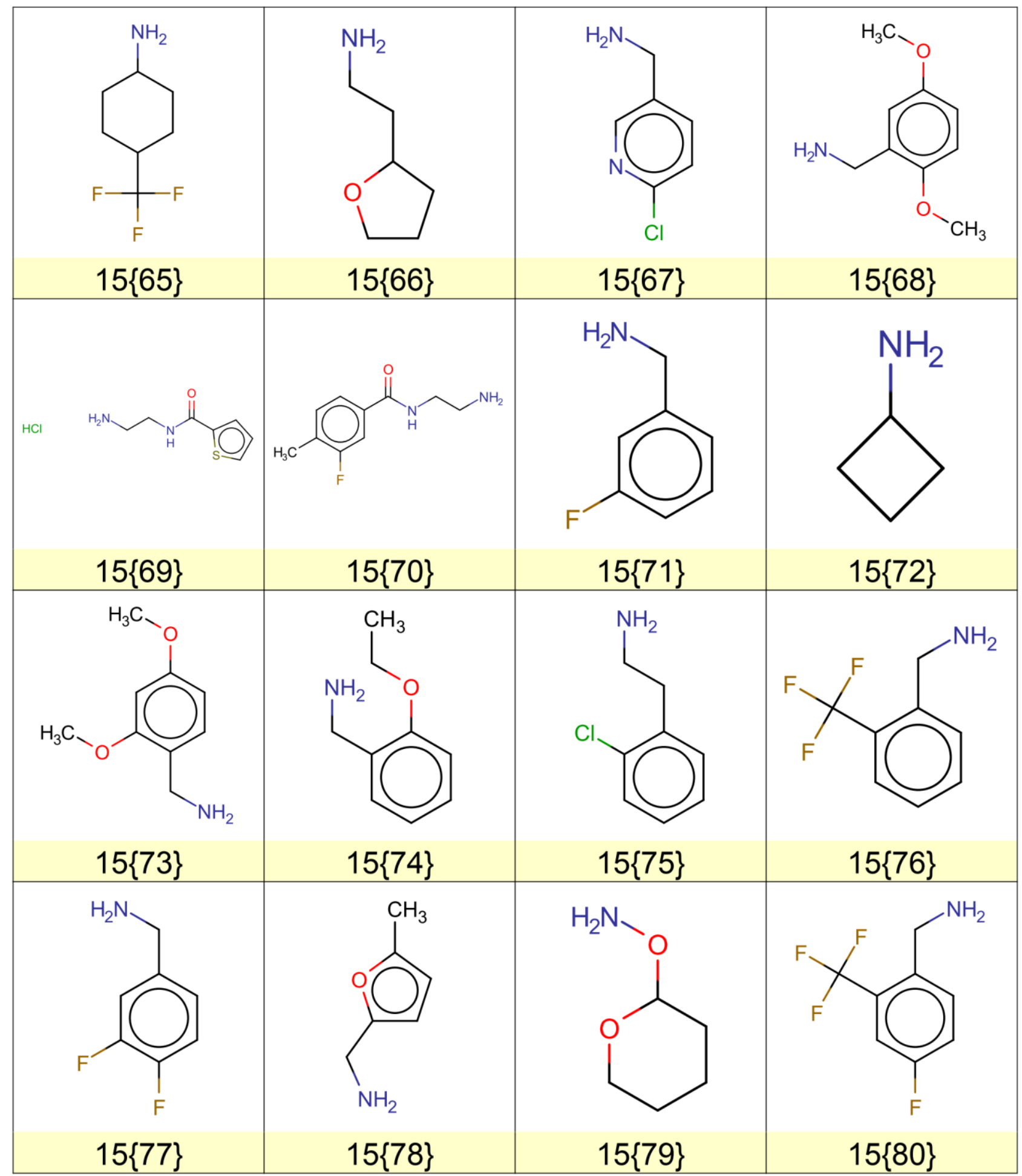




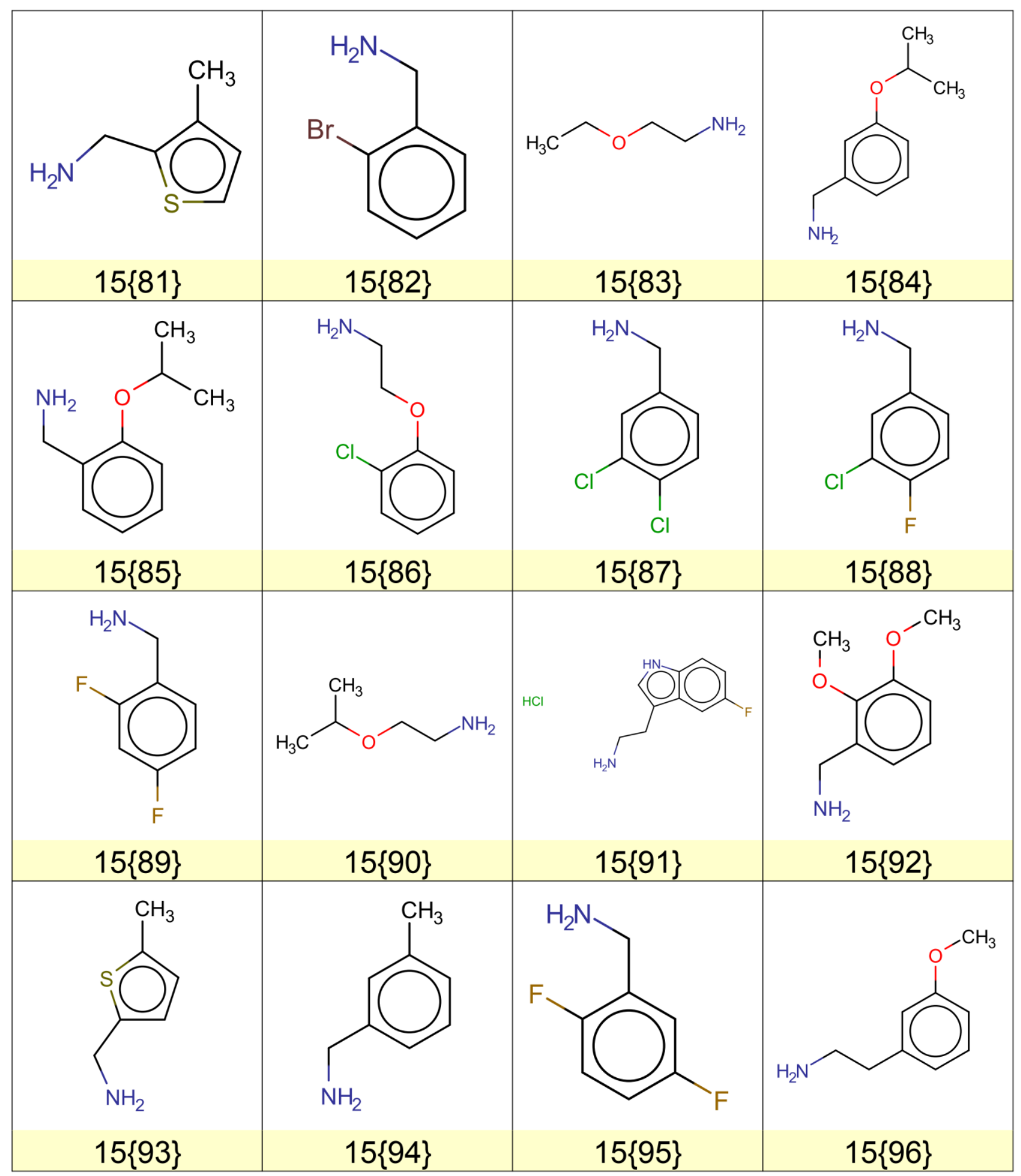




(n)




(15)




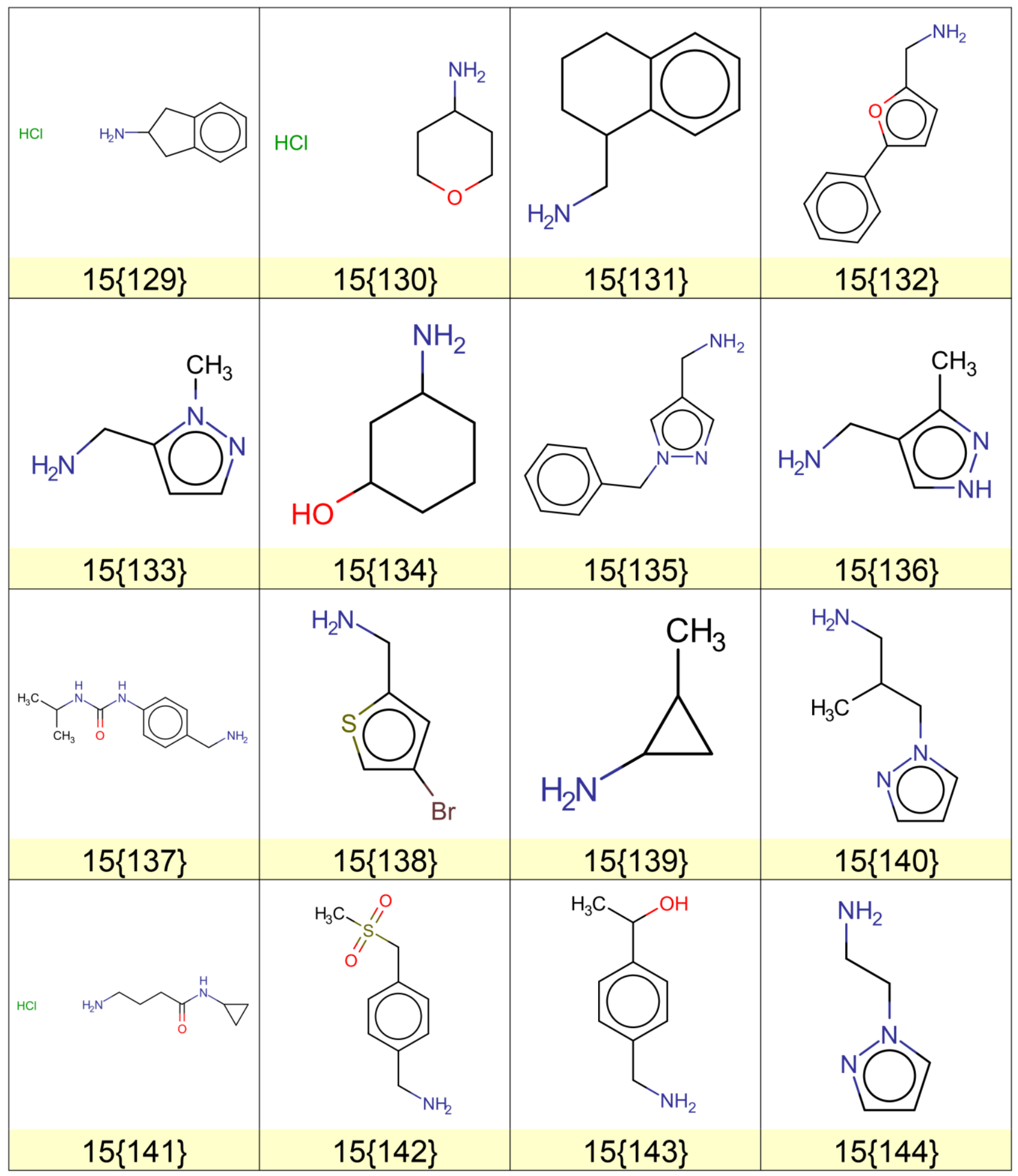




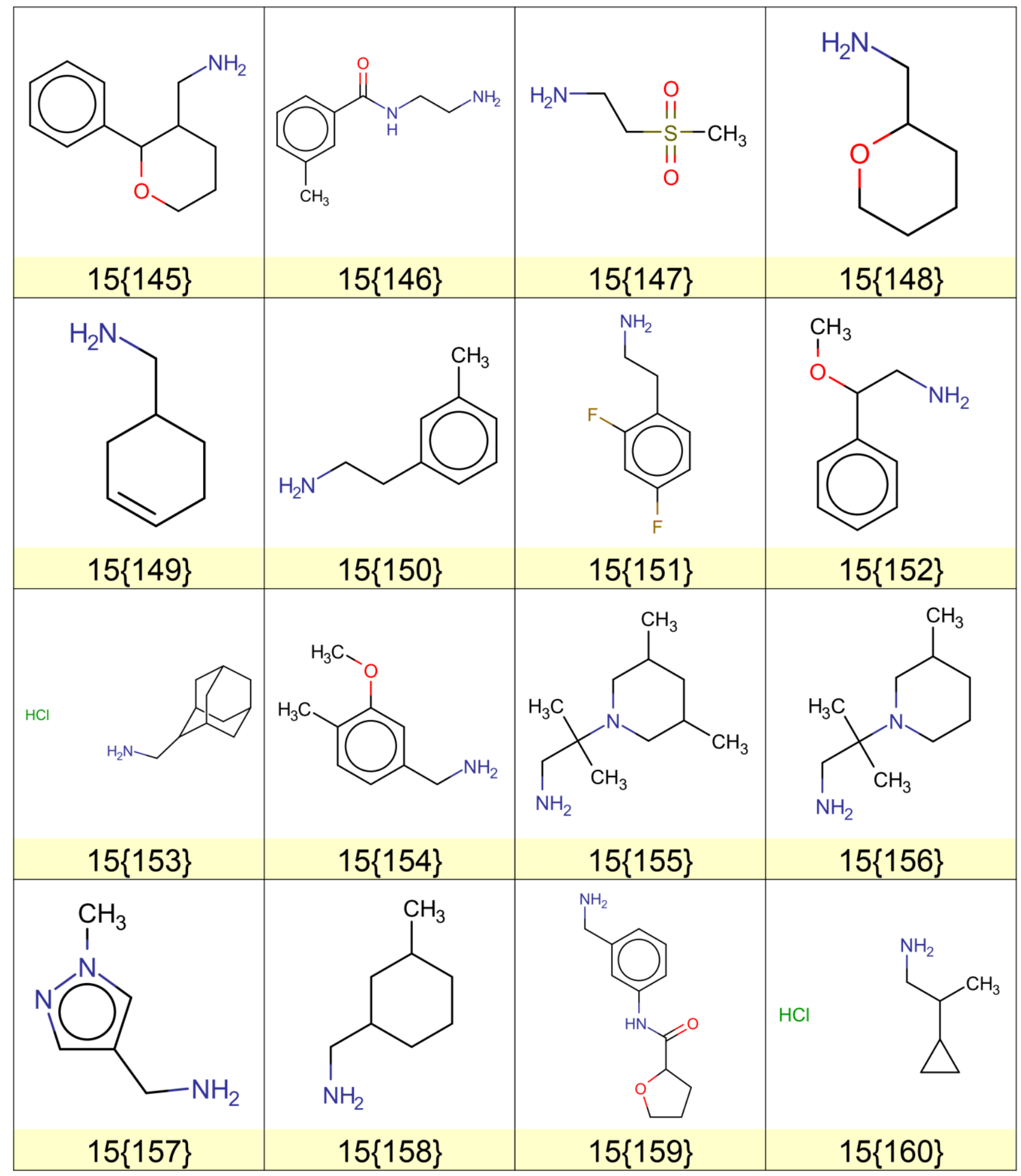




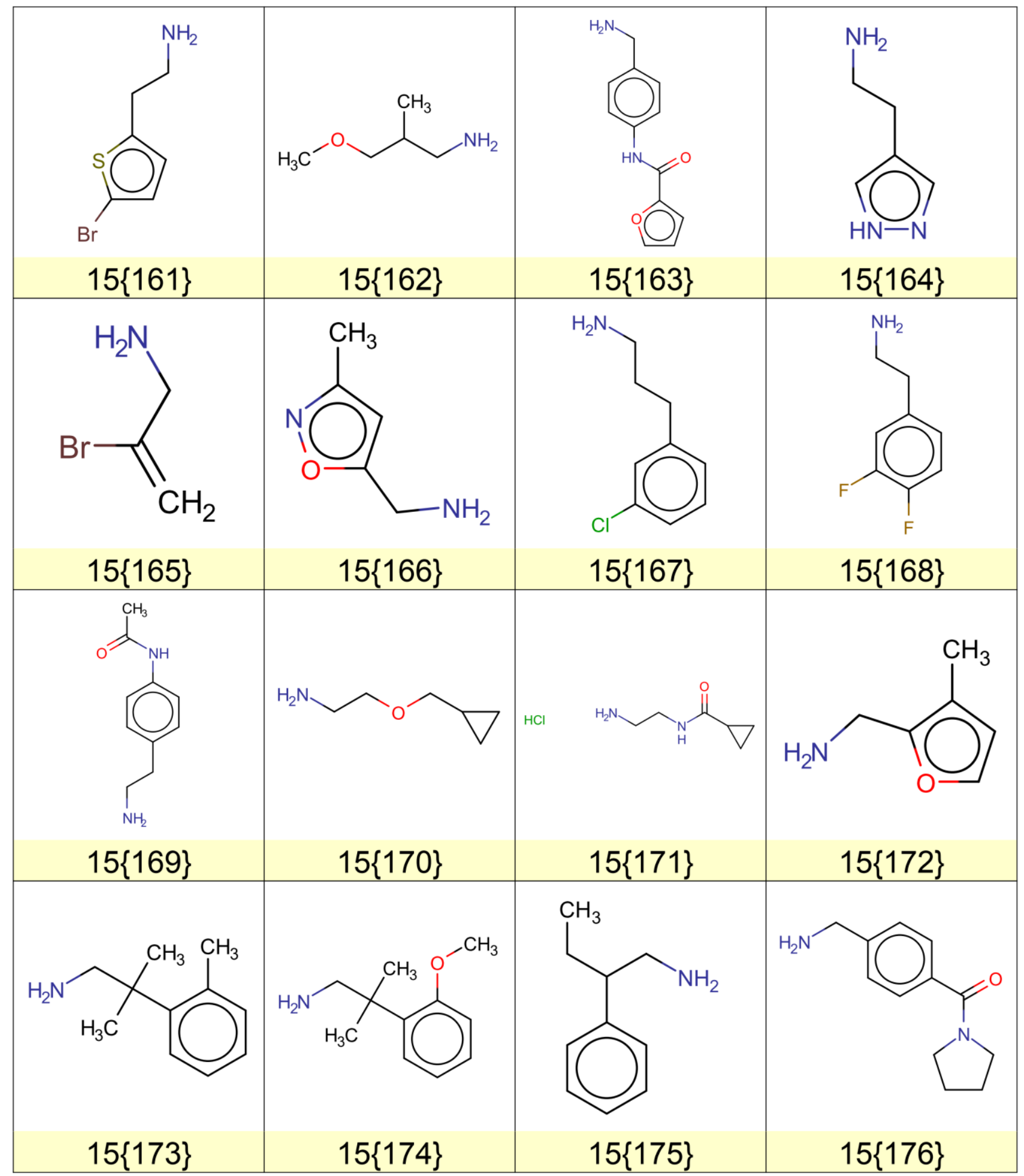




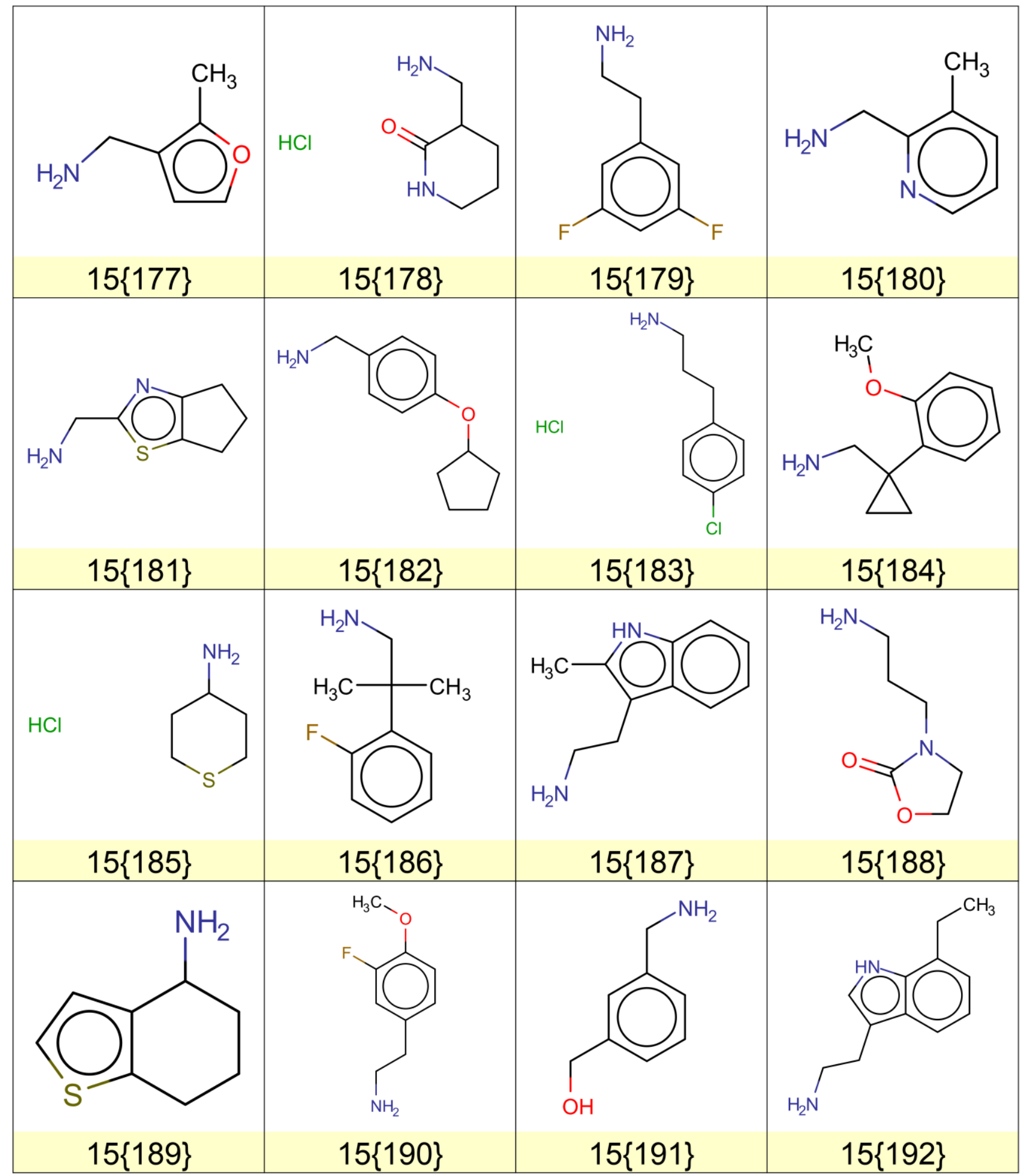




(15,




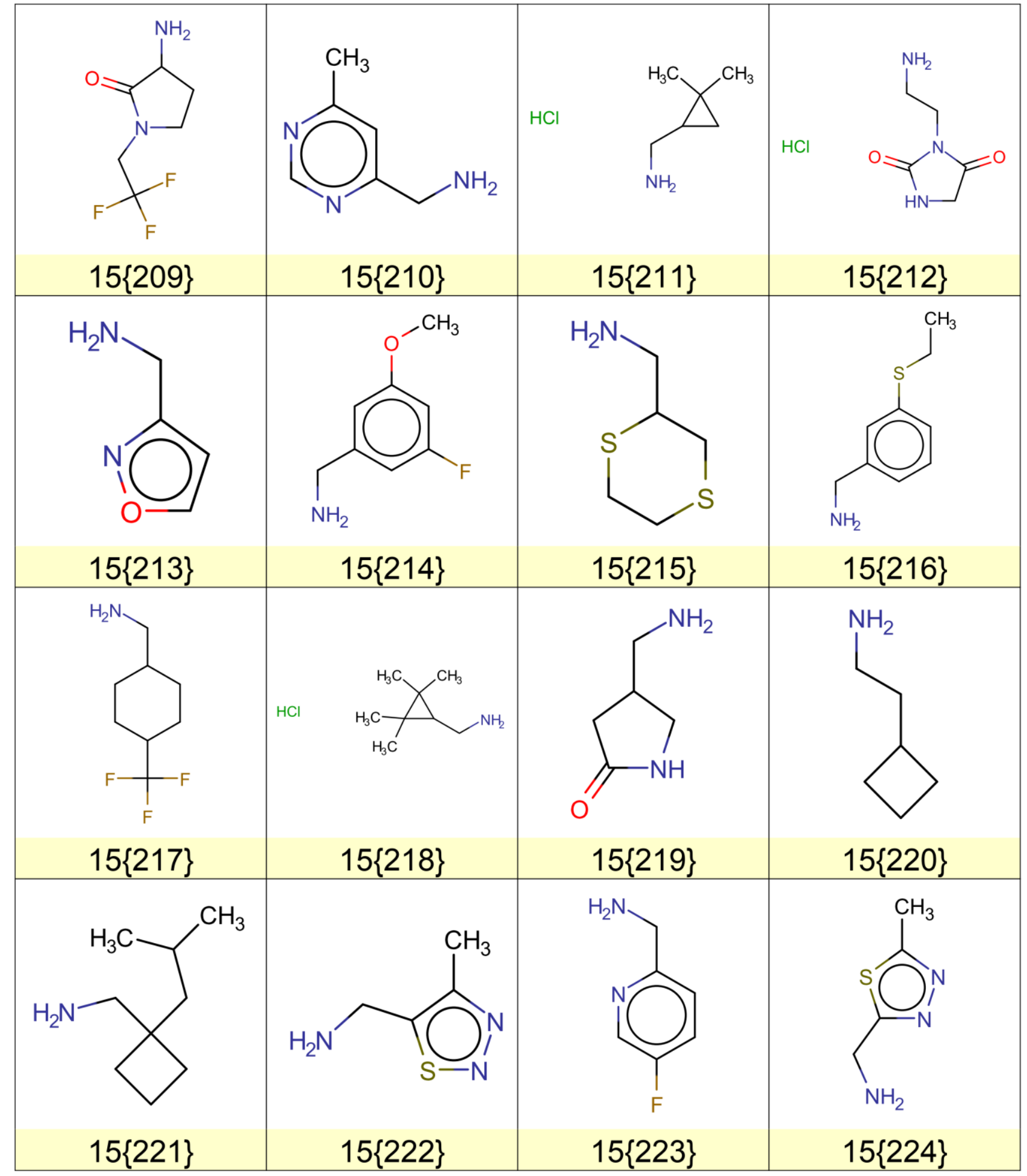




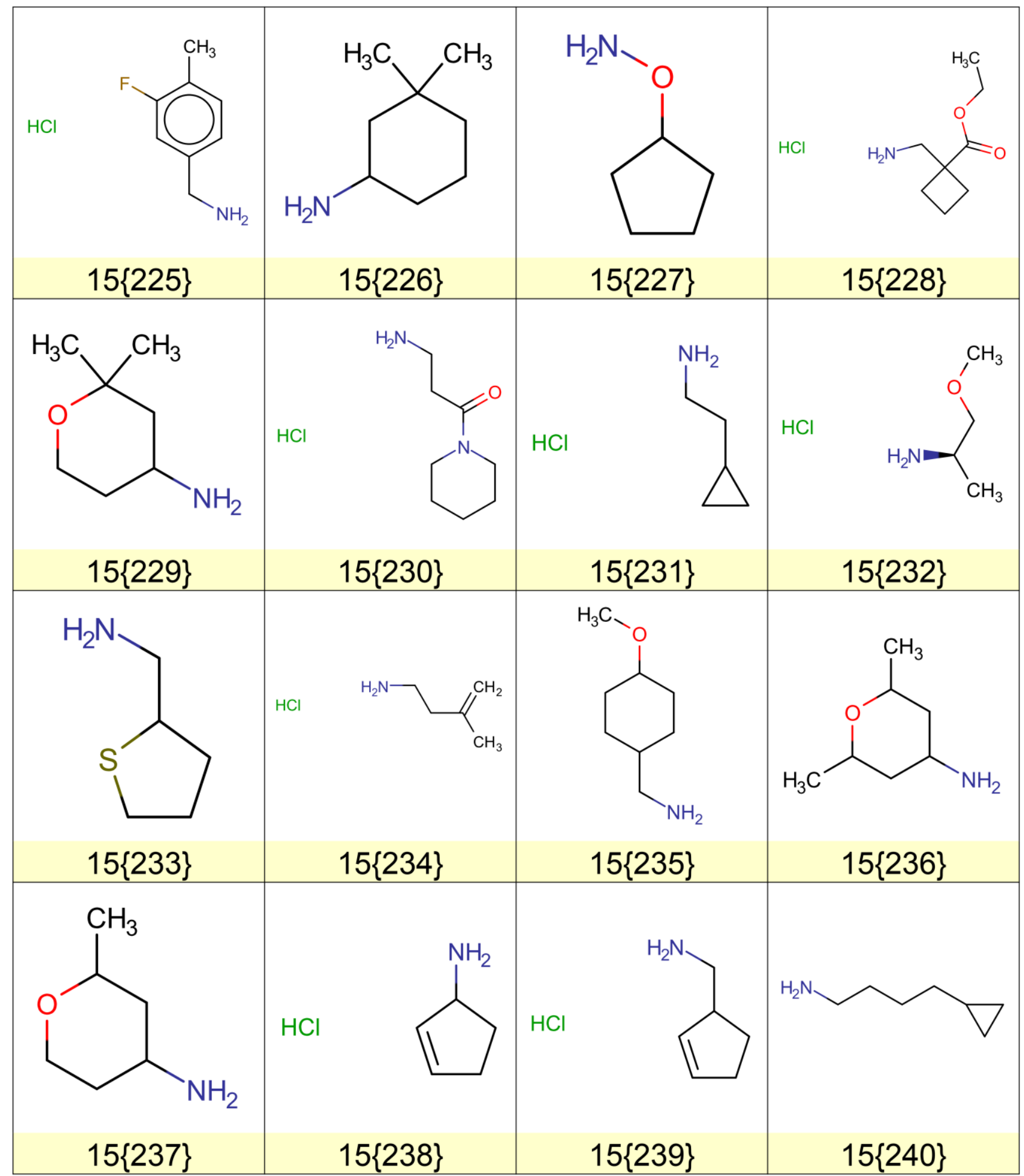




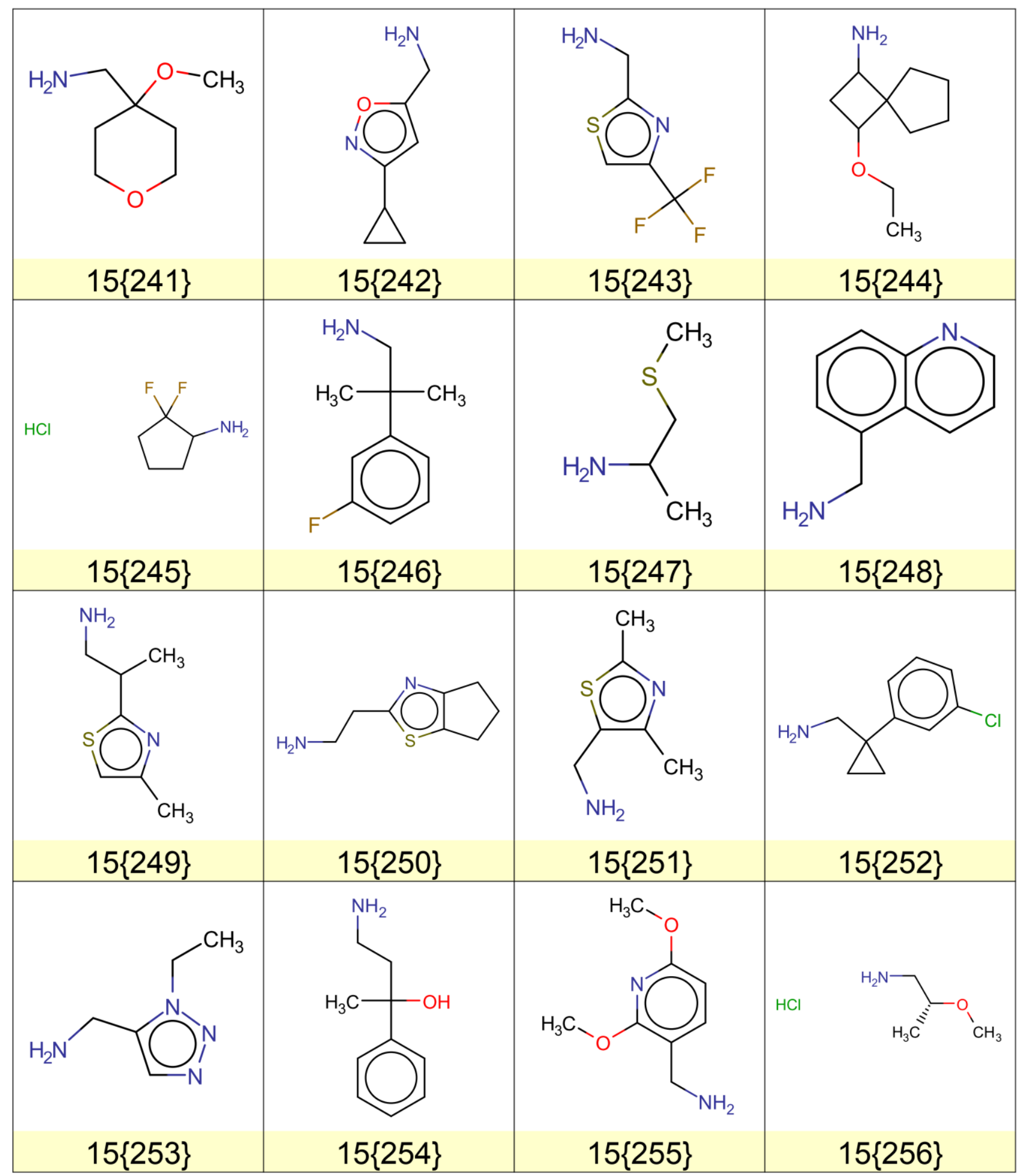




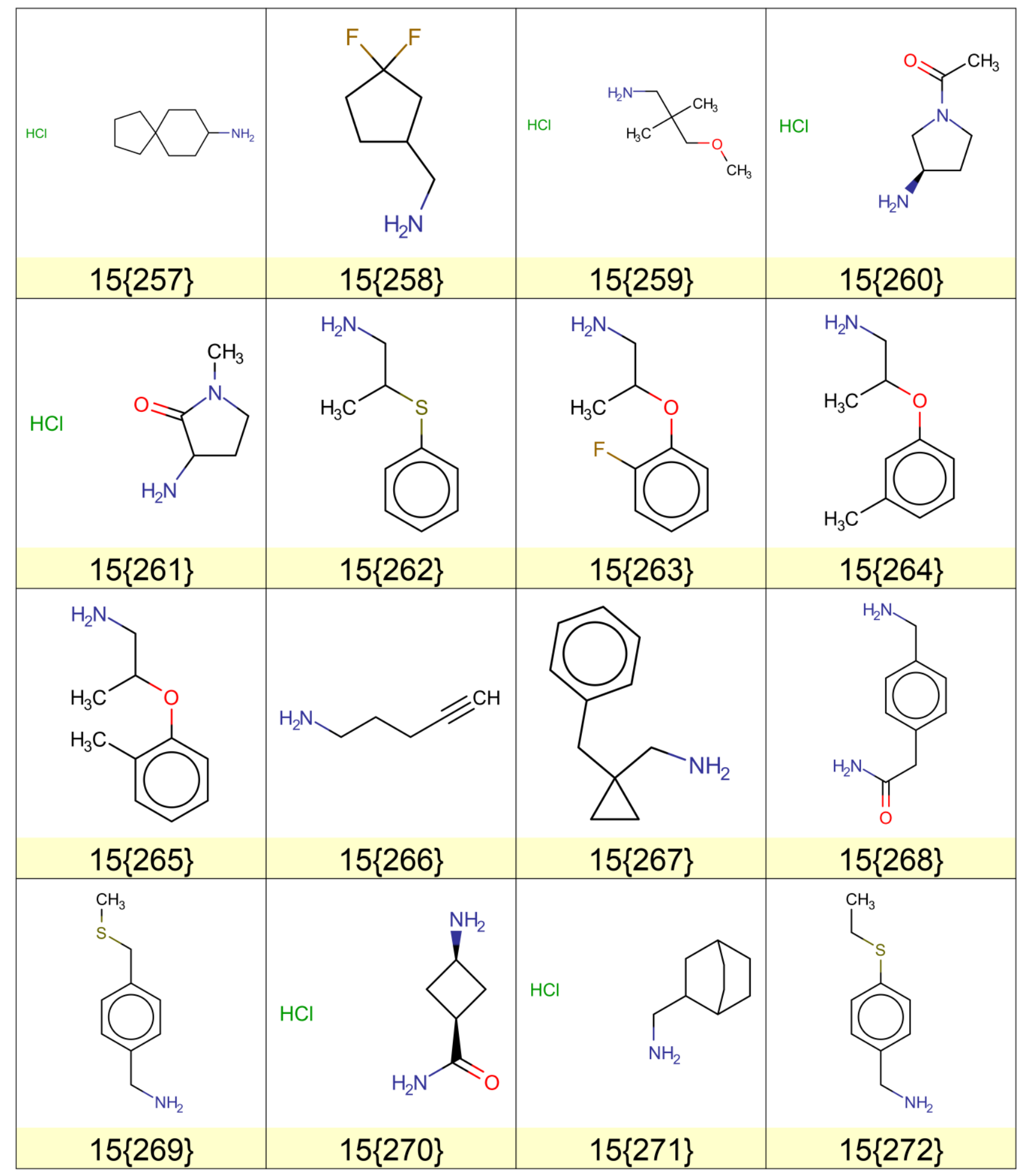




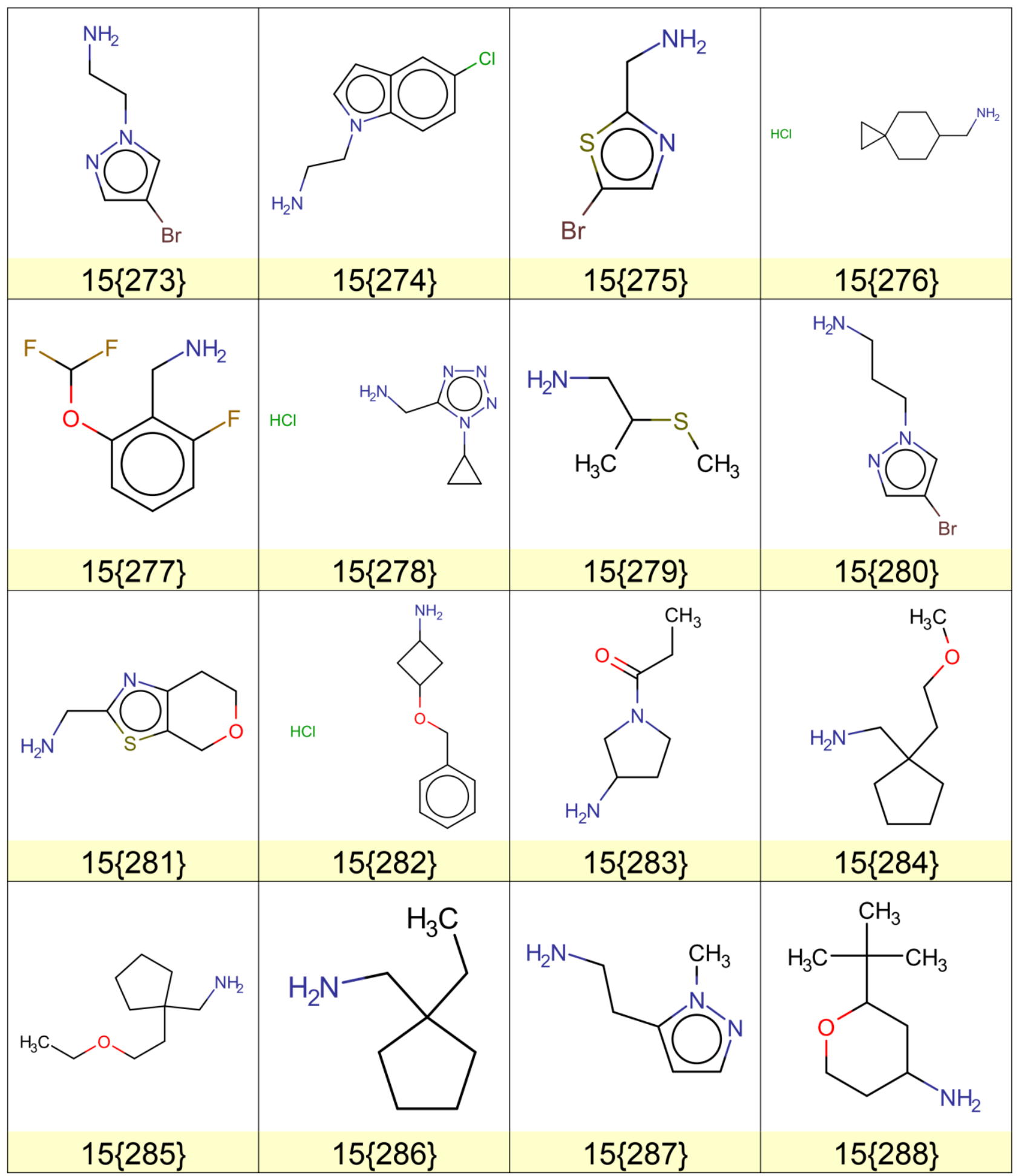




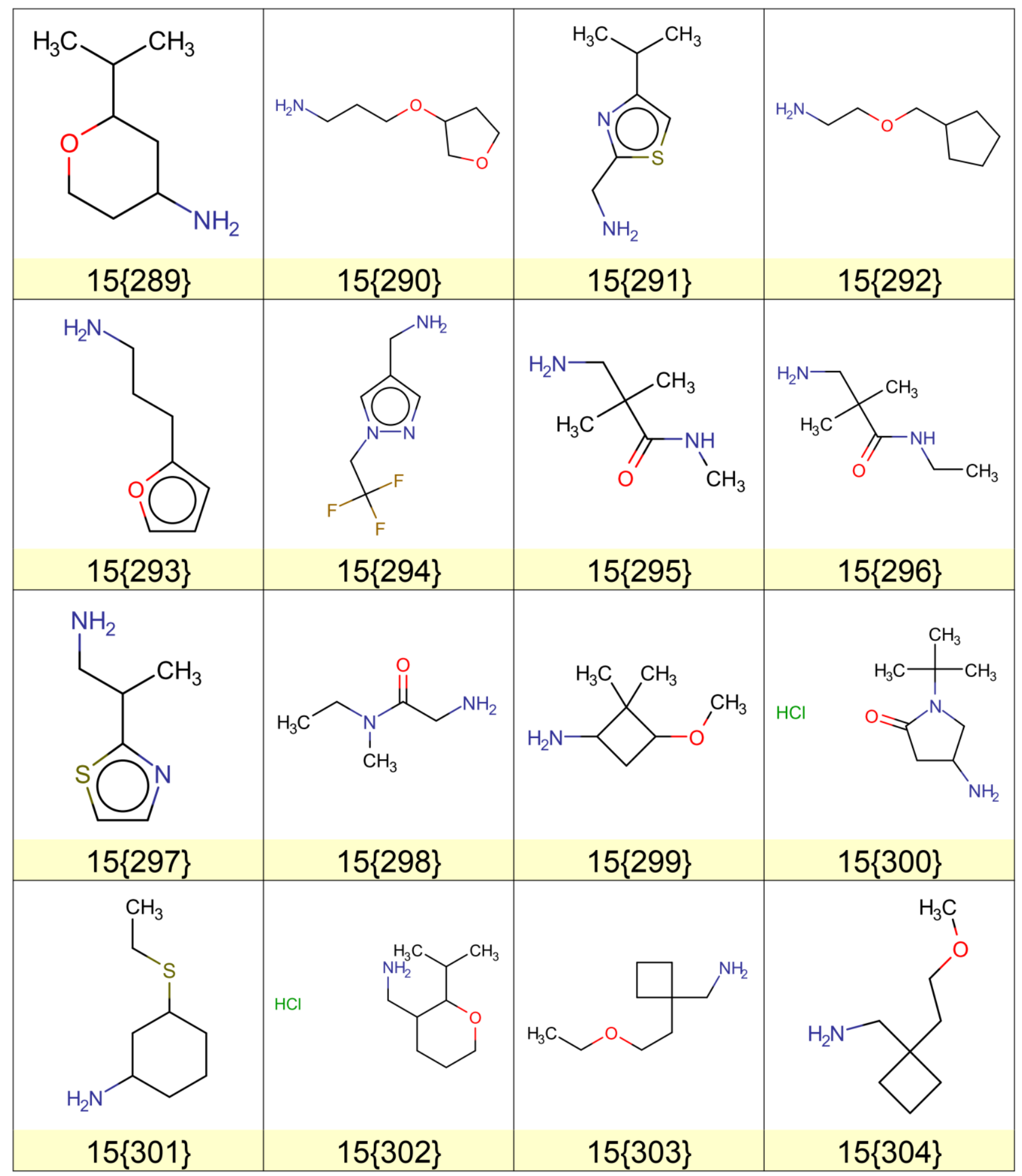




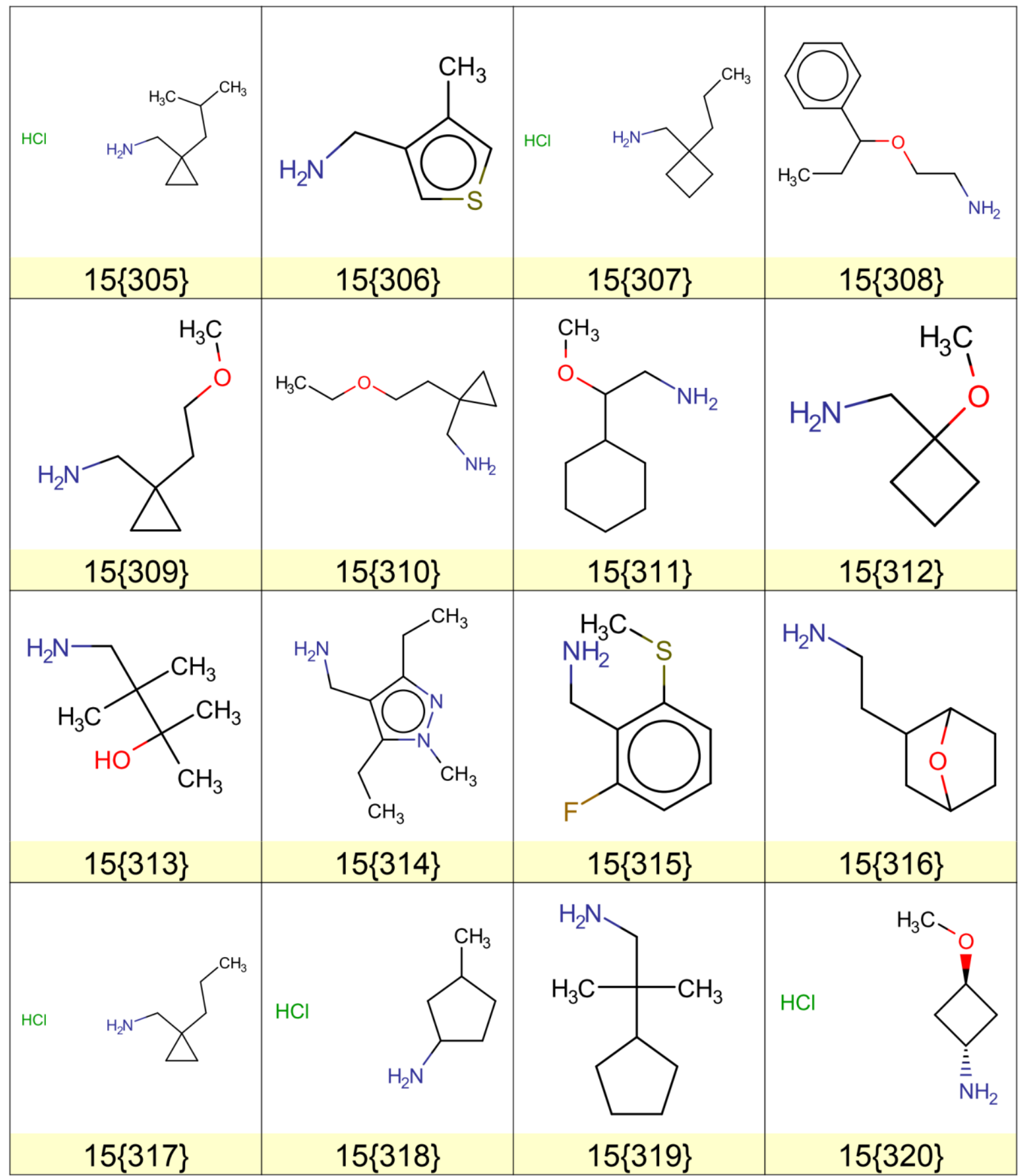




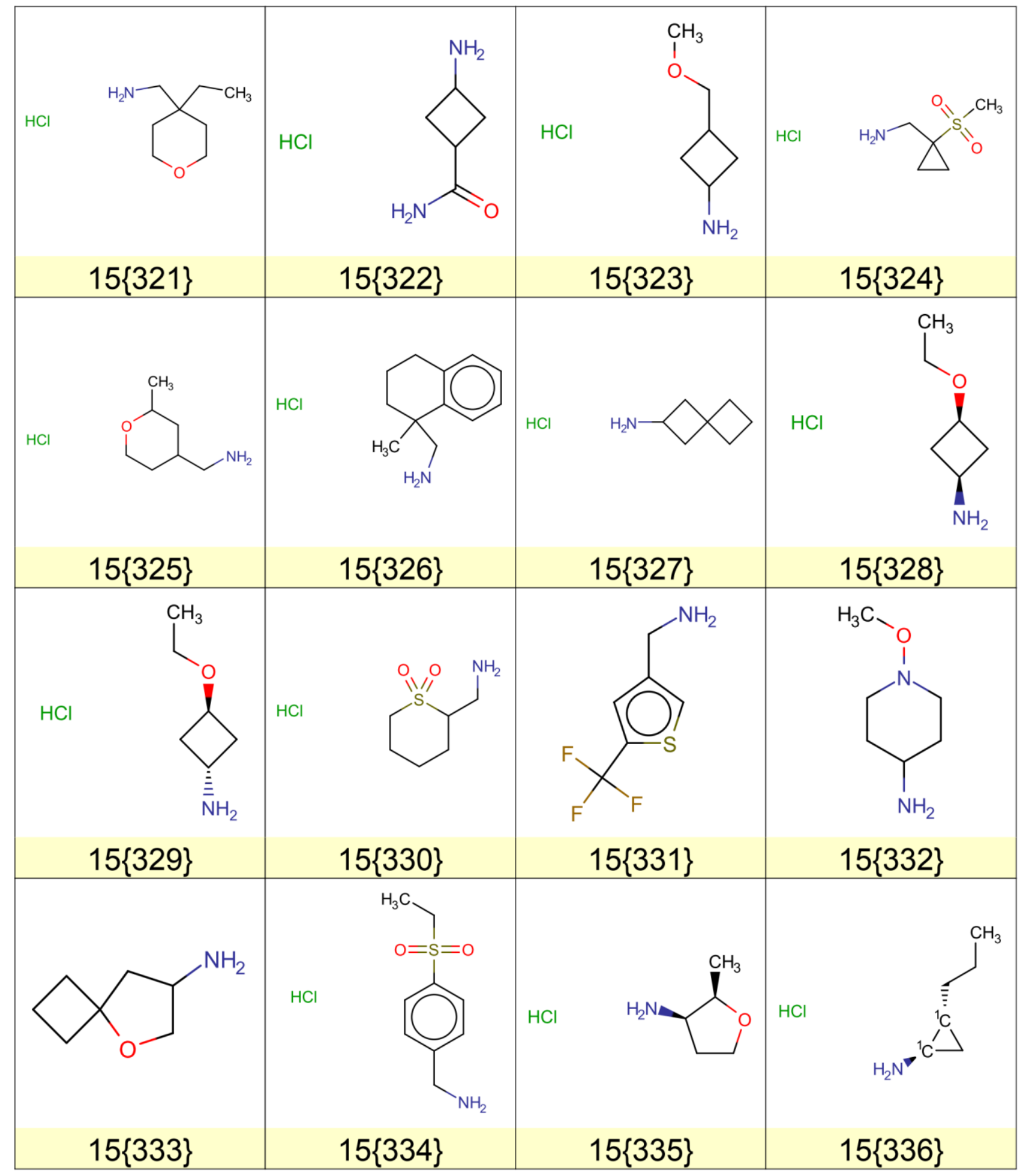




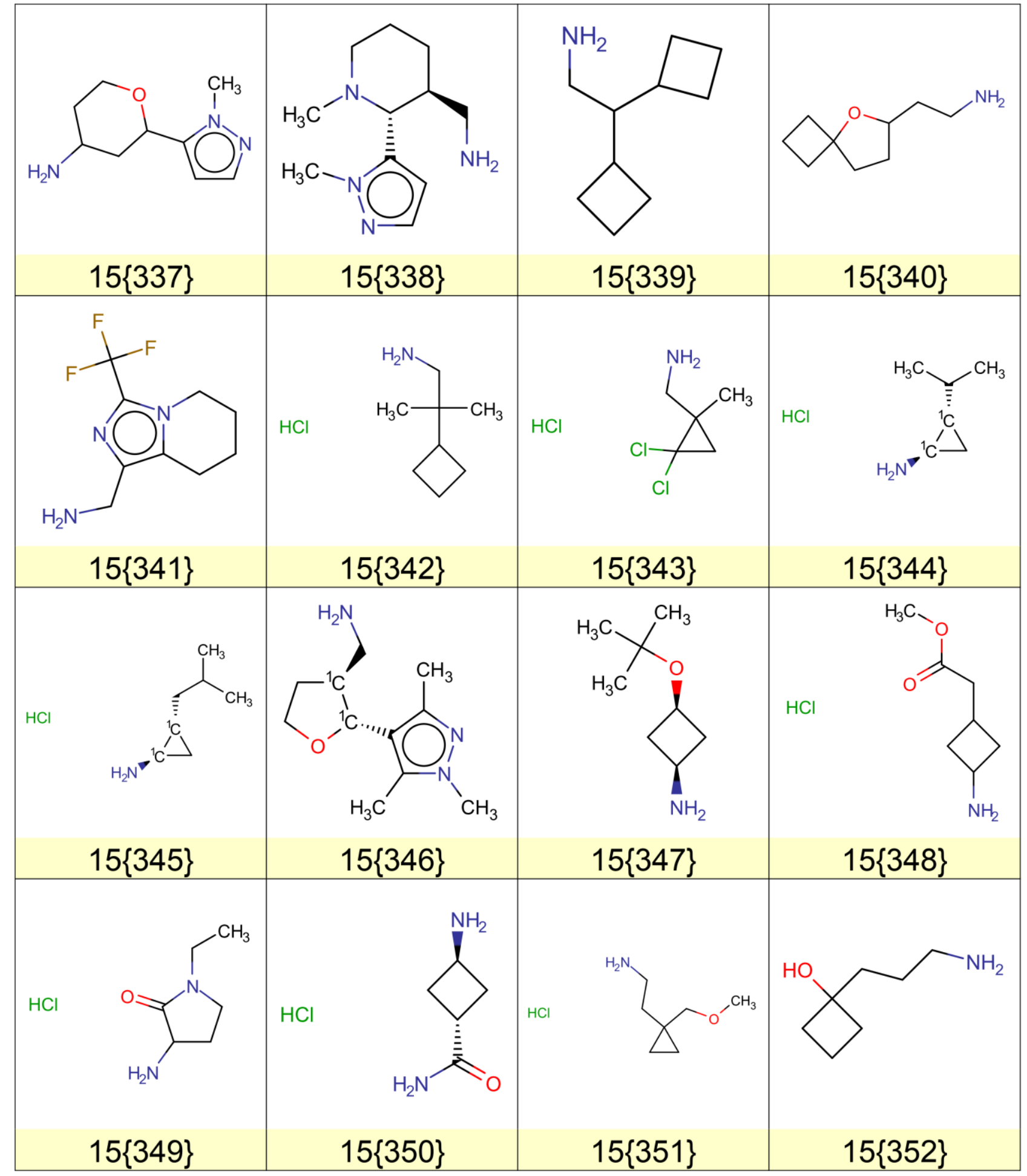




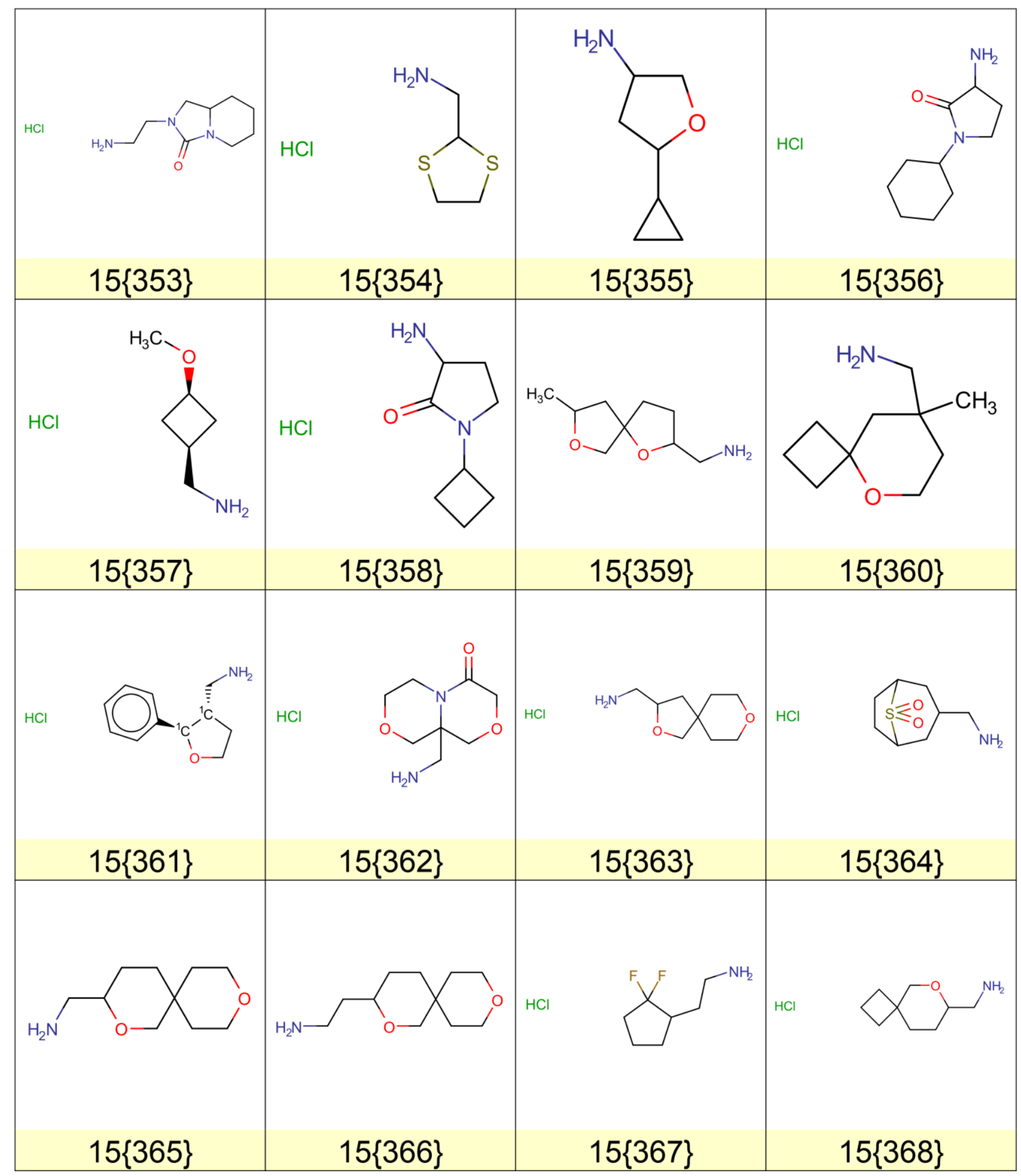




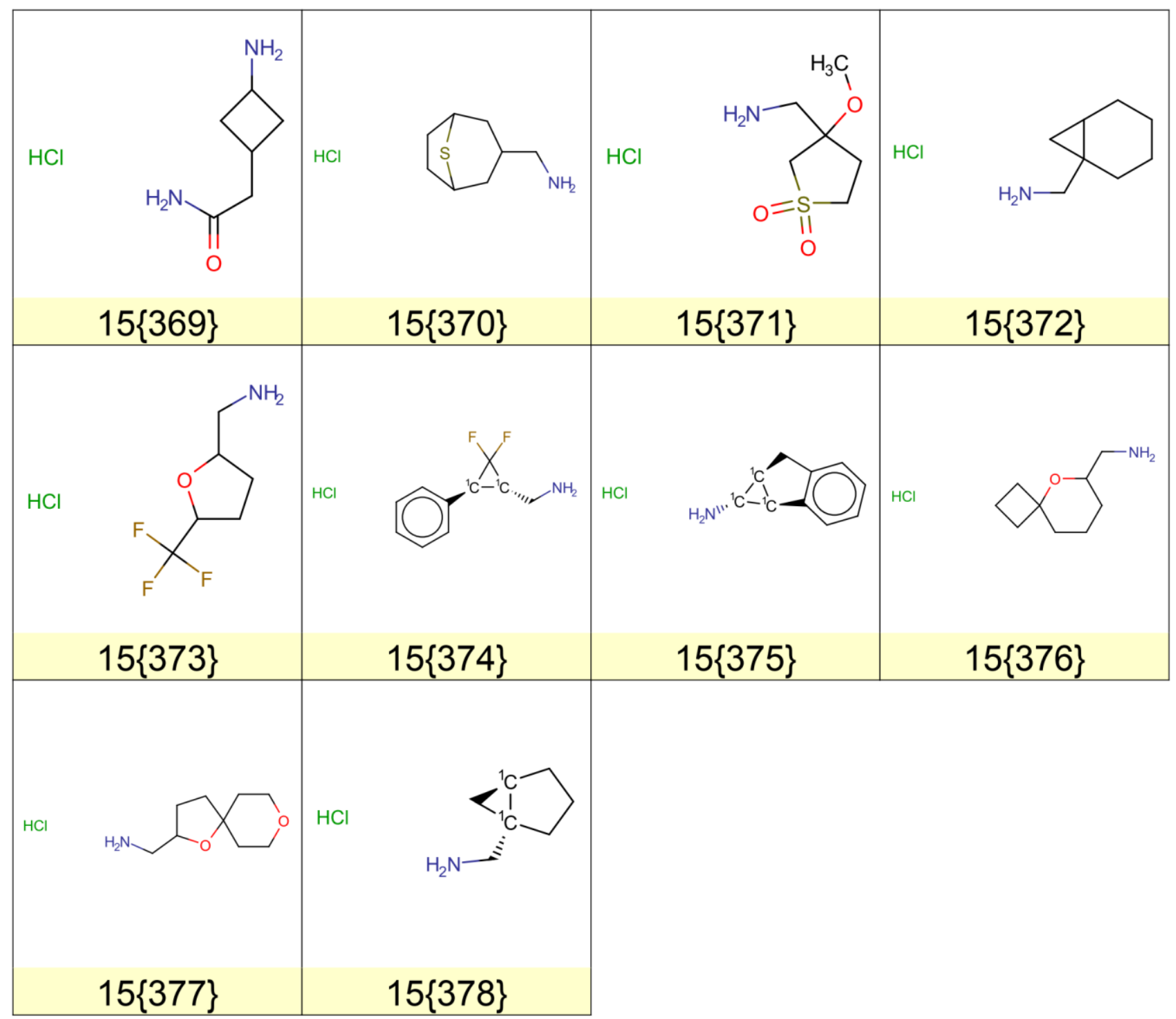


Table S1. SMILES strings for the regents $\mathbf{1 0 .}$

\begin{tabular}{|c|c|c|}
\hline \# & Reagent 10 & SMILE \\
\hline 1 & $\mathbf{1 0}\{1\}$ & $\mathrm{C}=\mathrm{CCN}=\mathrm{C}=\mathrm{S}$ \\
\hline 2 & $\mathbf{1 0}\{2\}$ & $\mathrm{CN}=\mathrm{C}=\mathrm{S}$ \\
\hline 3 & $\mathbf{1 0}\{3\}$ & $\mathrm{CCCCN}=\mathrm{C}=\mathrm{S}$ \\
\hline 4 & $10\{4\}$ & $\mathrm{CCN}=\mathrm{C}=\mathrm{S}$ \\
\hline 5 & $\mathbf{1 0}\{5\}$ & $\mathrm{S}=\mathrm{C}=\mathrm{NC} 1 \mathrm{ccccc} 1$ \\
\hline 6 & $10\{6\}$ & $\mathrm{S}=\mathrm{C}=\mathrm{NCC} 1 \mathrm{ccccc} 1$ \\
\hline 7 & $10\{7\}$ & $\mathrm{S}=\mathrm{C}=\mathrm{NC} 1 \mathrm{CCCCC} 1$ \\
\hline 8 & $\mathbf{1 0}\{8\}$ & $\mathrm{S}=\mathrm{C}=\mathrm{NCCN} 1 \mathrm{CCOCC} 1$ \\
\hline 9 & $\mathbf{1 0}\{9\}$ & $\mathrm{COCCN}=\mathrm{C}=\mathrm{S}$ \\
\hline 10 & $\mathbf{1 0}\{10\}$ & $\mathrm{CC}(\mathrm{C}) \mathrm{N}=\mathrm{C}=\mathrm{S}$ \\
\hline 11 & $\mathbf{1 0}\{11\}$ & $\mathrm{CC}(=\mathrm{C}) \mathrm{CN}=\mathrm{C}=\mathrm{S}$ \\
\hline 12 & $\mathbf{1 0}\{12\}$ & $\mathrm{CCOCCCN}=\mathrm{C}=\mathrm{S}$ \\
\hline 13 & $\mathbf{1 0}\{13\}$ & $\mathrm{COc} 1 \mathrm{ccc}(\mathrm{CCN}=\mathrm{C}=\mathrm{S}) \mathrm{cc} 1$ \\
\hline 14 & $\mathbf{1 0}\{14\}$ & $\mathrm{CCCN}=\mathrm{C}=\mathrm{S}$ \\
\hline 15 & $\mathbf{1 0}\{15\}$ & $\mathrm{S}=\mathrm{C}=\mathrm{NC} 1 \mathrm{CC} 1$ \\
\hline 16 & $\mathbf{1 0}\{16\}$ & $\operatorname{COCC}(\mathrm{C}) \mathrm{N}=\mathrm{C}=\mathrm{S}$ \\
\hline 17 & $\mathbf{1 0}\{17\}$ & $\mathrm{S}=\mathrm{C}=\mathrm{NC} 1 \mathrm{CC} 2 \mathrm{CCC} 1 \mathrm{C} 2$ \\
\hline 18 & $\mathbf{1 0}\{18\}$ & $\mathrm{S}=\mathrm{C}=\mathrm{NCCSc} 1 \operatorname{ccccc} 1$ \\
\hline 19 & $\mathbf{1 0}\{19\}$ & $\mathrm{S}=\mathrm{C}=\mathrm{NCc} 1 \mathrm{cccnc} 1$ \\
\hline 20 & $\mathbf{1 0}\{20\}$ & $\mathrm{CCN} 1 \mathrm{CCCC} 1 \mathrm{CN}=\mathrm{C}=\mathrm{S}$ \\
\hline 21 & $\mathbf{1 0}\{21\}$ & $\mathrm{CC}(\mathrm{C})(\mathrm{C}) \mathrm{N}=\mathrm{C}=\mathrm{S}$ \\
\hline 22 & $\mathbf{1 0}\{22\}$ & $\mathrm{S}=\mathrm{C}=\mathrm{NC} 1 \mathrm{CCCC} 1$ \\
\hline 23 & $\mathbf{1 0}\{23\}$ & $\mathrm{CC}(\mathrm{N}=\mathrm{C}=\mathrm{S}) \mathrm{c} 1 \mathrm{ccccc} 1$ \\
\hline 24 & $\mathbf{1 0}\{24\}$ & $\mathrm{CCC}(\mathrm{C}) \mathrm{N}=\mathrm{C}=\mathrm{S}$ \\
\hline 25 & $\mathbf{1 0}\{25\}$ & $\mathrm{S}=\mathrm{C}=\mathrm{NCC} 1 \mathrm{CC} 1$ \\
\hline
\end{tabular}




\begin{tabular}{|c|c|c|}
\hline 26 & $10\{26\}$ & $\mathrm{FC}(\mathrm{F})(\mathrm{F}) \mathrm{CN}=\mathrm{C}=\mathrm{S}$ \\
\hline 27 & $\mathbf{1 0}\{27\}$ & $\operatorname{CCOC}(=\mathrm{O}) \mathrm{N} 1 \mathrm{CCC}(\mathrm{CC} 1) \mathrm{N}=\mathrm{C}=\mathrm{S}$ \\
\hline 28 & $\mathbf{1 0}\{28\}$ & $\mathrm{CC}(\mathrm{N}=\mathrm{C}=\mathrm{S}) \mathrm{c} 1 \mathrm{ccc}(\mathrm{Br}) \mathrm{cc} 1$ \\
\hline 29 & $10\{29\}$ & $\mathrm{S}=\mathrm{C}=\mathrm{NC} 1 \mathrm{CCN}(\mathrm{Cc} 2 \operatorname{ccccc} 2) \mathrm{CC} 1$ \\
\hline 30 & $\mathbf{1 0}\{30\}$ & $\mathrm{Clc} 1 \mathrm{ccc}(\mathrm{CCN}=\mathrm{C}=\mathrm{S}) \mathrm{c}(\mathrm{Cl}) \mathrm{c} 1$ \\
\hline 31 & $\mathbf{1 0}\{31\}$ & $\mathrm{CCOCCN}=\mathrm{C}=\mathrm{S}$ \\
\hline 32 & $\mathbf{1 0}\{32\}$ & $\mathrm{S}=\mathrm{C}=\mathrm{NCC} 1 \mathrm{ccc} \operatorname{co} 1$ \\
\hline 33 & $\mathbf{1 0}\{33\}$ & $\mathrm{FC}(\mathrm{F})(\mathrm{F}) \mathrm{c} 1 \mathrm{cccc}(\mathrm{CN}=\mathrm{C}=\mathrm{S}) \mathrm{c} 1$ \\
\hline 34 & $10\{34\}$ & $\mathrm{S}=\mathrm{C}=\mathrm{NCC} 1 \mathrm{ccc} 2 \mathrm{OCCO} 2 \mathrm{c} 1$ \\
\hline 35 & $\mathbf{1 0}\{35\}$ & $\mathrm{CS}(=\mathrm{O})(=\mathrm{O}) \mathrm{NCCN}=\mathrm{C}=\mathrm{S}$ \\
\hline 36 & $10\{36\}$ & $\mathrm{S}=\mathrm{C}=\mathrm{NCC} 1 \mathrm{CCC} 1$ \\
\hline 37 & $\mathbf{1 0}\{37\}$ & $\mathrm{S}=\mathrm{C}=\mathrm{NCC} 1 \mathrm{cccnc} 1$ \\
\hline 38 & $\mathbf{1 0}\{38\}$ & $\mathrm{CC}(\mathrm{N}=\mathrm{C}=\mathrm{S}) \mathrm{c} 1 \mathrm{cccs} 1$ \\
\hline 39 & $\mathbf{1 0}\{39\}$ & $\mathrm{CS}(=\mathrm{O})(=\mathrm{O}) \mathrm{CCN}=\mathrm{C}=\mathrm{S}$ \\
\hline 40 & $10\{40\}$ & $\mathrm{S}=\mathrm{C}=\mathrm{NC} 1 \mathrm{CCOCC} 1$ \\
\hline 41 & $\mathbf{1 0}\{41\}$ & $\mathrm{S}=\mathrm{C}=\mathrm{NCC} 1 \mathrm{cccs} 1$ \\
\hline 42 & $\mathbf{1 0}\{42\}$ & $\mathrm{CC} 1(\mathrm{CC} 1) \mathrm{N}=\mathrm{C}=\mathrm{S}$ \\
\hline 43 & $\mathbf{1 0}\{43\}$ & $\mathrm{S}=\mathrm{C}=\mathrm{NC} 1 \mathrm{CCO} 2 \operatorname{ccccc} 12$ \\
\hline 44 & $\mathbf{1 0}\{44\}$ & $\mathrm{S}=\mathrm{C}=\mathrm{NC}(\mathrm{C} 1 \mathrm{CC} 1) \mathrm{C} 1 \mathrm{CC} 1$ \\
\hline 45 & $\mathbf{1 0}\{45\}$ & $\mathrm{FC}(\mathrm{F})(\mathrm{F}) \mathrm{Oc} 1 \mathrm{ccc}(\mathrm{CN}=\mathrm{C}=\mathrm{S}) \mathrm{cc} 1$ \\
\hline 46 & $10\{46\}$ & $\mathrm{S}=\mathrm{C}=\mathrm{NC} 1 \mathrm{CC} 2 \operatorname{ccccc} 2 \mathrm{C} 1$ \\
\hline 47 & $10\{47\}$ & $\mathrm{CC}(\mathrm{C})(\mathrm{C}) \mathrm{CN}=\mathrm{C}=\mathrm{S}$ \\
\hline 48 & $\mathbf{1 0}\{48\}$ & $\mathrm{CSCCN}=\mathrm{C}=\mathrm{S}$ \\
\hline 49 & $\mathbf{1 0}\{49\}$ & $\mathrm{CC}(\mathrm{Cc} 1 \operatorname{ccccc} 1) \mathrm{N}=\mathrm{C}=\mathrm{S}$ \\
\hline 50 & $\mathbf{1 0}\{50\}$ & $\operatorname{Brc} 1 \operatorname{ccc}(\mathrm{CN}=\mathrm{C}=\mathrm{S}) \operatorname{cc} 1$ \\
\hline 51 & $\mathbf{1 0}\{51\}$ & $\mathrm{S}=\mathrm{C}=\mathrm{NCC} 1 \mathrm{CCOC} 1$ \\
\hline 52 & $\mathbf{1 0}\{52\}$ & $\mathrm{S}=\mathrm{C}=\mathrm{NC} 1 \mathrm{CCC} 1$ \\
\hline 53 & $\mathbf{1 0}\{53\}$ & $\mathrm{Fc} 1 \mathrm{ccc}(\mathrm{CCN}=\mathrm{C}=\mathrm{S}) \mathrm{cc} 1$ \\
\hline
\end{tabular}


$\mathrm{S}=\mathrm{C}=\mathrm{NCC} 1 \mathrm{CCCCO} 1$

55

$10\{55\}$

$\mathrm{Cc} 1 \mathrm{cc}(\mathrm{CN}=\mathrm{C}=\mathrm{S})$ no1

56

$10\{56\}$

$\mathrm{S}=\mathrm{C}=\mathrm{NCc} 1 \mathrm{ccon} 1$

57

$10\{57\}$

$\mathrm{FC}(\mathrm{F}) \mathrm{CN}=\mathrm{C}=\mathrm{S}$

58

$10\{58\}$

$\mathrm{CC} 1(\mathrm{CCC} 1) \mathrm{N}=\mathrm{C}=\mathrm{S}$ 
Table S2. SMILES strings for the regents 11.

\begin{tabular}{|c|c|c|}
\hline \# & Reagent 11 & SMILE \\
\hline 1 & $\mathbf{1 1}\{1\}$ & $\mathrm{Cl} \cdot \mathrm{CNS}(=\mathrm{O})(=\mathrm{O}) \mathrm{c} 1 \mathrm{cccc} 2 \mathrm{CNCCc} 12$ \\
\hline 2 & $\mathbf{1 1}\{2\}$ & $\mathrm{Cl} . \mathrm{O}=\mathrm{C}(\mathrm{NC} 1 \mathrm{CC} 1) \mathrm{C}(=\mathrm{O}) \mathrm{N} 1 \mathrm{CCNCC} 1$ \\
\hline 3 & $\mathbf{1 1}\{3\}$ & $\mathrm{OC} 1(\mathrm{Cc} 2 \mathrm{nc}(\mathrm{no} 2) \mathrm{C} 2 \mathrm{CC} 2) \mathrm{CCCNC} 1$ \\
\hline 4 & $11\{4\}$ & $\mathrm{C} 1 \mathrm{CC} 2(\mathrm{C} 1) \mathrm{CNCCO} 2$ \\
\hline 5 & $\mathbf{1 1}\{5\}$ & Cl.Fc1cc(F)cc(c1)C1COCCN1 \\
\hline 6 & $11\{6\}$ & $\mathrm{Fc} 1 \mathrm{ccc} 2 \mathrm{CNCCOc} 2 \mathrm{c} 1$ \\
\hline 7 & $11\{7\}$ & $\mathrm{Cl} . \mathrm{CC} 1=\mathrm{C}(\mathrm{C}) \mathrm{CNCC} 1$ \\
\hline 8 & $\mathbf{1 1}\{8\}$ & C1C[1C@@H]2NCC3(CCOCC3)[1C@@H]2C1 \\
\hline 9 & $\mathbf{1 1}\{9\}$ & Clc1ccc2[nH]c(nc2c1)C1CCCN1 \\
\hline 10 & $\mathbf{1 1}\{10\}$ & Cl.Cn1 cccc1C(=O)N1CCNCC1 \\
\hline 11 & $\mathbf{1 1}\{11\}$ & $\mathrm{Cl} . \mathrm{CNC}(=\mathrm{O}) \mathrm{CNCc} 1 \mathrm{ccccc} 1$ \\
\hline 12 & $\mathbf{1 1}\{12\}$ & Cn1cnnc1C1CCCNC1 \\
\hline 13 & $\mathbf{1 1}\{13\}$ & $\mathrm{C} 1 \mathrm{CNC}(\mathrm{C} 1) \mathrm{C} 1 \mathrm{CCCO} 1$ \\
\hline 14 & $\mathbf{1 1}\{14\}$ & $\mathrm{CC}(\mathrm{CS}(\mathrm{C})(=\mathrm{O})=\mathrm{O}) \mathrm{NC} 1 \mathrm{CC} 1$ \\
\hline 15 & $\mathbf{1 1}\{15\}$ & Cl.COC1 $(\mathrm{CCNCC} 1) \mathrm{C}(\mathrm{F})(\mathrm{F}) \mathrm{F}$ \\
\hline 16 & $\mathbf{1 1}\{16\}$ & $\mathrm{O}=\mathrm{C} 1 \mathrm{NCCN} 1 \mathrm{C} 1 \mathrm{CCCNC} 1$ \\
\hline 17 & $\mathbf{1 1}\{17\}$ & COCCCNCCOC \\
\hline 18 & $\mathbf{1 1}\{18\}$ & $\mathrm{NC}(=\mathrm{O})[\mathrm{C} @ \mathrm{H}] 1 \mathrm{C}[\mathrm{C} @ @ \mathrm{H}](\mathrm{F}) \mathrm{CN} 1$ \\
\hline 19 & $\mathbf{1 1}\{19\}$ & $\mathrm{Cl} . \mathrm{COC} 1(\mathrm{CCNC} 1) \mathrm{C}(\mathrm{F})(\mathrm{F}) \mathrm{F}$ \\
\hline 20 & $\mathbf{1 1}\{20\}$ & $\mathrm{CCC} 1(\mathrm{CCNCC} 1) \mathrm{N} 1 \mathrm{CCOCC} 1$ \\
\hline 21 & $\mathbf{1 1}\{21\}$ & Cl.CNC1CCN $(\mathrm{CC} 1) \mathrm{S}(\mathrm{C})(=\mathrm{O})=\mathrm{O}$ \\
\hline 22 & $\mathbf{1 1}\{22\}$ & Cl.Cl.C1CNCCC(C1)n1ccnn1 \\
\hline 23 & $\mathbf{1 1}\{23\}$ & $\mathrm{C} 1 \mathrm{CCc} 2 \operatorname{cccc} 2 \mathrm{NC} 1$ \\
\hline 24 & $11\{24\}$ & $\mathrm{CC} 1(\mathrm{C}) \mathrm{CNCCC}(\mathrm{O}) \mathrm{C} 1$ \\
\hline 25 & $\mathbf{1 1}\{25\}$ & $\mathrm{C} 1 \mathrm{CNC}(\mathrm{C} 1) \mathrm{c} 1 \mathrm{cccnc} 1$ \\
\hline
\end{tabular}




\begin{tabular}{|c|c|c|}
\hline 26 & $\mathbf{1 1}\{26\}$ & Clc1 $1 \mathrm{ccc} 2 \mathrm{CNCCc} 2 \mathrm{c} 1$ \\
\hline 27 & $\mathbf{1 1}\{27\}$ & $\mathrm{C} 1 \mathrm{CC}(\mathrm{CCN} 1) \mathrm{c} 1 \mathrm{ccncc} 1$ \\
\hline 28 & $\mathbf{1 1}\{28\}$ & Cl.COC1CCCNC1 \\
\hline 29 & $\mathbf{1 1}\{29\}$ & $\mathrm{Cc} 1 \mathrm{ccc} 2 \mathrm{CNCCc} 2 \mathrm{c} 1$ \\
\hline 30 & $\mathbf{1 1}\{30\}$ & Cl.C1CC $=\mathrm{CCN} 1$ \\
\hline 31 & $\mathbf{1 1}\{31\}$ & $\mathrm{C} 1 \mathrm{CC}(\mathrm{CCN} 1) \mathrm{c} 1 \mathrm{ccccc} 1$ \\
\hline 32 & $\mathbf{1 1}\{32\}$ & $\mathrm{CC} 1 \mathrm{NCCc} 2 \mathrm{cccc}(\mathrm{F}) \mathrm{c} 12$ \\
\hline 33 & $\mathbf{1 1}\{33\}$ & $\mathrm{Cl} . \mathrm{O}=\mathrm{C} 1 \mathrm{CCNCCN} 1$ \\
\hline 34 & $\mathbf{1 1}\{34\}$ & $\mathrm{CCC} 1 \mathrm{NCC} 2 \mathrm{sccc} 12$ \\
\hline 35 & $\mathbf{1 1}\{35\}$ & $\mathrm{C} 1 \mathrm{CNCC}(\mathrm{C} 1) \mathrm{c} 1 \mathrm{ncc}[\mathrm{nH}] 1$ \\
\hline 36 & $\mathbf{1 1}\{36\}$ & $\mathrm{Cl} . \mathrm{O}[\mathrm{C} @ \mathrm{H}] 1 \mathrm{CCNC} 1$ \\
\hline 37 & $\mathbf{1 1}\{37\}$ & $\mathrm{COc} 1 \mathrm{ccc} 2 \mathrm{CNCCc} 2 \mathrm{c} 1$ \\
\hline 38 & $\mathbf{1 1}\{38\}$ & Cl.Cl.C1Cc2nc[nH]c2CN1 \\
\hline 39 & $\mathbf{1 1}\{39\}$ & $\mathrm{Fc} 1 \mathrm{ccc} 2 \mathrm{NCCc} 2 \mathrm{c} 1$ \\
\hline 40 & $\mathbf{1 1}\{40\}$ & Cl.Cl.C1Cc2[nH]ncc2CN1 \\
\hline 41 & $\mathbf{1 1}\{41\}$ & Cl.CNCc1 $1 \mathrm{ccc}(\mathrm{OC}) \mathrm{nc} 1$ \\
\hline 42 & $\mathbf{1 1}\{42\}$ & Cl.Cl.C1CNCC(C1)c1nnc2 $\operatorname{cccc} 12$ \\
\hline 43 & $\mathbf{1 1}\{43\}$ & Cl.CN[C@H]1C[C@H](C1)C(N)=O \\
\hline 44 & $\mathbf{1 1}\{44\}$ & $\mathrm{Cl} . \mathrm{O}=\mathrm{c} 1[\mathrm{nH}] \mathrm{c} 2 \mathrm{cccc}(\mathrm{N} 3 \mathrm{CCNCC} 3) \mathrm{c} 2[\mathrm{nH}] 1$ \\
\hline 45 & $\mathbf{1 1}\{45\}$ & $\mathrm{C}(\mathrm{Cc} 1 \mathrm{cnc} \mathrm{c} 1) \mathrm{N} 1 \mathrm{CCNCC} 1$ \\
\hline 46 & $\mathbf{1 1}\{46\}$ & $\mathrm{Cc} 1 \mathrm{ccc}(\mathrm{CNC} 2 \mathrm{CC} 2) \mathrm{o} 1$ \\
\hline 47 & $\mathbf{1 1}\{47\}$ & $\mathrm{Cl} . \mathrm{CC} 12 \mathrm{CNCC} 1(\mathrm{C}) \mathrm{C}(=\mathrm{O}) \mathrm{NC} 2=\mathrm{O}$ \\
\hline 48 & $\mathbf{1 1}\{48\}$ & Cc1cenc(c1)N1CCNCC1 \\
\hline 49 & $\mathbf{1 1}\{49\}$ & $\operatorname{CNCCS}(\mathrm{C})(=\mathrm{O})=\mathrm{O}$ \\
\hline 50 & $\mathbf{1 1}\{50\}$ & $\mathrm{CS}(=\mathrm{O})(=\mathrm{O}) \mathrm{NCC} 1 \mathrm{CCCNC} 1$ \\
\hline 51 & $\mathbf{1 1}\{51\}$ & $\mathrm{CCNCC} 1 \mathrm{CCOC} 1$ \\
\hline 52 & $\mathbf{1 1}\{52\}$ & $\mathrm{C} 1 \mathrm{CCN}(\mathrm{CC} 1) \mathrm{C} 1 \mathrm{CCNCC} 1$ \\
\hline 53 & $\mathbf{1 1}\{53\}$ & $\operatorname{CNCCNC}(\mathrm{C})=\mathrm{O}$ \\
\hline
\end{tabular}




\begin{tabular}{|c|c|c|}
\hline 54 & $\mathbf{1 1}\{54\}$ & $\mathrm{C} 1 \mathrm{CCC}(\mathrm{CC} 1) \mathrm{N} 1 \mathrm{CCNCC} 1$ \\
\hline 55 & $\mathbf{1 1}\{55\}$ & CNCc1ncen1C \\
\hline 56 & $\mathbf{1 1}\{56\}$ & Cn1nccc1C1CCCNC1 \\
\hline 57 & $\mathbf{1 1}\{57\}$ & $\mathrm{C} 1 \mathrm{CNc} 2 \mathrm{c}[\mathrm{nH}] \mathrm{nc} 2 \mathrm{C} 1$ \\
\hline 58 & $\mathbf{1 1}\{58\}$ & C[1C@@H]1CCNC[1C@H](C)O1 \\
\hline 59 & $\mathbf{1 1}\{59\}$ & Br.Oc1ccc2CNCc2c1 \\
\hline 60 & $11\{60\}$ & $\mathrm{Cl} . \mathrm{O}=\mathrm{C} 1 \mathrm{CC} 2 \mathrm{CCC}(\mathrm{CN} 1) \mathrm{N} 2$ \\
\hline 61 & $\mathbf{1 1}\{61\}$ & $\mathrm{C} 1 \mathrm{CC} 2(\mathrm{CN} 1) \mathrm{CCOCC} 2$ \\
\hline 62 & $\mathbf{1 1}\{62\}$ & $\mathrm{Cl} \cdot \mathrm{C} 1 \mathrm{CC} 2 \mathrm{NC} 1 \mathrm{CC}=\mathrm{C} 2$ \\
\hline 63 & $\mathbf{1 1}\{63\}$ & $\mathrm{Cl} . \mathrm{O}=\mathrm{C} 1 \mathrm{NCC} 2 \mathrm{CNCCN} 12$ \\
\hline 64 & $11\{64\}$ & $\mathrm{Cl} . \mathrm{FC} 1(\mathrm{~F}) \mathrm{CNCCOC} 1$ \\
\hline 65 & $\mathbf{1 1}\{65\}$ & $\mathrm{Cl} \cdot \mathrm{FC} 1=\mathrm{CCCNC} 1$ \\
\hline 66 & $11\{66\}$ & $\mathrm{Cl} . \mathrm{C} 1 \mathrm{NCC} 2 \mathrm{C} 1 \mathrm{C} 1 \mathrm{OC} 2 \mathrm{C}=\mathrm{C} 1$ \\
\hline 67 & $\mathbf{1 1}\{67\}$ & $\mathrm{Cl} . \mathrm{OCC} 1 \mathrm{CC} 2 \mathrm{CCC} 1 \mathrm{~N} 2$ \\
\hline 68 & $\mathbf{1 1}\{68\}$ & $\mathrm{C} 1 \mathrm{CNCC}(\mathrm{C} 1) \mathrm{c} 1 \mathrm{ccn}[\mathrm{nH}] 1$ \\
\hline 69 & $\mathbf{1 1}\{69\}$ & $\mathrm{CNCC}(\mathrm{C})(\mathrm{C}) \mathrm{CO}$ \\
\hline 70 & $\mathbf{1 1}\{70\}$ & $\mathrm{COc} 1 \mathrm{cccc}(\mathrm{c} 1) \mathrm{C} 1 \mathrm{CCNCC} 1$ \\
\hline 71 & $\mathbf{1 1}\{71\}$ & $\mathrm{CCn} 1 \mathrm{cc}(\mathrm{CNC}) \mathrm{cn} 1$ \\
\hline 72 & $\mathbf{1 1}\{72\}$ & CCNCc1ccoc1 \\
\hline 73 & $\mathbf{1 1}\{73\}$ & $\mathrm{C} 1 \mathrm{Cc} 2 \mathrm{cn}[\mathrm{nH}] \mathrm{c} 2 \mathrm{CN} 1$ \\
\hline 74 & $\mathbf{1 1}\{74\}$ & $\mathrm{Cc} 1 \mathrm{nc}(\mathrm{sc} 1 \mathrm{C}) \mathrm{N} 1 \mathrm{CCNCC} 1$ \\
\hline 75 & $\mathbf{1 1}\{75\}$ & $\mathrm{Cl} . \mathrm{Oc} 1 \mathrm{cccc}(\mathrm{c} 1) \mathrm{C}(=\mathrm{O}) \mathrm{N} 1 \mathrm{CCNCC} 1$ \\
\hline 76 & $\mathbf{1 1}\{76\}$ & $\mathrm{C} 1 \mathrm{Cn} 2 \mathrm{ccnc} 2 \mathrm{~N} 1$ \\
\hline 77 & $\mathbf{1 1}\{77\}$ & $\mathrm{C} 1 \mathrm{CC} 1 \mathrm{c} 1 \mathrm{ncc} 2 \mathrm{CNCC} 2 \mathrm{n} 1$ \\
\hline 78 & $\mathbf{1 1}\{78\}$ & Cl.COc1 $1 \mathrm{ccc} 2 \mathrm{CNCCCc} 2 \mathrm{c} 1$ \\
\hline 79 & $\mathbf{1 1}\{79\}$ & $\mathrm{Cl} . \mathrm{NC}(=\mathrm{O})[\mathrm{C} @ \mathrm{H}] 1 \mathrm{CCCN} 1$ \\
\hline 80 & $\mathbf{1 1}\{80\}$ & $\mathrm{Cl} . \mathrm{C}(\mathrm{OC} 1 \mathrm{CCNCC} 1) \mathrm{C} 1 \mathrm{CCCO} 1$ \\
\hline 81 & $\mathbf{1 1}\{81\}$ & $\mathrm{NC}(=\mathrm{O}) \mathrm{C} 1 \mathrm{CNCCO} 1$ \\
\hline
\end{tabular}




\begin{tabular}{|c|c|c|}
\hline 82 & $\mathbf{1 1}\{82\}$ & $\mathrm{C} 1 \mathrm{CCC} 2(\mathrm{C} 1) \mathrm{CCNC} 2$ \\
\hline 83 & $\mathbf{1 1}\{83\}$ & $\mathrm{CNC} 1 \mathrm{CCCc} 2 \mathrm{nc}(\mathrm{C}) \mathrm{sc} 12$ \\
\hline 84 & $\mathbf{1 1}\{84\}$ & $\mathrm{Cl} \cdot \mathrm{CNCC}(=\mathrm{O}) \mathrm{N} 1 \mathrm{CCCC} 1$ \\
\hline 85 & $\mathbf{1 1}\{85\}$ & Fc1 $\operatorname{ccc}(\mathrm{c} 1) \mathrm{N} 1 \mathrm{CCNCC} 1$ \\
\hline 86 & $\mathbf{1 1}\{86\}$ & OCC1CCCCCN1 \\
\hline 87 & $\mathbf{1 1}\{87\}$ & $\mathrm{CNCc} 1 \mathrm{ccoc} 1 \mathrm{C}$ \\
\hline 88 & $\mathbf{1 1}\{88\}$ & $\mathrm{C}(\mathrm{C} 1 \mathrm{CCOCC} 1) \mathrm{N} 1 \mathrm{CCNCC} 1$ \\
\hline 89 & $\mathbf{1 1}\{89\}$ & $\mathrm{CNC}(\mathrm{C}) \mathrm{c} 1 \mathrm{ccc} \operatorname{co} 1$ \\
\hline 90 & $11\{90\}$ & $\mathrm{C}(\mathrm{N} 1 \mathrm{CCNCC} 1) \mathrm{c} 1 \mathrm{cscn} 1$ \\
\hline 91 & $\mathbf{1 1}\{91\}$ & $\mathrm{CC} 1 \mathrm{NCCOc} 2 \operatorname{ccccc} 12$ \\
\hline 92 & $\mathbf{1 1}\{92\}$ & $\mathrm{C} 1 \mathrm{CNCC}(\mathrm{C} 1) \mathrm{c} 1 \mathrm{ccccc} 1$ \\
\hline 93 & $\mathbf{1 1}\{93\}$ & $\mathrm{C}(\mathrm{C} 1 \mathrm{CCCCC} 1) \mathrm{N} 1 \mathrm{CCNCC} 1$ \\
\hline 94 & $\mathbf{1 1}\{94\}$ & $\mathrm{CNc} 1 \mathrm{c}(\mathrm{C})$ noc $1 \mathrm{C}$ \\
\hline 95 & $\mathbf{1 1}\{95\}$ & $\mathrm{CNC}(\mathrm{C}) \mathrm{c} 1 \mathrm{sc}(\mathrm{C}) \mathrm{nc} 1 \mathrm{C}$ \\
\hline 96 & $\mathbf{1 1}\{96\}$ & $\mathrm{CC}(\mathrm{C}) \mathrm{OC} 1 \mathrm{CCNCC} 1$ \\
\hline 97 & $\mathbf{1 1}\{97\}$ & $\mathrm{Cl} . \mathrm{O}=\mathrm{C}(\mathrm{NC} 1 \mathrm{CC} 1) \mathrm{C} 1 \mathrm{CCCN} 1$ \\
\hline 98 & $\mathbf{1 1}\{98\}$ & $\mathrm{CNC}(\mathrm{C}) \mathrm{c} 1 \mathrm{ccco} 1$ \\
\hline 99 & $\mathbf{1 1}\{99\}$ & $\mathrm{C} 1 \mathrm{Cn} 2 \mathrm{cncc} 2 \mathrm{CN} 1$ \\
\hline 100 & $\mathbf{1 1}\{100\}$ & $\mathrm{Cl} . \mathrm{O}=\mathrm{S} 1 \mathrm{CCNCC} 1$ \\
\hline 101 & $\mathbf{1 1}\{101\}$ & Cl.CNCc1ccc(C)s1 \\
\hline 102 & $\mathbf{1 1}\{102\}$ & CNCc1senc1C \\
\hline 103 & $\mathbf{1 1}\{103\}$ & Cl.Cc $1 \mathrm{ccc} 2[\mathrm{nH}] \mathrm{c} 3 \mathrm{CCNCc} 3 \mathrm{c} 2 \mathrm{c} 1$ \\
\hline 104 & $\mathbf{1 1}\{104\}$ & $\mathrm{C} 1 \mathrm{CCC} 2(\mathrm{C} 1) \mathrm{CNCCOC} 2$ \\
\hline 105 & $\mathbf{1 1}\{105\}$ & $\mathrm{CN} 1 \mathrm{CCNCc} 2 \operatorname{ccccc} 12$ \\
\hline 106 & $\mathbf{1 1}\{106\}$ & $\mathrm{C} 1 \mathrm{Cc} 2 \operatorname{cccc} 2 \mathrm{CCN} 1$ \\
\hline 107 & $\mathbf{1 1}\{107\}$ & Cl.Fc1ccc(F)c2CNCCc12 \\
\hline 108 & $\mathbf{1 1}\{108\}$ & $\mathrm{O}=\mathrm{C} 1 \mathrm{CCCN} 1 \mathrm{C} 1 \mathrm{CCNCC} 1$ \\
\hline 109 & $\mathbf{1 1}\{109\}$ & Cl.Cn1nc2CCNCc2cc1=O \\
\hline
\end{tabular}




\begin{tabular}{|c|c|c|}
\hline 110 & $\mathbf{1 1}\{110\}$ & Cl.CNCC1(O)CCOCC 1 \\
\hline 111 & $\mathbf{1 1}\{111\}$ & $\mathrm{Cl} . \mathrm{CC} 1 \mathrm{CNCC}(\mathrm{C}) \mathrm{S} 1(=\mathrm{O})=\mathrm{O}$ \\
\hline 112 & $\mathbf{1 1}\{112\}$ & $\mathrm{Cl} . \mathrm{CCOC} 1(\mathrm{C}) \mathrm{CCNCC} 1$ \\
\hline 113 & $\mathbf{1 1}\{113\}$ & $\mathrm{FC}(\mathrm{F})(\mathrm{F}) \mathrm{CN} 1 \mathrm{CNCC} 1=\mathrm{O}$ \\
\hline 114 & $\mathbf{1 1}\{114\}$ & $\operatorname{Cl} . \mathrm{CS}(=\mathrm{O})(=\mathrm{O}) \mathrm{C} 1 \mathrm{CCCNCC} 1$ \\
\hline 115 & $\mathbf{1 1}\{115\}$ & $\mathrm{Cl} . \mathrm{O}=\mathrm{C} 1 \mathrm{Nc} 2 \mathrm{cccc} 2 \mathrm{C} 11 \mathrm{CCNC} 1$ \\
\hline 116 & $\mathbf{1 1}\{116\}$ & Cl.O[1C@@H]1CC[1C@@H]2CNC[1C@@H]2C1 \\
\hline 117 & $11\{117\}$ & $\mathrm{CC}(\mathrm{C})(\mathrm{CC} 1 \mathrm{CCCNC} 1) \mathrm{C}(\mathrm{N})=\mathrm{O}$ \\
\hline 118 & $\mathbf{1 1}\{118\}$ & Cl.C[1C@@H]1NCC[1C@@ $\mathrm{H}] 1 \mathrm{O}$ \\
\hline 119 & $\mathbf{1 1}\{119\}$ & $\mathrm{Cl} . \mathrm{CCNC} 1 \mathrm{CCNC} 1=\mathrm{O}$ \\
\hline 120 & $\mathbf{1 1}\{120\}$ & Cl.Cl.CN1CCNCC1CO \\
\hline 121 & $\mathbf{1 1}\{121\}$ & I.I.Cc1nnc(N2CCNCC2)n1C \\
\hline 122 & $\mathbf{1 1}\{122\}$ & $\mathrm{CCNCCO}$ \\
\hline 123 & $\mathbf{1 1}\{123\}$ & $\mathrm{Cl} . \mathrm{CC}(\mathrm{C})(\mathrm{C}) \mathrm{C} 1 \mathrm{CNCCO} 1$ \\
\hline 124 & $\mathbf{1 1}\{124\}$ & Cl.Cl.Cc1 $1 \mathrm{cc}(=\mathrm{O})[\mathrm{nH}] \mathrm{c}(\mathrm{n} 1) \mathrm{N} 1 \mathrm{CCNCC} 1$ \\
\hline 125 & $\mathbf{1 1}\{125\}$ & $\mathrm{CNCC} 1(\mathrm{O}) \mathrm{CCCC} 1$ \\
\hline 126 & $11\{126\}$ & $\mathrm{C}(\mathrm{N} 1 \mathrm{CCNCC} 1) \mathrm{c} 1 \mathrm{nc}[\mathrm{nH}] \mathrm{n} 1$ \\
\hline 127 & $\mathbf{1 1}\{127\}$ & $\mathrm{Cl} . \mathrm{NC}(=\mathrm{O})[\mathrm{C} @ @ \mathrm{H}] 1 \mathrm{CCCN} 1$ \\
\hline 128 & $\mathbf{1 1}\{128\}$ & $\mathrm{Cl} . \mathrm{C} 1 \mathrm{CC} 1 \mathrm{C} 1 \mathrm{CCCNC} 1$ \\
\hline 129 & $\mathbf{1 1}\{129\}$ & Cl.CNC[C@@ $@$ H $(\mathrm{C}) \mathrm{O}$ \\
\hline 130 & $\mathbf{1 1}\{130\}$ & Cl.CCC1CCCN1 \\
\hline 131 & $\mathbf{1 1}\{131\}$ & $\mathrm{Cl} . \mathrm{OCC} 1 \mathrm{NCC}=\mathrm{C} 1$ \\
\hline 132 & $\mathbf{1 1}\{132\}$ & $\mathrm{Cl} . \mathrm{CC}(\mathrm{C})(\mathrm{C}) \mathrm{C} 1=\mathrm{CCNCC} 1$ \\
\hline 133 & $\mathbf{1 1}\{133\}$ & $\mathrm{Cl} . \mathrm{CC} 1(\mathrm{C}) \mathrm{CCCN} 1$ \\
\hline 134 & $\mathbf{1 1}\{134\}$ & $\mathrm{Cl} . \mathrm{CN}(\mathrm{C}) \mathrm{C}(=\mathrm{O}) \mathrm{NC} 1 \mathrm{CCCNC} 1$ \\
\hline 135 & $11\{135\}$ & Cl.FC(F)(F)[C@@ @ $] 1 \mathrm{CCCNC1}$ \\
\hline 136 & $\mathbf{1 1}\{136\}$ & Cl.CC $(\mathrm{C}) \mathrm{NC}(=\mathrm{O})[\mathrm{C} @ @ \mathrm{H}] 1 \mathrm{CCCNC} 1$ \\
\hline 137 & $\mathbf{1 1}\{137\}$ & $\mathrm{CCN} 1 \mathrm{CCC} 2 \mathrm{CCC}(\mathrm{C} 1) \mathrm{N} 2$ \\
\hline
\end{tabular}




\begin{tabular}{|c|c|c|}
\hline 138 & $\mathbf{1 1}\{138\}$ & Clc1ecce $2 \mathrm{CCNCc} 12$ \\
\hline 139 & $\mathbf{1 1}\{139\}$ & $\mathrm{Fc} 1 \mathrm{cccc} 2 \mathrm{CCNc} 12$ \\
\hline 140 & $11\{140\}$ & Cl.CNc1 cnn(C)c1 \\
\hline 141 & $\mathbf{1 1}\{141\}$ & $\mathrm{Cl} . \mathrm{O}=\mathrm{C} 1 \mathrm{CC} 2(\mathrm{CN} 1) \mathrm{CCNCC} 2$ \\
\hline 142 & $\mathbf{1 1}\{142\}$ & $\mathrm{C} 1 \mathrm{CC}(\mathrm{CCN} 1) \mathrm{c} 1 \mathrm{cn}[\mathrm{nH}] \mathrm{c} 1$ \\
\hline 143 & $\mathbf{1 1}\{143\}$ & Cl.CNc1 cen(C)n1 \\
\hline 144 & $\mathbf{1 1}\{144\}$ & $\mathrm{C} 1 \mathrm{CN} 2 \mathrm{CCOCC} 2 \mathrm{CN} 1$ \\
\hline 145 & $\mathbf{1 1}\{145\}$ & $\mathrm{Cc} 1 \mathrm{ccc} 2 \mathrm{CCNCc} 12$ \\
\hline 146 & $11\{146\}$ & Cl.Cl.C1CNC(C1)c1cen[nH]1 \\
\hline 147 & $\mathbf{1 1}\{147\}$ & $\mathrm{Clc} 1 \mathrm{cnn}(\mathrm{c} 1) \mathrm{C} 1 \mathrm{CCNC} 1$ \\
\hline 148 & $\mathbf{1 1}\{148\}$ & $\mathrm{Cc} 1 \mathrm{csc}(\mathrm{n} 1) \mathrm{C} 1 \mathrm{CCNC} 1$ \\
\hline 149 & $\mathbf{1 1}\{149\}$ & $\mathrm{C} 1 \mathrm{CNC}(\mathrm{C} 1) \mathrm{c} 1 \mathrm{ccncc} 1$ \\
\hline 150 & $\mathbf{1 1}\{150\}$ & $\mathrm{CC} 1 \mathrm{CNCC}(=\mathrm{O}) \mathrm{N} 1$ \\
\hline 151 & $\mathbf{1 1}\{151\}$ & Cl.CC1CNCCOC1 \\
\hline 152 & $\mathbf{1 1}\{152\}$ & Cl.FC1CCNCC1 \\
\hline 153 & $\mathbf{1 1}\{153\}$ & $\mathrm{CN}(\mathrm{C}) \mathrm{C} 1 \mathrm{CCNC} 1$ \\
\hline 154 & $\mathbf{1 1}\{154\}$ & Cl.FC1(CCNCC1)C1CC1 \\
\hline 155 & $\mathbf{1 1}\{155\}$ & Cl.Clc1ccce2NCCc12 \\
\hline 156 & $\mathbf{1 1}\{156\}$ & Cl.FC(F)(F)[C@H]1CCCNC1 \\
\hline 157 & $\mathbf{1 1}\{157\}$ & $\mathrm{Cl} . \mathrm{CC} 1(\mathrm{~F}) \mathrm{CCCNC} 1$ \\
\hline 158 & $\mathbf{1 1}\{158\}$ & Cl.FC(F)C1CCNCC1 \\
\hline 159 & $\mathbf{1 1}\{159\}$ & Cl.Fc1cccenCCc12 \\
\hline 160 & $\mathbf{1 1}\{160\}$ & $\mathrm{Cl} . \mathrm{C} 1 \mathrm{CCC} 2(\mathrm{C} 1) \mathrm{CCNCC} 2$ \\
\hline 161 & $11\{161\}$ & $\mathrm{CNC}(\mathrm{C}) \mathrm{c} 1 \mathrm{cccnc} 1$ \\
\hline 162 & $\mathbf{1 1}\{162\}$ & $\mathrm{Cl} . \mathrm{C} 1 \mathrm{Cc} 2 \mathrm{nnc}(\mathrm{C} 3 \mathrm{CCCNC} 3) \mathrm{n} 2 \mathrm{Cl}$ \\
\hline 163 & $\mathbf{1 1}\{163\}$ & $\mathrm{Cc} 1 \mathrm{cccc}(\mathrm{n} 1) \mathrm{C} 1 \mathrm{CCCN} 1$ \\
\hline 164 & $\mathbf{1 1}\{164\}$ & $\mathrm{C} 1 \mathrm{C}[\mathrm{C} @ \mathrm{H}](\mathrm{CN} 1) \mathrm{Oc} 1 \mathrm{cccc} 1$ \\
\hline 165 & $\mathbf{1 1}\{165\}$ & $\mathrm{C}(\mathrm{C} 1 \mathrm{CCNCC} 1) \mathrm{N} 1 \mathrm{CCOCC} 1$ \\
\hline
\end{tabular}




\begin{tabular}{|c|c|c|}
\hline 166 & $\mathbf{1 1}\{166\}$ & Cl.OC1CCOC2(CCNCC2)C1 \\
\hline 167 & $\mathbf{1 1}\{167\}$ & Cl.Cl.C1CNC(C1)c1nccen1 \\
\hline 168 & $11\{168\}$ & $\mathrm{C} 1 \mathrm{COC}(\mathrm{CN} 1) \mathrm{c} 1 \mathrm{ccco} 1$ \\
\hline 169 & $\mathbf{1 1}\{169\}$ & $\mathrm{Cl} . \mathrm{C} 1 \mathrm{CC} 2(\mathrm{C} 1) \mathrm{CCNC} 2$ \\
\hline 170 & $\mathbf{1 1}\{170\}$ & $\mathrm{C} 1 \mathrm{CNCC}(\mathrm{C} 1) \mathrm{C} 1 \mathrm{CCOCC} 1$ \\
\hline 171 & $\mathbf{1 1}\{171\}$ & $\mathrm{C} 1 \mathrm{CC}(\mathrm{CN} 1) \mathrm{n} 1 \mathrm{ccc} 1$ \\
\hline 172 & $\mathbf{1 1}\{172\}$ & Cl.COC1CCCNCC1 \\
\hline 173 & $\mathbf{1 1}\{173\}$ & $\mathrm{Cl} . \mathrm{O}=\mathrm{C} 1 \mathrm{CNCCCN} 1$ \\
\hline 174 & $11\{174\}$ & Cl.FC(F)[C@@ @ $] 1 \mathrm{CCCN} 1$ \\
\hline 175 & $\mathbf{1 1}\{175\}$ & $\mathrm{Cn} 1 \mathrm{ccc}(\mathrm{n} 1) \mathrm{C} 1 \mathrm{CCCNC} 1$ \\
\hline 176 & $\mathbf{1 1}\{176\}$ & $\mathrm{CNCc} 1 \mathrm{cnc}(\mathrm{C}) \mathrm{s} 1$ \\
\hline 177 & $\mathbf{1 1}\{177\}$ & $\mathrm{CC}(\mathrm{C}) \mathrm{C}(=\mathrm{O}) \mathrm{N} 1 \mathrm{CCCNCC} 1$ \\
\hline 178 & $\mathbf{1 1}\{178\}$ & $\mathrm{CNCc} 1 \mathrm{ccoc} 1$ \\
\hline 179 & $\mathbf{1 1}\{179\}$ & $\mathrm{OC}[\mathrm{C} @ \mathrm{H}] 1[\mathrm{C} @ @ \mathrm{H}] 2 \mathrm{CNC}[\mathrm{C} @ \mathrm{H}] 12$ \\
\hline 180 & $\mathbf{1 1}\{180\}$ & $\mathrm{CNC}(\mathrm{C}) \mathrm{c} 1 \mathrm{cccc}(\mathrm{Cl}) \mathrm{c} 1$ \\
\hline 181 & $\mathbf{1 1}\{181\}$ & $\mathrm{CNc} 1 \mathrm{cccc}(\mathrm{C}) \mathrm{c} 1$ \\
\hline 182 & $\mathbf{1 1}\{182\}$ & $\mathrm{Cl} . \mathrm{CNCc} 1 \mathrm{ccc}(\mathrm{C}) \mathrm{c}(\mathrm{C}) \mathrm{c} 1$ \\
\hline 183 & $\mathbf{1 1}\{183\}$ & $\mathrm{Cl} . \mathrm{CC} 1(\mathrm{Cc} 2 \mathrm{ccccc} 2) \mathrm{CCCN} 1$ \\
\hline 184 & $\mathbf{1 1}\{184\}$ & $\mathrm{Cl} . \mathrm{COC} 1(\mathrm{CCCNC} 1) \mathrm{C}(\mathrm{F})(\mathrm{F}) \mathrm{F}$ \\
\hline 185 & $\mathbf{1 1}\{185\}$ & $\mathrm{CC} 1(\mathrm{C}) \mathrm{CNC} 1 \mathrm{c} 1 \mathrm{cccnc} 1$ \\
\hline 186 & $\mathbf{1 1}\{186\}$ & Cl.CCn1 $\operatorname{ccc}(\mathrm{CNC}) \mathrm{c} 1$ \\
\hline 187 & $\mathbf{1 1}\{187\}$ & $\mathrm{OC} 1(\mathrm{CCNC} 1) \mathrm{C} 1 \mathrm{CC} 1$ \\
\hline 188 & $\mathbf{1 1}\{188\}$ & $\mathrm{CCOC} 1 \mathrm{CC} 2(\mathrm{C} 1) \mathrm{CCNC} 2$ \\
\hline 189 & $\mathbf{1 1}\{189\}$ & $\mathrm{Cc} 1 \operatorname{ccc}(\mathrm{C} 2 \mathrm{CCCN} 2) \mathrm{c} 1 \mathrm{C}$ \\
\hline 190 & $\mathbf{1 1}\{190\}$ & $\mathrm{CC} 1(\mathrm{C}) \mathrm{CNCc} 2 \operatorname{ccccc} 2 \mathrm{O} 1$ \\
\hline 191 & $\mathbf{1 1}\{191\}$ & $\mathrm{CC} 1(\mathrm{C}) \mathrm{CNCC}(\mathrm{O} 1) \mathrm{C}(\mathrm{F})(\mathrm{F}) \mathrm{F}$ \\
\hline 192 & $\mathbf{1 1}\{192\}$ & CNCc1 $\operatorname{cccc}(\mathrm{C}) \mathrm{n} 1$ \\
\hline 193 & $\mathbf{1 1}\{193\}$ & Cl.COc1ccec1C1CCNC1 \\
\hline
\end{tabular}




\begin{tabular}{|c|c|c|}
\hline 194 & $\mathbf{1 1}\{194\}$ & $\mathrm{Cl} . \mathrm{O}=\mathrm{S} 1(=\mathrm{O}) \mathrm{CCNCc} 2 \operatorname{ccccc} 12$ \\
\hline 195 & $\mathbf{1 1}\{195\}$ & $\mathrm{Cl} . \mathrm{CCS}(=\mathrm{O})(=\mathrm{O}) \mathrm{C} 1 \mathrm{CNC} 1$ \\
\hline 196 & $\mathbf{1 1}\{196\}$ & Cl.CNc1 ccc $(\mathrm{OC}(\mathrm{C}) \mathrm{C}) \mathrm{cc} 1$ \\
\hline 197 & $\mathbf{1 1}\{197\}$ & $\mathrm{Cl} . \mathrm{CC} 1 \mathrm{CNC}(\mathrm{C})(\mathrm{C}) \mathrm{C} 1$ \\
\hline 198 & $\mathbf{1 1}\{198\}$ & $\mathrm{CC}(\mathrm{C}) \mathrm{c} 1 \mathrm{cc}([\mathrm{nH}] \mathrm{n} 1) \mathrm{C} 1 \mathrm{CCCNC} 1$ \\
\hline 199 & $\mathbf{1 1}\{199\}$ & CNCc1nnc2CCCn12 \\
\hline 200 & $11\{200\}$ & $\mathrm{CNCc} 1 \mathrm{cc} 2 \operatorname{ccccc} 2[\mathrm{nH}] 1$ \\
\hline 201 & $\mathbf{1 1}\{201\}$ & $\mathrm{CC} 1 \mathrm{CNc} 2 \mathrm{cccc} 2 \mathrm{O} 1$ \\
\hline 202 & $11\{202\}$ & $\mathrm{C} 1 \mathrm{CC} 1 \mathrm{c} 1 \mathrm{nc}(\mathrm{n}[\mathrm{nH}] 1) \mathrm{C} 1 \mathrm{CCCNC} 1$ \\
\hline 203 & $\mathbf{1 1}\{203\}$ & Cl.Cl.Cc1nc([nH]c1C)C1CCNCC1 \\
\hline 204 & $11\{204\}$ & $\mathrm{CNCC} 1(\mathrm{O}) \mathrm{CCC} 1$ \\
\hline 205 & $\mathbf{1 1}\{205\}$ & $\mathrm{Cl} . \mathrm{OC} 1 \mathrm{CCNCC} 1(\mathrm{~F}) \mathrm{F}$ \\
\hline 206 & $\mathbf{1 1}\{206\}$ & $\mathrm{CC} 1(\mathrm{C}) \mathrm{CNC} 1 \mathrm{c} 1 \mathrm{ccncc} 1$ \\
\hline 207 & $\mathbf{1 1}\{207\}$ & Cl.Cl.C1CC(CCN1)n1ccen1 \\
\hline 208 & $\mathbf{1 1}\{208\}$ & $\mathrm{C} 1 \mathrm{CN}(\mathrm{CCN} 1) \mathrm{C} 1 \mathrm{CCOCC} 1$ \\
\hline 209 & $\mathbf{1 1}\{209\}$ & CO[1C@@H]1CNC[1C@H]1O \\
\hline 210 & $\mathbf{1 1}\{210\}$ & Br.Oc1ccec2CNCCc12 \\
\hline 211 & $11\{211\}$ & $\mathrm{C} 1 \mathrm{CC}(\mathrm{CCN} 1) \mathrm{c} 1 \mathrm{ccco} 1$ \\
\hline 212 & $\mathbf{1 1}\{212\}$ & $\mathrm{Cl} . \mathrm{FC}(\mathrm{F})(\mathrm{F}) \mathrm{C} 1 \mathrm{CCNCCO} 1$ \\
\hline 213 & $\mathbf{1 1}\{213\}$ & $\mathrm{Cl} . \mathrm{CNC}(=\mathrm{O}) \mathrm{NC1CCNCC1}$ \\
\hline 214 & $\mathbf{1 1}\{214\}$ & $\mathrm{C} 1 \mathrm{CCC}(\mathrm{OC} 1) \mathrm{C} 1 \mathrm{CCNCC} 1$ \\
\hline 215 & $\mathbf{1 1}\{215\}$ & Cl.CCS $(=\mathrm{O})(=\mathrm{O}) \mathrm{CC}(\mathrm{C}) \mathrm{NC}$ \\
\hline 216 & $11\{216\}$ & CN[1C@H]1CCC[1C@@H]1O \\
\hline 217 & $11\{217\}$ & $\mathrm{Cl} . \mathrm{Oc} 1 \mathrm{ccc} 2 \mathrm{CNCCOc} 2 \mathrm{c} 1$ \\
\hline 218 & $\mathbf{1 1}\{218\}$ & Cc1cnce(c1)C1CCCN1 \\
\hline 219 & $\mathbf{1 1}\{219\}$ & $\mathrm{C} 1 \mathrm{CN}(\mathrm{CCN} 1) \mathrm{c} 1 \mathrm{cccs} 1$ \\
\hline 220 & $11\{220\}$ & $\operatorname{Cl} . \mathrm{CC} 1 \mathrm{CCS}(=\mathrm{O})(=\mathrm{O}) \mathrm{CCN} 1$ \\
\hline 221 & $11\{221\}$ & Cl.Cl.C1CNCC(C1)c1ccenc1 \\
\hline
\end{tabular}




\begin{tabular}{|c|c|c|}
\hline 222 & $\mathbf{1 1}\{222\}$ & Cl.Cl.C1CNCC(C1)c1cence1 \\
\hline 223 & $\mathbf{1 1}\{223\}$ & $\mathrm{CNCc} 1 \mathrm{nnc}(\mathrm{C}) \mathrm{s} 1$ \\
\hline 224 & $\mathbf{1 1}\{224\}$ & $\mathrm{CNCC}(\mathrm{C}) \mathrm{C}(=\mathrm{O}) \mathrm{NC}$ \\
\hline 225 & $\mathbf{1 1}\{225\}$ & $\mathrm{Cl} . \mathrm{O}=\mathrm{S}(=\mathrm{O})(\mathrm{C} 1 \mathrm{CC} 1) \mathrm{N} 1 \mathrm{CCNCC} 1$ \\
\hline 226 & $\mathbf{1 1}\{226\}$ & $\mathrm{Cl} . \mathrm{OCCNC}(=\mathrm{O}) \mathrm{C} 1 \mathrm{CCNCC} 1$ \\
\hline 227 & $\mathbf{1 1}\{227\}$ & COc1ccc(cc1F)C1CCNC1 \\
\hline 228 & $\mathbf{1 1}\{228\}$ & $\mathrm{CC}(\mathrm{C})(\mathrm{O}) \mathrm{CNC} 1 \mathrm{CC} 1$ \\
\hline 229 & $\mathbf{1 1}\{229\}$ & OCC $1(\mathrm{CCNC} 1) \mathrm{c} 1 \mathrm{ccccc} 1$ \\
\hline 230 & $\mathbf{1 1}\{230\}$ & $\mathrm{CN}(\mathrm{C} 1 \mathrm{CCNC} 1) \mathrm{c} 1 \mathrm{ccccc} 1$ \\
\hline 231 & $\mathbf{1 1}\{231\}$ & Cl.NC $(=\mathrm{O}) \mathrm{COC} 1 \mathrm{CCNCC} 1$ \\
\hline 232 & $\mathbf{1 1}\{232\}$ & CCNCc1occe1C \\
\hline 233 & $\mathbf{1 1}\{233\}$ & $\operatorname{Brc} 1 \mathrm{cncc}(\mathrm{c} 1) \mathrm{C} 1 \mathrm{CCCN} 1$ \\
\hline 234 & $\mathbf{1 1}\{234\}$ & Cl.CS $(=\mathrm{O})(=\mathrm{O}) \mathrm{CC} 1 \mathrm{CCNCC} 1$ \\
\hline 235 & $\mathbf{1 1}\{235\}$ & CNCc1csen 1 \\
\hline 236 & $\mathbf{1 1}\{236\}$ & Oc1cce $2 \mathrm{CCNCc} 2 \mathrm{c} 1$ \\
\hline 237 & $\mathbf{1 1}\{237\}$ & CNCc1occe1C \\
\hline 238 & $\mathbf{1 1}\{238\}$ & Cl.Clc1cnn(c1)C1CCNCC1 \\
\hline 239 & $\mathbf{1 1}\{239\}$ & $\mathrm{Cl} \cdot \mathrm{C} 1 \mathrm{NC} 2 \mathrm{CC} 1 \mathrm{C}=\mathrm{C} 2$ \\
\hline 240 & $\mathbf{1 1}\{240\}$ & $\mathrm{Cl} \cdot \mathrm{CC}(=\mathrm{O}) \mathrm{NC} 1 \mathrm{CCNCC} 1$ \\
\hline 241 & $\mathbf{1 1}\{241\}$ & $\mathrm{Cc} 1 \mathrm{cnn}(\mathrm{c} 1) \mathrm{C} 1 \mathrm{CCNC} 1$ \\
\hline 242 & $\mathbf{1 1}\{242\}$ & $\mathrm{CCNc} 1 \mathrm{cnn}(\mathrm{C}) \mathrm{c} 1$ \\
\hline 243 & $\mathbf{1 1}\{243\}$ & Cl.Cl.Cn1 $1 \mathrm{cc}(\mathrm{cn} 1) \mathrm{C} 1=\mathrm{CCCNC} 1$ \\
\hline 244 & $11\{244\}$ & Cl.Cl.CN1CC[1C@@H]2CC[1C@H](C1)N2 \\
\hline 245 & $\mathbf{1 1}\{245\}$ & $\mathrm{C}[\mathrm{C} @ \mathrm{H}] 1 \mathrm{COCCN} 1$ \\
\hline 246 & $\mathbf{1 1}\{246\}$ & Cl.N\#CC1CCCNC1 \\
\hline 247 & $11\{247\}$ & $\operatorname{Br} . C N C c 1 \operatorname{cccc}(=O)[\mathrm{nH}] 1$ \\
\hline 248 & $11\{248\}$ & Cl.Cl.C1CN[C@@H](C1)c1ncn[nH]1 \\
\hline 249 & $\mathbf{1 1}\{249\}$ & Cl.COC[C@H]1C[C@@H](O)CN1 \\
\hline
\end{tabular}




\begin{tabular}{|c|c|c|}
\hline 250 & $\mathbf{1 1}\{250\}$ & Cl.CC(C)[C@H]1CNCCN1C \\
\hline 251 & $\mathbf{1 1}\{251\}$ & $\mathrm{CN} 1 \mathrm{CCNCc} 2 \mathrm{cccnc} 12$ \\
\hline 252 & $\mathbf{1 1}\{252\}$ & $\mathrm{Cl} \cdot \mathrm{NC}(=\mathrm{O}) \mathrm{C} 1 \mathrm{NCC} 2 \operatorname{ccccc} 12$ \\
\hline 253 & $\mathbf{1 1}\{253\}$ & $\operatorname{Cl} . \operatorname{CC} 1 \mathrm{CCNCCS} 1(=\mathrm{O})=\mathrm{O}$ \\
\hline 254 & $\mathbf{1 1}\{254\}$ & Cl.O[1C@@H]1CNC[1C@H]1Oc1 ccccc1 \\
\hline 255 & $\mathbf{1 1}\{255\}$ & $\operatorname{Cl} . \mathrm{CC} 1 \mathrm{CNCCS}(=\mathrm{O})(=\mathrm{O}) \mathrm{C} 1$ \\
\hline 256 & $11\{256\}$ & $\mathrm{CC} 1(\mathrm{C}) \mathrm{CN}[1 \mathrm{C} @ \mathrm{H}] 2 \mathrm{COC}[1 \mathrm{C} @ \mathrm{H}] 12$ \\
\hline 257 & $\mathbf{1 1}\{257\}$ & Cl.Cl.C1CC(CCN1)c1nnc[nH]1 \\
\hline 258 & $\mathbf{1 1}\{258\}$ & $\mathrm{C} 1 \mathrm{CC}(\mathrm{CCN} 1) \mathrm{c} 1 \mathrm{ncc}[\mathrm{nH}] 1$ \\
\hline 259 & $\mathbf{1 1}\{259\}$ & $\mathrm{CC} 1 \mathrm{CNCCN}(\mathrm{C} 1) \mathrm{C} 1 \mathrm{CC} 1$ \\
\hline 260 & $\mathbf{1 1}\{260\}$ & $\mathrm{C} 1 \mathrm{CC}(\mathrm{CN} 1) \mathrm{N} 1 \mathrm{CCCCC} 1$ \\
\hline 261 & $11\{261\}$ & $\mathrm{C} 1 \mathrm{COC}(\mathrm{C} 1) \mathrm{C} 1 \mathrm{CCNCC} 1$ \\
\hline 262 & $11\{262\}$ & $\mathrm{CNC} 1 \mathrm{CCN}(\mathrm{C} 1) \mathrm{C}(\mathrm{C}) \mathrm{C}$ \\
\hline 263 & $11\{263\}$ & $\mathrm{CCc} 1 \mathrm{cccc}(\mathrm{NC}) \mathrm{c} 1$ \\
\hline 264 & $\mathbf{1 1}\{264\}$ & $\mathrm{CC} 1 \mathrm{NCCc} 2 \operatorname{ccccc} 12$ \\
\hline 265 & $\mathbf{1 1}\{265\}$ & $\mathrm{Cn} 1 \mathrm{cc}(\mathrm{CC} 2 \mathrm{CCNC} 2) \mathrm{cn} 1$ \\
\hline 266 & $11\{266\}$ & $\mathrm{Cl} . \mathrm{Fc} 1 \mathrm{cccc}(\mathrm{OC} 2 \mathrm{CCNCC} 2) \mathrm{c} 1$ \\
\hline 267 & $11\{267\}$ & $\mathrm{CNC}(\mathrm{C}) \mathrm{c} 1 \mathrm{ccncc} 1$ \\
\hline 268 & $\mathbf{1 1}\{268\}$ & $\mathrm{Cl} . \mathrm{C} 1 \mathrm{CNCc} 2 \operatorname{cssc} 2 \mathrm{C} 1$ \\
\hline 269 & $11\{269\}$ & Clc1ccce(c1)C1CCCN1 \\
\hline 270 & $11\{270\}$ & $\mathrm{CNC} 1 \mathrm{Cc} 2 \operatorname{ccccc} 2 \mathrm{C} 1$ \\
\hline 271 & $\mathbf{1 1}\{271\}$ & $\mathrm{CNC}(\mathrm{C}) \mathrm{c} 1 \mathrm{ccccc} 1 \mathrm{~F}$ \\
\hline 272 & $\mathbf{1 1}\{272\}$ & Cl.Cl.C1CNCC $(\mathrm{C} 1) \mathrm{c} 1 \mathrm{ccc} c \mathrm{n} 1$ \\
\hline 273 & $11\{273\}$ & $\operatorname{Cl} \cdot \operatorname{CCNCC}(\mathrm{N})=\mathrm{O}$ \\
\hline 274 & $11\{274\}$ & $\mathrm{C}(\mathrm{C} 1 \mathrm{CCNC} 1) \mathrm{N} 1 \mathrm{CCCC} 1$ \\
\hline 275 & $\mathbf{1 1}\{275\}$ & $\mathrm{C}(\mathrm{C} 1 \mathrm{CCNCC} 1) \mathrm{c} 1 \mathrm{cccnc} 1$ \\
\hline 276 & $11\{276\}$ & $\mathrm{CC} 1 \mathrm{CNCCN} 1 \mathrm{CC}(\mathrm{C})(\mathrm{C}) \mathrm{O}$ \\
\hline 277 & $\mathbf{1 1}\{277\}$ & $\mathrm{Cl} . \mathrm{OCC} 1(\mathrm{CCNC} 1) \mathrm{C}(\mathrm{F})(\mathrm{F}) \mathrm{F}$ \\
\hline
\end{tabular}




\begin{tabular}{|c|c|c|}
\hline 278 & $\mathbf{1 1}\{278\}$ & $\mathrm{C} 1 \mathrm{CC} 2(\mathrm{CCOC} 2) \mathrm{CN} 1$ \\
\hline 279 & $11\{279\}$ & $\mathrm{Cc} 1 \mathrm{cnc}(\mathrm{OC} 2 \mathrm{CCNCC} 2) \mathrm{nc} 1$ \\
\hline 280 & $11\{280\}$ & Fc1ccenc1N1CCCNCC1 \\
\hline 281 & $\mathbf{1 1}\{281\}$ & $\mathrm{C}(\mathrm{OC} 1 \mathrm{CCNCC} 1) \mathrm{C} 1 \mathrm{CC} 1$ \\
\hline 282 & $\mathbf{1 1}\{282\}$ & $\mathrm{CC}(=\mathrm{O}) \mathrm{N} 1 \mathrm{CCNCC} 11 \mathrm{CCCCC} 1$ \\
\hline 283 & $\mathbf{1 1}\{283\}$ & $\mathrm{Cl} . \mathrm{C} 1 \mathrm{CC} 2(\mathrm{C} 1) \mathrm{CNCc} 1 \mathrm{ccccc} 1 \mathrm{O} 2$ \\
\hline 284 & $\mathbf{1 1}\{284\}$ & $\mathrm{CC} 1(\mathrm{C}) \mathrm{CNCCO} 1$ \\
\hline 285 & $\mathbf{1 1}\{285\}$ & Cl.Cc1nc(no1)C1CC2CCC(C1)N2 \\
\hline 286 & $\mathbf{1 1}\{286\}$ & $\mathrm{Cl} . \mathrm{C} 1 \mathrm{OC} 2(\mathrm{CCNCC} 2) \mathrm{c} 2 \mathrm{cccc} 12$ \\
\hline 287 & $\mathbf{1 1}\{287\}$ & $\mathrm{O}=\mathrm{C}(\mathrm{N} 1 \mathrm{CCNCC} 1) \mathrm{c} 1 \mathrm{ccco} 1$ \\
\hline 288 & $\mathbf{1 1}\{288\}$ & $\mathrm{CC}(=\mathrm{O}) \mathrm{N} 1 \mathrm{CCCNCC} 1$ \\
\hline 289 & $\mathbf{1 1}\{289\}$ & $\mathrm{C} 1 \mathrm{CC}(\mathrm{CCN} 1) \mathrm{c} 1 \mathrm{nc} 2 \mathrm{ccccc} 2 \mathrm{~s} 1$ \\
\hline 290 & $\mathbf{1 1}\{290\}$ & $\mathrm{CC} 1 \mathrm{CNCCN} 1 \mathrm{C}(=\mathrm{O}) \mathrm{OC}(\mathrm{C})(\mathrm{C}) \mathrm{C}$ \\
\hline 291 & $\mathbf{1 1}\{291\}$ & $\mathrm{Fc} 1 \mathrm{ccc}(\mathrm{cc} 1) \mathrm{C}(=\mathrm{O}) \mathrm{N} 1 \mathrm{CCNCC} 1$ \\
\hline 292 & $11\{292\}$ & $\mathrm{C}[\mathrm{C} @ \mathrm{H}] 1 \mathrm{CNCCN} 1 \mathrm{C}(=\mathrm{O}) \mathrm{OC}(\mathrm{C})(\mathrm{C}) \mathrm{C}$ \\
\hline 293 & $\mathbf{1 1}\{293\}$ & $\begin{array}{l}\text { Cl.C1C }[\mathrm{C} @ @ \mathrm{H}] 2 \mathrm{C}[\mathrm{C} @ @ \mathrm{H}](\mathrm{C}[\mathrm{C} @ \mathrm{H}] 1 \mathrm{~N} 2) \mathrm{c} 1 \mathrm{ccccc} \\
1\end{array}$ \\
\hline 294 & $11\{294\}$ & Cl.Clc1eccec1N1CCNCC1 \\
\hline 295 & $\mathbf{1 1}\{295\}$ & $\mathrm{O}=\mathrm{C}(\mathrm{OCc} 1 \mathrm{ccccc} 1) \mathrm{N} 1 \mathrm{CCNCC} 1$ \\
\hline 296 & $11\{296\}$ & $\mathrm{C}[\mathrm{C} @ @ \mathrm{H}] 1 \mathrm{CNCCN} 1 \mathrm{C}(=\mathrm{O}) \mathrm{OC}(\mathrm{C})(\mathrm{C}) \mathrm{C}$ \\
\hline 297 & $\mathbf{1 1}\{297\}$ & $\mathrm{CC} 1(\mathrm{OCCO} 1) \mathrm{C} 1 \mathrm{CCNCC} 1$ \\
\hline 298 & $\mathbf{1 1}\{298\}$ & $\mathrm{CC} 1(\mathrm{OCCO} 1) \mathrm{C} 1 \mathrm{CCCNC} 1$ \\
\hline 299 & $\mathbf{1 1}\{299\}$ & $\mathrm{COC} 1(\mathrm{CO}) \mathrm{CCNCC} 1$ \\
\hline 300 & $11\{300\}$ & C1CNCCOC1 \\
\hline 301 & $11\{301\}$ & $\mathrm{FC}(\mathrm{F}) \mathrm{CN} 1 \mathrm{CCNCC} 1$ \\
\hline 302 & $\mathbf{1 1}\{302\}$ & $\mathrm{CNCCOc} 1 \mathrm{ccc}(\mathrm{F}) \mathrm{cc} 1$ \\
\hline 303 & $\mathbf{1 1}\{303\}$ & CC1CCCNC1C \\
\hline 304 & $11\{304\}$ & $\mathrm{CC}(\mathrm{C}) \mathrm{C}(=\mathrm{O}) \mathrm{NCC} 1 \mathrm{CCCNC} 1$ \\
\hline 305 & $\mathbf{1 1}\{305\}$ & CC1CCCNCC1 \\
\hline
\end{tabular}




\begin{tabular}{|c|c|c|}
\hline 306 & $11\{306\}$ & $\mathrm{CC}(\mathrm{C})(\mathrm{C}) \mathrm{OC}(=\mathrm{O}) \mathrm{NCC} 1 \mathrm{CCCNC} 1$ \\
\hline 307 & $11\{307\}$ & $\mathrm{CN}(\mathrm{CC} 1 \mathrm{CCNC} 1) \mathrm{C}(=\mathrm{O}) \mathrm{OC}(\mathrm{C})(\mathrm{C}) \mathrm{C}$ \\
\hline 308 & $\mathbf{1 1}\{308\}$ & $\mathrm{CC}(\mathrm{C})(\mathrm{C}) \mathrm{OC}(=\mathrm{O}) \mathrm{NCC} 1 \mathrm{CCNCC} 1$ \\
\hline 309 & $\mathbf{1 1}\{309\}$ & $\mathrm{O}=\mathrm{c} 1[\mathrm{nH}] \mathrm{c} 2 \mathrm{ccccc} 2 \mathrm{n} 1 \mathrm{C} 1 \mathrm{CCNCC} 1$ \\
\hline 310 & $\mathbf{1 1}\{310\}$ & $\operatorname{CC} 1 \mathrm{CNCCC} 1 \mathrm{NC}(=\mathrm{O}) \mathrm{OC}(\mathrm{C})(\mathrm{C}) \mathrm{C}$ \\
\hline 311 & $\mathbf{1 1}\{311\}$ & $\mathrm{CC}(\mathrm{C})(\mathrm{C}) \mathrm{OC}(=\mathrm{O}) \mathrm{N}[\mathrm{C} @ \mathrm{H}] 1 \mathrm{CCCNC} 1$ \\
\hline 312 & $\mathbf{1 1}\{312\}$ & $\mathrm{CC}(\mathrm{C})(\mathrm{C}) \mathrm{OC}(=\mathrm{O}) \mathrm{NC} 1 \mathrm{CCCNC} 1$ \\
\hline 313 & $\mathbf{1 1}\{313\}$ & $\mathrm{CC}(\mathrm{C})(\mathrm{C}) \mathrm{OC}(=\mathrm{O}) \mathrm{N} 1 \mathrm{C}[\mathrm{C} @ @ \mathrm{H}] 2 \mathrm{CNC}[\mathrm{C} @ @ \mathrm{H}] 2 \mathrm{C} 1$ \\
\hline 314 & $\mathbf{1 1}\{314\}$ & $\mathrm{CC}(\mathrm{C})(\mathrm{C}) \mathrm{OC}(=\mathrm{O}) \mathrm{N} 1 \mathrm{CCCNCC1}$ \\
\hline 315 & $\mathbf{1 1}\{315\}$ & $\mathrm{CC}(\mathrm{C})(\mathrm{C}) \mathrm{OC}(=\mathrm{O}) \mathrm{N} 1 \mathrm{C}[\mathrm{C} @ @ \mathrm{H}] 2 \mathrm{C}[\mathrm{C} @ \mathrm{H}] 1 \mathrm{CN} 2$ \\
\hline 316 & $\mathbf{1 1}\{316\}$ & $\mathrm{Fc} 1 \mathrm{cccc}(\mathrm{F}) \mathrm{c} 1 \mathrm{~N} 1 \mathrm{CCNCC} 1$ \\
\hline 317 & $\mathbf{1 1}\{317\}$ & $\mathrm{CNCc} 1 \mathrm{ccc}(\mathrm{C}) \mathrm{o} 1$ \\
\hline 318 & $\mathbf{1 1}\{318\}$ & $\mathrm{C} 1 \mathrm{Cn} 2 \mathrm{c}(\mathrm{CN} 1) \mathrm{nnc} 2-\mathrm{c} 1 \mathrm{ccccc} 1$ \\
\hline 319 & $\mathbf{1 1}\{319\}$ & $\mathrm{CNCC} 1 \mathrm{COCCO} 1$ \\
\hline 320 & $\mathbf{1 1}\{320\}$ & $\mathrm{COC}(=\mathrm{O}) \mathrm{C} 1 \mathrm{CCNCC} 1$ \\
\hline 321 & $\mathbf{1 1}\{321\}$ & OCC1CCNCC1 \\
\hline 322 & $\mathbf{1 1}\{322\}$ & $\mathrm{CNC} 1 \mathrm{CC} 1$ \\
\hline 323 & $\mathbf{1 1}\{323\}$ & $\mathrm{Cl} . \mathrm{O}=\mathrm{C} 1 \mathrm{CNC} 2 \operatorname{ccccc} 2 \mathrm{~N} 1$ \\
\hline 324 & $\mathbf{1 1}\{324\}$ & Cl.Cc1noc(n1)C1CCNCC1 \\
\hline 325 & $\mathbf{1 1}\{325\}$ & $\mathrm{Cl} . \mathrm{O}=\mathrm{c} 1[\mathrm{nH}] \mathrm{ncn} 1 \mathrm{C} 1 \mathrm{CCNCC} 1$ \\
\hline 326 & $\mathbf{1 1}\{326\}$ & $\mathrm{Cl} \cdot \mathrm{FC}(\mathrm{F})(\mathrm{F}) \mathrm{C} 1=\mathrm{CCNCC} 1$ \\
\hline 327 & $\mathbf{1 1}\{327\}$ & $\mathrm{C} 1 \mathrm{CNC}(\mathrm{C} 1) \mathrm{c} 1 \mathrm{ccsc} 1$ \\
\hline 328 & $\mathbf{1 1}\{328\}$ & C1COCCN1 \\
\hline 329 & $11\{329\}$ & Cl.FC(F)C1CCNCCO1 \\
\hline 330 & $11\{330\}$ & $\mathrm{Cl} . \mathrm{CNC} 1 \mathrm{CCCCN}(\mathrm{C}) \mathrm{C} 1=\mathrm{O}$ \\
\hline 331 & $11\{331\}$ & Cl.C1CC11CC2CCC(C1)N2 \\
\hline 332 & $\mathbf{1 1}\{332\}$ & Cl.CC1(CNCCO1)C(F)F \\
\hline 333 & $\mathbf{1 1}\{333\}$ & $\mathrm{CC} 1(\mathrm{C}) \mathrm{CNCCS} 1$ \\
\hline
\end{tabular}




\begin{tabular}{|c|c|c|}
\hline 334 & $11\{334\}$ & Cn1cnnc1N1CCNCC1 \\
\hline 335 & $\mathbf{1 1}\{335\}$ & $\mathrm{O}=\mathrm{C} 1 \mathrm{NC}(=\mathrm{O})[\mathrm{C} @ @ \mathrm{H}] 2 \mathrm{CNC}[\mathrm{C} @ \mathrm{H}] 12$ \\
\hline 336 & $11\{336\}$ & $\mathrm{Cl} . \mathrm{CS}(=\mathrm{O})(=\mathrm{O}) \mathrm{C} 1 \mathrm{CCNCC} 1$ \\
\hline 337 & $11\{337\}$ & $\mathrm{CNCC} 1 \mathrm{CCOC} 1$ \\
\hline 338 & $\mathbf{1 1}\{338\}$ & $\mathrm{CCC} 1 \mathrm{CNCC}(\mathrm{CC}) \mathrm{O} 1$ \\
\hline 339 & $\mathbf{1 1}\{339\}$ & $\mathrm{CNCc} 1 \mathrm{cccc}(\mathrm{F}) \mathrm{c} 1$ \\
\hline 340 & $11\{340\}$ & C1CCCNCC1 \\
\hline 341 & $\mathbf{1 1}\{341\}$ & $\mathrm{C} 1 \mathrm{CCNC} 1$ \\
\hline 342 & $11\{342\}$ & $\mathrm{CC} 1 \mathrm{CCCCN} 1$ \\
\hline 343 & $\mathbf{1 1}\{343\}$ & $\mathrm{C}(\mathrm{N} 1 \mathrm{CCNCC} 1) \mathrm{c} 1 \mathrm{ccccc} 1$ \\
\hline 344 & $11\{344\}$ & $\mathrm{O}=\mathrm{C}(\mathrm{C} 1 \mathrm{CC} 1) \mathrm{N} 1 \mathrm{CCNC} 1$ \\
\hline 345 & $\mathbf{1 1}\{345\}$ & C1CCCNCCC1 \\
\hline 346 & $11\{346\}$ & $\mathrm{CCNC}$ \\
\hline 347 & $11\{347\}$ & C1CCCCNCCC1 \\
\hline 348 & $\mathbf{1 1}\{348\}$ & $\mathrm{CC}(\mathrm{C}) \mathrm{C} 1 \mathrm{CNCCCO} 1$ \\
\hline 349 & $11\{349\}$ & $\mathrm{C \# CCC1CCCNC1}$ \\
\hline 350 & $11\{350\}$ & $\mathrm{CNCCC}=\mathrm{C}$ \\
\hline 351 & $\mathbf{1 1}\{351\}$ & $\mathrm{CCN} 1 \mathrm{CCNCC} 1=\mathrm{O}$ \\
\hline 352 & $\mathbf{1 1}\{352\}$ & Cl.C(C1CCNCC1)n1cenn1 \\
\hline 353 & $\mathbf{1 1}\{353\}$ & $\mathrm{Cl} . \mathrm{OCC} 1(\mathrm{O}) \mathrm{CCNCC} 1$ \\
\hline 354 & $\mathbf{1 1}\{354\}$ & $\mathrm{CC} 1 \mathrm{NCN}(\mathrm{C}) \mathrm{C} 1=\mathrm{O}$ \\
\hline 355 & $\mathbf{1 1}\{355\}$ & $\mathrm{CNCc} 1 \mathrm{cnn}(\mathrm{C}) \mathrm{c} 1$ \\
\hline 356 & $\mathbf{1 1}\{356\}$ & $\mathrm{CNCC} 1 \mathrm{CCC} 1$ \\
\hline 357 & $11\{357\}$ & COc1cccc1N1CCNCC1 \\
\hline 358 & $\mathbf{1 1}\{358\}$ & $\mathrm{CC}(\mathrm{C})(\mathrm{C}) \mathrm{OC}(=\mathrm{O}) \mathrm{NCC} 1 \mathrm{CCNC} 1$ \\
\hline 359 & $11\{359\}$ & $\mathrm{O}=\mathrm{c} 1 \mathrm{cccc} 2[\mathrm{C} @ \mathrm{H}] 3 \mathrm{CNC}[\mathrm{C} @ \mathrm{H}](\mathrm{C} 3) \mathrm{Cn} 12$ \\
\hline 360 & $\mathbf{1 1}\{360\}$ & $\mathrm{CC}(\mathrm{C})(\mathrm{C}) \mathrm{OC}(=\mathrm{O}) \mathrm{NC} 1 \mathrm{CCNCC} 1$ \\
\hline 361 & $\mathbf{1 1}\{361\}$ & $\mathrm{CC}(\mathrm{C})(\mathrm{C}) \mathrm{OC}(=\mathrm{O}) \mathrm{N} 1 \mathrm{CCNCC} 1$ \\
\hline
\end{tabular}




\begin{tabular}{|c|c|c|}
\hline 362 & $\mathbf{1 1}\{362\}$ & Cl.O[C@@H]1CCCNC1 \\
\hline 363 & $\mathbf{1 1}\{363\}$ & $\mathrm{COCC} 1(\mathrm{C}) \mathrm{CNCCO} 1$ \\
\hline 364 & $\mathbf{1 1}\{364\}$ & $\mathrm{CNCC} 1(\mathrm{C}) \mathrm{COC} 1$ \\
\hline 365 & $\mathbf{1 1}\{365\}$ & $\mathrm{CC} 1 \mathrm{CC}(\mathrm{CN} 1) \mathrm{c} 1 \mathrm{ccc}(\mathrm{C}) \mathrm{cc} 1$ \\
\hline 366 & $11\{366\}$ & $\mathrm{CNCC}(\mathrm{C})(\mathrm{C}) \mathrm{O}$ \\
\hline 367 & $\mathbf{1 1}\{367\}$ & $\mathrm{Cl} . \mathrm{CNCC}(=\mathrm{O}) \mathrm{N} 1 \mathrm{CCOCC} 1$ \\
\hline 368 & $\mathbf{1 1}\{368\}$ & $\mathrm{CS}(=\mathrm{O})(=\mathrm{O}) \mathrm{C} 1 \mathrm{CCCNC} 1$ \\
\hline 369 & $\mathbf{1 1}\{369\}$ & $\mathrm{CC} 1 \mathrm{CNCCO} 1$ \\
\hline 370 & $11\{370\}$ & $\mathrm{CNCC} 1 \mathrm{CCCOC} 1$ \\
\hline 371 & $\mathbf{1 1}\{371\}$ & $\mathrm{C} 1 \mathrm{CC} 2(\mathrm{CNCCO} 2) \mathrm{c} 2 \mathrm{ccccc} 12$ \\
\hline 372 & $\mathbf{1 1}\{372\}$ & Cl.C[1C@@H]1CNC[1C@H](O)C1 \\
\hline 373 & $\mathbf{1 1}\{373\}$ & $\mathrm{Cl} . \mathrm{CC}(\mathrm{C}) \mathrm{NC}(=\mathrm{O})[\mathrm{C} @ \mathrm{H}] 1 \mathrm{CCCNC} 1$ \\
\hline 374 & $\mathbf{1 1}\{374\}$ & Cc1noc(n1)C1CCNC1 \\
\hline 375 & $\mathbf{1 1}\{375\}$ & $\mathrm{C} 1 \mathrm{CN}(\mathrm{CCN} 1) \mathrm{c} 1 \mathrm{nccc} 1$ \\
\hline 376 & $11\{376\}$ & $\mathrm{COCC}(=\mathrm{O}) \mathrm{N} 1 \mathrm{CCNCC} 1$ \\
\hline 377 & $11\{377\}$ & Cl.Fc1ccec2OCCNCc12 \\
\hline 378 & $\mathbf{1 1}\{378\}$ & $\mathrm{Cc} 1 \mathrm{ccc}(\mathrm{C}) \mathrm{n} 1 \mathrm{C} 1 \mathrm{CCNCC} 1$ \\
\hline 379 & $\mathbf{1 1}\{379\}$ & Cl.OC1(CCCNCC1)C(F)(F)F \\
\hline 380 & $\mathbf{1 1}\{380\}$ & Cl.CNS $(=\mathrm{O})(=\mathrm{O}) \mathrm{N} 1 \mathrm{CCNCC} 1$ \\
\hline 381 & $\mathbf{1 1}\{381\}$ & Cl.COc1 cce2OCCNCc2c1 \\
\hline 382 & $\mathbf{1 1}\{382\}$ & $\mathrm{C} 1 \mathrm{CNCC}(\mathrm{C} 1) \mathrm{NC} 1 \mathrm{CCOCC} 1$ \\
\hline 383 & $\mathbf{1 1}\{383\}$ & Cl.C[C@@H]1CNCCN1S $(\mathrm{C})(=\mathrm{O})=\mathrm{O}$ \\
\hline 384 & $\mathbf{1 1}\{384\}$ & $\mathrm{Cl} . \mathrm{Cl} . \mathrm{O}=\mathrm{C} 1 \mathrm{CCCCN} 1 \mathrm{C} 1 \mathrm{CCCNC} 1$ \\
\hline 385 & $\mathbf{1 1}\{385\}$ & Cl.CNCc1nc(C)no1 \\
\hline 386 & $\mathbf{1 1}\{386\}$ & $\mathrm{CNCc} 1 \mathrm{ccc}(\mathrm{F}) \mathrm{cc} 1$ \\
\hline 387 & $\mathbf{1 1}\{387\}$ & $\mathrm{C}(\mathrm{C} 1 \mathrm{CCNCC} 1) \mathrm{c} 1 \mathrm{ccccc} 1$ \\
\hline 388 & $\mathbf{1 1}\{388\}$ & $\mathrm{CNCc} 1 \mathrm{ccc}(\mathrm{Cl}) \mathrm{s} 1$ \\
\hline 389 & $\mathbf{1 1}\{389\}$ & Cl.CNCc1cces1 \\
\hline
\end{tabular}




\begin{tabular}{|c|c|c|}
\hline 390 & $\mathbf{1 1}\{390\}$ & $\mathrm{Cl} . \mathrm{CS}(=\mathrm{O})(=\mathrm{O}) \mathrm{N} 1 \mathrm{CCNCC} 1$ \\
\hline 391 & $\mathbf{1 1}\{391\}$ & $\mathrm{CNCC} 1 \mathrm{CC} 1$ \\
\hline 392 & $\mathbf{1 1}\{392\}$ & $\mathrm{CC} 1 \mathrm{CNCC} 2(\mathrm{CCC} 2) \mathrm{O} 1$ \\
\hline 393 & $\mathbf{1 1}\{393\}$ & $\mathrm{C} 1 \mathrm{CC} 2(\mathrm{C} 1) \mathrm{CNCCOC} 2$ \\
\hline 394 & $\mathbf{1 1}\{394\}$ & $\mathrm{C} 1 \mathrm{CCC} 2(\mathrm{C} 1) \mathrm{CNCCO} 2$ \\
\hline 395 & $\mathbf{1 1}\{395\}$ & $\mathrm{C} 1 \mathrm{CC} 2(\mathrm{CO} 1) \mathrm{CNCCO} 2$ \\
\hline 396 & $\mathbf{1 1}\{396\}$ & $\mathrm{CNCCOC}$ \\
\hline 397 & $\mathbf{1 1}\{397\}$ & CSC1CCCCNC1 \\
\hline 398 & $\mathbf{1 1}\{398\}$ & $\mathrm{CC} 1 \mathrm{CNCC}(\mathrm{C}) \mathrm{O} 1$ \\
\hline 399 & $\mathbf{1 1}\{399\}$ & $\mathrm{Cl} . \mathrm{COCC} 1(\mathrm{C}) \mathrm{CCNC} 1$ \\
\hline 400 & $11\{400\}$ & $\operatorname{CNC} 1 \mathrm{CCS}(=\mathrm{O})(=\mathrm{O}) \mathrm{C} 1$ \\
\hline 401 & $\mathbf{1 1}\{401\}$ & $\mathrm{NC}(=\mathrm{O}) \mathrm{C} 1 \mathrm{CCNCC} 1$ \\
\hline 402 & $\mathbf{1 1}\{402\}$ & $\mathrm{CC} 1 \mathrm{CNCC} 2(\mathrm{CCCCC} 2) \mathrm{O} 1$ \\
\hline 403 & $\mathbf{1 1}\{403\}$ & $\mathrm{Cl} . \mathrm{CC} 12 \mathrm{CCNCC} 1 \mathrm{C} 2(\mathrm{Cl}) \mathrm{Cl}$ \\
\hline 404 & $\mathbf{1 1}\{404\}$ & $\mathrm{COC}(=\mathrm{O})[1 \mathrm{C} @] 12 \mathrm{CNC}[1 \mathrm{C} @ \mathrm{H}] 1 \mathrm{CCCC} 2$ \\
\hline 405 & $\mathbf{1 1}\{405\}$ & $\mathrm{C} 1 \mathrm{CCC} 2(\mathrm{CC} 1) \mathrm{CCCNC} 2$ \\
\hline 406 & $11\{406\}$ & CC1CCNCC11CCC1 \\
\hline 407 & $\mathbf{1 1}\{407\}$ & $\mathrm{C} 1 \mathrm{CC} 2(\mathrm{CN} 1) \mathrm{CCCCC} 2$ \\
\hline 408 & $\mathbf{1 1}\{408\}$ & $\mathrm{C} 1 \mathrm{CCC} 2(\mathrm{C} 1) \mathrm{CCCNC} 2$ \\
\hline 409 & $\mathbf{1 1}\{409\}$ & $\mathrm{C} 1 \mathrm{CCC} 2(\mathrm{CC} 1) \mathrm{CNCCS} 2$ \\
\hline 410 & $\mathbf{1 1}\{410\}$ & $\mathrm{Cl} . \mathrm{C} 1 \mathrm{CCC} 2(\mathrm{CC} 1) \mathrm{CCNCC} 2$ \\
\hline 411 & $\mathbf{1 1}\{411\}$ & CCC1CCCNC1 \\
\hline 412 & $\mathbf{1 1}\{412\}$ & $\mathrm{CONCC} 1 \mathrm{CC} 2 \mathrm{CCC} 1 \mathrm{C} 2$ \\
\hline 413 & $\mathbf{1 1}\{413\}$ & $\mathrm{CC} 1 \mathrm{CNCC} 2(\mathrm{CCCC} 2) \mathrm{O} 1$ \\
\hline 414 & $\mathbf{1 1}\{414\}$ & CNCc1nces1 \\
\hline 415 & $\mathbf{1 1}\{415\}$ & $\mathrm{CNC}[\mathrm{C} @ \mathrm{H}] 1 \mathrm{CCCO} 1$ \\
\hline 416 & $\mathbf{1 1}\{416\}$ & $\mathrm{C} 1 \mathrm{CNCCc} 2 \operatorname{ccccc} 2 \mathrm{C} 1$ \\
\hline 417 & $\mathbf{1 1}\{417\}$ & $\mathrm{COc} 1 \mathrm{ccc} 2 \mathrm{CCNCc} 12$ \\
\hline
\end{tabular}




\begin{tabular}{|c|c|c|}
\hline 418 & $\mathbf{1 1}\{418\}$ & Cl.FC1(F)CNCc2 $\operatorname{ccccc} 12$ \\
\hline 419 & $11\{419\}$ & $\mathrm{FC} 1(\mathrm{~F}) \mathrm{CC} 11 \mathrm{CC} 2 \mathrm{CCC}(\mathrm{C} 1) \mathrm{N} 2$ \\
\hline 420 & $\mathbf{1 1}\{420\}$ & $\mathrm{C} 1 \mathrm{COC}(\mathrm{CN} 1) \mathrm{c} 1 \mathrm{ccccc} 1$ \\
\hline 421 & $\mathbf{1 1}\{421\}$ & $\mathrm{C} 1 \mathrm{CC} 2(\mathrm{CN} 1) \mathrm{OCc} 1 \operatorname{ccccc} 21$ \\
\hline 422 & $\mathbf{1 1}\{422\}$ & $\mathrm{NC}(=\mathrm{O}) \mathrm{CC} 1 \mathrm{CCNCC} 1$ \\
\hline 423 & $\mathbf{1 1}\{423\}$ & $\mathrm{O}=\mathrm{C} 1 \mathrm{CNCCN} 1$ \\
\hline 424 & $\mathbf{1 1}\{424\}$ & OC1CCCNC1 \\
\hline 425 & $\mathbf{1 1}\{425\}$ & $\mathrm{Cl} . \mathrm{COCC} 1=\mathrm{CCNCC} 1$ \\
\hline 426 & $\mathbf{1 1}\{426\}$ & $\mathrm{Cl} \cdot \mathrm{COC}(=\mathrm{O}) \mathrm{C} 1 \mathrm{CC} 11 \mathrm{CCNCC} 1$ \\
\hline 427 & $\mathbf{1 1}\{427\}$ & $\mathrm{Cl} . \mathrm{CN} 1 \mathrm{CCC} 2(\mathrm{CCNCC} 2) \mathrm{C} 1=\mathrm{O}$ \\
\hline 428 & $11\{428\}$ & $\mathrm{O}=\mathrm{C} 1 \mathrm{NCCC} 2(\mathrm{CCNCC} 2) \mathrm{O} 1$ \\
\hline 429 & $\mathbf{1 1}\{429\}$ & $\mathrm{C} 1 \mathrm{CC} 2(\mathrm{CCO} 1) \mathrm{CCNCCO} 2$ \\
\hline 430 & $\mathbf{1 1}\{430\}$ & $\begin{array}{l}\mathrm{CCOC}(=\mathrm{O})[1 \mathrm{C} @ @ \mathrm{H}] 1[1 \mathrm{C} @ @ \mathrm{H}] 2 \mathrm{CCNC}[1 \mathrm{C} @ \mathrm{H}] 1 \\
2\end{array}$ \\
\hline 431 & $\mathbf{1 1}\{431\}$ & $\mathrm{C} 1 \mathrm{CC} 2(\mathrm{CS} 1) \mathrm{CNCCO} 2$ \\
\hline 432 & $\mathbf{1 1}\{432\}$ & $\mathrm{C} 1 \mathrm{CC} 2(\mathrm{CCO} 1) \mathrm{CNCCOC} 2$ \\
\hline 433 & $\mathbf{1 1}\{433\}$ & $\mathrm{CC} 1 \mathrm{CNCC} 2(\mathrm{CCOCC} 2) \mathrm{O} 1$ \\
\hline 434 & $\mathbf{1 1}\{434\}$ & $\mathrm{C} 1 \mathrm{COC} 2(\mathrm{CCOCC} 2) \mathrm{CN} 1$ \\
\hline 435 & $\mathbf{1 1}\{435\}$ & $\mathrm{COC}(=\mathrm{O})[1 \mathrm{C} @] 12 \mathrm{CNC}[1 \mathrm{C} @ \mathrm{H}] 1 \mathrm{COCC} 2$ \\
\hline 436 & $\mathbf{1 1}\{436\}$ & $\mathrm{COC}(=\mathrm{O})[1 \mathrm{C} @] 12 \mathrm{CCC}[1 \mathrm{C} @ \mathrm{H}] 1 \mathrm{NCC} 2$ \\
\hline 437 & $\mathbf{1 1}\{437\}$ & $\operatorname{Cl} . \mathrm{CCS}(=\mathrm{O})(=\mathrm{O}) \mathrm{N} 1 \mathrm{CCNCC} 1$ \\
\hline 438 & $\mathbf{1 1}\{438\}$ & $\mathrm{NC}(=\mathrm{O})[1 \mathrm{C} @] 12 \mathrm{CCC}[1 \mathrm{C} @ \mathrm{H}] 1 \mathrm{CNC} 2$ \\
\hline 439 & $\mathbf{1 1}\{439\}$ & $\mathrm{CNC} 1 \mathrm{CCCCC} 1$ \\
\hline 440 & $\mathbf{1 1}\{440\}$ & $\mathrm{CNC}(\mathrm{C}) \mathrm{CC \# N}$ \\
\hline 441 & $\mathbf{1 1}\{441\}$ & $\mathrm{Cl} . \mathrm{CC} 1(\mathrm{C}) \mathrm{CCNCCS} 1=\mathrm{O}$ \\
\hline 442 & $\mathbf{1 1}\{442\}$ & $\mathrm{Cc} 1 \mathrm{cccc}(\mathrm{c} 1) \mathrm{C} 1 \mathrm{CCNC} 1$ \\
\hline 443 & $\mathbf{1 1}\{443\}$ & C[C@H]1CNC[C@@H](C)O1 \\
\hline 444 & $\mathbf{1 1}\{444\}$ & $\mathrm{COc} 1 \mathrm{ccc}(\mathrm{cc} 1) \mathrm{C} 1 \mathrm{CNCCO} 1$ \\
\hline 445 & $\mathbf{1 1}\{445\}$ & $\mathrm{Cl} . \mathrm{COc} 1 \mathrm{cc} 2 \mathrm{CCNCc} 2 \mathrm{cc} 1 \mathrm{OC}$ \\
\hline
\end{tabular}




\begin{tabular}{|c|c|c|}
\hline 446 & $11\{446\}$ & $\mathrm{COC}(=\mathrm{O})[1 \mathrm{C} @ @ \mathrm{H}] 1 \mathrm{CNC}[1 \mathrm{C} @ \mathrm{H}] 1 \mathrm{C}$ \\
\hline 447 & $11\{447\}$ & $\mathrm{C} 1 \mathrm{Cc} 2 \operatorname{ccccc} 2 \mathrm{CN} 1$ \\
\hline 448 & $11\{448\}$ & $\mathrm{CCOC}(=\mathrm{O}) \mathrm{C} 1 \mathrm{CCNCC} 1$ \\
\hline 449 & $11\{449\}$ & $\mathrm{CC} 1(\mathrm{C}) \mathrm{CNCC}(\mathrm{C})(\mathrm{C}) \mathrm{O} 1$ \\
\hline 450 & $\mathbf{1 1}\{450\}$ & $\mathrm{C} 1 \mathrm{CN}(\mathrm{CCN} 1) \mathrm{c} 1 \mathrm{ccccc} 1$ \\
\hline 451 & $\mathbf{1 1}\{451\}$ & $\mathrm{CCOC}(=\mathrm{O}) \mathrm{N} 1 \mathrm{CCNCC} 1$ \\
\hline 452 & $\mathbf{1 1}\{452\}$ & $\mathrm{CCOC}(=\mathrm{O})[\mathrm{C} @ @ \mathrm{H}] 1 \mathrm{CCCNC} 1$ \\
\hline 453 & $\mathbf{1 1}\{453\}$ & $\mathrm{CCOC}(=\mathrm{O}) \mathrm{C} 1 \mathrm{CCCNC} 1$ \\
\hline 454 & $\mathbf{1 1}\{454\}$ & $\mathrm{CC} 1 \mathrm{OCCC} 11 \mathrm{CNCC}(\mathrm{C}) \mathrm{O} 1$ \\
\hline 455 & $\mathbf{1 1}\{455\}$ & $\mathrm{CC} 1 \mathrm{OCCC} 11 \mathrm{CNCCO} 1$ \\
\hline 456 & $11\{456\}$ & $\mathrm{C} 1 \mathrm{COC} 2(\mathrm{CCNCC} 2) \mathrm{OC} 1$ \\
\hline 457 & $\mathbf{1 1}\{457\}$ & $\mathrm{CCc} 1 \mathrm{ncc} 2 \mathrm{C} 3 \mathrm{CCC}(\mathrm{Cc} 2 \mathrm{n} 1) \mathrm{N} 3$ \\
\hline 458 & $\mathbf{1 1}\{458\}$ & $\begin{array}{l}\mathrm{CO}[1 \mathrm{C} @ @ \mathrm{H}] 1 \mathrm{CS}(=\mathrm{O})(=\mathrm{O})[1 \mathrm{C} @ \mathrm{H}] 2 \mathrm{CNC}[1 \mathrm{C} @ @ \mathrm{H} \\
] 12\end{array}$ \\
\hline 459 & $\mathbf{1 1}\{459\}$ & $\mathrm{CCNCC}$ \\
\hline 460 & $11\{460\}$ & $\mathrm{Fc} 1 \mathrm{ccc}(\mathrm{cc} 1) \mathrm{N} 1 \mathrm{CCNCC} 1$ \\
\hline 461 & $\mathbf{1 1}\{461\}$ & Cc1 $1 \mathrm{ccc}(\mathrm{cc} 1) \mathrm{N} 1 \mathrm{CCNCC} 1$ \\
\hline 462 & $11\{462\}$ & $\mathrm{Cc} 1 \mathrm{ccccc} 1 \mathrm{~N} 1 \mathrm{CCNCC} 1$ \\
\hline 463 & $\mathbf{1 1}\{463\}$ & $\mathrm{Cc} 1 \mathrm{ccc}(\mathrm{cc} 1 \mathrm{C}) \mathrm{N} 1 \mathrm{CCNCC} 1$ \\
\hline 464 & $11\{464\}$ & Cc1 $1 \mathrm{ccc}(\mathrm{c} 1) \mathrm{N} 1 \mathrm{CCNCC} 1$ \\
\hline 465 & $\mathbf{1 1}\{465\}$ & $\mathrm{CC} 1 \mathrm{CCNCC} 1 \mathrm{C}$ \\
\hline 466 & $11\{466\}$ & $\mathrm{C} 1 \mathrm{CNCC} 2(\mathrm{C} 1) \mathrm{Oc} 1 \mathrm{cccc} 1 \mathrm{C}=\mathrm{C} 2$ \\
\hline 467 & $11\{467\}$ & $\mathrm{FC}(\mathrm{F})(\mathrm{F}) \mathrm{CC} 1 \mathrm{CC} 2 \mathrm{CCC}(\mathrm{C} 1) \mathrm{N} 2$ \\
\hline 468 & $\mathbf{1 1}\{468\}$ & $\mathrm{NC}(=\mathrm{O})[1 \mathrm{C} @ @ \mathrm{H}] 1 \mathrm{CCO}[1 \mathrm{C} @ \mathrm{H}] 1 \mathrm{C} 1 \mathrm{CCNCC} 1$ \\
\hline 469 & $\mathbf{1 1}\{469\}$ & CC1CSCCN1 \\
\hline 470 & $\mathbf{1 1}\{470\}$ & $\mathrm{CC}(\mathrm{C})(\mathrm{C}) \mathrm{OC}(=\mathrm{O}) \mathrm{NC} 1 \mathrm{CCNC} 1$ \\
\hline 471 & $11\{471\}$ & $\mathrm{CC}(\mathrm{C})(\mathrm{C}) \mathrm{OC}(=\mathrm{O}) \mathrm{N}[\mathrm{C} @ \mathrm{H}] 1 \mathrm{CCNC} 1$ \\
\hline 472 & $\mathbf{1 1}\{472\}$ & $\mathrm{CC}(\mathrm{C})(\mathrm{C}) \mathrm{OC}(=\mathrm{O}) \mathrm{N}[\mathrm{C} @ @ \mathrm{H}] 1 \mathrm{CCNC} 1$ \\
\hline 473 & $11\{473\}$ & $\mathrm{CCO}[\mathrm{C} @ \mathrm{H}] 1 \mathrm{CCNC} 1$ \\
\hline
\end{tabular}




\begin{tabular}{|c|c|c|}
\hline 474 & $11\{474\}$ & CSC1CCNC1 \\
\hline 475 & $\mathbf{1 1}\{475\}$ & C1CSCCN1 \\
\hline 476 & $11\{476\}$ & $\mathrm{FC} 1(\mathrm{~F}) \mathrm{CCNCC} 1$ \\
\hline 477 & $\mathbf{1 1}\{477\}$ & COCC1CCCNC1 \\
\hline 478 & $\mathbf{1 1}\{478\}$ & $\mathrm{C} 1 \mathrm{CCNCC} 1$ \\
\hline 479 & $11\{479\}$ & $\mathrm{CC} 1 \mathrm{CCCNC} 1$ \\
\hline 480 & $\mathbf{1 1}\{480\}$ & CC1CCNCC1 \\
\hline 481 & $\mathbf{1 1}\{481\}$ & $\mathrm{C} 1 \mathrm{NC}[\mathrm{C} @ @ \mathrm{H}] 2 \mathrm{CC}=\mathrm{CC}[\mathrm{C} @ \mathrm{H}] 12$ \\
\hline 482 & $11\{482\}$ & $\mathrm{C} 1 \mathrm{CC} 2(\mathrm{CN} 1) \mathrm{OCCO} 2$ \\
\hline 483 & $\mathbf{1 1}\{483\}$ & $\mathrm{C} 1 \mathrm{CC} 2 \mathrm{CC} 1 \mathrm{CNC} 2$ \\
\hline 484 & $11\{484\}$ & CC1CCNCC11CCCCC1 \\
\hline 485 & $\mathbf{1 1}\{485\}$ & $\mathrm{C} 1 \mathrm{CCC} 2(\mathrm{CC} 1) \mathrm{CCCNCC} 2$ \\
\hline 486 & $11\{486\}$ & $\mathrm{CC}(\mathrm{C})(\mathrm{C}) \mathrm{OC}(=\mathrm{O}) \mathrm{N} 1 \mathrm{C} 2 \mathrm{CCC} 1 \mathrm{CNC} 2$ \\
\hline 487 & $\mathbf{1 1}\{487\}$ & $\mathrm{CC}(\mathrm{C})(\mathrm{C}) \mathrm{OC}(=\mathrm{O}) \mathrm{N} 1 \mathrm{CC} 2 \mathrm{CCC}(\mathrm{C} 1) \mathrm{N} 2$ \\
\hline 488 & $\mathbf{1 1}\{488\}$ & $\mathrm{CC}(\mathrm{C})(\mathrm{C}) \mathrm{OC}(=\mathrm{O})[1 \mathrm{C} @] 12 \mathrm{CNC}[1 \mathrm{C} @ \mathrm{H}] 1 \mathrm{COC} 2$ \\
\hline 489 & $\mathbf{1 1}\{489\}$ & $\mathrm{CC}(\mathrm{C})(\mathrm{C}) \mathrm{OC}(=\mathrm{O}) \mathrm{NCC} 12 \mathrm{CC}(\mathrm{C} 1) \mathrm{CN} 2$ \\
\hline 490 & $11\{490\}$ & $\mathrm{CC}(\mathrm{O}) \mathrm{C} 1 \mathrm{CCNCC} 1$ \\
\hline 491 & $\mathbf{1 1}\{491\}$ & $\mathrm{C} 1 \mathrm{CCN}(\mathrm{C} 1) \mathrm{C} 1 \mathrm{CCNC} 1$ \\
\hline 492 & $11\{492\}$ & $\mathrm{CNCC}(\mathrm{C})(\mathrm{O}) \mathrm{c} 1 \mathrm{ccccc} 1$ \\
\hline 493 & $\mathbf{1 1}\{493\}$ & $\mathrm{CC} 1 \mathrm{CC} 2 \mathrm{CCC}(\mathrm{C} 1) \mathrm{N} 2$ \\
\hline 494 & $\mathbf{1 1}\{494\}$ & COCC1CNCCO1 \\
\hline 495 & $\mathbf{1 1}\{495\}$ & $\mathrm{CC}(=\mathrm{O}) \mathrm{NCC} 1 \mathrm{CNCCO} 1$ \\
\hline 496 & $\mathbf{1 1}\{496\}$ & $\mathrm{CNC}(\mathrm{C}) \mathrm{C \# C}$ \\
\hline 497 & $\mathbf{1 1}\{497\}$ & $\mathrm{Cl} . \mathrm{O}=\mathrm{C} 1 \mathrm{NC}(=\mathrm{O}) \mathrm{N} 2 \mathrm{CCNCC} 12$ \\
\hline 498 & $\mathbf{1 1}\{498\}$ & Fc1cccc1N1CCNCC1 \\
\hline 499 & $\mathbf{1 1}\{499\}$ & C1CC11CCCNCC1 \\
\hline 500 & $\mathbf{1 1}\{500\}$ & $\mathrm{C} 1 \mathrm{CNCC}(\mathrm{C} 1) \mathrm{c} 1 \mathrm{cc} 2 \operatorname{ccccc} 2[\mathrm{nH}] 1$ \\
\hline 501 & $\mathbf{1 1}\{501\}$ & $\mathrm{CCNCC}(\mathrm{C})=\mathrm{C}$ \\
\hline
\end{tabular}




$\begin{array}{lll}502 & \mathbf{1 1}\{502\} & \text { C1CC2(CN1)Oc1cccc1O2 } \\ 503 & \mathbf{1 1}\{503\} & \text { C1C2CNCCCN2c2cccc12 } \\ 504 & \mathbf{1 1}\{504\} & \text { O=C1CNCCN1C1CC1 } \\ 505 & \mathbf{1 1}\{505\} & \text { CC1CNCC }(\mathrm{C}) \mathrm{C} 1 \\ 506 & \mathbf{1 1}\{506\} & \text { CNCC }=\mathrm{C} \\ 507 & \mathbf{1 1}\{507\} & \text { Cl.OC1(CCNC1)C(F)F } \\ 508 & \mathbf{1 1}\{508\} & \text { C1OCC11CCNC1 } \\ 509 & \mathbf{1 1}\{509\} & \text { Cl.C1CC2CCC1N2 } \\ 510 & \mathbf{1 1}\{510\} & \text { Cl.C1C2CNCC12 } \\ 511 & \mathbf{1 1}\{511\} & \text { Cl.CN(C)C }(=\mathrm{O}) \mathrm{C} 1 \mathrm{CCNC} 1\end{array}$


Table S3. SMILES strings for the regents 15.

\begin{tabular}{|c|c|c|}
\hline \# & Reagent 15 & SMILE \\
\hline 1 & $\mathbf{1 5}\{1\}$ & $\mathrm{CC}(\mathrm{C}) \mathrm{N}$ \\
\hline 2 & $15\{2\}$ & NCc1cccol \\
\hline 3 & $\mathbf{1 5}\{3\}$ & $\mathrm{NCc} 1 \mathrm{ccc} 2 \mathrm{OCOc} 2 \mathrm{c} 1$ \\
\hline 4 & $15\{4\}$ & $\mathrm{COCCN}$ \\
\hline 5 & $\mathbf{1 5}\{5\}$ & $\mathrm{CCCCCN}$ \\
\hline 6 & $15\{6\}$ & $\mathrm{NCc} 1 \operatorname{ccc}(\mathrm{Cl}) \operatorname{cc} 1$ \\
\hline 7 & $15\{7\}$ & NCc $1 \operatorname{cccc} 1$ \\
\hline 8 & $15\{8\}$ & NCC1CCCO1 \\
\hline 9 & $15\{9\}$ & $\mathrm{COCCCN}$ \\
\hline 10 & $\mathbf{1 5}\{10\}$ & $\mathrm{CC}(\mathrm{C}) \mathrm{CCN}$ \\
\hline 11 & $\mathbf{1 5}\{11\}$ & NCC1CCCCC1 \\
\hline 12 & $\mathbf{1 5}\{12\}$ & $\mathrm{CN}(\mathrm{C}) \mathrm{CCCN}$ \\
\hline 13 & $\mathbf{1 5}\{13\}$ & $\mathrm{NCC}=\mathrm{C}$ \\
\hline 14 & $\mathbf{1 5}\{14\}$ & $\mathrm{COc} 1 \mathrm{ccc}(\mathrm{CCN}) \mathrm{cc} 1$ \\
\hline 15 & $\mathbf{1 5}\{15\}$ & $\mathrm{NCc} 1 \mathrm{ccccc} 1 \mathrm{Cl}$ \\
\hline 16 & $\mathbf{1 5}\{16\}$ & $\mathrm{NCc} 1 \operatorname{ccc}(\mathrm{Cl}) \mathrm{c} 1$ \\
\hline 17 & $\mathbf{1 5}\{17\}$ & $\mathrm{NC} 1 \mathrm{CC} 1$ \\
\hline 18 & $\mathbf{1 5}\{18\}$ & $\mathrm{COc} 1 \mathrm{ccc}(\mathrm{CN}) \mathrm{cc} 1$ \\
\hline 19 & $\mathbf{1 5}\{19\}$ & $\mathrm{NC} 1 \mathrm{CCCC} 1$ \\
\hline 20 & $15\{20\}$ & $\mathrm{NCCc} 1 \mathrm{c}[\mathrm{nH}] \mathrm{c} 2 \operatorname{ccccc} 12$ \\
\hline 21 & $\mathbf{1 5}\{21\}$ & $\mathrm{NCCc} 1 \mathrm{ccc}(\mathrm{Cl}) \mathrm{cc} 1$ \\
\hline 22 & $\mathbf{1 5}\{22\}$ & $\mathrm{NCc} 1 \mathrm{ccc}(\mathrm{Cl}) \mathrm{cc} 1 \mathrm{Cl}$ \\
\hline 23 & $15\{23\}$ & NCCCe1 1 cecc 1 \\
\hline 24 & $15\{24\}$ & $\mathrm{COc} 1 \mathrm{ccc}(\mathrm{CN}) \mathrm{cc} 1 \mathrm{OC}$ \\
\hline 25 & $\mathbf{1 5}\{25\}$ & $\mathrm{NCCC} 1=\mathrm{CCCCC} 1$ \\
\hline
\end{tabular}




\begin{tabular}{|c|c|c|}
\hline 26 & $15\{26\}$ & COc1ccec1CN \\
\hline 27 & $15\{27\}$ & $\mathrm{CCCN}$ \\
\hline 28 & $\mathbf{1 5}\{28\}$ & NCCc1cce(F)cc1 \\
\hline 29 & $\mathbf{1 5}\{29\}$ & NCc1ccc(F)cc1 \\
\hline 30 & $15\{30\}$ & Cc1 $1 \mathrm{ccc}(\mathrm{CN}) \mathrm{cc} 1$ \\
\hline 31 & $15\{31\}$ & $\mathrm{COc} 1 \mathrm{cccc}(\mathrm{CN}) \mathrm{c} 1$ \\
\hline 32 & $\mathbf{1 5}\{32\}$ & NCc1 $1 \operatorname{ccs} 1$ \\
\hline 33 & $\mathbf{1 5}\{33\}$ & $\mathrm{NCc} 1 \mathrm{ccccc} 1 \mathrm{~F}$ \\
\hline 34 & $15\{34\}$ & $\mathrm{NCc} 1 \operatorname{cccc}(\mathrm{c} 1) \mathrm{C}(\mathrm{F})(\mathrm{F}) \mathrm{F}$ \\
\hline 35 & $\mathbf{1 5}\{35\}$ & $\mathrm{NCCc} 1 \mathrm{ccc}(\mathrm{cc} 1) \mathrm{C}(\mathrm{F})(\mathrm{F}) \mathrm{F}$ \\
\hline 36 & $15\{36\}$ & $\mathrm{CC}(=\mathrm{O}) \mathrm{NCCN}$ \\
\hline 37 & $\mathbf{1 5}\{37\}$ & $\mathrm{CN}(\mathrm{C}) \mathrm{C} 1(\mathrm{CN}) \mathrm{CCCC} 1$ \\
\hline 38 & $15\{38\}$ & NCCOc1cecc1 \\
\hline 39 & $\mathbf{1 5}\{39\}$ & $\mathrm{CC}(\mathrm{C})(\mathrm{CN}) \mathrm{N} 1 \mathrm{CCOCC} 1$ \\
\hline 40 & $15\{40\}$ & $\mathrm{CC}(\mathrm{C})(\mathrm{CN}) \mathrm{N} 1 \mathrm{CCCCC} 1$ \\
\hline 41 & $\mathbf{1 5}\{41\}$ & $\mathrm{COc} 1 \mathrm{ccccc} 1 \mathrm{CCN}$ \\
\hline 42 & $15\{42\}$ & NCCCn1 $1 \operatorname{ccc} 2 \operatorname{ccccc} 12$ \\
\hline 43 & $\mathbf{1 5}\{43\}$ & $\mathrm{NCc} 1 \operatorname{ccc}(\operatorname{cc} 1) \mathrm{C}(\mathrm{N})=\mathrm{O}$ \\
\hline 44 & $15\{44\}$ & $\mathrm{NCCCN} 1 \mathrm{CCCCCC} 1=\mathrm{O}$ \\
\hline 45 & $\mathbf{1 5}\{45\}$ & NCCc1cccs 1 \\
\hline 46 & $15\{46\}$ & NCCe1ccco1 \\
\hline 47 & $15\{47\}$ & $\mathrm{Cc} 1 \mathrm{ccccc} 1 \mathrm{CN}$ \\
\hline 48 & $\mathbf{1 5}\{48\}$ & $\mathrm{NCCc} 1 \mathrm{ccccc} 1 \mathrm{~F}$ \\
\hline 49 & $15\{49\}$ & $\mathrm{NCc} 1 \mathrm{ccc}(\mathrm{OC}(\mathrm{F}) \mathrm{F}) \mathrm{cc} 1$ \\
\hline 50 & $\mathbf{1 5}\{50\}$ & $\operatorname{CCCNC}(=\mathrm{O}) \mathrm{CN}$ \\
\hline 51 & $\mathbf{1 5}\{51\}$ & NCCc1ccc2OCOc2c1 \\
\hline 52 & $\mathbf{1 5}\{52\}$ & $\operatorname{COc} 1 \mathrm{ccc}(\mathrm{C}) \mathrm{cc} 1 \mathrm{CCN}$ \\
\hline 53 & $\mathbf{1 5}\{53\}$ & $\mathrm{NCCc} 1 \operatorname{cccc}(\mathrm{F}) \mathrm{c} 1$ \\
\hline
\end{tabular}




\begin{tabular}{|c|c|c|}
\hline 54 & $\mathbf{1 5}\{54\}$ & NCCCOCC1CCCO1 \\
\hline 55 & $\mathbf{1 5}\{55\}$ & $\mathrm{NCc} 1 \mathrm{ccc}(\mathrm{cc} 1) \mathrm{C}(\mathrm{F})(\mathrm{F}) \mathrm{F}$ \\
\hline 56 & $15\{56\}$ & $\mathrm{Cc} 1 \mathrm{nc}(\mathrm{CN}) \mathrm{cs} 1$ \\
\hline 57 & $\mathbf{1 5}\{57\}$ & $\mathrm{Cc} 1 \mathrm{cc}(\mathrm{CN}) \mathrm{ccc} 1 \mathrm{~F}$ \\
\hline 58 & $\mathbf{1 5}\{58\}$ & $\mathrm{NCc} 1 \mathrm{ccc}(\mathrm{OC}(\mathrm{F})(\mathrm{F}) \mathrm{F}) \mathrm{cc} 1$ \\
\hline 59 & $\mathbf{1 5}\{59\}$ & $\mathrm{COc} 1 \mathrm{ccc} 2 \operatorname{cc}(\mathrm{CN}) \operatorname{ccc} 2 \mathrm{c} 1$ \\
\hline 60 & $15\{60\}$ & $\mathrm{NCc} 1 \mathrm{cnn}(\mathrm{c} 1)-\mathrm{c} 1 \mathrm{cccc} 1$ \\
\hline 61 & $15\{61\}$ & $\mathrm{CN}(\mathrm{C}) \mathrm{S}(=\mathrm{O})(=\mathrm{O}) \mathrm{c} 1 \mathrm{cccc} 1 \mathrm{CN}$ \\
\hline 62 & $15\{62\}$ & $\mathrm{NC} 1 \mathrm{CCN}(\mathrm{CC} 1) \mathrm{C}(=\mathrm{O}) \mathrm{c} 1 \mathrm{ccco} 1$ \\
\hline 63 & $15\{63\}$ & NCC1CC1 \\
\hline 64 & $15\{64\}$ & $\mathrm{CN}(\mathrm{C}) \mathrm{C}(=\mathrm{O}) \mathrm{c} 1 \mathrm{ccc}(\mathrm{CN}) \mathrm{cc} 1$ \\
\hline 65 & $\mathbf{1 5}\{65\}$ & $\mathrm{NC} 1 \mathrm{CCC}(\mathrm{CC} 1) \mathrm{C}(\mathrm{F})(\mathrm{F}) \mathrm{F}$ \\
\hline 66 & $15\{66\}$ & NCCC1CCCO1 \\
\hline 67 & $15\{67\}$ & $\mathrm{NCc} 1 \mathrm{ccc}(\mathrm{Cl}) \mathrm{nc} 1$ \\
\hline 68 & $\mathbf{1 5}\{68\}$ & $\mathrm{COc} 1 \mathrm{ccc}(\mathrm{OC}) \mathrm{c}(\mathrm{CN}) \mathrm{c} 1$ \\
\hline 69 & $15\{69\}$ & Cl.NCCNC $(=\mathrm{O}) \mathrm{c} 1 \mathrm{cccs} 1$ \\
\hline 70 & $15\{70\}$ & $\mathrm{Cc} 1 \mathrm{ccc}(\mathrm{cc} 1 \mathrm{~F}) \mathrm{C}(=\mathrm{O}) \mathrm{NCCN}$ \\
\hline 71 & $\mathbf{1 5}\{71\}$ & $\mathrm{NCc} 1 \operatorname{ccc}(\mathrm{F}) \mathrm{c} 1$ \\
\hline 72 & $\mathbf{1 5}\{72\}$ & $\mathrm{NC} 1 \mathrm{CCC} 1$ \\
\hline 73 & $15\{73\}$ & $\mathrm{COc} 1 \mathrm{ccc}(\mathrm{CN}) \mathrm{c}(\mathrm{OC}) \mathrm{c} 1$ \\
\hline 74 & $\mathbf{1 5}\{74\}$ & $\mathrm{CCOc} 1 \mathrm{cccc} 1 \mathrm{CN}$ \\
\hline 75 & $\mathbf{1 5}\{75\}$ & $\mathrm{NCCc} 1 \mathrm{ccccc} 1 \mathrm{Cl}$ \\
\hline 76 & $15\{76\}$ & $\mathrm{NCc} 1 \operatorname{cccc} 1 \mathrm{C}(\mathrm{F})(\mathrm{F}) \mathrm{F}$ \\
\hline 77 & $\mathbf{1 5}\{77\}$ & $\mathrm{NCc} 1 \mathrm{ccc}(\mathrm{F}) \mathrm{c}(\mathrm{F}) \mathrm{c} 1$ \\
\hline 78 & $\mathbf{1 5}\{78\}$ & $\mathrm{Cc} 1 \mathrm{ccc}(\mathrm{CN}) \mathrm{o} 1$ \\
\hline 79 & $\mathbf{1 5}\{79\}$ & NOC1CCCCO1 \\
\hline 80 & $\mathbf{1 5}\{80\}$ & $\mathrm{NCc} 1 \operatorname{ccc}(\mathrm{F}) \operatorname{cc} 1 \mathrm{C}(\mathrm{F})(\mathrm{F}) \mathrm{F}$ \\
\hline 81 & $\mathbf{1 5}\{81\}$ & $\mathrm{Cc} 1 \mathrm{ccsc} 1 \mathrm{CN}$ \\
\hline
\end{tabular}




\begin{tabular}{|c|c|c|}
\hline 82 & $\mathbf{1 5}\{82\}$ & $\mathrm{NCc} 1 \mathrm{ccccc} 1 \mathrm{Br}$ \\
\hline 83 & $\mathbf{1 5}\{83\}$ & $\mathrm{CCOCCN}$ \\
\hline 84 & $\mathbf{1 5}\{84\}$ & $\mathrm{CC}(\mathrm{C}) \mathrm{Oc} 1 \mathrm{cccc}(\mathrm{CN}) \mathrm{c} 1$ \\
\hline 85 & $\mathbf{1 5}\{85\}$ & $\mathrm{CC}(\mathrm{C}) \mathrm{Oc} 1 \mathrm{ccccc} 1 \mathrm{CN}$ \\
\hline 86 & $15\{86\}$ & $\mathrm{NCCOc} 1 \mathrm{ccccc} 1 \mathrm{Cl}$ \\
\hline 87 & $15\{87\}$ & $\mathrm{NCc} 1 \mathrm{ccc}(\mathrm{Cl}) \mathrm{c}(\mathrm{Cl}) \mathrm{c} 1$ \\
\hline 88 & $\mathbf{1 5}\{88\}$ & $\mathrm{NCc} 1 \operatorname{ccc}(\mathrm{F}) \mathrm{c}(\mathrm{Cl}) \mathrm{c} 1$ \\
\hline 89 & $\mathbf{1 5}\{89\}$ & $\mathrm{NCc} 1 \mathrm{ccc}(\mathrm{F}) \mathrm{cc} 1 \mathrm{~F}$ \\
\hline 90 & $\mathbf{1 5}\{90\}$ & $\mathrm{CC}(\mathrm{C}) \mathrm{OCCN}$ \\
\hline 91 & $\mathbf{1 5}\{91\}$ & Cl.NCCc1c $[\mathrm{nH}] \mathrm{c} 2 \mathrm{ccc}(\mathrm{F}) \mathrm{cc} 12$ \\
\hline 92 & $\mathbf{1 5}\{92\}$ & $\mathrm{COc} 1 \mathrm{ccc}(\mathrm{CN}) \mathrm{c} 1 \mathrm{OC}$ \\
\hline 93 & $\mathbf{1 5}\{93\}$ & $\mathrm{Cc} 1 \mathrm{ccc}(\mathrm{CN}) \mathrm{s} 1$ \\
\hline 94 & $\mathbf{1 5}\{94\}$ & $\mathrm{Cc} 1 \mathrm{cccc}(\mathrm{CN}) \mathrm{c} 1$ \\
\hline 95 & $\mathbf{1 5}\{95\}$ & $\mathrm{NCc} 1 \mathrm{cc}(\mathrm{F}) \operatorname{ccc} 1 \mathrm{~F}$ \\
\hline 96 & $\mathbf{1 5}\{96\}$ & $\mathrm{COc} 1 \operatorname{ccc}(\mathrm{CCN}) \mathrm{c} 1$ \\
\hline 97 & $\mathbf{1 5}\{97\}$ & $\operatorname{CCNC}(=\mathrm{O}) \mathrm{c} 1 \mathrm{ccc}(\mathrm{CN}) \mathrm{cc} 1$ \\
\hline 98 & $\mathbf{1 5}\{98\}$ & $\mathrm{NC} 1 \mathrm{CCN}(\mathrm{CC} 1) \mathrm{C} 1 \mathrm{CCCC} 1$ \\
\hline 99 & $\mathbf{1 5}\{99\}$ & $\mathrm{CCOc} 1 \mathrm{ccc}(\mathrm{CN}) \mathrm{cc} 1$ \\
\hline 100 & $\mathbf{1 5}\{100\}$ & $\mathrm{Cc} 1 \mathrm{ccc}(\mathrm{CN}) \mathrm{c}(\mathrm{C}) \mathrm{c} 1$ \\
\hline 101 & $\mathbf{1 5}\{101\}$ & $\mathrm{Cc} 1 \mathrm{cc}(\mathrm{C}) \mathrm{c}(\mathrm{CN}) \mathrm{c}(\mathrm{C}) \mathrm{c} 1$ \\
\hline 102 & $\mathbf{1 5}\{102\}$ & $\mathrm{NCc} 1 \mathrm{ccsc} 1$ \\
\hline 103 & $\mathbf{1 5}\{103\}$ & $\mathrm{NCC} 1 \mathrm{CCOC} 1$ \\
\hline 104 & $\mathbf{1 5}\{104\}$ & $\mathrm{NCc} 1 \mathrm{ccc}(\mathrm{CN} 2 \mathrm{CCCC} 2=\mathrm{O}) \mathrm{cc} 1$ \\
\hline 105 & $\mathbf{1 5}\{105\}$ & $\mathrm{NCc} 1 \operatorname{ccccc} 1 \mathrm{CN} 1 \mathrm{CCCC} 1=\mathrm{O}$ \\
\hline 106 & $\mathbf{1 5}\{106\}$ & $\mathrm{COCc} 1 \mathrm{ccc}(\mathrm{CN}) \mathrm{cc} 1$ \\
\hline 107 & $\mathbf{1 5}\{107\}$ & $\operatorname{COCc} 1 \mathrm{cccc}(\mathrm{CN}) \mathrm{c} 1$ \\
\hline 108 & $\mathbf{1 5}\{108\}$ & $\mathrm{NCc} 1 \operatorname{cccc}(\mathrm{c} 1) \mathrm{C}(\mathrm{N})=\mathrm{O}$ \\
\hline 109 & $\mathbf{1 5}\{109\}$ & $\mathrm{CN}(\mathrm{C}) \mathrm{C}(=\mathrm{O}) \mathrm{c} 1 \operatorname{cccc}(\mathrm{CN}) \mathrm{c} 1$ \\
\hline
\end{tabular}




\begin{tabular}{|c|c|c|}
\hline 110 & $\mathbf{1 5}\{110\}$ & $\mathrm{CNC}(=\mathrm{O}) \mathrm{c} 1 \operatorname{cccc}(\mathrm{CN}) \mathrm{c} 1$ \\
\hline 111 & $\mathbf{1 5}\{111\}$ & $\mathrm{NCc} 1 \mathrm{cc}(\mathrm{Cl}) \mathrm{c} 2 \mathrm{OCCOc} 2 \mathrm{c} 1$ \\
\hline 112 & $\mathbf{1 5}\{112\}$ & $\mathrm{NCc} 1 \mathrm{c}(\mathrm{Cl}) \operatorname{cccc} 1 \mathrm{Cl}$ \\
\hline 113 & $\mathbf{1 5}\{113\}$ & $\mathrm{NCCc} 1 \mathrm{c}(\mathrm{F}) \mathrm{cccc} 1 \mathrm{~F}$ \\
\hline 114 & $15\{114\}$ & Cc1cce(CCN)o1 \\
\hline 115 & $\mathbf{1 5}\{115\}$ & $\mathrm{NCCN} 1 \mathrm{CCNC} 1=\mathrm{O}$ \\
\hline 116 & $\mathbf{1 5}\{116\}$ & $\operatorname{CCCC}(=\mathrm{O}) \mathrm{Nc} 1 \mathrm{ccc}(\mathrm{CN}) \mathrm{cc} 1$ \\
\hline 117 & $\mathbf{1 5}\{117\}$ & NCc1ccoc1 \\
\hline 118 & $\mathbf{1 5}\{118\}$ & NCCCC1CCCC1 \\
\hline 119 & $\mathbf{1 5}\{119\}$ & $\mathrm{NCc} 1 \mathrm{cccc}(\mathrm{c} 1) \mathrm{N} 1 \mathrm{CCCC} 1=\mathrm{O}$ \\
\hline 120 & $15\{120\}$ & $\mathrm{Cc} 1 \mathrm{cc}(\mathrm{CN}) \mathrm{no} 1$ \\
\hline 121 & $\mathbf{1 5}\{121\}$ & $\mathrm{NCC} 1 \mathrm{CCOCC} 1$ \\
\hline 122 & $\mathbf{1 5}\{122\}$ & Cl.NCc1 $1 \csc 2 \operatorname{ccccc} 12$ \\
\hline 123 & $\mathbf{1 5}\{123\}$ & $\mathrm{Cc} \operatorname{lnn}(\mathrm{C}) \mathrm{c}(\mathrm{C}) \mathrm{c} 1 \mathrm{CN}$ \\
\hline 124 & $15\{124\}$ & NCc1 $1 \mathrm{ccc}(\mathrm{cc} 1)-\mathrm{n} 1 \mathrm{cccn} 1$ \\
\hline 125 & $\mathbf{1 5}\{125\}$ & $\mathrm{CC}(\mathrm{CN}) \operatorname{Oc} 1 \mathrm{ccc}(\mathrm{C}) \mathrm{cc} 1$ \\
\hline 126 & $15\{126\}$ & Cl.NCC12CC3CC $(\mathrm{CC}(\mathrm{C} 3) \mathrm{C} 1) \mathrm{C} 2$ \\
\hline 127 & $\mathbf{1 5}\{127\}$ & Cl.NCC\#C \\
\hline 128 & $\mathbf{1 5}\{128\}$ & $\operatorname{NCc} 1 \operatorname{ccc}(\mathrm{Cn} 2 \operatorname{ccc} 2) \operatorname{cc} 1$ \\
\hline 129 & $\mathbf{1 5}\{129\}$ & Cl.NC1Cc2 $2 \mathrm{cccc} 2 \mathrm{C} 1$ \\
\hline 130 & $\mathbf{1 5}\{130\}$ & Cl.NC1CCOCC1 \\
\hline 131 & $\mathbf{1 5}\{131\}$ & NCC1CCCc2 $\operatorname{ccccc} 12$ \\
\hline 132 & $\mathbf{1 5}\{132\}$ & NCc1cec(o1)-c1 $1 \operatorname{cccc} 1$ \\
\hline 133 & $\mathbf{1 5}\{133\}$ & Cn1ncce1CN \\
\hline 134 & $\mathbf{1 5}\{134\}$ & $\mathrm{NC} 1 \mathrm{CCCC}(\mathrm{O}) \mathrm{C} 1$ \\
\hline 135 & $\mathbf{1 5}\{135\}$ & $\operatorname{NCc} 1 \operatorname{cnn}(\mathrm{Cc} 2 \operatorname{ccccc} 2) \mathrm{c} 1$ \\
\hline 136 & $\mathbf{1 5}\{136\}$ & $\mathrm{Cc} \ln [\mathrm{nH}] \mathrm{cc} 1 \mathrm{CN}$ \\
\hline 137 & $\mathbf{1 5}\{137\}$ & $\mathrm{CC}(\mathrm{C}) \mathrm{NC}(=\mathrm{O}) \mathrm{Nc} 1 \mathrm{ccc}(\mathrm{CN}) \mathrm{cc} 1$ \\
\hline
\end{tabular}




\begin{tabular}{|c|c|c|}
\hline 138 & $\mathbf{1 5}\{138\}$ & $\mathrm{NCc} 1 \mathrm{cc}(\mathrm{Br}) \mathrm{cs} 1$ \\
\hline 139 & $\mathbf{1 5}\{139\}$ & $\mathrm{CC} 1 \mathrm{CC} 1 \mathrm{~N}$ \\
\hline 140 & $15\{140\}$ & $\mathrm{CC}(\mathrm{CN}) \operatorname{Cn} 1 \mathrm{ccc} 1$ \\
\hline 141 & $15\{141\}$ & $\mathrm{Cl} \cdot \mathrm{NCCCC}(=\mathrm{O}) \mathrm{NC} 1 \mathrm{CC} 1$ \\
\hline 142 & $15\{142\}$ & $\mathrm{CS}(=\mathrm{O})(=\mathrm{O}) \mathrm{Cc} 1 \mathrm{ccc}(\mathrm{CN}) \mathrm{cc} 1$ \\
\hline 143 & $15\{143\}$ & $\mathrm{CC}(\mathrm{O}) \mathrm{c} 1 \mathrm{ccc}(\mathrm{CN}) \mathrm{cc} 1$ \\
\hline 144 & $15\{144\}$ & NCCn 1 ccen 1 \\
\hline 145 & $\mathbf{1 5}\{145\}$ & NCC1CCCOC1c1cecc 1 \\
\hline 146 & $15\{146\}$ & $\mathrm{Cc} 1 \operatorname{cccc}(\mathrm{c} 1) \mathrm{C}(=\mathrm{O}) \mathrm{NCCN}$ \\
\hline 147 & $15\{147\}$ & $\mathrm{CS}(=\mathrm{O})(=\mathrm{O}) \mathrm{CCN}$ \\
\hline 148 & $\mathbf{1 5}\{148\}$ & NCC1CCCCO1 \\
\hline 149 & $15\{149\}$ & $\mathrm{NCC} 1 \mathrm{CCC}=\mathrm{CC} 1$ \\
\hline 150 & $\mathbf{1 5}\{150\}$ & $\mathrm{Cc} 1 \mathrm{cccc}(\mathrm{CCN}) \mathrm{c} 1$ \\
\hline 151 & $\mathbf{1 5}\{151\}$ & $\mathrm{NCCc} 1 \mathrm{ccc}(\mathrm{F}) \mathrm{cc} 1 \mathrm{~F}$ \\
\hline 152 & $\mathbf{1 5}\{152\}$ & $\mathrm{COC}(\mathrm{CN}) \mathrm{c} 1 \mathrm{ccccc} 1$ \\
\hline 153 & $\mathbf{1 5}\{153\}$ & $\mathrm{Cl} . \mathrm{NCC} 1 \mathrm{C} 2 \mathrm{CC} 3 \mathrm{CC}(\mathrm{C} 2) \mathrm{CC} 1 \mathrm{C} 3$ \\
\hline 154 & $\mathbf{1 5}\{154\}$ & $\mathrm{COc} 1 \mathrm{cc}(\mathrm{CN}) \operatorname{ccc} 1 \mathrm{C}$ \\
\hline 155 & $\mathbf{1 5}\{155\}$ & $\mathrm{CC} 1 \mathrm{CC}(\mathrm{C}) \mathrm{CN}(\mathrm{C} 1) \mathrm{C}(\mathrm{C})(\mathrm{C}) \mathrm{CN}$ \\
\hline 156 & $15\{156\}$ & $\mathrm{CC} 1 \mathrm{CCCN}(\mathrm{C} 1) \mathrm{C}(\mathrm{C})(\mathrm{C}) \mathrm{CN}$ \\
\hline 157 & $\mathbf{1 5}\{157\}$ & $\mathrm{Cn} 1 \mathrm{cc}(\mathrm{CN}) \mathrm{cn} 1$ \\
\hline 158 & $15\{158\}$ & $\mathrm{CC} 1 \mathrm{CCCC}(\mathrm{CN}) \mathrm{C} 1$ \\
\hline 159 & $\mathbf{1 5}\{159\}$ & $\mathrm{NCc} 1 \operatorname{ccc}(\mathrm{NC}(=\mathrm{O}) \mathrm{C} 2 \mathrm{CCCO} 2) \mathrm{c} 1$ \\
\hline 160 & $15\{160\}$ & $\mathrm{Cl} . \mathrm{CC}(\mathrm{CN}) \mathrm{C} 1 \mathrm{CC} 1$ \\
\hline 161 & $15\{161\}$ & $\mathrm{NCCc} 1 \mathrm{ccc}(\mathrm{Br}) \mathrm{s} 1$ \\
\hline 162 & $15\{162\}$ & $\mathrm{COCC}(\mathrm{C}) \mathrm{CN}$ \\
\hline 163 & $15\{163\}$ & $\operatorname{NCc} 1 \operatorname{ccc}(\mathrm{NC}(=\mathrm{O}) \operatorname{c} 2 \operatorname{ccco} 2) \mathrm{cc} 1$ \\
\hline 164 & $15\{164\}$ & $\mathrm{NCCc} 1 \mathrm{cn}[\mathrm{nH}] \mathrm{c} 1$ \\
\hline 165 & $\mathbf{1 5}\{165\}$ & $\mathrm{NCC}(\mathrm{Br})=\mathrm{C}$ \\
\hline
\end{tabular}




\begin{tabular}{|c|c|c|}
\hline 166 & $\mathbf{1 5}\{166\}$ & $\mathrm{Cc} 1 \mathrm{cc}(\mathrm{CN}) \mathrm{on} 1$ \\
\hline 167 & $\mathbf{1 5}\{167\}$ & $\mathrm{NCCCc} 1 \mathrm{cccc}(\mathrm{Cl}) \mathrm{c} 1$ \\
\hline 168 & $\mathbf{1 5}\{168\}$ & $\operatorname{NCCc} 1 \mathrm{ccc}(\mathrm{F}) \mathrm{c}(\mathrm{F}) \mathrm{c} 1$ \\
\hline 169 & $\mathbf{1 5}\{169\}$ & $\mathrm{CC}(=\mathrm{O}) \mathrm{Nc} 1 \mathrm{ccc}(\mathrm{CCN}) \mathrm{cc} 1$ \\
\hline 170 & $\mathbf{1 5}\{170\}$ & NCCOCC1CC1 \\
\hline 171 & $\mathbf{1 5}\{171\}$ & Cl.NCCNC $(=\mathrm{O}) \mathrm{C} 1 \mathrm{CC} 1$ \\
\hline 172 & $\mathbf{1 5}\{172\}$ & $\mathrm{Cc} 1 \mathrm{ccoc} 1 \mathrm{CN}$ \\
\hline 173 & $\mathbf{1 5}\{173\}$ & $\mathrm{Cc} 1 \mathrm{ccccc} 1 \mathrm{C}(\mathrm{C})(\mathrm{C}) \mathrm{CN}$ \\
\hline 174 & $\mathbf{1 5}\{174\}$ & $\mathrm{COc} 1 \mathrm{cccc} 1 \mathrm{C}(\mathrm{C})(\mathrm{C}) \mathrm{CN}$ \\
\hline 175 & $\mathbf{1 5}\{175\}$ & $\mathrm{CCC}(\mathrm{CN}) \mathrm{c} 1 \mathrm{ccccc} 1$ \\
\hline 176 & $15\{176\}$ & $\mathrm{NCc} 1 \operatorname{ccc}(\mathrm{cc} 1) \mathrm{C}(=\mathrm{O}) \mathrm{N} 1 \mathrm{CCCC} 1$ \\
\hline 177 & $\mathbf{1 5}\{177\}$ & Cc1occc1CN \\
\hline 178 & $\mathbf{1 5}\{178\}$ & Cl.NCC1CCCNC $1=\mathrm{O}$ \\
\hline 179 & $\mathbf{1 5}\{179\}$ & $\mathrm{NCCc} 1 \mathrm{cc}(\mathrm{F}) \mathrm{cc}(\mathrm{F}) \mathrm{c} 1$ \\
\hline 180 & $\mathbf{1 5}\{180\}$ & Cc1ccenc1CN \\
\hline 181 & $\mathbf{1 5}\{181\}$ & $\mathrm{NCc} 1 \mathrm{nc} 2 \mathrm{CCCc} 2 \mathrm{~s} 1$ \\
\hline 182 & $\mathbf{1 5}\{182\}$ & $\mathrm{NCc} 1 \mathrm{ccc}(\mathrm{OC} 2 \mathrm{CCCC} 2) \mathrm{cc} 1$ \\
\hline 183 & $\mathbf{1 5}\{183\}$ & Cl.NCCCc1 $1 \mathrm{ccc}(\mathrm{Cl}) \mathrm{cc} 1$ \\
\hline 184 & $\mathbf{1 5}\{184\}$ & $\mathrm{COc} 1 \operatorname{cccc} 1 \mathrm{C} 1(\mathrm{CN}) \mathrm{CC} 1$ \\
\hline 185 & $\mathbf{1 5}\{185\}$ & Cl.NC1CCSCC1 \\
\hline 186 & $\mathbf{1 5}\{186\}$ & $\mathrm{CC}(\mathrm{C})(\mathrm{CN}) \mathrm{c} 1 \mathrm{ccccc} 1 \mathrm{~F}$ \\
\hline 187 & $\mathbf{1 5}\{187\}$ & $\mathrm{Cc} 1[\mathrm{nH}] \mathrm{c} 2 \mathrm{ccccc} 2 \mathrm{c} 1 \mathrm{CCN}$ \\
\hline 188 & $\mathbf{1 5}\{188\}$ & $\mathrm{NCCCN} 1 \mathrm{CCOC} 1=\mathrm{O}$ \\
\hline 189 & $\mathbf{1 5}\{189\}$ & NC1CCCc2sccc12 \\
\hline 190 & $\mathbf{1 5}\{190\}$ & $\mathrm{COc} 1 \mathrm{ccc}(\mathrm{CCN}) \mathrm{cc} 1 \mathrm{~F}$ \\
\hline 191 & $\mathbf{1 5}\{191\}$ & $\mathrm{NCc} 1 \operatorname{ccc}(\mathrm{CO}) \mathrm{c} 1$ \\
\hline 192 & $\mathbf{1 5}\{192\}$ & $\mathrm{CCc} 1 \mathrm{cccc} 2 \mathrm{c}(\mathrm{CCN}) \mathrm{c}[\mathrm{nH}] \mathrm{c} 12$ \\
\hline 193 & $\mathbf{1 5}\{193\}$ & Cl.NCc1 $1 \operatorname{ccc}(\mathrm{CN} 2 \mathrm{CCCC} 2=\mathrm{O}) \mathrm{c} 1$ \\
\hline
\end{tabular}




\begin{tabular}{|c|c|c|}
\hline 194 & $\mathbf{1 5}\{194\}$ & $\mathrm{CCn} 1 \mathrm{cc}(\mathrm{CN}) \mathrm{cn} 1$ \\
\hline 195 & $\mathbf{1 5}\{195\}$ & $\mathrm{Cc} 1 \mathrm{c}(\mathrm{CN}) \mathrm{cnn} 1 \mathrm{C}$ \\
\hline 196 & $\mathbf{1 5}\{196\}$ & $\mathrm{Cn} 1 \mathrm{ccc}(\mathrm{CN}) \mathrm{n} 1$ \\
\hline 197 & $\mathbf{1 5}\{197\}$ & Cl.NCC(F)F \\
\hline 198 & $\mathbf{1 5}\{198\}$ & $\mathrm{CC}(\mathrm{N}) \mathrm{c} 1 \mathrm{cnn}(\mathrm{C}) \mathrm{c} 1$ \\
\hline 199 & $\mathbf{1 5}\{199\}$ & $\mathrm{NCc} 1 \mathrm{cccc} 2[\mathrm{nH}] \mathrm{ccc} 12$ \\
\hline 200 & $15\{200\}$ & NCc1 $1 \operatorname{ccc}(\mathrm{c} 1)-\mathrm{n} 1 \mathrm{cncn} 1$ \\
\hline 201 & $15\{201\}$ & Cl.NC1CCCOC1 \\
\hline 202 & $\mathbf{1 5}\{202\}$ & $\mathrm{CCC} 1(\mathrm{CN}) \mathrm{CCC} 1$ \\
\hline 203 & $15\{203\}$ & $\mathrm{CC} 1(\mathrm{CN}) \mathrm{CCCO} 1$ \\
\hline 204 & $\mathbf{1 5}\{204\}$ & $\mathrm{Cl} \cdot \mathrm{CCN} 1 \mathrm{CC}(\mathrm{N}) \mathrm{CC} 1=\mathrm{O}$ \\
\hline 205 & $\mathbf{1 5}\{205\}$ & $\mathrm{Cl} . \mathrm{NCC} 1=\mathrm{CCCCC} 1$ \\
\hline 206 & $15\{206\}$ & Cl.NCCc1nc(no1)-c1 $1 \mathrm{ccc}(\mathrm{Cl}) \mathrm{cc} 1$ \\
\hline 207 & $\mathbf{1 5}\{207\}$ & $\mathrm{CC} 1(\mathrm{CN}) \mathrm{CCOC} 1$ \\
\hline 208 & $\mathbf{1 5}\{208\}$ & $\mathrm{CC}(\mathrm{C}) \mathrm{n} 1 \mathrm{ccc}(\mathrm{CN}) \mathrm{n} 1$ \\
\hline 209 & $\mathbf{1 5}\{209\}$ & $\mathrm{NC} 1 \mathrm{CCN}(\mathrm{CC}(\mathrm{F})(\mathrm{F}) \mathrm{F}) \mathrm{C} 1=\mathrm{O}$ \\
\hline 210 & $\mathbf{1 5}\{210\}$ & $\mathrm{Cc} 1 \mathrm{cc}(\mathrm{CN}) \mathrm{ncn} 1$ \\
\hline 211 & $\mathbf{1 5}\{211\}$ & $\mathrm{Cl} . \mathrm{CC} 1(\mathrm{C}) \mathrm{CC} 1 \mathrm{CN}$ \\
\hline 212 & $\mathbf{1 5}\{212\}$ & Cl.NCCN1C $(=\mathrm{O}) \mathrm{CNC} 1=\mathrm{O}$ \\
\hline 213 & $\mathbf{1 5}\{213\}$ & NCc1ccon1 \\
\hline 214 & $\mathbf{1 5}\{214\}$ & $\mathrm{COc} 1 \mathrm{cc}(\mathrm{F}) \mathrm{cc}(\mathrm{CN}) \mathrm{c} 1$ \\
\hline 215 & $\mathbf{1 5}\{215\}$ & NCC1CSCCS1 \\
\hline 216 & $\mathbf{1 5}\{216\}$ & $\mathrm{CCSc} 1 \operatorname{ccc}(\mathrm{CN}) \mathrm{c} 1$ \\
\hline 217 & $\mathbf{1 5}\{217\}$ & $\operatorname{NCC} 1 \mathrm{CCC}(\mathrm{CC} 1) \mathrm{C}(\mathrm{F})(\mathrm{F}) \mathrm{F}$ \\
\hline 218 & $\mathbf{1 5}\{218\}$ & $\mathrm{Cl} . \mathrm{CC} 1(\mathrm{C}) \mathrm{C}(\mathrm{CN}) \mathrm{C} 1(\mathrm{C}) \mathrm{C}$ \\
\hline 219 & $\mathbf{1 5}\{219\}$ & $\mathrm{NCC} 1 \mathrm{CNC}(=\mathrm{O}) \mathrm{C} 1$ \\
\hline 220 & $\mathbf{1 5}\{220\}$ & NCCC1CCC1 \\
\hline 221 & $\mathbf{1 5}\{221\}$ & $\mathrm{CC}(\mathrm{C}) \mathrm{CC} 1(\mathrm{CN}) \mathrm{CCC} 1$ \\
\hline
\end{tabular}




\begin{tabular}{|c|c|c|}
\hline 222 & $\mathbf{1 5}\{222\}$ & Cc1nnsc1CN \\
\hline 223 & $\mathbf{1 5}\{223\}$ & $\mathrm{NCc} 1 \mathrm{ccc}(\mathrm{F}) \mathrm{cn} 1$ \\
\hline 224 & $15\{224\}$ & $\mathrm{Cc} 1 \mathrm{nnc}(\mathrm{CN}) \mathrm{s} 1$ \\
\hline 225 & $\mathbf{1 5}\{225\}$ & Cl.Cc1ccc $(\mathrm{CN}) \mathrm{cc} 1 \mathrm{~F}$ \\
\hline 226 & $15\{226\}$ & $\mathrm{CC} 1(\mathrm{C}) \mathrm{CCCC}(\mathrm{N}) \mathrm{C} 1$ \\
\hline 227 & $\mathbf{1 5}\{227\}$ & NOC1CCCC1 \\
\hline 228 & $15\{228\}$ & $\mathrm{Cl} \cdot \mathrm{CCOC}(=\mathrm{O}) \mathrm{C} 1(\mathrm{CN}) \mathrm{CCC} 1$ \\
\hline 229 & $15\{229\}$ & $\mathrm{CC} 1(\mathrm{C}) \mathrm{CC}(\mathrm{N}) \mathrm{CCO} 1$ \\
\hline 230 & $15\{230\}$ & Cl.NCCC $(=\mathrm{O}) \mathrm{N} 1 \mathrm{CCCCC} 1$ \\
\hline 231 & $\mathbf{1 5}\{231\}$ & Cl.NCCC1CC1 \\
\hline 232 & $15\{232\}$ & Cl.COC[C@@H](C)N \\
\hline 233 & $15\{233\}$ & NCC1CCCS1 \\
\hline 234 & $15\{234\}$ & $\mathrm{Cl} . \mathrm{CC}(=\mathrm{C}) \mathrm{CCN}$ \\
\hline 235 & $\mathbf{1 5}\{235\}$ & $\mathrm{COC} 1 \mathrm{CCC}(\mathrm{CN}) \mathrm{CC} 1$ \\
\hline 236 & $15\{236\}$ & $\mathrm{CC} 1 \mathrm{CC}(\mathrm{N}) \mathrm{CC}(\mathrm{C}) \mathrm{O} 1$ \\
\hline 237 & $\mathbf{1 5}\{237\}$ & $\mathrm{CC} 1 \mathrm{CC}(\mathrm{N}) \mathrm{CCO} 1$ \\
\hline 238 & $\mathbf{1 5}\{238\}$ & $\mathrm{Cl} . \mathrm{NC} 1 \mathrm{CCC}=\mathrm{C} 1$ \\
\hline 239 & $\mathbf{1 5}\{239\}$ & Cl.NCC1CCC $=\mathrm{C} 1$ \\
\hline 240 & $15\{240\}$ & $\mathrm{NCCCCC} 1 \mathrm{CC} 1$ \\
\hline 241 & $\mathbf{1 5}\{241\}$ & $\mathrm{COC} 1(\mathrm{CN}) \mathrm{CCOCC} 1$ \\
\hline 242 & $15\{242\}$ & $\mathrm{NCc} 1 \mathrm{cc}(\mathrm{no} 1) \mathrm{C} 1 \mathrm{CC} 1$ \\
\hline 243 & $15\{243\}$ & $\mathrm{NCc} 1 \mathrm{nc}(\mathrm{cs} 1) \mathrm{C}(\mathrm{F})(\mathrm{F}) \mathrm{F}$ \\
\hline 244 & $15\{244\}$ & $\mathrm{CCOC} 1 \mathrm{CC}(\mathrm{N}) \mathrm{C} 11 \mathrm{CCCC} 1$ \\
\hline 245 & $\mathbf{1 5}\{245\}$ & Cl.NC1CCCC1(F)F \\
\hline 246 & $15\{246\}$ & $\mathrm{CC}(\mathrm{C})(\mathrm{CN}) \mathrm{c} 1 \mathrm{cccc}(\mathrm{F}) \mathrm{c} 1$ \\
\hline 247 & $15\{247\}$ & $\operatorname{CSCC}(\mathrm{C}) \mathrm{N}$ \\
\hline 248 & $\mathbf{1 5}\{248\}$ & NCc1ccec2nccce12 \\
\hline 249 & $\mathbf{1 5}\{249\}$ & $\mathrm{CC}(\mathrm{CN}) \mathrm{c} 1 \mathrm{nc}(\mathrm{C}) \mathrm{cs} 1$ \\
\hline
\end{tabular}




\begin{tabular}{|c|c|c|}
\hline 250 & $\mathbf{1 5}\{250\}$ & NCCc1nc2CCCc2s1 \\
\hline 251 & $15\{251\}$ & $\mathrm{Cc} 1 \mathrm{lnc}(\mathrm{C}) \mathrm{c}(\mathrm{CN}) \mathrm{s} 1$ \\
\hline 252 & $\mathbf{1 5}\{252\}$ & $\operatorname{NCC} 1(\mathrm{CC} 1) \mathrm{c} 1 \mathrm{cccc}(\mathrm{Cl}) \mathrm{c} 1$ \\
\hline 253 & $\mathbf{1 5}\{253\}$ & $\mathrm{CCn} 1 \mathrm{nncc} 1 \mathrm{CN}$ \\
\hline 254 & $\mathbf{1 5}\{254\}$ & $\mathrm{CC}(\mathrm{O})(\mathrm{CCN}) \mathrm{c} 1 \mathrm{ccccc} 1$ \\
\hline 255 & $\mathbf{1 5}\{255\}$ & $\mathrm{COc} 1 \mathrm{ccc}(\mathrm{CN}) \mathrm{c}(\mathrm{OC}) \mathrm{n} 1$ \\
\hline 256 & $15\{256\}$ & $\mathrm{Cl} . \mathrm{CO}[\mathrm{C} @ \mathrm{H}](\mathrm{C}) \mathrm{CN}$ \\
\hline 257 & $\mathbf{1 5}\{257\}$ & Cl.NC1CCC2(CCCC2)CC1 \\
\hline 258 & $\mathbf{1 5}\{258\}$ & $\operatorname{NCC} 1 \mathrm{CCC}(\mathrm{F})(\mathrm{F}) \mathrm{C} 1$ \\
\hline 259 & $15\{259\}$ & $\mathrm{Cl} . \mathrm{COCC}(\mathrm{C})(\mathrm{C}) \mathrm{CN}$ \\
\hline 260 & $15\{260\}$ & Cl.CC $(=\mathrm{O}) \mathrm{N} 1 \mathrm{CC}[\mathrm{C} @ @ \mathrm{H}](\mathrm{N}) \mathrm{C} 1$ \\
\hline 261 & $15\{261\}$ & $\mathrm{Cl} \cdot \mathrm{CN} 1 \mathrm{CCC}(\mathrm{N}) \mathrm{C} 1=\mathrm{O}$ \\
\hline 262 & $15\{262\}$ & $\mathrm{CC}(\mathrm{CN}) \mathrm{Sc} 1 \mathrm{ccccc} 1$ \\
\hline 263 & $15\{263\}$ & $\mathrm{CC}(\mathrm{CN}) \mathrm{Oc} 1 \mathrm{cccc} 1 \mathrm{~F}$ \\
\hline 264 & $15\{264\}$ & $\mathrm{CC}(\mathrm{CN}) \mathrm{Oc} 1 \mathrm{cccc}(\mathrm{C}) \mathrm{c} 1$ \\
\hline 265 & $15\{265\}$ & $\mathrm{CC}(\mathrm{CN}) \mathrm{Oc} 1 \mathrm{cccc} 1 \mathrm{C}$ \\
\hline 266 & $15\{266\}$ & $\mathrm{NCCCC} \# \mathrm{C}$ \\
\hline 267 & $15\{267\}$ & $\mathrm{NCC} 1(\mathrm{Cc} 2 \operatorname{ccccc} 2) \mathrm{CC} 1$ \\
\hline 268 & $\mathbf{1 5}\{268\}$ & $\mathrm{NCc} 1 \operatorname{ccc}(\mathrm{CC}(\mathrm{N})=\mathrm{O}) \mathrm{cc} 1$ \\
\hline 269 & $15\{269\}$ & $\mathrm{CSCc} 1 \operatorname{ccc}(\mathrm{CN}) \mathrm{cc} 1$ \\
\hline 270 & $15\{270\}$ & $\mathrm{Cl.N}[\mathrm{C} @ \mathrm{H}] 1 \mathrm{C}[\mathrm{C} @ \mathrm{H}](\mathrm{C} 1) \mathrm{C}(\mathrm{N})=\mathrm{O}$ \\
\hline 271 & $15\{271\}$ & Cl.NCC1CC2CCC1CC2 \\
\hline 272 & $15\{272\}$ & $\mathrm{CCSc} 1 \mathrm{ccc}(\mathrm{CN}) \mathrm{cc} 1$ \\
\hline 273 & $15\{273\}$ & NCCn1cc(Br)cn1 \\
\hline 274 & $\mathbf{1 5}\{274\}$ & $\mathrm{NCCn} 1 \mathrm{ccc} 2 \mathrm{cc}(\mathrm{Cl}) \operatorname{ccc} 12$ \\
\hline 275 & $\mathbf{1 5}\{275\}$ & $\mathrm{NCc} 1 \mathrm{ncc}(\mathrm{Br}) \mathrm{s} 1$ \\
\hline 276 & $15\{276\}$ & Cl.NCC1CCC2(CC2)CC1 \\
\hline 277 & $15\{277\}$ & $\mathrm{NCc} 1 \mathrm{c}(\mathrm{F}) \operatorname{cccc} 1 \mathrm{OC}(\mathrm{F}) \mathrm{F}$ \\
\hline
\end{tabular}




\begin{tabular}{|c|c|c|}
\hline 278 & $15\{278\}$ & Cl.NCc1nnnn1C1CC1 \\
\hline 279 & $15\{279\}$ & $\mathrm{CSC}(\mathrm{C}) \mathrm{CN}$ \\
\hline 280 & $\mathbf{1 5}\{280\}$ & $\mathrm{NCCCn} 1 \mathrm{cc}(\mathrm{Br}) \mathrm{cn} 1$ \\
\hline 281 & $\mathbf{1 5}\{281\}$ & $\mathrm{NCc} 1 \mathrm{nc} 2 \mathrm{CCOCc} 2 \mathrm{~s} 1$ \\
\hline 282 & $15\{282\}$ & Cl.NC1CC(C1)OCc1 ccccc1 \\
\hline 283 & $\mathbf{1 5}\{283\}$ & $\mathrm{CCC}(=\mathrm{O}) \mathrm{N} 1 \mathrm{CCC}(\mathrm{N}) \mathrm{C} 1$ \\
\hline 284 & $15\{284\}$ & $\mathrm{COCCC} 1(\mathrm{CN}) \mathrm{CCCC} 1$ \\
\hline 285 & $\mathbf{1 5}\{285\}$ & $\mathrm{CCOCCC} 1(\mathrm{CN}) \mathrm{CCCC} 1$ \\
\hline 286 & $15\{286\}$ & $\mathrm{CCC} 1(\mathrm{CN}) \mathrm{CCCC} 1$ \\
\hline 287 & $15\{287\}$ & Cn1ncec1CCN \\
\hline 288 & $\mathbf{1 5}\{288\}$ & $\mathrm{CC}(\mathrm{C})(\mathrm{C}) \mathrm{C} 1 \mathrm{CC}(\mathrm{N}) \mathrm{CCO} 1$ \\
\hline 289 & $\mathbf{1 5}\{289\}$ & $\mathrm{CC}(\mathrm{C}) \mathrm{C} 1 \mathrm{CC}(\mathrm{N}) \mathrm{CCO} 1$ \\
\hline 290 & $15\{290\}$ & NCCCOC1CCOC1 \\
\hline 291 & $15\{291\}$ & $\mathrm{CC}(\mathrm{C}) \mathrm{c} 1 \csc (\mathrm{CN}) \mathrm{n} 1$ \\
\hline 292 & $15\{292\}$ & NCCOCC1CCCC1 \\
\hline 293 & $\mathbf{1 5}\{293\}$ & NCCCc1cccol \\
\hline 294 & $15\{294\}$ & $\mathrm{NCc} 1 \mathrm{cnn}(\mathrm{CC}(\mathrm{F})(\mathrm{F}) \mathrm{F}) \mathrm{c} 1$ \\
\hline 295 & $\mathbf{1 5}\{295\}$ & $\mathrm{CNC}(=\mathrm{O}) \mathrm{C}(\mathrm{C})(\mathrm{C}) \mathrm{CN}$ \\
\hline 296 & $15\{296\}$ & $\mathrm{CCNC}(=\mathrm{O}) \mathrm{C}(\mathrm{C})(\mathrm{C}) \mathrm{CN}$ \\
\hline 297 & $\mathbf{1 5}\{297\}$ & $\mathrm{CC}(\mathrm{CN}) \mathrm{c} 1 \mathrm{nccs} 1$ \\
\hline 298 & $\mathbf{1 5}\{298\}$ & $\mathrm{CCN}(\mathrm{C}) \mathrm{C}(=\mathrm{O}) \mathrm{CN}$ \\
\hline 299 & $\mathbf{1 5}\{299\}$ & $\mathrm{COC} 1 \mathrm{CC}(\mathrm{N}) \mathrm{C} 1(\mathrm{C}) \mathrm{C}$ \\
\hline 300 & $\mathbf{1 5}\{300\}$ & $\mathrm{Cl} . \mathrm{CC}(\mathrm{C})(\mathrm{C}) \mathrm{N} 1 \mathrm{CC}(\mathrm{N}) \mathrm{CC} 1=\mathrm{O}$ \\
\hline 301 & $15\{301\}$ & $\operatorname{CCSC} 1 \mathrm{CCCC}(\mathrm{N}) \mathrm{C} 1$ \\
\hline 302 & $\mathbf{1 5}\{302\}$ & $\mathrm{Cl} . \mathrm{CC}(\mathrm{C}) \mathrm{C} 1 \mathrm{OCCCC} 1 \mathrm{CN}$ \\
\hline 303 & $15\{303\}$ & $\mathrm{CCOCCC} 1(\mathrm{CN}) \mathrm{CCC} 1$ \\
\hline 304 & $\mathbf{1 5}\{304\}$ & $\mathrm{COCCC} 1(\mathrm{CN}) \mathrm{CCC} 1$ \\
\hline 305 & $\mathbf{1 5}\{305\}$ & $\mathrm{Cl} . \mathrm{CC}(\mathrm{C}) \mathrm{CC} 1(\mathrm{CN}) \mathrm{CC} 1$ \\
\hline
\end{tabular}




\begin{tabular}{|c|c|c|}
\hline 306 & $15\{306\}$ & $\mathrm{Cc} 1 \mathrm{cscc} 1 \mathrm{CN}$ \\
\hline 307 & $15\{307\}$ & $\mathrm{Cl} . \mathrm{CCCC} 1(\mathrm{CN}) \mathrm{CCC} 1$ \\
\hline 308 & $\mathbf{1 5}\{308\}$ & $\mathrm{CCC}(\mathrm{OCCN}) \mathrm{c} 1 \mathrm{ccccc} 1$ \\
\hline 309 & $\mathbf{1 5}\{309\}$ & $\mathrm{COCCC} 1(\mathrm{CN}) \mathrm{CC} 1$ \\
\hline 310 & $\mathbf{1 5}\{310\}$ & $\mathrm{CCOCCC} 1(\mathrm{CN}) \mathrm{CC} 1$ \\
\hline 311 & $\mathbf{1 5}\{311\}$ & $\mathrm{COC}(\mathrm{CN}) \mathrm{C} 1 \mathrm{CCCCC} 1$ \\
\hline 312 & $\mathbf{1 5}\{312\}$ & $\mathrm{COC} 1(\mathrm{CN}) \mathrm{CCC} 1$ \\
\hline 313 & $\mathbf{1 5}\{313\}$ & $\mathrm{CC}(\mathrm{C})(\mathrm{O}) \mathrm{C}(\mathrm{C})(\mathrm{C}) \mathrm{CN}$ \\
\hline 314 & $15\{314\}$ & $\mathrm{CCc} \operatorname{lnn}(\mathrm{C}) \mathrm{c}(\mathrm{CC}) \mathrm{c} 1 \mathrm{CN}$ \\
\hline 315 & $\mathbf{1 5}\{315\}$ & $\mathrm{CSc} 1 \mathrm{cccc}(\mathrm{F}) \mathrm{c} 1 \mathrm{CN}$ \\
\hline 316 & $15\{316\}$ & $\mathrm{NCCC} 1 \mathrm{CC} 2 \mathrm{CCC} 1 \mathrm{O} 2$ \\
\hline 317 & $15\{317\}$ & Cl.CCCC1(CN)CC1 \\
\hline 318 & $\mathbf{1 5}\{318\}$ & Cl.CC1CCC $(\mathrm{N}) \mathrm{C} 1$ \\
\hline 319 & $\mathbf{1 5}\{319\}$ & $\mathrm{CC}(\mathrm{C})(\mathrm{CN}) \mathrm{C} 1 \mathrm{CCCC} 1$ \\
\hline 320 & $15\{320\}$ & Cl.CO[C@H]1C[C@H](N)C1 \\
\hline 321 & $\mathbf{1 5}\{321\}$ & Cl.CCC1(CN)CCOCC1 \\
\hline 322 & $\mathbf{1 5}\{322\}$ & $\mathrm{Cl} \cdot \mathrm{NC} 1 \mathrm{CC}(\mathrm{C} 1) \mathrm{C}(\mathrm{N})=\mathrm{O}$ \\
\hline 323 & $\mathbf{1 5}\{323\}$ & Cl.COCC1CC(N)C1 \\
\hline 324 & $15\{324\}$ & $\mathrm{Cl} . \mathrm{CS}(=\mathrm{O})(=\mathrm{O}) \mathrm{C} 1(\mathrm{CN}) \mathrm{CC} 1$ \\
\hline 325 & $\mathbf{1 5}\{325\}$ & $\mathrm{Cl} . \mathrm{CC} 1 \mathrm{CC}(\mathrm{CN}) \mathrm{CCO} 1$ \\
\hline 326 & $15\{326\}$ & $\mathrm{Cl} . \mathrm{CC} 1(\mathrm{CN}) \mathrm{CCCc} 2 \mathrm{cccc} 12$ \\
\hline 327 & $15\{327\}$ & $\mathrm{Cl} . \mathrm{NC} 1 \mathrm{CC} 2(\mathrm{CCC} 2) \mathrm{C} 1$ \\
\hline 328 & $\mathbf{1 5}\{328\}$ & Cl.CCO[C@@H]1C[C@H](N)C \\
\hline 329 & $15\{329\}$ & Cl.CCO $[\mathrm{C} @ \mathrm{H}] 1 \mathrm{C}[\mathrm{C} @ \mathrm{H}](\mathrm{N}) \mathrm{C} 1$ \\
\hline 330 & $\mathbf{1 5}\{330\}$ & $\operatorname{Cl} \cdot \operatorname{NCC} 1 \mathrm{CCCCS} 1(=\mathrm{O})=\mathrm{O}$ \\
\hline 331 & $15\{331\}$ & $\mathrm{NCc} 1 \csc (\mathrm{c} 1) \mathrm{C}(\mathrm{F})(\mathrm{F}) \mathrm{F}$ \\
\hline 332 & $\mathbf{1 5}\{332\}$ & $\mathrm{CON} 1 \mathrm{CCC}(\mathrm{N}) \mathrm{CC} 1$ \\
\hline 333 & $15\{333\}$ & $\mathrm{NC} 1 \mathrm{COC} 2(\mathrm{CCC} 2) \mathrm{C} 1$ \\
\hline
\end{tabular}




\begin{tabular}{|c|c|c|}
\hline 334 & $\mathbf{1 5}\{334\}$ & $\operatorname{Cl} . \mathrm{CCS}(=\mathrm{O})(=\mathrm{O}) \mathrm{c} 1 \mathrm{ccc}(\mathrm{CN}) \mathrm{cc} 1$ \\
\hline 335 & $\mathbf{1 5}\{335\}$ & Cl.C[C@H]1OCC[C@H]1N \\
\hline 336 & $15\{336\}$ & Cl.CCC[1C@@H]1C[1C@H]1N \\
\hline 337 & $15\{337\}$ & $\mathrm{Cn} 1 \mathrm{nccc} 1 \mathrm{C} 1 \mathrm{CC}(\mathrm{N}) \mathrm{CCO} 1$ \\
\hline 338 & $15\{338\}$ & CN1CCC $[\mathrm{C} @ @ \mathrm{H}](\mathrm{CN})[\mathrm{C} @ @ \mathrm{H}] 1 \mathrm{c} 1 \mathrm{ccnn} 1 \mathrm{C}$ \\
\hline 339 & $\mathbf{1 5}\{339\}$ & $\mathrm{NCC}(\mathrm{C} 1 \mathrm{CCC} 1) \mathrm{C} 1 \mathrm{CCC} 1$ \\
\hline 340 & $15\{340\}$ & $\mathrm{NCCC} 1 \mathrm{CCC} 2(\mathrm{CCC} 2) \mathrm{O} 1$ \\
\hline 341 & $15\{341\}$ & $\mathrm{NCc} 1 \mathrm{nc}(\mathrm{n} 2 \mathrm{CCCCc} 12) \mathrm{C}(\mathrm{F})(\mathrm{F}) \mathrm{F}$ \\
\hline 342 & $15\{342\}$ & $\mathrm{Cl} . \mathrm{CC}(\mathrm{C})(\mathrm{CN}) \mathrm{C} 1 \mathrm{CCC} 1$ \\
\hline 343 & $15\{343\}$ & $\mathrm{Cl} . \mathrm{CC} 1(\mathrm{CN}) \mathrm{CC} 1(\mathrm{Cl}) \mathrm{Cl}$ \\
\hline 344 & $15\{344\}$ & Cl.CC(C)[1C@@ $\mathrm{H}] 1 \mathrm{C}[1 \mathrm{C} @ \mathrm{H}] 1 \mathrm{~N}$ \\
\hline 345 & $\mathbf{1 5}\{345\}$ & Cl.CC(C)C[1C@@H]1C[1C@H]1N \\
\hline 346 & $15\{346\}$ & $\mathrm{Cc} 1 \mathrm{nn}(\mathrm{C}) \mathrm{c}(\mathrm{C}) \mathrm{c} 1[1 \mathrm{C} @ @ \mathrm{H}] 1 \mathrm{OCC}[1 \mathrm{C} @ \mathrm{H}] 1 \mathrm{CN}$ \\
\hline 347 & $15\{347\}$ & $\mathrm{CC}(\mathrm{C})(\mathrm{C}) \mathrm{O}[\mathrm{C} @ @ \mathrm{H}] 1 \mathrm{C}[\mathrm{C} @ \mathrm{H}](\mathrm{N}) \mathrm{C} 1$ \\
\hline 348 & $15\{348\}$ & $\operatorname{Cl} \cdot \mathrm{COC}(=\mathrm{O}) \mathrm{CC} 1 \mathrm{CC}(\mathrm{N}) \mathrm{C} 1$ \\
\hline 349 & $15\{349\}$ & Cl.CCN1CCC $(\mathrm{N}) \mathrm{C} 1=\mathrm{O}$ \\
\hline 350 & $\mathbf{1 5}\{350\}$ & Cl.N[C@H]1C[C@@H](C1)C(N)=O \\
\hline 351 & $\mathbf{1 5}\{351\}$ & $\mathrm{Cl} . \mathrm{COCC} 1(\mathrm{CCN}) \mathrm{CC} 1$ \\
\hline 352 & $\mathbf{1 5}\{352\}$ & $\operatorname{NCCCC} 1(\mathrm{O}) \mathrm{CCC} 1$ \\
\hline 353 & $\mathbf{1 5}\{353\}$ & Cl.NCCN1CC2CCCCN2C1=O \\
\hline 354 & $15\{354\}$ & Cl.NCC1SCCS1 \\
\hline 355 & $\mathbf{1 5}\{355\}$ & $\mathrm{NC} 1 \mathrm{COC}(\mathrm{C} 1) \mathrm{C} 1 \mathrm{CC} 1$ \\
\hline 356 & $15\{356\}$ & $\mathrm{Cl} . \mathrm{NC} 1 \mathrm{CCN}(\mathrm{C} 2 \mathrm{CCCCC} 2) \mathrm{C} 1=\mathrm{O}$ \\
\hline 357 & $\mathbf{1 5}\{357\}$ & Cl.CO[C@@H]1C[C@H](CN)C1 \\
\hline 358 & $\mathbf{1 5}\{358\}$ & Cl.NC1CCN $(\mathrm{C} 2 \mathrm{CCC} 2) \mathrm{C} 1=\mathrm{O}$ \\
\hline 359 & $\mathbf{1 5}\{359\}$ & $\mathrm{CC} 1 \mathrm{CC} 2(\mathrm{CCC}(\mathrm{CN}) \mathrm{O} 2) \mathrm{CO} 1$ \\
\hline 360 & $15\{360\}$ & $\mathrm{CC} 1(\mathrm{CN}) \mathrm{CCOC} 2(\mathrm{CCC} 2) \mathrm{C} 1$ \\
\hline 361 & $\mathbf{1 5}\{361\}$ & Cl.NC[1C@@H]1CCO[1C@H]1c1ccccc1 \\
\hline
\end{tabular}




\begin{tabular}{|c|c|c|}
\hline 362 & $\mathbf{1 5}\{362\}$ & Cl.NCC12COCCN1C $(=\mathrm{O}) \mathrm{COC} 2$ \\
\hline 363 & $\mathbf{1 5}\{363\}$ & Cl.NCC1CC2(CO1)CCOCC2 \\
\hline 364 & $\mathbf{1 5}\{364\}$ & Cl.NCC1CC2CCC $(\mathrm{C} 1) \mathrm{S} 2(=\mathrm{O})=\mathrm{O}$ \\
\hline 365 & $\mathbf{1 5}\{365\}$ & NCC1CCC2(CCOCC2)CO1 \\
\hline 366 & $15\{366\}$ & NCCC1CCC2(CCOCC2)CO1 \\
\hline 367 & $\mathbf{1 5}\{367\}$ & Cl.NCCC1CCCC1(F)F \\
\hline 368 & $\mathbf{1 5}\{368\}$ & Cl.NCC1CCC2(CCC2)CO1 \\
\hline 369 & $\mathbf{1 5}\{369\}$ & Cl. NC1CC $(\mathrm{CC}(\mathrm{N})=\mathrm{O}) \mathrm{C} 1$ \\
\hline 370 & $\mathbf{1 5}\{370\}$ & Cl.NCC1CC2CCC(C1)S2 \\
\hline 371 & $\mathbf{1 5}\{371\}$ & $\mathrm{Cl} \cdot \mathrm{COC} 1(\mathrm{CN}) \mathrm{CCS}(=\mathrm{O})(=\mathrm{O}) \mathrm{C} 1$ \\
\hline 372 & $\mathbf{1 5}\{372\}$ & Cl.NCC12CC1CCCC2 \\
\hline 373 & $\mathbf{1 5}\{373\}$ & Cl.NCC1CCC $(\mathrm{O} 1) \mathrm{C}(\mathrm{F})(\mathrm{F}) \mathrm{F}$ \\
\hline 374 & $\mathbf{1 5}\{374\}$ & Cl.NC[1C@@H]1[1C@@H](c2ccccs)C1(F)F \\
\hline 375 & $\mathbf{1 5}\{375\}$ & Cl.N[1C@@H]1[1C@H]2Cc3ccccs3[1C@@H]12 \\
\hline 376 & $\mathbf{1 5}\{376\}$ & Cl.NCC1CCCC2(CCC2)O1 \\
\hline 377 & $\mathbf{1 5}\{377\}$ & Cl.NCC1CCC2(CCOCC2)O1 \\
\hline 378 & $\mathbf{1 5}\{378\}$ & Cl.NC[1C@]12C[1C@H]1CCC2 \\
\hline
\end{tabular}


Table S4. Parallel synthesis of 5-aminotetrazoles 8

\begin{tabular}{|c|c|c|c|c|c|c|}
\hline \# & $\begin{array}{l}\text { Isothio- } \\
\text { cyanate }\end{array}$ & $\begin{array}{c}\text { Secondary } \\
\text { amine }\end{array}$ & Product & SMILE & $\begin{array}{c}\text { Yield, } \\
\text { mg }\end{array}$ & $\begin{array}{c}\text { Yield, } \\
\%\end{array}$ \\
\hline 1 & $10\{1\}$ & $11\{1\}$ & $8\{1,1\}$ & $\mathrm{CNS}(=\mathrm{O})(=\mathrm{O}) \mathrm{c} 1 \operatorname{cccc} 2 \mathrm{CN}(\mathrm{CCc} 12) \mathrm{c} 1 \mathrm{nnnn} 1 \mathrm{CC}=\mathrm{C}$ & 27 & 22 \\
\hline 2 & $10\{1\}$ & $11\{2\}$ & $\mathbf{8}\{1,2\}$ & $\mathrm{C}=\mathrm{CCn} 1 \mathrm{nnnc} 1 \mathrm{~N} 1 \mathrm{CCN}(\mathrm{CC} 1) \mathrm{C}(=\mathrm{O}) \mathrm{C}(=\mathrm{O}) \mathrm{NC} 1 \mathrm{CC} 1$ & 13 & 15 \\
\hline 3 & $10\{1\}$ & $11\{3\}$ & $\mathbf{8}\{1,3\}$ & $\mathrm{OC} 1(\mathrm{Cc} 2 \mathrm{nc}(\mathrm{no} 2) \mathrm{C} 2 \mathrm{CC} 2) \mathrm{CCCN}(\mathrm{C} 1) \mathrm{c} 1 \mathrm{nnnn} 1 \mathrm{CC}=\mathrm{C}$ & 0 & 0 \\
\hline 4 & $10\{2\}$ & $11\{4\}$ & $\mathbf{8}\{2,4\}$ & Cn1nnnc1N1CCOC2(CCC2)C1 & 0 & 0 \\
\hline 5 & $10\{2\}$ & $11\{5\}$ & $\mathbf{8}\{2,5\}$ & Cn1nnnc1N1CCOCC1c1cc(F)ce(F)c1 & 0 & 0 \\
\hline 6 & $10\{2\}$ & $11\{6\}$ & $\mathbf{8}\{2,6\}$ & Cn1nnnc1N1CCOc2ce(F)cce2C1 & 0 & 0 \\
\hline 7 & $\mathbf{1 0}\{2\}$ & $11\{7\}$ & $\mathbf{8}\{2,7\}$ & $\mathrm{CC} 1=\mathrm{C}(\mathrm{C}) \mathrm{CN}(\mathrm{CC} 1) \mathrm{c} 1 \mathrm{nnnnn} 1 \mathrm{C}$ & 0 & 0 \\
\hline 8 & $10\{2\}$ & $11\{8\}$ & $\mathbf{8}\{2,8\}$ & Cn1nnnc1N1CC2(CCOCC2)[1C@@H]2CCC[1C@H]12 & 43 & 34 \\
\hline 9 & $10\{2\}$ & $\mathbf{1 1}\{9\}$ & $\mathbf{8}\{2,9\}$ & Cn1nnnc1N1CCCC1c1nc2ce(Cl)ccc2[nH]1 & 43 & 21 \\
\hline 10 & $10\{3\}$ & $\mathbf{1 1}\{10\}$ & $8\{3,10\}$ & CCCCn1nnnc1N1CCN $(\mathrm{CC} 1) \mathrm{C}(=\mathrm{O}) \mathrm{c} 1 \mathrm{ccc} 1 \mathrm{C}$ & 27 & 31 \\
\hline 11 & $10\{3\}$ & $\mathbf{1 1}\{2\}$ & $\mathbf{8}\{3,2\}$ & $\mathrm{CCCCn} 1 \mathrm{nnn} 1 \mathrm{~N} 1 \mathrm{CCN}(\mathrm{CC} 1) \mathrm{C}(=\mathrm{O}) \mathrm{C}(=\mathrm{O}) \mathrm{NC} 1 \mathrm{CC} 1$ & 16 & 21 \\
\hline 12 & $10\{3\}$ & $11\{8\}$ & $\mathbf{8}\{3,8\}$ & CCCCn1nnnc1N1CC2(CCOCC2)[1C@@H]2CCC[1C@H]12 & 21 & 24 \\
\hline 13 & $\mathbf{1 0}\{3\}$ & $11\{3\}$ & $\mathbf{8}\{3,3\}$ & CCCCn1nnnc1N1CCCC $(\mathrm{O})(\mathrm{Cc} 2 \mathrm{nc}(\mathrm{no} 2) \mathrm{C} 2 \mathrm{CC} 2) \mathrm{C} 1$ & 0 & 0 \\
\hline 14 & $10\{3\}$ & $11\{11\}$ & $8\{3,11\}$ & $\mathrm{CCCCn} 1 \mathrm{nnn} 1 \mathrm{~N}(\mathrm{CC}(=\mathrm{O}) \mathrm{NC}) \mathrm{Cc} 1 \mathrm{ccccc} 1$ & 0 & 0 \\
\hline 15 & $10\{3\}$ & $\mathbf{1 1}\{12\}$ & $\mathbf{8}\{3,12\}$ & CCCCn1nnnc1N1CCCC(C1)c1nncn1C & 0 & 0 \\
\hline 16 & $10\{4\}$ & $11\{7\}$ & $\mathbf{8}\{4,7\}$ & $\mathrm{CCn} 1 \mathrm{nnnc} 1 \mathrm{~N} 1 \mathrm{CCC}(\mathrm{C})=\mathrm{C}(\mathrm{C}) \mathrm{C} 1$ & 0 & 0 \\
\hline 17 & $10\{4\}$ & $11\{8\}$ & $\mathbf{8}\{4,8\}$ & CCn1nnnc1N1CC2(CCOCC2)[1C@@H]2CCC[1C@H]12 & 26 & 29 \\
\hline
\end{tabular}




\begin{tabular}{|c|c|c|c|c|c|}
\hline 18 & $10\{4\}$ & $11\{2\}$ & $\mathbf{8}\{4,2\}$ & $\mathrm{CCn} 1 \mathrm{nnnc} 1 \mathrm{~N} 1 \mathrm{CCN}(\mathrm{CC} 1) \mathrm{C}(=\mathrm{O}) \mathrm{C}(=\mathrm{O}) \mathrm{NC} 1 \mathrm{CC} 1$ & 29 \\
\hline 19 & $10\{5\}$ & $11\{13\}$ & $\mathbf{8}\{5,13\}$ & $\mathrm{C}(\mathrm{c} 1 \mathrm{ccccc} 1) \mathrm{n} 1 \mathrm{nnn} \mathrm{c} 1 \mathrm{~N} 1 \mathrm{CCCC} 1 \mathrm{C} 1 \mathrm{CCCO} 1$ & 35 \\
\hline 20 & $10\{5\}$ & $11\{14\}$ & $\mathbf{8}\{5,14\}$ & $\mathrm{CC}(\mathrm{CS}(\mathrm{C})(=\mathrm{O})=\mathrm{O}) \mathrm{N}(\mathrm{C} 1 \mathrm{CC} 1) \mathrm{c} 1 \mathrm{nnnn} 1 \mathrm{Cc} 1 \mathrm{ccccc} 1$ & 15 \\
\hline 21 & $10\{5\}$ & $\mathbf{1 1}\{15\}$ & $\mathbf{8}\{5,15\}$ & $\mathrm{COC} 1(\mathrm{CCN}(\mathrm{CC} 1) \mathrm{c} 1 \mathrm{nnnn} 1 \mathrm{Cc} 1 \mathrm{cccc} 1) \mathrm{C}(\mathrm{F})(\mathrm{F}) \mathrm{F}$ & 0 \\
\hline 22 & $\mathbf{1 0}\{5\}$ & $\mathbf{1 1}\{8\}$ & $\mathbf{8}\{5,8\}$ & $\begin{array}{l}\mathrm{C}(\mathrm{c} 1 \mathrm{ccccc} 1) \mathrm{n} 1 \mathrm{nnn} 1 \mathrm{~N} 1 \mathrm{CC} 2(\mathrm{CCOCC} 2)[1 \mathrm{C} @ @ \mathrm{H}] 2 \mathrm{CCC}[1 \mathrm{C} @ \\
\mathrm{H}] 12\end{array}$ & 0 \\
\hline 23 & $10\{5\}$ & $11\{12\}$ & $\mathbf{8}\{5,12\}$ & Cn1 cnnc1C1CCCN(C1)c1nnnn1Cc1 1 cccc 1 & 0 \\
\hline 24 & $10\{6\}$ & $\mathbf{1 1}\{16\}$ & $8\{6,16\}$ & $\mathrm{O}=\mathrm{C} 1 \mathrm{NCCN} 1 \mathrm{C} 1 \mathrm{CCCN}(\mathrm{C} 1) \mathrm{c} 1 \mathrm{nnnnn} 1 \mathrm{CCc} 1 \mathrm{ccccc} 1$ & 29 \\
\hline 25 & $10\{6\}$ & $\mathbf{1 1}\{13\}$ & $\mathbf{8}\{6,13\}$ & $\mathrm{C}(\mathrm{Cn} 1 \mathrm{nnnn} 1 \mathrm{~N} 1 \mathrm{CCCC} 1 \mathrm{C} 1 \mathrm{CCCO} 1) \mathrm{c} 1 \mathrm{cccc} 1$ & 5 \\
\hline 26 & $10\{6\}$ & $\mathbf{1 1}\{14\}$ & $\mathbf{8}\{6,14\}$ & $\operatorname{CC}(\operatorname{CS}(\mathrm{C})(=\mathrm{O})=\mathrm{O}) \mathrm{N}(\mathrm{C} 1 \mathrm{CC} 1) \mathrm{c} 1 \mathrm{nnnn} 1 \mathrm{CCc} 1 \mathrm{ccccc} 1$ & 0 \\
\hline 27 & $10\{6\}$ & $\mathbf{1 1}\{17\}$ & $8\{6,17\}$ & $\operatorname{COCCCN}(\mathrm{CCOC}) \mathrm{c} 1 \mathrm{nnnnn} 1 \mathrm{CCc} 1 \mathrm{ccccc} 1$ & 0 \\
\hline 28 & $10\{6\}$ & $\mathbf{1 1}\{18\}$ & $8\{6,18\}$ & $\mathrm{NC}(=\mathrm{O})[\mathrm{C} @ @ \mathrm{H}] 1 \mathrm{C}[\mathrm{C} @ @ \mathrm{H}](\mathrm{F}) \mathrm{CN} 1 \mathrm{c} 1 \mathrm{nnnn} 1 \mathrm{CCc} 1 \mathrm{ccccc} 1$ & 0 \\
\hline 29 & $10\{6\}$ & $\mathbf{1 1}\{12\}$ & $\mathbf{8}\{6,12\}$ & $\mathrm{Cn} 1 \mathrm{cnn} \operatorname{c} 1 \mathrm{C} 1 \mathrm{CCCN}(\mathrm{C} 1) \mathrm{c} 1 \mathrm{nnnn} 1 \mathrm{CCc} 1 \mathrm{ccccc} 1$ & 0 \\
\hline 30 & $10\{6\}$ & $11\{4\}$ & $\mathbf{8}\{6,4\}$ & $\mathrm{C}(\mathrm{Cn} 1 \mathrm{nnnn} 1 \mathrm{~N} 1 \mathrm{CCOC} 2(\mathrm{CCC} 2) \mathrm{C} 1) \mathrm{c} 1 \mathrm{ccccc} 1$ & 0 \\
\hline 31 & $10\{7\}$ & $\mathbf{1 1}\{19\}$ & $\mathbf{8}\{7,19\}$ & $\mathrm{COC} 1(\mathrm{CCN}(\mathrm{C} 1) \mathrm{c} 1 \mathrm{nnnn} 1 \mathrm{C} 1 \mathrm{CCCCC} 1) \mathrm{C}(\mathrm{F})(\mathrm{F}) \mathrm{F}$ & 14 \\
\hline 32 & $10\{7\}$ & $\mathbf{1 1}\{20\}$ & $\mathbf{8}\{7,20\}$ & $\mathrm{CCC} 1(\mathrm{CCN}(\mathrm{CC} 1) \mathrm{c} 1 \mathrm{nnnn} 1 \mathrm{C} 1 \mathrm{CCCCC} 1) \mathrm{N} 1 \mathrm{CCOCC} 1$ & 20 \\
\hline 33 & $10\{7\}$ & $\mathbf{1 1}\{21\}$ & $\mathbf{8}\{7,21\}$ & $\mathrm{CN}(\mathrm{C} 1 \mathrm{CCN}(\mathrm{CC} 1) \mathrm{S}(\mathrm{C})(=\mathrm{O})=\mathrm{O}) \mathrm{c} 1 \mathrm{nnnn} 1 \mathrm{C} 1 \mathrm{CCCCC} 1$ & 26 \\
\hline 34 & $10\{7\}$ & $\mathbf{1 1}\{22\}$ & $\mathbf{8}\{7,22\}$ & $\mathrm{C} 1 \mathrm{CCC}(\mathrm{CC} 1) \mathrm{n} 1 \mathrm{nnnn} 1 \mathrm{~N} 1 \mathrm{CCCC}(\mathrm{CC} 1) \mathrm{n} 1 \mathrm{ccnn} 1$ & 0 \\
\hline 35 & $10\{8\}$ & $11\{23\}$ & $\mathbf{8}\{8,23\}$ & $\mathrm{C}(\mathrm{Cn} 1 \mathrm{nnnn} 1 \mathrm{~N} 1 \mathrm{CCCC} 2 \mathrm{ccccc} 12) \mathrm{N} 1 \mathrm{CCOCC} 1$ & 19 \\
\hline 36 & $\mathbf{1 0}\{8\}$ & $\mathbf{1 1}\{24\}$ & $\mathbf{8}\{8,24\}$ & $\mathrm{CC} 1(\mathrm{C}) \mathrm{CC}(\mathrm{O}) \mathrm{CCN}(\mathrm{C} 1) \mathrm{c} 1 \mathrm{nnnn} 1 \mathrm{CCN} 1 \mathrm{CCOCC} 1$ & 0 \\
\hline 37 & $10\{8\}$ & $11\{25\}$ & $\mathbf{8}\{8,25\}$ & $\mathrm{C}(\mathrm{Cn} 1 \mathrm{nnnn} 1 \mathrm{~N} 1 \mathrm{CCCC} 1 \mathrm{c} 1 \mathrm{cccnc} 1) \mathrm{N} 1 \mathrm{CCOCC} 1$ & 0 \\
\hline 38 & $10\{8\}$ & $11\{26\}$ & $\mathbf{8}\{8,26\}$ & $\mathrm{Clc} 1 \mathrm{ccc} 2 \mathrm{CN}(\mathrm{CCc} 2 \mathrm{c} 1) \mathrm{c} 1 \mathrm{nnnn} 1 \mathrm{CCN} 1 \mathrm{CCOCC} 1$ & 0 \\
\hline
\end{tabular}




\begin{tabular}{|c|c|c|c|c|c|}
\hline 39 & $\mathbf{1 0}\{8\}$ & $\mathbf{1 1}\{27\}$ & $\mathbf{8}\{8,27\}$ & $\mathrm{C}(\mathrm{Cn} 1 \mathrm{nnnn} 1 \mathrm{~N} 1 \mathrm{CCC}(\mathrm{CC} 1) \mathrm{c} 1 \mathrm{ccncc} 1) \mathrm{N} 1 \mathrm{CCOCC} 1$ & 0 \\
\hline 40 & $10\{8\}$ & $\mathbf{1 1}\{28\}$ & $\mathbf{8}\{8,28\}$ & $\mathrm{COC} 1 \mathrm{CCCN}(\mathrm{C} 1) \mathrm{c} 1 \mathrm{nnnn} 1 \mathrm{CCN} 1 \mathrm{CCOCC} 1$ & 0 \\
\hline 41 & $10\{8\}$ & $11\{29\}$ & $\mathbf{8}\{8,29\}$ & $\mathrm{Cc} 1 \mathrm{ccc} 2 \mathrm{CN}(\mathrm{CCc} 2 \mathrm{c} 1) \mathrm{c} 1 \mathrm{nnnn} 1 \mathrm{CCN} 1 \mathrm{CCOCC} 1$ & 0 \\
\hline 42 & $\mathbf{1 0}\{8\}$ & $\mathbf{1 1}\{30\}$ & $\mathbf{8}\{8,30\}$ & $\mathrm{C}(\mathrm{Cn} 1 \mathrm{nnn} 1 \mathrm{~N} 1 \mathrm{CCC}=\mathrm{CC} 1) \mathrm{N} 1 \mathrm{CCOCC} 1$ & 0 \\
\hline 43 & $10\{8\}$ & $11\{31\}$ & $\mathbf{8}\{8,31\}$ & $\mathrm{C}(\mathrm{Cn} 1 \mathrm{nnnn} 1 \mathrm{~N} 1 \mathrm{CCC}(\mathrm{CC} 1) \mathrm{c} 1 \mathrm{ccccc} 1) \mathrm{N} 1 \mathrm{CCOCC} 1$ & 0 \\
\hline 44 & $\mathbf{1 0}\{8\}$ & $\mathbf{1 1}\{32\}$ & $\mathbf{8}\{8,32\}$ & $\mathrm{CC} 1 \mathrm{~N}(\mathrm{CCc} 2 \mathrm{cccc}(\mathrm{F}) \mathrm{c} 12) \mathrm{c} 1 \mathrm{nnnn} 1 \mathrm{CCN} 1 \mathrm{CCOCC} 1$ & 0 \\
\hline 45 & $10\{8\}$ & $\mathbf{1 1}\{33\}$ & $\mathbf{8}\{8,33\}$ & $\mathrm{O}=\mathrm{C} 1 \mathrm{CCN}(\mathrm{CCN} 1) \mathrm{c} 1 \mathrm{nnnn} 1 \mathrm{CCN} 1 \mathrm{CCOCC} 1$ & 0 \\
\hline 46 & $\mathbf{1 0}\{8\}$ & $\mathbf{1 1}\{34\}$ & $\mathbf{8}\{8,34\}$ & $\mathrm{CCC} 1 \mathrm{~N}(\mathrm{CC} 2 \mathrm{sccc} 12) \mathrm{c} 1 \mathrm{nnnn} 1 \mathrm{CCN} 1 \mathrm{CCOCC} 1$ & 0 \\
\hline 47 & $10\{8\}$ & $11\{35\}$ & $\mathbf{8}\{8,35\}$ & $\mathrm{C}(\mathrm{Cn} 1 \mathrm{nnnn} 1 \mathrm{~N} 1 \mathrm{CCCC}(\mathrm{C} 1) \mathrm{c} 1 \mathrm{ncc}[\mathrm{nH}] 1) \mathrm{N} 1 \mathrm{CCOCC} 1$ & 0 \\
\hline 48 & $\mathbf{1 0}\{8\}$ & $\mathbf{1 1}\{36\}$ & $\mathbf{8}\{8,36\}$ & $\mathrm{O}[\mathrm{C} @ \mathrm{H}] 1 \mathrm{CCN}(\mathrm{C} 1) \mathrm{c} 1 \mathrm{nnnn} 1 \mathrm{CCN} 1 \mathrm{CCOCC} 1$ & 0 \\
\hline 49 & $10\{8\}$ & $11\{37\}$ & $\mathbf{8}\{8,37\}$ & $\mathrm{COc} 1 \mathrm{ccc} 2 \mathrm{CN}(\mathrm{CCc} 2 \mathrm{c} 1) \mathrm{c} 1 \mathrm{nnnn} 1 \mathrm{CCN} 1 \mathrm{CCOCC} 1$ & 0 \\
\hline 50 & $\mathbf{1 0}\{8\}$ & $\mathbf{1 1}\{38\}$ & $\mathbf{8}\{8,38\}$ & $\mathrm{C}(\mathrm{Cn} 1 \mathrm{nnnn} 1 \mathrm{~N} 1 \mathrm{CCc} 2 \mathrm{nc}[\mathrm{nH}] \mathrm{c} 2 \mathrm{C} 1) \mathrm{N} 1 \mathrm{CCOCC} 1$ & 0 \\
\hline 51 & $10\{8\}$ & $11\{39\}$ & $\mathbf{8}\{8,39\}$ & Fc1ccc2N(CCc2c1)c1nnnn1CCN1CCOCC 1 & 0 \\
\hline 52 & $10\{9\}$ & $\mathbf{1 1}\{4\}$ & $8\{9,4\}$ & COCCn1nnnc1N1CCOC2(CCC2)C1 & 29 \\
\hline 53 & $10\{9\}$ & $11\{7\}$ & $\mathbf{8}\{9,7\}$ & $\mathrm{COCCn} 1 \mathrm{nnn} 1 \mathrm{~N} 1 \mathrm{CCC}(\mathrm{C})=\mathrm{C}(\mathrm{C}) \mathrm{C} 1$ & 21 \\
\hline 54 & $10\{9\}$ & $11\{5\}$ & $\mathbf{8}\{9,5\}$ & COCCn1nnnc1N1CCOCC1c1ce(F)cc(F)c1 & 0 \\
\hline 55 & $10\{9\}$ & $\mathbf{1 1}\{6\}$ & $\mathbf{8}\{9,6\}$ & COCCn1nnnc1N1CCOc2cc(F)ccc2C1 & 0 \\
\hline 56 & $\mathbf{1 0}\{10\}$ & $11\{40\}$ & $\mathbf{8}\{10,40\}$ & $\mathrm{CC}(\mathrm{C}) \mathrm{n} 1 \mathrm{nnnc} 1 \mathrm{~N} 1 \mathrm{CCc} 2[\mathrm{nH}] \mathrm{ncc} 2 \mathrm{C} 1$ & 0 \\
\hline 57 & $\mathbf{1 0}\{10\}$ & $\mathbf{1 1}\{41\}$ & $\mathbf{8}\{10,41\}$ & $\operatorname{COc} 1 \mathrm{ccc}(\mathrm{CN}(\mathrm{C}) \mathrm{c} 2 \mathrm{nnnn} 2 \mathrm{C}(\mathrm{C}) \mathrm{C}) \mathrm{cn} 1$ & 0 \\
\hline 58 & $\mathbf{1 0}\{10\}$ & $\mathbf{1 1}\{42\}$ & $\mathbf{8}\{10,42\}$ & $\mathrm{CC}(\mathrm{C}) \mathrm{n} 1 \mathrm{nnnn} 1 \mathrm{~N} 1 \mathrm{CCCC}(\mathrm{C} 1) \mathrm{c} 1 \mathrm{nnc} 2 \mathrm{ccc} \mathrm{c} 12$ & 0 \\
\hline 59 & $\mathbf{1 0}\{10\}$ & $\mathbf{1 1}\{43\}$ & $\mathbf{8}\{10,43\}$ & $\mathrm{CC}(\mathrm{C}) \mathrm{n} 1 \mathrm{nnnc} 1 \mathrm{~N}(\mathrm{C})[\mathrm{C} @ \mathrm{H}] 1 \mathrm{C}[\mathrm{C} @ \mathrm{H}](\mathrm{C} 1) \mathrm{C}(\mathrm{N})=\mathrm{O}$ & 16 \\
\hline
\end{tabular}




\begin{tabular}{|c|c|c|c|c|c|c|}
\hline 60 & $\mathbf{1 0}\{10\}$ & $11\{2\}$ & $\mathbf{8}\{10,2\}$ & $\mathrm{CC}(\mathrm{C}) \mathrm{n} 1 \mathrm{nnnc} 1 \mathrm{~N} 1 \mathrm{CCN}(\mathrm{CC} 1) \mathrm{C}(=\mathrm{O}) \mathrm{C}(=\mathrm{O}) \mathrm{NC} 1 \mathrm{CC} 1$ & 19 & 18 \\
\hline 61 & $10\{10\}$ & $11\{44\}$ & $\mathbf{8}\{10,44\}$ & $\mathrm{CC}(\mathrm{C}) \mathrm{n} 1 \mathrm{nnn} 1 \mathrm{~N} 1 \mathrm{CCN}(\mathrm{CC} 1) \mathrm{c} 1 \mathrm{cccc} 2[\mathrm{nH}] \mathrm{c}(=\mathrm{O})[\mathrm{nH}] \mathrm{c} 12$ & 5 & 3 \\
\hline 62 & $10\{10\}$ & $11\{45\}$ & $\mathbf{8}\{10,45\}$ & $\mathrm{CC}(\mathrm{C}) \mathrm{n} 1 \mathrm{nnnn} 1 \mathrm{~N} 1 \mathrm{CCN}(\mathrm{CCc} 2 \mathrm{cnccn} 2) \mathrm{CC} 1$ & 16 & 8 \\
\hline 63 & $\mathbf{1 0}\{10\}$ & $\mathbf{1 1}\{46\}$ & $\mathbf{8}\{10,46\}$ & $\mathrm{CC}(\mathrm{C}) \mathrm{n} 1 \mathrm{nnnnc} 1 \mathrm{~N}(\mathrm{Cc} 1 \mathrm{ccc}(\mathrm{C}) \mathrm{o} 1) \mathrm{C} 1 \mathrm{CC} 1$ & 15 & 7 \\
\hline 64 & $10\{10\}$ & $\mathbf{1 1}\{47\}$ & $\mathbf{8}\{10,47\}$ & $\mathrm{CC}(\mathrm{C}) \mathrm{n} 1 \mathrm{nnnc} 1 \mathrm{~N} 1 \mathrm{CC} 2(\mathrm{C}) \mathrm{C}(=\mathrm{O}) \mathrm{NC}(=\mathrm{O}) \mathrm{C} 2(\mathrm{C}) \mathrm{C} 1$ & 26 & 13 \\
\hline 65 & $\mathbf{1 0}\{10\}$ & $11\{1\}$ & $\mathbf{8}\{10,1\}$ & $\mathrm{CNS}(=\mathrm{O})(=\mathrm{O}) \mathrm{c} 1 \mathrm{cccc} 2 \mathrm{CN}(\mathrm{CCc} 12) \mathrm{c} 1 \mathrm{nnnn} 1 \mathrm{C}(\mathrm{C}) \mathrm{C}$ & 17 & 13 \\
\hline 66 & $\mathbf{1 0}\{10\}$ & $\mathbf{1 1}\{48\}$ & $\mathbf{8}\{10,48\}$ & $\mathrm{CC}(\mathrm{C}) \mathrm{n} 1 \mathrm{nnnnc} 1 \mathrm{~N} 1 \mathrm{CCN}(\mathrm{CC} 1) \mathrm{c} 1 \mathrm{cc}(\mathrm{C}) \mathrm{ccn} 1$ & 9 & 4 \\
\hline 67 & $\mathbf{1 0}\{10\}$ & $\mathbf{1 1}\{49\}$ & $\mathbf{8}\{10,49\}$ & $\mathrm{CC}(\mathrm{C}) \mathrm{n} 1 \mathrm{nnnn} 1 \mathrm{~N}(\mathrm{C}) \mathrm{CCS}(\mathrm{C})(=\mathrm{O})=\mathrm{O}$ & 11 & 5 \\
\hline 68 & $\mathbf{1 0}\{10\}$ & $\mathbf{1 1}\{50\}$ & $\mathbf{8}\{10,50\}$ & $\mathrm{CC}(\mathrm{C}) \mathrm{n} 1 \mathrm{nnnn} 1 \mathrm{~N} 1 \mathrm{CCCC}(\mathrm{CNS}(\mathrm{C})(=\mathrm{O})=\mathrm{O}) \mathrm{C} 1$ & 51 & 29 \\
\hline 69 & $10\{10\}$ & $\mathbf{1 1}\{51\}$ & $\mathbf{8}\{10,51\}$ & $\mathrm{CCN}(\mathrm{CC} 1 \mathrm{CCOC} 1) \mathrm{c} 1 \mathrm{nnnn} 1 \mathrm{C}(\mathrm{C}) \mathrm{C}$ & 43 & 22 \\
\hline 70 & $\mathbf{1 0}\{10\}$ & $\mathbf{1 1}\{52\}$ & $\mathbf{8}\{10,52\}$ & $\mathrm{CC}(\mathrm{C}) \mathrm{n} 1 \mathrm{nnnn} 1 \mathrm{~N} 1 \mathrm{CCC}(\mathrm{CC} 1) \mathrm{N} 1 \mathrm{CCCCC} 1$ & 16 & 8 \\
\hline 71 & $\mathbf{1 0}\{10\}$ & $\mathbf{1 1}\{53\}$ & $\mathbf{8}\{10,53\}$ & $\mathrm{CC}(\mathrm{C}) \mathrm{n} 1 \mathrm{nnnc} 1 \mathrm{~N}(\mathrm{C}) \mathrm{CCNC}(\mathrm{C})=\mathrm{O}$ & 19 & 10 \\
\hline 72 & $\mathbf{1 0}\{10\}$ & $\mathbf{1 1}\{54\}$ & $\mathbf{8}\{10,54\}$ & $\mathrm{CC}(\mathrm{C}) \mathrm{n} 1 \mathrm{nnnn} 1 \mathrm{~N} 1 \mathrm{CCN}(\mathrm{CC} 1) \mathrm{C} 1 \mathrm{CCCCC} 1$ & 0 & 0 \\
\hline 73 & $\mathbf{1 0}\{10\}$ & $\mathbf{1 1}\{55\}$ & $\mathbf{8}\{10,55\}$ & $\mathrm{CC}(\mathrm{C}) \mathrm{n} 1 \mathrm{nnn} 1 \mathrm{~N}(\mathrm{C}) \mathrm{Cc} 1 \mathrm{nccn} 1 \mathrm{C}$ & 0 & 0 \\
\hline 74 & $\mathbf{1 0}\{10\}$ & $\mathbf{1 1}\{56\}$ & $\mathbf{8}\{10,56\}$ & $\mathrm{CC}(\mathrm{C}) \mathrm{n} 1 \mathrm{nnnnc} 1 \mathrm{~N} 1 \mathrm{CCCC}(\mathrm{C} 1) \mathrm{c} 1 \mathrm{ccnn} 1 \mathrm{C}$ & 0 & 0 \\
\hline 75 & $\mathbf{1 0}\{11\}$ & $11\{2\}$ & $\mathbf{8}\{11,2\}$ & $\mathrm{CC}(=\mathrm{C}) \mathrm{Cn} 1 \mathrm{nnn} n \mathrm{~N} 1 \mathrm{CCN}(\mathrm{CC} 1) \mathrm{C}(=\mathrm{O}) \mathrm{C}(=\mathrm{O}) \mathrm{NC} 1 \mathrm{CC} 1$ & 17 & 19 \\
\hline 76 & $\mathbf{1 0}\{11\}$ & $\mathbf{1 1}\{12\}$ & $\mathbf{8}\{11,12\}$ & $\mathrm{CC}(=\mathrm{C}) \mathrm{Cn} 1 \mathrm{nnnnc} 1 \mathrm{~N} 1 \mathrm{CCCC}(\mathrm{C} 1) \mathrm{c} 1 \mathrm{nncn} 1 \mathrm{C}$ & 0 & 0 \\
\hline 77 & $\mathbf{1 0}\{12\}$ & $11\{6\}$ & $\mathbf{8}\{12,6\}$ & CCOCCCn1nnnc1N1CCOc2ce(F)cce2C1 & 29 & 27 \\
\hline 78 & $\mathbf{1 0}\{12\}$ & $11\{8\}$ & $\mathbf{8}\{12,8\}$ & $\begin{array}{l}\mathrm{CCOCCCn} 1 \mathrm{nnn} 1 \mathrm{~N} 1 \mathrm{CC} 2(\mathrm{CCOCC} 2)[1 \mathrm{C} @ @ \mathrm{H}] 2 \mathrm{CCC}[1 \mathrm{C} @ \mathrm{H}] \\
12\end{array}$ & 9 & 10 \\
\hline 79 & $\mathbf{1 0}\{12\}$ & $11\{7\}$ & $\mathbf{8}\{12,7\}$ & 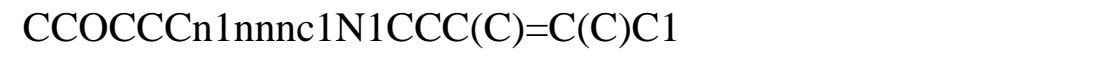 & 11 & 12 \\
\hline 80 & $\mathbf{1 0}\{12\}$ & $11\{10\}$ & $\mathbf{8}\{12,10\}$ & CCOCCCn1nnnc1N1CCN $(\mathrm{CC} 1) \mathrm{C}(=\mathrm{O}) \mathrm{c} 1 \mathrm{ccc} 1 \mathrm{C}$ & 0 & 0 \\
\hline
\end{tabular}




\begin{tabular}{|c|c|c|c|c|c|}
\hline 81 & $\mathbf{1 0}\{13\}$ & $11\{57\}$ & $\mathbf{8}\{13,57\}$ & $\mathrm{COc} 1 \mathrm{ccc}(\mathrm{CCn} 2 \mathrm{nnnn} 2 \mathrm{~N} 2 \mathrm{CCCc} 3 \mathrm{n}[\mathrm{nH}] \mathrm{cc} 23) \mathrm{cc} 1$ & 5 \\
\hline 82 & $\mathbf{1 0}\{13\}$ & $\mathbf{1 1}\{58\}$ & $\mathbf{8}\{13,58\}$ & $\begin{array}{l}\mathrm{COc} 1 \mathrm{ccc}(\mathrm{CCn} 2 \mathrm{nnn} 22 \mathrm{~N} 2 \mathrm{CC}[1 \mathrm{C} @ @ \mathrm{H}](\mathrm{C}) \mathrm{O}[1 \mathrm{C} @ @ \mathrm{H}](\mathrm{C}) \mathrm{C} 2) \mathrm{c} \\
\mathrm{c} 1\end{array}$ & 34 \\
\hline 83 & $\mathbf{1 0}\{13\}$ & $\mathbf{1 1}\{59\}$ & $\mathbf{8}\{13,59\}$ & $\mathrm{COc} 1 \mathrm{ccc}(\mathrm{CCn} 2 \mathrm{nnnn} 2 \mathrm{~N} 2 \mathrm{Cc} 3 \mathrm{ccc}(\mathrm{O}) \mathrm{cc} 3 \mathrm{C} 2) \mathrm{cc} 1$ & 11 \\
\hline 84 & $\mathbf{1 0}\{13\}$ & $\mathbf{1 1}\{60\}$ & $\mathbf{8}\{13,60\}$ & $\mathrm{COc} 1 \mathrm{ccc}(\mathrm{CCn} 2 \mathrm{nnn} 22 \mathrm{~N} 2 \mathrm{C} 3 \mathrm{CCC} 2 \mathrm{CC}(=\mathrm{O}) \mathrm{NC} 3) \mathrm{cc} 1$ & 7 \\
\hline 85 & $\mathbf{1 0}\{13\}$ & $11\{61\}$ & $\mathbf{8}\{13,61\}$ & $\mathrm{COc} 1 \mathrm{ccc}(\mathrm{CCn} 2 \mathrm{nnnc} 2 \mathrm{~N} 2 \mathrm{CCC} 3(\mathrm{C} 2) \mathrm{CCOCC} 3) \mathrm{cc} 1$ & 48 \\
\hline 86 & $\mathbf{1 0}\{13\}$ & $\mathbf{1 1}\{62\}$ & $\mathbf{8}\{13,62\}$ & $\mathrm{COc} 1 \mathrm{ccc}(\mathrm{CCn} 2 \mathrm{nnn} \mathrm{c} 2 \mathrm{~N} 2 \mathrm{C} 3 \mathrm{CCC} 2 \mathrm{C}=\mathrm{CC} 3) \mathrm{cc} 1$ & 30 \\
\hline 87 & $\mathbf{1 0}\{13\}$ & $11\{63\}$ & $\mathbf{8}\{13,63\}$ & $\mathrm{COc} 1 \mathrm{ccc}(\mathrm{CCn} 2 \mathrm{nnnn} 2 \mathrm{~N} 2 \mathrm{CCN} 3 \mathrm{C}(\mathrm{CNC} 3=\mathrm{O}) \mathrm{C} 2) \mathrm{cc} 1$ & 10 \\
\hline 88 & $\mathbf{1 0}\{13\}$ & $\mathbf{1 1}\{17\}$ & $\mathbf{8}\{13,17\}$ & $\operatorname{COCCCN}(\mathrm{CCOC}) \mathrm{c} 1 \mathrm{nnnn} 1 \mathrm{CCc} 1 \mathrm{ccc}(\mathrm{OC}) \mathrm{cc} 1$ & 8 \\
\hline 89 & $\mathbf{1 0}\{13\}$ & $\mathbf{1 1}\{64\}$ & $\mathbf{8}\{13,64\}$ & $\mathrm{COc} 1 \mathrm{ccc}(\mathrm{CCn} 2 \mathrm{nnn} n 2 \mathrm{~N} 2 \mathrm{CCOCC}(\mathrm{F})(\mathrm{F}) \mathrm{C} 2) \mathrm{cc} 1$ & 0 \\
\hline 90 & $\mathbf{1 0}\{13\}$ & $\mathbf{1 1}\{65\}$ & $\mathbf{8}\{13,65\}$ & $\mathrm{COc} 1 \mathrm{ccc}(\mathrm{CCn} 2 \mathrm{nnn} \operatorname{2N} 2 \mathrm{CCC}=\mathrm{C}(\mathrm{F}) \mathrm{C} 2) \mathrm{cc} 1$ & 0 \\
\hline 91 & $\mathbf{1 0}\{13\}$ & $11\{66\}$ & $\mathbf{8}\{13,66\}$ & $\mathrm{COc} 1 \mathrm{ccc}(\mathrm{CCn} 2 \mathrm{nnnn} 2 \mathrm{~N} 2 \mathrm{CC} 3 \mathrm{C} 4 \mathrm{OC}(\mathrm{C}=\mathrm{C} 4) \mathrm{C} 3 \mathrm{C} 2) \mathrm{cc} 1$ & 0 \\
\hline 92 & $\mathbf{1 0}\{13\}$ & $\mathbf{1 1}\{13\}$ & $\mathbf{8}\{13,13\}$ & $\mathrm{COc} 1 \mathrm{ccc}(\mathrm{CCn} 2 \mathrm{nnnc} 2 \mathrm{~N} 2 \mathrm{CCCC} 2 \mathrm{C} 2 \mathrm{CCCO} 2) \mathrm{cc} 1$ & 0 \\
\hline 93 & $\mathbf{1 0}\{13\}$ & $\mathbf{1 1}\{67\}$ & $\mathbf{8}\{13,67\}$ & $\mathrm{COc} 1 \mathrm{ccc}(\mathrm{CCn} 2 \mathrm{nnnn} 2 \mathrm{~N} 2 \mathrm{C} 3 \mathrm{CCC} 2 \mathrm{C}(\mathrm{CO}) \mathrm{C} 3) \mathrm{cc} 1$ & 0 \\
\hline 94 & $\mathbf{1 0}\{14\}$ & $11\{8\}$ & $\mathbf{8}\{14,8\}$ & CCCn1nnnc1N1CC2(CCOCC2)[1C@@ @ $] 2 C C C[1 \mathrm{C} @ H] 12$ & 0 \\
\hline 95 & $\mathbf{1 0}\{14\}$ & $11\{7\}$ & $\mathbf{8}\{14,7\}$ & $\mathrm{CCCn} 1 \mathrm{nnnc} 1 \mathrm{~N} 1 \mathrm{CCC}(\mathrm{C})=\mathrm{C}(\mathrm{C}) \mathrm{C} 1$ & 0 \\
\hline 96 & $\mathbf{1 0}\{14\}$ & $\mathbf{1 1}\{12\}$ & $\mathbf{8}\{14,12\}$ & CCCn1nnnc1N1CCCC(C1)c1nncn1C & 4 \\
\hline 97 & $\mathbf{1 0}\{14\}$ & $11\{2\}$ & $\mathbf{8}\{14,2\}$ & $\mathrm{CCCn} 1 \mathrm{nnn} 1 \mathrm{~N} 1 \mathrm{CCN}(\mathrm{CC} 1) \mathrm{C}(=\mathrm{O}) \mathrm{C}(=\mathrm{O}) \mathrm{NC} 1 \mathrm{CC} 1$ & 30 \\
\hline 98 & $\mathbf{1 0}\{15\}$ & $11\{68\}$ & $\mathbf{8}\{15,68\}$ & $\mathrm{C} 1 \mathrm{CC} 1 \mathrm{n} 1 \mathrm{nnnn} 1 \mathrm{~N} 1 \mathrm{CCCC}(\mathrm{C} 1) \mathrm{c} 1 \mathrm{ccn}[\mathrm{nH}] 1$ & 0 \\
\hline 99 & $\mathbf{1 0}\{15\}$ & $11\{1\}$ & $\mathbf{8}\{15,1\}$ & $\mathrm{CNS}(=\mathrm{O})(=\mathrm{O}) \mathrm{c} 1 \mathrm{cccc} 2 \mathrm{CN}(\mathrm{CCc} 12) \mathrm{c} 1 \mathrm{nnnn} 1 \mathrm{C} 1 \mathrm{CC} 1$ & 8 \\
\hline 100 & $\mathbf{1 0}\{15\}$ & $11\{69\}$ & $\mathbf{8}\{15,69\}$ & $\mathrm{CN}(\mathrm{CC}(\mathrm{C})(\mathrm{C}) \mathrm{CO}) \mathrm{c} 1 \mathrm{nnnn} 1 \mathrm{C} 1 \mathrm{CC} 1$ & 10 \\
\hline 101 & $\mathbf{1 0}\{15\}$ & $11\{70\}$ & $\mathbf{8}\{15,70\}$ & $\mathrm{COc} 1 \mathrm{cccc}(\mathrm{c} 1) \mathrm{C} 1 \mathrm{CCN}(\mathrm{CC} 1) \mathrm{c} 1 \mathrm{nnnn} 1 \mathrm{C} 1 \mathrm{CC} 1$ & 13 \\
\hline
\end{tabular}




\begin{tabular}{|c|c|c|c|c|c|}
\hline 102 & $\mathbf{1 0}\{15\}$ & $11\{7\}$ & $\mathbf{8}\{15,7\}$ & $\mathrm{CC} 1=\mathrm{C}(\mathrm{C}) \mathrm{CN}(\mathrm{CC} 1) \mathrm{c} 1 \mathrm{nnnn} 1 \mathrm{C} 1 \mathrm{CC} 1$ & 36 \\
\hline 103 & $\mathbf{1 0}\{15\}$ & $11\{2\}$ & $\mathbf{8}\{15,2\}$ & $\mathrm{O}=\mathrm{C}(\mathrm{NC} 1 \mathrm{CC} 1) \mathrm{C}(=\mathrm{O}) \mathrm{N} 1 \mathrm{CCN}(\mathrm{CC} 1) \mathrm{c} 1 \mathrm{nnnn} 1 \mathrm{C} 1 \mathrm{CC} 1$ & 20 \\
\hline 104 & $\mathbf{1 0}\{15\}$ & $\mathbf{1 1}\{8\}$ & $\mathbf{8}\{15,8\}$ & C1CC1n1nnnc1N1CC2(CCOCC2)[1C@@H]2CCC[1C@H]12 & 66 \\
\hline 105 & $\mathbf{1 0}\{15\}$ & $\mathbf{1 1}\{71\}$ & $\mathbf{8}\{15,71\}$ & $\mathrm{CCn} 1 \mathrm{cc}(\mathrm{CN}(\mathrm{C}) \mathrm{c} 2 \mathrm{nnnn} 2 \mathrm{C} 2 \mathrm{CC} 2) \mathrm{cn} 1$ & 62 \\
\hline 106 & $\mathbf{1 0}\{15\}$ & $\mathbf{1 1}\{72\}$ & $\mathbf{8}\{15,72\}$ & $\mathrm{CCN}(\mathrm{Cc} 1 \mathrm{ccoc} 1) \mathrm{c} 1 \mathrm{nnnn} 1 \mathrm{C} 1 \mathrm{CC} 1$ & 87 \\
\hline 107 & $\mathbf{1 0}\{16\}$ & $\mathbf{1 1}\{73\}$ & $\mathbf{8}\{16,73\}$ & $\mathrm{COCC}(\mathrm{C}) \mathrm{n} 1 \mathrm{nnn} \mathrm{n} 1 \mathrm{~N} 1 \mathrm{CCc} 2 \mathrm{cn}[\mathrm{nH}] \mathrm{c} 2 \mathrm{C} 1$ & 0 \\
\hline 108 & $\mathbf{1 0}\{16\}$ & $\mathbf{1 1}\{74\}$ & $\mathbf{8}\{16,74\}$ & $\mathrm{COCC}(\mathrm{C}) \mathrm{n} 1 \mathrm{nnn} \mathrm{c} 1 \mathrm{~N} 1 \mathrm{CCN}(\mathrm{CC} 1) \mathrm{c} 1 \mathrm{nc}(\mathrm{C}) \mathrm{c}(\mathrm{C}) \mathrm{s} 1$ & 0 \\
\hline 109 & $\mathbf{1 0}\{16\}$ & $\mathbf{1 1}\{75\}$ & $\mathbf{8}\{16,75\}$ & $\mathrm{COCC}(\mathrm{C}) \mathrm{n} 1 \mathrm{nnn} \mathrm{c} 1 \mathrm{~N} 1 \mathrm{CCN}(\mathrm{CC} 1) \mathrm{C}(=\mathrm{O}) \mathrm{c} 1 \mathrm{cccc}(\mathrm{O}) \mathrm{c} 1$ & 7 \\
\hline 110 & $\mathbf{1 0}\{16\}$ & $\mathbf{1 1}\{76\}$ & $\mathbf{8}\{16,76\}$ & $\mathrm{COCC}(\mathrm{C}) \mathrm{n} 1 \mathrm{nnnn} 1 \mathrm{~N} 1 \mathrm{CCn} 2 \mathrm{ccnc} 12$ & 8 \\
\hline 111 & $\mathbf{1 0}\{16\}$ & $\mathbf{1 1}\{77\}$ & $\mathbf{8}\{16,77\}$ & $\mathrm{COCC}(\mathrm{C}) \mathrm{n} 1 \mathrm{nnnnc} 1 \mathrm{~N} 1 \mathrm{CC} 2 \mathrm{nc}(\mathrm{ncc} 2 \mathrm{C} 1) \mathrm{C} 1 \mathrm{CC} 1$ & 17 \\
\hline 112 & $\mathbf{1 0}\{16\}$ & $\mathbf{1 1}\{48\}$ & $\mathbf{8}\{16,48\}$ & $\mathrm{COCC}(\mathrm{C}) \mathrm{n} 1 \mathrm{nnnnc} 1 \mathrm{~N} 1 \mathrm{CCN}(\mathrm{CC} 1) \mathrm{c} 1 \mathrm{cc}(\mathrm{C}) \mathrm{ccn} 1$ & 8 \\
\hline 113 & $\mathbf{1 0}\{16\}$ & $\mathbf{1 1}\{78\}$ & $\mathbf{8}\{16,78\}$ & $\mathrm{COCC}(\mathrm{C}) \mathrm{n} 1 \mathrm{nnn} \mathrm{c} 1 \mathrm{~N} 1 \mathrm{CCCc} 2 \mathrm{cc}(\mathrm{OC}) \mathrm{ccc} 2 \mathrm{C} 1$ & 0 \\
\hline 114 & $\mathbf{1 0}\{16\}$ & $\mathbf{1 1}\{79\}$ & $\mathbf{8}\{16,79\}$ & $\mathrm{COCC}(\mathrm{C}) n 1 \mathrm{nnn}$ 1N1CCC $[\mathrm{C} @ @ \mathrm{H}] 1 \mathrm{C}(\mathrm{N})=\mathrm{O}$ & 0 \\
\hline 115 & $\mathbf{1 0}\{17\}$ & $\mathbf{1 1}\{15\}$ & $\mathbf{8}\{17,15\}$ & $\mathrm{COC} 1(\mathrm{CCN}(\mathrm{CC} 1) \mathrm{c} 1 \mathrm{nnnn} 1 \mathrm{C} 1 \mathrm{CC} 2 \mathrm{CCC} 1 \mathrm{C} 2) \mathrm{C}(\mathrm{F})(\mathrm{F}) \mathrm{F}$ & 29 \\
\hline 116 & $\mathbf{1 0}\{17\}$ & $\mathbf{1 1}\{80\}$ & $\mathbf{8}\{17,80\}$ & $\mathrm{C}(\mathrm{OC} 1 \mathrm{CCN}(\mathrm{CC} 1) \mathrm{c} 1 \mathrm{nnnn} 1 \mathrm{C} 1 \mathrm{CC} 2 \mathrm{CCC} 1 \mathrm{C} 2) \mathrm{C} 1 \mathrm{CCCO} 1$ & 33 \\
\hline 117 & $\mathbf{1 0}\{18\}$ & $\mathbf{1 1}\{81\}$ & $\mathbf{8}\{18,81\}$ & $\mathrm{NC}(=\mathrm{O}) \mathrm{C} 1 \mathrm{CN}(\mathrm{CCO} 1) \mathrm{c} 1 \mathrm{nnnn} 1 \mathrm{CCSc} 1 \mathrm{ccccc} 1$ & 0 \\
\hline 118 & $\mathbf{1 0}\{19\}$ & $\mathbf{1 1}\{36\}$ & $\mathbf{8}\{19,36\}$ & $\mathrm{O}[\mathrm{C} @ \mathrm{H}] 1 \mathrm{CCN}(\mathrm{C} 1) \mathrm{c} 1 \mathrm{nnnn} 1 \mathrm{Cc} 1 \mathrm{ccc} n \mathrm{c} 1$ & 0 \\
\hline 119 & $\mathbf{1 0}\{19\}$ & $\mathbf{1 1}\{82\}$ & $\mathbf{8}\{19,82\}$ & $\mathrm{C}(\mathrm{c} 1 \mathrm{cccnc} 1) \mathrm{n} 1 \mathrm{nnn} \mathrm{c} 1 \mathrm{~N} 1 \mathrm{CCC} 2(\mathrm{CCCC} 2) \mathrm{C} 1$ & 0 \\
\hline 120 & $\mathbf{1 0}\{19\}$ & $\mathbf{1 1}\{83\}$ & $\mathbf{8}\{19,83\}$ & $\mathrm{CN}(\mathrm{C} 1 \mathrm{CCCc} 2 \mathrm{nc}(\mathrm{C}) \mathrm{sc} 12) \mathrm{c} 1 \mathrm{nnnn} 1 \mathrm{Cc} 1 \mathrm{ccc} n \mathrm{1}$ & 0 \\
\hline 121 & $\mathbf{1 0}\{19\}$ & $11\{84\}$ & $\mathbf{8}\{19,84\}$ & $\mathrm{CN}(\mathrm{CC}(=\mathrm{O}) \mathrm{N} 1 \mathrm{CCCC} 1) \mathrm{c} 1 \mathrm{nnnn} 1 \mathrm{Cc} 1 \mathrm{cccnc} 1$ & 0 \\
\hline 122 & $\mathbf{1 0}\{19\}$ & $\mathbf{1 1}\{85\}$ & $\mathbf{8}\{19,85\}$ & $\mathrm{Fc} 1 \mathrm{cccc}(\mathrm{c} 1) \mathrm{N} 1 \mathrm{CCN}(\mathrm{CC} 1) \mathrm{c} 1 \mathrm{nnnn} 1 \mathrm{Cc} 1 \mathrm{cccnc} 1$ & 0 \\
\hline
\end{tabular}




\begin{tabular}{|c|c|c|c|c|c|}
\hline 123 & $\mathbf{1 0}\{19\}$ & $\mathbf{1 1}\{86\}$ & $\mathbf{8}\{19,86\}$ & OCC1CCCCCN1c1nnnn1Cc1cecnc1 & 0 \\
\hline 124 & $\mathbf{1 0}\{19\}$ & $\mathbf{1 1}\{87\}$ & $\mathbf{8}\{19,87\}$ & $\mathrm{CN}(\mathrm{Cc} 1 \mathrm{ccoc} 1 \mathrm{C}) \mathrm{c} 1 \mathrm{nnnn} 1 \mathrm{Cc} 1 \mathrm{ccc} n \mathrm{1}$ & 0 \\
\hline 125 & $\mathbf{1 0}\{19\}$ & $\mathbf{1 1}\{88\}$ & $\mathbf{8}\{19,88\}$ & $\mathrm{C}(\mathrm{C} 1 \mathrm{CCOCC} 1) \mathrm{N} 1 \mathrm{CCN}(\mathrm{CC} 1) \mathrm{c} 1 \mathrm{nnnn} 1 \mathrm{Cc} 1 \mathrm{ccc} n \mathrm{l}$ & 0 \\
\hline 126 & $\mathbf{1 0}\{19\}$ & $\mathbf{1 1}\{78\}$ & $\mathbf{8}\{19,78\}$ & $\mathrm{COc} 1 \mathrm{ccc} 2 \mathrm{CN}(\mathrm{CCCc} 2 \mathrm{c} 1) \mathrm{c} 1 \mathrm{nnnn} 1 \mathrm{Cc} 1 \mathrm{cccnc} 1$ & 0 \\
\hline 127 & $\mathbf{1 0}\{19\}$ & $\mathbf{1 1}\{89\}$ & $\mathbf{8}\{19,89\}$ & $\mathrm{CC}(\mathrm{N}(\mathrm{C}) \mathrm{c} 1 \mathrm{nnnn} 1 \mathrm{Cc} 1 \mathrm{cccnc} 1) \mathrm{c} 1 \mathrm{ccc} \mathrm{c} 1$ & 0 \\
\hline 128 & $\mathbf{1 0}\{19\}$ & $\mathbf{1 1}\{90\}$ & $\mathbf{8}\{19,90\}$ & $\mathrm{C}(\mathrm{N} 1 \mathrm{CCN}(\mathrm{CC} 1) \mathrm{c} 1 \mathrm{nnnn} 1 \mathrm{Cc} 1 \mathrm{ccc} n \mathrm{c} 1) \mathrm{c} 1 \mathrm{cscn} 1$ & 0 \\
\hline 129 & $\mathbf{1 0}\{19\}$ & $\mathbf{1 1}\{91\}$ & $\mathbf{8}\{19,91\}$ & $\mathrm{CC} 1 \mathrm{~N}(\mathrm{CCOc} 2 \mathrm{ccccc} 12) \mathrm{c} 1 \mathrm{nnnn} 1 \mathrm{Cc} 1 \mathrm{ccc} n \mathrm{c} 1$ & 0 \\
\hline 130 & $\mathbf{1 0}\{19\}$ & $\mathbf{1 1}\{92\}$ & $\mathbf{8}\{19,92\}$ & $\mathrm{C}(\mathrm{c} 1 \mathrm{cccnc} 1) \mathrm{n} 1 \mathrm{nnn} \mathrm{c} 1 \mathrm{~N} 1 \mathrm{CCCC}(\mathrm{C} 1) \mathrm{c} 1 \mathrm{ccccc} 1$ & 0 \\
\hline 131 & $\mathbf{1 0}\{19\}$ & $\mathbf{1 1}\{93\}$ & $\mathbf{8}\{19,93\}$ & $\mathrm{C}(\mathrm{C} 1 \mathrm{CCCCC} 1) \mathrm{N} 1 \mathrm{CCN}(\mathrm{CC} 1) \mathrm{c} 1 \mathrm{nnnn} 1 \mathrm{Cc} 1 \mathrm{cccnc} 1$ & 0 \\
\hline 132 & $\mathbf{1 0}\{19\}$ & $\mathbf{1 1}\{94\}$ & $\mathbf{8}\{19,94\}$ & $\mathrm{CN}(\mathrm{c} 1 \mathrm{nnnn} 1 \mathrm{Cc} 1 \mathrm{cccnc} 1) \mathrm{c} 1 \mathrm{c}(\mathrm{C}) \operatorname{noc} 1 \mathrm{C}$ & 0 \\
\hline 133 & $\mathbf{1 0}\{19\}$ & $\mathbf{1 1}\{95\}$ & $\mathbf{8}\{19,95\}$ & $\mathrm{CC}(\mathrm{N}(\mathrm{C}) \mathrm{c} 1 \mathrm{nnnnn} 1 \mathrm{Cc} 1 \mathrm{cccnc} 1) \mathrm{c} 1 \mathrm{sc}(\mathrm{C}) \mathrm{nc} 1 \mathrm{C}$ & 0 \\
\hline 134 & $\mathbf{1 0}\{19\}$ & $\mathbf{1 1}\{96\}$ & $\mathbf{8}\{19,96\}$ & $\mathrm{CC}(\mathrm{C}) \mathrm{OC} 1 \mathrm{CCN}(\mathrm{CC} 1) \mathrm{c} 1 \mathrm{nnnn} 1 \mathrm{Cc} 1 \mathrm{cccnc} 1$ & 0 \\
\hline 135 & $\mathbf{1 0}\{19\}$ & $\mathbf{1 1}\{97\}$ & $\mathbf{8}\{19,97\}$ & $\mathrm{O}=\mathrm{C}(\mathrm{NC} 1 \mathrm{CC} 1) \mathrm{C} 1 \mathrm{CCCN} 1 \mathrm{c} 1 \mathrm{nnnn} 1 \mathrm{Cc} 1 \mathrm{ccc} n \mathrm{c} 1$ & 0 \\
\hline 136 & $\mathbf{1 0}\{19\}$ & $\mathbf{1 1}\{98\}$ & $\mathbf{8}\{19,98\}$ & $\mathrm{CC}(\mathrm{N}(\mathrm{C}) \mathrm{c} 1 \mathrm{nnnn} 1 \mathrm{Cc} 1 \mathrm{cccnc} 1) \mathrm{c} 1 \mathrm{ccco} 1$ & 0 \\
\hline 137 & $\mathbf{1 0}\{19\}$ & $\mathbf{1 1}\{99\}$ & $\mathbf{8}\{19,99\}$ & $\mathrm{C}(\mathrm{c} 1 \mathrm{ccc} n \mathrm{c} 1) \mathrm{n} 1 \mathrm{nnn} \mathrm{c} 1 \mathrm{~N} 1 \mathrm{CCn} 2 \mathrm{cncc} 2 \mathrm{C} 1$ & 0 \\
\hline 138 & $\mathbf{1 0}\{19\}$ & $\mathbf{1 1}\{100\}$ & $\mathbf{8}\{19,100\}$ & $\mathrm{O}=\mathrm{S} 1 \mathrm{CCN}(\mathrm{CC} 1) \mathrm{c} 1 \mathrm{nnnn} 1 \mathrm{Cc} 1 \mathrm{cccnc} 1$ & 0 \\
\hline 139 & $\mathbf{1 0}\{19\}$ & $\mathbf{1 1}\{101\}$ & $\mathbf{8}\{19,101\}$ & $\mathrm{CN}(\mathrm{Cc} 1 \mathrm{ccc}(\mathrm{C}) \mathrm{s} 1) \mathrm{c} 1 \mathrm{nnnn} 1 \mathrm{Cc} 1 \mathrm{cccnc} 1$ & 0 \\
\hline 140 & $\mathbf{1 0}\{19\}$ & $\mathbf{1 1}\{102\}$ & $\mathbf{8}\{19,102\}$ & $\mathrm{CN}(\mathrm{Cc} 1 \mathrm{scnc} 1 \mathrm{C}) \mathrm{c} 1 \mathrm{nnnn} 1 \mathrm{Cc} 1 \mathrm{ccc} n \mathrm{c} 1$ & 0 \\
\hline 141 & $\mathbf{1 0}\{19\}$ & $\mathbf{1 1}\{103\}$ & $\mathbf{8}\{19,103\}$ & $\mathrm{Cc} 1 \mathrm{ccc} 2[\mathrm{nH}] \mathrm{c} 3 \mathrm{CCN}(\mathrm{Cc} 3 \mathrm{c} 2 \mathrm{c} 1) \mathrm{c} 1 \mathrm{nnnn} 1 \mathrm{Cc} 1 \mathrm{ccc} n \mathrm{l}$ & 0 \\
\hline 142 & $10\{20\}$ & $\mathbf{1 1}\{79\}$ & $\mathbf{8}\{20,79\}$ & CCN1CCCC1Cn1nnnc1N1CCC[C@@H]1C(N)=O & 0 \\
\hline 143 & $\mathbf{1 0}\{20\}$ & $\mathbf{1 1}\{104\}$ & $\mathbf{8}\{20,104\}$ & CCN1CCCC1Cn1nnnc1N1CCOCC2(CCCC2)C1 & 0 \\
\hline
\end{tabular}




\begin{tabular}{|c|c|c|c|c|c|}
\hline 144 & $\mathbf{1 0}\{20\}$ & $\mathbf{1 1}\{105\}$ & $\mathbf{8}\{20,105\}$ & CCN1CCCC1Cn1nnnc1N1CCN(C)c2eccec2C1 & 0 \\
\hline 145 & $\mathbf{1 0}\{20\}$ & $\mathbf{1 1}\{38\}$ & $\mathbf{8}\{20,38\}$ & CCN1CCCC1Cn1nnnc1N1CCc2nc[nH]c2C1 & 0 \\
\hline 146 & $\mathbf{1 0}\{20\}$ & $\mathbf{1 1}\{106\}$ & $\mathbf{8}\{20,106\}$ & CCN1CCCC1Cn1nnnc1N1CCc2eccec2CC1 & 0 \\
\hline 147 & $10\{20\}$ & $\mathbf{1 1}\{40\}$ & $\mathbf{8}\{20,40\}$ & CCN1CCCC1Cn1nnnc1N1CCc2[nH]ncc2C1 & 0 \\
\hline 148 & $\mathbf{1 0}\{20\}$ & $\mathbf{1 1}\{107\}$ & $\mathbf{8}\{20,107\}$ & CCN1CCCC1Cn1nnnc1N1CCc2c(F)ccc(F)c2C1 & 0 \\
\hline 149 & $\mathbf{1 0}\{20\}$ & $\mathbf{1 1}\{108\}$ & $\mathbf{8}\{20,108\}$ & $\mathrm{CCN} 1 \mathrm{CCCC} 1 \mathrm{Cn} 1 \mathrm{nnn} \mathrm{n} 1 \mathrm{~N} 1 \mathrm{CCC}(\mathrm{CC} 1) \mathrm{N} 1 \mathrm{CCCC} 1=\mathrm{O}$ & 0 \\
\hline 150 & $\mathbf{1 0}\{20\}$ & $\mathbf{1 1}\{109\}$ & $\mathbf{8}\{20,109\}$ & CCN1CCCC1Cn1nnnc1N1CCc2nn(C)c $(=\mathrm{O}) \mathrm{cc} 2 \mathrm{C} 1$ & 0 \\
\hline 151 & $\mathbf{1 0}\{21\}$ & $\mathbf{1 1}\{110\}$ & $\mathbf{8}\{21,110\}$ & $\mathrm{CN}(\mathrm{CC} 1(\mathrm{O}) \mathrm{CCOCC} 1) \mathrm{c} 1 \mathrm{nnnn} 1 \mathrm{C}(\mathrm{C})(\mathrm{C}) \mathrm{C}$ & 0 \\
\hline 152 & $\mathbf{1 0}\{21\}$ & $\mathbf{1 1}\{111\}$ & $\mathbf{8}\{21,111\}$ & $\mathrm{CC} 1 \mathrm{CN}(\mathrm{CC}(\mathrm{C}) \mathrm{S} 1(=\mathrm{O})=\mathrm{O}) \mathrm{c} 1 \mathrm{nnnn} 1 \mathrm{C}(\mathrm{C})(\mathrm{C}) \mathrm{C}$ & 0 \\
\hline 153 & $\mathbf{1 0}\{21\}$ & $\mathbf{1 1}\{112\}$ & $\mathbf{8}\{21,112\}$ & $\mathrm{CCOC} 1(\mathrm{C}) \mathrm{CCN}(\mathrm{CC} 1) \mathrm{c} 1 \mathrm{nnnn} 1 \mathrm{C}(\mathrm{C})(\mathrm{C}) \mathrm{C}$ & 0 \\
\hline 154 & $\mathbf{1 0}\{21\}$ & $\mathbf{1 1}\{113\}$ & $\mathbf{8}\{21,113\}$ & $\mathrm{CC}(\mathrm{C})(\mathrm{C}) \mathrm{n} 1 \mathrm{nnnn} 1 \mathrm{~N} 1 \mathrm{CN}(\mathrm{CC}(\mathrm{F})(\mathrm{F}) \mathrm{F}) \mathrm{C}(=\mathrm{O}) \mathrm{C} 1$ & 0 \\
\hline 155 & $\mathbf{1 0}\{21\}$ & $\mathbf{1 1}\{114\}$ & $\mathbf{8}\{21,114\}$ & $\mathrm{CC}(\mathrm{C})(\mathrm{C}) \mathrm{n} 1 \mathrm{nnnc} 1 \mathrm{~N} 1 \mathrm{CCCC}(\mathrm{CC} 1) \mathrm{S}(\mathrm{C})(=\mathrm{O})=\mathrm{O}$ & 0 \\
\hline 156 & $\mathbf{1 0}\{21\}$ & $\mathbf{1 1}\{115\}$ & $\mathbf{8}\{21,115\}$ & $\mathrm{CC}(\mathrm{C})(\mathrm{C}) \mathrm{n} 1 \mathrm{nnnnc} 1 \mathrm{~N} 1 \mathrm{CCC} 2(\mathrm{C} 1) \mathrm{C}(=\mathrm{O}) \mathrm{Nc} 1 \mathrm{cccc} 21$ & 28 \\
\hline 157 & $\mathbf{1 0}\{21\}$ & $\mathbf{1 1}\{116\}$ & $\mathbf{8}\{21,116\}$ & $\begin{array}{l}\mathrm{CC}(\mathrm{C})(\mathrm{C}) n 1 n n n c 1 \mathrm{~N} 1 \mathrm{C}[1 \mathrm{C} @ @ \mathrm{H}] 2 \mathrm{CC}[1 \mathrm{C} @ \mathrm{H}](\mathrm{O}) \mathrm{C}[1 \mathrm{C} @ @ \mathrm{H}] \\
2 \mathrm{C} 1\end{array}$ & 31 \\
\hline 158 & $\mathbf{1 0}\{21\}$ & $\mathbf{1 1}\{117\}$ & $\mathbf{8}\{21,117\}$ & $\mathrm{CC}(\mathrm{C})(\mathrm{C}) \mathrm{n} 1 \mathrm{nnn} \mathrm{n} 1 \mathrm{~N} 1 \mathrm{CCCC}(\mathrm{CC}(\mathrm{C})(\mathrm{C}) \mathrm{C}(\mathrm{N})=\mathrm{O}) \mathrm{C} 1$ & 8 \\
\hline 159 & $\mathbf{1 0}\{21\}$ & $\mathbf{1 1}\{118\}$ & $\mathbf{8}\{21,118\}$ & C[1C@@H]1[1C@H](O)CCN1c1nnnn1C(C)(C)C & 38 \\
\hline 160 & $\mathbf{1 0}\{21\}$ & $\mathbf{1 1}\{119\}$ & $\mathbf{8}\{21,119\}$ & $\mathrm{CCN}(\mathrm{C} 1 \mathrm{CCNC} 1=\mathrm{O}) \mathrm{c} 1 \mathrm{nnnn} 1 \mathrm{C}(\mathrm{C})(\mathrm{C}) \mathrm{C}$ & 0 \\
\hline 161 & $\mathbf{1 0}\{21\}$ & $\mathbf{1 1}\{120\}$ & $\mathbf{8}\{21,120\}$ & $\mathrm{CN} 1 \mathrm{CCN}(\mathrm{CC} 1 \mathrm{CO}) \mathrm{c} 1 \mathrm{nnnn} 1 \mathrm{C}(\mathrm{C})(\mathrm{C}) \mathrm{C}$ & 0 \\
\hline 162 & $\mathbf{1 0}\{21\}$ & $\mathbf{1 1}\{121\}$ & $\mathbf{8}\{21,121\}$ & Cc1nnc(N2CCN $(\mathrm{CC} 2) \mathrm{c} 2 \mathrm{nnnn} 2 \mathrm{C}(\mathrm{C})(\mathrm{C}) \mathrm{C}) \mathrm{n} 1 \mathrm{C}$ & 0 \\
\hline 163 & $\mathbf{1 0}\{21\}$ & $\mathbf{1 1}\{122\}$ & $\mathbf{8}\{21,122\}$ & $\mathrm{CCN}(\mathrm{CCO}) \mathrm{c} 1 \mathrm{nnnn} 1 \mathrm{C}(\mathrm{C})(\mathrm{C}) \mathrm{C}$ & 0 \\
\hline 164 & $\mathbf{1 0}\{21\}$ & $\mathbf{1 1}\{123\}$ & $\mathbf{8}\{21,123\}$ & $\mathrm{CC}(\mathrm{C})(\mathrm{C}) \mathrm{C} 1 \mathrm{CN}(\mathrm{CCO} 1) \mathrm{c} 1 \mathrm{nnnn} 1 \mathrm{C}(\mathrm{C})(\mathrm{C}) \mathrm{C}$ & 0 \\
\hline
\end{tabular}




\begin{tabular}{|c|c|c|c|c|c|}
\hline 165 & $\mathbf{1 0}\{21\}$ & $\mathbf{1 1}\{124\}$ & $\mathbf{8}\{21,124\}$ & $\operatorname{Cc} 1 \mathrm{cc}(=\mathrm{O})[\mathrm{nH}] \mathrm{c}(\mathrm{n} 1) \mathrm{N} 1 \mathrm{CCN}(\mathrm{CC} 1) \mathrm{c} 1 \mathrm{nnnn} 1 \mathrm{C}(\mathrm{C})(\mathrm{C}) \mathrm{C}$ & 0 \\
\hline 166 & $\mathbf{1 0}\{22\}$ & $\mathbf{1 1}\{12\}$ & $\mathbf{8}\{22,12\}$ & Cn1cnnc1C1CCCN(C1)c1nnnn1C1CCCC1 & 0 \\
\hline 167 & $\mathbf{1 0}\{22\}$ & $\mathbf{1 1}\{2\}$ & $\mathbf{8}\{22,2\}$ & $\mathrm{O}=\mathrm{C}(\mathrm{NC} 1 \mathrm{CC} 1) \mathrm{C}(=\mathrm{O}) \mathrm{N} 1 \mathrm{CCN}(\mathrm{CC} 1) \mathrm{c} 1 \mathrm{nnnn} 1 \mathrm{C} 1 \mathrm{CCCC} 1$ & 24 \\
\hline 168 & $\mathbf{1 0}\{22\}$ & $\mathbf{1 1}\{10\}$ & $\mathbf{8}\{22,10\}$ & $\mathrm{Cn} 1 \operatorname{ccc} 1 \mathrm{C}(=\mathrm{O}) \mathrm{N} 1 \mathrm{CCN}(\mathrm{CC} 1) \mathrm{c} 1 \mathrm{nnnn} 1 \mathrm{C} 1 \mathrm{CCCC} 1$ & 9 \\
\hline 169 & $\mathbf{1 0}\{22\}$ & $\mathbf{1 1}\{100\}$ & $\mathbf{8}\{22,100\}$ & $\mathrm{O}=\mathrm{S} 1 \mathrm{CCN}(\mathrm{CC} 1) \mathrm{c} 1 \mathrm{nnnn} 1 \mathrm{C} 1 \mathrm{CCCC} 1$ & 0 \\
\hline 170 & $10\{23\}$ & $\mathbf{1 1}\{38\}$ & $\mathbf{8}\{23,38\}$ & $\mathrm{CC}(\mathrm{c} 1 \mathrm{ccccc} 1) \mathrm{n} 1 \mathrm{nnn} \mathrm{c} 1 \mathrm{~N} 1 \mathrm{CCc} 2 \mathrm{nc}[\mathrm{nH}] \mathrm{c} 2 \mathrm{C} 1$ & 0 \\
\hline 171 & $\mathbf{1 0}\{23\}$ & $\mathbf{1 1}\{125\}$ & $\mathbf{8}\{23,125\}$ & $\mathrm{CC}(\mathrm{c} 1 \mathrm{ccccc} 1) \mathrm{n} 1 \mathrm{nnnnc} 1 \mathrm{~N}(\mathrm{C}) \mathrm{CC} 1(\mathrm{O}) \mathrm{CCCC} 1$ & 0 \\
\hline 172 & $10\{23\}$ & $\mathbf{1 1}\{94\}$ & $\mathbf{8}\{23,94\}$ & $\mathrm{CC}(\mathrm{c} 1 \mathrm{ccccc} 1) \mathrm{n} 1 \mathrm{nnnc} 1 \mathrm{~N}(\mathrm{C}) \mathrm{c} 1 \mathrm{c}(\mathrm{C})$ noc $1 \mathrm{C}$ & 33 \\
\hline 173 & $\mathbf{1 0}\{23\}$ & $11\{126\}$ & $\mathbf{8}\{23,126\}$ & $\mathrm{CC}(\mathrm{c} 1 \mathrm{ccccc} 1) \mathrm{n} 1 \mathrm{nnnn} 1 \mathrm{~N} 1 \mathrm{CCN}(\mathrm{Cc} 2 \mathrm{nc}[\mathrm{nH}] \mathrm{n} 2) \mathrm{CC} 1$ & 21 \\
\hline 174 & $\mathbf{1 0}\{23\}$ & $\mathbf{1 1}\{108\}$ & $\mathbf{8}\{23,108\}$ & $\mathrm{CC}(\mathrm{c} 1 \mathrm{ccccc} 1) \mathrm{n} 1 \mathrm{nnn} \mathrm{c} 1 \mathrm{~N} 1 \mathrm{CCC}(\mathrm{CC} 1) \mathrm{N} 1 \mathrm{CCCC} 1=\mathrm{O}$ & 45 \\
\hline 175 & $\mathbf{1 0}\{23\}$ & $\mathbf{1 1}\{99\}$ & $\mathbf{8}\{23,99\}$ & $\mathrm{CC}(\mathrm{c} 1 \mathrm{ccccc} 1) \mathrm{n} 1 \mathrm{nnnn} 1 \mathrm{~N} 1 \mathrm{CCn} 2 \mathrm{cncc} 2 \mathrm{C} 1$ & 0 \\
\hline 176 & $\mathbf{1 0}\{23\}$ & $11\{127\}$ & $\mathbf{8}\{23,127\}$ & $\mathrm{CC}(\mathrm{c} 1 \mathrm{ccccc} 1) \mathrm{n} 1 \mathrm{nnn} \mathrm{c} 1 \mathrm{~N} 1 \mathrm{CCC}[\mathrm{C} @ \mathrm{H}] 1 \mathrm{C}(\mathrm{N})=\mathrm{O}$ & 0 \\
\hline 177 & $\mathbf{1 0}\{24\}$ & $\mathbf{1 1}\{12\}$ & $\mathbf{8}\{24,12\}$ & $\mathrm{CCC}(\mathrm{C}) \mathrm{n} 1 \mathrm{nnnn} 1 \mathrm{~N} 1 \mathrm{CCCC}(\mathrm{C} 1) \mathrm{c} 1 \mathrm{nnn} \mathrm{cn} 1 \mathrm{C}$ & 0 \\
\hline 178 & $\mathbf{1 0}\{25\}$ & $11\{8\}$ & $\mathbf{8}\{25,8\}$ & $\begin{array}{l}\mathrm{C}(\mathrm{C} 1 \mathrm{CC} 1) n 1 \mathrm{nnn} 1 \mathrm{~N} 1 \mathrm{CC} 2(\mathrm{CCOCC} 2)[1 \mathrm{C} @ @ \mathrm{H}] 2 \mathrm{CCC}[1 \mathrm{C} @ \mathrm{H} \\
] 12\end{array}$ & 0 \\
\hline 179 & $\mathbf{1 0}\{25\}$ & $\mathbf{1 1}\{1\}$ & $\mathbf{8}\{25,1\}$ & $\mathrm{CNS}(=\mathrm{O})(=\mathrm{O}) \mathrm{c} 1 \operatorname{ccc} 2 \mathrm{CN}(\mathrm{CCc} 12) \mathrm{c} 1 \mathrm{nnnn} 1 \mathrm{CC} 1 \mathrm{CC} 1$ & 26 \\
\hline 180 & $\mathbf{1 0}\{26\}$ & $11\{7\}$ & $\mathbf{8}\{26,7\}$ & $\mathrm{CC} 1=\mathrm{C}(\mathrm{C}) \mathrm{CN}(\mathrm{CC} 1) \mathrm{c} 1 \mathrm{nnnn} 1 \mathrm{CC}(\mathrm{F})(\mathrm{F}) \mathrm{F}$ & 0 \\
\hline 181 & $\mathbf{1 0}\{26\}$ & $11\{4\}$ & $\mathbf{8}\{26,4\}$ & $\mathrm{FC}(\mathrm{F})(\mathrm{F}) \mathrm{Cn} 1 \mathrm{nnnc} 1 \mathrm{~N} 1 \mathrm{CCOC} 2(\mathrm{CCC} 2) \mathrm{C} 1$ & 0 \\
\hline 182 & $\mathbf{1 0}\{26\}$ & $\mathbf{1 1}\{10\}$ & $\mathbf{8}\{26,10\}$ & $\mathrm{Cn} 1 \operatorname{ccc} 1 \mathrm{C}(=\mathrm{O}) \mathrm{N} 1 \mathrm{CCN}(\mathrm{CC} 1) \mathrm{c} 1 \mathrm{nnnn} 1 \mathrm{CC}(\mathrm{F})(\mathrm{F}) \mathrm{F}$ & 0 \\
\hline 183 & $\mathbf{1 0}\{26\}$ & $\mathbf{1 1}\{5\}$ & $\mathbf{8}\{26,5\}$ & $\mathrm{Fc} 1 \mathrm{cc}(\mathrm{F}) \mathrm{cc}(\mathrm{c} 1) \mathrm{C} 1 \mathrm{COCCN} 1 \mathrm{c} 1 \mathrm{nnnn} 1 \mathrm{CC}(\mathrm{F})(\mathrm{F}) \mathrm{F}$ & 0 \\
\hline 184 & $\mathbf{1 0}\{26\}$ & $11\{6\}$ & $\mathbf{8}\{26,6\}$ & $\mathrm{Fc} 1 \mathrm{ccc} 2 \mathrm{CN}(\mathrm{CCOc} 2 \mathrm{c} 1) \mathrm{c} 1 \mathrm{nnnn} 1 \mathrm{CC}(\mathrm{F})(\mathrm{F}) \mathrm{F}$ & 0 \\
\hline 185 & $\mathbf{1 0}\{26\}$ & $\mathbf{1 1}\{8\}$ & $\mathbf{8}\{26,8\}$ & $\begin{array}{l}\mathrm{FC}(\mathrm{F})(\mathrm{F}) \mathrm{Cn} 1 \mathrm{nnn} \mathrm{n} 1 \mathrm{~N} 1 \mathrm{CC} 2(\mathrm{CCOCC} 2)[1 \mathrm{C} @ \mathrm{H}] 2 \mathrm{CCC}[1 \mathrm{C} @ \mathrm{H} \\
] 12\end{array}$ & 27 \\
\hline
\end{tabular}




\begin{tabular}{|c|c|c|c|c|c|}
\hline 186 & $\mathbf{1 0}\{27\}$ & $\mathbf{1 1}\{128\}$ & $\mathbf{8}\{27,128\}$ & $\mathrm{CCOC}(=\mathrm{O}) \mathrm{N} 1 \mathrm{CCC}(\mathrm{CC} 1) \mathrm{n} 1 \mathrm{nnn} \mathrm{n} 1 \mathrm{~N} 1 \mathrm{CCCC}(\mathrm{C} 1) \mathrm{C} 1 \mathrm{CC} 1$ & 13 \\
\hline 187 & $\mathbf{1 0}\{27\}$ & $11\{7\}$ & $\mathbf{8}\{27,7\}$ & $\mathrm{CCOC}(=\mathrm{O}) \mathrm{N} 1 \mathrm{CCC}(\mathrm{CC} 1) \mathrm{n} 1 \mathrm{nnn} \mathrm{n} 1 \mathrm{~N} 1 \mathrm{CCC}(\mathrm{C})=\mathrm{C}(\mathrm{C}) \mathrm{C} 1$ & 0 \\
\hline 188 & $\mathbf{1 0}\{28\}$ & $\mathbf{1 1}\{129\}$ & $\mathbf{8}\{28,129\}$ & $\mathrm{C}[\mathrm{C} @ @ \mathrm{H}](\mathrm{O}) \mathrm{CN}(\mathrm{C}) \mathrm{c} 1 \mathrm{nnnn} 1 \mathrm{C}(\mathrm{C}) \mathrm{c} 1 \mathrm{ccc}(\mathrm{Br}) \mathrm{cc} 1$ & 13 \\
\hline 189 & $\mathbf{1 0}\{29\}$ & $\mathbf{1 1}\{130\}$ & $\mathbf{8}\{29,130\}$ & CCC1CCCN1c1nnnn1C1CCN(Cc2 $\operatorname{ccccc} 2) \mathrm{CC} 1$ & 21 \\
\hline 190 & $\mathbf{1 0}\{30\}$ & $\mathbf{1 1}\{36\}$ & $\mathbf{8}\{30,36\}$ & $\mathrm{O}[\mathrm{C} @ \mathrm{H}] 1 \mathrm{CCN}(\mathrm{C} 1) \mathrm{c} 1 \mathrm{nnnn} 1 \mathrm{CCc} 1 \mathrm{ccc}(\mathrm{Cl}) \mathrm{cc} 1 \mathrm{Cl}$ & 24 \\
\hline 191 & $\mathbf{1 0}\{30\}$ & $\mathbf{1 1}\{131\}$ & $\mathbf{8}\{30,131\}$ & OCC1C $=\mathrm{CCN} 1 \mathrm{c} 1 \mathrm{nnnn} 1 \mathrm{CCc} 1 \mathrm{ccc}(\mathrm{Cl}) \mathrm{cc} 1 \mathrm{Cl}$ & 0 \\
\hline 192 & $\mathbf{1 0}\{31\}$ & $\mathbf{1 1}\{7\}$ & $\mathbf{8}\{31,7\}$ & $\mathrm{CCOCCn} 1 \mathrm{nnn} \mathrm{c} 1 \mathrm{~N} 1 \mathrm{CCC}(\mathrm{C})=\mathrm{C}(\mathrm{C}) \mathrm{C} 1$ & 0 \\
\hline 193 & $\mathbf{1 0}\{32\}$ & $\mathbf{1 1}\{132\}$ & $\mathbf{8}\{32,132\}$ & $\mathrm{CC}(\mathrm{C})(\mathrm{C}) \mathrm{C} 1=\mathrm{CCN}(\mathrm{CC} 1) \mathrm{c} 1 \mathrm{nnnn} 1 \mathrm{CCc} 1 \mathrm{ccc} n 1$ & 0 \\
\hline 194 & $\mathbf{1 0}\{32\}$ & $\mathbf{1 1}\{133\}$ & $\mathbf{8}\{32,133\}$ & $\mathrm{CC} 1(\mathrm{C}) \mathrm{CCCN} 1 \mathrm{c} 1 \mathrm{nnnn} 1 \mathrm{CCc} 1 \mathrm{ccc} n 1$ & 16 \\
\hline 195 & $\mathbf{1 0}\{32\}$ & $\mathbf{1 1}\{104\}$ & $\mathbf{8}\{32,104\}$ & $\mathrm{C}(\mathrm{Cn} 1 \mathrm{nnnn} 1 \mathrm{~N} 1 \mathrm{CCOCC} 2(\mathrm{CCCC} 2) \mathrm{C} 1) \mathrm{c} 1 \mathrm{cccc} 1$ & 0 \\
\hline 196 & $\mathbf{1 0}\{32\}$ & $\mathbf{1 1}\{134\}$ & $\mathbf{8}\{32,134\}$ & $\mathrm{CN}(\mathrm{C}) \mathrm{C}(=\mathrm{O}) \mathrm{NC} 1 \mathrm{CCCN}(\mathrm{C} 1) \mathrm{c} 1 \mathrm{nnnn} 1 \mathrm{CCc} 1 \mathrm{ccc} n 1$ & 0 \\
\hline 197 & $\mathbf{1 0}\{32\}$ & $\mathbf{1 1}\{135\}$ & $\mathbf{8}\{32,135\}$ & $\mathrm{FC}(\mathrm{F})(\mathrm{F})[\mathrm{C} @ @ \mathrm{H}] 1 \mathrm{CCCN}(\mathrm{C} 1) \mathrm{c} 1 \mathrm{nnnn} 1 \mathrm{CCc} 1 \mathrm{ccc} n 1$ & 0 \\
\hline 198 & $\mathbf{1 0}\{32\}$ & $\mathbf{1 1}\{136\}$ & $\mathbf{8}\{32,136\}$ & $\mathrm{CC}(\mathrm{C}) \mathrm{NC}(=\mathrm{O})[\mathrm{C} @ @ \mathrm{H}] 1 \mathrm{CCCN}(\mathrm{C} 1) \mathrm{c} 1 \mathrm{nnnn} 1 \mathrm{CCc} 1 \mathrm{ccc} n 1$ & 0 \\
\hline 199 & $\mathbf{1 0}\{32\}$ & $\mathbf{1 1}\{137\}$ & $\mathbf{8}\{32,137\}$ & CCN1CCC2CCC(C1)N2c1nnnn1CCc1cccen1 & 0 \\
\hline 200 & $\mathbf{1 0}\{32\}$ & $\mathbf{1 1}\{99\}$ & $\mathbf{8}\{32,99\}$ & $\mathrm{C}(\mathrm{Cn} 1 \mathrm{nnn} \mathrm{n} 1 \mathrm{~N} 1 \mathrm{CCn} 2 \mathrm{cncc} 2 \mathrm{C} 1) \mathrm{c} 1 \mathrm{ccc} n 1$ & 0 \\
\hline 201 & $\mathbf{1 0}\{32\}$ & $\mathbf{1 1}\{116\}$ & $\mathbf{8}\{32,116\}$ & $\begin{array}{l}\mathrm{O}[1 \mathrm{C} @ \mathrm{H}] 1 \mathrm{CC}[1 \mathrm{C} @ \mathrm{H}] 2 \mathrm{CN}(\mathrm{C}[1 \mathrm{C} @ @ \mathrm{H}] 2 \mathrm{C} 1) \mathrm{c} 1 \mathrm{nnnn} 1 \mathrm{CC} \\
\mathrm{c} 1 \mathrm{ccc} n 1\end{array}$ & 0 \\
\hline 202 & $\mathbf{1 0}\{32\}$ & $\mathbf{1 1}\{138\}$ & $\mathbf{8}\{32,138\}$ & Clc1ecceCCN(Cc12)c1nnnn1CCc1cccen1 & 0 \\
\hline 203 & $\mathbf{1 0}\{32\}$ & $\mathbf{1 1}\{139\}$ & $\mathbf{8}\{32,139\}$ & Fc1 $1 \mathrm{ccc} 2 \mathrm{CCN}(\mathrm{c} 3 \mathrm{nnnnn} 3 \mathrm{CCc} 3 \mathrm{cccc} n 3) \mathrm{c} 12$ & 0 \\
\hline 204 & $\mathbf{1 0}\{32\}$ & $\mathbf{1 1}\{140\}$ & $\mathbf{8}\{32,140\}$ & $\mathrm{CN}(\mathrm{c} 1 \mathrm{cnn}(\mathrm{C}) \mathrm{c} 1) \mathrm{c} 1 \mathrm{nnnn} 1 \mathrm{CCc} 1 \mathrm{ccc} n 1$ & 0 \\
\hline 205 & $\mathbf{1 0}\{32\}$ & $\mathbf{1 1}\{141\}$ & $\mathbf{8}\{32,141\}$ & $\mathrm{O}=\mathrm{C} 1 \mathrm{CC} 2(\mathrm{CN} 1) \mathrm{CCN}(\mathrm{CC} 2) \mathrm{c} 1 \mathrm{nnnn} 1 \mathrm{CCc} 1 \mathrm{ccc} n 1$ & 0 \\
\hline 206 & $\mathbf{1 0}\{32\}$ & $\mathbf{1 1}\{142\}$ & $\mathbf{8}\{32,142\}$ & $\mathrm{C}(\mathrm{Cn} 1 \mathrm{nnn} \mathrm{n} 1 \mathrm{~N} 1 \mathrm{CCC}(\mathrm{CC} 1) \mathrm{c} 1 \mathrm{cn}[\mathrm{nH}] \mathrm{c} 1) \mathrm{c} 1 \mathrm{ccc} n 1$ & 0 \\
\hline
\end{tabular}




\begin{tabular}{|c|c|c|c|c|c|}
\hline 207 & $\mathbf{1 0}\{32\}$ & $\mathbf{1 1}\{143\}$ & $\mathbf{8}\{32,143\}$ & $\mathrm{CN}(\mathrm{c} 1 \mathrm{ccn}(\mathrm{C}) \mathrm{n} 1) \mathrm{c} 1 \mathrm{nnnn} 1 \mathrm{CC} 1 \mathrm{ccc} n 1$ & 0 \\
\hline 208 & $\mathbf{1 0}\{32\}$ & $\mathbf{1 1}\{144\}$ & $\mathbf{8}\{32,144\}$ & $\mathrm{C}(\mathrm{Cn} 1 \mathrm{nnn} n \mathrm{1N} 1 \mathrm{CCN} 2 \mathrm{CCOCC} 2 \mathrm{C} 1) \mathrm{c} 1 \mathrm{cccc} 1$ & 0 \\
\hline 209 & $\mathbf{1 0}\{32\}$ & $\mathbf{1 1}\{145\}$ & $\mathbf{8}\{32,145\}$ & $\mathrm{Cc} 1 \mathrm{ccc} 2 \mathrm{CCN}(\mathrm{Cc} 12) \mathrm{c} 1 \mathrm{nnnn} 1 \mathrm{CCc} 1 \mathrm{ccc} n 1$ & 0 \\
\hline 210 & $\mathbf{1 0}\{32\}$ & $11\{4\}$ & $\mathbf{8}\{32,4\}$ & $\mathrm{C}(\mathrm{Cn} 1 \mathrm{nnn} \mathrm{n} 1 \mathrm{~N} 1 \mathrm{CCOC} 2(\mathrm{CCC} 2) \mathrm{C} 1) \mathrm{c} 1 \mathrm{cccc} 1$ & 0 \\
\hline 211 & $\mathbf{1 0}\{32\}$ & $\mathbf{1 1}\{146\}$ & $\mathbf{8}\{32,146\}$ & $\mathrm{C}(\mathrm{Cn} 1 \mathrm{nnn} \operatorname{c} 1 \mathrm{~N} 1 \mathrm{CCCC} 1 \mathrm{c} 1 \mathrm{ccn}[\mathrm{nH}] 1) \mathrm{c} 1 \mathrm{ccc} n 1$ & 0 \\
\hline 212 & $\mathbf{1 0}\{32\}$ & $\mathbf{1 1}\{147\}$ & $\mathbf{8}\{32,147\}$ & Clc1cnn(c1)C1CCN(C1)c1nnnn1CCc1 cccen1 & 0 \\
\hline 213 & $\mathbf{1 0}\{32\}$ & $\mathbf{1 1}\{148\}$ & $\mathbf{8}\{32,148\}$ & $\mathrm{Cc} 1 \csc (\mathrm{n} 1) \mathrm{C} 1 \mathrm{CCN}(\mathrm{C} 1) \mathrm{c} 1 \mathrm{nnnn} 1 \mathrm{CCc} 1 \mathrm{ccc} n 1$ & 0 \\
\hline 214 & $\mathbf{1 0}\{32\}$ & $\mathbf{1 1}\{149\}$ & $\mathbf{8}\{32,149\}$ & $\mathrm{C}(\mathrm{Cn} 1 \mathrm{nnn} \mathrm{c} 1 \mathrm{~N} 1 \mathrm{CCCC} 1 \mathrm{c} 1 \mathrm{ccncc} 1) \mathrm{c} 1 \mathrm{ccc} \mathrm{1}$ & 0 \\
\hline 215 & $\mathbf{1 0}\{33\}$ & $\mathbf{1 1}\{150\}$ & $\mathbf{8}\{33,150\}$ & $\mathrm{CC} 1 \mathrm{CN}(\mathrm{CC}(=\mathrm{O}) \mathrm{N} 1) \mathrm{c} 1 \mathrm{nnnnn} 1 \mathrm{Cc} 1 \mathrm{cccc}(\mathrm{c} 1) \mathrm{C}(\mathrm{F})(\mathrm{F}) \mathrm{F}$ & 19 \\
\hline 216 & $\mathbf{1 0}\{33\}$ & $\mathbf{1 1}\{143\}$ & $\mathbf{8}\{33,143\}$ & $\mathrm{CN}(\mathrm{c} 1 \mathrm{ccn}(\mathrm{C}) \mathrm{n} 1) \mathrm{c} 1 \mathrm{nnnn} 1 \mathrm{Cc} 1 \mathrm{cccc}(\mathrm{c} 1) \mathrm{C}(\mathrm{F})(\mathrm{F}) \mathrm{F}$ & 0 \\
\hline 217 & $\mathbf{1 0}\{33\}$ & $\mathbf{1 1}\{99\}$ & $\mathbf{8}\{33,99\}$ & $\mathrm{FC}(\mathrm{F})(\mathrm{F}) \mathrm{c} 1 \mathrm{cccc}(\mathrm{Cn} 2 \mathrm{nnn} \mathrm{n} 2 \mathrm{~N} 2 \mathrm{CCn} 3 \mathrm{cncc} 3 \mathrm{C} 2) \mathrm{c} 1$ & 0 \\
\hline 218 & $\mathbf{1 0}\{33\}$ & $\mathbf{1 1}\{140\}$ & $\mathbf{8}\{33,140\}$ & $\mathrm{CN}(\mathrm{c} 1 \mathrm{cnn}(\mathrm{C}) \mathrm{c} 1) \mathrm{c} 1 \mathrm{nnnn} 1 \mathrm{Cc} 1 \mathrm{cccc}(\mathrm{c} 1) \mathrm{C}(\mathrm{F})(\mathrm{F}) \mathrm{F}$ & 0 \\
\hline 219 & $\mathbf{1 0}\{33\}$ & $\mathbf{1 1}\{73\}$ & $\mathbf{8}\{33,73\}$ & $\mathrm{FC}(\mathrm{F})(\mathrm{F}) \mathrm{c} 1 \mathrm{cccc}(\mathrm{Cn} 2 \mathrm{nnnc} 2 \mathrm{~N} 2 \mathrm{CCc} 3 \mathrm{cn}[\mathrm{nH}] \mathrm{c} 3 \mathrm{C} 2) \mathrm{c} 1$ & 0 \\
\hline 220 & $\mathbf{1 0}\{34\}$ & $\mathbf{1 1}\{151\}$ & $\mathbf{8}\{34,151\}$ & $\mathrm{CC} 1 \mathrm{COCCN}(\mathrm{C} 1) \mathrm{c} 1 \mathrm{nnnn} 1 \mathrm{CCc} 1 \mathrm{ccc} 2 \mathrm{OCCOc} 2 \mathrm{c} 1$ & 24 \\
\hline 221 & $\mathbf{1 0}\{34\}$ & $\mathbf{1 1}\{152\}$ & $\mathbf{8}\{34,152\}$ & $\mathrm{FC} 1 \mathrm{CCN}(\mathrm{CC} 1) \mathrm{c} 1 \mathrm{nnnn} 1 \mathrm{CCc} 1 \mathrm{ccc} 2 \mathrm{OCCOc} 2 \mathrm{c} 1$ & 21 \\
\hline 222 & $\mathbf{1 0}\{34\}$ & $\mathbf{1 1}\{153\}$ & $\mathbf{8}\{34,153\}$ & $\mathrm{CN}(\mathrm{C}) \mathrm{C} 1 \mathrm{CCN}(\mathrm{C} 1) \mathrm{c} 1 \mathrm{nnnn} 1 \mathrm{CCc} 1 \mathrm{ccc} 2 \mathrm{OCCOc} 2 \mathrm{c} 1$ & 0 \\
\hline 223 & $\mathbf{1 0}\{35\}$ & $\mathbf{1 1}\{154\}$ & $\mathbf{8}\{35,154\}$ & $\mathrm{CS}(=\mathrm{O})(=\mathrm{O}) \mathrm{NCC} 1 \mathrm{nnn} \mathrm{c} 1 \mathrm{~N} 1 \mathrm{CCC}(\mathrm{F})(\mathrm{CC} 1) \mathrm{C} 1 \mathrm{CC} 1$ & 0 \\
\hline 224 & $\mathbf{1 0}\{35\}$ & $\mathbf{1 1}\{155\}$ & $\mathbf{8}\{35,155\}$ & $\mathrm{CS}(=\mathrm{O})(=\mathrm{O}) \mathrm{NCCn} 1 \mathrm{nnnc} 1 \mathrm{~N} 1 \mathrm{CCc} 2 \mathrm{c} 1 \mathrm{cccc} 2 \mathrm{Cl}$ & 10 \\
\hline 225 & $\mathbf{1 0}\{35\}$ & $\mathbf{1 1}\{156\}$ & $\mathbf{8}\{35,156\}$ & $\mathrm{CS}(=\mathrm{O})(=\mathrm{O}) \mathrm{NCCn} 1 \mathrm{nnnc1N} 1 \mathrm{CCC}[\mathrm{C} @ @ \mathrm{H}](\mathrm{C} 1) \mathrm{C}(\mathrm{F})(\mathrm{F}) \mathrm{F}$ & 0 \\
\hline 226 & $\mathbf{1 0}\{35\}$ & $\mathbf{1 1}\{157\}$ & $\mathbf{8}\{35,157\}$ & $\mathrm{CC} 1(\mathrm{~F}) \mathrm{CCCN}(\mathrm{C} 1) \mathrm{c} 1 \mathrm{nnnn} 1 \mathrm{CCNS}(\mathrm{C})(=\mathrm{O})=\mathrm{O}$ & 0 \\
\hline 227 & $\mathbf{1 0}\{35\}$ & $\mathbf{1 1}\{158\}$ & $\mathbf{8}\{35,158\}$ & $\mathrm{CS}(=\mathrm{O})(=\mathrm{O}) \mathrm{NCCn} 1 \mathrm{nnnc} 1 \mathrm{~N} 1 \mathrm{CCC}(\mathrm{CC} 1) \mathrm{C}(\mathrm{F}) \mathrm{F}$ & 0 \\
\hline
\end{tabular}




\begin{tabular}{|c|c|c|c|c|c|}
\hline 228 & $\mathbf{1 0}\{35\}$ & $\mathbf{1 1}\{159\}$ & $\mathbf{8}\{35,159\}$ & $\mathrm{CS}(=\mathrm{O})(=\mathrm{O}) \mathrm{NCCn} 1 \mathrm{nnn} \operatorname{c} 1 \mathrm{~N} 1 \mathrm{CCc} 2 \mathrm{c} 1 \mathrm{ccc} 2 \mathrm{~F}$ & 0 \\
\hline 229 & $\mathbf{1 0}\{35\}$ & $\mathbf{1 1}\{133\}$ & $\mathbf{8}\{35,133\}$ & $\mathrm{CC} 1(\mathrm{C}) \mathrm{CCCN} 1 \mathrm{c} 1 \mathrm{nnnn} 1 \mathrm{CCNS}(\mathrm{C})(=\mathrm{O})=\mathrm{O}$ & 0 \\
\hline 230 & $\mathbf{1 0}\{35\}$ & $\mathbf{1 1}\{160\}$ & $\mathbf{8}\{35,160\}$ & $\mathrm{CS}(=\mathrm{O})(=\mathrm{O}) \mathrm{NCCn} 1 \mathrm{nnnc} 1 \mathrm{~N} 1 \mathrm{CCC} 2(\mathrm{CCCC} 2) \mathrm{CC} 1$ & 0 \\
\hline 231 & $\mathbf{1 0}\{36\}$ & $\mathbf{1 1}\{161\}$ & $\mathbf{8}\{36,161\}$ & $\mathrm{CC}(\mathrm{N}(\mathrm{C}) \mathrm{c} 1 \mathrm{nnnn} 1 \mathrm{CC} 1 \mathrm{CCC} 1) \mathrm{c} 1 \mathrm{cccnc} 1$ & 0 \\
\hline 232 & $\mathbf{1 0}\{36\}$ & $\mathbf{1 1}\{162\}$ & $\mathbf{8}\{36,162\}$ & $\mathrm{C}(\mathrm{C} 1 \mathrm{CCC} 1) \mathrm{n} 1 \mathrm{nnn} \mathrm{c} 1 \mathrm{~N} 1 \mathrm{CCCC}(\mathrm{C} 1) \mathrm{c} 1 \mathrm{nnc} 2 \mathrm{CCCn} 12$ & 0 \\
\hline 233 & $\mathbf{1 0}\{36\}$ & $\mathbf{1 1}\{163\}$ & $\mathbf{8}\{36,163\}$ & Cc1ccc(n1)C1CCCN1c1nnnn1CC1CCC1 & 15 \\
\hline 234 & $\mathbf{1 0}\{36\}$ & $\mathbf{1 1}\{164\}$ & $\mathbf{8}\{36,164\}$ & $\mathrm{C}(\mathrm{C} 1 \mathrm{CCC} 1) \mathrm{n} 1 \mathrm{nnn} n 1 \mathrm{~N} 1 \mathrm{CC}[\mathrm{C} @ \mathrm{H}](\mathrm{C} 1) \mathrm{Oc} 1 \mathrm{ccccc} 1$ & 117 \\
\hline 235 & $\mathbf{1 0}\{36\}$ & $\mathbf{1 1}\{165\}$ & $\mathbf{8}\{36,165\}$ & $\mathrm{C}(\mathrm{C} 1 \mathrm{CCN}(\mathrm{CC} 1) \mathrm{c} 1 \mathrm{nnnn} 1 \mathrm{CC} 1 \mathrm{CCC} 1) \mathrm{N} 1 \mathrm{CCOCC} 1$ & 53 \\
\hline 236 & $\mathbf{1 0}\{36\}$ & $\mathbf{1 1}\{94\}$ & $\mathbf{8}\{36,94\}$ & $\mathrm{CN}(\mathrm{c} 1 \mathrm{nnnn} 1 \mathrm{CC} 1 \mathrm{CCC} 1) \mathrm{c} 1 \mathrm{c}(\mathrm{C})$ noc $1 \mathrm{C}$ & 15 \\
\hline 237 & $\mathbf{1 0}\{37\}$ & $\mathbf{1 1}\{166\}$ & $\mathbf{8}\{37,166\}$ & OC1CCOC2 $(\mathrm{CCN}(\mathrm{CC} 2) \mathrm{c} 2 \mathrm{nnnn} 2 \mathrm{CCc} 2 \mathrm{cccnc} 2) \mathrm{C} 1$ & 0 \\
\hline 238 & $\mathbf{1 0}\{37\}$ & $\mathbf{1 1}\{59\}$ & $\mathbf{8}\{37,59\}$ & Oc1cce $2 \mathrm{CN}(\mathrm{Cc} 2 \mathrm{c} 1) \mathrm{c} 1 \mathrm{nnnn} 1 \mathrm{CCc} 1 \mathrm{ccc} n \mathrm{1}$ & 0 \\
\hline 239 & $\mathbf{1 0}\{37\}$ & $\mathbf{1 1}\{167\}$ & $\mathbf{8}\{37,167\}$ & $\mathrm{C}(\mathrm{Cn} 1 \mathrm{nnnn} 1 \mathrm{~N} 1 \mathrm{CCCC} 1 \mathrm{c} 1 \mathrm{nccc} 1) \mathrm{c} 1 \mathrm{cccnc} 1$ & 0 \\
\hline 240 & $\mathbf{1 0}\{37\}$ & $\mathbf{1 1}\{168\}$ & $\mathbf{8}\{37,168\}$ & $\mathrm{C}(\mathrm{Cn} 1 \mathrm{nnn} \mathrm{1} 1 \mathrm{~N} 1 \mathrm{CCOC}(\mathrm{C} 1) \mathrm{c} 1 \mathrm{ccco} 1) \mathrm{c} 1 \mathrm{cccnc} 1$ & 0 \\
\hline 241 & $\mathbf{1 0}\{37\}$ & $\mathbf{1 1}\{169\}$ & $\mathbf{8}\{37,169\}$ & $\mathrm{C}(\mathrm{Cn} 1 \mathrm{nnn} \mathrm{n} 1 \mathrm{~N} 1 \mathrm{CCC} 2(\mathrm{CCC} 2) \mathrm{C} 1) \mathrm{c} 1 \mathrm{ccc} n \mathrm{c} 1$ & 7 \\
\hline 242 & $\mathbf{1 0}\{37\}$ & $11\{64\}$ & $\mathbf{8}\{37,64\}$ & $\mathrm{FC} 1(\mathrm{~F}) \mathrm{COCCN}(\mathrm{C} 1) \mathrm{c} 1 \mathrm{nnnn} 1 \mathrm{CCc} 1 \mathrm{cccnc} 1$ & 0 \\
\hline 243 & $\mathbf{1 0}\{37\}$ & $\mathbf{1 1}\{104\}$ & $\mathbf{8}\{37,104\}$ & $\mathrm{C}(\mathrm{Cn} 1 \mathrm{nnnn} 1 \mathrm{~N} 1 \mathrm{CCOCC} 2(\mathrm{CCCC} 2) \mathrm{C} 1) \mathrm{c} 1 \mathrm{ccc} n \mathrm{c} 1$ & 0 \\
\hline 244 & $\mathbf{1 0}\{37\}$ & $\mathbf{1 1}\{170\}$ & $\mathbf{8}\{37,170\}$ & $\mathrm{C}(\mathrm{Cn} 1 \mathrm{nnn} \mathrm{n} 1 \mathrm{~N} 1 \mathrm{CCCC}(\mathrm{C} 1) \mathrm{C} 1 \mathrm{CCOCC} 1) \mathrm{c} 1 \mathrm{cccnc} 1$ & 0 \\
\hline 245 & $\mathbf{1 0}\{37\}$ & $\mathbf{1 1}\{171\}$ & $\mathbf{8}\{37,171\}$ & $\mathrm{C}(\mathrm{Cn} 1 \mathrm{nnn} \mathrm{n} 1 \mathrm{~N} 1 \mathrm{CCC}(\mathrm{C} 1) \mathrm{n} 1 \mathrm{cccn} 1) \mathrm{c} 1 \mathrm{ccc} \mathrm{c} 1$ & 0 \\
\hline 246 & $\mathbf{1 0}\{37\}$ & $\mathbf{1 1}\{135\}$ & $\mathbf{8}\{37,135\}$ & $\mathrm{FC}(\mathrm{F})(\mathrm{F})[\mathrm{C} @ @ \mathrm{H}] 1 \mathrm{CCCN}(\mathrm{C} 1) \mathrm{c} 1 \mathrm{nnnn} 1 \mathrm{CCc} 1 \mathrm{ccc} c 1$ & 0 \\
\hline 247 & $\mathbf{1 0}\{37\}$ & $\mathbf{1 1}\{172\}$ & $\mathbf{8}\{37,172\}$ & COC1CCCN(CC1)c1nnnn1CCc1ccenc1 & 0 \\
\hline 248 & $\mathbf{1 0}\{37\}$ & $\mathbf{1 1}\{109\}$ & $\mathbf{8}\{37,109\}$ & $\mathrm{Cn} 1 \mathrm{nc} 2 \mathrm{CCN}(\mathrm{Cc} 2 \mathrm{cc} 1=\mathrm{O}) \mathrm{c} 1 \mathrm{nnnn} 1 \mathrm{CC} 1 \mathrm{cccnc} 1$ & 0 \\
\hline
\end{tabular}




\begin{tabular}{|c|c|c|c|c|c|}
\hline 249 & $\mathbf{1 0}\{37\}$ & $\mathbf{1 1}\{173\}$ & $\mathbf{8}\{37,173\}$ & $\mathrm{O}=\mathrm{C} 1 \mathrm{CN}(\mathrm{CCCN} 1) \mathrm{c} 1 \mathrm{nnnn} 1 \mathrm{CCc} 1 \mathrm{ccc} n \mathrm{c} 1$ & 0 \\
\hline 250 & $\mathbf{1 0}\{37\}$ & $\mathbf{1 1}\{174\}$ & $\mathbf{8}\{37,174\}$ & FC(F)[C@@ $@$ ]1CCCN1c1nnnn1CCc1cccnc1 & 0 \\
\hline 251 & $\mathbf{1 0}\{37\}$ & $\mathbf{1 1}\{175\}$ & $\mathbf{8}\{37,175\}$ & $\mathrm{Cn} 1 \mathrm{ccc}(\mathrm{n} 1) \mathrm{C} 1 \mathrm{CCCN}(\mathrm{C} 1) \mathrm{c} 1 \mathrm{nnnn} 1 \mathrm{CCc} 1 \mathrm{ccc} n \mathrm{c} 1$ & 0 \\
\hline 252 & $\mathbf{1 0}\{38\}$ & $\mathbf{1 1}\{176\}$ & $\mathbf{8}\{38,176\}$ & $\mathrm{CC}(\mathrm{c} 1 \mathrm{cccs} 1) \mathrm{n} 1 \mathrm{nnn} c 1 \mathrm{~N}(\mathrm{C}) \mathrm{Cc} 1 \mathrm{cnc}(\mathrm{C}) \mathrm{s} 1$ & 0 \\
\hline 253 & $\mathbf{1 0}\{38\}$ & $\mathbf{1 1}\{177\}$ & $\mathbf{8}\{38,177\}$ & $\mathrm{CC}(\mathrm{C}) \mathrm{C}(=\mathrm{O}) \mathrm{N} 1 \mathrm{CCCN}(\mathrm{CC} 1) \mathrm{c} 1 \mathrm{nnnn} 1 \mathrm{C}(\mathrm{C}) \mathrm{c} 1 \mathrm{cccs} 1$ & 0 \\
\hline 254 & $\mathbf{1 0}\{38\}$ & $\mathbf{1 1}\{178\}$ & $\mathbf{8}\{38,178\}$ & $\mathrm{CC}(\mathrm{c} 1 \mathrm{cccs} 1) \mathrm{n} 1 \mathrm{nnn} \operatorname{1N}(\mathrm{C}) \mathrm{Cc} 1 \mathrm{ccoc} 1$ & 0 \\
\hline 255 & $\mathbf{1 0}\{38\}$ & $\mathbf{1 1}\{142\}$ & $\mathbf{8}\{38,142\}$ & $\mathrm{CC}(\mathrm{c} 1 \mathrm{cccs} 1) \mathrm{n} 1 \mathrm{nnn} \mathrm{c} 1 \mathrm{~N} 1 \mathrm{CCC}(\mathrm{CC} 1) \mathrm{c} 1 \mathrm{cn}[\mathrm{nH}] \mathrm{c} 1$ & 0 \\
\hline 256 & $\mathbf{1 0}\{38\}$ & $\mathbf{1 1}\{179\}$ & $\mathbf{8}\{38,179\}$ & $\mathrm{CC}(\mathrm{c} 1 \mathrm{cccs} 1) n 1 \mathrm{nnnc1N} 1 \mathrm{C}[\mathrm{C} @ \mathrm{H}] 2[\mathrm{C} @ \mathrm{H}](\mathrm{CO})[\mathrm{C} @ \mathrm{H}] 2 \mathrm{C} 1$ & 0 \\
\hline 257 & $\mathbf{1 0}\{38\}$ & $\mathbf{1 1}\{84\}$ & $\mathbf{8}\{38,84\}$ & $\mathrm{CC}(\mathrm{c} 1 \mathrm{cccs} 1) \mathrm{n} 1 \mathrm{nnn} \operatorname{c} 1 \mathrm{~N}(\mathrm{C}) \mathrm{CC}(=\mathrm{O}) \mathrm{N} 1 \mathrm{CCCC} 1$ & 0 \\
\hline 258 & $\mathbf{1 0}\{39\}$ & $11\{7\}$ & $\mathbf{8}\{39,7\}$ & $\mathrm{CC} 1=\mathrm{C}(\mathrm{C}) \mathrm{CN}(\mathrm{CC} 1) \mathrm{c} 1 \mathrm{nnnn} 1 \mathrm{CCS}(\mathrm{C})(=\mathrm{O})=\mathrm{O}$ & 0 \\
\hline 259 & $\mathbf{1 0}\{39\}$ & $\mathbf{1 1}\{180\}$ & $\mathbf{8}\{39,180\}$ & $\mathrm{CC}(\mathrm{N}(\mathrm{C}) \mathrm{c} 1 \mathrm{nnnn} 1 \mathrm{CCS}(\mathrm{C})(=\mathrm{O})=\mathrm{O}) \mathrm{c} 1 \operatorname{ccc}(\mathrm{Cl}) \mathrm{c} 1$ & 0 \\
\hline 260 & $\mathbf{1 0}\{39\}$ & $\mathbf{1 1}\{181\}$ & $\mathbf{8}\{39,181\}$ & $\mathrm{CN}(\mathrm{c} 1 \mathrm{nnnn} 1 \mathrm{CCS}(\mathrm{C})(=\mathrm{O})=\mathrm{O}) \mathrm{c} 1 \mathrm{cccc}(\mathrm{C}) \mathrm{c} 1$ & 0 \\
\hline 261 & $\mathbf{1 0}\{39\}$ & $\mathbf{1 1}\{182\}$ & $\mathbf{8}\{39,182\}$ & $\mathrm{CN}(\mathrm{Cc} 1 \mathrm{ccc}(\mathrm{C}) \mathrm{c}(\mathrm{C}) \mathrm{c} 1) \mathrm{c} 1 \mathrm{nnnn} 1 \mathrm{CCS}(\mathrm{C})(=\mathrm{O})=\mathrm{O}$ & 0 \\
\hline 262 & $\mathbf{1 0}\{39\}$ & $\mathbf{1 1}\{183\}$ & $\mathbf{8}\{39,183\}$ & $\mathrm{CC} 1(\mathrm{Cc} 2 \operatorname{cccc} 2) \mathrm{CCCN} 1 \mathrm{c} 1 \mathrm{nnnn} 1 \mathrm{CCS}(\mathrm{C})(=\mathrm{O})=\mathrm{O}$ & 0 \\
\hline 263 & $\mathbf{1 0}\{39\}$ & $11\{6\}$ & $\mathbf{8}\{39,6\}$ & $\mathrm{CS}(=\mathrm{O})(=\mathrm{O}) \mathrm{CCn} 1 \mathrm{nnn} \operatorname{c} 1 \mathrm{~N} 1 \mathrm{CCOc} 2 \mathrm{cc}(\mathrm{F}) \operatorname{ccc} 2 \mathrm{C} 1$ & 0 \\
\hline 264 & $\mathbf{1 0}\{40\}$ & $\mathbf{1 1}\{184\}$ & $\mathbf{8}\{40,184\}$ & $\mathrm{COC} 1(\mathrm{CCCN}(\mathrm{C} 1) \mathrm{c} 1 \mathrm{nnnn} 1 \mathrm{C} 1 \mathrm{CCOCC} 1) \mathrm{C}(\mathrm{F})(\mathrm{F}) \mathrm{F}$ & 0 \\
\hline 265 & $\mathbf{1 0}\{40\}$ & $\mathbf{1 1}\{185\}$ & $\mathbf{8}\{40,185\}$ & $\mathrm{CC} 1(\mathrm{C}) \mathrm{CN}(\mathrm{C} 1 \mathrm{c} 1 \mathrm{ccc} n \mathrm{c} 1) \mathrm{c} 1 \mathrm{nnnn} 1 \mathrm{C} 1 \mathrm{CCOCC} 1$ & 0 \\
\hline 266 & $\mathbf{1 0}\{40\}$ & $\mathbf{1 1}\{186\}$ & $\mathbf{8}\{40,186\}$ & $\mathrm{CCn} 1 \mathrm{ccc}(\mathrm{CN}(\mathrm{C}) \mathrm{c} 2 \mathrm{nnnn} 2 \mathrm{C} 2 \mathrm{CCOCC} 2) \mathrm{c} 1$ & 0 \\
\hline 267 & $\mathbf{1 0}\{40\}$ & $\mathbf{1 1}\{187\}$ & $\mathbf{8}\{40,187\}$ & OC1(CCN(C1)c1nnnn1C1CCOCC1)C1CC1 & 32 \\
\hline 268 & $\mathbf{1 0}\{40\}$ & $\mathbf{1 1}\{188\}$ & $\mathbf{8}\{40,188\}$ & $\mathrm{CCOC} 1 \mathrm{CC} 2(\mathrm{C} 1) \mathrm{CCN}(\mathrm{C} 2) \mathrm{c} 1 \mathrm{nnnn} 1 \mathrm{C} 1 \mathrm{CCOCC} 1$ & 112 \\
\hline 269 & $\mathbf{1 0}\{40\}$ & $\mathbf{1 1}\{189\}$ & $\mathbf{8}\{40,189\}$ & $\mathrm{Cc} 1 \mathrm{ccc}(\mathrm{C} 2 \mathrm{CCCN} 2 \mathrm{c} 2 \mathrm{nnnn} 2 \mathrm{C} 2 \mathrm{CCOCC} 2) \mathrm{c} 1 \mathrm{C}$ & 66 \\
\hline
\end{tabular}




\begin{tabular}{|c|c|c|c|c|c|}
\hline 270 & $\mathbf{1 0}\{40\}$ & $\mathbf{1 1}\{115\}$ & $\mathbf{8}\{40,115\}$ & $\mathrm{O}=\mathrm{C} 1 \mathrm{Nc} 2 \operatorname{ccccc} 2 \mathrm{C} 11 \mathrm{CCN}(\mathrm{C} 1) \mathrm{c} 1 \mathrm{nnnn} 1 \mathrm{C} 1 \mathrm{CCOCC} 1$ & 27 \\
\hline 271 & $\mathbf{1 0}\{40\}$ & $\mathbf{1 1}\{190\}$ & $\mathbf{8}\{40,190\}$ & $\mathrm{CC} 1(\mathrm{C}) \mathrm{CN}(\mathrm{Cc} 2 \operatorname{ccccc} 2 \mathrm{O} 1) \mathrm{c} 1 \mathrm{nnnn} 1 \mathrm{C} 1 \mathrm{CCOCC} 1$ & 41 \\
\hline 272 & $\mathbf{1 0}\{40\}$ & $\mathbf{1 1}\{191\}$ & $\mathbf{8}\{40,191\}$ & $\mathrm{CC} 1(\mathrm{C}) \mathrm{CN}(\mathrm{CC}(\mathrm{O} 1) \mathrm{C}(\mathrm{F})(\mathrm{F}) \mathrm{F}) \mathrm{c} 1 \mathrm{nnnn} 1 \mathrm{C} 1 \mathrm{CCOCC} 1$ & 35 \\
\hline 273 & $\mathbf{1 0}\{40\}$ & $\mathbf{1 1}\{192\}$ & $\mathbf{8}\{40,192\}$ & $\mathrm{CN}(\mathrm{Cc} 1 \operatorname{ccc}(\mathrm{C}) \mathrm{n} 1) \mathrm{c} 1 \mathrm{nnnn} 1 \mathrm{C} 1 \mathrm{CCOCC} 1$ & 84 \\
\hline 274 & $\mathbf{1 0}\{40\}$ & $\mathbf{1 1}\{193\}$ & $\mathbf{8}\{40,193\}$ & COc1cecc $1 \mathrm{C} 1 \mathrm{CCN}(\mathrm{C} 1) \mathrm{c} 1 \mathrm{nnnn} 1 \mathrm{C} 1 \mathrm{CCOCC} 1$ & 45 \\
\hline 275 & $\mathbf{1 0}\{40\}$ & $\mathbf{1 1}\{194\}$ & $\mathbf{8}\{40,194\}$ & $\mathrm{O}=\mathrm{S} 1(=\mathrm{O}) \mathrm{CCN}(\mathrm{Cc} 2 \mathrm{ccccc} 12) \mathrm{c} 1 \mathrm{nnnn} 1 \mathrm{C} 1 \mathrm{CCOCC} 1$ & 113 \\
\hline 276 & $\mathbf{1 0}\{40\}$ & $\mathbf{1 1}\{195\}$ & $\mathbf{8}\{40,195\}$ & $\operatorname{CCS}(=\mathrm{O})(=\mathrm{O}) \mathrm{C} 1 \mathrm{CN}(\mathrm{C} 1) \mathrm{c} 1 \mathrm{nnnn} 1 \mathrm{C} 1 \mathrm{CCOCC} 1$ & 18 \\
\hline 277 & $\mathbf{1 0}\{40\}$ & $\mathbf{1 1}\{196\}$ & $\mathbf{8}\{40,196\}$ & $\mathrm{CC}(\mathrm{C}) \mathrm{Oc} 1 \mathrm{ccc}(\mathrm{cc} 1) \mathrm{N}(\mathrm{C}) \mathrm{c} 1 \mathrm{nnnn} 1 \mathrm{C} 1 \mathrm{CCOCC} 1$ & 0 \\
\hline 278 & $\mathbf{1 0}\{40\}$ & $\mathbf{1 1}\{197\}$ & $\mathbf{8}\{40,197\}$ & $\mathrm{CC} 1 \mathrm{CN}(\mathrm{c} 2 \mathrm{nnnn} 2 \mathrm{C} 2 \mathrm{CCOCC} 2) \mathrm{C}(\mathrm{C})(\mathrm{C}) \mathrm{C} 1$ & 0 \\
\hline 279 & $\mathbf{1 0}\{40\}$ & $\mathbf{1 1}\{198\}$ & $\mathbf{8}\{40,198\}$ & $\mathrm{CC}(\mathrm{C}) \mathrm{c} 1 \mathrm{cc}([\mathrm{nH}] \mathrm{n} 1) \mathrm{C} 1 \mathrm{CCCN}(\mathrm{C} 1) \mathrm{c} 1 \mathrm{nnnn} 1 \mathrm{C} 1 \mathrm{CCOCC} 1$ & 0 \\
\hline 280 & $\mathbf{1 0}\{40\}$ & $\mathbf{1 1}\{199\}$ & $\mathbf{8}\{40,199\}$ & $\mathrm{CN}(\mathrm{Cc} 1 \mathrm{nn} \mathrm{2} 2 \mathrm{CC} \mathrm{C} 12) \mathrm{c} 1 \mathrm{nnnn} 1 \mathrm{C} 1 \mathrm{CCOCC} 1$ & 0 \\
\hline 281 & $\mathbf{1 0}\{40\}$ & $\mathbf{1 1}\{143\}$ & $\mathbf{8}\{40,143\}$ & $\mathrm{CN}(\mathrm{c} 1 \mathrm{ccn}(\mathrm{C}) \mathrm{n} 1) \mathrm{c} 1 \mathrm{nnnn} 1 \mathrm{C} 1 \mathrm{CCOCC} 1$ & 0 \\
\hline 282 & $\mathbf{1 0}\{40\}$ & $\mathbf{1 1}\{200\}$ & $\mathbf{8}\{40,200\}$ & $\mathrm{CN}(\mathrm{Cc} 1 \mathrm{cc} 2 \operatorname{cccc} 2[\mathrm{nH}] 1) \mathrm{c} 1 \mathrm{nnnn} 1 \mathrm{C} 1 \mathrm{CCOCC} 1$ & 0 \\
\hline 283 & $\mathbf{1 0}\{40\}$ & $\mathbf{1 1}\{201\}$ & $\mathbf{8}\{40,201\}$ & $\mathrm{CC} 1 \mathrm{CN}(\mathrm{c} 2 \mathrm{nnnn} 2 \mathrm{C} 2 \mathrm{CCOCC} 2) \mathrm{c} 2 \mathrm{ccccc} 2 \mathrm{O} 1$ & 0 \\
\hline 284 & $\mathbf{1 0}\{40\}$ & $\mathbf{1 1}\{202\}$ & $\mathbf{8}\{40,202\}$ & $\mathrm{C} 1 \mathrm{CC} 1 \mathrm{c} 1 \mathrm{nc}(\mathrm{n}[\mathrm{nH}] 1) \mathrm{C} 1 \mathrm{CCCN}(\mathrm{C} 1) \mathrm{c} 1 \mathrm{nnnn} 1 \mathrm{C} 1 \mathrm{CCOCC} 1$ & 0 \\
\hline 285 & $\mathbf{1 0}\{40\}$ & $\mathbf{1 1}\{203\}$ & $\mathbf{8}\{40,203\}$ & $\mathrm{Cc} 1 \mathrm{nc}([\mathrm{nH}] \mathrm{c} 1 \mathrm{C}) \mathrm{C} 1 \mathrm{CCN}(\mathrm{CC} 1) \mathrm{c} 1 \mathrm{nnnn} 1 \mathrm{C} 1 \mathrm{CCOCC} 1$ & 0 \\
\hline 286 & $\mathbf{1 0}\{40\}$ & $\mathbf{1 1}\{204\}$ & $\mathbf{8}\{40,204\}$ & $\mathrm{CN}(\mathrm{CC} 1(\mathrm{O}) \mathrm{CCC} 1) \mathrm{c} 1 \mathrm{nnnn} 1 \mathrm{C} 1 \mathrm{CCOCC} 1$ & 0 \\
\hline 287 & $\mathbf{1 0}\{41\}$ & $\mathbf{1 1}\{205\}$ & $\mathbf{8}\{41,205\}$ & $\mathrm{OC} 1 \mathrm{CCN}(\mathrm{CC} 1(\mathrm{~F}) \mathrm{F}) \mathrm{c} 1 \mathrm{nnnn} 1 \mathrm{CCc} 1 \mathrm{cccs} 1$ & 0 \\
\hline 288 & $\mathbf{1 0}\{41\}$ & $\mathbf{1 1}\{206\}$ & $\mathbf{8}\{41,206\}$ & $\mathrm{CC} 1(\mathrm{C}) \mathrm{CN}(\mathrm{C} 1 \mathrm{c} 1 \mathrm{ccncc} 1) \mathrm{c} 1 \mathrm{nnnn} 1 \mathrm{CCc} 1 \mathrm{cccs} 1$ & 0 \\
\hline 289 & $\mathbf{1 0}\{41\}$ & $\mathbf{1 1}\{207\}$ & $\mathbf{8}\{41,207\}$ & $\mathrm{C}(\mathrm{Cn} 1 \mathrm{nnn} \mathrm{n} 1 \mathrm{~N} 1 \mathrm{CCC}(\mathrm{CC} 1) \mathrm{n} 1 \mathrm{ccc} 1) \mathrm{c} 1 \mathrm{cccs} 1$ & 0 \\
\hline 290 & $\mathbf{1 0}\{41\}$ & $\mathbf{1 1}\{208\}$ & $\mathbf{8}\{41,208\}$ & $\mathrm{C}(\mathrm{Cn} 1 \mathrm{nnn} \mathrm{n} 1 \mathrm{~N} 1 \mathrm{CCN}(\mathrm{CC} 1) \mathrm{C} 1 \mathrm{CCOCC} 1) \mathrm{c} 1 \mathrm{cccs} 1$ & 36 \\
\hline
\end{tabular}




\begin{tabular}{|c|c|c|c|c|c|}
\hline 291 & $\mathbf{1 0}\{41\}$ & $\mathbf{1 1}\{147\}$ & $\mathbf{8}\{41,147\}$ & Clc1cnn(c1)C1CCN(C1)c1nnnn1CCc1 cccs1 & 70 \\
\hline 292 & $\mathbf{1 0}\{41\}$ & $\mathbf{1 1}\{148\}$ & $\mathbf{8}\{41,148\}$ & $\mathrm{Cc} 1 \mathrm{csc}(\mathrm{n} 1) \mathrm{C} 1 \mathrm{CCN}(\mathrm{C} 1) \mathrm{c} 1 \mathrm{nnnn} 1 \mathrm{CCc} 1 \mathrm{cccs} 1$ & 62 \\
\hline 293 & $\mathbf{1 0}\{41\}$ & $\mathbf{1 1}\{209\}$ & $\mathbf{8}\{41,209\}$ & $\mathrm{CO}[1 \mathrm{C} @ @ \mathrm{H}] 1 \mathrm{CN}(\mathrm{C}[1 \mathrm{C} @ \mathrm{H}] 1 \mathrm{O}) \mathrm{c} 1 \mathrm{nnnn} 1 \mathrm{CCc} 1 \mathrm{cccs} 1$ & 48 \\
\hline 294 & $\mathbf{1 0}\{41\}$ & $\mathbf{1 1}\{210\}$ & $\mathbf{8}\{41,210\}$ & Oc1ccc $2 \mathrm{CN}(\mathrm{CCc} 12) \mathrm{c} 1 \mathrm{nnnn} 1 \mathrm{CCc} 1 \mathrm{cccs} 1$ & 71 \\
\hline 295 & $\mathbf{1 0}\{41\}$ & $\mathbf{1 1}\{211\}$ & $\mathbf{8}\{41,211\}$ & $\mathrm{C}(\mathrm{Cn} 1 \mathrm{nnnn} 1 \mathrm{~N} 1 \mathrm{CCC}(\mathrm{CC} 1) \mathrm{c} 1 \mathrm{ccco} 1) \mathrm{c} 1 \mathrm{cccs} 1$ & 40 \\
\hline 296 & $\mathbf{1 0}\{41\}$ & $\mathbf{1 1}\{212\}$ & $\mathbf{8}\{41,212\}$ & $\mathrm{FC}(\mathrm{F})(\mathrm{F}) \mathrm{C} 1 \mathrm{CCN}(\mathrm{CCO} 1) \mathrm{c} 1 \mathrm{nnnn} 1 \mathrm{CCc} 1 \mathrm{cccs} 1$ & 41 \\
\hline 297 & $\mathbf{1 0}\{41\}$ & $\mathbf{1 1}\{213\}$ & $\mathbf{8}\{41,213\}$ & $\mathrm{CNC}(=\mathrm{O}) \mathrm{NC} 1 \mathrm{CCN}(\mathrm{CC} 1) \mathrm{c} 1 \mathrm{nnnn} 1 \mathrm{CCc} 1 \mathrm{cccs} 1$ & 46 \\
\hline 298 & $\mathbf{1 0}\{41\}$ & $\mathbf{1 1}\{214\}$ & $\mathbf{8}\{41,214\}$ & $\mathrm{C}(\mathrm{Cn} 1 \mathrm{nnn} \mathrm{n} 1 \mathrm{~N} 1 \mathrm{CCC}(\mathrm{CC} 1) \mathrm{C} 1 \mathrm{CCCCO} 1) \mathrm{c} 1 \mathrm{cccs} 1$ & 54 \\
\hline 299 & $\mathbf{1 0}\{41\}$ & $\mathbf{1 1}\{215\}$ & $\mathbf{8}\{41,215\}$ & $\operatorname{CCS}(=\mathrm{O})(=\mathrm{O}) \mathrm{CC}(\mathrm{C}) \mathrm{N}(\mathrm{C}) \mathrm{c} 1 \mathrm{nnnn} 1 \mathrm{CCc} 1 \mathrm{cccs} 1$ & 14 \\
\hline 300 & $\mathbf{1 0}\{41\}$ & $\mathbf{1 1}\{216\}$ & $\mathbf{8}\{41,216\}$ & $\mathrm{CN}([1 \mathrm{C} @ @ \mathrm{H}] 1 \mathrm{CCC}[1 \mathrm{C} @ \mathrm{H}] 1 \mathrm{O}) \mathrm{c} 1 \mathrm{nnnn} 1 \mathrm{CCc} 1 \mathrm{cccs} 1$ & 18 \\
\hline 301 & $\mathbf{1 0}\{41\}$ & $\mathbf{1 1}\{217\}$ & $\mathbf{8}\{41,217\}$ & Oc1 $1 \mathrm{cc} 2 \mathrm{CN}(\mathrm{CCOc} 2 \mathrm{c} 1) \mathrm{c} 1 \mathrm{nnnn} 1 \mathrm{CCc} 1 \mathrm{cccs} 1$ & 37 \\
\hline 302 & $\mathbf{1 0}\{41\}$ & $\mathbf{1 1}\{218\}$ & $\mathbf{8}\{41,218\}$ & Cc1cncc(c1)C1CCCN1c1nnnn1CCc1cces1 & 0 \\
\hline 303 & $\mathbf{1 0}\{41\}$ & $\mathbf{1 1}\{16\}$ & $\mathbf{8}\{41,16\}$ & $\mathrm{O}=\mathrm{C} 1 \mathrm{NCCN} 1 \mathrm{C} 1 \mathrm{CCCN}(\mathrm{C} 1) \mathrm{c} 1 \mathrm{nnnn} 1 \mathrm{CCc} 1 \mathrm{cccs} 1$ & 0 \\
\hline 304 & $\mathbf{1 0}\{41\}$ & $\mathbf{1 1}\{219\}$ & $\mathbf{8}\{41,219\}$ & $\mathrm{C}(\mathrm{Cn} 1 \mathrm{nnn} n \mathrm{~N} 1 \mathrm{CCN}(\mathrm{CC} 1) \mathrm{c} 1 \mathrm{cccs} 1) \mathrm{c} 1 \mathrm{cccs} 1$ & 0 \\
\hline 305 & $\mathbf{1 0}\{41\}$ & $\mathbf{1 1}\{146\}$ & $\mathbf{8}\{41,146\}$ & $\mathrm{C}(\mathrm{Cn} 1 \mathrm{nnnn} 1 \mathrm{~N} 1 \mathrm{CCCC} 1 \mathrm{c} 1 \mathrm{ccn}[\mathrm{nH}] 1) \mathrm{c} 1 \mathrm{cccs} 1$ & 0 \\
\hline 306 & $\mathbf{1 0}\{41\}$ & $\mathbf{1 1}\{220\}$ & $\mathbf{8}\{41,220\}$ & $\operatorname{CC} 1 \mathrm{CCS}(=\mathrm{O})(=\mathrm{O}) \mathrm{CCN} 1 \mathrm{c} 1 \mathrm{nnnn} 1 \mathrm{CCc} 1 \mathrm{cccs} 1$ & 0 \\
\hline 307 & $\mathbf{1 0}\{41\}$ & $11\{68\}$ & $\mathbf{8}\{41,68\}$ & $\mathrm{C}(\mathrm{Cn} 1 \mathrm{nnnnc} 1 \mathrm{~N} 1 \mathrm{CCCC}(\mathrm{C} 1) \mathrm{c} 1 \mathrm{ccn}[\mathrm{nH}] 1) \mathrm{c} 1 \mathrm{cccs} 1$ & 0 \\
\hline 308 & $\mathbf{1 0}\{41\}$ & $\mathbf{1 1}\{221\}$ & $\mathbf{8}\{41,221\}$ & $\mathrm{C}(\mathrm{Cn} 1 \mathrm{nnn} \mathrm{c} 1 \mathrm{~N} 1 \mathrm{CCCC}(\mathrm{C} 1) \mathrm{c} 1 \mathrm{ccc} n \mathrm{c} 1) \mathrm{c} 1 \mathrm{cccs} 1$ & 0 \\
\hline 309 & $\mathbf{1 0}\{41\}$ & $\mathbf{1 1}\{222\}$ & $\mathbf{8}\{41,222\}$ & $\mathrm{C}(\mathrm{Cn} 1 \mathrm{nnn} \operatorname{c} 1 \mathrm{~N} 1 \mathrm{CCCC}(\mathrm{C} 1) \mathrm{c} 1 \mathrm{ccncc} 1) \mathrm{c} 1 \mathrm{cccs} 1$ & 0 \\
\hline 310 & $\mathbf{1 0}\{42\}$ & $\mathbf{1 1}\{223\}$ & $\mathbf{8}\{42,223\}$ & $\mathrm{CN}(\mathrm{Cc} 1 \mathrm{nnc}(\mathrm{C}) \mathrm{s} 1) \mathrm{c} 1 \mathrm{nnnn} 1 \mathrm{C} 1(\mathrm{C}) \mathrm{CC} 1$ & 0 \\
\hline 311 & $\mathbf{1 0}\{42\}$ & $\mathbf{1 1}\{224\}$ & $\mathbf{8}\{42,224\}$ & $\mathrm{CNC}(=\mathrm{O}) \mathrm{C}(\mathrm{C}) \mathrm{CN}(\mathrm{C}) \mathrm{c} 1 \mathrm{nnnn} 1 \mathrm{C} 1(\mathrm{C}) \mathrm{CC} 1$ & 0 \\
\hline
\end{tabular}




\begin{tabular}{|c|c|c|c|c|c|}
\hline 312 & $\mathbf{1 0}\{42\}$ & $\mathbf{1 1}\{225\}$ & $\mathbf{8}\{42,225\}$ & $\mathrm{CC} 1(\mathrm{CC} 1) \mathrm{n} 1 \mathrm{nnn} n \mathrm{~N} 1 \mathrm{CCN}(\mathrm{CC} 1) \mathrm{S}(=\mathrm{O})(=\mathrm{O}) \mathrm{C} 1 \mathrm{CC} 1$ & 10 \\
\hline 313 & $\mathbf{1 0}\{42\}$ & $\mathbf{1 1}\{226\}$ & $\mathbf{8}\{42,226\}$ & $\mathrm{CC} 1(\mathrm{CC} 1) \mathrm{n} 1 \mathrm{nnnc} 1 \mathrm{~N} 1 \mathrm{CCC}(\mathrm{CC} 1) \mathrm{C}(=\mathrm{O}) \mathrm{NCCO}$ & 41 \\
\hline 314 & $\mathbf{1 0}\{42\}$ & $\mathbf{1 1}\{227\}$ & $\mathbf{8}\{42,227\}$ & $\mathrm{COc} 1 \mathrm{ccc}(\mathrm{cc} 1 \mathrm{~F}) \mathrm{C} 1 \mathrm{CCN}(\mathrm{C} 1) \mathrm{c} 1 \mathrm{nnnn} 1 \mathrm{C} 1(\mathrm{C}) \mathrm{CC} 1$ & 93 \\
\hline 315 & $\mathbf{1 0}\{42\}$ & $\mathbf{1 1}\{228\}$ & $\mathbf{8}\{42,228\}$ & $\mathrm{CC}(\mathrm{C})(\mathrm{O}) \mathrm{CN}(\mathrm{C} 1 \mathrm{CC} 1) \mathrm{c} 1 \mathrm{nnnn} 1 \mathrm{C} 1(\mathrm{C}) \mathrm{CC} 1$ & 4 \\
\hline 316 & $\mathbf{1 0}\{42\}$ & $\mathbf{1 1}\{229\}$ & $\mathbf{8}\{42,229\}$ & $\mathrm{CC} 1(\mathrm{CC} 1) \mathrm{n} 1 \mathrm{nnnnc} 1 \mathrm{~N} 1 \mathrm{CCC}(\mathrm{CO})(\mathrm{C} 1) \mathrm{c} 1 \mathrm{ccccc} 1$ & 6 \\
\hline 317 & $\mathbf{1 0}\{42\}$ & $\mathbf{1 1}\{230\}$ & $\mathbf{8}\{42,230\}$ & $\mathrm{CN}(\mathrm{C} 1 \mathrm{CCN}(\mathrm{C} 1) \mathrm{c} 1 \mathrm{nnnn} 1 \mathrm{C} 1(\mathrm{C}) \mathrm{CC} 1) \mathrm{c} 1 \mathrm{ccccc} 1$ & 24 \\
\hline 318 & $\mathbf{1 0}\{42\}$ & $\mathbf{1 1}\{193\}$ & $\mathbf{8}\{42,193\}$ & $\mathrm{COc} 1 \mathrm{ccccc} 1 \mathrm{C} 1 \mathrm{CCN}(\mathrm{C} 1) \mathrm{c} 1 \mathrm{nnnn} 1 \mathrm{C} 1(\mathrm{C}) \mathrm{CC} 1$ & 51 \\
\hline 319 & $\mathbf{1 0}\{42\}$ & $\mathbf{1 1}\{231\}$ & $\mathbf{8}\{42,231\}$ & $\mathrm{CC} 1(\mathrm{CC} 1) \mathrm{n} 1 \mathrm{nnnc} 1 \mathrm{~N} 1 \mathrm{CCC}(\mathrm{CC} 1) \mathrm{OCC}(\mathrm{N})=\mathrm{O}$ & 83 \\
\hline 320 & $\mathbf{1 0}\{42\}$ & $\mathbf{1 1}\{232\}$ & $\mathbf{8}\{42,232\}$ & $\mathrm{CCN}(\mathrm{Cc} 1 \mathrm{loccc} 1 \mathrm{C}) \mathrm{c} 1 \mathrm{nnnn} 1 \mathrm{C} 1(\mathrm{C}) \mathrm{CC} 1$ & 29 \\
\hline 321 & $\mathbf{1 0}\{42\}$ & $\mathbf{1 1}\{233\}$ & $\mathbf{8}\{42,233\}$ & $\mathrm{CC} 1(\mathrm{CC} 1) \mathrm{n} 1 \mathrm{nnnc} 1 \mathrm{~N} 1 \mathrm{CCCC} 1 \mathrm{c} 1 \mathrm{cncc}(\mathrm{Br}) \mathrm{c} 1$ & 6 \\
\hline 322 & $\mathbf{1 0}\{42\}$ & $\mathbf{1 1}\{234\}$ & $\mathbf{8}\{42,234\}$ & $\mathrm{CC} 1(\mathrm{CC} 1) \mathrm{n} 1 \mathrm{nnnc} 1 \mathrm{~N} 1 \mathrm{CCC}(\mathrm{CS}(\mathrm{C})(=\mathrm{O})=\mathrm{O}) \mathrm{CC} 1$ & 75 \\
\hline 323 & $\mathbf{1 0}\{43\}$ & $\mathbf{1 1}\{235\}$ & $\mathbf{8}\{43,235\}$ & $\mathrm{CN}(\mathrm{Cc} 1 \mathrm{cscn} 1) \mathrm{c} 1 \mathrm{nnnn} 1 \mathrm{C} 1 \mathrm{CCOc} 2 \mathrm{cccc} 12$ & 0 \\
\hline 324 & $\mathbf{1 0}\{43\}$ & $\mathbf{1 1}\{178\}$ & $\mathbf{8}\{43,178\}$ & $\mathrm{CN}(\mathrm{Cc} 1 \mathrm{ccoc} 1) \mathrm{c} 1 \mathrm{nnnn} 1 \mathrm{C} 1 \mathrm{CCOc} 2 \mathrm{cccc} 12$ & 0 \\
\hline 325 & $\mathbf{1 0}\{43\}$ & $\mathbf{1 1}\{176\}$ & $\mathbf{8}\{43,176\}$ & $\mathrm{CN}(\mathrm{Cc} 1 \mathrm{cnc}(\mathrm{C}) \mathrm{s} 1) \mathrm{c} 1 \mathrm{nnnn} 1 \mathrm{C} 1 \mathrm{CCOc} 2 \mathrm{cccc} 12$ & 0 \\
\hline 326 & $\mathbf{1 0}\{43\}$ & $11\{236\}$ & $\mathbf{8}\{43,236\}$ & Oc1cce $2 \mathrm{CCN}(\mathrm{Cc} 2 \mathrm{c} 1) \mathrm{c} 1 \mathrm{nnnn} 1 \mathrm{C} 1 \mathrm{CCOc} 2 \mathrm{cccc} 12$ & 25 \\
\hline 327 & $\mathbf{1 0}\{43\}$ & $\mathbf{1 1}\{237\}$ & $\mathbf{8}\{43,237\}$ & $\mathrm{CN}(\mathrm{Cc} 1 \mathrm{loccc} 1 \mathrm{C}) \mathrm{c} 1 \mathrm{nnnn} 1 \mathrm{C} 1 \mathrm{CCOc} 2 \mathrm{cccc} 12$ & 26 \\
\hline 328 & $\mathbf{1 0}\{43\}$ & $\mathbf{1 1}\{99\}$ & $\mathbf{8}\{43,99\}$ & $\mathrm{C} 1 \mathrm{CC}(\mathrm{c} 2 \mathrm{ccccc} 2 \mathrm{O} 1) \mathrm{n} 1 \mathrm{nnn} n 1 \mathrm{~N} 1 \mathrm{CCn} 2 \mathrm{cncc} 2 \mathrm{C} 1$ & 0 \\
\hline 329 & $\mathbf{1 0}\{43\}$ & $\mathbf{1 1}\{94\}$ & $\mathbf{8}\{43,94\}$ & $\mathrm{CN}(\mathrm{c} 1 \mathrm{nnnn} 1 \mathrm{C} 1 \mathrm{CCOc} 2 \mathrm{cccc} 12) \mathrm{c} 1 \mathrm{c}(\mathrm{C}) \operatorname{noc} 1 \mathrm{C}$ & 0 \\
\hline 330 & $\mathbf{1 0}\{44\}$ & $\mathbf{1 1}\{238\}$ & $\mathbf{8}\{44,238\}$ & $\mathrm{Clc} 1 \mathrm{cnn}(\mathrm{c} 1) \mathrm{C} 1 \mathrm{CCN}(\mathrm{CC} 1) \mathrm{c} 1 \mathrm{nnnn} 1 \mathrm{C}(\mathrm{C} 1 \mathrm{CC} 1) \mathrm{C} 1 \mathrm{CC} 1$ & 0 \\
\hline 331 & $\mathbf{1 0}\{44\}$ & $\mathbf{1 1}\{73\}$ & $\mathbf{8}\{44,73\}$ & $\mathrm{C} 1 \mathrm{CC} 1 \mathrm{C}(\mathrm{C} 1 \mathrm{CC} 1) \mathrm{n} 1 \mathrm{nnnc} 1 \mathrm{~N} 1 \mathrm{CCc} 2 \mathrm{cn}[\mathrm{nH}] \mathrm{c} 2 \mathrm{C} 1$ & 0 \\
\hline 332 & $\mathbf{1 0}\{44\}$ & $\mathbf{1 1}\{142\}$ & $\mathbf{8}\{44,142\}$ & $\mathrm{C} 1 \mathrm{CC} 1 \mathrm{C}(\mathrm{C} 1 \mathrm{CC} 1) \mathrm{n} 1 \mathrm{nnnnc} 1 \mathrm{~N} 1 \mathrm{CCC}(\mathrm{CC} 1) \mathrm{c} 1 \mathrm{cn}[\mathrm{nH}] \mathrm{c} 1$ & 0 \\
\hline
\end{tabular}




\begin{tabular}{|c|c|c|c|c|c|}
\hline 333 & $\mathbf{1 0}\{44\}$ & $\mathbf{1 1}\{148\}$ & $\mathbf{8}\{44,148\}$ & $\mathrm{Cc} 1 \mathrm{csc}(\mathrm{n} 1) \mathrm{C} 1 \mathrm{CCN}(\mathrm{C} 1) \mathrm{c} 1 \mathrm{nnnn} 1 \mathrm{C}(\mathrm{C} 1 \mathrm{CC} 1) \mathrm{C} 1 \mathrm{CC} 1$ & 19 \\
\hline 334 & $\mathbf{1 0}\{45\}$ & $\mathbf{1 1}\{239\}$ & $\mathbf{8}\{45,239\}$ & $\mathrm{FC}(\mathrm{F})(\mathrm{F}) \mathrm{Oc} 1 \mathrm{ccc}(\mathrm{Cn} 2 \mathrm{nnn} \mathrm{c} 2 \mathrm{~N} 2 \mathrm{CC} 3 \mathrm{CC} 2 \mathrm{C}=\mathrm{C} 3) \mathrm{cc} 1$ & 0 \\
\hline 335 & $\mathbf{1 0}\{46\}$ & $\mathbf{1 1}\{240\}$ & $\mathbf{8}\{46,240\}$ & $\mathrm{CC}(=\mathrm{O}) \mathrm{NC} 1 \mathrm{CCN}(\mathrm{CC} 1) \mathrm{c} 1 \mathrm{nnnn} 1 \mathrm{C} 1 \mathrm{CCc} 2 \operatorname{ccccc} 2 \mathrm{C} 1$ & 24 \\
\hline 336 & $\mathbf{1 0}\{46\}$ & $\mathbf{1 1}\{84\}$ & $\mathbf{8}\{46,84\}$ & $\mathrm{CN}(\mathrm{CC}(=\mathrm{O}) \mathrm{N} 1 \mathrm{CCCC} 1) \mathrm{c} 1 \mathrm{nnnn} 1 \mathrm{C} 1 \mathrm{CCc} 2 \operatorname{ccccc} 2 \mathrm{C} 1$ & 0 \\
\hline 337 & $\mathbf{1 0}\{46\}$ & $\mathbf{1 1}\{79\}$ & $\mathbf{8}\{46,79\}$ & $\mathrm{NC}(=\mathrm{O})[\mathrm{C} @ \mathrm{H}] 1 \mathrm{CCCN} 1 \mathrm{c} 1 \mathrm{nnnn} 1 \mathrm{C} 1 \mathrm{CCc} 2 \mathrm{ccccc} 2 \mathrm{C} 1$ & 0 \\
\hline 338 & $\mathbf{1 0}\{46\}$ & $\mathbf{1 1}\{40\}$ & $\mathbf{8}\{46,40\}$ & C1Cc2 2 ccce2CC1n1nnnc1N1CCc2[nH]ncc2C1 & 0 \\
\hline 339 & $\mathbf{1 0}\{46\}$ & $\mathbf{1 1}\{241\}$ & $\mathbf{8}\{46,241\}$ & Cc1cnn(c1)C1CCN(C1)c1nnnn1C1CCc2eccec2C1 & 0 \\
\hline 340 & $\mathbf{1 0}\{46\}$ & $\mathbf{1 1}\{116\}$ & $\mathbf{8}\{46,116\}$ & $\begin{array}{l}\text { O[1C@@ } \mathrm{H}] 1 \mathrm{CC}[1 \mathrm{C} @ \mathrm{H}] 2 \mathrm{CN}(\mathrm{C}[1 \mathrm{C} @ @ \mathrm{H}] 2 \mathrm{C} 1) \mathrm{c} 1 n n n n 1 \mathrm{C} 1 \\
\mathrm{CCc} 2 \mathrm{cccc} 2 \mathrm{C} 1\end{array}$ & 0 \\
\hline 341 & $\mathbf{1 0}\{46\}$ & $\mathbf{1 1}\{143\}$ & $\mathbf{8}\{46,143\}$ & $\mathrm{CN}(\mathrm{c} 1 \mathrm{ccn}(\mathrm{C}) \mathrm{n} 1) \mathrm{c} 1 \mathrm{nnnn} 1 \mathrm{C} 1 \mathrm{CCc} 2 \operatorname{ccccc} 2 \mathrm{C} 1$ & 0 \\
\hline 342 & $10\{46\}$ & $\mathbf{1 1}\{81\}$ & $\mathbf{8}\{46,81\}$ & $\mathrm{NC}(=\mathrm{O}) \mathrm{C} 1 \mathrm{CN}(\mathrm{CCO} 1) \mathrm{c} 1 \mathrm{nnnn} 1 \mathrm{C} 1 \mathrm{CCc} 2 \operatorname{cccc} 2 \mathrm{C} 1$ & 0 \\
\hline 343 & $\mathbf{1 0}\{47\}$ & $\mathbf{1 1}\{10\}$ & $\mathbf{8}\{47,10\}$ & $\mathrm{Cn} 1 \operatorname{cccc} 1 \mathrm{C}(=\mathrm{O}) \mathrm{N} 1 \mathrm{CCN}(\mathrm{CC} 1) \mathrm{c} 1 \mathrm{nnnn} 1 \mathrm{CC}(\mathrm{C})(\mathrm{C}) \mathrm{C}$ & 0 \\
\hline 344 & $\mathbf{1 0}\{47\}$ & $\mathbf{1 1}\{12\}$ & $\mathbf{8}\{47,12\}$ & Cn1cnnc1C1CCCN(C1)c1nnnn1CC(C)(C)C & 0 \\
\hline 345 & $\mathbf{1 0}\{48\}$ & $11\{7\}$ & $\mathbf{8}\{48,7\}$ & $\mathrm{CSCCn} 1 \mathrm{nnn} \mathrm{1N} 1 \mathrm{CCC}(\mathrm{C})=\mathrm{C}(\mathrm{C}) \mathrm{C} 1$ & 0 \\
\hline 346 & $\mathbf{1 0}\{48\}$ & $\mathbf{1 1}\{8\}$ & $\mathbf{8}\{48,8\}$ & CSCCn1nnnc1N1CC2(CCOCC2)[1C@@ @ $] 2 C C C[1 \mathrm{C} @ \mathrm{H}] 12$ & 20 \\
\hline 347 & $\mathbf{1 0}\{49\}$ & $\mathbf{1 1}\{81\}$ & $\mathbf{8}\{49,81\}$ & $\mathrm{CC}(\mathrm{Cc} 1 \mathrm{ccccc} 1) \mathrm{n} 1 \mathrm{nnn} \operatorname{c} 1 \mathrm{~N} 1 \mathrm{CCOC}(\mathrm{C} 1) \mathrm{C}(\mathrm{N})=\mathrm{O}$ & 0 \\
\hline 348 & $\mathbf{1 0}\{49\}$ & $\mathbf{1 1}\{143\}$ & $\mathbf{8}\{49,143\}$ & $\mathrm{CC}(\mathrm{Cc} 1 \mathrm{ccccc} 1) \mathrm{n} 1 \mathrm{nnnn} 1 \mathrm{~N}(\mathrm{C}) \mathrm{c} 1 \mathrm{ccn}(\mathrm{C}) \mathrm{n} 1$ & 22 \\
\hline 349 & $\mathbf{1 0}\{49\}$ & $\mathbf{1 1}\{242\}$ & $\mathbf{8}\{49,242\}$ & $\mathrm{CCN}(\mathrm{c} 1 \mathrm{cnn}(\mathrm{C}) \mathrm{c} 1) \mathrm{c} 1 \mathrm{nnnn} 1 \mathrm{C}(\mathrm{C}) \mathrm{Cc} 1 \mathrm{ccccc} 1$ & 12 \\
\hline 350 & $\mathbf{1 0}\{49\}$ & $\mathbf{1 1}\{143\}$ & $\mathbf{8}\{49,143\}$ & $\mathrm{CC}(\mathrm{Cc} 1 \mathrm{ccccc} 1) \mathrm{n} 1 \mathrm{nnnc} 1 \mathrm{~N}(\mathrm{C}) \mathrm{c} 1 \mathrm{ccn}(\mathrm{C}) \mathrm{n} 1$ & 12 \\
\hline 351 & $\mathbf{1 0}\{49\}$ & $\mathbf{1 1}\{242\}$ & $\mathbf{8}\{49,242\}$ & $\mathrm{CCN}(\mathrm{c} 1 \mathrm{cnn}(\mathrm{C}) \mathrm{c} 1) \mathrm{c} 1 \mathrm{nnnn} 1 \mathrm{C}(\mathrm{C}) \mathrm{Cc} 1 \mathrm{ccccc} 1$ & 29 \\
\hline 352 & $\mathbf{1 0}\{49\}$ & $\mathbf{1 1}\{242\}$ & $\mathbf{8}\{49,242\}$ & $\mathrm{CCN}(\mathrm{c} 1 \mathrm{cnn}(\mathrm{C}) \mathrm{c} 1) \mathrm{c} 1 \mathrm{nnnnn} 1 \mathrm{C}(\mathrm{C}) \mathrm{Cc} 1 \mathrm{ccccc} 1$ & 0 \\
\hline 353 & $\mathbf{1 0}\{49\}$ & $\mathbf{1 1}\{140\}$ & $\mathbf{8}\{49,140\}$ & $\mathrm{CC}(\mathrm{Cc} 1 \mathrm{ccccc} 1) \mathrm{n} 1 \mathrm{nnn} \operatorname{c} 1 \mathrm{~N}(\mathrm{C}) \mathrm{c} 1 \mathrm{cnn}(\mathrm{C}) \mathrm{c} 1$ & 0 \\
\hline
\end{tabular}




\begin{tabular}{|c|c|c|c|c|c|}
\hline 354 & $\mathbf{1 0}\{49\}$ & $\mathbf{1 1}\{243\}$ & $\mathbf{8}\{49,243\}$ & $\mathrm{CC}(\mathrm{Cc} 1 \mathrm{ccccc} 1) \mathrm{n} 1 \mathrm{nnnn} 1 \mathrm{~N} 1 \mathrm{CCC}=\mathrm{C}(\mathrm{C} 1) \mathrm{c} 1 \mathrm{cnn}(\mathrm{C}) \mathrm{c} 1$ & 0 \\
\hline 355 & $\mathbf{1 0}\{49\}$ & $\mathbf{1 1}\{143\}$ & $\mathbf{8}\{49,143\}$ & $\mathrm{CC}(\mathrm{Cc} 1 \mathrm{ccccc} 1) \mathrm{n} 1 \mathrm{nnnn} 1 \mathrm{~N}(\mathrm{C}) \mathrm{c} 1 \mathrm{ccn}(\mathrm{C}) \mathrm{n} 1$ & 0 \\
\hline 356 & $\mathbf{1 0}\{49\}$ & $\mathbf{1 1}\{73\}$ & $\mathbf{8}\{49,73\}$ & $\mathrm{CC}(\mathrm{Cc} 1 \mathrm{ccccc} 1) \mathrm{n} 1 \mathrm{nnnn} 1 \mathrm{~N} 1 \mathrm{CCc} 2 \mathrm{cn}[\mathrm{nH}] \mathrm{c} 2 \mathrm{C} 1$ & 0 \\
\hline 357 & $\mathbf{1 0}\{49\}$ & $\mathbf{1 1}\{99\}$ & $\mathbf{8}\{49,99\}$ & $\mathrm{CC}(\mathrm{Cc} 1 \mathrm{ccccc} 1) \mathrm{n} 1 \mathrm{nnn} \mathrm{c} 1 \mathrm{~N} 1 \mathrm{CCn} 2 \mathrm{cnc} 2 \mathrm{C} 1$ & 0 \\
\hline 358 & $\mathbf{1 0}\{49\}$ & $\mathbf{1 1}\{79\}$ & $\mathbf{8}\{49,79\}$ & $\mathrm{CC}(\mathrm{Cc} 1 \mathrm{ccccc} 1) \mathrm{n} 1 \mathrm{nnn} n 1 \mathrm{~N} 1 \mathrm{CCC}[\mathrm{C} @ @ \mathrm{H}] 1 \mathrm{C}(\mathrm{N})=\mathrm{O}$ & 0 \\
\hline 359 & $\mathbf{1 0}\{49\}$ & $11\{244\}$ & $\mathbf{8}\{49,244\}$ & $\begin{array}{l}\mathrm{CC}(\mathrm{Cc} 1 \mathrm{ccccc} 1) n 1 \mathrm{nnnc} 1 \mathrm{~N} 1[1 \mathrm{C} @ @ \mathrm{H}] 2 \mathrm{CC}[1 \mathrm{C} @ \mathrm{H}] 1 \mathrm{CN}(\mathrm{C}) \mathrm{CC} \\
2\end{array}$ & 0 \\
\hline 360 & $\mathbf{1 0}\{49\}$ & $\mathbf{1 1}\{140\}$ & $8\{49,140\}$ & $\mathrm{CC}(\mathrm{Cc} 1 \mathrm{ccccc} 1) \mathrm{n} 1 \mathrm{nnnn} 1 \mathrm{~N}(\mathrm{C}) \mathrm{c} 1 \mathrm{cnn}(\mathrm{C}) \mathrm{c} 1$ & 0 \\
\hline 361 & $\mathbf{1 0}\{50\}$ & $\mathbf{1 1}\{245\}$ & $\mathbf{8}\{50,245\}$ & $\mathrm{C}[\mathrm{C} @ \mathrm{H}] 1 \mathrm{COCCN} 1 \mathrm{c} 1 \mathrm{nnnn} 1 \mathrm{Cc} 1 \mathrm{ccc}(\mathrm{Br}) \mathrm{cc} 1$ & 7 \\
\hline 362 & $\mathbf{1 0}\{50\}$ & $\mathbf{1 1}\{118\}$ & $\mathbf{8}\{50,118\}$ & $\mathrm{C}[1 \mathrm{C} @ @ \mathrm{H}] 1[1 \mathrm{C} @ \mathrm{H}](\mathrm{O}) \mathrm{CCN} 1 \mathrm{c} 1 \mathrm{nnnn} 1 \mathrm{Cc} 1 \mathrm{ccc}(\mathrm{Br}) \mathrm{cc} 1$ & 15 \\
\hline 363 & $\mathbf{1 0}\{50\}$ & $\mathbf{1 1}\{246\}$ & $8\{50,246\}$ & $\operatorname{Brc} 1 \mathrm{ccc}(\mathrm{Cn} 2 \mathrm{nnnc} 2 \mathrm{~N} 2 \mathrm{CCCC}(\mathrm{C} 2) \mathrm{C \# N}) \mathrm{cc} 1$ & 0 \\
\hline 364 & $\mathbf{1 0}\{51\}$ & $\mathbf{1 1}\{247\}$ & $\mathbf{8}\{51,247\}$ & $\mathrm{CN}(\mathrm{Cc} 1 \mathrm{cccc}(=\mathrm{O})[\mathrm{nH}] 1) \mathrm{c} 1 \mathrm{nnnn} 1 \mathrm{CC} 1 \mathrm{CCOC} 1$ & 0 \\
\hline 365 & $\mathbf{1 0}\{51\}$ & $\mathbf{1 1}\{248\}$ & $\mathbf{8}\{51,248\}$ & $\mathrm{C}(\mathrm{C} 1 \mathrm{CCOC} 1) \mathrm{n} 1 \mathrm{nnnc} 1 \mathrm{~N} 1 \mathrm{CCC}[\mathrm{C} @ \mathrm{H}] 1 \mathrm{c} 1 \mathrm{ncn}[\mathrm{nH}] 1$ & 0 \\
\hline 366 & $\mathbf{1 0}\{51\}$ & $\mathbf{1 1}\{249\}$ & $\mathbf{8}\{51,249\}$ & COC[C@H]1C[C@@H](O)CN1c1nnnn1CC1CCOC1 & 0 \\
\hline 367 & $\mathbf{1 0}\{51\}$ & $\mathbf{1 1}\{244\}$ & $\mathbf{8}\{51,244\}$ & CN1CC[1C@@H]2CC[1C@H](C1)N2c1nnnn1CC1CCOC1 & 0 \\
\hline 368 & $\mathbf{1 0}\{51\}$ & $\mathbf{1 1}\{250\}$ & $\mathbf{8}\{51,250\}$ & $\mathrm{CC}(\mathrm{C})[\mathrm{C} @ \mathrm{H}] 1 \mathrm{CN}(\mathrm{CCN} 1 \mathrm{C}) \mathrm{c} 1 \mathrm{nnnn} 1 \mathrm{CC} 1 \mathrm{CCOC} 1$ & 0 \\
\hline 369 & $\mathbf{1 0}\{51\}$ & $\mathbf{1 1}\{43\}$ & $\mathbf{8}\{51,43\}$ & $\mathrm{CN}([\mathrm{C} @ \mathrm{H}] 1 \mathrm{C}[\mathrm{C} @ \mathrm{H}](\mathrm{C} 1) \mathrm{C}(\mathrm{N})=\mathrm{O}) \mathrm{c} 1 \mathrm{nnnn} 1 \mathrm{CC} 1 \mathrm{CCOC} 1$ & 39 \\
\hline 370 & $\mathbf{1 0}\{51\}$ & $\mathbf{1 1}\{251\}$ & $\mathbf{8}\{51,251\}$ & $\mathrm{CN} 1 \mathrm{CCN}(\mathrm{Cc} 2 \mathrm{cccnc} 12) \mathrm{c} 1 \mathrm{nnnn} 1 \mathrm{CC} 1 \mathrm{CCOC} 1$ & 93 \\
\hline 371 & $\mathbf{1 0}\{52\}$ & $\mathbf{1 1}\{252\}$ & $\mathbf{8}\{52,252\}$ & $\mathrm{NC}(=\mathrm{O}) \mathrm{C} 1 \mathrm{~N}(\mathrm{CCc} 2 \operatorname{ccccc} 12) \mathrm{c} 1 \mathrm{nnnn} 1 \mathrm{C} 1 \mathrm{CCC} 1$ & 0 \\
\hline 372 & $\mathbf{1 0}\{52\}$ & $\mathbf{1 1}\{222\}$ & $\mathbf{8}\{52,222\}$ & $\mathrm{C} 1 \mathrm{CC}(\mathrm{C} 1) \mathrm{n} 1 \mathrm{nnn} \mathrm{c} 1 \mathrm{~N} 1 \mathrm{CCCC}(\mathrm{C} 1) \mathrm{c} 1 \mathrm{ccncc} 1$ & 0 \\
\hline 373 & $\mathbf{1 0}\{52\}$ & $\mathbf{1 1}\{1\}$ & $\mathbf{8}\{52,1\}$ & $\mathrm{CNS}(=\mathrm{O})(=\mathrm{O}) \mathrm{c} 1 \mathrm{ccc} 2 \mathrm{CN}(\mathrm{CCc} 12) \mathrm{c} 1 \mathrm{nnnn} 1 \mathrm{C} 1 \mathrm{CCC} 1$ & 14 \\
\hline 374 & $\mathbf{1 0}\{52\}$ & $\mathbf{1 1}\{253\}$ & $\mathbf{8}\{52,253\}$ & $\operatorname{CC} 1 \mathrm{CCN}(\operatorname{CCS} 1(=\mathrm{O})=\mathrm{O}) \mathrm{c} 1 \mathrm{nnnn} 1 \mathrm{C} 1 \mathrm{CCC} 1$ & 18 \\
\hline
\end{tabular}




\begin{tabular}{|c|c|c|c|c|c|}
\hline 375 & $\mathbf{1 0}\{52\}$ & $\mathbf{1 1}\{254\}$ & $\mathbf{8}\{52,254\}$ & $\mathrm{O}[1 \mathrm{C} @ @ \mathrm{H}] 1 \mathrm{CN}(\mathrm{C}[1 \mathrm{C} @ \mathrm{H}] 1 \mathrm{Oc} 1 \mathrm{cccc} 1) \mathrm{c} 1 \mathrm{nnnn} 1 \mathrm{C} 1 \mathrm{CCC} 1$ & 27 \\
\hline 376 & $\mathbf{1 0}\{52\}$ & $\mathbf{1 1}\{255\}$ & $\mathbf{8}\{52,255\}$ & $\operatorname{CC} 1 \mathrm{CN}(\mathrm{CCS}(=\mathrm{O})(=\mathrm{O}) \mathrm{C} 1) \mathrm{c} 1 \mathrm{nnnn} 1 \mathrm{C} 1 \mathrm{CCC} 1$ & 21 \\
\hline 377 & $\mathbf{1 0}\{52\}$ & $\mathbf{1 1}\{256\}$ & $\mathbf{8}\{52,256\}$ & $\mathrm{CC} 1(\mathrm{C}) \mathrm{CN}([1 \mathrm{C} @ @ \mathrm{H}] 2 \mathrm{COC}[1 \mathrm{C} @ \mathrm{H}] 12) \mathrm{c} 1 \mathrm{nnnn} 1 \mathrm{C} 1 \mathrm{CCC} 1$ & 43 \\
\hline 378 & $\mathbf{1 0}\{52\}$ & $\mathbf{1 1}\{220\}$ & $\mathbf{8}\{52,220\}$ & $\operatorname{CC} 1 \mathrm{CCS}(=\mathrm{O})(=\mathrm{O}) \mathrm{CCN} 1 \mathrm{c} 1 \mathrm{nnnn} 1 \mathrm{C} 1 \mathrm{CCC} 1$ & 8 \\
\hline 379 & $\mathbf{1 0}\{53\}$ & $\mathbf{1 1}\{127\}$ & $\mathbf{8}\{53,127\}$ & $\mathrm{NC}(=\mathrm{O})[\mathrm{C} @ @ \mathrm{H}] 1 \mathrm{CCCN} 1 \mathrm{c} 1 \mathrm{nnnn} 1 \mathrm{CCc} 1 \mathrm{ccc}(\mathrm{F}) \mathrm{cc} 1$ & 0 \\
\hline 380 & $\mathbf{1 0}\{53\}$ & $\mathbf{1 1}\{257\}$ & $\mathbf{8}\{53,257\}$ & $\mathrm{Fc} 1 \mathrm{ccc}(\mathrm{CCn} 2 \mathrm{nnnc} 2 \mathrm{~N} 2 \mathrm{CCC}(\mathrm{CC} 2) \mathrm{c} 2 \mathrm{nnc}[\mathrm{nH}] 2) \mathrm{cc} 1$ & 0 \\
\hline 381 & $\mathbf{1 0}\{53\}$ & $\mathbf{1 1}\{258\}$ & $\mathbf{8}\{53,258\}$ & Fc1 $1 \mathrm{ccc}(\mathrm{CCn} 2 \mathrm{nnnn} 2 \mathrm{~N} 2 \mathrm{CCC}(\mathrm{CC} 2) \mathrm{c} 2 \mathrm{ncc}[\mathrm{nH}] 2) \mathrm{cc} 1$ & 0 \\
\hline 382 & $\mathbf{1 0}\{53\}$ & $\mathbf{1 1}\{259\}$ & $\mathbf{8}\{53,259\}$ & $\mathrm{CC} 1 \mathrm{CN}(\mathrm{CCN}(\mathrm{C} 1) \mathrm{c} 1 \mathrm{nnnn} 1 \mathrm{CCc} 1 \mathrm{ccc}(\mathrm{F}) \mathrm{cc} 1) \mathrm{C} 1 \mathrm{CC} 1$ & 0 \\
\hline 383 & $\mathbf{1 0}\{53\}$ & $\mathbf{1 1}\{260\}$ & $\mathbf{8}\{53,260\}$ & $\mathrm{Fc} 1 \mathrm{ccc}(\mathrm{CCn} 2 \mathrm{nnnn} 2 \mathrm{~N} 2 \mathrm{CCC}(\mathrm{C} 2) \mathrm{N} 2 \mathrm{CCCCC} 2) \mathrm{cc} 1$ & 0 \\
\hline 384 & $\mathbf{1 0}\{53\}$ & $\mathbf{1 1}\{205\}$ & $\mathbf{8}\{53,205\}$ & $\mathrm{OC} 1 \mathrm{CCN}(\mathrm{CC} 1(\mathrm{~F}) \mathrm{F}) \mathrm{c} 1 \mathrm{nnnn} 1 \mathrm{CCc} 1 \mathrm{ccc}(\mathrm{F}) \mathrm{cc} 1$ & 6 \\
\hline 385 & $\mathbf{1 0}\{53\}$ & $\mathbf{1 1}\{68\}$ & $\mathbf{8}\{53,68\}$ & $\mathrm{Fc} 1 \mathrm{ccc}(\mathrm{CCn} 2 \mathrm{nnnn} 2 \mathrm{~N} 2 \mathrm{CCCC}(\mathrm{C} 2) \mathrm{c} 2 \mathrm{ccn}[\mathrm{nH}] 2) \mathrm{cc} 1$ & 0 \\
\hline 386 & $\mathbf{1 0}\{53\}$ & $\mathbf{1 1}\{236\}$ & $\mathbf{8}\{53,236\}$ & Oc1cce $2 \mathrm{CCN}(\mathrm{Cc} 2 \mathrm{c} 1) \mathrm{c} 1 \mathrm{nnnn} 1 \mathrm{CCc} 1 \mathrm{ccc}(\mathrm{F}) \mathrm{cc} 1$ & 47 \\
\hline 387 & $\mathbf{1 0}\{53\}$ & $11\{4\}$ & $\mathbf{8}\{53,4\}$ & Fc1 $1 \mathrm{cc}(\mathrm{CCn} 2 \mathrm{nnn} \mathrm{2} 2 \mathrm{~N} 2 \mathrm{CCOC} 3(\mathrm{CCC} 3) \mathrm{C} 2) \mathrm{cc} 1$ & 0 \\
\hline 388 & $\mathbf{1 0}\{53\}$ & $\mathbf{1 1}\{57\}$ & $\mathbf{8}\{53,57\}$ & $\mathrm{Fc} 1 \mathrm{ccc}(\mathrm{CCn} 2 \mathrm{nnnn} 2 \mathrm{~N} 2 \mathrm{CCCc} 3 \mathrm{n}[\mathrm{nH}] \mathrm{cc} 23) \mathrm{cc} 1$ & 0 \\
\hline 389 & $\mathbf{1 0}\{53\}$ & $\mathbf{1 1}\{261\}$ & $\mathbf{8}\{53,261\}$ & $\mathrm{Fc} 1 \mathrm{ccc}(\mathrm{CCn} 2 \mathrm{nnnn} 2 \mathrm{~N} 2 \mathrm{CCC}(\mathrm{CC} 2) \mathrm{C} 2 \mathrm{CCCO} 2) \mathrm{cc} 1$ & 41 \\
\hline 390 & $\mathbf{1 0}\{53\}$ & $\mathbf{1 1}\{140\}$ & $\mathbf{8}\{53,140\}$ & $\mathrm{CN}(\mathrm{c} 1 \mathrm{cnn}(\mathrm{C}) \mathrm{c} 1) \mathrm{c} 1 \mathrm{nnnn} 1 \mathrm{CCc} 1 \mathrm{ccc}(\mathrm{F}) \mathrm{cc} 1$ & 0 \\
\hline 391 & $\mathbf{1 0}\{53\}$ & $\mathbf{1 1}\{73\}$ & $\mathbf{8}\{53,73\}$ & Fc1 ccc $(\mathrm{CCn} 2 \mathrm{nnnn} 2 \mathrm{~N} 2 \mathrm{CCc} 3 \mathrm{cn}[\mathrm{nH}] \mathrm{c} 3 \mathrm{C} 2) \mathrm{cc} 1$ & 6 \\
\hline 392 & $\mathbf{1 0}\{53\}$ & $\mathbf{1 1}\{116\}$ & $\mathbf{8}\{53,116\}$ & $\begin{array}{l}\mathrm{O}[1 \mathrm{C} @ @ \mathrm{H}] 1 \mathrm{CC}[1 \mathrm{C} @ @ \mathrm{H}] 2 \mathrm{CN}(\mathrm{C}[1 \mathrm{C} @ @ \mathrm{H}] 2 \mathrm{C} 1) \mathrm{c} 1 \mathrm{nnnn} 1 \mathrm{CC} \\
\mathrm{c} 1 \mathrm{ccc}(\mathrm{F}) \mathrm{cc} 1\end{array}$ & 23 \\
\hline 393 & $\mathbf{1 0}\{53\}$ & $\mathbf{1 1}\{249\}$ & $\mathbf{8}\{53,249\}$ & COC[C@H]1C[C@@H](O)CN1c1nnnn1CCc1ccc(F)cc1 & 11 \\
\hline 394 & $\mathbf{1 0}\{54\}$ & $\mathbf{1 1}\{27\}$ & $\mathbf{8}\{54,27\}$ & $\mathrm{C}(\mathrm{C} 1 \mathrm{CCCCO} 1) \mathrm{n} 1 \mathrm{nnnn} 1 \mathrm{~N} 1 \mathrm{CCC}(\mathrm{CC} 1) \mathrm{c} 1 \mathrm{ccncc} 1$ & 0 \\
\hline 395 & $\mathbf{1 0}\{54\}$ & $\mathbf{1 1}\{90\}$ & $\mathbf{8}\{54,90\}$ & $\mathrm{C}(\mathrm{N} 1 \mathrm{CCN}(\mathrm{CC} 1) \mathrm{c} 1 \mathrm{nnnn} 1 \mathrm{CC} 1 \mathrm{CCCCO} 1) \mathrm{c} 1 \mathrm{cscn} 1$ & 12 \\
\hline
\end{tabular}




\begin{tabular}{|c|c|c|c|c|c|}
\hline 396 & $\mathbf{1 0}\{55\}$ & $\mathbf{1 1}\{262\}$ & $\mathbf{8}\{55,262\}$ & $\mathrm{CC}(\mathrm{C}) \mathrm{N} 1 \mathrm{CCC}(\mathrm{C} 1) \mathrm{N}(\mathrm{C}) \mathrm{c} 1 \mathrm{nnnn} 1 \mathrm{Cc} 1 \mathrm{cc}(\mathrm{C}) \mathrm{on} 1$ & 0 \\
\hline 397 & $\mathbf{1 0}\{55\}$ & $\mathbf{1 1}\{156\}$ & $\mathbf{8}\{55,156\}$ & Cc1cc(Cn2nnnc2N2CCC[C@@ $@ \mathrm{H}](\mathrm{C} 2) \mathrm{C}(\mathrm{F})(\mathrm{F}) \mathrm{F}) \mathrm{no} 1$ & 0 \\
\hline 398 & $\mathbf{1 0}\{55\}$ & $\mathbf{1 1}\{263\}$ & $\mathbf{8}\{55,263\}$ & $\operatorname{CCc} 1 \operatorname{cccc}(\mathrm{c} 1) \mathrm{N}(\mathrm{C}) \mathrm{c} 1 \mathrm{nnnn} 1 \mathrm{Cc} 1 \mathrm{cc}(\mathrm{C}) \mathrm{on} 1$ & 41 \\
\hline 399 & $\mathbf{1 0}\{55\}$ & $\mathbf{1 1}\{264\}$ & $\mathbf{8}\{55,264\}$ & $\mathrm{CC} 1 \mathrm{~N}(\mathrm{CCc} 2 \operatorname{ccccc} 12) \mathrm{c} 1 \mathrm{nnnn} 1 \mathrm{Cc} 1 \mathrm{cc}(\mathrm{C})$ on 1 & 35 \\
\hline 400 & $\mathbf{1 0}\{55\}$ & $\mathbf{1 1}\{265\}$ & $\mathbf{8}\{55,265\}$ & $\mathrm{Cc} 1 \mathrm{cc}(\mathrm{Cn} 2 \mathrm{nnnn} 2 \mathrm{~N} 2 \mathrm{CCC}(\mathrm{Cc} 3 \mathrm{cnn}(\mathrm{C}) \mathrm{c} 3) \mathrm{C} 2) \mathrm{no} 1$ & 25 \\
\hline 401 & $\mathbf{1 0}\{56\}$ & $\mathbf{1 1}\{266\}$ & $\mathbf{8}\{56,266\}$ & $\mathrm{Fc} 1 \mathrm{cccc}(\mathrm{OC} 2 \mathrm{CCN}(\mathrm{CC} 2) \mathrm{c} 2 \mathrm{nnnn} 2 \mathrm{Cc} 2 \mathrm{ccon} 2) \mathrm{c} 1$ & 0 \\
\hline 402 & $\mathbf{1 0}\{56\}$ & $\mathbf{1 1}\{101\}$ & $\mathbf{8}\{56,101\}$ & $\mathrm{CN}(\mathrm{Cc} 1 \mathrm{ccc}(\mathrm{C}) \mathrm{s} 1) \mathrm{c} 1 \mathrm{nnnn} 1 \mathrm{Cc} 1 \mathrm{ccon} 1$ & 0 \\
\hline 403 & $\mathbf{1 0}\{56\}$ & $\mathbf{1 1}\{267\}$ & $\mathbf{8}\{56,267\}$ & $\mathrm{CC}(\mathrm{N}(\mathrm{C}) \mathrm{c} 1 \mathrm{nnnn} 1 \mathrm{Cc} 1 \mathrm{ccon} 1) \mathrm{c} 1 \mathrm{ccncc} 1$ & 0 \\
\hline 404 & $\mathbf{1 0}\{56\}$ & $\mathbf{1 1}\{268\}$ & $\mathbf{8}\{56,268\}$ & $\mathrm{C}(\mathrm{c} 1 \mathrm{ccon} 1) \mathrm{n} 1 \mathrm{nnn} c 1 \mathrm{~N} 1 \mathrm{CCCc} 2 \mathrm{sccc} 2 \mathrm{C} 1$ & 0 \\
\hline 405 & $\mathbf{1 0}\{56\}$ & $\mathbf{1 1}\{94\}$ & $\mathbf{8}\{56,94\}$ & $\mathrm{CN}(\mathrm{c} 1 \mathrm{nnnn} 1 \mathrm{Cc} 1 \mathrm{ccon} 1) \mathrm{c} 1 \mathrm{c}(\mathrm{C}) \operatorname{noc} 1 \mathrm{C}$ & 0 \\
\hline 406 & $\mathbf{1 0}\{56\}$ & $\mathbf{1 1}\{176\}$ & $\mathbf{8}\{56,176\}$ & $\mathrm{CN}(\mathrm{Cc} 1 \mathrm{cnc}(\mathrm{C}) \mathrm{s} 1) \mathrm{c} 1 \mathrm{nnnn} 1 \mathrm{Cc} 1 \mathrm{ccon} 1$ & 0 \\
\hline 407 & $\mathbf{1 0}\{56\}$ & $\mathbf{1 1}\{269\}$ & $\mathbf{8}\{56,269\}$ & Clc1ccc(c1)C1CCCN1c1nnnn1Cc1ccon1 & 0 \\
\hline 408 & $\mathbf{1 0}\{56\}$ & $11\{6\}$ & $\mathbf{8}\{56,6\}$ & Fc1cce $2 \mathrm{CN}(\mathrm{CCOc} 2 \mathrm{c} 1) \mathrm{c} 1 \mathrm{nnnn} 1 \mathrm{Cc} 1 \mathrm{ccon} 1$ & 0 \\
\hline 409 & $\mathbf{1 0}\{56\}$ & $\mathbf{1 1}\{270\}$ & $\mathbf{8}\{56,270\}$ & $\mathrm{CN}(\mathrm{C} 1 \mathrm{Cc} 2 \operatorname{ccccc} 2 \mathrm{C} 1) \mathrm{c} 1 \mathrm{nnnn} 1 \mathrm{Cc} 1 \mathrm{ccon} 1$ & 21 \\
\hline 410 & $\mathbf{1 0}\{56\}$ & $\mathbf{1 1}\{165\}$ & $\mathbf{8}\{56,165\}$ & $\mathrm{C}(\mathrm{C} 1 \mathrm{CCN}(\mathrm{CC} 1) \mathrm{c} 1 \mathrm{nnnn} 1 \mathrm{Cc} 1 \mathrm{ccon} 1) \mathrm{N} 1 \mathrm{CCOCC} 1$ & 20 \\
\hline 411 & $\mathbf{1 0}\{56\}$ & $\mathbf{1 1}\{48\}$ & $\mathbf{8}\{56,48\}$ & Cc1cenc(c1)N1CCN(CC1)c1nnnn1Cc1ccon1 & 8 \\
\hline 412 & $\mathbf{1 0}\{56\}$ & $\mathbf{1 1}\{271\}$ & $\mathbf{8}\{56,271\}$ & $\mathrm{CC}(\mathrm{N}(\mathrm{C}) \mathrm{c} 1 \mathrm{nnnn} 1 \mathrm{Cc} 1 \mathrm{ccon} 1) \mathrm{c} 1 \mathrm{ccccc} 1 \mathrm{~F}$ & 59 \\
\hline 413 & $\mathbf{1 0}\{56\}$ & $11\{4\}$ & $\mathbf{8}\{56,4\}$ & $\mathrm{C}(\mathrm{c} 1 \mathrm{ccon} 1) \mathrm{n} 1 \mathrm{nnn} \mathrm{n} 1 \mathrm{~N} 1 \mathrm{CCOC} 2(\mathrm{CCC} 2) \mathrm{C} 1$ & 18 \\
\hline 414 & $\mathbf{1 0}\{56\}$ & $\mathbf{1 1}\{108\}$ & $\mathbf{8}\{56,108\}$ & $\mathrm{O}=\mathrm{C} 1 \mathrm{CCCN} 1 \mathrm{C} 1 \mathrm{CCN}(\mathrm{CC} 1) \mathrm{c} 1 \mathrm{nnnn} 1 \mathrm{Cc} 1 \mathrm{ccon} 1$ & 89 \\
\hline 415 & $\mathbf{1 0}\{57\}$ & $11\{7\}$ & $\mathbf{8}\{57,7\}$ & $\mathrm{CC} 1=\mathrm{C}(\mathrm{C}) \mathrm{CN}(\mathrm{CC} 1) \mathrm{c} 1 \mathrm{nnnn} 1 \mathrm{CC}(\mathrm{F}) \mathrm{F}$ & 0 \\
\hline 416 & $\mathbf{1 0}\{57\}$ & $\mathbf{1 1}\{4\}$ & $\mathbf{8}\{57,4\}$ & $\mathrm{FC}(\mathrm{F}) \mathrm{Cn} 1 \mathrm{nnn} \mathrm{1N} 1 \mathrm{CCOC} 2(\mathrm{CCC} 2) \mathrm{C} 1$ & 0 \\
\hline
\end{tabular}




\begin{tabular}{|c|c|c|c|c|c|}
\hline 417 & $\mathbf{1 0}\{57\}$ & $11\{2\}$ & $\mathbf{8}\{57,2\}$ & $\mathrm{FC}(\mathrm{F}) \mathrm{Cn} 1 \mathrm{nnnc} 1 \mathrm{~N} 1 \mathrm{CCN}(\mathrm{CC} 1) \mathrm{C}(=\mathrm{O}) \mathrm{C}(=\mathrm{O}) \mathrm{NC} 1 \mathrm{CC} 1$ & 0 \\
\hline 418 & $\mathbf{1 0}\{57\}$ & $11\{8\}$ & $\mathbf{8}\{57,8\}$ & FC(F)Cn1nnnc1N1CC2(CCOCC2)[1C@@H]2CCC[1C@H]12 & 28 \\
\hline 419 & $\mathbf{1 0}\{58\}$ & $11\{272\}$ & $\mathbf{8}\{58,272\}$ & $\mathrm{CC} 1(\mathrm{CCC} 1) \mathrm{n} 1 \mathrm{nnnn} 1 \mathrm{~N} 1 \mathrm{CCCC}(\mathrm{C} 1) \mathrm{c} 1 \mathrm{ccc} n 1$ & 0 \\
\hline 420 & $\mathbf{1 0}\{58\}$ & $11\{273\}$ & $\mathbf{8}\{58,273\}$ & $\mathrm{CCN}(\mathrm{CC}(\mathrm{N})=\mathrm{O}) \mathrm{c} 1 \mathrm{nnnn} 1 \mathrm{C} 1(\mathrm{C}) \mathrm{CCC} 1$ & 0 \\
\hline 421 & $\mathbf{1 0}\{58\}$ & $11\{274\}$ & $\mathbf{8}\{58,274\}$ & $\mathrm{CC} 1(\mathrm{CCC} 1) \mathrm{n} 1 \mathrm{nnnc} 1 \mathrm{~N} 1 \mathrm{CCC}(\mathrm{CN} 2 \mathrm{CCCC} 2) \mathrm{C} 1$ & 0 \\
\hline 422 & $\mathbf{1 0}\{58\}$ & $11\{127\}$ & $\mathbf{8}\{58,127\}$ & $\mathrm{CC} 1(\mathrm{CCC} 1) \mathrm{n} 1 \mathrm{nnnc} 1 \mathrm{~N} 1 \mathrm{CCC}[\mathrm{C} @ \mathrm{H}] 1 \mathrm{C}(\mathrm{N})=\mathrm{O}$ & 0 \\
\hline 423 & $\mathbf{1 0}\{58\}$ & $11\{275\}$ & $\mathbf{8}\{58,275\}$ & $\mathrm{CC} 1(\mathrm{CCC} 1) \mathrm{n} 1 \mathrm{nnn} 1 \mathrm{~N} 1 \mathrm{CCC}(\mathrm{Cc} 2 \mathrm{cccnc} 2) \mathrm{CC} 1$ & 0 \\
\hline 424 & $\mathbf{1 0}\{58\}$ & $\mathbf{1 1}\{276\}$ & $\mathbf{8}\{58,276\}$ & $\mathrm{CC} 1 \mathrm{CN}(\mathrm{CCN} 1 \mathrm{CC}(\mathrm{C})(\mathrm{C}) \mathrm{O}) \mathrm{c} 1 \mathrm{nnnn} 1 \mathrm{C} 1(\mathrm{C}) \mathrm{CCC} 1$ & 17 \\
\hline 425 & $\mathbf{1 0}\{58\}$ & $11\{277\}$ & $\mathbf{8}\{58,277\}$ & $\mathrm{CC} 1(\mathrm{CCC} 1) \mathrm{n} 1 \mathrm{nnnc} 1 \mathrm{~N} 1 \mathrm{CCC}(\mathrm{CO})(\mathrm{C} 1) \mathrm{C}(\mathrm{F})(\mathrm{F}) \mathrm{F}$ & 38 \\
\hline 426 & $\mathbf{1 0}\{58\}$ & $\mathbf{1 1}\{278\}$ & $\mathbf{8}\{58,278\}$ & $\mathrm{CC} 1(\mathrm{CCC} 1) \mathrm{n} 1 \mathrm{nnnc} 1 \mathrm{~N} 1 \mathrm{CCC} 2(\mathrm{CCOC} 2) \mathrm{C} 1$ & 21 \\
\hline 427 & $\mathbf{1 0}\{58\}$ & $\mathbf{1 1}\{187\}$ & $\mathbf{8}\{58,187\}$ & $\mathrm{CC} 1(\mathrm{CCC} 1) \mathrm{n} 1 \mathrm{nnnc} 1 \mathrm{~N} 1 \mathrm{CCC}(\mathrm{O})(\mathrm{C} 1) \mathrm{C} 1 \mathrm{CC} 1$ & 15 \\
\hline 428 & $\mathbf{1 0}\{58\}$ & $11\{279\}$ & $\mathbf{8}\{58,279\}$ & $\mathrm{Cc} 1 \mathrm{cnc}(\mathrm{OC} 2 \mathrm{CCN}(\mathrm{CC} 2) \mathrm{c} 2 \mathrm{nnnn} 2 \mathrm{C} 2(\mathrm{C}) \mathrm{CCC} 2) \mathrm{nc} 1$ & 19 \\
\hline 429 & $\mathbf{1 0}\{58\}$ & $11\{280\}$ & $\mathbf{8}\{58,280\}$ & $\mathrm{CC} 1(\mathrm{CCC} 1) \mathrm{n} 1 \mathrm{nnnc} 1 \mathrm{~N} 1 \mathrm{CCCN}(\mathrm{CC} 1) \mathrm{c} 1 \mathrm{ncccc} 1 \mathrm{~F}$ & 106 \\
\hline 430 & $\mathbf{1 0}\{58\}$ & $\mathbf{1 1}\{192\}$ & $\mathbf{8}\{58,192\}$ & $\mathrm{CN}(\mathrm{Cc} 1 \operatorname{ccc}(\mathrm{C}) \mathrm{n} 1) \mathrm{c} 1 \mathrm{nnnn} 1 \mathrm{C} 1(\mathrm{C}) \mathrm{CCC} 1$ & 51 \\
\hline
\end{tabular}


Table S5. Parallel synthesis of 5-aminotetrazoles 9

\begin{tabular}{|c|c|c|c|c|c|c|}
\hline \# & $\begin{array}{l}\text { Primary } \\
\text { amine }\end{array}$ & $\begin{array}{l}\text { Secondary } \\
\text { amine }\end{array}$ & Product & SMILE & $\begin{array}{l}\text { Yield, } \\
\text { mg }\end{array}$ & $\begin{array}{l}\text { Yield, } \\
\%\end{array}$ \\
\hline 1 & $\mathbf{1 5}\{1\}$ & $\mathbf{1 1}\{281\}$ & $\mathbf{9}\{1,281\}$ & $\mathrm{CC}(\mathrm{C}) \mathrm{n} 1 \mathrm{nnn} n 1 \mathrm{~N} 1 \mathrm{CCC}(\mathrm{CC} 1) \mathrm{OCC} 1 \mathrm{CC} 1$ & 11 & 5 \\
\hline 2 & $15\{2\}$ & $\mathbf{1 1}\{282\}$ & $\mathbf{9}\{2,282\}$ & $\begin{array}{l}\mathrm{CC}(=\mathrm{O}) \mathrm{N} 1 \mathrm{CCN}(\mathrm{CC} 11 \mathrm{CCCCC} 1) \mathrm{c} 1 \mathrm{nnnn} 1 \mathrm{Cc} 1 \mathrm{ccc} \\
\mathrm{o} 1\end{array}$ & 0 & 0 \\
\hline 3 & $15\{2\}$ & $\mathbf{1 1}\{283\}$ & $\mathbf{9}\{2,283\}$ & $\mathrm{C}(\mathrm{c} 1 \mathrm{ccco} 1) \mathrm{n} 1 \mathrm{nnn} \mathrm{c} 1 \mathrm{~N} 1 \mathrm{Cc} 2 \mathrm{cccc} 2 \mathrm{OC} 2(\mathrm{CCC} 2) \mathrm{C} 1$ & 0 & 0 \\
\hline 4 & $15\{2\}$ & $\mathbf{1 1}\{284\}$ & $\mathbf{9}\{2,284\}$ & $\mathrm{CC} 1(\mathrm{C}) \mathrm{CN}(\mathrm{CCO} 1) \mathrm{c} 1 \mathrm{nnnn} 1 \mathrm{Cc} 1 \mathrm{ccco} 1$ & 9 & 4 \\
\hline 5 & $15\{2\}$ & $\mathbf{1 1}\{285\}$ & $\mathbf{9}\{2,285\}$ & ${ }_{1} \mathrm{Cc} 1 \mathrm{nc}(\mathrm{no} 1) \mathrm{C} 1 \mathrm{CC} 2 \mathrm{CCC}(\mathrm{C} 1) \mathrm{N} 2 \mathrm{c} 1 \mathrm{nnnn} 1 \mathrm{Cc} 1 \mathrm{ccco}$ & 0 & 0 \\
\hline 6 & $15\{2\}$ & $\mathbf{1 1}\{286\}$ & $\mathbf{9}\{2,286\}$ & C(c1ccco1)n1nnnc1N1CCC2(CC1)OCc1ccece21 & 0 & 0 \\
\hline 7 & $15\{2\}$ & $\mathbf{1 1}\{287\}$ & $\mathbf{9}\{2,287\}$ & $\mathrm{O}=\mathrm{C}(\mathrm{N} 1 \mathrm{CCN}(\mathrm{CC} 1) \mathrm{c} 1 \mathrm{nnnn} 1 \mathrm{Cc} 1 \mathrm{ccco} 1) \mathrm{c} 1 \mathrm{ccco} 1$ & 0 & 0 \\
\hline 8 & $15\{3\}$ & $\mathbf{1 1}\{288\}$ & $\mathbf{9}\{3,288\}$ & $\begin{array}{l}\mathrm{CC}(=\mathrm{O}) \mathrm{N} 1 \mathrm{CCCN}(\mathrm{CC} 1) \mathrm{c} 1 \mathrm{nnnn} 1 \mathrm{Cc} 1 \mathrm{ccc} 2 \mathrm{OCOc} 2 \\
\mathrm{c} 1\end{array}$ & 0 & 0 \\
\hline 9 & $15\{4\}$ & $\mathbf{1 1}\{289\}$ & $\mathbf{9}\{4,289\}$ & COCCn1nnnc1N1CCC(CC1)c1nc2cccce2s1 & 111 & 46 \\
\hline 10 & $15\{4\}$ & $\mathbf{1 1}\{290\}$ & $\mathbf{9}\{4,290\}$ & C $\mathrm{COCCn1nnnc1 \textrm {N } 1 \mathrm { CCN } ( \mathrm { C } ( \mathrm { C } ) \mathrm { C } 1 ) \mathrm { C } ( = \mathrm { O } ) \mathrm { OC } ( \mathrm { C } ) ( \mathrm { C } )}$ & 18 & 10 \\
\hline 11 & $\mathbf{1 5}\{4\}$ & $\mathbf{1 1}\{291\}$ & $\mathbf{9}\{4,291\}$ & COCCn1nnnc1N1CCN(CC1)C(=O)c1ccc(F)cc1 & 70 & 33 \\
\hline 12 & $15\{4\}$ & $\mathbf{1 1}\{292\}$ & $\mathbf{9}\{4,292\}$ & $\begin{array}{l}\text { COCCn1nnnc1N1CCN }([\mathrm{C} @ @ \mathrm{H}](\mathrm{C}) \mathrm{C} 1) \mathrm{C}(=\mathrm{O}) \mathrm{O} \\
\mathrm{C}(\mathrm{C})(\mathrm{C}) \mathrm{C}\end{array}$ & 26 & 12 \\
\hline 13 & $15\{4\}$ & $\mathbf{1 1}\{293\}$ & $\mathbf{9}\{4,293\}$ & $\begin{array}{l}\text { COCCn1nnnc1N1[C@H]2CC[C@@H]1C[C@@ } \\
\mathrm{H}](\mathrm{C} 2) \mathrm{c} 1 \mathrm{cccc} 1\end{array}$ & 0 & 0 \\
\hline 14 & $15\{4\}$ & $\mathbf{1 1}\{287\}$ & $\mathbf{9}\{4,287\}$ & COCCn1nnnc1N1CCN(CC1)C(=O)c1ccco1 & 40 & 22 \\
\hline 15 & $15\{4\}$ & $\mathbf{1 1}\{294\}$ & $\mathbf{9}\{4,294\}$ & COCCn1nnnc1N1CCN(CC1)c1cccc1Cl & 6 & 3 \\
\hline 16 & $15\{4\}$ & $\mathbf{1 1}\{295\}$ & $\mathbf{9}\{4,295\}$ & COCCn1nnnc1N1CCN(CC1)C(=O)OCc1ccccc1 & 38 & 17 \\
\hline 17 & $15\{4\}$ & $\mathbf{1 1}\{296\}$ & $\mathbf{9}\{4,296\}$ & $\begin{array}{l}\text { COCCn1nnnc1N1CCN }([\mathrm{C} @ \mathrm{H}](\mathrm{C}) \mathrm{C} 1) \mathrm{C}(=\mathrm{O}) \mathrm{OC}( \\
\mathrm{C})(\mathrm{C}) \mathrm{C} \\
\quad \mathrm{S} 137\end{array}$ & 12 & 6 \\
\hline
\end{tabular}




\begin{tabular}{|c|c|c|c|c|c|}
\hline 18 & $15\{5\}$ & $11\{297\}$ & $\mathbf{9}\{5,297\}$ & CCCCCn1nnnc1N1CCC(CC1)C1(C)OCCO1 & 7 \\
\hline 19 & $15\{5\}$ & $11\{298\}$ & $\mathbf{9}\{5,298\}$ & CCCCCn1nnnc1N1CCCC(C1)C1(C)OCCO1 & 8 \\
\hline 20 & $15\{6\}$ & $11\{299\}$ & $\mathbf{9}\{6,299\}$ & $\mathrm{COC} 1(\mathrm{CO}) \mathrm{CCN}(\mathrm{CC} 1) \mathrm{c} 1 \mathrm{nnnn} 1 \mathrm{Cc} 1 \mathrm{ccc}(\mathrm{Cl}) \mathrm{cc} 1$ & 46 \\
\hline 21 & $15\{6\}$ & $\mathbf{1 1}\{300\}$ & $\mathbf{9}\{6,300\}$ & $\mathrm{Clc} 1 \mathrm{ccc}(\mathrm{Cn} 2 \mathrm{nnnn} 2 \mathrm{~N} 2 \mathrm{CCCOCC} 2) \mathrm{cc} 1$ & 54 \\
\hline 22 & $15\{7\}$ & $11\{301\}$ & $\mathbf{9}\{7,301\}$ & $\mathrm{FC}(\mathrm{F}) \mathrm{CN} 1 \mathrm{CCN}(\mathrm{CC} 1) \mathrm{c} 1 \mathrm{nnnn} 1 \mathrm{Cc} 1 \mathrm{ccccc} 1$ & 15 \\
\hline 23 & $15\{8\}$ & $11\{302\}$ & $\mathbf{9}\{8,302\}$ & $\mathrm{CN}(\mathrm{CCOc} 1 \mathrm{ccc}(\mathrm{F}) \mathrm{cc} 1) \mathrm{c} 1 \mathrm{nnnn} 1 \mathrm{CC} 1 \mathrm{CCCO} 1$ & 56 \\
\hline 24 & $15\{8\}$ & $11\{303\}$ & $\mathbf{9}\{8,303\}$ & $\mathrm{CC} 1 \mathrm{CCCN}(\mathrm{C} 1 \mathrm{C}) \mathrm{c} 1 \mathrm{nnnn} 1 \mathrm{CC} 1 \mathrm{CCCO} 1$ & 26 \\
\hline 25 & $15\{9\}$ & $11\{297\}$ & $\mathbf{9}\{9,297\}$ & COCCCn1nnnc1N1CCC(CC1)C1(C)OCCO1 & 26 \\
\hline 26 & $15\{9\}$ & $11\{298\}$ & $\mathbf{9}\{9,298\}$ & COCCCn1nnnc1N1CCCC(C1)C1(C)OCCO1 & 24 \\
\hline 27 & $15\{10\}$ & $11\{298\}$ & $\mathbf{9}\{10,298\}$ & $\mathrm{CC}(\mathrm{C}) \mathrm{CCn} 1 \mathrm{nnnn} 1 \mathrm{~N} 1 \mathrm{CCCC}(\mathrm{C} 1) \mathrm{C} 1(\mathrm{C}) \mathrm{OCCO} 1$ & 29 \\
\hline 28 & $\mathbf{1 5}\{10\}$ & $\mathbf{1 1}\{297\}$ & $\mathbf{9}\{10,297\}$ & $\mathrm{CC}(\mathrm{C}) \mathrm{CCn} 1 \mathrm{nnn} \mathrm{c} 1 \mathrm{~N} 1 \mathrm{CCC}(\mathrm{CC} 1) \mathrm{C} 1(\mathrm{C}) \mathrm{OCCO} 1$ & 34 \\
\hline 29 & $15\{11\}$ & $11\{304\}$ & $\mathbf{9}\{11,304\}$ & $\begin{array}{l}\mathrm{CC}(\mathrm{C}) \mathrm{C}(=\mathrm{O}) \mathrm{NCC} 1 \mathrm{CCCN}(\mathrm{C} 1) \mathrm{c} 1 \mathrm{nnnn} 1 \mathrm{CC} 1 \mathrm{CCC} \\
\mathrm{CC} 1\end{array}$ & 20 \\
\hline 30 & $\mathbf{1 5}\{12\}$ & $\mathbf{1 1}\{305\}$ & $\mathbf{9}\{12,305\}$ & $\mathrm{CC} 1 \mathrm{CCCN}(\mathrm{CC} 1) \mathrm{c} 1 \mathrm{nnnn} 1 \mathrm{CCCN}(\mathrm{C}) \mathrm{C}$ & 0 \\
\hline 31 & $15\{13\}$ & $11\{306\}$ & $\mathbf{9}\{13,306\}$ & 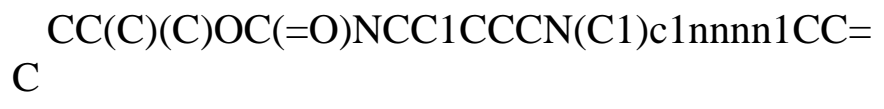 & 0 \\
\hline 32 & $15\{13\}$ & $11\{307\}$ & $\mathbf{9}\{13,307\}$ & $\begin{array}{l}\mathrm{CN}(\mathrm{CC} 1 \mathrm{CCN}(\mathrm{C} 1) \mathrm{c} 1 \mathrm{nnnn} 1 \mathrm{CC}=\mathrm{C}) \mathrm{C}(=\mathrm{O}) \mathrm{OC}(\mathrm{C})( \\
\mathrm{C}) \mathrm{C}\end{array}$ & 19 \\
\hline 33 & $15\{13\}$ & $11\{308\}$ & $\mathbf{9}\{13,308\}$ & ${ }_{\mathrm{C}}^{\mathrm{CC}(\mathrm{C})(\mathrm{C}) \mathrm{OC}(=\mathrm{O}) \mathrm{NCC} 1 \mathrm{CCN}(\mathrm{CC} 1) \mathrm{c} 1 \mathrm{nnnn} 1 \mathrm{CC}=}$ & 0 \\
\hline 34 & $15\{13\}$ & $11\{307\}$ & $\mathbf{9}\{13,307\}$ & $\begin{array}{l}\mathrm{CN}(\mathrm{CC} 1 \mathrm{CCN}(\mathrm{C} 1) \mathrm{c} 1 \mathrm{nnnn} 1 \mathrm{CC}=\mathrm{C}) \mathrm{C}(=\mathrm{O}) \mathrm{OC}(\mathrm{C})( \\
\mathrm{C}) \mathrm{C}\end{array}$ & 0 \\
\hline 35 & $15\{13\}$ & $\mathbf{1 1}\{309\}$ & $\mathbf{9}\{13,309\}$ & 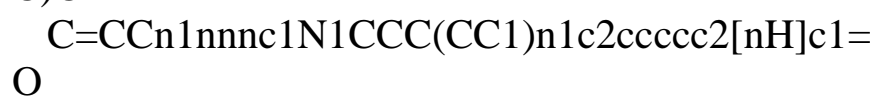 & 56 \\
\hline 36 & $15\{13\}$ & $11\{310\}$ & $\mathbf{9}\{13,310\}$ & ${ }_{\mathrm{C}}^{\mathrm{CC} 1 \mathrm{CN}(\mathrm{CCC} 1 \mathrm{NC}(=\mathrm{O}) \mathrm{OC}(\mathrm{C})(\mathrm{C}) \mathrm{C}) \mathrm{c} 1 \mathrm{nnnn} 1 \mathrm{CC}=}$ & 0 \\
\hline 37 & $\mathbf{1 5}\{13\}$ & $\mathbf{1 1}\{309\}$ & $\mathbf{9}\{13,309\}$ & $\mathrm{C}=\mathrm{CCn} 1 \mathrm{nnnn} 1 \mathrm{~N} 1 \mathrm{CCC}(\mathrm{CC} 1) \mathrm{n} 1 \mathrm{c} 2 \operatorname{ccccc} 2[\mathrm{nH}] \mathrm{c} 1=$ & 0 \\
\hline
\end{tabular}




\begin{tabular}{|c|c|c|c|c|c|}
\hline 38 & $\mathbf{1 5}\{13\}$ & $\mathbf{1 1}\{311\}$ & $\mathbf{9}\{13,311\}$ & $\begin{aligned} \mathrm{CC}(\mathrm{C})(\mathrm{C}) \mathrm{OC}(=\mathrm{O}) \mathrm{N}[\mathrm{C} @ \mathrm{H}] 1 \mathrm{CCCN}(\mathrm{C} 1) \mathrm{c} 1 \mathrm{nnnn} 1 \\
\mathrm{CC}=\mathrm{C}\end{aligned}$ & 0 \\
\hline 39 & $\mathbf{1 5}\{13\}$ & $\mathbf{1 1}\{306\}$ & $\mathbf{9}\{13,306\}$ & 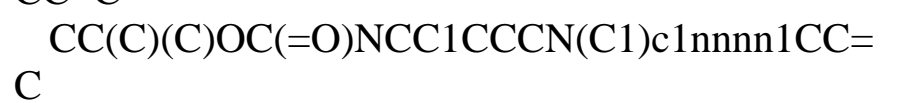 & 16 \\
\hline 40 & $\mathbf{1 5}\{13\}$ & $\mathbf{1 1}\{311\}$ & $\mathbf{9}\{13,311\}$ & $\begin{array}{l}\mathrm{CC}(\mathrm{C})(\mathrm{C}) \mathrm{OC}(=\mathrm{O}) \mathrm{N}[\mathrm{C} @ \mathrm{H}] 1 \mathrm{CCCN}(\mathrm{C} 1) \mathrm{c} 1 \mathrm{nnnn} 1 \\
\mathrm{CC}=\mathrm{C}\end{array}$ & 25 \\
\hline 41 & $\mathbf{1 5}\{13\}$ & $\mathbf{1 1}\{312\}$ & $\mathbf{9}\{13,312\}$ & $\mathrm{CC}(\mathrm{C})(\mathrm{C}) \mathrm{OC}(=\mathrm{O}) \mathrm{NC} 1 \mathrm{CCCN}(\mathrm{C} 1) \mathrm{c} 1 \mathrm{nnnn} 1 \mathrm{CC}=\mathrm{C}$ & 0 \\
\hline 42 & $\mathbf{1 5}\{13\}$ & $\mathbf{1 1}\{313\}$ & $\mathbf{9}\{13,313\}$ & $\begin{array}{l}\mathrm{CC}(\mathrm{C})(\mathrm{C}) \mathrm{OC}(=\mathrm{O}) \mathrm{N} 1 \mathrm{C}[\mathrm{C} @ \mathrm{H}] 2 \mathrm{CN}(\mathrm{C}[\mathrm{C} @ \mathrm{H}] 2 \mathrm{C} 1) \\
\text { c1nnnn1CC}=\mathrm{C}\end{array}$ & 0 \\
\hline 43 & $\mathbf{1 5}\{13\}$ & $\mathbf{1 1}\{313\}$ & $\mathbf{9}\{13,313\}$ & $\begin{array}{l}\mathrm{CC}(\mathrm{C})(\mathrm{C}) \mathrm{OC}(=\mathrm{O}) \mathrm{N} 1 \mathrm{C}[\mathrm{C} @ \mathrm{H}] 2 \mathrm{CN}(\mathrm{C}[\mathrm{C} @ \mathrm{H}] 2 \mathrm{C} 1) \\
\text { c1nnnn1CC=C }\end{array}$ & 30 \\
\hline 44 & $\mathbf{1 5}\{13\}$ & $\mathbf{1 1}\{314\}$ & $\mathbf{9}\{13,314\}$ & $\mathrm{CC}(\mathrm{C})(\mathrm{C}) \mathrm{OC}(=\mathrm{O}) \mathrm{N} 1 \mathrm{CCCN}(\mathrm{CC} 1) \mathrm{c} 1 \mathrm{nnnn} 1 \mathrm{CC}=\mathrm{C}$ & 0 \\
\hline 45 & $\mathbf{1 5}\{13\}$ & $\mathbf{1 1}\{315\}$ & $\mathbf{9}\{13,315\}$ & $\begin{array}{l}\mathrm{CC}(\mathrm{C})(\mathrm{C}) \mathrm{OC}(=\mathrm{O}) \mathrm{N} 1 \mathrm{C}[\mathrm{C} @ @ \mathrm{H}] 2 \mathrm{C}[\mathrm{C} @ \mathrm{H}] 1 \mathrm{CN} 2 \mathrm{c} \\
\text { 1nnnn1CC=C }\end{array}$ & 0 \\
\hline 46 & $\mathbf{1 5}\{13\}$ & $\mathbf{1 1}\{314\}$ & $\mathbf{9}\{13,314\}$ & $\mathrm{CC}(\mathrm{C})(\mathrm{C}) \mathrm{OC}(=\mathrm{O}) \mathrm{N} 1 \mathrm{CCCN}(\mathrm{CC} 1) \mathrm{c} 1 \mathrm{nnnn} 1 \mathrm{CC}=\mathrm{C}$ & 9 \\
\hline 47 & $15\{14\}$ & $\mathbf{1 1}\{300\}$ & $\mathbf{9}\{14,300\}$ & $\mathrm{COc} 1 \mathrm{ccc}(\mathrm{CCn} 2 \mathrm{nnn} \mathrm{c} 2 \mathrm{~N} 2 \mathrm{CCCOCC} 2) \mathrm{cc} 1$ & 53 \\
\hline 48 & $15\{14\}$ & $\mathbf{1 1}\{299\}$ & $\mathbf{9}\{14,299\}$ & $\mathrm{COc} 1 \mathrm{ccc}(\mathrm{CCn} 2 \mathrm{nnn} \mathrm{2} 2 \mathrm{~N} 2 \mathrm{CCC}(\mathrm{CO})(\mathrm{CC} 2) \mathrm{OC}) \mathrm{cc} 1$ & 32 \\
\hline 49 & $\mathbf{1 5}\{15\}$ & $\mathbf{1 1}\{110\}$ & $\mathbf{9}\{15,110\}$ & $\mathrm{CN}(\mathrm{CC} 1(\mathrm{O}) \mathrm{CCOCC} 1) \mathrm{c} 1 \mathrm{nnnn} 1 \mathrm{Cc} 1 \mathrm{ccccc} 1 \mathrm{Cl}$ & 0 \\
\hline 50 & $\mathbf{1 5}\{15\}$ & $\mathbf{1 1}\{102\}$ & $\mathbf{9}\{15,102\}$ & $\mathrm{CN}(\mathrm{Cc} 1 \mathrm{scnc} 1 \mathrm{C}) \mathrm{c} 1 \mathrm{nnnn} 1 \mathrm{Cc} 1 \mathrm{cccc} 1 \mathrm{Cl}$ & 0 \\
\hline 51 & $15\{15\}$ & $\mathbf{1 1}\{299\}$ & $\mathbf{9}\{15,299\}$ & $\mathrm{COC} 1(\mathrm{CO}) \mathrm{CCN}(\mathrm{CC} 1) \mathrm{c} 1 \mathrm{nnnn} 1 \mathrm{Cc} 1 \mathrm{cccc} 1 \mathrm{Cl}$ & 52 \\
\hline 52 & $\mathbf{1 5}\{15\}$ & $\mathbf{1 1}\{213\}$ & $\mathbf{9}\{15,213\}$ & $\mathrm{CNC}(=\mathrm{O}) \mathrm{NC} 1 \mathrm{CCN}(\mathrm{CC} 1) \mathrm{c} 1 \mathrm{nnnn} 1 \mathrm{Cc} 1 \mathrm{ccccc} 1 \mathrm{Cl}$ & 0 \\
\hline 53 & $\mathbf{1 5}\{15\}$ & $\mathbf{1 1}\{300\}$ & $\mathbf{9}\{15,300\}$ & Clc1eccc1Cn1nnnc1N1CCCOCC1 & 27 \\
\hline 54 & $15\{16\}$ & $\mathbf{1 1}\{299\}$ & $\mathbf{9}\{16,299\}$ & $\mathrm{COC} 1(\mathrm{CO}) \mathrm{CCN}(\mathrm{CC} 1) \mathrm{c} 1 \mathrm{nnnn} 1 \mathrm{Cc} 1 \mathrm{cccc}(\mathrm{Cl}) \mathrm{c} 1$ & 53 \\
\hline 55 & $15\{16\}$ & $\mathbf{1 1}\{300\}$ & $\mathbf{9}\{16,300\}$ & Clc1ccce(Cn2nnnc2N2CCCOCC2)c1 & 45 \\
\hline 56 & $15\{17\}$ & $\mathbf{1 1}\{316\}$ & $\mathbf{9}\{17,316\}$ & $\mathrm{Fc} 1 \mathrm{cccc}(\mathrm{F}) \mathrm{c} 1 \mathrm{~N} 1 \mathrm{CCN}(\mathrm{CC} 1) \mathrm{c} 1 \mathrm{nnnn} 1 \mathrm{C} 1 \mathrm{CC} 1$ & 18 \\
\hline
\end{tabular}




\begin{tabular}{|c|c|c|c|c|c|}
\hline 57 & $\mathbf{1 5}\{18\}$ & $\mathbf{1 1}\{299\}$ & $\mathbf{9}\{18,299\}$ & $\mathrm{COc} 1 \mathrm{ccc}(\mathrm{Cn} 2 \mathrm{nnn} 2 \mathrm{~N} 2 \mathrm{CCC}(\mathrm{CO})(\mathrm{CC} 2) \mathrm{OC}) \mathrm{cc} 1$ & 46 \\
\hline 58 & $\mathbf{1 5}\{18\}$ & $\mathbf{1 1}\{300\}$ & $\mathbf{9}\{18,300\}$ & $\mathrm{COc} 1 \mathrm{ccc}(\mathrm{Cn} 2 \mathrm{nnn} \mathrm{c} 2 \mathrm{~N} 2 \mathrm{CCCOCC} 2) \mathrm{cc} 1$ & 63 \\
\hline 59 & $\mathbf{1 5}\{19\}$ & $\mathbf{1 1}\{317\}$ & $\mathbf{9}\{19,317\}$ & $\mathrm{CN}(\mathrm{Cc} 1 \mathrm{ccc}(\mathrm{C}) \mathrm{o} 1) \mathrm{c} 1 \mathrm{nnnn} 1 \mathrm{C} 1 \mathrm{CCCC} 1$ & 18 \\
\hline 60 & $\mathbf{1 5}\{19\}$ & $\mathbf{1 1}\{288\}$ & $\mathbf{9}\{19,288\}$ & $\mathrm{CC}(=\mathrm{O}) \mathrm{N} 1 \mathrm{CCCN}(\mathrm{CC} 1) \mathrm{c} 1 \mathrm{nnnn} 1 \mathrm{C} 1 \mathrm{CCCC} 1$ & 29 \\
\hline 61 & $\mathbf{1 5}\{19\}$ & $\mathbf{1 1}\{318\}$ & $\mathbf{9}\{19,318\}$ & $\begin{array}{l}\text { C1CCC }(\mathrm{C} 1) n 1 \mathrm{nnnc} 1 \mathrm{~N} 1 \mathrm{CCn} 2 \mathrm{c}(\mathrm{C} 1) \mathrm{nnc} 2- \\
\mathrm{c} 1 \mathrm{ccccc} 1\end{array}$ & 0 \\
\hline 62 & $15\{20\}$ & $\mathbf{1 1}\{319\}$ & $\mathbf{9}\{20,319\}$ & $\mathrm{CN}(\mathrm{CC} 1 \mathrm{COCCO} 1) \mathrm{c} 1 \mathrm{nnnn} 1 \mathrm{CCc} 1 \mathrm{c}[\mathrm{nH}] \mathrm{c} 2 \mathrm{ccccc} 12$ & 20 \\
\hline 63 & $15\{20\}$ & $\mathbf{1 1}\{300\}$ & $\mathbf{9}\{20,300\}$ & $\mathrm{C}(\mathrm{Cn} 1 \mathrm{nnn} \mathrm{c} 1 \mathrm{~N} 1 \mathrm{CCCOCC} 1) \mathrm{c} 1 \mathrm{c}[\mathrm{nH}] \mathrm{c} 2 \mathrm{ccccc} 12$ & 0 \\
\hline 64 & $\mathbf{1 5}\{21\}$ & $\mathbf{1 1}\{300\}$ & $\mathbf{9}\{21,300\}$ & $\mathrm{Clc} 1 \mathrm{ccc}(\mathrm{CCn} 2 \mathrm{nnn} \mathrm{2} 2 \mathrm{~N} 2 \mathrm{CCCOCC} 2) \mathrm{cc} 1$ & 52 \\
\hline 65 & $\mathbf{1 5}\{21\}$ & $\mathbf{1 1}\{320\}$ & $\mathbf{9}\{21,320\}$ & $\mathrm{COC}(=\mathrm{O}) \mathrm{C} 1 \mathrm{CCN}(\mathrm{CC} 1) \mathrm{c} 1 \mathrm{nnnn} 1 \mathrm{CCc} 1 \mathrm{ccc}(\mathrm{Cl}) \mathrm{cc} 1$ & 38 \\
\hline 66 & $\mathbf{1 5}\{22\}$ & $\mathbf{1 1}\{321\}$ & $\mathbf{9}\{22,321\}$ & OCC1CCN(CC1)c1nnnn1Cc1ccc $(\mathrm{Cl}) \mathrm{cc} 1 \mathrm{Cl}$ & 11 \\
\hline 67 & $15\{22\}$ & $\mathbf{1 1}\{300\}$ & $\mathbf{9}\{22,300\}$ & $\mathrm{Clc} 1 \mathrm{ccc}(\mathrm{Cn} 2 \mathrm{nnnn} 2 \mathrm{~N} 2 \mathrm{CCCOCC} 2) \mathrm{c}(\mathrm{Cl}) \mathrm{c} 1$ & 39 \\
\hline 68 & $\mathbf{1 5}\{23\}$ & $\mathbf{1 1}\{300\}$ & $\mathbf{9}\{23,300\}$ & $\mathrm{C}(\mathrm{Cc} 1 \mathrm{ccccc} 1) \mathrm{Cn} 1 \mathrm{nnn} \mathrm{c} 1 \mathrm{~N} 1 \mathrm{CCCOCC} 1$ & 32 \\
\hline 69 & $15\{23\}$ & $\mathbf{1 1}\{299\}$ & $\mathbf{9}\{23,299\}$ & $\mathrm{COC} 1(\mathrm{CO}) \mathrm{CCN}(\mathrm{CC} 1) \mathrm{c} 1 \mathrm{nnnn} 1 \mathrm{CCC} 1 \mathrm{ccccc} 1$ & 47 \\
\hline 70 & $15\{24\}$ & $\mathbf{1 1}\{322\}$ & $\mathbf{9}\{24,322\}$ & $\mathrm{COc} 1 \mathrm{ccc}(\mathrm{Cn} 2 \mathrm{nnn} \mathrm{c} 2 \mathrm{~N}(\mathrm{C}) \mathrm{C} 2 \mathrm{CC} 2) \mathrm{cc} 1 \mathrm{OC}$ & 13 \\
\hline 71 & $15\{24\}$ & $11\{300\}$ & $\mathbf{9}\{24,300\}$ & COc1ccc $(\mathrm{Cn} 2 \mathrm{nnn} \mathrm{c} 2 \mathrm{~N} 2 \mathrm{CCCOCC} 2) \mathrm{cc} 1 \mathrm{OC}$ & 11 \\
\hline 72 & $15\{25\}$ & $\mathbf{1 1}\{147\}$ & $\mathbf{9}\{25,147\}$ & ${ }_{1} \mathrm{Clc} 1 \mathrm{cnn}(\mathrm{c} 1) \mathrm{C} 1 \mathrm{CCN}(\mathrm{C} 1) \mathrm{c} 1 \mathrm{nnnn} 1 \mathrm{CCC} 1=\mathrm{CCCCC}$ & 63 \\
\hline 73 & $\mathbf{1 5}\{25\}$ & $\mathbf{1 1}\{323\}$ & $\mathbf{9}\{25,323\}$ & ${ }_{1} \mathrm{O}=\mathrm{C} 1 \mathrm{CN}(\mathrm{Cc} 2 \operatorname{ccc} c 2 \mathrm{~N} 1) \mathrm{c} 1 \mathrm{nnnn} 1 \mathrm{CCC} 1=\mathrm{CCCCC}$ & 0 \\
\hline 74 & $\mathbf{1 5}\{25\}$ & $\mathbf{1 1}\{324\}$ & $\mathbf{9}\{25,324\}$ & $\begin{array}{l}\mathrm{Cc} 1 \mathrm{noc}(\mathrm{n} 1) \mathrm{C} 1 \mathrm{CCN}(\mathrm{CC} 1) \mathrm{c} 1 \mathrm{nnnn} 1 \mathrm{CCC} 1=\mathrm{CCCC} \\
\mathrm{C} 1\end{array}$ & 20 \\
\hline 75 & $\mathbf{1 5}\{25\}$ & $\mathbf{1 1}\{325\}$ & $\mathbf{9}\{25,325\}$ & $\begin{array}{l}\mathrm{O}=\mathrm{c} 1[\mathrm{nH}] \mathrm{ncn} 1 \mathrm{C} 1 \mathrm{CCN}(\mathrm{CC} 1) \mathrm{c} 1 \mathrm{nnnn} 1 \mathrm{CCC} 1=\mathrm{CC} \\
\mathrm{CCC} 1\end{array}$ & 0 \\
\hline 76 & $\mathbf{1 5}\{25\}$ & $\mathbf{1 1}\{288\}$ & $\mathbf{9}\{25,288\}$ & $\mathrm{CC}(=\mathrm{O}) \mathrm{N} 1 \mathrm{CCCN}(\mathrm{CC} 1) \mathrm{c} 1 \mathrm{nnnn} 1 \mathrm{CCC} 1=\mathrm{CCCCC} 1$ & 26 \\
\hline
\end{tabular}




\begin{tabular}{|c|c|c|c|c|c|}
\hline 77 & $15\{26\}$ & $11\{326\}$ & $\mathbf{9}\{26,326\}$ & COc1cccc1Cn1nnnc1N1CCC $(=\mathrm{CC} 1) \mathrm{C}(\mathrm{F})(\mathrm{F}) \mathrm{F}$ & 13 \\
\hline 78 & $15\{26\}$ & $11\{299\}$ & $\mathbf{9}\{26,299\}$ & COc1cccc1Cn1nnnc1N1CCC(CO)(CC1)OC & 45 \\
\hline 79 & $15\{26\}$ & $11\{300\}$ & $\mathbf{9}\{26,300\}$ & COc1cccc1 $1 \mathrm{Cn} 1 \mathrm{nnnn} 1 \mathrm{~N} 1 \mathrm{CCCOCC} 1$ & 49 \\
\hline 80 & $15\{27\}$ & $11\{327\}$ & $\mathbf{9}\{27,327\}$ & CCCn1nnnc1N1CCCC1c1cesc1 & 35 \\
\hline 81 & $15\{28\}$ & $11\{299\}$ & $\mathbf{9}\{28,299\}$ & $\mathrm{COC} 1(\mathrm{CO}) \mathrm{CCN}(\mathrm{CC} 1) \mathrm{c} 1 \mathrm{nnnn} 1 \mathrm{CCc} 1 \mathrm{ccc}(\mathrm{F}) \mathrm{cc} 1$ & 0 \\
\hline 82 & $15\{28\}$ & $11\{300\}$ & $\mathbf{9}\{28,300\}$ & $\mathrm{Fc} 1 \mathrm{ccc}(\mathrm{CCn} 2 \mathrm{nnnn} 2 \mathrm{~N} 2 \mathrm{CCCOCC} 2) \mathrm{cc} 1$ & 28 \\
\hline 83 & $15\{29\}$ & $11\{328\}$ & $\mathbf{9}\{29,328\}$ & Fc1 $1 \mathrm{ccc}(\mathrm{Cn} 2 \mathrm{nnnn} 2 \mathrm{~N} 2 \mathrm{CCOCC} 2) \mathrm{cc} 1$ & 41 \\
\hline 84 & $15\{30\}$ & $11\{299\}$ & $\mathbf{9}\{30,299\}$ & $\mathrm{COC} 1(\mathrm{CO}) \mathrm{CCN}(\mathrm{CC} 1) \mathrm{c} 1 \mathrm{nnnn} 1 \mathrm{Cc} 1 \mathrm{ccc}(\mathrm{C}) \mathrm{cc} 1$ & 26 \\
\hline 85 & $\mathbf{1 5}\{30\}$ & $11\{300\}$ & $\mathbf{9}\{30,300\}$ & $\mathrm{Cc} 1 \mathrm{ccc}(\mathrm{Cn} 2 \mathrm{nnnn} 2 \mathrm{~N} 2 \mathrm{CCCOCC} 2) \mathrm{cc} 1$ & 50 \\
\hline 86 & $\mathbf{1 5}\{31\}$ & $11\{329\}$ & $\mathbf{9}\{31,329\}$ & $\mathrm{COc} 1 \mathrm{cccc}(\mathrm{Cn} 2 \mathrm{nnnc} 2 \mathrm{~N} 2 \mathrm{CCOC}(\mathrm{CC} 2) \mathrm{C}(\mathrm{F}) \mathrm{F}) \mathrm{c} 1$ & 13 \\
\hline 87 & $\mathbf{1 5}\{31\}$ & $11\{330\}$ & $\mathbf{9}\{31,330\}$ & ${ }_{1} \operatorname{COc} 1 \operatorname{cccc}(\operatorname{Cn} 2 \operatorname{nnn} 22 \mathrm{~N}(\mathrm{C}) \mathrm{C} 2 \mathrm{CCCCN}(\mathrm{C}) \mathrm{C} 2=\mathrm{O}) \mathrm{c}$ & 0 \\
\hline 88 & $15\{31\}$ & $11\{331\}$ & $\mathbf{9}\{31,331\}$ & ${ }_{1}^{\mathrm{COc} 1 \mathrm{cccc}(\mathrm{Cn} 2 \mathrm{nnnn} 2 \mathrm{~N} 2 \mathrm{C} 3 \mathrm{CCC} 2 \mathrm{CC} 2(\mathrm{CC} 2) \mathrm{C} 3) \mathrm{c}}$ & 0 \\
\hline 89 & $15\{31\}$ & $11\{299\}$ & $\mathbf{9}\{31,299\}$ & $\mathrm{COc} 1 \mathrm{cccc}(\mathrm{Cn} 2 \mathrm{nnnn} 2 \mathrm{~N} 2 \mathrm{CCC}(\mathrm{CO})(\mathrm{CC} 2) \mathrm{OC}) \mathrm{c} 1$ & 39 \\
\hline 90 & $15\{31\}$ & $11\{332\}$ & $\mathbf{9}\{31,332\}$ & $\mathrm{COc} 1 \mathrm{cccc}(\mathrm{Cn} 2 \mathrm{nnn} \mathrm{c} 2 \mathrm{~N} 2 \mathrm{CCOC}(\mathrm{C})(\mathrm{C} 2) \mathrm{C}(\mathrm{F}) \mathrm{F}) \mathrm{c} 1$ & 0 \\
\hline 91 & $15\{31\}$ & $11\{300\}$ & $\mathbf{9}\{31,300\}$ & $\mathrm{COc} 1 \mathrm{cccc}(\mathrm{Cn} 2 \mathrm{nnnn} 2 \mathrm{~N} 2 \mathrm{CCCOCC} 2) \mathrm{c} 1$ & 54 \\
\hline 92 & $15\{32\}$ & $11\{287\}$ & $\mathbf{9}\{32,287\}$ & $\mathrm{O}=\mathrm{C}(\mathrm{N} 1 \mathrm{CCN}(\mathrm{CC} 1) \mathrm{c} 1 \mathrm{nnnn} 1 \mathrm{Cc} 1 \mathrm{cccs} 1) \mathrm{c} 1 \mathrm{ccco} 1$ & 0 \\
\hline 93 & $15\{32\}$ & $11\{333\}$ & $\mathbf{9}\{32,333\}$ & $\mathrm{CC} 1(\mathrm{C}) \mathrm{CN}(\mathrm{CCS} 1) \mathrm{c} 1 \mathrm{nnnn} 1 \mathrm{Cc} 1 \mathrm{cccs} 1$ & 23 \\
\hline 94 & $\mathbf{1 5}\{33\}$ & $11\{334\}$ & $\mathbf{9}\{33,334\}$ & Cn1cnnc1N1CCN(CC1)c1nnnn1Cc1cccce1F & 0 \\
\hline 95 & $15\{33\}$ & $\mathbf{1 1}\{335\}$ & $\mathbf{9}\{33,335\}$ & $\begin{array}{l}\text { Fc1cccc1Cn1nnnc1N1C }[\mathrm{C} @ \mathrm{H}] 2[\mathrm{C} @ @ \mathrm{H}](\mathrm{C} 1) \mathrm{C} \\
(=\mathrm{O}) \mathrm{NC} 2=\mathrm{O}\end{array}$ & 0 \\
\hline 96 & $15\{33\}$ & $11\{336\}$ & $\mathbf{9}\{33,336\}$ & $\mathrm{CS}(=\mathrm{O})(=\mathrm{O}) \mathrm{C} 1 \mathrm{CCN}(\mathrm{CC} 1) \mathrm{c} 1 \mathrm{nnnn} 1 \mathrm{Cc} 1 \mathrm{ccccc} 1 \mathrm{~F}$ & 0 \\
\hline
\end{tabular}




\begin{tabular}{|c|c|c|c|c|c|}
\hline 97 & $15\{33\}$ & $11\{109\}$ & $\mathbf{9}\{33,109\}$ & $\mathrm{Cn} 1 \mathrm{nc} 2 \mathrm{CCN}(\mathrm{Cc} 2 \mathrm{cc} 1=\mathrm{O}) \mathrm{c} 1 \mathrm{nnnn} 1 \mathrm{Cc} 1 \mathrm{cccc} 1 \mathrm{~F}$ & 0 \\
\hline 98 & $15\{33\}$ & $11\{321\}$ & $\mathbf{9}\{33,321\}$ & $\mathrm{OCC} 1 \mathrm{CCN}(\mathrm{CC} 1) \mathrm{c} 1 \mathrm{nnnn} 1 \mathrm{Cc} 1 \mathrm{ccccc} 1 \mathrm{~F}$ & 29 \\
\hline 99 & $15\{34\}$ & $11\{337\}$ & $\mathbf{9}\{34,337\}$ & $\mathrm{CN}(\mathrm{CC} 1 \mathrm{CCOC} 1) \mathrm{c} 1 \mathrm{nnnn} 1 \mathrm{Cc} 1 \mathrm{ccc}(\mathrm{c} 1) \mathrm{C}(\mathrm{F})(\mathrm{F}) \mathrm{F}$ & 31 \\
\hline 100 & $15\{34\}$ & $11\{300\}$ & $\mathbf{9}\{34,300\}$ & $\mathrm{FC}(\mathrm{F})(\mathrm{F}) \mathrm{c} 1 \mathrm{cccc}(\mathrm{Cn} 2 \mathrm{nnnn} 2 \mathrm{~N} 2 \mathrm{CCCOCC} 2) \mathrm{c} 1$ & 66 \\
\hline 101 & $15\{35\}$ & $11\{328\}$ & $\mathbf{9}\{35,328\}$ & $\mathrm{FC}(\mathrm{F})(\mathrm{F}) \mathrm{c} 1 \mathrm{ccc}(\mathrm{CCn} 2 \mathrm{nnnc} 2 \mathrm{~N} 2 \mathrm{CCOCC} 2) \mathrm{cc} 1$ & 39 \\
\hline 102 & $15\{36\}$ & $\mathbf{1 1}\{338\}$ & $\mathbf{9}\{36,338\}$ & $\mathrm{CCC} 1 \mathrm{CN}(\mathrm{CC}(\mathrm{CC}) \mathrm{O} 1) \mathrm{c} 1 \mathrm{nnnn} 1 \mathrm{CCNC}(\mathrm{C})=\mathrm{O}$ & 42 \\
\hline 103 & $15\{36\}$ & $11\{339\}$ & $\mathbf{9}\{36,339\}$ & $\mathrm{CN}(\mathrm{Cc} 1 \mathrm{cccc}(\mathrm{F}) \mathrm{c} 1) \mathrm{c} 1 \mathrm{nnnn} 1 \mathrm{CCNC}(\mathrm{C})=\mathrm{O}$ & 44 \\
\hline 104 & $15\{37\}$ & $\mathbf{1 1}\{340\}$ & $\mathbf{9}\{37,340\}$ & $\mathrm{CN}(\mathrm{C}) \mathrm{C} 1(\mathrm{Cn} 2 \mathrm{nnnn} 2 \mathrm{~N} 2 \mathrm{CCCCCC} 2) \mathrm{CCCC} 1$ & 0 \\
\hline 105 & $15\{38\}$ & $11\{341\}$ & $\mathbf{9}\{38,341\}$ & $\mathrm{C}(\mathrm{Cn} 1 \mathrm{nnnn} 1 \mathrm{~N} 1 \mathrm{CCCC} 1) \mathrm{Oc} 1 \mathrm{ccccc} 1$ & 0 \\
\hline 106 & $15\{38\}$ & $11\{342\}$ & $\mathbf{9}\{38,342\}$ & CC1CCCCN1c1nnnn1CCOc1ccecc1 & 20 \\
\hline 107 & $\mathbf{1 5}\{38\}$ & $\mathbf{1 1}\{343\}$ & $\mathbf{9}\{38,343\}$ & $\mathrm{C}(\mathrm{Cn} 1 \mathrm{nnn} \operatorname{c} 1 \mathrm{~N} 1 \mathrm{CCN}(\mathrm{Cc} 2 \mathrm{ccccc} 2) \mathrm{CC} 1) \mathrm{Oc} 1 \mathrm{ccccc} 1$ & 0 \\
\hline 108 & $\mathbf{1 5}\{38\}$ & $\mathbf{1 1}\{343\}$ & $\mathbf{9}\{38,343\}$ & $\mathrm{C}(\mathrm{Cn} 1 \mathrm{nnn} 1 \mathrm{~N} 1 \mathrm{CCN}(\mathrm{Cc} 2 \mathrm{ccccc} 2) \mathrm{CC} 1) \mathrm{Oc} 1 \mathrm{ccccc} 1$ & 17 \\
\hline 109 & $\mathbf{1 5}\{38\}$ & $\mathbf{1 1}\{341\}$ & $\mathbf{9}\{38,341\}$ & $\mathrm{C}(\mathrm{Cn} 1 \mathrm{nnnn} 1 \mathrm{~N} 1 \mathrm{CCCC} 1) \mathrm{Oc} 1 \mathrm{ccccc} 1$ & 36 \\
\hline 110 & $15\{39\}$ & $\mathbf{1 1}\{158\}$ & $\mathbf{9}\{39,158\}$ & $\begin{array}{l}\mathrm{CC}(\mathrm{C})(\mathrm{Cn} 1 \mathrm{nnnn} 1 \mathrm{~N} 1 \mathrm{CCC}(\mathrm{CC} 1) \mathrm{C}(\mathrm{F}) \mathrm{F}) \mathrm{N} 1 \mathrm{CCOC} \\
\mathrm{C} 1\end{array}$ & 13 \\
\hline 111 & $15\{39\}$ & $11\{344\}$ & $\mathbf{9}\{39,344\}$ & $\begin{array}{l}\mathrm{CC}(\mathrm{C})(\mathrm{Cn} 1 \mathrm{nnnn} 1 \mathrm{~N} 1 \mathrm{CCN}(\mathrm{C} 1) \mathrm{C}(=\mathrm{O}) \mathrm{C} 1 \mathrm{CC} 1) \mathrm{N} 1 \mathrm{C} \\
\mathrm{COCC} 1\end{array}$ & 0 \\
\hline 112 & $15\{39\}$ & $11\{345\}$ & $\mathbf{9}\{39,345\}$ & CC(C)(Cn1nnnc1N1CCCCCCC1)N1CCOCC1 & 0 \\
\hline 113 & $15\{40\}$ & $11\{346\}$ & $\mathbf{9}\{40,346\}$ & $\mathrm{CCN}(\mathrm{C}) \mathrm{c} 1 \mathrm{nnnn} 1 \mathrm{CC}(\mathrm{C})(\mathrm{C}) \mathrm{N} 1 \mathrm{CCCCC} 1$ & 0 \\
\hline 114 & $15\{41\}$ & $11\{299\}$ & $\mathbf{9}\{41,299\}$ & COc1cccc1CCn1nnnc1N1CCC(CO)(CC1)OC & 35 \\
\hline 115 & $15\{41\}$ & $11\{300\}$ & $\mathbf{9}\{41,300\}$ & COc1ccccc1CCn1nnnc1N1CCCOCC1 & 56 \\
\hline 116 & $\mathbf{1 5}\{42\}$ & $\mathbf{1 1}\{300\}$ & $\mathbf{9}\{42,300\}$ & $\mathrm{C}(\mathrm{Cn} 1 \mathrm{nnnnc} 1 \mathrm{~N} 1 \mathrm{CCCOCC} 1) \mathrm{Cn} 1 \mathrm{ccc} 2 \mathrm{cccc} 12$ & 7 \\
\hline 117 & $15\{42\}$ & $11\{337\}$ & $\mathbf{9}\{42,337\}$ & $\mathrm{CN}(\mathrm{CC} 1 \mathrm{CCOC} 1) \mathrm{c} 1 \mathrm{nnnn} 1 \mathrm{CCCn} 1 \mathrm{ccc} 2 \operatorname{ccccc} 12$ & 5 \\
\hline
\end{tabular}




\begin{tabular}{|c|c|c|c|c|c|c|}
\hline 118 & $15\{43\}$ & $11\{347\}$ & $\mathbf{9}\{43,347\}$ & $\mathrm{NC}(=\mathrm{O}) \mathrm{c} 1 \mathrm{ccc}(\mathrm{Cn} 2 \mathrm{nnnn} 2 \mathrm{~N} 2 \mathrm{CCCCCCCC} 2) \mathrm{cc} 1$ & 32 & 17 \\
\hline 119 & $15\{43\}$ & $11\{106\}$ & $\mathbf{9}\{43,106\}$ & $\mathrm{NC}(=\mathrm{O}) \mathrm{c} 1 \mathrm{ccc}(\mathrm{Cn} 2 \mathrm{nnnn} 2 \mathrm{~N} 2 \mathrm{CC} 3 \mathrm{cccc} 3 \mathrm{CC} 2) \mathrm{cc} 1$ & 49 & 24 \\
\hline 120 & $15\{43\}$ & $11\{320\}$ & $\mathbf{9}\{43,320\}$ & $\underset{)=\mathrm{O}}{\mathrm{COC}(=\mathrm{O}) \mathrm{C} 1 \mathrm{CCN}(\mathrm{CC} 1) \mathrm{c} 1 \mathrm{nnnnn} 1 \mathrm{Cc} 1 \mathrm{ccc}(\mathrm{cc} 1) \mathrm{C}(\mathrm{N}}$ & 17 & 8 \\
\hline 121 & $15\{43\}$ & $11\{348\}$ & $\mathbf{9}\{43,348\}$ & $\begin{aligned} & \mathrm{CC}(\mathrm{C}) \mathrm{C} 1 \mathrm{CN}(\mathrm{CCCO} 1) \mathrm{c} 1 \mathrm{nnnn} 1 \mathrm{Cc} 1 \mathrm{ccc}(\mathrm{cc} 1) \mathrm{C}(\mathrm{N}) \\
= & \mathrm{O}\end{aligned}$ & 0 & 0 \\
\hline 122 & $\mathbf{1 5}\{43\}$ & $11\{338\}$ & $\mathbf{9}\{43,338\}$ & $\begin{aligned} & \mathrm{CCC} 1 \mathrm{CN}(\mathrm{CC}(\mathrm{CC}) \mathrm{O} 1) \mathrm{c} 1 \mathrm{nnnn} 1 \mathrm{Cc} 1 \mathrm{ccc}(\mathrm{cc} 1) \mathrm{C}(\mathrm{N}) \\
= & \mathrm{O}\end{aligned}$ & 31 & 15 \\
\hline 123 & $15\{44\}$ & $11\{349\}$ & $\mathbf{9}\{44,349\}$ & $\begin{array}{l}\mathrm{O}=\mathrm{C} 1 \mathrm{CCCCCN} 1 \mathrm{CCCn} 1 \mathrm{nnn} 1 \mathrm{~N} 1 \mathrm{CCCC}(\mathrm{CC} \# \mathrm{C}) \\
\mathrm{C} 1\end{array}$ & 43 & 22 \\
\hline 124 & $\mathbf{1 5}\{44\}$ & $\mathbf{1 1}\{350\}$ & $\mathbf{9}\{44,350\}$ & $\mathrm{CN}(\mathrm{CCC}=\mathrm{C}) \mathrm{c} 1 \mathrm{nnnn} 1 \mathrm{CCCN} 1 \mathrm{CCCCCC} 1=\mathrm{O}$ & 53 & 24 \\
\hline 125 & $15\{44\}$ & $\mathbf{1 1}\{351\}$ & $\mathbf{9}\{44,351\}$ & $\begin{aligned} & \mathrm{CCN} 1 \mathrm{CCN}(\mathrm{CC} 1=\mathrm{O}) \mathrm{c} 1 \mathrm{nnnn} 1 \mathrm{CCCN} 1 \mathrm{CCCCCC} 1 \\
= & \mathrm{O}\end{aligned}$ & 36 & 18 \\
\hline 126 & $15\{45\}$ & $11\{297\}$ & $\mathbf{9}\{45,297\}$ & $\mathrm{CC} 1(\mathrm{OCCO} 1) \mathrm{C} 1 \mathrm{CCN}(\mathrm{CC} 1) \mathrm{c} 1 \mathrm{nnnnn} 1 \mathrm{CCc} 1 \mathrm{cccs} 1$ & 39 & 19 \\
\hline 127 & $\mathbf{1 5}\{45\}$ & $11\{298\}$ & $\mathbf{9}\{45,298\}$ & $\mathrm{CC} 1(\mathrm{OCCO} 1) \mathrm{C} 1 \mathrm{CCCN}(\mathrm{C} 1) \mathrm{c} 1 \mathrm{nnnn} 1 \mathrm{CCc} 1 \mathrm{cccs} 1$ & 23 & 12 \\
\hline 128 & $15\{46\}$ & $11\{297\}$ & $\mathbf{9}\{46,297\}$ & $\mathrm{CC} 1(\mathrm{OCCO} 1) \mathrm{C} 1 \mathrm{CCN}(\mathrm{CC} 1) \mathrm{c} 1 \mathrm{nnnn} 1 \mathrm{CCc} 1 \mathrm{ccco} 1$ & 0 & 0 \\
\hline 129 & $15\{46\}$ & $11\{298\}$ & $\mathbf{9}\{46,298\}$ & $\mathrm{CC} 1(\mathrm{OCCO} 1) \mathrm{C} 1 \mathrm{CCCN}(\mathrm{C} 1) \mathrm{c} 1 \mathrm{nnnn} 1 \mathrm{CCc} 1 \mathrm{ccco} 1$ & 26 & 13 \\
\hline 130 & $15\{47\}$ & $\mathbf{1 1}\{343\}$ & $\mathbf{9}\{47,343\}$ & $\mathrm{Cc} 1 \mathrm{ccccc} 1 \mathrm{Cn} 1 \mathrm{nnnc} 1 \mathrm{~N} 1 \mathrm{CCN}(\mathrm{Cc} 2 \mathrm{ccccc} 2) \mathrm{CC} 1$ & 0 & 0 \\
\hline 131 & $15\{47\}$ & $11\{341\}$ & $\mathbf{9}\{47,341\}$ & Cc1cccc1Cn1nnnc1N1CCCC1 & 36 & 17 \\
\hline 132 & $15\{47\}$ & $11\{352\}$ & $\mathbf{9}\{47,352\}$ & Cc1cccc1Cn1nnnc1N1CCC(Cn2cenn2)CC1 & 0 & 0 \\
\hline 133 & $15\{47\}$ & $11\{341\}$ & $\mathbf{9}\{47,341\}$ & Cc1cccc1Cn1nnnc1N1CCCC 1 & 0 & 0 \\
\hline 134 & $15\{47\}$ & $11\{343\}$ & $\mathbf{9}\{47,343\}$ & Cc1cccc1Cn1nnnc1N1CCN $(\mathrm{Cc} 2 \operatorname{ccccc} 2) \mathrm{CC} 1$ & 0 & 0 \\
\hline 135 & $15\{48\}$ & $11\{299\}$ & $\mathbf{9}\{48,299\}$ & $\mathrm{COC} 1(\mathrm{CO}) \mathrm{CCN}(\mathrm{CC} 1) \mathrm{c} 1 \mathrm{nnnn} 1 \mathrm{CCc} 1 \mathrm{ccccc} 1 \mathrm{~F}$ & 33 & 17 \\
\hline 136 & $15\{48\}$ & $11\{300\}$ & $\mathbf{9}\{48,300\}$ & Fc1eccc1CCn1nnnc1N1CCCOCC1 & 49 & 24 \\
\hline 137 & $\mathbf{1 5}\{48\}$ & $\mathbf{1 1}\{353\}$ & $\mathbf{9}\{48,353\}$ & OCC1(O)CCN(CC1)c1nnnn1CCc1 1 cccc1F & 27 & 13 \\
\hline
\end{tabular}




\begin{tabular}{|c|c|c|c|c|c|}
\hline 138 & $15\{49\}$ & $11\{354\}$ & $\mathbf{9}\{49,354\}$ & $\mathrm{CC} 1 \mathrm{~N}(\mathrm{CN}(\mathrm{C}) \mathrm{C} 1=\mathrm{O}) \mathrm{c} 1 \mathrm{nnnn} 1 \mathrm{Cc} 1 \mathrm{ccc}(\mathrm{OC}(\mathrm{F}) \mathrm{F}) \mathrm{cc} 1$ & 0 \\
\hline 139 & $\mathbf{1 5}\{50\}$ & $\mathbf{1 1}\{298\}$ & $\mathbf{9}\{50,298\}$ & $\begin{array}{l}\mathrm{CCCNC}(=\mathrm{O}) \mathrm{Cn} 1 \mathrm{nnnn} 1 \mathrm{~N} 1 \mathrm{CCCC}(\mathrm{C} 1) \mathrm{C} 1(\mathrm{C}) \mathrm{OCC} \\
\mathrm{O} 1\end{array}$ & 0 \\
\hline 140 & $15\{50\}$ & $\mathbf{1 1}\{297\}$ & $\mathbf{9}\{50,297\}$ & $\begin{array}{l}\mathrm{CCCNC}(=\mathrm{O}) \mathrm{Cn} 1 \mathrm{nnnn} 1 \mathrm{~N} 1 \mathrm{CCC}(\mathrm{CC} 1) \mathrm{C} 1(\mathrm{C}) \mathrm{OCC} \\
\mathrm{O} 1\end{array}$ & 0 \\
\hline 141 & $\mathbf{1 5}\{51\}$ & $\mathbf{1 1}\{355\}$ & $\mathbf{9}\{51,355\}$ & $\mathrm{CN}(\mathrm{Cc} 1 \mathrm{cnn}(\mathrm{C}) \mathrm{c} 1) \mathrm{c} 1 \mathrm{nnnn} 1 \mathrm{CC} 1 \mathrm{ccc} 2 \mathrm{OCOc} 2 \mathrm{c} 1$ & 44 \\
\hline 142 & $\mathbf{1 5}\{52\}$ & $11\{300\}$ & $\mathbf{9}\{52,300\}$ & COc1cec(C)ce1CCn1nnnc1N1CCCOCC1 & 56 \\
\hline 143 & $15\{52\}$ & $\mathbf{1 1}\{319\}$ & $\mathbf{9}\{52,319\}$ & COc1cce(C)cc1CCn1nnnc1N(C)CC1COCCO1 & 67 \\
\hline 144 & $\mathbf{1 5}\{53\}$ & $11\{300\}$ & $\mathbf{9}\{53,300\}$ & $\mathrm{Fc} 1 \mathrm{cccc}(\mathrm{CCn} 2 \mathrm{nnn} 2 \mathrm{~N} 2 \mathrm{CCCOCC} 2) \mathrm{c} 1$ & 37 \\
\hline 145 & $15\{53\}$ & $11\{299\}$ & $\mathbf{9}\{53,299\}$ & $\mathrm{COC} 1(\mathrm{CO}) \mathrm{CCN}(\mathrm{CC} 1) \mathrm{c} 1 \mathrm{nnnn} 1 \mathrm{CCc} 1 \mathrm{ccc}(\mathrm{F}) \mathrm{c} 1$ & 45 \\
\hline 146 & $15\{54\}$ & $\mathbf{1 1}\{319\}$ & $\mathbf{9}\{54,319\}$ & $\mathrm{CN}(\mathrm{CC} 1 \mathrm{COCCO} 1) \mathrm{c} 1 \mathrm{nnnn} 1 \mathrm{CCCOCC} 1 \mathrm{CCCO} 1$ & 38 \\
\hline 147 & $\mathbf{1 5}\{55\}$ & $\mathbf{1 1}\{300\}$ & $\mathbf{9}\{55,300\}$ & $\mathrm{FC}(\mathrm{F})(\mathrm{F}) \mathrm{c} 1 \mathrm{ccc}(\mathrm{Cn} 2 \mathrm{nnn} \mathrm{c} 2 \mathrm{~N} 2 \mathrm{CCCOCC} 2) \mathrm{cc} 1$ & 64 \\
\hline 148 & $15\{55\}$ & $\mathbf{1 1}\{337\}$ & $\mathbf{9}\{55,337\}$ & $\mathrm{CN}(\mathrm{CC} 1 \mathrm{CCOC} 1) \mathrm{c} 1 \mathrm{nnnn} 1 \mathrm{Cc} 1 \mathrm{ccc}(\mathrm{cc} 1) \mathrm{C}(\mathrm{F})(\mathrm{F}) \mathrm{F}$ & 60 \\
\hline 149 & $15\{56\}$ & $\mathbf{1 1}\{338\}$ & $\mathbf{9}\{56,338\}$ & $\mathrm{CCC} 1 \mathrm{CN}(\mathrm{CC}(\mathrm{CC}) \mathrm{O} 1) \mathrm{c} 1 \mathrm{nnnn} 1 \mathrm{Cc} 1 \mathrm{csc}(\mathrm{C}) \mathrm{n} 1$ & 28 \\
\hline 150 & $15\{57\}$ & $11\{300\}$ & $\mathbf{9}\{57,300\}$ & $\mathrm{Cc} 1 \mathrm{cc}(\mathrm{Cn} 2 \mathrm{nnnn} 2 \mathrm{~N} 2 \mathrm{CCCOCC} 2) \mathrm{ccc} 1 \mathrm{~F}$ & 65 \\
\hline 151 & $15\{57\}$ & $11\{261\}$ & $\mathbf{9}\{57,261\}$ & $\mathrm{Cc} 1 \mathrm{cc}(\mathrm{Cn} 2 \mathrm{nnnn} 2 \mathrm{~N} 2 \mathrm{CCC}(\mathrm{CC} 2) \mathrm{C} 2 \mathrm{CCCO} 2) \mathrm{ccc} 1 \mathrm{~F}$ & 65 \\
\hline 152 & $\mathbf{1 5}\{57\}$ & $11\{299\}$ & $\mathbf{9}\{57,299\}$ & $\mathrm{COC} 1(\mathrm{CO}) \mathrm{CCN}(\mathrm{CC} 1) \mathrm{c} 1 \mathrm{nnnn} 1 \mathrm{Cc} 1 \mathrm{ccc}(\mathrm{F}) \mathrm{c}(\mathrm{C}) \mathrm{c} 1$ & 54 \\
\hline 153 & $\mathbf{1 5}\{58\}$ & $11\{328\}$ & $\mathbf{9}\{58,328\}$ & $\mathrm{FC}(\mathrm{F})(\mathrm{F}) \mathrm{Oc} 1 \mathrm{ccc}(\mathrm{Cn} 2 \mathrm{nnn} n 2 \mathrm{~N} 2 \mathrm{CCOCC} 2) \mathrm{cc} 1$ & 39 \\
\hline 154 & $\mathbf{1 5}\{58\}$ & $11\{300\}$ & $\mathbf{9}\{58,300\}$ & $\mathrm{FC}(\mathrm{F})(\mathrm{F}) \mathrm{Oc} 1 \mathrm{ccc}(\mathrm{Cn} 2 \mathrm{nnn} 2 \mathrm{~N} 2 \mathrm{CCCOCC} 2) \mathrm{cc} 1$ & 24 \\
\hline 155 & $\mathbf{1 5}\{59\}$ & $11\{300\}$ & $\mathbf{9}\{59,300\}$ & $\mathrm{COc} 1 \mathrm{ccc} 2 \mathrm{cc}(\mathrm{Cn} 3 \mathrm{nnn} \mathrm{c} 3 \mathrm{~N} 3 \mathrm{CCCOCC} 3) \mathrm{ccc} 2 \mathrm{c} 1$ & 59 \\
\hline 156 & $15\{60\}$ & $11\{346\}$ & $\mathbf{9}\{60,346\}$ & $\mathrm{CCN}(\mathrm{C}) \mathrm{c} 1 \mathrm{nnnn} 1 \mathrm{Cc} 1 \mathrm{cnn}(\mathrm{c} 1)-\mathrm{c} 1 \mathrm{ccccc} 1$ & 0 \\
\hline 157 & $15\{60\}$ & $11\{354\}$ & $\mathbf{9}\{60,354\}$ & $\begin{array}{l}\mathrm{CC} 1 \mathrm{~N}(\mathrm{CN}(\mathrm{C}) \mathrm{C} 1=\mathrm{O}) \mathrm{c} 1 \mathrm{nnnn} 1 \mathrm{Cc} 1 \mathrm{cnn}(\mathrm{c} 1)- \\
\mathrm{c} 1 \operatorname{ccccc} 1\end{array}$ & 0 \\
\hline
\end{tabular}




\begin{tabular}{|c|c|c|c|c|c|}
\hline 158 & $15\{61\}$ & $\mathbf{1 1}\{322\}$ & $\mathbf{9}\{61,322\}$ & $\mathrm{CN}(\mathrm{C}) \mathrm{S}(=\mathrm{O})(=\mathrm{O}) \mathrm{c} 1 \mathrm{ccccc} 1 \mathrm{Cn} 1 \mathrm{nnn} c 1 \mathrm{~N}(\mathrm{C}) \mathrm{C} 1 \mathrm{CC} 1$ & 37 \\
\hline 159 & $15\{61\}$ & $\mathbf{1 1}\{346\}$ & $\mathbf{9}\{61,346\}$ & $\mathrm{CCN}(\mathrm{C}) \mathrm{c} 1 \mathrm{nnnn} 1 \mathrm{Cc} 1 \operatorname{ccccc} 1 \mathrm{~S}(=\mathrm{O})(=\mathrm{O}) \mathrm{N}(\mathrm{C}) \mathrm{C}$ & 40 \\
\hline 160 & $\mathbf{1 5}\{62\}$ & $\mathbf{1 1}\{322\}$ & $\mathbf{9}\{62,322\}$ & ${ }_{1}^{\mathrm{CN}}(\mathrm{C} 1 \mathrm{CC} 1) \mathrm{c} 1 \mathrm{nnnn} 1 \mathrm{C} 1 \mathrm{CCN}(\mathrm{CC} 1) \mathrm{C}(=\mathrm{O}) \mathrm{c} 1 \mathrm{ccco}$ & 40 \\
\hline 161 & $\mathbf{1 5}\{62\}$ & $\mathbf{1 1}\{356\}$ & $\mathbf{9}\{62,356\}$ & $\begin{array}{l}\mathrm{CN}(\mathrm{CC} 1 \mathrm{CCC} 1) \mathrm{c} 1 \mathrm{nnnn} 1 \mathrm{C} 1 \mathrm{CCN}(\mathrm{CC} 1) \mathrm{C}(=\mathrm{O}) \mathrm{c} 1 \mathrm{cc} \\
\mathrm{co} 1\end{array}$ & 38 \\
\hline 162 & $15\{63\}$ & $\mathbf{1 1}\{318\}$ & $\mathbf{9}\{63,318\}$ & $\mathrm{C}(\mathrm{C} 1 \mathrm{CC} 1) \mathrm{n} 1 \mathrm{nnn} c 1 \mathrm{~N} 1 \mathrm{CCn} 2 \mathrm{c}(\mathrm{C} 1) \mathrm{nnn} 2-\mathrm{c} 1 \mathrm{ccccc} 1$ & 0 \\
\hline 163 & $15\{63\}$ & $\mathbf{1 1}\{357\}$ & $\mathbf{9}\{63,357\}$ & COc1cccc1N1CCN(CC1)c1nnnn1CC1CC1 & 40 \\
\hline 164 & $\mathbf{1 5}\{63\}$ & $\mathbf{1 1}\{311\}$ & $\mathbf{9}\{63,311\}$ & $\begin{array}{l}\mathrm{CC}(\mathrm{C})(\mathrm{C}) \mathrm{OC}(=\mathrm{O}) \mathrm{N}[\mathrm{C} @ \mathrm{H}] 1 \mathrm{CCCN}(\mathrm{C} 1) \mathrm{c} 1 \mathrm{nnnn} 1 \\
\mathrm{CC} 1 \mathrm{CC} 1\end{array}$ & 0 \\
\hline 165 & $15\{63\}$ & $\mathbf{1 1}\{307\}$ & $\mathbf{9}\{63,307\}$ & $\begin{array}{l}\mathrm{CN}(\mathrm{CC} 1 \mathrm{CCN}(\mathrm{C} 1) \mathrm{c} 1 \mathrm{nnnn} 1 \mathrm{CC} 1 \mathrm{CC} 1) \mathrm{C}(=\mathrm{O}) \mathrm{OC}(\mathrm{C} \\
)(\mathrm{C}) \mathrm{C}\end{array}$ & 4 \\
\hline 166 & $15\{63\}$ & $\mathbf{1 1}\{309\}$ & $\mathbf{9}\{63,309\}$ & $\begin{array}{l}\mathrm{O}=\mathrm{c} 1[\mathrm{nH}] \mathrm{c} 2 \operatorname{cccc} 2 \mathrm{n} 1 \mathrm{C} 1 \mathrm{CCN}(\mathrm{CC} 1) \mathrm{c} 1 \mathrm{nnnn} 1 \mathrm{CC} 1 \\
\mathrm{CC} 1\end{array}$ & 0 \\
\hline 167 & $15\{63\}$ & $\mathbf{1 1}\{358\}$ & $\mathbf{9}\{63,358\}$ & $\begin{array}{l}\mathrm{CC}(\mathrm{C})(\mathrm{C}) \mathrm{OC}(=\mathrm{O}) \mathrm{NCC} 1 \mathrm{CCN}(\mathrm{C} 1) \mathrm{c} 1 \mathrm{nnnn} 1 \mathrm{CC} 1 \mathrm{C} \\
\mathrm{C} 1\end{array}$ & 39 \\
\hline 168 & $15\{63\}$ & $\mathbf{1 1}\{313\}$ & $\mathbf{9}\{63,313\}$ & $\begin{array}{l}\mathrm{CC}(\mathrm{C})(\mathrm{C}) \mathrm{OC}(=\mathrm{O}) \mathrm{N} 1 \mathrm{C}[\mathrm{C} @ \mathrm{H}] 2 \mathrm{CN}(\mathrm{C}[\mathrm{C} @ \mathrm{H}] 2 \mathrm{C} 1) \\
\text { c1nnnn1CC1CC1 }\end{array}$ & 22 \\
\hline 169 & $15\{63\}$ & $\mathbf{1 1}\{314\}$ & $\mathbf{9}\{63,314\}$ & $\begin{array}{l}\mathrm{CC}(\mathrm{C})(\mathrm{C}) \mathrm{OC}(=\mathrm{O}) \mathrm{N} 1 \mathrm{CCCN}(\mathrm{CC} 1) \mathrm{c} 1 \mathrm{nnnn} 1 \mathrm{CC} 1 \mathrm{C} \\
\mathrm{C} 1\end{array}$ & 43 \\
\hline 170 & $15\{63\}$ & $\mathbf{1 1}\{310\}$ & $\mathbf{9}\{63,310\}$ & $\begin{array}{l}\mathrm{CC} 1 \mathrm{CN}(\mathrm{CCC} 1 \mathrm{NC}(=\mathrm{O}) \mathrm{OC}(\mathrm{C})(\mathrm{C}) \mathrm{C}) \mathrm{c} 1 \mathrm{nnnn} 1 \mathrm{CC} 1 \\
\mathrm{CC} 1\end{array}$ & 32 \\
\hline 171 & $15\{63\}$ & $\mathbf{1 1}\{315\}$ & $\mathbf{9}\{63,315\}$ & $\begin{array}{l}\mathrm{CC}(\mathrm{C})(\mathrm{C}) \mathrm{OC}(=\mathrm{O}) \mathrm{N} 1 \mathrm{C}[\mathrm{C} @ @ \mathrm{H}] 2 \mathrm{C}[\mathrm{C} @ \mathrm{H}] 1 \mathrm{CN} 2 \mathrm{c} \\
\text { 1nnnn1CC1CC1 }\end{array}$ & 0 \\
\hline 172 & $15\{63\}$ & $\mathbf{1 1}\{312\}$ & $\mathbf{9}\{63,312\}$ & $\begin{array}{l}\mathrm{CC}(\mathrm{C})(\mathrm{C}) \mathrm{OC}(=\mathrm{O}) \mathrm{NC} 1 \mathrm{CCCN}(\mathrm{C} 1) \mathrm{c} 1 \mathrm{nnnn} 1 \mathrm{CC} 1 \mathrm{C} \\
\mathrm{C} 1\end{array}$ & 0 \\
\hline 173 & $15\{63\}$ & $\mathbf{1 1}\{359\}$ & $\mathbf{9}\{63,359\}$ & $\begin{array}{l}\mathrm{O}=\mathrm{c} 1 \mathrm{cccc} 2[\mathrm{C} @ @ \mathrm{H}] 3 \mathrm{C}[\mathrm{C} @ @ \mathrm{H}](\mathrm{CN}(\mathrm{C} 3) \mathrm{c} 3 \mathrm{nnnn} \\
3 \mathrm{CC} 3 \mathrm{CC} 3) \mathrm{Cn} 12\end{array}$ & 0 \\
\hline 174 & $15\{63\}$ & $\mathbf{1 1}\{360\}$ & $\mathbf{9}\{63,360\}$ & $\begin{array}{l}\mathrm{CC}(\mathrm{C})(\mathrm{C}) \mathrm{OC}(=\mathrm{O}) \mathrm{NC} 1 \mathrm{CCN}(\mathrm{CC} 1) \mathrm{c} 1 \mathrm{nnnn} 1 \mathrm{CC} 1 \mathrm{C} \\
\mathrm{C} 1\end{array}$ & 22 \\
\hline 175 & $15\{63\}$ & $\mathbf{1 1}\{361\}$ & $\mathbf{9}\{63,361\}$ & ${ }_{1} \mathrm{CC}(\mathrm{C})(\mathrm{C}) \mathrm{OC}(=\mathrm{O}) \mathrm{N} 1 \mathrm{CCN}(\mathrm{CC} 1) \mathrm{c} 1 \mathrm{nnnn} 1 \mathrm{CC} 1 \mathrm{CC}$ & 0 \\
\hline
\end{tabular}




\begin{tabular}{|c|c|c|c|c|c|}
\hline 176 & $15\{63\}$ & $11\{327\}$ & $\mathbf{9}\{63,327\}$ & $\mathrm{C}(\mathrm{C} 1 \mathrm{CC} 1) \mathrm{n} 1 \mathrm{nnnc} 1 \mathrm{~N} 1 \mathrm{CCCC} 1 \mathrm{c} 1 \mathrm{ccsc} 1$ & 106 \\
\hline 177 & $15\{63\}$ & $11\{306\}$ & $\mathbf{9}\{63,306\}$ & $\begin{array}{l}\mathrm{CC}(\mathrm{C})(\mathrm{C}) \mathrm{OC}(=\mathrm{O}) \mathrm{NCC} 1 \mathrm{CCCN}(\mathrm{C} 1) \mathrm{c} 1 \mathrm{nnnn} 1 \mathrm{CC} 1 \\
\mathrm{CC} 1\end{array}$ & 37 \\
\hline 178 & $15\{63\}$ & $11\{308\}$ & $\mathbf{9}\{63,308\}$ & $\begin{array}{l}\mathrm{CC}(\mathrm{C})(\mathrm{C}) \mathrm{OC}(=\mathrm{O}) \mathrm{NCC} 1 \mathrm{CCN}(\mathrm{CC} 1) \mathrm{c} 1 \mathrm{nnnn} 1 \mathrm{CC} 1 \\
\mathrm{CC} 1\end{array}$ & 21 \\
\hline 179 & $15\{64\}$ & $11\{322\}$ & $\mathbf{9}\{64,322\}$ & $\mathrm{CN}(\mathrm{C}) \mathrm{C}(=\mathrm{O}) \mathrm{c} 1 \mathrm{ccc}(\mathrm{Cn} 2 \mathrm{nnnn} 2 \mathrm{~N}(\mathrm{C}) \mathrm{C} 2 \mathrm{CC} 2) \mathrm{cc} 1$ & 26 \\
\hline 180 & $15\{64\}$ & $11\{356\}$ & $\mathbf{9}\{64,356\}$ & ${ }_{1}^{\mathrm{CN}}(\mathrm{C}) \mathrm{C}(=\mathrm{O}) \mathrm{c} 1 \mathrm{ccc}(\mathrm{Cn} 2 \mathrm{nnn} \operatorname{c} 2 \mathrm{~N}(\mathrm{C}) \mathrm{CC} 2 \mathrm{CCC} 2) \mathrm{cc}$ & 32 \\
\hline 181 & $15\{65\}$ & $11\{355\}$ & $\mathbf{9}\{65,355\}$ & ${ }_{\mathrm{F}}^{\mathrm{CN}(\mathrm{Cc} 1 \mathrm{cnn}(\mathrm{C}) \mathrm{c} 1) \mathrm{c} 1 \mathrm{nnnn} 1 \mathrm{C} 1 \mathrm{CCC}(\mathrm{CC} 1) \mathrm{C}(\mathrm{F})(\mathrm{F})}$ & 17 \\
\hline 182 & $15\{66\}$ & $11\{338\}$ & $\mathbf{9}\{66,338\}$ & CCC1CN(CC(CC)O1)c1nnnn1CCC1CCCO1 & 62 \\
\hline 183 & $15\{67\}$ & $11\{300\}$ & $\mathbf{9}\{67,300\}$ & Clc1ccc(Cn2nnnc2N2CCCOCC2)cn1 & 11 \\
\hline 184 & $15\{67\}$ & $11\{338\}$ & $\mathbf{9}\{67,338\}$ & $\mathrm{CCC} 1 \mathrm{CN}(\mathrm{CC}(\mathrm{CC}) \mathrm{O} 1) \mathrm{c} 1 \mathrm{nnnn} 1 \mathrm{Cc} 1 \mathrm{ccc}(\mathrm{Cl}) \mathrm{nc} 1$ & 26 \\
\hline 185 & $15\{68\}$ & $11\{322\}$ & $\mathbf{9}\{68,322\}$ & $\mathrm{COc} 1 \mathrm{ccc}(\mathrm{OC}) \mathrm{c}(\mathrm{Cn} 2 \mathrm{nnnn} 2 \mathrm{~N}(\mathrm{C}) \mathrm{C} 2 \mathrm{CC} 2) \mathrm{c} 1$ & 43 \\
\hline 186 & $15\{68\}$ & $11\{300\}$ & $\mathbf{9}\{68,300\}$ & $\mathrm{COc} 1 \mathrm{ccc}(\mathrm{OC}) \mathrm{c}(\mathrm{Cn} 2 \mathrm{nnnn} 2 \mathrm{~N} 2 \mathrm{CCCOCC} 2) \mathrm{c} 1$ & 72 \\
\hline 187 & $15\{69\}$ & $\mathbf{1 1}\{65\}$ & $\mathbf{9}\{69,65\}$ & $\mathrm{FC} 1=\mathrm{CCCN}(\mathrm{C} 1) \mathrm{c} 1 \mathrm{nnnn} 1 \mathrm{CCNC}(=\mathrm{O}) \mathrm{c} 1 \mathrm{cccs} 1$ & 0 \\
\hline 188 & $15\{70\}$ & $11\{362\}$ & $\mathbf{9}\{70,362\}$ & $\begin{array}{l}\text { Cc1ccc(cc1F)C }(=O) N C C n 1 n n n c 1 N 1 C C C[C @ @ ~ \\
\mathrm{H}](\mathrm{O}) \mathrm{C} 1\end{array}$ & 0 \\
\hline 189 & $15\{71\}$ & $11\{297\}$ & $\mathbf{9}\{71,297\}$ & 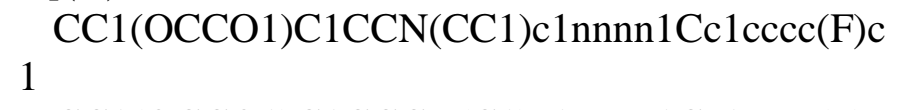 & 0 \\
\hline 190 & $15\{71\}$ & $11\{298\}$ & $\mathbf{9}\{71,298\}$ & ${ }_{1}^{\mathrm{CC} 1(\mathrm{OCCO} 1) \mathrm{C} 1 \mathrm{CCCN}(\mathrm{C} 1) \mathrm{c} 1 \mathrm{nnnn} 1 \mathrm{Cc} 1 \mathrm{cccc}(\mathrm{F}) \mathrm{c}}$ & 25 \\
\hline 191 & $15\{72\}$ & $11\{318\}$ & $\mathbf{9}\{72,318\}$ & C1CC(C1)n1nnnc1N1CCn2c(C1)nnc2-c1 1ccccc1 & 0 \\
\hline 192 & $15\{73\}$ & $11\{322\}$ & $\mathbf{9}\{73,322\}$ & $\mathrm{COc} 1 \mathrm{ccc}(\mathrm{Cn} 2 \mathrm{nnnc} 2 \mathrm{~N}(\mathrm{C}) \mathrm{C} 2 \mathrm{CC} 2) \mathrm{c}(\mathrm{OC}) \mathrm{c} 1$ & 0 \\
\hline 193 & $15\{73\}$ & $11\{300\}$ & $\mathbf{9}\{73,300\}$ & $\mathrm{COc} 1 \mathrm{ccc}(\mathrm{Cn} 2 \mathrm{nnnn} 2 \mathrm{~N} 2 \mathrm{CCCOCC} 2) \mathrm{c}(\mathrm{OC}) \mathrm{c} 1$ & 0 \\
\hline 194 & $\mathbf{1 5}\{74\}$ & $11\{299\}$ & $\mathbf{9}\{74,299\}$ & CCOc1cccc1 $1 \mathrm{Cn} 1 \mathrm{nnnn} 1 \mathrm{~N} 1 \mathrm{CCC}(\mathrm{CO})(\mathrm{CC} 1) \mathrm{OC}$ & 41 \\
\hline 195 & $\mathbf{1 5}\{74\}$ & $11\{300\}$ & $\mathbf{9}\{74,300\}$ & CCOc1cccc1Cn1nnnc1N1CCCOCC1 & 44 \\
\hline
\end{tabular}




\begin{tabular}{|c|c|c|c|c|c|}
\hline 196 & $15\{75\}$ & $11\{320\}$ & $\mathbf{9}\{75,320\}$ & $\operatorname{COC}(=\mathrm{O}) \mathrm{C} 1 \mathrm{CCN}(\mathrm{CC} 1) \mathrm{c} 1 \mathrm{nnnnn} 1 \mathrm{CC} 1 \mathrm{ccccc} 1 \mathrm{Cl}$ & 54 \\
\hline 197 & $\mathbf{1 5}\{75\}$ & $\mathbf{1 1}\{335\}$ & $\mathbf{9}\{75,335\}$ & $\begin{array}{l}\text { Clc1ccccc1CCn1nnnc1N1C }[\mathrm{C} @ \mathrm{H}] 2[\mathrm{C} @ @ \mathrm{H}](\mathrm{C} \\
\text { 1)C(=O)NC2=O }\end{array}$ & 0 \\
\hline 198 & $15\{75\}$ & $11\{300\}$ & $\mathbf{9}\{75,300\}$ & Clc1cccc1CCn1nnnc1N1CCCOCC1 & 42 \\
\hline 199 & $15\{76\}$ & $11\{337\}$ & $\mathbf{9}\{76,337\}$ & $\mathrm{CN}(\mathrm{CC} 1 \mathrm{CCOC} 1) \mathrm{c} 1 \mathrm{nnnn} 1 \mathrm{Cc} 1 \mathrm{ccccc} 1 \mathrm{C}(\mathrm{F})(\mathrm{F}) \mathrm{F}$ & 42 \\
\hline 200 & $15\{77\}$ & $\mathbf{1 1}\{363\}$ & $\mathbf{9}\{77,363\}$ & $\mathrm{COCC} 1(\mathrm{C}) \mathrm{CN}(\mathrm{CCO} 1) \mathrm{c} 1 \mathrm{nnnn} 1 \mathrm{Cc} 1 \mathrm{ccc}(\mathrm{F}) \mathrm{c}(\mathrm{F}) \mathrm{c} 1$ & 72 \\
\hline 201 & $15\{77\}$ & $11\{364\}$ & $\mathbf{9}\{77,364\}$ & $\mathrm{CN}(\mathrm{CC} 1(\mathrm{C}) \mathrm{COC} 1) \mathrm{c} 1 \mathrm{nnnn} 1 \mathrm{Cc} 1 \mathrm{ccc}(\mathrm{F}) \mathrm{c}(\mathrm{F}) \mathrm{c} 1$ & 0 \\
\hline 202 & $15\{77\}$ & $\mathbf{1 1}\{299\}$ & $\mathbf{9}\{77,299\}$ & $\mathrm{COC} 1(\mathrm{CO}) \mathrm{CCN}(\mathrm{CC} 1) \mathrm{c} 1 \mathrm{nnnn} 1 \mathrm{Cc} 1 \mathrm{ccc}(\mathrm{F}) \mathrm{c}(\mathrm{F}) \mathrm{c} 1$ & 50 \\
\hline 203 & $\mathbf{1 5}\{78\}$ & $\mathbf{1 1}\{298\}$ & $\mathbf{9}\{78,298\}$ & ${ }_{1}^{\mathrm{Cc} 1 \mathrm{ccc}(\mathrm{Cn} 2 \mathrm{nnnn} 2 \mathrm{~N} 2 \mathrm{CCCC}(\mathrm{C} 2) \mathrm{C} 2(\mathrm{C}) \mathrm{OCCO} 2) \mathrm{o}}$ & 31 \\
\hline 204 & $15\{78\}$ & $11\{297\}$ & $\mathbf{9}\{78,297\}$ & 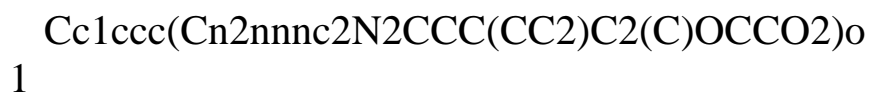 & 23 \\
\hline 205 & $15\{79\}$ & $\mathbf{1 1}\{365\}$ & $\mathbf{9}\{79,365\}$ & $\mathrm{CC} 1 \mathrm{CC}(\mathrm{CN} 1 \mathrm{c} 1 \mathrm{nnnn} 1 \mathrm{OC} 1 \mathrm{CCCCO} 1) \mathrm{c} 1 \mathrm{ccc}(\mathrm{C}) \mathrm{cc} 1$ & 0 \\
\hline 206 & $15\{79\}$ & $\mathbf{1 1}\{327\}$ & $\mathbf{9}\{79,327\}$ & $\mathrm{C} 1 \mathrm{CC}(\mathrm{N}(\mathrm{C} 1) \mathrm{c} 1 \mathrm{nnnn} 1 \mathrm{OC} 1 \mathrm{CCCCO} 1) \mathrm{c} 1 \mathrm{ccsc} 1$ & 0 \\
\hline 207 & $\mathbf{1 5}\{80\}$ & $11\{328\}$ & $\mathbf{9}\{80,328\}$ & $\mathrm{Fc} 1 \mathrm{ccc}(\mathrm{Cn} 2 \mathrm{nnnn} 2 \mathrm{~N} 2 \mathrm{CCOCC} 2) \mathrm{c}(\mathrm{c} 1) \mathrm{C}(\mathrm{F})(\mathrm{F}) \mathrm{F}$ & 18 \\
\hline 208 & $\mathbf{1 5}\{81\}$ & $\mathbf{1 1}\{298\}$ & $\mathbf{9}\{81,298\}$ & Cc1ccsc1Cn1nnnc1N1CCCC(C1)C1(C)OCCO1 & 23 \\
\hline 209 & $\mathbf{1 5}\{81\}$ & $11\{366\}$ & $\mathbf{9}\{81,366\}$ & $\mathrm{CN}(\mathrm{CC}(\mathrm{C})(\mathrm{C}) \mathrm{O}) \mathrm{c} 1 \mathrm{nnnn} 1 \mathrm{Cc} 1 \mathrm{sccc} 1 \mathrm{C}$ & 0 \\
\hline 210 & $\mathbf{1 5}\{81\}$ & $\mathbf{1 1}\{297\}$ & $\mathbf{9}\{81,297\}$ & Cc1ccsc1Cn1nnnc1N1CCC(CC1)C1(C)OCCO1 & 38 \\
\hline 211 & $\mathbf{1 5}\{81\}$ & $\mathbf{1 1}\{367\}$ & $\mathbf{9}\{81,367\}$ & $\mathrm{CN}(\mathrm{CC}(=\mathrm{O}) \mathrm{N} 1 \mathrm{CCOCC} 1) \mathrm{c} 1 \mathrm{nnnn} 1 \mathrm{Cc} 1 \mathrm{secc} 1 \mathrm{C}$ & 0 \\
\hline 212 & $\mathbf{1 5}\{81\}$ & $11\{368\}$ & $\mathbf{9}\{81,368\}$ & $\mathrm{Cc} 1 \mathrm{ccsc} 1 \mathrm{Cn} 1 \mathrm{nnn} \operatorname{1N} 1 \mathrm{CCCC}(\mathrm{C} 1) \mathrm{S}(\mathrm{C})(=\mathrm{O})=\mathrm{O}$ & 0 \\
\hline 213 & $\mathbf{1 5}\{82\}$ & $\mathbf{1 1}\{369\}$ & $\mathbf{9}\{82,369\}$ & $\mathrm{CC} 1 \mathrm{CN}(\mathrm{CCO} 1) \mathrm{c} 1 \mathrm{nnnn} 1 \mathrm{Cc} 1 \mathrm{ccccc} 1 \mathrm{Br}$ & 33 \\
\hline 214 & $\mathbf{1 5}\{83\}$ & $\mathbf{1 1}\{298\}$ & $\mathbf{9}\{83,298\}$ & $\mathrm{CCOCCn} 1 \mathrm{nnnc} 1 \mathrm{~N} 1 \mathrm{CCCC}(\mathrm{C} 1) \mathrm{C} 1(\mathrm{C}) \mathrm{OCCO} 1$ & 41 \\
\hline 215 & $\mathbf{1 5}\{83\}$ & $11\{297\}$ & $\mathbf{9}\{83,297\}$ & CCOCCn1nnnc1N1CCC(CC1)C1(C)OCCO1 & 28 \\
\hline
\end{tabular}




\begin{tabular}{|c|c|c|c|c|c|}
\hline 216 & $\mathbf{1 5}\{84\}$ & $\mathbf{1 1}\{319\}$ & $\mathbf{9}\{84,319\}$ & $\mathrm{CC}(\mathrm{C}) \mathrm{Oc} 1 \mathrm{cccc}(\mathrm{Cn} 2 \mathrm{nnn} \mathrm{2} 2 \mathrm{~N}(\mathrm{C}) \mathrm{CC} 2 \mathrm{COCCO} 2) \mathrm{c} 1$ & 70 \\
\hline 217 & $\mathbf{1 5}\{85\}$ & $\mathbf{1 1}\{319\}$ & $\mathbf{9}\{85,319\}$ & $\mathrm{CC}(\mathrm{C}) \mathrm{Oc} 1 \mathrm{ccccc} 1 \mathrm{Cn} 1 \mathrm{nnn} n 1 \mathrm{~N}(\mathrm{C}) \mathrm{CC} 1 \mathrm{COCCO} 1$ & 56 \\
\hline 218 & $\mathbf{1 5}\{86\}$ & $\mathbf{1 1}\{337\}$ & $\mathbf{9}\{86,337\}$ & $\mathrm{CN}(\mathrm{CC} 1 \mathrm{CCOC} 1) \mathrm{c} 1 \mathrm{nnnn} 1 \mathrm{CCOc} 1 \mathrm{cccc} 1 \mathrm{Cl}$ & 0 \\
\hline 219 & $\mathbf{1 5}\{87\}$ & $\mathbf{1 1}\{321\}$ & $\mathbf{9}\{87,321\}$ & OCC1CCN(CC1)c1nnnn1Cc1 $\operatorname{ccc}(\mathrm{Cl}) \mathrm{c}(\mathrm{Cl}) \mathrm{c} 1$ & 44 \\
\hline 220 & $\mathbf{1 5}\{88\}$ & $\mathbf{1 1}\{319\}$ & $\mathbf{9}\{88,319\}$ & $\mathrm{CN}(\mathrm{CC} 1 \mathrm{COCCO} 1) \mathrm{c} 1 \mathrm{nnnn} 1 \mathrm{Cc} 1 \mathrm{ccc}(\mathrm{F}) \mathrm{c}(\mathrm{Cl}) \mathrm{c} 1$ & 41 \\
\hline 221 & $\mathbf{1 5}\{89\}$ & $\mathbf{1 1}\{110\}$ & $\mathbf{9}\{89,110\}$ & $\mathrm{CN}(\mathrm{CC} 1(\mathrm{O}) \mathrm{CCOCC} 1) \mathrm{c} 1 \mathrm{nnnn} 1 \mathrm{Cc} 1 \mathrm{ccc}(\mathrm{F}) \mathrm{cc} 1 \mathrm{~F}$ & 0 \\
\hline 222 & $\mathbf{1 5}\{89\}$ & $\mathbf{1 1}\{370\}$ & $\mathbf{9}\{89,370\}$ & $\mathrm{CN}(\mathrm{CC} 1 \mathrm{CCCOC} 1) \mathrm{c} 1 \mathrm{nnnn} 1 \mathrm{Cc} 1 \mathrm{ccc}(\mathrm{F}) \mathrm{cc} 1 \mathrm{~F}$ & 35 \\
\hline 223 & $\mathbf{1 5}\{89\}$ & $\mathbf{1 1}\{299\}$ & $\mathbf{9}\{89,299\}$ & $\mathrm{COC} 1(\mathrm{CO}) \mathrm{CCN}(\mathrm{CC} 1) \mathrm{c} 1 \mathrm{nnnn} 1 \mathrm{Cc} 1 \mathrm{ccc}(\mathrm{F}) \mathrm{cc} 1 \mathrm{~F}$ & 33 \\
\hline 224 & $\mathbf{1 5}\{90\}$ & $\mathbf{1 1}\{371\}$ & $\mathbf{9}\{90,371\}$ & $\mathrm{CC}(\mathrm{C}) \mathrm{OCC} 1 \mathrm{nnnn} 1 \mathrm{~N} 1 \mathrm{CCOC} 2(\mathrm{CCc} 3 \operatorname{cccc} 23) \mathrm{C} 1$ & 73 \\
\hline 225 & $\mathbf{1 5}\{91\}$ & $\mathbf{1 1}\{372\}$ & $\mathbf{9}\{91,372\}$ & $\begin{array}{l}\mathrm{C}[1 \mathrm{C} @ @ \mathrm{H}] 1 \mathrm{C}[1 \mathrm{C} @ \mathrm{H}](\mathrm{O}) \mathrm{CN}(\mathrm{C} 1) \mathrm{c} 1 \mathrm{nnnn} 1 \mathrm{CCc} 1 \\
\mathrm{c}[\mathrm{nH}] \mathrm{c} 2 \mathrm{ccc}(\mathrm{F}) \mathrm{cc} 12\end{array}$ & 0 \\
\hline 226 & $\mathbf{1 5}\{92\}$ & $\mathbf{1 1}\{322\}$ & $\mathbf{9}\{92,322\}$ & COc1cccc(Cn2nnnc2N(C)C2CC2)c1OC & 43 \\
\hline 227 & $\mathbf{1 5}\{93\}$ & $\mathbf{1 1}\{344\}$ & $\mathbf{9}\{93,344\}$ & $\mathrm{Cc} 1 \mathrm{ccc}(\mathrm{Cn} 2 \mathrm{nnn} \operatorname{coN} 2 \mathrm{CCN}(\mathrm{C} 2) \mathrm{C}(=\mathrm{O}) \mathrm{C} 2 \mathrm{CC} 2) \mathrm{s} 1$ & 0 \\
\hline 228 & $\mathbf{1 5}\{93\}$ & $\mathbf{1 1}\{367\}$ & $\mathbf{9}\{93,367\}$ & $\mathrm{CN}(\mathrm{CC}(=\mathrm{O}) \mathrm{N} 1 \mathrm{CCOCC} 1) \mathrm{c} 1 \mathrm{nnnn} 1 \mathrm{Cc} 1 \mathrm{ccc}(\mathrm{C}) \mathrm{s} 1$ & 0 \\
\hline 229 & $\mathbf{1 5}\{93\}$ & $\mathbf{1 1}\{297\}$ & $\mathbf{9}\{93,297\}$ & ${ }_{1}^{\mathrm{Cc} 1 \mathrm{ccc}(\mathrm{Cn} 2 \mathrm{nnnn} 2 \mathrm{~N} 2 \mathrm{CCC}(\mathrm{CC} 2) \mathrm{C} 2(\mathrm{C}) \mathrm{OCCO} 2) \mathrm{s}}$ & 39 \\
\hline 230 & $\mathbf{1 5}\{93\}$ & $\mathbf{1 1}\{373\}$ & $\mathbf{9}\{93,373\}$ & $\begin{array}{l}\mathrm{CC}(\mathrm{C}) \mathrm{NC}(=\mathrm{O})[\mathrm{C} @ \mathrm{H}] 1 \mathrm{CCCN}(\mathrm{C} 1) \mathrm{c} 1 \mathrm{nnnn} 1 \mathrm{Cc} 1 \mathrm{cc} \\
\mathrm{c}(\mathrm{C}) \mathrm{s} 1\end{array}$ & 0 \\
\hline 231 & $\mathbf{1 5}\{93\}$ & $\mathbf{1 1}\{298\}$ & $\mathbf{9}\{93,298\}$ & 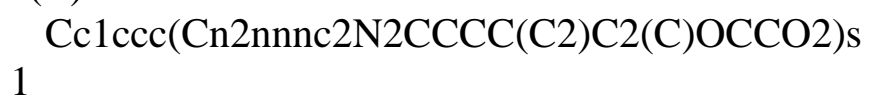 & 0 \\
\hline 232 & $\mathbf{1 5}\{93\}$ & $\mathbf{1 1}\{374\}$ & $\mathbf{9}\{93,374\}$ & Cc1noc(n1)C1CCN(C1)c1nnnn1Cc1ccc(C)s1 & 0 \\
\hline 233 & $\mathbf{1 5}\{94\}$ & $\mathbf{1 1}\{299\}$ & $\mathbf{9}\{94,299\}$ & $\mathrm{COC} 1(\mathrm{CO}) \mathrm{CCN}(\mathrm{CC} 1) \mathrm{c} 1 \mathrm{nnnn} 1 \mathrm{Cc} 1 \mathrm{cccc}(\mathrm{C}) \mathrm{c} 1$ & 34 \\
\hline 234 & $\mathbf{1 5}\{94\}$ & $\mathbf{1 1}\{375\}$ & $\mathbf{9}\{94,375\}$ & $\mathrm{Cc} 1 \mathrm{cccc}(\mathrm{Cn} 2 \mathrm{nnnn} 2 \mathrm{~N} 2 \mathrm{CCN}(\mathrm{CC} 2) \mathrm{c} 2 \mathrm{nccc} 2) \mathrm{c} 1$ & 48 \\
\hline 235 & $\mathbf{1 5}\{95\}$ & $\mathbf{1 1}\{299\}$ & $\mathbf{9}\{95,299\}$ & $\mathrm{COC} 1(\mathrm{CO}) \mathrm{CCN}(\mathrm{CC} 1) \mathrm{c} 1 \mathrm{nnnn} 1 \mathrm{Cc} 1 \mathrm{cc}(\mathrm{F}) \mathrm{ccc} 1 \mathrm{~F}$ & 17 \\
\hline
\end{tabular}




\begin{tabular}{|c|c|c|c|c|c|c|}
\hline 236 & $\mathbf{1 5}\{96\}$ & $\mathbf{1 1}\{299\}$ & $\mathbf{9}\{96,299\}$ & $\mathrm{COc} 1 \mathrm{cccc}(\mathrm{CCn} 2 \mathrm{nnnn} 2 \mathrm{~N} 2 \mathrm{CCC}(\mathrm{CO})(\mathrm{CC} 2) \mathrm{OC}) \mathrm{c} 1$ & 42 & 21 \\
\hline 237 & $\mathbf{1 5}\{97\}$ & $\mathbf{1 1}\{356\}$ & $\mathbf{9}\{97,356\}$ & $\mathrm{CCNC}(=\mathrm{O}) \mathrm{c} 1 \mathrm{ccc}(\mathrm{Cn} 2 \mathrm{nnn} \mathrm{c} 2 \mathrm{~N}(\mathrm{C}) \mathrm{CC} 2 \mathrm{CCC} 2) \mathrm{cc} 1$ & 0 & 0 \\
\hline 238 & $\mathbf{1 5}\{98\}$ & $\mathbf{1 1}\{349\}$ & $\mathbf{9}\{98,349\}$ & $\begin{array}{l}\text { C\#CCC1CCCN }(\mathrm{C} 1) \mathrm{c} 1 \mathrm{nnnn} 1 \mathrm{C} 1 \mathrm{CCN}(\mathrm{CC} 1) \mathrm{C} 1 \mathrm{CC} \\
\mathrm{CC} 1\end{array}$ & 0 & 0 \\
\hline 239 & $\mathbf{1 5}\{98\}$ & $\mathbf{1 1}\{350\}$ & $\mathbf{9}\{98,350\}$ & CN(CCC=C)c1nnnn1C1CCN(CC1)C1CCCC1 & 0 & 0 \\
\hline 240 & $\mathbf{1 5}\{99\}$ & $\mathbf{1 1}\{299\}$ & $\mathbf{9}\{99,299\}$ & $\mathrm{CCOc} 1 \mathrm{ccc}(\mathrm{Cn} 2 \mathrm{nnnn} 2 \mathrm{~N} 2 \mathrm{CCC}(\mathrm{CO})(\mathrm{CC} 2) \mathrm{OC}) \mathrm{cc} 1$ & 58 & 24 \\
\hline 241 & $\mathbf{1 5}\{100\}$ & $\mathbf{1 1}\{299\}$ & $\mathbf{9}\{100,299\}$ & $\mathrm{COC} 1(\mathrm{CO}) \mathrm{CCN}(\mathrm{CC} 1) \mathrm{c} 1 \mathrm{nnnn} 1 \mathrm{Cc} 1 \mathrm{ccc}(\mathrm{C}) \mathrm{cc} 1 \mathrm{C}$ & 31 & 16 \\
\hline 242 & $\mathbf{1 5}\{100\}$ & $\mathbf{1 1}\{376\}$ & $\mathbf{9}\{100,376\}$ & ${ }_{\mathrm{C}}^{\mathrm{COCC}(=\mathrm{O}) \mathrm{N} 1 \mathrm{CCN}(\mathrm{CC} 1) \mathrm{c} 1 \mathrm{nnnn} 1 \mathrm{Cc} 1 \mathrm{ccc}(\mathrm{C}) \mathrm{cc} 1}$ & 11 & 6 \\
\hline 243 & $\mathbf{1 5}\{101\}$ & $\mathbf{1 1}\{299\}$ & $\mathbf{9}\{101,299\}$ & $\mathrm{C}^{\mathrm{COC} 1(\mathrm{CO}) \mathrm{CCN}(\mathrm{CC} 1) \mathrm{c} 1 \mathrm{nnnnn} 1 \mathrm{Cc} 1 \mathrm{c}(\mathrm{C}) \mathrm{cc}(\mathrm{C}) \mathrm{cc} 1}$ & 14 & 7 \\
\hline 244 & $\mathbf{1 5}\{102\}$ & $\mathbf{1 1}\{377\}$ & $\mathbf{9}\{102,377\}$ & Fc1 $1 \mathrm{ccc} 2 \mathrm{OCCN}(\mathrm{Cc} 12) \mathrm{c} 1 \mathrm{nnnn} 1 \mathrm{Cc} 1 \mathrm{ccsc} 1$ & 0 & 0 \\
\hline 245 & $\mathbf{1 5}\{102\}$ & $\mathbf{1 1}\{378\}$ & $\mathbf{9}\{102,378\}$ & $\mathrm{Cc} 1 \mathrm{ccc}(\mathrm{C}) \mathrm{n} 1 \mathrm{C} 1 \mathrm{CCN}(\mathrm{CC} 1) \mathrm{c} 1 \mathrm{nnnnn} 1 \mathrm{Cc} 1 \mathrm{ccsc} 1$ & 0 & 0 \\
\hline 246 & $\mathbf{1 5}\{102\}$ & $\mathbf{1 1}\{255\}$ & $\mathbf{9}\{102,255\}$ & $\mathrm{CC} 1 \mathrm{CN}(\mathrm{CCS}(=\mathrm{O})(=\mathrm{O}) \mathrm{C} 1) \mathrm{c} 1 \mathrm{nnnn} 1 \mathrm{Cc} 1 \mathrm{ccsc} 1$ & 0 & 0 \\
\hline 247 & $\mathbf{1 5}\{102\}$ & $\mathbf{1 1}\{379\}$ & $\mathbf{9}\{102,379\}$ & $\mathrm{OC} 1(\mathrm{CCCN}(\mathrm{CC} 1) \mathrm{c} 1 \mathrm{nnnn} 1 \mathrm{Cc} 1 \mathrm{ccsc} 1) \mathrm{C}(\mathrm{F})(\mathrm{F}) \mathrm{F}$ & 0 & 0 \\
\hline 248 & $\mathbf{1 5}\{102\}$ & $\mathbf{1 1}\{184\}$ & $\mathbf{9}\{102,184\}$ & $\mathrm{COC} 1(\mathrm{CCCN}(\mathrm{C} 1) \mathrm{c} 1 \mathrm{nnnn} 1 \mathrm{Cc} 1 \mathrm{ccsc} 1) \mathrm{C}(\mathrm{F})(\mathrm{F}) \mathrm{F}$ & 0 & 0 \\
\hline 249 & $\mathbf{1 5}\{102\}$ & $\mathbf{1 1}\{380\}$ & $\mathbf{9}\{102,380\}$ & $\mathrm{CNS}(=\mathrm{O})(=\mathrm{O}) \mathrm{N} 1 \mathrm{CCN}(\mathrm{CC} 1) \mathrm{c} 1 \mathrm{nnnn} 1 \mathrm{Cc} 1 \mathrm{ccsc} 1$ & 0 & 0 \\
\hline 250 & $\mathbf{1 5}\{102\}$ & $\mathbf{1 1}\{381\}$ & $\mathbf{9}\{102,381\}$ & $\mathrm{COc} 1 \mathrm{ccc} 2 \mathrm{OCCN}(\mathrm{Cc} 2 \mathrm{c} 1) \mathrm{c} 1 \mathrm{nnnn} 1 \mathrm{Cc} 1 \mathrm{ccsc} 1$ & 0 & 0 \\
\hline 251 & $\mathbf{1 5}\{102\}$ & $\mathbf{1 1}\{382\}$ & $\mathbf{9}\{102,382\}$ & $\mathrm{C}(\mathrm{c} 1 \mathrm{ccsc} 1) \mathrm{n} 1 \mathrm{nnn} \mathrm{c} 1 \mathrm{~N} 1 \mathrm{CCCC}(\mathrm{C} 1) \mathrm{NC} 1 \mathrm{CCOCC} 1$ & 0 & 0 \\
\hline 252 & $\mathbf{1 5}\{102\}$ & $\mathbf{1 1}\{287\}$ & $\mathbf{9}\{102,287\}$ & $\mathrm{O}=\mathrm{C}(\mathrm{N} 1 \mathrm{CCN}(\mathrm{CC} 1) \mathrm{c} 1 \mathrm{nnnn} 1 \mathrm{Cc} 1 \mathrm{ccsc} 1) \mathrm{c} 1 \mathrm{ccco} 1$ & 65 & 23 \\
\hline 253 & $\mathbf{1 5}\{102\}$ & $\mathbf{1 1}\{383\}$ & $\mathbf{9}\{102,383\}$ & $\begin{array}{l}\mathrm{C}[\mathrm{C} @ @ \mathrm{H}] 1 \mathrm{CN}(\mathrm{CCN} 1 \mathrm{~S}(\mathrm{C})(=\mathrm{O})=\mathrm{O}) \mathrm{c} 1 \mathrm{nnnn} 1 \mathrm{Cc} 1 \\
\operatorname{ccsc} 1\end{array}$ & 11 & 5 \\
\hline 254 & $\mathbf{1 5}\{102\}$ & $\mathbf{1 1}\{384\}$ & $\mathbf{9}\{102,384\}$ & O=C1CCCCN1C1CCCN(C1)c1nnnn1Cc1cesc1 & 0 & 0 \\
\hline 255 & $\mathbf{1 5}\{102\}$ & $\mathbf{1 1}\{385\}$ & $\mathbf{9}\{102,385\}$ & $\mathrm{CN}(\mathrm{Cc} 1 \mathrm{nc}(\mathrm{C}) \mathrm{no1}) \mathrm{c} 1 \mathrm{nnnn} 1 \mathrm{Cc} 1 \mathrm{ccsc} 1$ & 0 & 0 \\
\hline
\end{tabular}




\begin{tabular}{|c|c|c|c|c|c|}
\hline 256 & $\mathbf{1 5}\{102\}$ & $\mathbf{1 1}\{356\}$ & $\mathbf{9}\{102,356\}$ & $\mathrm{CN}(\mathrm{CC} 1 \mathrm{CCC} 1) \mathrm{c} 1 \mathrm{nnnn} 1 \mathrm{Cc} 1 \mathrm{ccsc} 1$ & 58 \\
\hline 257 & $\mathbf{1 5}\{103\}$ & $\mathbf{1 1}\{293\}$ & $\mathbf{9}\{103,293\}$ & $\begin{array}{l}\mathrm{C}(\mathrm{C} 1 \mathrm{CCOC} 1) n 1 \mathrm{nnnc1N} 1[\mathrm{C} @ \mathrm{H}] 2 \mathrm{CC}[\mathrm{C} @ @ \mathrm{H}] 1 \\
\mathrm{C}[\mathrm{C} @ @ \mathrm{H}](\mathrm{C} 2) \mathrm{c} 1 \mathrm{cccc} 1\end{array}$ & 0 \\
\hline 258 & $\mathbf{1 5}\{103\}$ & $\mathbf{1 1}\{386\}$ & $\mathbf{9}\{103,386\}$ & $\mathrm{CN}(\mathrm{Cc} 1 \mathrm{ccc}(\mathrm{F}) \mathrm{cc} 1) \mathrm{c} 1 \mathrm{nnnn} 1 \mathrm{CC} 1 \mathrm{CCOC} 1$ & 80 \\
\hline 259 & $\mathbf{1 5}\{103\}$ & $\mathbf{1 1}\{387\}$ & $\mathbf{9}\{103,387\}$ & $\mathrm{C}(\mathrm{C} 1 \mathrm{CCOC} 1) \mathrm{n} 1 \mathrm{nnn} \mathrm{c} 1 \mathrm{~N} 1 \mathrm{CCC}(\mathrm{Cc} 2 \mathrm{cccc} 2) \mathrm{CC} 1$ & 0 \\
\hline 260 & $\mathbf{1 5}\{103\}$ & $\mathbf{1 1}\{388\}$ & $\mathbf{9}\{103,388\}$ & $\mathrm{CN}(\mathrm{Cc} 1 \mathrm{ccc}(\mathrm{Cl}) \mathrm{s} 1) \mathrm{c} 1 \mathrm{nnnn} 1 \mathrm{CC} 1 \mathrm{CCOC} 1$ & 0 \\
\hline 261 & $\mathbf{1 5}\{103\}$ & $\mathbf{1 1}\{317\}$ & $\mathbf{9}\{103,317\}$ & $\mathrm{CN}(\mathrm{Cc} 1 \mathrm{ccc}(\mathrm{C}) \mathrm{o} 1) \mathrm{c} 1 \mathrm{nnnn} 1 \mathrm{CC} 1 \mathrm{CCOC} 1$ & 0 \\
\hline 262 & $\mathbf{1 5}\{103\}$ & $\mathbf{1 1}\{389\}$ & $\mathbf{9}\{103,389\}$ & $\mathrm{CN}(\mathrm{Cc} 1 \mathrm{cccs} 1) \mathrm{c} 1 \mathrm{nnnn} 1 \mathrm{CC} 1 \mathrm{CCOC} 1$ & 22 \\
\hline 263 & $\mathbf{1 5}\{103\}$ & $\mathbf{1 1}\{390\}$ & $\mathbf{9}\{103,390\}$ & $\mathrm{CS}(=\mathrm{O})(=\mathrm{O}) \mathrm{N} 1 \mathrm{CCN}(\mathrm{CC} 1) \mathrm{c} 1 \mathrm{nnnn} 1 \mathrm{CC} 1 \mathrm{CCOC} 1$ & 7 \\
\hline 264 & $\mathbf{1 5}\{104\}$ & $\mathbf{1 1}\{391\}$ & $\mathbf{9}\{104,391\}$ & ${ }_{1} \mathrm{CN}(\mathrm{CC} 1 \mathrm{CC} 1) \mathrm{c} 1 \mathrm{nnnn} 1 \mathrm{Cc} 1 \mathrm{ccc}(\mathrm{CN} 2 \mathrm{CCCC} 2=\mathrm{O}) \mathrm{cc}$ & 41 \\
\hline 265 & $\mathbf{1 5}\{104\}$ & $\mathbf{1 1}\{322\}$ & $\mathbf{9}\{104,322\}$ & $\mathrm{CN}(\mathrm{C} 1 \mathrm{CC} 1) \mathrm{c} 1 \mathrm{nnnn} 1 \mathrm{Cc} 1 \mathrm{ccc}(\mathrm{CN} 2 \mathrm{CCCC} 2=\mathrm{O}) \mathrm{cc} 1$ & 53 \\
\hline 266 & $\mathbf{1 5}\{105\}$ & $\mathbf{1 1}\{322\}$ & $\mathbf{9}\{105,322\}$ & $\mathrm{CN}(\mathrm{C} 1 \mathrm{CC} 1) \mathrm{c} 1 \mathrm{nnnn} 1 \mathrm{Cc} 1 \mathrm{ccccc} 1 \mathrm{CN} 1 \mathrm{CCCC} 1=\mathrm{O}$ & 19 \\
\hline 267 & $\mathbf{1 5}\{105\}$ & $\mathbf{1 1}\{391\}$ & $\mathbf{9}\{105,391\}$ & $\mathrm{CN}(\mathrm{CC} 1 \mathrm{CC} 1) \mathrm{c} 1 \mathrm{nnnn} 1 \mathrm{Cc} 1 \mathrm{cccc} 1 \mathrm{CN} 1 \mathrm{CCCC} 1=\mathrm{O}$ & 41 \\
\hline 268 & $\mathbf{1 5}\{106\}$ & $\mathbf{1 1}\{392\}$ & $\mathbf{9}\{106,392\}$ & $\begin{array}{l}\mathrm{COCc} 1 \mathrm{ccc}(\mathrm{Cn} 2 \mathrm{nnn} n \mathrm{2N} 2 \mathrm{CC}(\mathrm{C}) \mathrm{OC} 3(\mathrm{CCC} 3) \mathrm{C} 2) \mathrm{c} \\
\mathrm{c} 1\end{array}$ & 48 \\
\hline 269 & $\mathbf{1 5}\{106\}$ & $\mathbf{1 1}\{61\}$ & $\mathbf{9}\{106,61\}$ & COCc1ccc(Cn2nnnc2N2CCC3(C2)CCOCC3)cc1 & 23 \\
\hline 270 & $\mathbf{1 5}\{106\}$ & $\mathbf{1 1}\{393\}$ & $\mathbf{9}\{106,393\}$ & $\mathrm{COCc} 1 \mathrm{ccc}(\mathrm{Cn} 2 \mathrm{nnnn} 2 \mathrm{~N} 2 \mathrm{CCOCC} 3(\mathrm{CCC} 3) \mathrm{C} 2) \mathrm{cc} 1$ & 37 \\
\hline 271 & $\mathbf{1 5}\{106\}$ & $\mathbf{1 1}\{394\}$ & $\mathbf{9}\{106,394\}$ & $\mathrm{COCc} 1 \mathrm{ccc}(\mathrm{Cn} 2 \mathrm{nnn} 2 \mathrm{~N} 2 \mathrm{CCOC} 3(\mathrm{CCCC} 3) \mathrm{C} 2) \mathrm{cc} 1$ & 37 \\
\hline 272 & $\mathbf{1 5}\{106\}$ & $\mathbf{1 1}\{395\}$ & $\mathbf{9}\{106,395\}$ & $\mathrm{COCc} 1 \mathrm{ccc}(\mathrm{Cn} 2 \mathrm{nnnn} 2 \mathrm{~N} 2 \mathrm{CCOC} 3(\mathrm{CCOC} 3) \mathrm{C} 2) \mathrm{cc} 1$ & 20 \\
\hline 273 & $\mathbf{1 5}\{107\}$ & $\mathbf{1 1}\{299\}$ & $\mathbf{9}\{107,299\}$ & $\mathrm{COCc} 1 \mathrm{cccc}(\mathrm{Cn} 2 \mathrm{nnn} \mathrm{2} 2 \mathrm{~N} 2 \mathrm{CCC}(\mathrm{CO})(\mathrm{CC} 2) \mathrm{OC}) \mathrm{c} 1$ & 14 \\
\hline 274 & $\mathbf{1 5}\{108\}$ & $\mathbf{1 1}\{338\}$ & $\mathbf{9}\{108,338\}$ & $\begin{aligned} & \mathrm{CCC} 1 \mathrm{CN}(\mathrm{CC}(\mathrm{CC}) \mathrm{O} 1) \mathrm{c} 1 \mathrm{nnnn} 1 \mathrm{Cc} 1 \mathrm{cccc}(\mathrm{c} 1) \mathrm{C}(\mathrm{N}) \\
= & \mathrm{O}\end{aligned}$ & 32 \\
\hline 275 & $\mathbf{1 5}\{108\}$ & $\mathbf{1 1}\{320\}$ & $\mathbf{9}\{108,320\}$ & $\begin{array}{l}\mathrm{COC}(=\mathrm{O}) \mathrm{C} 1 \mathrm{CCN}(\mathrm{CC} 1) \mathrm{c} 1 \mathrm{nnnnn} 1 \mathrm{Cc} 1 \mathrm{cccc}(\mathrm{c} 1) \mathrm{C}(\mathrm{N} \\
)=\mathrm{O}\end{array}$ & 27 \\
\hline
\end{tabular}




\begin{tabular}{|c|c|c|c|c|c|}
\hline 276 & $\mathbf{1 5}\{109\}$ & $\mathbf{1 1}\{322\}$ & $\mathbf{9}\{109,322\}$ & $\mathrm{CN}(\mathrm{C}) \mathrm{C}(=\mathrm{O}) \mathrm{c} 1 \operatorname{cccc}(\mathrm{Cn} 2 \mathrm{nnn} \mathrm{c} 2 \mathrm{~N}(\mathrm{C}) \mathrm{C} 2 \mathrm{CC} 2) \mathrm{c} 1$ & 26 \\
\hline 277 & $\mathbf{1 5}\{109\}$ & $\mathbf{1 1}\{356\}$ & $\mathbf{9}\{109,356\}$ & $\mathrm{CN}(\mathrm{C}) \mathrm{C}(=\mathrm{O}) \mathrm{c} 1 \mathrm{cccc}(\mathrm{Cn} 2 \mathrm{nnnn} 2 \mathrm{~N}(\mathrm{C}) \mathrm{CC} 2 \mathrm{CCC} 2) \mathrm{c}$ & 44 \\
\hline 278 & $\mathbf{1 5}\{110\}$ & $\mathbf{1 1}\{333\}$ & $\mathbf{9}\{110,333\}$ & $\mathrm{CNC}(=\mathrm{O}) \mathrm{c} 1 \operatorname{ccc}(\mathrm{Cn} 2 \mathrm{nnn} n \mathrm{2} 2 \mathrm{~N} 2 \mathrm{CCSC}(\mathrm{C})(\mathrm{C}) \mathrm{C} 2) \mathrm{c} 1$ & 87 \\
\hline 279 & $\mathbf{1 5}\{111\}$ & $\mathbf{1 1}\{328\}$ & $\mathbf{9}\{111,328\}$ & Clc1cc(Cn2nnnc2N2CCOCC2)cc2OCCOc12 & 57 \\
\hline 280 & $\mathbf{1 5}\{111\}$ & $\mathbf{1 1}\{396\}$ & $\mathbf{9}\{111,396\}$ & $\operatorname{COCCN}(\mathrm{C}) \mathrm{c} 1 \mathrm{nnnn} 1 \mathrm{Cc} 1 \mathrm{cc}(\mathrm{Cl}) \mathrm{c} 2 \mathrm{OCCOc} 2 \mathrm{c} 1$ & 55 \\
\hline 281 & $\mathbf{1 5}\{112\}$ & $\mathbf{1 1}\{321\}$ & $\mathbf{9}\{112,321\}$ & OCC1CCN(CC1)c1nnnn1Cc1c $(\mathrm{Cl}) \operatorname{cccc} 1 \mathrm{Cl}$ & 42 \\
\hline 282 & $\mathbf{1 5}\{113\}$ & $\mathbf{1 1}\{319\}$ & $\mathbf{9}\{113,319\}$ & $\mathrm{CN}(\mathrm{CC} 1 \mathrm{COCCO} 1) \mathrm{c} 1 \mathrm{nnnn} 1 \mathrm{CCc} 1 \mathrm{c}(\mathrm{F}) \mathrm{cccc} 1 \mathrm{~F}$ & 0 \\
\hline 283 & $\mathbf{1 5}\{114\}$ & $\mathbf{1 1}\{297\}$ & $\mathbf{9}\{114,297\}$ & $\begin{array}{l}\mathrm{Cc} 1 \mathrm{ccc}(\mathrm{CCn} 2 \mathrm{nnn} 22 \mathrm{~N} 2 \mathrm{CCC}(\mathrm{CC} 2) \mathrm{C} 2(\mathrm{C}) \mathrm{OCCO} 2) \\
\mathrm{o} 1\end{array}$ & 20 \\
\hline 284 & $\mathbf{1 5}\{114\}$ & $\mathbf{1 1}\{298\}$ & $\mathbf{9}\{114,298\}$ & $\begin{array}{l}\mathrm{Cc} 1 \mathrm{ccc}(\mathrm{CCn} 2 \mathrm{nnn} \operatorname{2N} 2 \mathrm{CCCC}(\mathrm{C} 2) \mathrm{C} 2(\mathrm{C}) \mathrm{OCCO} 2) \\
\mathrm{o} 1\end{array}$ & 29 \\
\hline 285 & $\mathbf{1 5}\{115\}$ & $\mathbf{1 1}\{302\}$ & $\mathbf{9}\{115,302\}$ & $\mathrm{CN}(\mathrm{CCOc} 1 \mathrm{ccc}(\mathrm{F}) \mathrm{cc} 1) \mathrm{c} 1 \mathrm{nnnn} 1 \mathrm{CCN} 1 \mathrm{CCNC} 1=\mathrm{O}$ & 40 \\
\hline 286 & $\mathbf{1 5}\{115\}$ & $\mathbf{1 1}\{327\}$ & $\mathbf{9}\{115,327\}$ & $\mathrm{O}=\mathrm{C} 1 \mathrm{NCCN} 1 \mathrm{CCn} 1 \mathrm{nnn} \mathrm{c} 1 \mathrm{~N} 1 \mathrm{CCCC} 1 \mathrm{c} 1 \mathrm{ccsc} 1$ & 27 \\
\hline 287 & $\mathbf{1 5}\{115\}$ & $\mathbf{1 1}\{349\}$ & $\mathbf{9}\{115,349\}$ & $\mathrm{O}=\mathrm{C} 1 \mathrm{NCCN} 1 \mathrm{CCn} 1 \mathrm{nnn} \mathrm{c} 1 \mathrm{~N} 1 \mathrm{CCCC}(\mathrm{CC} \# \mathrm{C}) \mathrm{C} 1$ & 25 \\
\hline 288 & $\mathbf{1 5}\{116\}$ & $\mathbf{1 1}\{322\}$ & $\mathbf{9}\{116,322\}$ & $\mathrm{CCCC}(=\mathrm{O}) \mathrm{Nc} 1 \mathrm{ccc}(\mathrm{Cn} 2 \mathrm{nnn} n \mathrm{2N}(\mathrm{C}) \mathrm{C} 2 \mathrm{CC} 2) \mathrm{cc} 1$ & 32 \\
\hline 289 & $\mathbf{1 5}\{117\}$ & $\mathbf{1 1}\{340\}$ & $\mathbf{9}\{117,340\}$ & $\mathrm{C}(\mathrm{c} 1 \mathrm{ccoc} 1) \mathrm{n} 1 \mathrm{nnn} \mathrm{c} 1 \mathrm{~N} 1 \mathrm{CCCCCC} 1$ & 33 \\
\hline 290 & $\mathbf{1 5}\{117\}$ & $\mathbf{1 1}\{397\}$ & $\mathbf{9}\{117,397\}$ & CSC1CCCCN(C1)c1nnnn1Cc1ccoc1 & 48 \\
\hline 291 & $\mathbf{1 5}\{118\}$ & $\mathbf{1 1}\{288\}$ & $\mathbf{9}\{118,288\}$ & $\mathrm{CC}(=\mathrm{O}) \mathrm{N} 1 \mathrm{CCCN}(\mathrm{CC} 1) \mathrm{c} 1 \mathrm{nnnn} 1 \mathrm{CCCC} 1 \mathrm{CCCC} 1$ & 52 \\
\hline 292 & $\mathbf{1 5}\{119\}$ & $\mathbf{1 1}\{322\}$ & $\mathbf{9}\{119,322\}$ & $\mathrm{CN}(\mathrm{C} 1 \mathrm{CC} 1) \mathrm{c} 1 \mathrm{nnnn} 1 \mathrm{Cc} 1 \mathrm{cccc}(\mathrm{c} 1) \mathrm{N} 1 \mathrm{CCCC} 1=\mathrm{O}$ & 48 \\
\hline 293 & $\mathbf{1 5}\{120\}$ & $\mathbf{1 1}\{344\}$ & $\mathbf{9}\{120,344\}$ & $\mathrm{Cc} 1 \mathrm{cc}(\mathrm{Cn} 2 \mathrm{nnn} n 2 \mathrm{~N} 2 \mathrm{CCN}(\mathrm{C} 2) \mathrm{C}(=\mathrm{O}) \mathrm{C} 2 \mathrm{CC} 2) \mathrm{no} 1$ & 0 \\
\hline 294 & $\mathbf{1 5}\{120\}$ & $\mathbf{1 1}\{298\}$ & $\mathbf{9}\{120,298\}$ & ${ }_{1} \mathrm{Cc} 1 \mathrm{cc}(\mathrm{Cn} 2 \mathrm{nnn} n \mathrm{2N} 2 \mathrm{CCCC}(\mathrm{C} 2) \mathrm{C} 2(\mathrm{C}) \mathrm{OCCO} 2) \mathrm{no}$ & 0 \\
\hline 295 & $\mathbf{1 5}\{120\}$ & $\mathbf{1 1}\{297\}$ & $\mathbf{9}\{120,297\}$ & ${ }_{1} \mathrm{Cc} 1 \mathrm{cc}(\mathrm{Cn} 2 \mathrm{nnn} n 2 \mathrm{~N} 2 \mathrm{CCC}(\mathrm{CC} 2) \mathrm{C} 2(\mathrm{C}) \mathrm{OCCO} 2) \mathrm{no}$ & 22 \\
\hline
\end{tabular}




\begin{tabular}{|c|c|c|c|c|c|}
\hline 296 & $\mathbf{1 5}\{121\}$ & $\mathbf{1 1}\{398\}$ & $\mathbf{9}\{121,398\}$ & $\mathrm{CC} 1 \mathrm{CN}(\mathrm{CC}(\mathrm{C}) \mathrm{O} 1) \mathrm{c} 1 \mathrm{nnnn} 1 \mathrm{CC} 1 \mathrm{CCOCC} 1$ & 29 \\
\hline 297 & $\mathbf{1 5}\{121\}$ & $\mathbf{1 1}\{302\}$ & $\mathbf{9}\{121,302\}$ & $\mathrm{CN}(\mathrm{CCOc} 1 \mathrm{ccc}(\mathrm{F}) \mathrm{cc} 1) \mathrm{c} 1 \mathrm{nnnn} 1 \mathrm{CC} 1 \mathrm{CCOCC} 1$ & 71 \\
\hline 298 & $\mathbf{1 5}\{121\}$ & $\mathbf{1 1}\{114\}$ & $\mathbf{9}\{121,114\}$ & $\begin{array}{l}\mathrm{CS}(=\mathrm{O})(=\mathrm{O}) \mathrm{C} 1 \mathrm{CCCN}(\mathrm{CC} 1) \mathrm{c} 1 \mathrm{nnnn} 1 \mathrm{CC} 1 \mathrm{CCOC} \\
\mathrm{C} 1\end{array}$ & 22 \\
\hline 299 & $\mathbf{1 5}\{121\}$ & $\mathbf{1 1}\{349\}$ & $\mathbf{9}\{121,349\}$ & C\#CCC1CCCN(C1)c1nnnn1CC1CCOCC1 & 45 \\
\hline 300 & $\mathbf{1 5}\{121\}$ & $\mathbf{1 1}\{399\}$ & $\mathbf{9}\{121,399\}$ & $\mathrm{COCC} 1(\mathrm{C}) \mathrm{CCN}(\mathrm{C} 1) \mathrm{c} 1 \mathrm{nnnn} 1 \mathrm{CC} 1 \mathrm{CCOCC} 1$ & 28 \\
\hline 301 & $\mathbf{1 5}\{122\}$ & $\mathbf{1 1}\{385\}$ & $\mathbf{9}\{122,385\}$ & $\mathrm{CN}(\mathrm{Cc} 1 \mathrm{nc}(\mathrm{C}) \mathrm{no1}) \mathrm{c} 1 \mathrm{nnnn} 1 \mathrm{Cc} 1 \mathrm{csc} 2 \mathrm{cccc} 12$ & 0 \\
\hline 302 & $\mathbf{1 5}\{123\}$ & $\mathbf{1 1}\{400\}$ & $\mathbf{9}\{123,400\}$ & $\underset{1 \mathrm{C}}{\mathrm{CN}}(\mathrm{C} 1 \mathrm{CCS}(=\mathrm{O})(=\mathrm{O}) \mathrm{C} 1) \mathrm{c} 1 \mathrm{nnnn} 1 \mathrm{Cc} 1 \mathrm{c}(\mathrm{C}) \mathrm{nn}(\mathrm{C}) \mathrm{c}$ & 0 \\
\hline 303 & $\mathbf{1 5}\{124\}$ & $\mathbf{1 1}\{322\}$ & $\mathbf{9}\{124,322\}$ & $\mathrm{CN}(\mathrm{C} 1 \mathrm{CC} 1) \mathrm{c} 1 \mathrm{nnnn} 1 \mathrm{Cc} 1 \mathrm{ccc}(\mathrm{cc} 1)-\mathrm{n} 1 \mathrm{cccn} 1$ & 41 \\
\hline 304 & $\mathbf{1 5}\{125\}$ & $\mathbf{1 1}\{319\}$ & $\mathbf{9}\{125,319\}$ & $\mathrm{CC}(\mathrm{Cn} 1 \mathrm{nnnnc} 1 \mathrm{~N}(\mathrm{C}) \mathrm{CC} 1 \mathrm{COCCO} 1) \mathrm{Oc} 1 \mathrm{ccc}(\mathrm{C}) \mathrm{cc} 1$ & 40 \\
\hline 305 & $\mathbf{1 5}\{126\}$ & $\mathbf{1 1}\{401\}$ & $\mathbf{9}\{126,401\}$ & $\begin{array}{l}\mathrm{NC}(=\mathrm{O}) \mathrm{C} 1 \mathrm{CCN}(\mathrm{CC} 1) \mathrm{c} 1 \mathrm{nnnn} 1 \mathrm{CC} 12 \mathrm{CC} 3 \mathrm{CC}(\mathrm{CC}( \\
\mathrm{C} 3) \mathrm{C} 1) \mathrm{C} 2\end{array}$ & 0 \\
\hline 306 & $\mathbf{1 5}\{127\}$ & $\mathbf{1 1}\{290\}$ & $\mathbf{9}\{127,290\}$ & $\mathrm{CC} 1 \mathrm{CN}(\mathrm{CCN} 1 \mathrm{C}(=\mathrm{O}) \mathrm{OC}(\mathrm{C})(\mathrm{C}) \mathrm{C}) \mathrm{c} 1 \mathrm{nnnn} 1 \mathrm{CC \# C}$ & 0 \\
\hline 307 & $\mathbf{1 5}\{127\}$ & $\mathbf{1 1}\{295\}$ & $\mathbf{9}\{127,295\}$ & $\mathrm{O}=\mathrm{C}(\mathrm{OCc} 1 \mathrm{ccccc} 1) \mathrm{N} 1 \mathrm{CCN}(\mathrm{CC} 1) \mathrm{c} 1 \mathrm{nnnn} 1 \mathrm{CC} \# \mathrm{C}$ & 0 \\
\hline 308 & $\mathbf{1 5}\{127\}$ & $\mathbf{1 1}\{292\}$ & $\mathbf{9}\{127,292\}$ & $\begin{array}{l}\mathrm{C}[\mathrm{C} @ \mathrm{H}] 1 \mathrm{CN}(\mathrm{CCN} 1 \mathrm{C}(=\mathrm{O}) \mathrm{OC}(\mathrm{C})(\mathrm{C}) \mathrm{C}) \mathrm{c} 1 \mathrm{nnnn} 1 \\
\mathrm{CC \# C}\end{array}$ & 0 \\
\hline 309 & $\mathbf{1 5}\{127\}$ & $\mathbf{1 1}\{296\}$ & $\mathbf{9}\{127,296\}$ & $\begin{array}{l}\mathrm{C}[\mathrm{C} @ @ \mathrm{H}] 1 \mathrm{CN}(\mathrm{CCN} 1 \mathrm{C}(=\mathrm{O}) \mathrm{OC}(\mathrm{C})(\mathrm{C}) \mathrm{C}) \mathrm{c} 1 \mathrm{nnnn} \\
1 \mathrm{CC} \# \mathrm{C}\end{array}$ & 0 \\
\hline 310 & $\mathbf{1 5}\{127\}$ & $\mathbf{1 1}\{289\}$ & $\mathbf{9}\{127,289\}$ & C\#CCn1nnnc1N1CCC(CC1)c1nc2cccce2s1 & 0 \\
\hline 311 & $\mathbf{1 5}\{128\}$ & $\mathbf{1 1}\{322\}$ & $\mathbf{9}\{128,322\}$ & $\mathrm{CN}(\mathrm{C} 1 \mathrm{CC} 1) \mathrm{c} 1 \mathrm{nnnn} 1 \mathrm{Cc} 1 \mathrm{ccc}(\mathrm{Cn} 2 \mathrm{cccn} 2) \mathrm{cc} 1$ & 41 \\
\hline 312 & $\mathbf{1 5}\{129\}$ & $\mathbf{1 1}\{288\}$ & $\mathbf{9}\{129,288\}$ & $\mathrm{CC}(=\mathrm{O}) \mathrm{N} 1 \mathrm{CCCN}(\mathrm{CC} 1) \mathrm{c} 1 \mathrm{nnnn} 1 \mathrm{C} 1 \mathrm{Cc} 2 \operatorname{cccc} 2 \mathrm{C} 1$ & 29 \\
\hline 313 & $\mathbf{1 5}\{130\}$ & $\mathbf{1 1}\{302\}$ & $\mathbf{9}\{130,302\}$ & $\mathrm{CN}(\mathrm{CCOc} 1 \mathrm{ccc}(\mathrm{F}) \mathrm{cc} 1) \mathrm{c} 1 \mathrm{nnnn} 1 \mathrm{C} 1 \mathrm{CCOCC} 1$ & 0 \\
\hline 314 & $\mathbf{1 5}\{130\}$ & $\mathbf{1 1}\{327\}$ & $\mathbf{9}\{130,327\}$ & $\mathrm{C} 1 \mathrm{CC}(\mathrm{N}(\mathrm{C} 1) \mathrm{c} 1 \mathrm{nnnn} 1 \mathrm{C} 1 \mathrm{CCOCC} 1) \mathrm{c} 1 \mathrm{ccsc} 1$ & 30 \\
\hline 315 & $\mathbf{1 5}\{130\}$ & $\mathbf{1 1}\{365\}$ & $\mathbf{9}\{130,365\}$ & $\mathrm{CC} 1 \mathrm{CC}(\mathrm{CN} 1 \mathrm{c} 1 \mathrm{nnnn} 1 \mathrm{C} 1 \mathrm{CCOCC} 1) \mathrm{c} 1 \mathrm{ccc}(\mathrm{C}) \mathrm{cc} 1$ & 27 \\
\hline
\end{tabular}




\begin{tabular}{|c|c|c|c|c|c|}
\hline 316 & $15\{131\}$ & $11\{401\}$ & $\mathbf{9}\{131,401\}$ & $2^{\mathrm{NC}(=\mathrm{O}) \mathrm{C} 1 \mathrm{CCN}(\mathrm{CC} 1) \mathrm{c} 1 \mathrm{nnnn} 1 \mathrm{CC} 1 \mathrm{CCC} 2 \mathrm{cccc} 1}$ & 0 \\
\hline 317 & $15\{132\}$ & $\mathbf{1 1}\{321\}$ & $\mathbf{9}\{132,321\}$ & OCC1CCN(CC1)c1nnnn1Cc1ccc(o1)-c1 1cccce1 & 0 \\
\hline 318 & $15\{133\}$ & $11\{402\}$ & $\mathbf{9}\{133,402\}$ & $\mathrm{CC} 1 \mathrm{CN}(\mathrm{CC} 2(\mathrm{CCCCC} 2) \mathrm{O} 1) \mathrm{c} 1 \mathrm{nnnn} 1 \mathrm{Cc} 1 \mathrm{ccnn} 1 \mathrm{C}$ & 8 \\
\hline 319 & $\mathbf{1 5}\{133\}$ & $\mathbf{1 1}\{403\}$ & $\mathbf{9}\{133,403\}$ & $\mathrm{Cn} 1 \mathrm{nccc} 1 \mathrm{Cn} 1 \mathrm{nnn}$ 1N1CCC2(C)C(C1)C2(Cl)Cl & 5 \\
\hline 320 & $\mathbf{1 5}\{133\}$ & $11\{404\}$ & $\mathbf{9}\{133,404\}$ & $\begin{array}{l}\mathrm{COC}(=\mathrm{O})[1 \mathrm{C} @] 12 \mathrm{CN}(\mathrm{C}[1 \mathrm{C} @ \mathrm{H}] 1 \mathrm{CCCC} 2) \mathrm{c} 1 \mathrm{nnn} \\
\text { n1Cc1ccnn1C }\end{array}$ & 45 \\
\hline 321 & $\mathbf{1 5}\{133\}$ & $\mathbf{1 1}\{405\}$ & $\mathbf{9}\{133,405\}$ & Cn1nccc1Cn1nnnc1N1CCCC2(CCCCC2)C1 & 30 \\
\hline 322 & $\mathbf{1 5}\{133\}$ & $11\{406\}$ & $\mathbf{9}\{133,406\}$ & $\mathrm{CC} 1 \mathrm{CCN}(\mathrm{CC} 11 \mathrm{CCC} 1) \mathrm{c} 1 \mathrm{nnnn} 1 \mathrm{Cc} 1 \mathrm{ccnn} 1 \mathrm{C}$ & 36 \\
\hline 323 & $\mathbf{1 5}\{133\}$ & $\mathbf{1 1}\{407\}$ & $\mathbf{9}\{133,407\}$ & Cn1ncce1Cn1nnnc1N1CCC2(C1)CCCCC2 & 50 \\
\hline 324 & $\mathbf{1 5}\{133\}$ & $\mathbf{1 1}\{408\}$ & $\mathbf{9}\{133,408\}$ & Cn1ncce1Cn1nnnc1N1CCCC2(CCCC2)C1 & 36 \\
\hline 325 & $\mathbf{1 5}\{133\}$ & $\mathbf{1 1}\{409\}$ & $\mathbf{9}\{133,409\}$ & Cn1ncce1Cn1nnnc1N1CCSC2(CCCCC2)C1 & 11 \\
\hline 326 & $\mathbf{1 5}\{133\}$ & $\mathbf{1 1}\{410\}$ & $\mathbf{9}\{133,410\}$ & Cn1nccc1Cn1nnnc1N1CCC2(CCCCC2)CC1 & 11 \\
\hline 327 & $\mathbf{1 5}\{133\}$ & $\mathbf{1 1}\{411\}$ & $\mathbf{9}\{133,411\}$ & $\mathrm{CCC} 1 \mathrm{CCCN}(\mathrm{C} 1) \mathrm{c} 1 \mathrm{nnnnn} 1 \mathrm{Cc} 1 \mathrm{ccnn} 1 \mathrm{C}$ & 39 \\
\hline 328 & $\mathbf{1 5}\{133\}$ & $\mathbf{1 1}\{412\}$ & $\mathbf{9}\{133,412\}$ & $\mathrm{CON}(\mathrm{CC} 1 \mathrm{CC} 2 \mathrm{CCC} 1 \mathrm{C} 2) \mathrm{c} 1 \mathrm{nnnn} 1 \mathrm{Cc} 1 \mathrm{ccnn} 1 \mathrm{C}$ & 0 \\
\hline 329 & $\mathbf{1 5}\{133\}$ & $\mathbf{1 1}\{413\}$ & $\mathbf{9}\{133,413\}$ & $\mathrm{CC} 1 \mathrm{CN}(\mathrm{CC} 2(\mathrm{CCCC} 2) \mathrm{O} 1) \mathrm{c} 1 \mathrm{nnnn} 1 \mathrm{Cc} 1 \mathrm{ccnn} 1 \mathrm{C}$ & 17 \\
\hline 330 & $15\{134\}$ & $\mathbf{1 1}\{414\}$ & $\mathbf{9}\{134,414\}$ & $\mathrm{CN}(\mathrm{Cc} 1 \mathrm{nccs} 1) \mathrm{c} 1 \mathrm{nnnn} 1 \mathrm{C} 1 \mathrm{CCCC}(\mathrm{O}) \mathrm{C} 1$ & 2 \\
\hline 331 & $\mathbf{1 5}\{135\}$ & $11\{322\}$ & $\mathbf{9}\{135,322\}$ & $\mathrm{CN}(\mathrm{C} 1 \mathrm{CC} 1) \mathrm{c} 1 \mathrm{nnnn} 1 \mathrm{Cc} 1 \mathrm{cnn}(\mathrm{Cc} 2 \mathrm{ccccc} 2) \mathrm{c} 1$ & 9 \\
\hline 332 & $15\{136\}$ & $11\{284\}$ & $\mathbf{9}\{136,284\}$ & $\mathrm{Cc} 1 \mathrm{n}[\mathrm{nH}] \mathrm{cc} 1 \mathrm{Cn} 1 \mathrm{nnnn} 1 \mathrm{~N} 1 \mathrm{CCOC}(\mathrm{C})(\mathrm{C}) \mathrm{C} 1$ & 0 \\
\hline 333 & $15\{137\}$ & $\mathbf{1 1}\{391\}$ & $\mathbf{9}\{137,391\}$ & $\underset{\mathrm{c} 1}{\mathrm{CC}(\mathrm{C}) \mathrm{NC}(=\mathrm{O}) \mathrm{Nc} 1 \mathrm{ccc}(\mathrm{Cn} 2 \mathrm{nnnc} 2 \mathrm{~N}(\mathrm{C}) \mathrm{CC} 2 \mathrm{CC} 2) \mathrm{c}}$ & 0 \\
\hline 334 & $15\{137\}$ & $11\{322\}$ & $\mathbf{9}\{137,322\}$ & ${ }_{1} \mathrm{CC}(\mathrm{C}) \mathrm{NC}(=\mathrm{O}) \mathrm{Nc} 1 \mathrm{ccc}(\mathrm{Cn} 2 \mathrm{nnnn} 2 \mathrm{~N}(\mathrm{C}) \mathrm{C} 2 \mathrm{CC} 2) \mathrm{cc}$ & 0 \\
\hline 335 & $\mathbf{1 5}\{138\}$ & $\mathbf{1 1}\{321\}$ & $\mathbf{9}\{138,321\}$ & OCC1CCN(CC1)c1nnnn1Cc1cc(Br)cs1 & 34 \\
\hline
\end{tabular}




\begin{tabular}{|c|c|c|c|c|c|}
\hline 336 & $\mathbf{1 5}\{139\}$ & $\mathbf{1 1}\{415\}$ & $\mathbf{9}\{139,415\}$ & CC1CC1n1nnnc1N(C)C[C@H]1CCCO1 & 11 \\
\hline 337 & $\mathbf{1 5}\{139\}$ & $\mathbf{1 1}\{318\}$ & $\mathbf{9}\{139,318\}$ & CC1CC1n1nnnc1N1CCn2c(C1)nnc2-c1cccce1 & 23 \\
\hline 338 & $\mathbf{1 5}\{140\}$ & $\mathbf{1 1}\{338\}$ & $\mathbf{9}\{140,338\}$ & $\mathrm{CCC} 1 \mathrm{CN}(\mathrm{CC}(\mathrm{CC}) \mathrm{O} 1) \mathrm{c} 1 \mathrm{nnnn} 1 \mathrm{CC}(\mathrm{C}) \mathrm{Cn} 1 \mathrm{ccc} 1$ & 50 \\
\hline 339 & $\mathbf{1 5}\{141\}$ & $\mathbf{1 1}\{349\}$ & $\mathbf{9}\{141,349\}$ & $\mathrm{O}=\mathrm{C}(\mathrm{CCCn} 1 \mathrm{nnn} \mathrm{c} 1 \mathrm{~N} 1 \mathrm{CCCC}(\mathrm{CC} \# \mathrm{C}) \mathrm{C} 1) \mathrm{NC} 1 \mathrm{CC} 1$ & 12 \\
\hline 340 & $\mathbf{1 5}\{142\}$ & $\mathbf{1 1}\{322\}$ & $\mathbf{9}\{142,322\}$ & $\mathrm{CN}(\mathrm{C} 1 \mathrm{CC} 1) \mathrm{c} 1 \mathrm{nnnn} 1 \mathrm{Cc} 1 \mathrm{ccc}(\mathrm{CS}(\mathrm{C})(=\mathrm{O})=\mathrm{O}) \mathrm{cc} 1$ & 30 \\
\hline 341 & $\mathbf{1 5}\{142\}$ & $\mathbf{1 1}\{356\}$ & $\mathbf{9}\{142,356\}$ & $\begin{array}{l}\mathrm{CN}(\mathrm{CC} 1 \mathrm{CCC} 1) \mathrm{c} 1 \mathrm{nnnn} 1 \mathrm{Cc} 1 \operatorname{ccc}(\mathrm{CS}(\mathrm{C})(=\mathrm{O})=\mathrm{O}) \mathrm{c} \\
\mathrm{c} 1\end{array}$ & 47 \\
\hline 342 & $\mathbf{1 5}\{143\}$ & $\mathbf{1 1}\{338\}$ & $\mathbf{9}\{143,338\}$ & ${ }_{\mathrm{O}}^{\mathrm{CCC} 1 \mathrm{CN}(\mathrm{CC}(\mathrm{CC}) \mathrm{O} 1) \mathrm{c} 1 \mathrm{nnnn} 1 \mathrm{Cc} 1 \mathrm{ccc}(\mathrm{cc} 1) \mathrm{C}(\mathrm{C})}$ & 30 \\
\hline 343 & $\mathbf{1 5}\{144\}$ & $\mathbf{1 1}\{298\}$ & $\mathbf{9}\{144,298\}$ & CC1(OCCO1)C1CCCN(C1)c1nnnn1CCn1ccen1 & 10 \\
\hline 344 & $\mathbf{1 5}\{144\}$ & $\mathbf{1 1}\{297\}$ & $\mathbf{9}\{144,297\}$ & $\mathrm{CC} 1(\mathrm{OCCO} 1) \mathrm{C} 1 \mathrm{CCN}(\mathrm{CC} 1) \mathrm{c} 1 \mathrm{nnnn} 1 \mathrm{CCn} 1 \mathrm{ccc} 1$ & 31 \\
\hline 345 & $\mathbf{1 5}\{145\}$ & $\mathbf{1 1}\{322\}$ & $\mathbf{9}\{145,322\}$ & $\mathrm{CN}(\mathrm{C} 1 \mathrm{CC} 1) \mathrm{c} 1 \mathrm{nnnn} 1 \mathrm{CC} 1 \mathrm{CCCOC} 1 \mathrm{c} 1 \mathrm{ccccc} 1$ & 27 \\
\hline 346 & $\mathbf{1 5}\{146\}$ & $\mathbf{1 1}\{322\}$ & $\mathbf{9}\{146,322\}$ & $\mathrm{CN}(\mathrm{C} 1 \mathrm{CC} 1) \mathrm{c} 1 \mathrm{nnnnn} 1 \mathrm{CCNC}(=\mathrm{O}) \mathrm{c} 1 \mathrm{cccc}(\mathrm{C}) \mathrm{c} 1$ & 66 \\
\hline 347 & $\mathbf{1 5}\{147\}$ & $\mathbf{1 1}\{416\}$ & $\mathbf{9}\{147,416\}$ & $\mathrm{CS}(=\mathrm{O})(=\mathrm{O}) \mathrm{CCn} 1 \mathrm{nnn} \operatorname{c} 1 \mathrm{~N} 1 \mathrm{CCCc} 2 \operatorname{ccccc} 2 \mathrm{CC} 1$ & 0 \\
\hline 348 & $\mathbf{1 5}\{147\}$ & $\mathbf{1 1}\{417\}$ & $\mathbf{9}\{147,417\}$ & $\operatorname{COc} 1 \operatorname{ccc} 2 \mathrm{CCN}(\mathrm{Cc} 12) \mathrm{c} 1 \mathrm{nnnn} 1 \mathrm{CCS}(\mathrm{C})(=\mathrm{O})=\mathrm{O}$ & 0 \\
\hline 349 & $\mathbf{1 5}\{147\}$ & $\mathbf{1 1}\{405\}$ & $\mathbf{9}\{147,405\}$ & $\mathrm{CS}(=\mathrm{O})(=\mathrm{O}) \mathrm{CCn} 1 \mathrm{nnn} \mathrm{c} 1 \mathrm{~N} 1 \mathrm{CCCC} 2(\mathrm{CCCCC} 2) \mathrm{C} 1$ & 0 \\
\hline 350 & $\mathbf{1 5}\{147\}$ & $\mathbf{1 1}\{418\}$ & $\mathbf{9}\{147,418\}$ & $\mathrm{CS}(=\mathrm{O})(=\mathrm{O}) \mathrm{CCn} 1 \mathrm{nnn} \operatorname{1N} 1 \mathrm{Cc} 2 \operatorname{cccc} 2 \mathrm{C}(\mathrm{F})(\mathrm{F}) \mathrm{C} 1$ & 0 \\
\hline 351 & $\mathbf{1 5}\{147\}$ & $\mathbf{1 1}\{419\}$ & $\mathbf{9}\{147,419\}$ & $\begin{array}{l}\mathrm{CS}(=\mathrm{O})(=\mathrm{O}) \mathrm{CCn} 1 \mathrm{nnn} \mathrm{c} 1 \mathrm{~N} 1 \mathrm{C} 2 \mathrm{CCC} 1 \mathrm{CC} 1(\mathrm{CC} 1(\mathrm{~F}) \\
\mathrm{F}) \mathrm{C} 2\end{array}$ & 0 \\
\hline 352 & $\mathbf{1 5}\{147\}$ & $\mathbf{1 1}\{420\}$ & $\mathbf{9}\{147,420\}$ & $\mathrm{CS}(=\mathrm{O})(=\mathrm{O}) \mathrm{CCn} 1 \mathrm{nnnnc1N} 1 \mathrm{CCOC}(\mathrm{C} 1) \mathrm{c} 1 \mathrm{ccccc} 1$ & 0 \\
\hline 353 & $\mathbf{1 5}\{147\}$ & $\mathbf{1 1}\{421\}$ & $\mathbf{9}\{147,421\}$ & ${ }_{21} \mathrm{CS}(=\mathrm{O})(=\mathrm{O}) \mathrm{CCn} 1 \mathrm{nnnn} 1 \mathrm{~N} 1 \mathrm{CCC} 2(\mathrm{C} 1) \mathrm{OCc} 1 \mathrm{ccccc}$ & 24 \\
\hline 354 & $\mathbf{1 5}\{147\}$ & $\mathbf{1 1}\{387\}$ & $\mathbf{9}\{147,387\}$ & $\mathrm{CS}(=\mathrm{O})(=\mathrm{O}) \mathrm{CCn} 1 \mathrm{nnnn} 1 \mathrm{~N} 1 \mathrm{CCC}(\mathrm{Cc} 2 \mathrm{ccccc} 2) \mathrm{CC} 1$ & 35 \\
\hline 355 & $\mathbf{1 5}\{148\}$ & $\mathbf{1 1}\{349\}$ & $\mathbf{9}\{148,349\}$ & C\#CCC1CCCN(C1)c1nnnn1CC1CCCCO1 & 45 \\
\hline
\end{tabular}




\begin{tabular}{|c|c|c|c|c|c|}
\hline 356 & $15\{149\}$ & $11\{304\}$ & $\mathbf{9}\{149,304\}$ & $\begin{aligned} & \mathrm{CC}(\mathrm{C}) \mathrm{C}(=\mathrm{O}) \mathrm{NCC} 1 \mathrm{CCCN}(\mathrm{C} 1) \mathrm{c} 1 \mathrm{nnnn} 1 \mathrm{CC} 1 \mathrm{CCC} \\
= & \mathrm{CC} 1\end{aligned}$ & 26 \\
\hline 357 & $\mathbf{1 5}\{150\}$ & $11\{299\}$ & $\mathbf{9}\{150,299\}$ & $\mathrm{COC} 1(\mathrm{CO}) \mathrm{CCN}(\mathrm{CC} 1) \mathrm{c} 1 \mathrm{nnnn} 1 \mathrm{CCc} 1 \mathrm{cccc}(\mathrm{C}) \mathrm{c} 1$ & 31 \\
\hline 358 & $\mathbf{1 5}\{151\}$ & $11\{319\}$ & $\mathbf{9}\{151,319\}$ & $\mathrm{CN}(\mathrm{CC} 1 \mathrm{COCCO} 1) \mathrm{c} 1 \mathrm{nnnn} 1 \mathrm{CCc} 1 \mathrm{ccc}(\mathrm{F}) \mathrm{cc} 1 \mathrm{~F}$ & 74 \\
\hline 359 & $\mathbf{1 5}\{152\}$ & $\mathbf{1 1}\{299\}$ & $\mathbf{9}\{152,299\}$ & $\mathrm{COC}(\mathrm{Cn} 1 \mathrm{nnnn} 1 \mathrm{~N} 1 \mathrm{CCC}(\mathrm{CO})(\mathrm{CC} 1) \mathrm{OC}) \mathrm{c} 1 \mathrm{ccccc} 1$ & 0 \\
\hline 360 & $\mathbf{1 5}\{153\}$ & $11\{401\}$ & $\mathbf{9}\{153,401\}$ & $\begin{array}{l}\mathrm{NC}(=\mathrm{O}) \mathrm{C} 1 \mathrm{CCN}(\mathrm{CC} 1) \mathrm{c} 1 \mathrm{nnnn} 1 \mathrm{CC} 1 \mathrm{C} 2 \mathrm{CC} 3 \mathrm{CC}(\mathrm{C} \\
\text { 2) } \mathrm{CC} 1 \mathrm{C} 3\end{array}$ & 0 \\
\hline 361 & $\mathbf{1 5}\{154\}$ & $11\{299\}$ & $\mathbf{9}\{154,299\}$ & $\mathrm{COc} 1 \mathrm{cc}(\mathrm{Cn} 2 \mathrm{nnnn} 2 \mathrm{~N} 2 \mathrm{CCC}(\mathrm{CO})(\mathrm{CC} 2) \mathrm{OC}) \mathrm{ccc} 1 \mathrm{C}$ & 36 \\
\hline 362 & $\mathbf{1 5}\{155\}$ & $11\{328\}$ & $\mathbf{9}\{155,328\}$ & 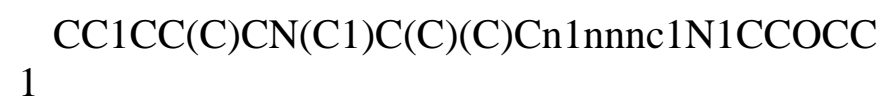 & 0 \\
\hline 363 & $15\{156\}$ & $11\{355\}$ & $\mathbf{9}\{156,355\}$ & $\begin{array}{l}\mathrm{CC} 1 \mathrm{CCCN}(\mathrm{C} 1) \mathrm{C}(\mathrm{C})(\mathrm{C}) \mathrm{Cn} 1 \mathrm{nnnn} 1 \mathrm{~N}(\mathrm{C}) \mathrm{Cc} 1 \mathrm{cnn}(\mathrm{C} \\
) \mathrm{c} 1\end{array}$ & 0 \\
\hline 364 & $15\{157\}$ & $11\{298\}$ & $\mathbf{9}\{157,298\}$ & 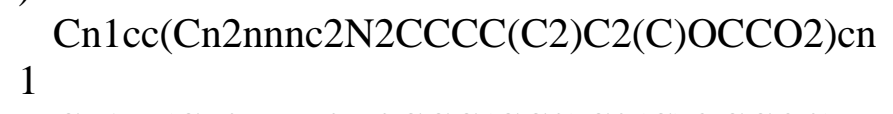 & 35 \\
\hline 365 & $\mathbf{1 5}\{157\}$ & $11\{297\}$ & $\mathbf{9}\{157,297\}$ & 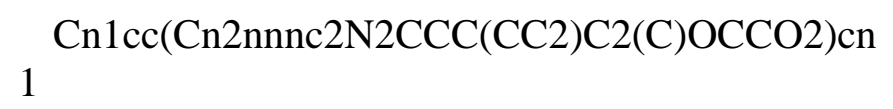 & 25 \\
\hline 366 & $\mathbf{1 5}\{158\}$ & $11\{422\}$ & $\mathbf{9}\{158,422\}$ & 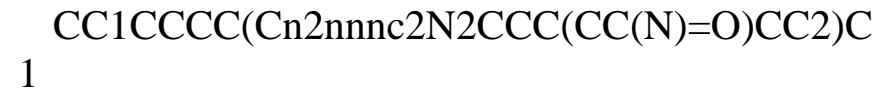 & 0 \\
\hline 367 & $\mathbf{1 5}\{158\}$ & $11\{321\}$ & $\mathbf{9}\{158,321\}$ & $\mathrm{CC} 1 \mathrm{CCCC}(\mathrm{Cn} 2 \mathrm{nnnn} 2 \mathrm{~N} 2 \mathrm{CCC}(\mathrm{CO}) \mathrm{CC} 2) \mathrm{C} 1$ & 37 \\
\hline 368 & $\mathbf{1 5}\{158\}$ & $11\{401\}$ & $\mathbf{9}\{158,401\}$ & $\mathrm{CC} 1 \mathrm{CCCC}(\mathrm{Cn} 2 \mathrm{nnn} 2 \mathrm{~N} 2 \mathrm{CCC}(\mathrm{CC} 2) \mathrm{C}(\mathrm{N})=\mathrm{O}) \mathrm{C} 1$ & 23 \\
\hline 369 & $\mathbf{1 5}\{158\}$ & $11\{328\}$ & $\mathbf{9}\{158,328\}$ & $\mathrm{CC} 1 \mathrm{CCCC}(\mathrm{Cn} 2 \mathrm{nnnn} 2 \mathrm{~N} 2 \mathrm{CCOCC} 2) \mathrm{C} 1$ & 37 \\
\hline 370 & $\mathbf{1 5}\{158\}$ & $11\{423\}$ & $\mathbf{9}\{158,423\}$ & $\mathrm{CC} 1 \mathrm{CCCC}(\mathrm{Cn} 2 \mathrm{nnn} \mathrm{n} 2 \mathrm{~N} 2 \mathrm{CCNC}(=\mathrm{O}) \mathrm{C} 2) \mathrm{C} 1$ & 0 \\
\hline 371 & $\mathbf{1 5}\{158\}$ & $11\{424\}$ & $\mathbf{9}\{158,424\}$ & $\mathrm{CC} 1 \mathrm{CCCC}(\mathrm{Cn} 2 \mathrm{nnn} \mathrm{c} 2 \mathrm{~N} 2 \mathrm{CCCC}(\mathrm{O}) \mathrm{C} 2) \mathrm{C} 1$ & 0 \\
\hline 372 & $\mathbf{1 5}\{158\}$ & $11\{299\}$ & $\mathbf{9}\{158,299\}$ & $\mathrm{COC} 1(\mathrm{CO}) \mathrm{CCN}(\mathrm{CC} 1) \mathrm{c} 1 \mathrm{nnnn} 1 \mathrm{CC} 1 \mathrm{CCCC}(\mathrm{C}) \mathrm{C} 1$ & 12 \\
\hline 373 & $15\{159\}$ & $11\{346\}$ & $\mathbf{9}\{159,346\}$ & $\mathrm{CCN}(\mathrm{C}) \mathrm{c} 1 \mathrm{nnnnn} 1 \mathrm{Cc} 1 \mathrm{cccc}(\mathrm{NC}(=\mathrm{O}) \mathrm{C} 2 \mathrm{CCCO} 2) \mathrm{c} 1$ & 31 \\
\hline 374 & $15\{160\}$ & $11\{304\}$ & $\mathbf{9}\{160,304\}$ & $\begin{array}{l}\mathrm{CC}(\mathrm{Cn} 1 \mathrm{nnnn} 1 \mathrm{~N} 1 \mathrm{CCCC}(\mathrm{CNC}(=\mathrm{O}) \mathrm{C}(\mathrm{C}) \mathrm{C}) \mathrm{C} 1) \mathrm{C} 1 \\
\mathrm{CC} 1\end{array}$ & 27 \\
\hline
\end{tabular}




\begin{tabular}{|c|c|c|c|c|c|}
\hline 375 & $\mathbf{1 5}\{161\}$ & $\mathbf{1 1}\{328\}$ & $\mathbf{9}\{161,328\}$ & $\operatorname{Brc} 1 \mathrm{ccc}(\mathrm{CCn} 2 \mathrm{nnn} \mathrm{2} 2 \mathrm{~N} 2 \mathrm{CCOCC} 2) \mathrm{s} 1$ & 34 \\
\hline 376 & $\mathbf{1 5}\{162\}$ & $\mathbf{1 1}\{298\}$ & $\mathbf{9}\{162,298\}$ & $\mathrm{COCC}(\mathrm{C}) \mathrm{Cn} 1 \mathrm{nnn} \mathrm{c} 1 \mathrm{~N} 1 \mathrm{CCCC}(\mathrm{C} 1) \mathrm{C} 1(\mathrm{C}) \mathrm{OCCO} 1$ & 20 \\
\hline 377 & $\mathbf{1 5}\{162\}$ & $\mathbf{1 1}\{297\}$ & $\mathbf{9}\{162,297\}$ & $\mathrm{COCC}(\mathrm{C}) \mathrm{Cn} 1 \mathrm{nnnn} 1 \mathrm{~N} 1 \mathrm{CCC}(\mathrm{CC} 1) \mathrm{C} 1(\mathrm{C}) \mathrm{OCCO} 1$ & 26 \\
\hline 378 & $\mathbf{1 5}\{163\}$ & $\mathbf{1 1}\{322\}$ & $\mathbf{9}\{163,322\}$ & ${ }_{1}^{\mathrm{CN}}(\mathrm{C} 1 \mathrm{CC} 1) \mathrm{c} 1 \mathrm{nnnn} 1 \mathrm{Cc} 1 \mathrm{ccc}(\mathrm{NC}(=\mathrm{O}) \mathrm{c} 2 \mathrm{ccco} 2) \mathrm{cc}$ & 22 \\
\hline 379 & $\mathbf{1 5}\{163\}$ & $\mathbf{1 1}\{346\}$ & $\mathbf{9}\{163,346\}$ & $\mathrm{CCN}(\mathrm{C}) \mathrm{c} 1 \mathrm{nnnn} 1 \mathrm{Cc} 1 \mathrm{ccc}(\mathrm{NC}(=\mathrm{O}) \mathrm{c} 2 \mathrm{ccco} 2) \mathrm{cc} 1$ & 0 \\
\hline 380 & $\mathbf{1 5}\{164\}$ & $\mathbf{1 1}\{284\}$ & $\mathbf{9}\{164,284\}$ & $\mathrm{CC} 1(\mathrm{C}) \mathrm{CN}(\mathrm{CCO} 1) \mathrm{c} 1 \mathrm{nnnn} 1 \mathrm{CCc} 1 \mathrm{cn}[\mathrm{nH}] \mathrm{c} 1$ & 13 \\
\hline 381 & $\mathbf{1 5}\{165\}$ & $\mathbf{1 1}\{299\}$ & $\mathbf{9}\{165,299\}$ & $\mathrm{COC} 1(\mathrm{CO}) \mathrm{CCN}(\mathrm{CC} 1) \mathrm{c} 1 \mathrm{nnnn} 1 \mathrm{CC}(\mathrm{Br})=\mathrm{C}$ & 66 \\
\hline 382 & $\mathbf{1 5}\{166\}$ & $\mathbf{1 1}\{78\}$ & $\mathbf{9}\{166,78\}$ & COc1cce2CN(CCCc2c1)c1nnnn1Cc1cc(C)no1 & 0 \\
\hline 383 & $\mathbf{1 5}\{166\}$ & $\mathbf{1 1}\{340\}$ & $\mathbf{9}\{166,340\}$ & Cc1cc(Cn2nnnc2N2CCCCCC2)on1 & 44 \\
\hline 384 & $\mathbf{1 5}\{166\}$ & $\mathbf{1 1}\{103\}$ & $\mathbf{9}\{166,103\}$ & $\begin{array}{l}\mathrm{Cc} 1 \mathrm{cc}(\mathrm{Cn} 2 \mathrm{nnn} n 2 \mathrm{~N} 2 \mathrm{CCc} 3[\mathrm{nH}] \mathrm{c} 4 \operatorname{ccc}(\mathrm{C}) \mathrm{cc} 4 \mathrm{c} 3 \mathrm{C} 2) \\
\text { on } 1\end{array}$ & 0 \\
\hline 385 & $\mathbf{1 5}\{166\}$ & $\mathbf{1 1}\{425\}$ & $\mathbf{9}\{166,425\}$ & COCC1=CCN(CC1)c1nnnn1Cc1cc(C)no1 & 11 \\
\hline 386 & $\mathbf{1 5}\{167\}$ & $\mathbf{1 1}\{401\}$ & $\mathbf{9}\{167,401\}$ & $\mathrm{NC}(=\mathrm{O}) \mathrm{C} 1 \mathrm{CCN}(\mathrm{CC} 1) \mathrm{c} 1 \mathrm{nnnnn} 1 \mathrm{CCCc} 1 \mathrm{ccc}(\mathrm{Cl}) \mathrm{c} 1$ & 25 \\
\hline 387 & $\mathbf{1 5}\{168\}$ & $\mathbf{1 1}\{319\}$ & $\mathbf{9}\{168,319\}$ & $\mathrm{CN}(\mathrm{CC} 1 \mathrm{COCCO} 1) \mathrm{c} 1 \mathrm{nnnn} 1 \mathrm{CCc} 1 \mathrm{ccc}(\mathrm{F}) \mathrm{c}(\mathrm{F}) \mathrm{c} 1$ & 59 \\
\hline 388 & $\mathbf{1 5}\{169\}$ & $\mathbf{1 1}\{322\}$ & $\mathbf{9}\{169,322\}$ & $\mathrm{CN}(\mathrm{C} 1 \mathrm{CC} 1) \mathrm{c} 1 \mathrm{nnnn} 1 \mathrm{CCc} 1 \mathrm{ccc}(\mathrm{NC}(\mathrm{C})=\mathrm{O}) \mathrm{cc} 1$ & 32 \\
\hline 389 & $\mathbf{1 5}\{170\}$ & $\mathbf{1 1}\{338\}$ & $\mathbf{9}\{170,338\}$ & $\mathrm{CCC} 1 \mathrm{CN}(\mathrm{CC}(\mathrm{CC}) \mathrm{O} 1) \mathrm{c} 1 \mathrm{nnnn} 1 \mathrm{CCOCC} 1 \mathrm{CC} 1$ & 35 \\
\hline 390 & $\mathbf{1 5}\{171\}$ & $\mathbf{1 1}\{302\}$ & $\mathbf{9}\{171,302\}$ & 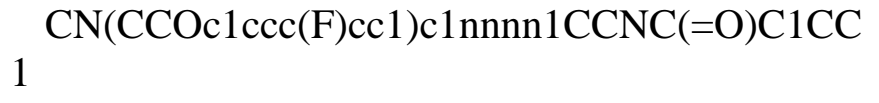 & 0 \\
\hline 391 & $\mathbf{1 5}\{171\}$ & $\mathbf{1 1}\{349\}$ & $\mathbf{9}\{171,349\}$ & $\mathrm{O}=\mathrm{C}(\mathrm{NCCn} 1 \mathrm{nnn} \mathrm{1} 1 \mathrm{~N} 1 \mathrm{CCCC}(\mathrm{CC} \# \mathrm{C}) \mathrm{C} 1) \mathrm{C} 1 \mathrm{CC} 1$ & 0 \\
\hline 392 & $\mathbf{1 5}\{172\}$ & $\mathbf{1 1}\{426\}$ & $\mathbf{9}\{172,426\}$ & $\underset{C}{\mathrm{COC}(=\mathrm{O}) \mathrm{C} 1 \mathrm{CC} 11 \mathrm{CCN}(\mathrm{CC} 1) \mathrm{c} 1 \mathrm{nnnn} 1 \mathrm{Cc} 1 \mathrm{occc} 1}$ & 0 \\
\hline 393 & $\mathbf{1 5}\{172\}$ & $\mathbf{1 1}\{427\}$ & $\mathbf{9}\{172,427\}$ & ${ }_{\mathrm{O}}^{\mathrm{CN} 1 \mathrm{CCC} 2(\mathrm{CCN}(\mathrm{CC} 2) \mathrm{c} 2 \mathrm{nnnn} 2 \mathrm{Cc} 2 \mathrm{occc} 2 \mathrm{C}) \mathrm{C} 1=}$ & 0 \\
\hline 394 & $\mathbf{1 5}\{172\}$ & $\mathbf{1 1}\{428\}$ & $\mathbf{9}\{172,428\}$ & ${ }_{1}^{\mathrm{Cc} 1 \mathrm{ccoc} 1 \mathrm{Cn} 1 \mathrm{nnnn} 1 \mathrm{~N} 1 \mathrm{CCC} 2(\mathrm{CCNC}(=\mathrm{O}) \mathrm{O} 2) \mathrm{CC}}$ & 27 \\
\hline
\end{tabular}




\begin{tabular}{|c|c|c|c|c|c|}
\hline 395 & $\mathbf{1 5}\{172\}$ & $\mathbf{1 1}\{429\}$ & $\mathbf{9}\{172,429\}$ & Cc1ccoc1Cn1nnnc1N1CCOC2(CCOCC2)CC1 & 32 \\
\hline 396 & $\mathbf{1 5}\{172\}$ & $\mathbf{1 1}\{430\}$ & $\mathbf{9}\{172,430\}$ & $\begin{array}{l}\mathrm{CCOC}(=\mathrm{O})[1 \mathrm{C} @ @ \mathrm{H}] 1[1 \mathrm{C} @ @ \mathrm{H}] 2 \mathrm{CCN}(\mathrm{C}[1 \mathrm{C} @ \\
\mathrm{H}] 12) \mathrm{c} 1 \mathrm{nnnn} 1 \mathrm{Cc} 1 \mathrm{occ} 1 \mathrm{C}\end{array}$ & 27 \\
\hline 397 & $\mathbf{1 5}\{172\}$ & $\mathbf{1 1}\{419\}$ & $\mathbf{9}\{172,419\}$ & $2 \mathrm{Cc} 1 \mathrm{ccoc} 1 \mathrm{Cn} 1 \mathrm{nnn} n 1 \mathrm{~N} 1 \mathrm{C} 2 \mathrm{CCC} 1 \mathrm{CC} 1(\mathrm{CC} 1(\mathrm{~F}) \mathrm{F}) \mathrm{C}$ & 39 \\
\hline 398 & $\mathbf{1 5}\{172\}$ & $\mathbf{1 1}\{63\}$ & $\mathbf{9}\{172,63\}$ & Cc1ccoc1Cn1nnnc1N1CCN2C(CNC2=O)C1 & 0 \\
\hline 399 & $\mathbf{1 5}\{172\}$ & $\mathbf{1 1}\{431\}$ & $\mathbf{9}\{172,431\}$ & Cc1ccoc1Cn1nnnc1N1CCOC2(CCSC2)C1 & 0 \\
\hline 400 & $\mathbf{1 5}\{172\}$ & $\mathbf{1 1}\{432\}$ & $\mathbf{9}\{172,432\}$ & Cc1ccoc1Cn1nnnc1N1CCOCC2(CCOCC2)C1 & 33 \\
\hline 401 & $\mathbf{1 5}\{172\}$ & $\mathbf{1 1}\{433\}$ & $\mathbf{9}\{172,433\}$ & $\mathrm{CC} 1 \mathrm{CN}(\mathrm{CC} 2(\mathrm{CCOCC} 2) \mathrm{O} 1) \mathrm{c} 1 \mathrm{nnnn} 1 \mathrm{Cc} 1 \mathrm{occc} 1 \mathrm{C}$ & 32 \\
\hline 402 & $\mathbf{1 5}\{172\}$ & $\mathbf{1 1}\{434\}$ & $\mathbf{9}\{172,434\}$ & Cc1ccoc1Cn1nnnc1N1CCOC2(CCOCC2)C1 & 29 \\
\hline 403 & $\mathbf{1 5}\{172\}$ & $\mathbf{1 1}\{435\}$ & $\mathbf{9}\{172,435\}$ & $\begin{array}{l}\mathrm{COC}(=\mathrm{O})[1 \mathrm{C} @] 12 \mathrm{CN}(\mathrm{C}[1 \mathrm{C} @ \mathrm{H}] 1 \mathrm{COCC} 2) \mathrm{c} 1 \mathrm{nn} \\
\mathrm{nn} 1 \mathrm{Cc} 1 \mathrm{occc} 1 \mathrm{C}\end{array}$ & 25 \\
\hline 404 & $\mathbf{1 5}\{172\}$ & $\mathbf{1 1}\{436\}$ & $\mathbf{9}\{172,436\}$ & $\begin{array}{l}\mathrm{COC}(=\mathrm{O})[1 \mathrm{C} @] 12 \mathrm{CCC}[1 \mathrm{C} @ \mathrm{H}] 1 \mathrm{~N}(\mathrm{CC} 2) \mathrm{c} 1 \mathrm{nnnn} \\
1 \mathrm{Cc} 1 \mathrm{occc} 1 \mathrm{C}\end{array}$ & 25 \\
\hline 405 & $\mathbf{1 5}\{172\}$ & $\mathbf{1 1}\{437\}$ & $\mathbf{9}\{172,437\}$ & $\mathrm{CCS}(=\mathrm{O})(=\mathrm{O}) \mathrm{N} 1 \mathrm{CCN}(\mathrm{CC} 1) \mathrm{c} 1 \mathrm{nnnn} 1 \mathrm{Cc} 1 \mathrm{occc} 1 \mathrm{C}$ & 0 \\
\hline 406 & $\mathbf{1 5}\{172\}$ & $\mathbf{1 1}\{438\}$ & $\mathbf{9}\{172,438\}$ & $\begin{array}{l}\text { Cc1ccoc1Cn1nnnc1N1C[1C@@ } @ \text { H } 2 \text { CCC }[1 \mathrm{C} @ \\
@] 2(\mathrm{C} 1) \mathrm{C}(\mathrm{N})=\mathrm{O}\end{array}$ & 31 \\
\hline 407 & $\mathbf{1 5}\{172\}$ & $\mathbf{1 1}\{421\}$ & $\mathbf{9}\{172,421\}$ & Cc1ccoc1Cn1nnnc1N1CCC2(C1)OCc1cccc21 & 34 \\
\hline 408 & $\mathbf{1 5}\{172\}$ & $\mathbf{1 1}\{104\}$ & $\mathbf{9}\{172,104\}$ & Cc1ccoc1Cn1nnnc1N1CCOCC2(CCCC2)C1 & 0 \\
\hline 409 & $\mathbf{1 5}\{173\}$ & $\mathbf{1 1}\{321\}$ & $\mathbf{9}\{173,321\}$ & $\mathrm{Cc} 1 \mathrm{cccc} 1 \mathrm{C}(\mathrm{C})(\mathrm{C}) \mathrm{Cn} 1 \mathrm{nnn} \mathrm{1N} 1 \mathrm{CCC}(\mathrm{CO}) \mathrm{CC} 1$ & 21 \\
\hline 410 & $\mathbf{1 5}\{173\}$ & $\mathbf{1 1}\{401\}$ & $\mathbf{9}\{173,401\}$ & $\begin{aligned} & \mathrm{Cc} 1 \operatorname{ccccc} 1 \mathrm{C}(\mathrm{C})(\mathrm{C}) \mathrm{Cn} 1 \mathrm{nnnn} 1 \mathrm{~N} 1 \mathrm{CCC}(\mathrm{CC} 1) \mathrm{C}(\mathrm{N}) \\
= & \mathrm{O}\end{aligned}$ & 33 \\
\hline 411 & $\mathbf{1 5}\{174\}$ & $\mathbf{1 1}\{321\}$ & $\mathbf{9}\{174,321\}$ & $\mathrm{COc} 1 \mathrm{ccccc} 1 \mathrm{C}(\mathrm{C})(\mathrm{C}) \mathrm{Cn} 1 \mathrm{nnn} 1 \mathrm{~N} 1 \mathrm{CCC}(\mathrm{CO}) \mathrm{CC} 1$ & 19 \\
\hline 412 & $\mathbf{1 5}\{175\}$ & $\mathbf{1 1}\{299\}$ & $\mathbf{9}\{175,299\}$ & $\mathrm{CCC}(\mathrm{Cn} 1 \mathrm{nnn} n \mathrm{1N} 1 \mathrm{CCC}(\mathrm{CO})(\mathrm{CC} 1) \mathrm{OC}) \mathrm{c} 1 \mathrm{ccccc} 1$ & 64 \\
\hline 413 & $\mathbf{1 5}\{176\}$ & $\mathbf{1 1}\{391\}$ & $\mathbf{9}\{176,391\}$ & $\begin{array}{l}\mathrm{CN}(\mathrm{CC} 1 \mathrm{CC} 1) \mathrm{c} 1 \mathrm{nnnn} 1 \mathrm{Cc} 1 \mathrm{ccc}(\mathrm{cc} 1) \mathrm{C}(=\mathrm{O}) \mathrm{N} 1 \mathrm{CCC} \\
\mathrm{C} 1\end{array}$ & 22 \\
\hline 414 & $\mathbf{1 5}\{176\}$ & $\mathbf{1 1}\{322\}$ & $\mathbf{9}\{176,322\}$ & $\mathrm{CN}(\mathrm{C} 1 \mathrm{CC} 1) \mathrm{c} 1 \mathrm{nnnn} 1 \mathrm{Cc} 1 \mathrm{ccc}(\mathrm{cc} 1) \mathrm{C}(=\mathrm{O}) \mathrm{N} 1 \mathrm{CCCC}$ & 39 \\
\hline
\end{tabular}


1

\begin{tabular}{|c|c|c|c|c|c|}
\hline 415 & $\mathbf{1 5}\{177\}$ & $\mathbf{1 1}\{298\}$ & $\mathbf{9}\{177,298\}$ & Cc1occc1Cn1nnnc1N1CCCC(C1)C1(C)OCCO1 & 13 \\
\hline 416 & $\mathbf{1 5}\{177\}$ & $\mathbf{1 1}\{297\}$ & $\mathbf{9}\{177,297\}$ & Cc1occc1Cn1nnnc1N1CCC(CC1)C1(C)OCCO1 & 0 \\
\hline 417 & $\mathbf{1 5}\{178\}$ & $\mathbf{1 1}\{302\}$ & $\mathbf{9}\{178,302\}$ & $\mathrm{CN}(\mathrm{CCOc} 1 \mathrm{ccc}(\mathrm{F}) \mathrm{cc} 1) \mathrm{c} 1 \mathrm{nnnn} 1 \mathrm{CC} 1 \mathrm{CCCNC} 1=\mathrm{O}$ & 16 \\
\hline 418 & $\mathbf{1 5}\{179\}$ & $\mathbf{1 1}\{319\}$ & $\mathbf{9}\{179,319\}$ & $\mathrm{CN}(\mathrm{CC} 1 \mathrm{COCCO} 1) \mathrm{c} 1 \mathrm{nnnn} 1 \mathrm{CCc} 1 \mathrm{cc}(\mathrm{F}) \mathrm{cc}(\mathrm{F}) \mathrm{c} 1$ & 45 \\
\hline 419 & $\mathbf{1 5}\{180\}$ & $\mathbf{1 1}\{439\}$ & $\mathbf{9}\{180,439\}$ & $\mathrm{CN}(\mathrm{C} 1 \mathrm{CCCCC} 1) \mathrm{c} 1 \mathrm{nnnn} 1 \mathrm{Cc} 1 \mathrm{nccc} 1 \mathrm{C}$ & 0 \\
\hline 420 & $\mathbf{1 5}\{181\}$ & $\mathbf{1 1}\{338\}$ & $\mathbf{9}\{181,338\}$ & $\mathrm{CCC} 1 \mathrm{CN}(\mathrm{CC}(\mathrm{CC}) \mathrm{O} 1) \mathrm{c} 1 \mathrm{nnnn} 1 \mathrm{Cc} 1 \mathrm{nc} 2 \mathrm{CCCc} 2 \mathrm{~s} 1$ & 0 \\
\hline 421 & $\mathbf{1 5}\{182\}$ & $\mathbf{1 1}\{328\}$ & $\mathbf{9}\{182,328\}$ & $\mathrm{C}(\mathrm{c} 1 \mathrm{ccc}(\mathrm{OC} 2 \mathrm{CCCC} 2) \mathrm{cc} 1) \mathrm{n} 1 \mathrm{nnn} c 1 \mathrm{~N} 1 \mathrm{CCOCC} 1$ & 0 \\
\hline 422 & $\mathbf{1 5}\{183\}$ & $\mathbf{1 1}\{328\}$ & $\mathbf{9}\{183,328\}$ & Clc1ccc(CCCn2nnnc2N2CCOCC2)cc1 & 0 \\
\hline 423 & $\mathbf{1 5}\{183\}$ & $\mathbf{1 1}\{328\}$ & $\mathbf{9}\{183,328\}$ & Clc1ccc(CCCn2nnnc2N2CCOCC2)cc1 & 0 \\
\hline 424 & $\mathbf{1 5}\{184\}$ & $\mathbf{1 1}\{337\}$ & $\mathbf{9}\{184,337\}$ & $\mathrm{COc} 1 \mathrm{ccccc} 1 \mathrm{C} 1(\mathrm{Cn} 2 \mathrm{nnnn} 2 \mathrm{~N}(\mathrm{C}) \mathrm{CC} 2 \mathrm{CCOC} 2) \mathrm{CC} 1$ & 47 \\
\hline 425 & $\mathbf{1 5}\{185\}$ & $\mathbf{1 1}\{349\}$ & $\mathbf{9}\{185,349\}$ & C\#CCC1CCCN(C1)c1nnnn1C1CCSCC1 & 32 \\
\hline 426 & $\mathbf{1 5}\{185\}$ & $\mathbf{1 1}\{338\}$ & $\mathbf{9}\{185,338\}$ & CCC1CN(CC(CC)O1)c1nnnn1C1CCSCC1 & 0 \\
\hline 427 & $\mathbf{1 5}\{185\}$ & $\mathbf{1 1}\{327\}$ & $\mathbf{9}\{185,327\}$ & $\mathrm{C} 1 \mathrm{CC}(\mathrm{N}(\mathrm{C} 1) \mathrm{c} 1 \mathrm{nnnn} 1 \mathrm{C} 1 \mathrm{CCSCC} 1) \mathrm{c} 1 \mathrm{ccsc} 1$ & 37 \\
\hline 428 & $\mathbf{1 5}\{186\}$ & $\mathbf{1 1}\{401\}$ & $\mathbf{9}\{186,401\}$ & $\underset{1 \mathrm{~F}}{\mathrm{CC}}(\mathrm{C})(\mathrm{Cn} 1 \mathrm{nnnnc} 1 \mathrm{~N} 1 \mathrm{CCC}(\mathrm{CC} 1) \mathrm{C}(\mathrm{N})=\mathrm{O}) \mathrm{c} 1 \mathrm{ccccc}$ & 24 \\
\hline 429 & $\mathbf{1 5}\{187\}$ & $\mathbf{1 1}\{337\}$ & $\mathbf{9}\{187,337\}$ & ${ }_{12}^{\mathrm{CN}(\mathrm{CC} 1 \mathrm{CCOC} 1) \mathrm{c} 1 \mathrm{nnnn} 1 \mathrm{CC} 1 \mathrm{c}(\mathrm{C})[\mathrm{nH}] \mathrm{c} 2 \mathrm{ccccc}}$ & 26 \\
\hline 430 & $\mathbf{1 5}\{188\}$ & $\mathbf{1 1}\{349\}$ & $\mathbf{9}\{188,349\}$ & $\mathrm{O}=\mathrm{C} 1 \mathrm{OCCN} 1 \mathrm{CCCn} 1 \mathrm{nnn} \mathrm{c} 1 \mathrm{~N} 1 \mathrm{CCCC}(\mathrm{CC} \# \mathrm{C}) \mathrm{C} 1$ & 29 \\
\hline 431 & $\mathbf{1 5}\{188\}$ & $\mathbf{1 1}\{338\}$ & $\mathbf{9}\{188,338\}$ & $\mathrm{O}$ OCC1CN(CC $(\mathrm{CC}) \mathrm{O} 1) \mathrm{c} 1 \mathrm{nnnn} 1 \mathrm{CCCN} 1 \mathrm{CCOC} 1=$ & 60 \\
\hline 432 & $\mathbf{1 5}\{189\}$ & $\mathbf{1 1}\{440\}$ & $\mathbf{9}\{189,440\}$ & $\mathrm{CC}(\mathrm{CC} \# \mathrm{~N}) \mathrm{N}(\mathrm{C}) \mathrm{c} 1 \mathrm{nnnn} 1 \mathrm{C} 1 \mathrm{CCCc} 2 \mathrm{sccc} 12$ & 0 \\
\hline 433 & $\mathbf{1 5}\{190\}$ & $\mathbf{1 1}\{341\}$ & $\mathbf{9}\{190,341\}$ & $\mathrm{COc} 1 \mathrm{ccc}(\mathrm{CCn} 2 \mathrm{nnn} \operatorname{2N} 2 \mathrm{CCCC} 2) \mathrm{cc} 1 \mathrm{~F}$ & 78 \\
\hline
\end{tabular}




\begin{tabular}{|c|c|c|c|c|c|}
\hline 434 & $\mathbf{1 5}\{191\}$ & $\mathbf{1 1}\{338\}$ & $\mathbf{9}\{191,338\}$ & $\mathrm{CCC} 1 \mathrm{CN}(\mathrm{CC}(\mathrm{CC}) \mathrm{O} 1) \mathrm{c} 1 \mathrm{nnnn} 1 \mathrm{Cc} 1 \mathrm{cccc}(\mathrm{CO}) \mathrm{c} 1$ & 20 \\
\hline 435 & $\mathbf{1 5}\{192\}$ & $\mathbf{1 1}\{328\}$ & $\mathbf{9}\{192,328\}$ & $\mathrm{CCc} 1 \mathrm{cccc} 2 \mathrm{c}(\mathrm{CCn} 3 \mathrm{nnn} \mathrm{c} 3 \mathrm{~N} 3 \mathrm{CCOCC} 3) \mathrm{c}[\mathrm{nH}] \mathrm{c} 12$ & 9 \\
\hline 436 & $\mathbf{1 5}\{193\}$ & $\mathbf{1 1}\{350\}$ & $\mathbf{9}\{193,350\}$ & $\mathrm{CN}(\mathrm{CCC}=\mathrm{C}) \mathrm{c} 1 \mathrm{nnnn} 1 \mathrm{Cc} 1 \mathrm{ccc}(\mathrm{CN} 2 \mathrm{CCCC} 2=\mathrm{O}) \mathrm{c} 1$ & 23 \\
\hline 437 & $\mathbf{1 5}\{194\}$ & $\mathbf{1 1}\{297\}$ & $\mathbf{9}\{194,297\}$ & $\begin{array}{l}\mathrm{CCn} 1 \mathrm{cc}(\mathrm{Cn} 2 \mathrm{nnn} \mathrm{n} 2 \mathrm{~N} 2 \mathrm{CCC}(\mathrm{CC} 2) \mathrm{C} 2(\mathrm{C}) \mathrm{OCCO} 2) \mathrm{c} \\
\mathrm{n} 1\end{array}$ & 33 \\
\hline 438 & $\mathbf{1 5}\{194\}$ & $\mathbf{1 1}\{298\}$ & $\mathbf{9}\{194,298\}$ & $\begin{array}{l}\mathrm{CCn} 1 \mathrm{cc}(\mathrm{Cn} 2 \mathrm{nnnn} 2 \mathrm{~N} 2 \mathrm{CCCC}(\mathrm{C} 2) \mathrm{C} 2(\mathrm{C}) \mathrm{OCCO} 2) \mathrm{c} \\
\mathrm{n} 1\end{array}$ & 26 \\
\hline 439 & $\mathbf{1 5}\{195\}$ & $\mathbf{1 1}\{441\}$ & $\mathbf{9}\{195,441\}$ & $\mathrm{Cc} 1 \mathrm{c}(\mathrm{Cn} 2 \mathrm{nnnnc} 2 \mathrm{~N} 2 \mathrm{CCS}(=\mathrm{O}) \mathrm{C}(\mathrm{C})(\mathrm{C}) \mathrm{CC} 2) \operatorname{cnn} 1 \mathrm{C}$ & 0 \\
\hline 440 & $\mathbf{1 5}\{195\}$ & $\mathbf{1 1}\{326\}$ & $\mathbf{9}\{195,326\}$ & $\mathrm{Cc} 1 \mathrm{c}(\mathrm{Cn} 2 \mathrm{nnnn} 2 \mathrm{~N} 2 \mathrm{CCC}(=\mathrm{CC} 2) \mathrm{C}(\mathrm{F})(\mathrm{F}) \mathrm{F}) \mathrm{cnn} 1 \mathrm{C}$ & 0 \\
\hline 441 & $\mathbf{1 5}\{195\}$ & $\mathbf{1 1}\{442\}$ & $\mathbf{9}\{195,442\}$ & $\mathrm{Cc} 1 \mathrm{c}(\mathrm{Cn} 2 \mathrm{nnnn} 2 \mathrm{~N} 2 \mathrm{CCC}(\mathrm{C} 2) \mathrm{c} 2 \operatorname{cccc}(\mathrm{C}) \mathrm{c} 2) \mathrm{cnn} 1 \mathrm{C}$ & 0 \\
\hline 442 & $\mathbf{1 5}\{195\}$ & $\mathbf{1 1}\{182\}$ & $\mathbf{9}\{195,182\}$ & $\mathrm{CN}(\mathrm{Cc} 1 \mathrm{ccc}(\mathrm{C}) \mathrm{c}(\mathrm{C}) \mathrm{c} 1) \mathrm{c} 1 \mathrm{nnnn} 1 \mathrm{Cc} 1 \mathrm{cnn}(\mathrm{C}) \mathrm{c} 1 \mathrm{C}$ & 0 \\
\hline 443 & $\mathbf{1 5}\{195\}$ & $\mathbf{1 1}\{443\}$ & $\mathbf{9}\{195,443\}$ & $\begin{array}{l}\text { C[C@H]1CN(C[C@@ @ }](C) O 1) c 1 n n n n 1 C c 1 c n n \\
(\mathrm{C}) \mathrm{c} 1 \mathrm{C}\end{array}$ & 17 \\
\hline 444 & $\mathbf{1 5}\{196\}$ & $\mathbf{1 1}\{297\}$ & $\mathbf{9}\{196,297\}$ & ${ }_{1} \mathrm{Cn} 1 \mathrm{ccc}(\mathrm{Cn} 2 \mathrm{nnn} \operatorname{c} 2 \mathrm{~N} 2 \mathrm{CCC}(\mathrm{CC} 2) \mathrm{C} 2(\mathrm{C}) \mathrm{OCCO} 2) \mathrm{n}$ & 0 \\
\hline 445 & $\mathbf{1 5}\{196\}$ & $\mathbf{1 1}\{298\}$ & $\mathbf{9}\{196,298\}$ & ${ }_{1} \mathrm{Cn} 1 \mathrm{ccc}(\mathrm{Cn} 2 \mathrm{nnnnc} 2 \mathrm{~N} 2 \mathrm{CCCC}(\mathrm{C} 2) \mathrm{C} 2(\mathrm{C}) \mathrm{OCCO} 2) \mathrm{n}$ & 42 \\
\hline 446 & $\mathbf{1 5}\{197\}$ & $\mathbf{1 1}\{105\}$ & $\mathbf{9}\{197,105\}$ & CN1CCN(Cc2cccc12)c1nnnn1CC(F)F & 0 \\
\hline 447 & $\mathbf{1 5}\{197\}$ & $\mathbf{1 1}\{444\}$ & $\mathbf{9}\{197,444\}$ & $\mathrm{COc} 1 \mathrm{ccc}(\mathrm{cc} 1) \mathrm{C} 1 \mathrm{CN}(\mathrm{CCO} 1) \mathrm{c} 1 \mathrm{nnnn} 1 \mathrm{CC}(\mathrm{F}) \mathrm{F}$ & 0 \\
\hline 448 & $\mathbf{1 5}\{197\}$ & $\mathbf{1 1}\{445\}$ & $\mathbf{9}\{197,445\}$ & $\mathrm{COc} 1 \mathrm{cc} 2 \mathrm{CCN}(\mathrm{Cc} 2 \mathrm{cc} 1 \mathrm{OC}) \mathrm{c} 1 \mathrm{nnnn} 1 \mathrm{CC}(\mathrm{F}) \mathrm{F}$ & 0 \\
\hline 449 & $\mathbf{1 5}\{198\}$ & $\mathbf{1 1}\{297\}$ & $\mathbf{9}\{198,297\}$ & $\begin{array}{l}\mathrm{CC}(\mathrm{c} 1 \mathrm{cnn}(\mathrm{C}) \mathrm{c} 1) \mathrm{n} 1 \mathrm{nnnn} 1 \mathrm{~N} 1 \mathrm{CCC}(\mathrm{CC} 1) \mathrm{C} 1(\mathrm{C}) \mathrm{OC} \\
\mathrm{CO} 1\end{array}$ & 0 \\
\hline 450 & $\mathbf{1 5}\{198\}$ & $\mathbf{1 1}\{298\}$ & $\mathbf{9}\{198,298\}$ & $\begin{array}{l}\mathrm{CC}(\mathrm{c} 1 \mathrm{cnn}(\mathrm{C}) \mathrm{c} 1) \mathrm{n} 1 \mathrm{nnnn} 1 \mathrm{~N} 1 \mathrm{CCCC}(\mathrm{C} 1) \mathrm{C} 1(\mathrm{C}) \mathrm{OC} \\
\mathrm{CO} 1\end{array}$ & 0 \\
\hline 451 & $\mathbf{1 5}\{199\}$ & $\mathbf{1 1}\{446\}$ & $\mathbf{9}\{199,446\}$ & $\begin{array}{l}\mathrm{COC}(=\mathrm{O})[1 \mathrm{C} @ @ \mathrm{H}] 1 \mathrm{CN}(\mathrm{C}[1 \mathrm{C} @ \mathrm{H}] 1 \mathrm{C}) \mathrm{c} 1 \mathrm{nnnn} 1 \\
\mathrm{Cc} 1 \mathrm{cccc} 2[\mathrm{nH}] \mathrm{ccc} 12\end{array}$ & 16 \\
\hline 452 & $\mathbf{1 5}\{199\}$ & $\mathbf{1 1}\{96\}$ & $\mathbf{9}\{199,96\}$ & ${ }_{12}^{\mathrm{CC}(\mathrm{C}) \mathrm{OC} 1 \mathrm{CCN}(\mathrm{CC} 1) \mathrm{c} 1 \mathrm{nnnn} 1 \mathrm{Cc} 1 \mathrm{ccc} 2[\mathrm{nH}] \mathrm{ccc}}$ & 20 \\
\hline
\end{tabular}




\begin{tabular}{|c|c|c|c|c|c|}
\hline 453 & $\mathbf{1 5}\{199\}$ & $\mathbf{1 1}\{299\}$ & $\mathbf{9}\{199,299\}$ & ${ }_{12}^{\mathrm{COC} 1(\mathrm{CO}) \mathrm{CCN}(\mathrm{CC} 1) \mathrm{c} 1 \mathrm{nnnn} 1 \mathrm{Cc} 1 \mathrm{ccc} 2[\mathrm{nH}] \mathrm{ccc}}$ & 3 \\
\hline 454 & $\mathbf{1 5}\{199\}$ & $\mathbf{1 1}\{320\}$ & $\mathbf{9}\{199,320\}$ & $\begin{array}{l}\mathrm{COC}(=\mathrm{O}) \mathrm{C} 1 \mathrm{CCN}(\mathrm{CC} 1) \mathrm{c} 1 \mathrm{nnnn} 1 \mathrm{Cc} 1 \mathrm{cccc} 2[\mathrm{nH}] \mathrm{cc} \\
\mathrm{c} 12\end{array}$ & 12 \\
\hline 455 & $\mathbf{1 5}\{200\}$ & $\mathbf{1 1}\{356\}$ & $\mathbf{9}\{200,356\}$ & CN(CC1CCC1)c1nnnn1Cc1ccce(c1)-n1cncn1 & 35 \\
\hline 456 & $\mathbf{1 5}\{201\}$ & $\mathbf{1 1}\{327\}$ & $\mathbf{9}\{201,327\}$ & $\mathrm{C} 1 \mathrm{CC}(\mathrm{N}(\mathrm{C} 1) \mathrm{c} 1 \mathrm{nnnn} 1 \mathrm{C} 1 \mathrm{CCCOC} 1) \mathrm{c} 1 \mathrm{ccsc} 1$ & 10 \\
\hline 457 & $\mathbf{1 5}\{201\}$ & $\mathbf{1 1}\{302\}$ & $\mathbf{9}\{201,302\}$ & $\mathrm{CN}(\mathrm{CCOc} 1 \mathrm{ccc}(\mathrm{F}) \mathrm{cc} 1) \mathrm{c} 1 \mathrm{nnnn} 1 \mathrm{C} 1 \mathrm{CCCOC} 1$ & 0 \\
\hline 458 & $\mathbf{1 5}\{201\}$ & $\mathbf{1 1}\{349\}$ & $\mathbf{9}\{201,349\}$ & 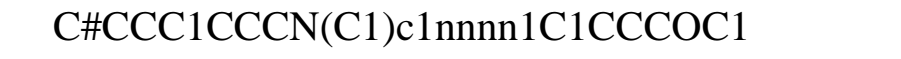 & 35 \\
\hline 459 & $\mathbf{1 5}\{202\}$ & $\mathbf{1 1}\{304\}$ & $\mathbf{9}\{202,304\}$ & $\begin{array}{l}\mathrm{CCC} 1(\mathrm{Cn} 2 \mathrm{nnn} \mathrm{n} 2 \mathrm{~N} 2 \mathrm{CCCC}(\mathrm{CNC}(=\mathrm{O}) \mathrm{C}(\mathrm{C}) \mathrm{C}) \mathrm{C} 2) \\
\mathrm{CCC} 1\end{array}$ & 34 \\
\hline 460 & $\mathbf{1 5}\{203\}$ & $\mathbf{1 1}\{338\}$ & $\mathbf{9}\{203,338\}$ & CCC1CN(CC(CC)O1)c1nnnn1CC1(C)CCCO1 & 15 \\
\hline 461 & $\mathbf{1 5}\{203\}$ & $\mathbf{1 1}\{339\}$ & $\mathbf{9}\{203,339\}$ & $\mathrm{CN}(\mathrm{Cc} 1 \mathrm{cccc}(\mathrm{F}) \mathrm{c} 1) \mathrm{c} 1 \mathrm{nnnn} 1 \mathrm{CC} 1(\mathrm{C}) \mathrm{CCCO} 1$ & 29 \\
\hline 462 & $\mathbf{1 5}\{204\}$ & $\mathbf{1 1}\{447\}$ & $\mathbf{9}\{204,447\}$ & $\mathrm{CCN} 1 \mathrm{CC}(\mathrm{CC} 1=\mathrm{O}) \mathrm{n} 1 \mathrm{nnnnc} 1 \mathrm{~N} 1 \mathrm{CCc} 2 \operatorname{ccccc} 2 \mathrm{C} 1$ & 9 \\
\hline 463 & $\mathbf{1 5}\{204\}$ & $\mathbf{1 1}\{448\}$ & $\mathbf{9}\{204,448\}$ & $\begin{array}{l}\mathrm{CCOC}(=\mathrm{O}) \mathrm{C} 1 \mathrm{CCN}(\mathrm{CC} 1) \mathrm{c} 1 \mathrm{nnnn} 1 \mathrm{C} 1 \mathrm{CN}(\mathrm{CC}) \mathrm{C}(= \\
\mathrm{O}) \mathrm{C} 1\end{array}$ & 22 \\
\hline 464 & $\mathbf{1 5}\{204\}$ & $\mathbf{1 1}\{449\}$ & $\mathbf{9}\{204,449\}$ & $\begin{array}{l}\mathrm{CCN} 1 \mathrm{CC}(\mathrm{CC} 1=\mathrm{O}) \mathrm{n} 1 \mathrm{nnnn} 1 \mathrm{~N} 1 \mathrm{CC}(\mathrm{C})(\mathrm{C}) \mathrm{OC}(\mathrm{C})(\mathrm{C} \\
) \mathrm{C} 1\end{array}$ & 0 \\
\hline 465 & $\mathbf{1 5}\{204\}$ & $\mathbf{1 1}\{106\}$ & $\mathbf{9}\{204,106\}$ & $\mathrm{CCN} 1 \mathrm{CC}(\mathrm{CC} 1=\mathrm{O}) \mathrm{n} 1 \mathrm{nnn} \mathrm{c} 1 \mathrm{~N} 1 \mathrm{CC} 2 \mathrm{ccccc} 2 \mathrm{CC} 1$ & 0 \\
\hline 466 & $\mathbf{1 5}\{204\}$ & $\mathbf{1 1}\{450\}$ & $\mathbf{9}\{204,450\}$ & ${ }_{1} \mathrm{CCN} 1 \mathrm{CC}(\mathrm{CC} 1=\mathrm{O}) \mathrm{n} 1 \mathrm{nnnnc} 1 \mathrm{~N} 1 \mathrm{CCN}(\mathrm{CC} 1) \mathrm{c} 1 \mathrm{ccccc}$ & 0 \\
\hline 467 & $\mathbf{1 5}\{204\}$ & $\mathbf{1 1}\{451\}$ & $\mathbf{9}\{204,451\}$ & $\begin{array}{l}\mathrm{CCOC}(=\mathrm{O}) \mathrm{N} 1 \mathrm{CCN}(\mathrm{CC} 1) \mathrm{c} 1 \mathrm{nnnn} 1 \mathrm{C} 1 \mathrm{CN}(\mathrm{CC}) \mathrm{C}(= \\
\mathrm{O}) \mathrm{C} 1\end{array}$ & 0 \\
\hline 468 & $\mathbf{1 5}\{204\}$ & $\mathbf{1 1}\{349\}$ & $\mathbf{9}\{204,349\}$ & $\mathrm{CCN} 1 \mathrm{CC}(\mathrm{CC} 1=\mathrm{O}) \mathrm{n} 1 \mathrm{nnn} \mathrm{c} 1 \mathrm{~N} 1 \mathrm{CCCC}(\mathrm{CC} \# \mathrm{C}) \mathrm{C} 1$ & 0 \\
\hline 469 & $\mathbf{1 5}\{204\}$ & $\mathbf{1 1}\{452\}$ & $\mathbf{9}\{204,452\}$ & $\begin{array}{l}\mathrm{CCOC}(=\mathrm{O})[\mathrm{C} @ @ \mathrm{H}] 1 \mathrm{CCCN}(\mathrm{C} 1) \mathrm{c} 1 \mathrm{nnnn} 1 \mathrm{C} 1 \mathrm{CN}( \\
\mathrm{CC}) \mathrm{C}(=\mathrm{O}) \mathrm{C} 1\end{array}$ & 0 \\
\hline 470 & $\mathbf{1 5}\{204\}$ & $\mathbf{1 1}\{453\}$ & $\mathbf{9}\{204,453\}$ & $\begin{array}{l}\mathrm{CCOC}(=\mathrm{O}) \mathrm{C} 1 \mathrm{CCCN}(\mathrm{C} 1) \mathrm{c} 1 \mathrm{nnnn} 1 \mathrm{C} 1 \mathrm{CN}(\mathrm{CC}) \mathrm{C}(= \\
\mathrm{O}) \mathrm{C} 1\end{array}$ & 0 \\
\hline 471 & $\mathbf{1 5}\{204\}$ & $\mathbf{1 1}\{302\}$ & $\mathbf{9}\{204,302\}$ & ${ }_{1} \mathrm{CCN} 1 \mathrm{CC}(\mathrm{CC} 1=\mathrm{O}) \mathrm{n} 1 \mathrm{nnnc} 1 \mathrm{~N}(\mathrm{C}) \mathrm{CCOc} 1 \mathrm{ccc}(\mathrm{F}) \mathrm{cc}$ & 16 \\
\hline
\end{tabular}




\begin{tabular}{|c|c|c|c|c|c|}
\hline 472 & $\mathbf{1 5}\{204\}$ & $\mathbf{1 1}\{327\}$ & $\mathbf{9}\{204,327\}$ & $\mathrm{CCN} 1 \mathrm{CC}(\mathrm{CC} 1=\mathrm{O}) \mathrm{n} 1 \mathrm{nnnnc} 1 \mathrm{~N} 1 \mathrm{CCCC} 1 \mathrm{c} 1 \mathrm{ccsc} 1$ & 32 \\
\hline 473 & $\mathbf{1 5}\{205\}$ & $\mathbf{1 1}\{435\}$ & $\mathbf{9}\{205,435\}$ & $\begin{aligned} \mathrm{COC}(=\mathrm{O})[1 \mathrm{C} @] 12 \mathrm{CN}(\mathrm{C}[1 \mathrm{C} @ \mathrm{H}] 1 \mathrm{COCC} 2) \mathrm{c} 1 \mathrm{nn} \\
\mathrm{nn} 1 \mathrm{CC} 1=\mathrm{CCCCC} 1\end{aligned}$ & 15 \\
\hline 474 & $\mathbf{1 5}\{205\}$ & $\mathbf{1 1}\{454\}$ & $\mathbf{9}\{205,454\}$ & $\begin{array}{l}\mathrm{CC} 1 \mathrm{OCCC} 11 \mathrm{CN}(\mathrm{CC}(\mathrm{C}) \mathrm{O} 1) \mathrm{c} 1 \mathrm{nnnn} 1 \mathrm{CC} 1=\mathrm{CCCC} \\
\mathrm{C} 1\end{array}$ & 12 \\
\hline 475 & $\mathbf{1 5}\{205\}$ & $\mathbf{1 1}\{438\}$ & $\mathbf{9}\{205,438\}$ & $\begin{array}{l}\mathrm{NC}(=\mathrm{O})[1 \mathrm{C} @] 12 \mathrm{CCC}[1 \mathrm{C} @ \mathrm{H}] 1 \mathrm{CN}(\mathrm{C} 2) \mathrm{c} 1 \mathrm{nnnn} 1 \\
\mathrm{CC} 1=\mathrm{CCCCC} 1\end{array}$ & 28 \\
\hline 476 & $\mathbf{1 5}\{205\}$ & $\mathbf{1 1}\{433\}$ & $\mathbf{9}\{205,433\}$ & $\begin{array}{l}\mathrm{CC} 1 \mathrm{CN}(\mathrm{CC} 2(\mathrm{CCOCC} 2) \mathrm{O} 1) \mathrm{c} 1 \mathrm{nnnn} 1 \mathrm{CC} 1=\mathrm{CCCC} \\
\mathrm{C} 1\end{array}$ & 31 \\
\hline 477 & $\mathbf{1 5}\{205\}$ & $\mathbf{1 1}\{455\}$ & $\mathbf{9}\{205,455\}$ & CC1OCCC11CN(CCO1)c1nnnn1CC1=CCCCC1 & 0 \\
\hline 478 & $\mathbf{1 5}\{205\}$ & $\mathbf{1 1}\{335\}$ & $\mathbf{9}\{205,335\}$ & $\begin{array}{l}\mathrm{O}=\mathrm{C} 1 \mathrm{NC}(=\mathrm{O})[\mathrm{C} @ @ \mathrm{H}] 2 \mathrm{CN}(\mathrm{C}[\mathrm{C} @ \mathrm{H}] 12) \mathrm{c} 1 \mathrm{nnnn} \\
1 \mathrm{CC} 1=\mathrm{CCCCC} 1\end{array}$ & 0 \\
\hline 479 & $\mathbf{1 5}\{205\}$ & $\mathbf{1 1}\{432\}$ & $\mathbf{9}\{205,432\}$ & $\begin{array}{l}\mathrm{C}(\mathrm{C} 1=\mathrm{CCCCC} 1) \mathrm{n} 1 \mathrm{nnn} \mathrm{c} 1 \mathrm{~N} 1 \mathrm{CCOCC} 2(\mathrm{CCOCC} 2) \\
\mathrm{C} 1\end{array}$ & 25 \\
\hline 480 & $\mathbf{1 5}\{205\}$ & $\mathbf{1 1}\{429\}$ & $\mathbf{9}\{205,429\}$ & $\begin{array}{l}\mathrm{C}(\mathrm{C} 1=\mathrm{CCCCC} 1) \mathrm{n} 1 \mathrm{nnn} \mathrm{n} 1 \mathrm{~N} 1 \mathrm{CCOC} 2(\mathrm{CCOCC} 2) \mathrm{C} \\
\mathrm{C} 1\end{array}$ & 0 \\
\hline 481 & $\mathbf{1 5}\{205\}$ & $\mathbf{1 1}\{188\}$ & $\mathbf{9}\{205,188\}$ & ${ }_{1} \mathrm{CCOC} 1 \mathrm{CC} 2(\mathrm{C} 1) \mathrm{CCN}(\mathrm{C} 2) \mathrm{c} 1 \mathrm{nnnn} 1 \mathrm{CC} 1=\mathrm{CCCCC}$ & 32 \\
\hline 482 & $\mathbf{1 5}\{205\}$ & $\mathbf{1 1}\{431\}$ & $\mathbf{9}\{205,431\}$ & $\mathrm{C}(\mathrm{C} 1=\mathrm{CCCCC} 1) \mathrm{n} 1 \mathrm{nnnn} 1 \mathrm{~N} 1 \mathrm{CCOC} 2(\mathrm{CCSC} 2) \mathrm{C} 1$ & 12 \\
\hline 483 & $\mathbf{1 5}\{205\}$ & $\mathbf{1 1}\{428\}$ & $\mathbf{9}\{205,428\}$ & $\begin{array}{l}\mathrm{O}=\mathrm{C} 1 \mathrm{NCCC} 2(\mathrm{CCN}(\mathrm{CC} 2) \mathrm{c} 2 \mathrm{nnnn} 2 \mathrm{CC} 2=\mathrm{CCCCC} \\
\text { 2) } \mathrm{O} 1\end{array}$ & 16 \\
\hline 484 & $\mathbf{1 5}\{205\}$ & $\mathbf{1 1}\{434\}$ & $\mathbf{9}\{205,434\}$ & ${ }_{1}^{\mathrm{C}(\mathrm{C} 1=\mathrm{CCCCC} 1) \mathrm{n} 1 \mathrm{nnnc} 1 \mathrm{~N} 1 \mathrm{CCOC} 2(\mathrm{CCOCC} 2) \mathrm{C}}$ & 10 \\
\hline 485 & $\mathbf{1 5}\{205\}$ & $\mathbf{1 1}\{304\}$ & $\mathbf{9}\{205,304\}$ & $\begin{array}{l}\mathrm{CC}(\mathrm{C}) \mathrm{C}(=\mathrm{O}) \mathrm{NCC} 1 \mathrm{CCCN}(\mathrm{C} 1) \mathrm{c} 1 \mathrm{nnnn} 1 \mathrm{CC} 1=\mathrm{CC} \\
\mathrm{CCC} 1\end{array}$ & 8 \\
\hline 486 & $\mathbf{1 5}\{205\}$ & $\mathbf{1 1}\{456\}$ & $\mathbf{9}\{205,456\}$ & ${ }_{2}^{\mathrm{C}(\mathrm{C} 1=\mathrm{CCCCC} 1) \mathrm{n} 1 \mathrm{nnnc} 1 \mathrm{~N} 1 \mathrm{CCC} 2(\mathrm{CC} 1) \mathrm{OCCCO}}$ & 29 \\
\hline 487 & $\mathbf{1 5}\{206\}$ & $\mathbf{1 1}\{341\}$ & $\mathbf{9}\{206,341\}$ & Clc1cce(cc1)-c1noc(CCn2nnnc2N2CCCC2)n1 & 25 \\
\hline 488 & $\mathbf{1 5}\{207\}$ & $\mathbf{1 1}\{302\}$ & $\mathbf{9}\{207,302\}$ & $\mathrm{CN}(\mathrm{CCOc} 1 \mathrm{ccc}(\mathrm{F}) \mathrm{cc} 1) \mathrm{c} 1 \mathrm{nnnn} 1 \mathrm{CC} 1(\mathrm{C}) \mathrm{CCOC} 1$ & 17 \\
\hline 489 & $\mathbf{1 5}\{208\}$ & $\mathbf{1 1}\{338\}$ & $\mathbf{9}\{208,338\}$ & $\mathrm{CCC} 1 \mathrm{CN}(\mathrm{CC}(\mathrm{CC}) \mathrm{O} 1) \mathrm{c} 1 \mathrm{nnnn} 1 \mathrm{Cc} 1 \mathrm{ccn}(\mathrm{n} 1) \mathrm{C}(\mathrm{C}) \mathrm{C}$ & 61 \\
\hline 490 & $\mathbf{1 5}\{209\}$ & $\mathbf{1 1}\{350\}$ & $\mathbf{9}\{209,350\}$ & $\mathrm{CN}(\mathrm{CCC}=\mathrm{C}) \mathrm{c} 1 \mathrm{nnnn} 1 \mathrm{C} 1 \mathrm{CCN}(\mathrm{CC}(\mathrm{F})(\mathrm{F}) \mathrm{F}) \mathrm{C} 1=\mathrm{O}$ & 18 \\
\hline
\end{tabular}




\begin{tabular}{|c|c|c|c|c|c|}
\hline 491 & $\mathbf{1 5}\{210\}$ & $11\{298\}$ & $\mathbf{9}\{210,298\}$ & $\begin{array}{l}\mathrm{Cc} 1 \mathrm{cc}(\mathrm{Cn} 2 \mathrm{nnnn} 2 \mathrm{~N} 2 \mathrm{CCCC}(\mathrm{C} 2) \mathrm{C} 2(\mathrm{C}) \mathrm{OCCO} 2) \mathrm{nc} \\
\mathrm{n} 1\end{array}$ & 0 \\
\hline 492 & $15\{210\}$ & $11\{297\}$ & $\mathbf{9}\{210,297\}$ & $\begin{array}{l}\mathrm{Cc} 1 \mathrm{cc}(\mathrm{Cn} 2 \mathrm{nnn} n 2 \mathrm{~N} 2 \mathrm{CCC}(\mathrm{CC} 2) \mathrm{C} 2(\mathrm{C}) \mathrm{OCCO} 2) \mathrm{nc} \\
\mathrm{n} 1\end{array}$ & 0 \\
\hline 493 & $15\{211\}$ & $\mathbf{1 1}\{457\}$ & $\mathbf{9}\{211,457\}$ & ${ }_{\mathrm{C}}^{\mathrm{CC}} \mathrm{lncc} 2 \mathrm{C} 3 \mathrm{CCC}(\mathrm{Cc} 2 \mathrm{n} 1) \mathrm{N} 3 \mathrm{c} 1 \mathrm{nnnn} 1 \mathrm{CC} 1 \mathrm{CC} 1(\mathrm{C}$ & 8 \\
\hline 494 & $\mathbf{1 5}\{211\}$ & $11\{359\}$ & $\mathbf{9}\{211,359\}$ & $\begin{array}{l}\mathrm{CC} 1(\mathrm{C}) \mathrm{CC} 1 \mathrm{Cn} 1 \mathrm{nnn} n 1 \mathrm{~N} 1 \mathrm{C}[\mathrm{C} @ @ \mathrm{H}] 2 \mathrm{C}[\mathrm{C} @ \mathrm{H}](\mathrm{C} \\
\text { 1)c1 } \mathrm{cccc}(=\mathrm{O}) \mathrm{n} 1 \mathrm{C} 2\end{array}$ & 27 \\
\hline 495 & $\mathbf{1 5}\{211\}$ & $\mathbf{1 1}\{458\}$ & $\mathbf{9}\{211,458\}$ & $\begin{array}{l}\mathrm{CO}[1 \mathrm{C} @ @ \mathrm{H}] 1 \mathrm{CS}(=\mathrm{O})(=\mathrm{O})[1 \mathrm{C} @ \mathrm{H}] 2 \mathrm{CN}(\mathrm{C}[1 \mathrm{C} \\
@ \mathrm{H}] 12) \mathrm{c} 1 \mathrm{nnnn} 1 \mathrm{CC} 1 \mathrm{CC} 1(\mathrm{C}) \mathrm{C}\end{array}$ & 20 \\
\hline 496 & $\mathbf{1 5}\{212\}$ & $11\{327\}$ & $\mathbf{9}\{212,327\}$ & $\mathrm{O}=\mathrm{C} 1 \mathrm{CNC}(=\mathrm{O}) \mathrm{N} 1 \mathrm{CCn} 1 \mathrm{nnnn} 1 \mathrm{~N} 1 \mathrm{CCCC} 1 \mathrm{c} 1 \mathrm{ccsc} 1$ & 57 \\
\hline 497 & $\mathbf{1 5}\{212\}$ & $\mathbf{1 1}\{349\}$ & $\mathbf{9}\{212,349\}$ & $\begin{array}{l}\mathrm{O}=\mathrm{C} 1 \mathrm{CNC}(=\mathrm{O}) \mathrm{N} 1 \mathrm{CCn} 1 \mathrm{nnn} \mathrm{1} 1 \mathrm{~N} 1 \mathrm{CCCC}(\mathrm{CC} \# \mathrm{C}) \\
\mathrm{C} 1\end{array}$ & 21 \\
\hline 498 & $\mathbf{1 5}\{213\}$ & $11\{297\}$ & $\mathbf{9}\{213,297\}$ & $\mathrm{CC} 1(\mathrm{OCCO} 1) \mathrm{C} 1 \mathrm{CCN}(\mathrm{CC} 1) \mathrm{c} 1 \mathrm{nnnn} 1 \mathrm{Cc} 1 \mathrm{ccon} 1$ & 0 \\
\hline 499 & $15\{213\}$ & $\mathbf{1 1}\{298\}$ & $\mathbf{9}\{213,298\}$ & $\mathrm{CC} 1(\mathrm{OCCO} 1) \mathrm{C} 1 \mathrm{CCCN}(\mathrm{C} 1) \mathrm{c} 1 \mathrm{nnnn} 1 \mathrm{Cc} 1 \mathrm{ccon} 1$ & 0 \\
\hline 500 & $15\{214\}$ & $11\{320\}$ & $\mathbf{9}\{214,320\}$ & ${ }_{\mathrm{c} 1}^{\mathrm{COC}(=\mathrm{O}) \mathrm{C} 1 \mathrm{CCN}(\mathrm{CC} 1) \mathrm{c} 1 \mathrm{nnnn} 1 \mathrm{Cc} 1 \mathrm{cc}(\mathrm{F}) \mathrm{cc}(\mathrm{OC})}$ & 43 \\
\hline 501 & $\mathbf{1 5}\{215\}$ & $11\{369\}$ & $\mathbf{9}\{215,369\}$ & $\mathrm{CC} 1 \mathrm{CN}(\mathrm{CCO} 1) \mathrm{c} 1 \mathrm{nnnn} 1 \mathrm{CC} 1 \mathrm{CSCCS} 1$ & 30 \\
\hline 502 & $15\{216\}$ & $11\{319\}$ & $\mathbf{9}\{216,319\}$ & $\mathrm{CCSc} 1 \mathrm{cccc}(\mathrm{Cn} 2 \mathrm{nnnn} 2 \mathrm{~N}(\mathrm{C}) \mathrm{CC} 2 \mathrm{COCCO} 2) \mathrm{c} 1$ & 57 \\
\hline 503 & $15\{217\}$ & $\mathbf{1 1}\{337\}$ & $\mathbf{9}\{217,337\}$ & ${ }_{\mathrm{F}}^{\mathrm{CN}(\mathrm{CC} 1 \mathrm{CCOC} 1) \mathrm{c} 1 \mathrm{nnnn} 1 \mathrm{CC} 1 \mathrm{CCC}(\mathrm{CC} 1) \mathrm{C}(\mathrm{F})(\mathrm{F})}$ & 49 \\
\hline 504 & $\mathbf{1 5}\{218\}$ & $11\{288\}$ & $\mathbf{9}\{218,288\}$ & $\begin{array}{l}\mathrm{CC}(=\mathrm{O}) \mathrm{N} 1 \mathrm{CCCN}(\mathrm{CC} 1) \mathrm{c} 1 \mathrm{nnnn} 1 \mathrm{CC} 1 \mathrm{C}(\mathrm{C})(\mathrm{C}) \mathrm{C} 1( \\
\mathrm{C}) \mathrm{C}\end{array}$ & 12 \\
\hline 505 & $\mathbf{1 5}\{218\}$ & $\mathbf{1 1}\{395\}$ & $\mathbf{9}\{218,395\}$ & $\begin{array}{l}\mathrm{CC} 1(\mathrm{C}) \mathrm{C}(\mathrm{Cn} 2 \mathrm{nnn} \mathrm{c} 2 \mathrm{~N} 2 \mathrm{CCOC} 3(\mathrm{CCOC} 3) \mathrm{C} 2) \mathrm{C} 1( \\
\mathrm{C}) \mathrm{C}\end{array}$ & 0 \\
\hline 506 & $15\{219\}$ & $11\{327\}$ & $\mathbf{9}\{219,327\}$ & $\mathrm{O}=\mathrm{C} 1 \mathrm{CC}(\mathrm{Cn} 2 \mathrm{nnn} \mathrm{c} 2 \mathrm{~N} 2 \mathrm{CCCC} 2 \mathrm{c} 2 \mathrm{ccsc} 2) \mathrm{CN} 1$ & 32 \\
\hline 507 & $15\{219\}$ & $\mathbf{1 1}\{365\}$ & $\mathbf{9}\{219,365\}$ & $\begin{array}{l}\mathrm{CC} 1 \mathrm{CC}(\mathrm{CN} 1 \mathrm{c} 1 \mathrm{nnnn} 1 \mathrm{CC} 1 \mathrm{CNC}(=\mathrm{O}) \mathrm{C} 1) \mathrm{c} 1 \mathrm{ccc}(\mathrm{C}) \\
\mathrm{cc} 1\end{array}$ & 12 \\
\hline 508 & $15\{219\}$ & $11\{302\}$ & $\mathbf{9}\{219,302\}$ & $\mathrm{CN}(\mathrm{CCOc} 1 \mathrm{ccc}(\mathrm{F}) \mathrm{cc} 1) \mathrm{c} 1 \mathrm{nnnn} 1 \mathrm{CC} 1 \mathrm{CNC}(=\mathrm{O}) \mathrm{C} 1$ & 0 \\
\hline 509 & $15\{220\}$ & $11\{304\}$ & $\mathbf{9}\{220,304\}$ & $\begin{array}{l}\mathrm{CC}(\mathrm{C}) \mathrm{C}(=\mathrm{O}) \mathrm{NCC} 1 \mathrm{CCCN}(\mathrm{C} 1) \mathrm{c} 1 \mathrm{nnnn} 1 \mathrm{CCC} 1 \mathrm{CC} \\
\mathrm{C} 1\end{array}$ & 28 \\
\hline
\end{tabular}




\begin{tabular}{|c|c|c|c|c|c|c|}
\hline 510 & $15\{220\}$ & $\mathbf{1 1}\{288\}$ & $\mathbf{9}\{220,288\}$ & $\mathrm{CC}(=\mathrm{O}) \mathrm{N} 1 \mathrm{CCCN}(\mathrm{CC} 1) \mathrm{c} 1 \mathrm{nnnn} 1 \mathrm{CCC} 1 \mathrm{CCC} 1$ & 32 & 16 \\
\hline 511 & $15\{221\}$ & $11\{288\}$ & $\mathbf{9}\{221,288\}$ & $\begin{array}{l}\mathrm{CC}(\mathrm{C}) \mathrm{CC} 1(\mathrm{Cn} 2 \mathrm{nnn} n 2 \mathrm{~N} 2 \mathrm{CCCN}(\mathrm{CC} 2) \mathrm{C}(\mathrm{C})=\mathrm{O}) \mathrm{C} \\
\mathrm{CC} 1\end{array}$ & 27 & 13 \\
\hline 512 & $15\{221\}$ & $\mathbf{1 1}\{395\}$ & $\mathbf{9}\{221,395\}$ & $\begin{array}{l}\mathrm{CC}(\mathrm{C}) \mathrm{CC} 1(\mathrm{Cn} 2 \mathrm{nnnn} 2 \mathrm{~N} 2 \mathrm{CCOC} 3(\mathrm{CCOC} 3) \mathrm{C} 2) \mathrm{C} \\
\mathrm{CC} 1\end{array}$ & 0 & 0 \\
\hline 513 & $15\{222\}$ & $\mathbf{1 1}\{411\}$ & $\mathbf{9}\{222,411\}$ & $\mathrm{CCC} 1 \mathrm{CCCN}(\mathrm{C} 1) \mathrm{c} 1 \mathrm{nnnn} 1 \mathrm{Cc} 1 \mathrm{snnc} 1 \mathrm{C}$ & 10 & 5 \\
\hline 514 & $15\{223\}$ & $11\{333\}$ & $\mathbf{9}\{223,333\}$ & $\mathrm{CC} 1(\mathrm{C}) \mathrm{CN}(\mathrm{CCS} 1) \mathrm{c} 1 \mathrm{nnnn} 1 \mathrm{Cc} 1 \mathrm{ccc}(\mathrm{F}) \mathrm{cn} 1$ & 12 & 6 \\
\hline 515 & $15\{224\}$ & $\mathbf{1 1}\{459\}$ & $\mathbf{9}\{224,459\}$ & $\mathrm{CCN}(\mathrm{CC}) \mathrm{c} 1 \mathrm{nnnn} 1 \mathrm{Cc} 1 \mathrm{nnc}(\mathrm{C}) \mathrm{s} 1$ & 9 & 4 \\
\hline 516 & $15\{225\}$ & $11\{366\}$ & $\mathbf{9}\{225,366\}$ & $\mathrm{CN}(\mathrm{CC}(\mathrm{C})(\mathrm{C}) \mathrm{O}) \mathrm{c} 1 \mathrm{nnnn} 1 \mathrm{Cc} 1 \mathrm{ccc}(\mathrm{C}) \mathrm{c}(\mathrm{F}) \mathrm{c} 1$ & 0 & 0 \\
\hline 517 & $15\{225\}$ & $11\{399\}$ & $\mathbf{9}\{225,399\}$ & $\mathrm{COCC} 1(\mathrm{C}) \mathrm{CCN}(\mathrm{C} 1) \mathrm{c} 1 \mathrm{nnnn} 1 \mathrm{Cc} 1 \mathrm{ccc}(\mathrm{C}) \mathrm{c}(\mathrm{F}) \mathrm{c} 1$ & 33 & 15 \\
\hline 518 & $\mathbf{1 5}\{225\}$ & $11\{367\}$ & $\mathbf{9}\{225,367\}$ & $\begin{array}{l}\mathrm{CN}(\mathrm{CC}(=\mathrm{O}) \mathrm{N} 1 \mathrm{CCOCC} 1) \mathrm{c} 1 \mathrm{nnnn} 1 \mathrm{Cc} 1 \mathrm{ccc}(\mathrm{C}) \mathrm{c}(\mathrm{F}) \\
\mathrm{c} 1\end{array}$ & 0 & 0 \\
\hline 519 & $15\{226\}$ & $11\{288\}$ & $\mathbf{9}\{226,288\}$ & $\begin{array}{l}\mathrm{CC}(=\mathrm{O}) \mathrm{N} 1 \mathrm{CCCN}(\mathrm{CC} 1) \mathrm{c} 1 \mathrm{nnnn} 1 \mathrm{C} 1 \mathrm{CCCC}(\mathrm{C})(\mathrm{C}) \\
\mathrm{C} 1\end{array}$ & 21 & 9 \\
\hline 520 & $\mathbf{1 5}\{227\}$ & $11\{460\}$ & $\mathbf{9}\{227,460\}$ & Fc1ccc(cc1)N1CCN(CC1)c1nnnn1OC1CCCC1 & 0 & 0 \\
\hline 521 & $15\{227\}$ & $11\{446\}$ & $\mathbf{9}\{227,446\}$ & $\begin{array}{l}\mathrm{COC}(=\mathrm{O})[1 \mathrm{C} @ @ \mathrm{H}] 1 \mathrm{CN}(\mathrm{C}[1 \mathrm{C} @ \mathrm{H}] 1 \mathrm{C}) \mathrm{c} 1 \mathrm{nnnn} 1 \\
\mathrm{OC} 1 \mathrm{CCCC} 1\end{array}$ & 0 & 0 \\
\hline 522 & $15\{227\}$ & $11\{461\}$ & $\mathbf{9}\{227,461\}$ & Cc1ccc(cc1)N1CCN(CC1)c1nnnn1OC1CCCC1 & 0 & 0 \\
\hline 523 & $15\{227\}$ & $11\{462\}$ & $\mathbf{9}\{227,462\}$ & Cc1cccce1N1CCN(CC1)c1nnnn1OC1CCCC1 & 0 & 0 \\
\hline 524 & $15\{227\}$ & $11\{463\}$ & $\mathbf{9}\{227,463\}$ & $\mathrm{Cc} 1 \mathrm{ccc}(\mathrm{cc} 1 \mathrm{C}) \mathrm{N} 1 \mathrm{CCN}(\mathrm{CC} 1) \mathrm{c} 1 \mathrm{nnnn} 1 \mathrm{OC} 1 \mathrm{CCCC} 1$ & 0 & 0 \\
\hline 525 & $15\{227\}$ & $11\{464\}$ & $\mathbf{9}\{227,464\}$ & Cc1ccce(c1)N1CCN(CC1)c1nnnn1OC1CCCC1 & 0 & 0 \\
\hline 526 & $15\{227\}$ & $\mathbf{1 1}\{465\}$ & $\mathbf{9}\{227,465\}$ & $\mathrm{CC} 1 \mathrm{CCN}(\mathrm{CC} 1 \mathrm{C}) \mathrm{c} 1 \mathrm{nnnn} 1 \mathrm{OC} 1 \mathrm{CCCC} 1$ & 0 & 0 \\
\hline 527 & $15\{228\}$ & $\mathbf{1 1}\{355\}$ & $\mathbf{9}\{228,355\}$ & ${ }_{1} \mathrm{CCOC}(=\mathrm{O}) \mathrm{C} 1(\mathrm{Cn} 2 \mathrm{nnnn} 2 \mathrm{~N}(\mathrm{C}) \mathrm{Cc} 2 \mathrm{cnn}(\mathrm{C}) \mathrm{c} 2) \mathrm{CCC}$ & 21 & 11 \\
\hline 528 & $15\{229\}$ & $11\{327\}$ & $\mathbf{9}\{229,327\}$ & $\mathrm{CC} 1(\mathrm{C}) \mathrm{CC}(\mathrm{CCO} 1) \mathrm{n} 1 \mathrm{nnnn} 1 \mathrm{~N} 1 \mathrm{CCCC} 1 \mathrm{c} 1 \mathrm{ccsc} 1$ & 180 & 92 \\
\hline 529 & $15\{229\}$ & $11\{349\}$ & $\mathbf{9}\{229,349\}$ & $\mathrm{CC} 1(\mathrm{C}) \mathrm{CC}(\mathrm{CCO} 1) \mathrm{n} 1 \mathrm{nnnc} 1 \mathrm{~N} 1 \mathrm{CCCC}(\mathrm{CC} \# \mathrm{C}) \mathrm{C} 1$ & 37 & 18 \\
\hline
\end{tabular}




\begin{tabular}{|c|c|c|c|c|c|}
\hline 530 & $\mathbf{1 5}\{229\}$ & $\mathbf{1 1}\{338\}$ & $\mathbf{9}\{229,338\}$ & 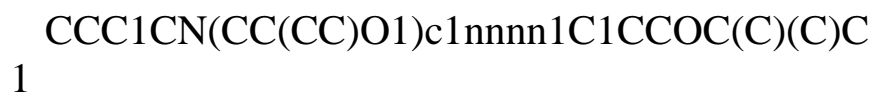 & 42 \\
\hline 531 & $\mathbf{1 5}\{230\}$ & $\mathbf{1 1}\{349\}$ & $\mathbf{9}\{230,349\}$ & $\begin{array}{l}\mathrm{O}=\mathrm{C}(\mathrm{CCn} 1 \mathrm{nnn} n \mathrm{1N} 1 \mathrm{CCCC}(\mathrm{CC} \# \mathrm{C}) \mathrm{C} 1) \mathrm{N} 1 \mathrm{CCCC} \\
\mathrm{C} 1\end{array}$ & 29 \\
\hline 532 & $\mathbf{1 5}\{230\}$ & $\mathbf{1 1}\{350\}$ & $\mathbf{9}\{230,350\}$ & $\mathrm{CN}(\mathrm{CCC}=\mathrm{C}) \mathrm{c} 1 \mathrm{nnnn} 1 \mathrm{CCC}(=\mathrm{O}) \mathrm{N} 1 \mathrm{CCCCC} 1$ & 18 \\
\hline 533 & $\mathbf{1 5}\{231\}$ & $\mathbf{1 1}\{349\}$ & $\mathbf{9}\{231,349\}$ & C\#CCC1CCCN(C1)c1nnnn1CCC1CC1 & 37 \\
\hline 534 & $\mathbf{1 5}\{231\}$ & $\mathbf{1 1}\{288\}$ & $\mathbf{9}\{231,288\}$ & $\mathrm{CC}(=\mathrm{O}) \mathrm{N} 1 \mathrm{CCCN}(\mathrm{CC} 1) \mathrm{c} 1 \mathrm{nnnn} 1 \mathrm{CCC} 1 \mathrm{CC} 1$ & 25 \\
\hline 535 & $\mathbf{1 5}\{231\}$ & $\mathbf{1 1}\{318\}$ & $\mathbf{9}\{231,318\}$ & $\begin{array}{l}\mathrm{C}(\mathrm{Cn} 1 \mathrm{nnn} \mathrm{n} 1 \mathrm{~N} 1 \mathrm{CCn} 2 \mathrm{c}(\mathrm{C} 1) \mathrm{nn} \mathrm{c} 2- \\
\mathrm{c} 1 \mathrm{ccccc} 1) \mathrm{C} 1 \mathrm{CC} 1\end{array}$ & 0 \\
\hline 536 & $\mathbf{1 5}\{232\}$ & $\mathbf{1 1}\{466\}$ & $\mathbf{9}\{232,466\}$ & $\begin{array}{l}\text { COC }[\mathrm{C} @ @ \mathrm{H}](\mathrm{C}) \mathrm{n} 1 \mathrm{nnnn} 1 \mathrm{~N} 1 \mathrm{CCCC} 2(\mathrm{C} 1) \mathrm{Oc} 1 \mathrm{ccc} \\
\mathrm{cc} 1 \mathrm{C}=\mathrm{C} 2\end{array}$ & 26 \\
\hline 537 & $\mathbf{1 5}\{232\}$ & $\mathbf{1 1}\{315\}$ & $\mathbf{9}\{232,315\}$ & $\begin{array}{l}\mathrm{COC}[\mathrm{C} @ @ \mathrm{H}](\mathrm{C}) \mathrm{n} 1 \mathrm{nnn} \mathrm{n} 1 \mathrm{~N} 1 \mathrm{C}[\mathrm{C} @ \mathrm{H}] 2 \mathrm{C}[\mathrm{C} @ \\
\mathrm{H}] 1 \mathrm{CN} 2 \mathrm{C}(=\mathrm{O}) \mathrm{OC}(\mathrm{C})(\mathrm{C}) \mathrm{C}\end{array}$ & 20 \\
\hline 538 & $\mathbf{1 5}\{232\}$ & $\mathbf{1 1}\{467\}$ & $\mathbf{9}\{232,467\}$ & $\begin{array}{l}\mathrm{COC}[\mathrm{C} @ @ \mathrm{H}](\mathrm{C}) \mathrm{n} 1 \mathrm{nnn} c 1 \mathrm{~N} 1 \mathrm{C} 2 \mathrm{CCC} 1 \mathrm{CC}(\mathrm{CC}(\mathrm{F}) \\
(\mathrm{F}) \mathrm{F}) \mathrm{C} 2\end{array}$ & 33 \\
\hline 539 & $\mathbf{1 5}\{233\}$ & $\mathbf{1 1}\{349\}$ & $\mathbf{9}\{233,349\}$ & C\#CCC1CCCN(C1)c1nnnn1CC1CCCS1 & 29 \\
\hline 540 & $\mathbf{1 5}\{234\}$ & $\mathbf{1 1}\{468\}$ & $\mathbf{9}\{234,468\}$ & $\begin{array}{l}\mathrm{CC}(=\mathrm{C}) \mathrm{CCn} 1 \mathrm{nnn} n 1 \mathrm{~N} 1 \mathrm{CCC}(\mathrm{CC} 1)[1 \mathrm{C} @ @ \mathrm{H}] 1 \mathrm{OC} \\
\mathrm{C}[1 \mathrm{C} @ \mathrm{H}] 1 \mathrm{C}(\mathrm{N})=\mathrm{O}\end{array}$ & 21 \\
\hline 541 & $\mathbf{1 5}\{235\}$ & $\mathbf{1 1}\{469\}$ & $\mathbf{9}\{235,469\}$ & $\mathrm{COC} 1 \mathrm{CCC}(\mathrm{Cn} 2 \mathrm{nnn} n 2 \mathrm{~N} 2 \mathrm{CCSCC} 2 \mathrm{C}) \mathrm{CC} 1$ & 12 \\
\hline 542 & $15\{236\}$ & $\mathbf{1 1}\{349\}$ & $\mathbf{9}\{236,349\}$ & $\mathrm{CC} 1 \mathrm{CC}(\mathrm{CC}(\mathrm{C}) \mathrm{O} 1) \mathrm{n} 1 \mathrm{nnnnc} 1 \mathrm{~N} 1 \mathrm{CCCC}(\mathrm{CC} \# \mathrm{C}) \mathrm{C} 1$ & 0 \\
\hline 543 & $\mathbf{1 5}\{236\}$ & $\mathbf{1 1}\{327\}$ & $\mathbf{9}\{236,327\}$ & $\mathrm{CC} 1 \mathrm{CC}(\mathrm{CC}(\mathrm{C}) \mathrm{O} 1) \mathrm{n} 1 \mathrm{nnn} \mathrm{n} 1 \mathrm{~N} 1 \mathrm{CCCC} 1 \mathrm{c} 1 \mathrm{ccsc} 1$ & 40 \\
\hline 544 & $\mathbf{1 5}\{236\}$ & $\mathbf{1 1}\{338\}$ & $\mathbf{9}\{236,338\}$ & ${ }_{1} \mathrm{CCC} 1 \mathrm{CN}(\mathrm{CC}(\mathrm{CC}) \mathrm{O} 1) \mathrm{c} 1 \mathrm{nnnn} 1 \mathrm{C} 1 \mathrm{CC}(\mathrm{C}) \mathrm{OC}(\mathrm{C}) \mathrm{C}$ & 34 \\
\hline 545 & $\mathbf{1 5}\{237\}$ & $\mathbf{1 1}\{302\}$ & $\mathbf{9}\{237,302\}$ & CC1CC(CCO1)n1nnnc1N(C)CCOc1ccc(F)cc1 & 31 \\
\hline 546 & $\mathbf{1 5}\{238\}$ & $\mathbf{1 1}\{318\}$ & $\mathbf{9}\{238,318\}$ & $\begin{array}{l}\mathrm{C} 1 \mathrm{CC}(\mathrm{C}=\mathrm{C} 1) \mathrm{n} 1 \mathrm{nnn} \mathrm{c} 1 \mathrm{~N} 1 \mathrm{CCn} 2 \mathrm{c}(\mathrm{C} 1) \mathrm{nnc} 2- \\
\mathrm{c} 1 \mathrm{ccccc} 1\end{array}$ & 0 \\
\hline 547 & $\mathbf{1 5}\{239\}$ & $\mathbf{1 1}\{298\}$ & $\mathbf{9}\{239,298\}$ & 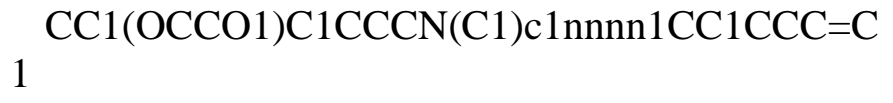 & 28 \\
\hline 548 & $\mathbf{1 5}\{239\}$ & $\mathbf{1 1}\{468\}$ & $\mathbf{9}\{239,468\}$ & $\begin{array}{l}\mathrm{NC}(=\mathrm{O})[1 \mathrm{C} @ @ \mathrm{H}] 1 \mathrm{CCO}[1 \mathrm{C} @ \mathrm{H}] 1 \mathrm{C} 1 \mathrm{CCN}(\mathrm{CC} 1) \\
\mathrm{c} 1 \mathrm{nnnn} 1 \mathrm{CC} 1 \mathrm{CCC}=\mathrm{C} 1\end{array}$ & 43 \\
\hline
\end{tabular}




\begin{tabular}{|c|c|c|c|c|c|}
\hline 549 & $\mathbf{1 5}\{239\}$ & $\mathbf{1 1}\{470\}$ & $\mathbf{9}\{239,470\}$ & $\begin{array}{l}\mathrm{CC}(\mathrm{C})(\mathrm{C}) \mathrm{OC}(=\mathrm{O}) \mathrm{NC} 1 \mathrm{CCN}(\mathrm{C} 1) \mathrm{c} 1 \mathrm{nnnn} 1 \mathrm{CC} 1 \mathrm{CC} \\
\mathrm{C}=\mathrm{C} 1\end{array}$ & 52 \\
\hline 550 & $\mathbf{1 5}\{239\}$ & $\mathbf{1 1}\{471\}$ & $\mathbf{9}\{239,471\}$ & $\begin{array}{l}\mathrm{CC}(\mathrm{C})(\mathrm{C}) \mathrm{OC}(=\mathrm{O}) \mathrm{N}[\mathrm{C} @ \mathrm{H}] 1 \mathrm{CCN}(\mathrm{C} 1) \mathrm{c} 1 \mathrm{nnnn} 1 \mathrm{C} \\
\mathrm{C} 1 \mathrm{CCC}=\mathrm{C} 1\end{array}$ & 0 \\
\hline 551 & $\mathbf{1 5}\{239\}$ & $\mathbf{1 1}\{360\}$ & $\mathbf{9}\{239,360\}$ & $\begin{array}{l}\mathrm{CC}(\mathrm{C})(\mathrm{C}) \mathrm{OC}(=\mathrm{O}) \mathrm{NC} 1 \mathrm{CCN}(\mathrm{CC} 1) \mathrm{c} 1 \mathrm{nnnn} 1 \mathrm{CC} 1 \mathrm{C} \\
\mathrm{CC}=\mathrm{C} 1\end{array}$ & 35 \\
\hline 552 & $\mathbf{1 5}\{239\}$ & $\mathbf{1 1}\{297\}$ & $\mathbf{9}\{239,297\}$ & ${ }_{1} \mathrm{CC} 1(\mathrm{OCCO} 1) \mathrm{C} 1 \mathrm{CCN}(\mathrm{CC} 1) \mathrm{c} 1 \mathrm{nnnn} 1 \mathrm{CC} 1 \mathrm{CCC}=\mathrm{C}$ & 53 \\
\hline 553 & $\mathbf{1 5}\{239\}$ & $\mathbf{1 1}\{287\}$ & $\mathbf{9}\{239,287\}$ & $\begin{array}{l}\mathrm{O}=\mathrm{C}(\mathrm{N} 1 \mathrm{CCN}(\mathrm{CC} 1) \mathrm{c} 1 \mathrm{nnnn} 1 \mathrm{CC} 1 \mathrm{CCC}=\mathrm{C} 1) \mathrm{c} 1 \mathrm{ccc} \\
\mathrm{o} 1\end{array}$ & 22 \\
\hline 554 & $\mathbf{1 5}\{239\}$ & $\mathbf{1 1}\{359\}$ & $\mathbf{9}\{239,359\}$ & $\begin{array}{l}\mathrm{O}=\mathrm{c} 1 \mathrm{cccc} 2[\mathrm{C} @ @ \mathrm{H}] 3 \mathrm{C}[\mathrm{C} @ @ \mathrm{H}](\mathrm{CN}(\mathrm{C} 3) \mathrm{c} 3 \mathrm{nnnn} \\
\text { 3CC3CCC=C3)Cn12 }\end{array}$ & 17 \\
\hline 555 & $\mathbf{1 5}\{239\}$ & $\mathbf{1 1}\{320\}$ & $\mathbf{9}\{239,320\}$ & $\mathrm{COC}(=\mathrm{O}) \mathrm{C} 1 \mathrm{CCN}(\mathrm{CC} 1) \mathrm{c} 1 \mathrm{nnnn} 1 \mathrm{CC} 1 \mathrm{CCC}=\mathrm{C} 1$ & 20 \\
\hline 556 & $\mathbf{1 5}\{239\}$ & $\mathbf{1 1}\{358\}$ & $\mathbf{9}\{239,358\}$ & $\begin{array}{l}\mathrm{CC}(\mathrm{C})(\mathrm{C}) \mathrm{OC}(=\mathrm{O}) \mathrm{NCC} 1 \mathrm{CCN}(\mathrm{C} 1) \mathrm{c} 1 \mathrm{nnnnn} 1 \mathrm{CC} 1 \mathrm{C} \\
\mathrm{CC}=\mathrm{C} 1\end{array}$ & 108 \\
\hline 557 & $\mathbf{1 5}\{239\}$ & $\mathbf{1 1}\{472\}$ & $\mathbf{9}\{239,472\}$ & $\begin{array}{l}\mathrm{CC}(\mathrm{C})(\mathrm{C}) \mathrm{OC}(=\mathrm{O}) \mathrm{N}[\mathrm{C} @ @ \mathrm{H}] 1 \mathrm{CCN}(\mathrm{C} 1) \mathrm{c} 1 \mathrm{nnnn} 1 \\
\mathrm{CC} 1 \mathrm{CCC}=\mathrm{C} 1\end{array}$ & 44 \\
\hline 558 & $\mathbf{1 5}\{239\}$ & $\mathbf{1 1}\{375\}$ & $\mathbf{9}\{239,375\}$ & $\mathrm{C}(\mathrm{C} 1 \mathrm{CCC}=\mathrm{C} 1) \mathrm{n} 1 \mathrm{nnn} \mathrm{c} 1 \mathrm{~N} 1 \mathrm{CCN}(\mathrm{CC} 1) \mathrm{c} 1 \mathrm{ncccn} 1$ & 26 \\
\hline 559 & $\mathbf{1 5}\{240\}$ & $\mathbf{1 1}\{304\}$ & $\mathbf{9}\{240,304\}$ & $\begin{array}{l}\mathrm{CC}(\mathrm{C}) \mathrm{C}(=\mathrm{O}) \mathrm{NCC} 1 \mathrm{CCCN}(\mathrm{C} 1) \mathrm{c} 1 \mathrm{nnnn} 1 \mathrm{CCCCC} 1 \\
\mathrm{CC} 1\end{array}$ & 16 \\
\hline 560 & $\mathbf{1 5}\{241\}$ & $\mathbf{1 1}\{349\}$ & $\mathbf{9}\{241,349\}$ & COC1(Cn2nnnc2N2CCCC(CC\#C)C2)CCOCC1 & 33 \\
\hline 561 & $\mathbf{1 5}\{241\}$ & $\mathbf{1 1}\{327\}$ & $\mathbf{9}\{241,327\}$ & $\mathrm{COC} 1(\mathrm{Cn} 2 \mathrm{nnn} \mathrm{n} 2 \mathrm{~N} 2 \mathrm{CCCC} 2 \mathrm{c} 2 \mathrm{ccsc} 2) \mathrm{CCOCC} 1$ & 61 \\
\hline 562 & $\mathbf{1 5}\{241\}$ & $\mathbf{1 1}\{338\}$ & $\mathbf{9}\{241,338\}$ & ${ }_{\mathrm{C}}^{\mathrm{CCC} 1 \mathrm{CN}(\mathrm{CC}(\mathrm{CC}) \mathrm{O} 1) \mathrm{c} 1 \mathrm{nnnn} 1 \mathrm{CC} 1(\mathrm{CCOCC} 1) \mathrm{O}}$ & 49 \\
\hline 563 & $15\{242\}$ & $\mathbf{1 1}\{338\}$ & $\mathbf{9}\{242,338\}$ & ${ }_{1} \mathrm{CCC} 1 \mathrm{CN}(\mathrm{CC}(\mathrm{CC}) \mathrm{O} 1) \mathrm{c} 1 \mathrm{nnnn} 1 \mathrm{Cc} 1 \mathrm{cc}(\mathrm{no} 1) \mathrm{C} 1 \mathrm{CC}$ & 55 \\
\hline 564 & $\mathbf{1 5}\{243\}$ & $11\{322\}$ & $\mathbf{9}\{243,322\}$ & CN(C1CC1)c1nnnn1Cc1nc(cs1)C(F)(F)F & 43 \\
\hline 565 & $15\{244\}$ & $\mathbf{1 1}\{473\}$ & $\mathbf{9}\{244,473\}$ & $\begin{array}{l}\text { CCOC1CC(n2nnnc2N2CC }[\mathrm{C} @ @ \mathrm{H}](\mathrm{C} 2) \mathrm{OCC}) \mathrm{C} \\
11 \mathrm{CCCC} 1\end{array}$ & 43 \\
\hline 566 & $\mathbf{1 5}\{244\}$ & $\mathbf{1 1}\{474\}$ & $\mathbf{9}\{244,474\}$ & CCOC1CC(n2nnnc2N2CCC(C2)SC)C11CCCC1 & 45 \\
\hline 567 & $\mathbf{1 5}\{245\}$ & $\mathbf{1 1}\{288\}$ & $\mathbf{9}\{245,288\}$ & $\mathrm{CC}(=\mathrm{O}) \mathrm{N} 1 \mathrm{CCCN}(\mathrm{CC} 1) \mathrm{c} 1 \mathrm{nnnn} 1 \mathrm{C} 1 \mathrm{CCCC} 1(\mathrm{~F}) \mathrm{F}$ & 0 \\
\hline
\end{tabular}




\begin{tabular}{|c|c|c|c|c|c|}
\hline 568 & $\mathbf{1 5}\{246\}$ & $\mathbf{1 1}\{401\}$ & $\mathbf{9}\{246,401\}$ & $\begin{array}{l}\mathrm{CC}(\mathrm{C})(\mathrm{Cn} 1 \mathrm{nnn} \mathrm{c} 1 \mathrm{~N} 1 \mathrm{CCC}(\mathrm{CC} 1) \mathrm{C}(\mathrm{N})=\mathrm{O}) \mathrm{c} 1 \mathrm{cccc}( \\
\mathrm{F}) \mathrm{c} 1\end{array}$ & 0 \\
\hline 569 & $\mathbf{1 5}\{247\}$ & $\mathbf{1 1}\{298\}$ & $\mathbf{9}\{247,298\}$ & CSCC(C)n1nnnc1N1CCCC(C1)C1(C)OCCO1 & 12 \\
\hline 570 & $15\{247\}$ & $\mathbf{1 1}\{297\}$ & $\mathbf{9}\{247,297\}$ & $\mathrm{CSCC}(\mathrm{C}) \mathrm{n} 1 \mathrm{nnn} \mathrm{n} 1 \mathrm{~N} 1 \mathrm{CCC}(\mathrm{CC} 1) \mathrm{C} 1(\mathrm{C}) \mathrm{OCCO} 1$ & 14 \\
\hline 571 & $\mathbf{1 5}\{248\}$ & $\mathbf{1 1}\{284\}$ & $\mathbf{9}\{248,284\}$ & $\mathrm{CC} 1(\mathrm{C}) \mathrm{CN}(\mathrm{CCO} 1) \mathrm{c} 1 \mathrm{nnnn} 1 \mathrm{Cc} 1 \mathrm{cccc} 2 \mathrm{nccc} 12$ & 5 \\
\hline 572 & $\mathbf{1 5}\{248\}$ & $\mathbf{1 1}\{351\}$ & $\mathbf{9}\{248,351\}$ & $\mathrm{CCN} 1 \mathrm{CCN}(\mathrm{CC} 1=\mathrm{O}) \mathrm{c} 1 \mathrm{nnnn} 1 \mathrm{Cc} 1 \mathrm{cccc} 2 \mathrm{ncccc} 12$ & 0 \\
\hline 573 & $\mathbf{1 5}\{248\}$ & $\mathbf{1 1}\{475\}$ & $\mathbf{9}\{248,475\}$ & $\mathrm{C}(\mathrm{c} 1 \mathrm{ccc} 2 \mathrm{ncccc} 12) \mathrm{n} 1 \mathrm{nnnn} 1 \mathrm{~N} 1 \mathrm{CCSCC} 1$ & 11 \\
\hline 574 & $\mathbf{1 5}\{248\}$ & $\mathbf{1 1}\{476\}$ & $\mathbf{9}\{248,476\}$ & $\mathrm{FC} 1(\mathrm{~F}) \mathrm{CCN}(\mathrm{CC} 1) \mathrm{c} 1 \mathrm{nnnn} 1 \mathrm{Cc} 1 \mathrm{cccc} 2 \mathrm{ncccc} 12$ & 6 \\
\hline 575 & $15\{248\}$ & $\mathbf{1 1}\{477\}$ & $\mathbf{9}\{248,477\}$ & $\operatorname{COCC} 1 \mathrm{CCCN}(\mathrm{C} 1) \mathrm{c} 1 \mathrm{nnnn} 1 \mathrm{Cc} 1 \mathrm{cccc} 2 \mathrm{ncccc} 12$ & 14 \\
\hline 576 & $\mathbf{1 5}\{248\}$ & $\mathbf{1 1}\{478\}$ & $\mathbf{9}\{248,478\}$ & $\mathrm{C}(\mathrm{c} 1 \mathrm{ccc} 2 \mathrm{nccc} 12) \mathrm{n} 1 \mathrm{nnnn} 1 \mathrm{~N} 1 \mathrm{CCCCC} 1$ & 9 \\
\hline 577 & $\mathbf{1 5}\{248\}$ & $\mathbf{1 1}\{401\}$ & $\mathbf{9}\{248,401\}$ & $\mathrm{NC}(=\mathrm{O}) \mathrm{C} 1 \mathrm{CCN}(\mathrm{CC} 1) \mathrm{c} 1 \mathrm{nnnn} 1 \mathrm{Cc} 1 \mathrm{cccc} 2 \mathrm{ncccc} 12$ & 13 \\
\hline 578 & $\mathbf{1 5}\{248\}$ & $\mathbf{1 1}\{443\}$ & $\mathbf{9}\{248,443\}$ & $\begin{array}{l}\mathrm{C}[\mathrm{C} @ \mathrm{H}] 1 \mathrm{CN}(\mathrm{C}[\mathrm{C} @ @ \mathrm{H}](\mathrm{C}) \mathrm{O} 1) \mathrm{c} 1 \mathrm{nnnn} 1 \mathrm{Cc} 1 \mathrm{ccc} \\
\mathrm{c} 2 \mathrm{ncccc} 12\end{array}$ & 10 \\
\hline 579 & $\mathbf{1 5}\{249\}$ & $\mathbf{1 1}\{479\}$ & $\mathbf{9}\{249,479\}$ & $\mathrm{CC}(\mathrm{Cn} 1 \mathrm{nnnc} 1 \mathrm{~N} 1 \mathrm{CCCC}(\mathrm{C}) \mathrm{C} 1) \mathrm{c} 1 \mathrm{nc}(\mathrm{C}) \mathrm{cs} 1$ & 29 \\
\hline 580 & $\mathbf{1 5}\{249\}$ & $\mathbf{1 1}\{422\}$ & $\mathbf{9}\{249,422\}$ & ${ }_{1} \mathrm{CC}(\mathrm{Cn} 1 \mathrm{nnnnc} 1 \mathrm{~N} 1 \mathrm{CCC}(\mathrm{CC}(\mathrm{N})=\mathrm{O}) \mathrm{CC} 1) \mathrm{c} 1 \mathrm{nc}(\mathrm{C}) \mathrm{cs}$ & 32 \\
\hline 581 & $\mathbf{1 5}\{249\}$ & $\mathbf{1 1}\{341\}$ & $\mathbf{9}\{249,341\}$ & CC(Cn1nnnc1N1CCCC1)c1nc(C)cs1 & 22 \\
\hline 582 & $\mathbf{1 5}\{249\}$ & $\mathbf{1 1}\{478\}$ & $\mathbf{9}\{249,478\}$ & $\mathrm{CC}(\mathrm{Cn} 1 \mathrm{nnn} n 1 \mathrm{~N} 1 \mathrm{CCCCC} 1) \mathrm{c} 1 \mathrm{nc}(\mathrm{C}) \mathrm{cs} 1$ & 19 \\
\hline 583 & $\mathbf{1 5}\{249\}$ & $\mathbf{1 1}\{443\}$ & $\mathbf{9}\{249,443\}$ & $\begin{array}{l}\mathrm{CC}(\mathrm{Cn} 1 \mathrm{nnn} n 1 \mathrm{~N} 1 \mathrm{C}[\mathrm{C} @ \mathrm{H}](\mathrm{C}) \mathrm{O}[\mathrm{C} @ \mathrm{H}](\mathrm{C}) \mathrm{C} 1) \mathrm{c} 1 \mathrm{n} \\
\mathrm{c}(\mathrm{C}) \mathrm{cs} 1\end{array}$ & 38 \\
\hline 584 & $\mathbf{1 5}\{249\}$ & $\mathbf{1 1}\{480\}$ & $\mathbf{9}\{249,480\}$ & $\mathrm{CC}(\mathrm{Cn} 1 \mathrm{nnn} \mathrm{c} 1 \mathrm{~N} 1 \mathrm{CCC}(\mathrm{C}) \mathrm{CC} 1) \mathrm{c} 1 \mathrm{nc}(\mathrm{C}) \mathrm{cs} 1$ & 28 \\
\hline 585 & $\mathbf{1 5}\{249\}$ & $\mathbf{1 1}\{284\}$ & $\mathbf{9}\{249,284\}$ & $\mathrm{CC}(\mathrm{Cn} 1 \mathrm{nnn} \mathrm{c} 1 \mathrm{~N} 1 \mathrm{CCOC}(\mathrm{C})(\mathrm{C}) \mathrm{C} 1) \mathrm{c} 1 \mathrm{nc}(\mathrm{C}) \mathrm{cs} 1$ & 13 \\
\hline 586 & $\mathbf{1 5}\{250\}$ & $\mathbf{1 1}\{278\}$ & $\mathbf{9}\{250,278\}$ & ${ }_{1}^{\mathrm{C}(\mathrm{Cn} 1 \mathrm{nnnn} 1 \mathrm{~N} 1 \mathrm{CCC} 2(\mathrm{CCOC} 2) \mathrm{C} 1) \mathrm{c} 1 \mathrm{nc} 2 \mathrm{CCC} 2 \mathrm{~s}}$ & 22 \\
\hline 587 & $\mathbf{1 5}\{250\}$ & $11\{4\}$ & $\mathbf{9}\{250,4\}$ & ${ }_{1} \mathrm{C}(\mathrm{Cn} 1 \mathrm{nnnn} 1 \mathrm{~N} 1 \mathrm{CCOC} 2(\mathrm{CCC} 2) \mathrm{C} 1) \mathrm{c} 1 \mathrm{nc} 2 \mathrm{CCC} 2 \mathrm{~s}$ & 0 \\
\hline
\end{tabular}




\begin{tabular}{|c|c|c|c|c|c|c|}
\hline 588 & $\mathbf{1 5}\{250\}$ & $\mathbf{1 1}\{179\}$ & $\mathbf{9}\{250,179\}$ & $\begin{array}{l}\text { OC[C@H]1[C@@ } @ \mathrm{H}] 2 \mathrm{CN}(\mathrm{C}[\mathrm{C} @ \mathrm{H}] 12) \mathrm{c} 1 \mathrm{nnnn} 1 \mathrm{C} \\
\mathrm{Cc} 1 \mathrm{nc} 2 \mathrm{CCCc} 2 \mathrm{~s} 1\end{array}$ & 0 & 0 \\
\hline 589 & $\mathbf{1 5}\{250\}$ & $\mathbf{1 1}\{481\}$ & $\mathbf{9}\{250,481\}$ & $\begin{array}{l}\mathrm{C}(\mathrm{Cn} 1 \mathrm{nnn} n 1 \mathrm{~N} 1 \mathrm{C}[\mathrm{C} @ \mathrm{H}] 2 \mathrm{CC}=\mathrm{CC}[\mathrm{C} @ \mathrm{H}] 2 \mathrm{C} 1) \mathrm{c} 1 \\
\text { nc2CCCc2s1 }\end{array}$ & 21 & 11 \\
\hline 590 & $\mathbf{1 5}\{250\}$ & $\mathbf{1 1}\{482\}$ & $\mathbf{9}\{250,482\}$ & ${ }_{1} \mathrm{C}(\mathrm{Cn} 1 \mathrm{nnnn} 1 \mathrm{~N} 1 \mathrm{CCC} 2(\mathrm{C} 1) \mathrm{OCCO} 2) \mathrm{c} 1 \mathrm{nc} 2 \mathrm{CCC} 2 \mathrm{~s}$ & 0 & 0 \\
\hline 591 & $\mathbf{1 5}\{250\}$ & $\mathbf{1 1}\{483\}$ & $\mathbf{9}\{250,483\}$ & $\mathrm{C}(\mathrm{Cn} 1 \mathrm{nnn} \mathrm{c} 1 \mathrm{~N} 1 \mathrm{CC} 2 \mathrm{CCC}(\mathrm{C} 2) \mathrm{C} 1) \mathrm{c} 1 \mathrm{nc} 2 \mathrm{CCCc} 2 \mathrm{~s} 1$ & 0 & 0 \\
\hline 592 & $\mathbf{1 5}\{251\}$ & $\mathbf{1 1}\{338\}$ & $\mathbf{9}\{251,338\}$ & $\mathrm{CCC} 1 \mathrm{CN}(\mathrm{CC}(\mathrm{CC}) \mathrm{O} 1) \mathrm{c} 1 \mathrm{nnnn} 1 \mathrm{Cc} 1 \mathrm{sc}(\mathrm{C}) \mathrm{nc} 1 \mathrm{C}$ & 22 & 11 \\
\hline 593 & $\mathbf{1 5}\{252\}$ & $\mathbf{1 1}\{321\}$ & $\mathbf{9}\{252,321\}$ & ${ }_{1} \mathrm{OCC} 1 \mathrm{CCN}(\mathrm{CC} 1) \mathrm{c} 1 \mathrm{nnnnn} 1 \mathrm{CC} 1(\mathrm{CC} 1) \mathrm{c} 1 \mathrm{cccc}(\mathrm{Cl}) \mathrm{c}$ & 19 & 10 \\
\hline 594 & $\mathbf{1 5}\{252\}$ & $\mathbf{1 1}\{328\}$ & $\mathbf{9}\{252,328\}$ & Clc1cccc(c1)C1(Cn2nnnc2N2CCOCC2)CC1 & 0 & 0 \\
\hline 595 & $\mathbf{1 5}\{253\}$ & $\mathbf{1 1}\{405\}$ & $\mathbf{9}\{253,405\}$ & CCn1nncc1Cn1nnnc1N1CCCC2(CCCCC2)C1 & 10 & 5 \\
\hline 596 & $\mathbf{1 5}\{254\}$ & $\mathbf{1 1}\{355\}$ & $\mathbf{9}\{254,355\}$ & $\mathrm{CN}(\mathrm{Cc} 1 \mathrm{cnn}(\mathrm{C}) \mathrm{c} 1) \mathrm{c} 1 \mathrm{nnnn} 1 \mathrm{CCC}(\mathrm{C})(\mathrm{O}) \mathrm{c} 1 \mathrm{ccccc} 1$ & 0 & 0 \\
\hline 597 & $\mathbf{1 5}\{254\}$ & $\mathbf{1 1}\{355\}$ & $\mathbf{9}\{254,355\}$ & $\mathrm{CN}(\mathrm{Cc} 1 \mathrm{cnn}(\mathrm{C}) \mathrm{c} 1) \mathrm{c} 1 \mathrm{nnnn} 1 \mathrm{CCC}(\mathrm{C})(\mathrm{O}) \mathrm{c} 1 \mathrm{ccccc} 1$ & 0 & 0 \\
\hline 598 & $\mathbf{1 5}\{255\}$ & $\mathbf{1 1}\{322\}$ & $\mathbf{9}\{255,322\}$ & $\mathrm{COc} 1 \mathrm{ccc}(\mathrm{Cn} 2 \mathrm{nnnc} 2 \mathrm{~N}(\mathrm{C}) \mathrm{C} 2 \mathrm{CC} 2) \mathrm{c}(\mathrm{OC}) \mathrm{n} 1$ & 0 & 0 \\
\hline 599 & $15\{256\}$ & $\mathbf{1 1}\{466\}$ & $\mathbf{9}\{256,466\}$ & $\begin{array}{l}\mathrm{CO}[\mathrm{C} @ \mathrm{H}](\mathrm{C}) \mathrm{Cn} 1 \mathrm{nnn} \mathrm{c} 1 \mathrm{~N} 1 \mathrm{CCCC} 2(\mathrm{C} 1) \mathrm{Oc} 1 \mathrm{ccccc} \\
1 \mathrm{C}=\mathrm{C} 2\end{array}$ & 18 & 9 \\
\hline 600 & $\mathbf{1 5}\{257\}$ & $\mathbf{1 1}\{288\}$ & $\mathbf{9}\{257,288\}$ & $\begin{array}{l}\mathrm{CC}(=\mathrm{O}) \mathrm{N} 1 \mathrm{CCCN}(\mathrm{CC} 1) \mathrm{c} 1 \mathrm{nnnn} 1 \mathrm{C} 1 \mathrm{CCC} 2(\mathrm{CCCC} \\
\text { 2) } \mathrm{CC} 1\end{array}$ & 23 & 11 \\
\hline 601 & $\mathbf{1 5}\{258\}$ & $\mathbf{1 1}\{322\}$ & $\mathbf{9}\{258,322\}$ & $\mathrm{CN}(\mathrm{C} 1 \mathrm{CC} 1) \mathrm{c} 1 \mathrm{nnnn} 1 \mathrm{CC} 1 \mathrm{CCC}(\mathrm{F})(\mathrm{F}) \mathrm{C} 1$ & 43 & 20 \\
\hline 602 & $\mathbf{1 5}\{258\}$ & $\mathbf{1 1}\{391\}$ & $\mathbf{9}\{258,391\}$ & $\mathrm{CN}(\mathrm{CC} 1 \mathrm{CC} 1) \mathrm{c} 1 \mathrm{nnnn} 1 \mathrm{CC} 1 \mathrm{CCC}(\mathrm{F})(\mathrm{F}) \mathrm{C} 1$ & 35 & 17 \\
\hline 603 & $\mathbf{1 5}\{259\}$ & $\mathbf{1 1}\{391\}$ & $\mathbf{9}\{259,391\}$ & $\mathrm{COCC}(\mathrm{C})(\mathrm{C}) \mathrm{Cn} 1 \mathrm{nnnnc} 1 \mathrm{~N}(\mathrm{C}) \mathrm{CC} 1 \mathrm{CC} 1$ & 10 & 5 \\
\hline 604 & $\mathbf{1 5}\{259\}$ & $\mathbf{1 1}\{337\}$ & $\mathbf{9}\{259,337\}$ & $\mathrm{COCC}(\mathrm{C})(\mathrm{C}) \mathrm{Cn} 1 \mathrm{nnn} \mathrm{c} 1 \mathrm{~N}(\mathrm{C}) \mathrm{CC} 1 \mathrm{CCOC} 1$ & 37 & 18 \\
\hline 605 & $\mathbf{1 5}\{259\}$ & $\mathbf{1 1}\{300\}$ & $\mathbf{9}\{259,300\}$ & $\mathrm{COCC}(\mathrm{C})(\mathrm{C}) \mathrm{Cn} 1 \mathrm{nnnn} 1 \mathrm{~N} 1 \mathrm{CCCOCC} 1$ & 14 & 7 \\
\hline 606 & $\mathbf{1 5}\{260\}$ & $\mathbf{1 1}\{484\}$ & $\mathbf{9}\{260,484\}$ & $\begin{array}{l}\mathrm{CC} 1 \mathrm{CCN}(\mathrm{CC} 11 \mathrm{CCCCC} 1) \mathrm{c} 1 \mathrm{nnnn} 1[\mathrm{C} @ \mathrm{H}] 1 \mathrm{CC} \\
\mathrm{N}(\mathrm{C} 1) \mathrm{C}(\mathrm{C})=\mathrm{O}\end{array}$ & 22 & 11 \\
\hline 607 & $\mathbf{1 5}\{260\}$ & $\mathbf{1 1}\{485\}$ & $\mathbf{9}\{260,485\}$ & $\mathrm{CC}(=\mathrm{O}) \mathrm{N} 1 \mathrm{CC}[\mathrm{C} @ \mathrm{H}](\mathrm{C} 1) \mathrm{n} 1 \mathrm{nnnc1N} 1 \mathrm{CCCC} 2(\mathrm{C}$ & 18 & 9 \\
\hline
\end{tabular}




\section{$\mathrm{CCCC} 2) \mathrm{CC} 1$}

\begin{tabular}{|c|c|c|c|c|c|}
\hline 608 & $\mathbf{1 5}\{261\}$ & $\mathbf{1 1}\{365\}$ & $\mathbf{9}\{261,365\}$ & $\begin{array}{l}\mathrm{CC} 1 \mathrm{CC}(\mathrm{CN} 1 \mathrm{c} 1 \mathrm{nnnn} 1 \mathrm{C} 1 \mathrm{CCN}(\mathrm{C}) \mathrm{C} 1=\mathrm{O}) \mathrm{c} 1 \mathrm{ccc}(\mathrm{C}) \\
\mathrm{cc} 1\end{array}$ & 0 \\
\hline 609 & $\mathbf{1 5}\{261\}$ & $\mathbf{1 1}\{327\}$ & $\mathbf{9}\{261,327\}$ & CN1CCC $(\mathrm{C} 1=0) n 1 \mathrm{nnnc1N1CCCC1c1ccsc1}$ & 0 \\
\hline 610 & $\mathbf{1 5}\{261\}$ & $\mathbf{1 1}\{302\}$ & $\mathbf{9}\{261,302\}$ & $\mathrm{CN}(\mathrm{CCOc} 1 \mathrm{ccc}(\mathrm{F}) \mathrm{cc} 1) \mathrm{c} 1 \mathrm{nnnn} 1 \mathrm{C} 1 \mathrm{CCN}(\mathrm{C}) \mathrm{C} 1=\mathrm{O}$ & 0 \\
\hline 611 & $\mathbf{1 5}\{262\}$ & $\mathbf{1 1}\{319\}$ & $\mathbf{9}\{262,319\}$ & $\mathrm{CC}(\mathrm{Cn} 1 \mathrm{nnnn} 1 \mathrm{~N}(\mathrm{C}) \mathrm{CC} 1 \mathrm{COCCO} 1) \mathrm{Sc} 1 \mathrm{cccc} 1$ & 65 \\
\hline 612 & $\mathbf{1 5}\{263\}$ & $\mathbf{1 1}\{341\}$ & $\mathbf{9}\{263,341\}$ & $\mathrm{CC}(\mathrm{Cn} 1 \mathrm{nnn} \mathrm{c} 1 \mathrm{~N} 1 \mathrm{CCCC} 1) \mathrm{Oc} 1 \mathrm{cccc} 1 \mathrm{~F}$ & 73 \\
\hline 613 & $15\{264\}$ & $\mathbf{1 1}\{319\}$ & $\mathbf{9}\{264,319\}$ & $\mathrm{CC}(\mathrm{Cn} 1 \mathrm{nnn} \operatorname{1N}(\mathrm{C}) \mathrm{CC} 1 \mathrm{COCCO} 1) \mathrm{Oc} 1 \mathrm{cccc}(\mathrm{C}) \mathrm{c} 1$ & 0 \\
\hline 614 & $15\{265\}$ & $\mathbf{1 1}\{319\}$ & $\mathbf{9}\{265,319\}$ & $\mathrm{CC}(\mathrm{Cn} 1 \mathrm{nnn} \operatorname{c} 1 \mathrm{~N}(\mathrm{C}) \mathrm{CC} 1 \mathrm{COCCO} 1) \mathrm{Oc} 1 \mathrm{cccc} 1 \mathrm{C}$ & 55 \\
\hline 615 & $\mathbf{1 5}\{266\}$ & $\mathbf{1 1}\{486\}$ & $\mathbf{9}\{266,486\}$ & $\begin{array}{l}\mathrm{CC}(\mathrm{C})(\mathrm{C}) \mathrm{OC}(=\mathrm{O}) \mathrm{N} 1 \mathrm{C} 2 \mathrm{CCC} 1 \mathrm{CN}(\mathrm{C} 2) \mathrm{c} 1 \mathrm{nnnn} 1 \mathrm{C} \\
\mathrm{CCC} \# \mathrm{C}\end{array}$ & 8 \\
\hline 616 & $\mathbf{1 5}\{266\}$ & $\mathbf{1 1}\{487\}$ & $\mathbf{9}\{266,487\}$ & $\begin{array}{l}\mathrm{CC}(\mathrm{C})(\mathrm{C}) \mathrm{OC}(=\mathrm{O}) \mathrm{N} 1 \mathrm{CC} 2 \mathrm{CCC}(\mathrm{C} 1) \mathrm{N} 2 \mathrm{c} 1 \mathrm{nnnnn} 1 \mathrm{C} \\
\mathrm{CCC} \# \mathrm{C}\end{array}$ & 13 \\
\hline 617 & $\mathbf{1 5}\{266\}$ & $\mathbf{1 1}\{488\}$ & $\mathbf{9}\{266,488\}$ & $\begin{array}{l}\mathrm{CC}(\mathrm{C})(\mathrm{C}) \mathrm{OC}(=\mathrm{O})[1 \mathrm{C} @] 12 \mathrm{COC}[1 \mathrm{C} @ \mathrm{H}] 1 \mathrm{CN}(\mathrm{C} 2 \\
) \mathrm{c} 1 \mathrm{nnnn} 1 \mathrm{CCCC} \mathrm{C}\end{array}$ & 0 \\
\hline 618 & $\mathbf{1 5}\{266\}$ & $\mathbf{1 1}\{313\}$ & $\mathbf{9}\{266,313\}$ & $\begin{array}{l}\mathrm{CC}(\mathrm{C})(\mathrm{C}) \mathrm{OC}(=\mathrm{O}) \mathrm{N} 1 \mathrm{C}[\mathrm{C} @ \mathrm{H}] 2 \mathrm{CN}(\mathrm{C}[\mathrm{C} @ \mathrm{H}] 2 \mathrm{C} 1) \\
\text { c1nnnn1CCCC\#C }\end{array}$ & 40 \\
\hline 619 & $\mathbf{1 5}\{266\}$ & $\mathbf{1 1}\{489\}$ & $\mathbf{9}\{266,489\}$ & $\begin{array}{l}\mathrm{CC}(\mathrm{C})(\mathrm{C}) \mathrm{OC}(=\mathrm{O}) \mathrm{NCC} 12 \mathrm{CC}(\mathrm{C} 1) \mathrm{CN} 2 \mathrm{c} 1 \mathrm{nnnn} 1 \mathrm{C} \\
\mathrm{CCC} \mathrm{C}\end{array}$ & 0 \\
\hline 620 & $\mathbf{1 5}\{267\}$ & $\mathbf{1 1}\{490\}$ & $\mathbf{9}\{267,490\}$ & ${ }_{1} \mathrm{CC}(\mathrm{O}) \mathrm{C} 1 \mathrm{CCN}(\mathrm{CC} 1) \mathrm{c} 1 \mathrm{nnnn} 1 \mathrm{CC} 1(\mathrm{Cc} 2 \mathrm{cccc} 2) \mathrm{CC}$ & 62 \\
\hline 621 & $\mathbf{1 5}\{268\}$ & $\mathbf{1 1}\{356\}$ & $\mathbf{9}\{268,356\}$ & $\mathrm{CN}(\mathrm{CC} 1 \mathrm{CCC} 1) \mathrm{c} 1 \mathrm{nnnn} 1 \mathrm{Cc} 1 \mathrm{ccc}(\mathrm{CC}(\mathrm{N})=\mathrm{O}) \mathrm{cc} 1$ & 30 \\
\hline 622 & $\mathbf{1 5}\{268\}$ & $\mathbf{1 1}\{333\}$ & $\mathbf{9}\{268,333\}$ & $\mathrm{CC} 1(\mathrm{C}) \mathrm{CN}(\mathrm{CCS} 1) \mathrm{c} 1 \mathrm{nnnnn} 1 \mathrm{Cc} 1 \mathrm{ccc}(\mathrm{CC}(\mathrm{N})=\mathrm{O}) \mathrm{cc} 1$ & 25 \\
\hline 623 & $\mathbf{1 5}\{269\}$ & $\mathbf{1 1}\{319\}$ & $\mathbf{9}\{269,319\}$ & $\mathrm{CSCc} 1 \mathrm{ccc}(\mathrm{Cn} 2 \mathrm{nnnn} 2 \mathrm{~N}(\mathrm{C}) \mathrm{CC} 2 \mathrm{COCCO} 2) \mathrm{cc} 1$ & 45 \\
\hline 624 & $\mathbf{1 5}\{270\}$ & $\mathbf{1 1}\{485\}$ & $\mathbf{9}\{270,485\}$ & $\begin{array}{l}\mathrm{NC}(=\mathrm{O})[\mathrm{C} @ \mathrm{H}] 1 \mathrm{C}[\mathrm{C} @ \mathrm{H}](\mathrm{C} 1) \mathrm{n} 1 \mathrm{nnn} \mathrm{n} 1 \mathrm{~N} 1 \mathrm{CCCC} \\
2(\mathrm{CCCCC}) \mathrm{CC} 1\end{array}$ & 35 \\
\hline 625 & $\mathbf{1 5}\{270\}$ & $\mathbf{1 1}\{365\}$ & $\mathbf{9}\{270,365\}$ & $\begin{array}{l}\mathrm{CC} 1 \mathrm{CC}(\mathrm{CN} 1 \mathrm{c} 1 \mathrm{nnnn} 1[\mathrm{C} @ @ \mathrm{H}] 1 \mathrm{C}[\mathrm{C} @ @ \mathrm{H}](\mathrm{C} 1) \\
\mathrm{C}(\mathrm{N})=\mathrm{O}) \mathrm{c} 1 \mathrm{ccc}(\mathrm{C}) \mathrm{cc} 1\end{array}$ & 34 \\
\hline
\end{tabular}




\begin{tabular}{|c|c|c|c|c|c|}
\hline 626 & $\mathbf{1 5}\{270\}$ & $\mathbf{1 1}\{302\}$ & $\mathbf{9}\{270,302\}$ & $\begin{array}{l}\mathrm{CN}(\mathrm{CCOc} 1 \mathrm{ccc}(\mathrm{F}) \mathrm{cc} 1) \mathrm{c} 1 \mathrm{nnnn} 1[\mathrm{C} @ @ \mathrm{H}] 1 \mathrm{C}[\mathrm{C} @ \\
@ \mathrm{H}](\mathrm{C} 1) \mathrm{C}(\mathrm{N})=\mathrm{O}\end{array}$ & 21 \\
\hline 627 & $\mathbf{1 5}\{270\}$ & $\mathbf{1 1}\{26\}$ & $\mathbf{9}\{270,26\}$ & $\begin{array}{l}\mathrm{NC}(=\mathrm{O})[\mathrm{C} @ \mathrm{H}] 1 \mathrm{C}[\mathrm{C} @ \mathrm{H}](\mathrm{C} 1) n 1 \mathrm{nnn} c 1 \mathrm{~N} 1 \mathrm{CCc} 2 \mathrm{c} \\
\mathrm{c}(\mathrm{Cl}) \mathrm{ccc} 2 \mathrm{C} 1\end{array}$ & 10 \\
\hline 628 & $\mathbf{1 5}\{270\}$ & $\mathbf{1 1}\{484\}$ & $\mathbf{9}\{270,484\}$ & $\begin{array}{l}\mathrm{CC} 1 \mathrm{CCN}(\mathrm{CC} 11 \mathrm{CCCCC} 1) \mathrm{c} 1 \mathrm{nnnn} 1[\mathrm{C} @ @ \mathrm{H}] 1 \mathrm{C}[ \\
\mathrm{C} @ @ \mathrm{H}](\mathrm{C} 1) \mathrm{C}(\mathrm{N})=\mathrm{O}\end{array}$ & 0 \\
\hline 629 & $15\{271\}$ & $\mathbf{1 1}\{288\}$ & $\mathbf{9}\{271,288\}$ & $\begin{array}{l}\mathrm{CC}(=\mathrm{O}) \mathrm{N} 1 \mathrm{CCCN}(\mathrm{CC} 1) \mathrm{c} 1 \mathrm{nnnn} 1 \mathrm{CC} 1 \mathrm{CC} 2 \mathrm{CCC} 1 \mathrm{C} \\
\mathrm{C} 2\end{array}$ & 30 \\
\hline 630 & $\mathbf{1 5}\{271\}$ & $\mathbf{1 1}\{395\}$ & $\mathbf{9}\{271,395\}$ & $\begin{array}{l}\mathrm{C}(\mathrm{C} 1 \mathrm{CC} 2 \mathrm{CCC} 1 \mathrm{CC} 2) n 1 \mathrm{nnnc} 1 \mathrm{~N} 1 \mathrm{CCOC} 2(\mathrm{CCOC} \\
2) \mathrm{C} 1\end{array}$ & 0 \\
\hline 631 & $\mathbf{1 5}\{272\}$ & $\mathbf{1 1}\{319\}$ & $\mathbf{9}\{272,319\}$ & CCSc1ccc(Cn2nnnc2N(C)CC2COCCO2)cc1 & 66 \\
\hline 632 & $\mathbf{1 5}\{273\}$ & $\mathbf{1 1}\{356\}$ & $\mathbf{9}\{273,356\}$ & $\mathrm{CN}(\mathrm{CC} 1 \mathrm{CCC} 1) \mathrm{c} 1 \mathrm{nnnn} 1 \mathrm{CCn} 1 \mathrm{cc}(\mathrm{Br}) \mathrm{cn} 1$ & 57 \\
\hline 633 & $\mathbf{1 5}\{274\}$ & $\mathbf{1 1}\{328\}$ & $\mathbf{9}\{274,328\}$ & Clc1ccc2n(CCn3nnnc3N3CCOCC3) $\operatorname{ccc} 2 \mathrm{c} 1$ & 23 \\
\hline 634 & $\mathbf{1 5}\{275\}$ & $\mathbf{1 1}\{322\}$ & $\mathbf{9}\{275,322\}$ & $\mathrm{CN}(\mathrm{C} 1 \mathrm{CC} 1) \mathrm{c} 1 \mathrm{nnnn} 1 \mathrm{Cc} 1 \mathrm{ncc}(\mathrm{Br}) \mathrm{s} 1$ & 0 \\
\hline 635 & $\mathbf{1 5}\{276\}$ & $\mathbf{1 1}\{288\}$ & $\mathbf{9}\{276,288\}$ & $\begin{array}{l}\mathrm{CC}(=\mathrm{O}) \mathrm{N} 1 \mathrm{CCCN}(\mathrm{CC} 1) \mathrm{c} 1 \mathrm{nnnn} 1 \mathrm{CC} 1 \mathrm{CCC} 2(\mathrm{CC} 2) \\
\mathrm{CC} 1\end{array}$ & 13 \\
\hline 636 & $15\{277\}$ & $\mathbf{1 1}\{341\}$ & $\mathbf{9}\{277,341\}$ & FC(F)Oc1ccce(F)c1Cn1nnnc1N1CCCC1 & 36 \\
\hline 637 & $\mathbf{1 5}\{278\}$ & $\mathbf{1 1}\{349\}$ & $\mathbf{9}\{278,349\}$ & C\#CCC1CCCN(C1)c1nnnn1Cc1nnnn1C1CC1 & 0 \\
\hline 638 & $\mathbf{1 5}\{278\}$ & $\mathbf{1 1}\{327\}$ & $\mathbf{9}\{278,327\}$ & C(c1nnnn1C1CC1)n1nnnc1N1CCCC1c1ccsc1 & 0 \\
\hline 639 & $\mathbf{1 5}\{279\}$ & $\mathbf{1 1}\{491\}$ & $\mathbf{9}\{279,491\}$ & $\mathrm{CSC}(\mathrm{C}) \mathrm{Cn} 1 \mathrm{nnn} \mathrm{c} 1 \mathrm{~N} 1 \mathrm{CCC}(\mathrm{C} 1) \mathrm{N} 1 \mathrm{CCCC} 1$ & 0 \\
\hline 640 & $\mathbf{1 5}\{280\}$ & $\mathbf{1 1}\{322\}$ & $\mathbf{9}\{280,322\}$ & $\mathrm{CN}(\mathrm{C} 1 \mathrm{CC} 1) \mathrm{c} 1 \mathrm{nnnn} 1 \mathrm{CCCn} 1 \mathrm{cc}(\mathrm{Br}) \mathrm{cn} 1$ & 39 \\
\hline 641 & $\mathbf{1 5}\{280\}$ & $\mathbf{1 1}\{391\}$ & $\mathbf{9}\{280,391\}$ & $\mathrm{CN}(\mathrm{CC} 1 \mathrm{CC} 1) \mathrm{c} 1 \mathrm{nnnn} 1 \mathrm{CCCn} 1 \mathrm{cc}(\mathrm{Br}) \mathrm{cn} 1$ & 80 \\
\hline 642 & $\mathbf{1 5}\{281\}$ & $\mathbf{1 1}\{345\}$ & $\mathbf{9}\{281,345\}$ & $\mathrm{C}(\mathrm{c} 1 \mathrm{nc} 2 \mathrm{CCOCc} 2 \mathrm{~s} 1) \mathrm{n} 1 \mathrm{nnnn} 1 \mathrm{~N} 1 \mathrm{CCCCCCC} 1$ & 27 \\
\hline 643 & $\mathbf{1 5}\{282\}$ & $\mathbf{1 1}\{350\}$ & $\mathbf{9}\{282,350\}$ & $\mathrm{CN}(\mathrm{CCC}=\mathrm{C}) \mathrm{c} 1 \mathrm{nnnn} 1 \mathrm{C} 1 \mathrm{CC}(\mathrm{C} 1) \mathrm{OCc} 1 \mathrm{ccccc} 1$ & 33 \\
\hline 644 & $\mathbf{1 5}\{283\}$ & $\mathbf{1 1}\{338\}$ & $\mathbf{9}\{283,338\}$ & $\begin{array}{l}\mathrm{CCC} 1 \mathrm{CN}(\mathrm{CC}(\mathrm{CC}) \mathrm{O} 1) \mathrm{c} 1 \mathrm{nnnn} 1 \mathrm{C} 1 \mathrm{CCN}(\mathrm{C} 1) \mathrm{C}(=\mathrm{O} \\
) \mathrm{CC}\end{array}$ & 38 \\
\hline 645 & $\mathbf{1 5}\{284\}$ & $\mathbf{1 1}\{355\}$ & $\mathbf{9}\{284,355\}$ & $\mathrm{COCCC} 1(\mathrm{Cn} 2 \mathrm{nnnc} 2 \mathrm{~N}(\mathrm{C}) \mathrm{Cc} 2 \mathrm{cnn}(\mathrm{C}) \mathrm{c} 2) \mathrm{CCCC} 1$ & 42 \\
\hline
\end{tabular}




\begin{tabular}{|c|c|c|c|c|c|}
\hline 646 & $\mathbf{1 5}\{285\}$ & $\mathbf{1 1}\{355\}$ & $\mathbf{9}\{285,355\}$ & $\mathrm{CCOCCC} 1(\mathrm{Cn} 2 \mathrm{nnnc} 2 \mathrm{~N}(\mathrm{C}) \mathrm{Cc} 2 \mathrm{cnn}(\mathrm{C}) \mathrm{c} 2) \mathrm{CCCC} 1$ & 33 \\
\hline 647 & $\mathbf{1 5}\{286\}$ & $\mathbf{1 1}\{288\}$ & $\mathbf{9}\{286,288\}$ & ${ }_{1} \mathrm{CCC} 1(\mathrm{Cn} 2 \mathrm{nnn} n \mathrm{2N} 2 \mathrm{CCCN}(\mathrm{CC} 2) \mathrm{C}(\mathrm{C})=\mathrm{O}) \mathrm{CCCC}$ & 29 \\
\hline 648 & $\mathbf{1 5}\{287\}$ & $\mathbf{1 1}\{492\}$ & $\mathbf{9}\{287,492\}$ & $\mathrm{CN}(\mathrm{CC}(\mathrm{C})(\mathrm{O}) \mathrm{c} 1 \mathrm{ccccc} 1) \mathrm{c} 1 \mathrm{nnnn} 1 \mathrm{CCc} 1 \mathrm{ccnn} 1 \mathrm{C}$ & 0 \\
\hline 649 & $\mathbf{1 5}\{287\}$ & $\mathbf{1 1}\{297\}$ & $\mathbf{9}\{287,297\}$ & 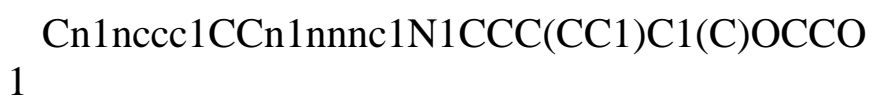 & 20 \\
\hline 650 & $\mathbf{1 5}\{287\}$ & $\mathbf{1 1}\{492\}$ & $\mathbf{9}\{287,492\}$ & $\mathrm{CN}(\mathrm{CC}(\mathrm{C})(\mathrm{O}) \mathrm{c} 1 \mathrm{ccccc} 1) \mathrm{c} 1 \mathrm{nnnn} 1 \mathrm{CCc} 1 \mathrm{ccnn} 1 \mathrm{C}$ & 20 \\
\hline 651 & $\mathbf{1 5}\{287\}$ & $\mathbf{1 1}\{298\}$ & $\mathbf{9}\{287,298\}$ & 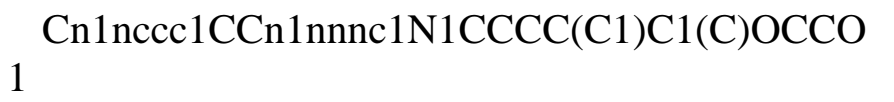 & 21 \\
\hline 652 & $\mathbf{1 5}\{288\}$ & $\mathbf{1 1}\{319\}$ & $\mathbf{9}\{288,319\}$ & ${ }_{) \mathrm{C}}^{\mathrm{CN}(\mathrm{CC} 1 \mathrm{COCCO} 1) \mathrm{c} 1 \mathrm{nnnn} 1 \mathrm{C} 1 \mathrm{CCOC}(\mathrm{C} 1) \mathrm{C}(\mathrm{C})(\mathrm{C}}$ & 38 \\
\hline 653 & $\mathbf{1 5}\{289\}$ & $\mathbf{1 1}\{338\}$ & $\mathbf{9}\{289,338\}$ & $\begin{array}{l}\mathrm{CCC} 1 \mathrm{CN}(\mathrm{CC}(\mathrm{CC}) \mathrm{O} 1) \mathrm{c} 1 \mathrm{nnnn} 1 \mathrm{C} 1 \mathrm{CCOC}(\mathrm{C} 1) \mathrm{C}(\mathrm{C} \\
\mathrm{C}\end{array}$ & 26 \\
\hline 654 & $\mathbf{1 5}\{290\}$ & $\mathbf{1 1}\{338\}$ & $\mathbf{9}\{290,338\}$ & CCC1CN(CC(CC)O1)c1nnnn1CCCOC1CCOC1 & 29 \\
\hline 655 & $\mathbf{1 5}\{291\}$ & $\mathbf{1 1}\{328\}$ & $\mathbf{9}\{291,328\}$ & $\mathrm{CC}(\mathrm{C}) \mathrm{c} 1 \mathrm{csc}(\mathrm{Cn} 2 \mathrm{nnn} \mathrm{2} 2 \mathrm{~N} 2 \mathrm{CCOCC} 2) \mathrm{n} 1$ & 0 \\
\hline 656 & $\mathbf{1 5}\{292\}$ & $\mathbf{1 1}\{288\}$ & $\mathbf{9}\{292,288\}$ & ${ }_{1} \mathrm{CC}(=\mathrm{O}) \mathrm{N} 1 \mathrm{CCCN}(\mathrm{CC} 1) \mathrm{c} 1 \mathrm{nnnn} 1 \mathrm{CCOCC} 1 \mathrm{CCCC}$ & 53 \\
\hline 657 & $\mathbf{1 5}\{293\}$ & $\mathbf{1 1}\{297\}$ & $\mathbf{9}\{293,297\}$ & ${ }_{1} \mathrm{CC} 1(\mathrm{OCCO} 1) \mathrm{C} 1 \mathrm{CCN}(\mathrm{CC} 1) \mathrm{c} 1 \mathrm{nnnn} 1 \mathrm{CCC} 1 \mathrm{ccco}$ & 31 \\
\hline 658 & $\mathbf{1 5}\{293\}$ & $\mathbf{1 1}\{298\}$ & $\mathbf{9}\{293,298\}$ & 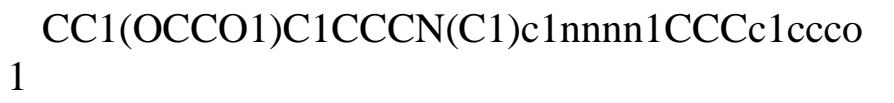 & 22 \\
\hline 659 & $\mathbf{1 5}\{294\}$ & $\mathbf{1 1}\{322\}$ & $\mathbf{9}\{294,322\}$ & CN(C1CC1)c1nnnn1Cc1cnn(CC(F)(F)F)c1 & 60 \\
\hline 660 & $\mathbf{1 5}\{294\}$ & $\mathbf{1 1}\{356\}$ & $\mathbf{9}\{294,356\}$ & $\mathrm{CN}(\mathrm{CC} 1 \mathrm{CCC} 1) \mathrm{c} 1 \mathrm{nnnn} 1 \mathrm{Cc} 1 \mathrm{cnn}(\mathrm{CC}(\mathrm{F})(\mathrm{F}) \mathrm{F}) \mathrm{c} 1$ & 49 \\
\hline 661 & $\mathbf{1 5}\{295\}$ & $\mathbf{1 1}\{339\}$ & $\mathbf{9}\{295,339\}$ & $\mathrm{CNC}(=\mathrm{O}) \mathrm{C}(\mathrm{C})(\mathrm{C}) \mathrm{Cn} 1 \mathrm{nnnnc} 1 \mathrm{~N}(\mathrm{C}) \mathrm{Cc} 1 \mathrm{cccc}(\mathrm{F}) \mathrm{c} 1$ & 0 \\
\hline 662 & $\mathbf{1 5}\{296\}$ & $\mathbf{1 1}\{339\}$ & $\mathbf{9}\{296,339\}$ & $\mathrm{CCNC}(=\mathrm{O}) \mathrm{C}(\mathrm{C})(\mathrm{C}) \mathrm{Cn} 1 \mathrm{nnnnc} 1 \mathrm{~N}(\mathrm{C}) \mathrm{Cc} 1 \mathrm{cccc}(\mathrm{F}) \mathrm{c} 1$ & 30 \\
\hline 663 & $\mathbf{1 5}\{296\}$ & $\mathbf{1 1}\{338\}$ & $\mathbf{9}\{296,338\}$ & $\begin{array}{l}\mathrm{CCNC}(=\mathrm{O}) \mathrm{C}(\mathrm{C})(\mathrm{C}) \mathrm{Cn} 1 \mathrm{nnnn} 1 \mathrm{~N} 1 \mathrm{CC}(\mathrm{CC}) \mathrm{OC}(\mathrm{CC}) \\
\mathrm{C} 1\end{array}$ & 0 \\
\hline 664 & $\mathbf{1 5}\{297\}$ & $\mathbf{1 1}\{338\}$ & $\mathbf{9}\{297,338\}$ & CCC1CN(CC(CC)O1)c1nnnn1CC(C)c1nccs1 & 0 \\
\hline
\end{tabular}




\begin{tabular}{|c|c|c|c|c|c|}
\hline 665 & $\mathbf{1 5}\{298\}$ & $\mathbf{1 1}\{303\}$ & $\mathbf{9}\{298,303\}$ & $\mathrm{CCN}(\mathrm{C}) \mathrm{C}(=\mathrm{O}) \mathrm{Cn} 1 \mathrm{nnnn} 1 \mathrm{~N} 1 \mathrm{CCCC}(\mathrm{C}) \mathrm{C} 1 \mathrm{C}$ & 46 \\
\hline 666 & $\mathbf{1 5}\{298\}$ & $\mathbf{1 1}\{417\}$ & $\mathbf{9}\{298,417\}$ & $\mathrm{CCN}(\mathrm{C}) \mathrm{C}(=\mathrm{O}) \mathrm{Cn} 1 \mathrm{nnn} \operatorname{c} 1 \mathrm{~N} 1 \mathrm{CCc} 2 \operatorname{ccc}(\mathrm{OC}) \mathrm{c} 2 \mathrm{C} 1$ & 11 \\
\hline 667 & $\mathbf{1 5}\{299\}$ & $\mathbf{1 1}\{338\}$ & $\mathbf{9}\{299,338\}$ & ${ }_{\mathrm{C}}^{\mathrm{CCC} 1 \mathrm{CN}(\mathrm{CC}(\mathrm{CC}) \mathrm{O} 1) \mathrm{c} 1 \mathrm{nnnn} 1 \mathrm{C} 1 \mathrm{CC}(\mathrm{OC}) \mathrm{C} 1(\mathrm{C})}$ & 26 \\
\hline 668 & $\mathbf{1 5}\{299\}$ & $\mathbf{1 1}\{327\}$ & $\mathbf{9}\{299,327\}$ & COC1CC(n2nnnc2N2CCCC2c2ccsc2)C1(C)C & 56 \\
\hline 669 & $\mathbf{1 5}\{299\}$ & $\mathbf{1 1}\{349\}$ & $\mathbf{9}\{299,349\}$ & $\mathrm{COC} 1 \mathrm{CC}(\mathrm{n} 2 \mathrm{nnn} \mathrm{c} 2 \mathrm{~N} 2 \mathrm{CCCC}(\mathrm{CC} \# \mathrm{C}) \mathrm{C} 2) \mathrm{C} 1(\mathrm{C}) \mathrm{C}$ & 0 \\
\hline 670 & $\mathbf{1 5}\{300\}$ & $\mathbf{1 1}\{493\}$ & $\mathbf{9}\{300,493\}$ & $\begin{array}{l}\mathrm{CC} 1 \mathrm{CC} 2 \mathrm{CCC}(\mathrm{C} 1) \mathrm{N} 2 \mathrm{c} 1 \mathrm{nnnn} 1 \mathrm{C} 1 \mathrm{CN}(\mathrm{C}(=\mathrm{O}) \mathrm{C} 1) \mathrm{C} \\
(\mathrm{C})(\mathrm{C}) \mathrm{C}\end{array}$ & 9 \\
\hline 671 & $\mathbf{1 5}\{301\}$ & $\mathbf{1 1}\{321\}$ & $\mathbf{9}\{301,321\}$ & CCSC1CCCC(C1)n1nnnc1N1CCC(CO)CC1 & 0 \\
\hline 672 & $\mathbf{1 5}\{302\}$ & $\mathbf{1 1}\{355\}$ & $\mathbf{9}\{302,355\}$ & $\mathrm{CC}(\mathrm{C}) \mathrm{C} 1 \mathrm{OCCCC} 1 \mathrm{Cn} 1 \mathrm{nnn} \mathrm{c} 1 \mathrm{~N}(\mathrm{C}) \mathrm{Cc} 1 \mathrm{cnn}(\mathrm{C}) \mathrm{c} 1$ & 24 \\
\hline 673 & $\mathbf{1 5}\{303\}$ & $\mathbf{1 1}\{355\}$ & $\mathbf{9}\{303,355\}$ & $\mathrm{CCOCCC} 1(\mathrm{Cn} 2 \mathrm{nnnn} 2 \mathrm{~N}(\mathrm{C}) \mathrm{Cc} 2 \mathrm{cnn}(\mathrm{C}) \mathrm{c} 2) \mathrm{CCC} 1$ & 28 \\
\hline 674 & $\mathbf{1 5}\{304\}$ & $\mathbf{1 1}\{349\}$ & $\mathbf{9}\{304,349\}$ & $\mathrm{COCCC} 1(\mathrm{Cn} 2 \mathrm{nnn} \mathrm{c} 2 \mathrm{~N} 2 \mathrm{CCCC}(\mathrm{CC} \# \mathrm{C}) \mathrm{C} 2) \mathrm{CCC} 1$ & 0 \\
\hline 675 & $\mathbf{1 5}\{304\}$ & $\mathbf{1 1}\{288\}$ & $\mathbf{9}\{304,288\}$ & $\begin{array}{l}\mathrm{COCCC} 1(\mathrm{Cn} 2 \mathrm{nnn} \operatorname{2N} 2 \mathrm{CCCN}(\mathrm{CC} 2) \mathrm{C}(\mathrm{C})=\mathrm{O}) \mathrm{CC} \\
\mathrm{C} 1\end{array}$ & 52 \\
\hline 676 & $\mathbf{1 5}\{305\}$ & $\mathbf{1 1}\{288\}$ & $\mathbf{9}\{305,288\}$ & $\begin{array}{l}\mathrm{CC}(\mathrm{C}) \mathrm{CC} 1(\mathrm{Cn} 2 \mathrm{nnn} \mathrm{n} 2 \mathrm{~N} 2 \mathrm{CCCN}(\mathrm{CC} 2) \mathrm{C}(\mathrm{C})=\mathrm{O}) \mathrm{C} \\
\mathrm{C} 1\end{array}$ & 0 \\
\hline 677 & $\mathbf{1 5}\{306\}$ & $\mathbf{1 1}\{494\}$ & $\mathbf{9}\{306,494\}$ & COCC1CN(CCO1)c1nnnn1Cc1cscc1C & 0 \\
\hline 678 & $\mathbf{1 5}\{306\}$ & $\mathbf{1 1}\{147\}$ & $\mathbf{9}\{306,147\}$ & Cc1cscc1Cn1nnnc1N1CCC (C1)n1ce(Cl)cn1 & 47 \\
\hline 679 & $\mathbf{1 5}\{306\}$ & $\mathbf{1 1}\{323\}$ & $\mathbf{9}\{306,323\}$ & Cc1cscc1Cn1nnnc1N1Cc2 $\operatorname{cccc} 2 \mathrm{NC}(=\mathrm{O}) \mathrm{C} 1$ & 0 \\
\hline 680 & $\mathbf{1 5}\{306\}$ & $\mathbf{1 1}\{390\}$ & $\mathbf{9}\{306,390\}$ & Cc1 $1 \sec 1 \mathrm{Cn} 1 \mathrm{nnnn} 1 \mathrm{~N} 1 \mathrm{CCN}(\mathrm{CC} 1) \mathrm{S}(\mathrm{C})(=\mathrm{O})=\mathrm{O}$ & 0 \\
\hline 681 & $\mathbf{1 5}\{306\}$ & $\mathbf{1 1}\{495\}$ & $\mathbf{9}\{306,495\}$ & $\mathrm{CC}(=\mathrm{O}) \mathrm{NCC} 1 \mathrm{CN}(\mathrm{CCO} 1) \mathrm{c} 1 \mathrm{nnnn} 1 \mathrm{Cc} 1 \mathrm{cscc} 1 \mathrm{C}$ & 28 \\
\hline 682 & $\mathbf{1 5}\{306\}$ & $\mathbf{1 1}\{176\}$ & $\mathbf{9}\{306,176\}$ & $\mathrm{CN}(\mathrm{Cc} 1 \mathrm{cnc}(\mathrm{C}) \mathrm{s} 1) \mathrm{c} 1 \mathrm{nnnn} 1 \mathrm{Cc} 1 \mathrm{cscc} 1 \mathrm{C}$ & 0 \\
\hline 683 & $\mathbf{1 5}\{306\}$ & $\mathbf{1 1}\{496\}$ & $\mathbf{9}\{306,496\}$ & $\mathrm{CC}(\mathrm{C} \# \mathrm{C}) \mathrm{N}(\mathrm{C}) \mathrm{c} 1 \mathrm{nnnn} 1 \mathrm{Cc} 1 \mathrm{cscc} 1 \mathrm{C}$ & 0 \\
\hline 684 & $\mathbf{1 5}\{306\}$ & $\mathbf{1 1}\{178\}$ & $\mathbf{9}\{306,178\}$ & $\mathrm{CN}(\mathrm{Cc} 1 \mathrm{ccoc} 1) \mathrm{c} 1 \mathrm{nnnn} 1 \mathrm{Cc} 1 \mathrm{cscc} 1 \mathrm{C}$ & 19 \\
\hline
\end{tabular}




\begin{tabular}{|c|c|c|c|c|c|}
\hline 685 & $\mathbf{1 5}\{306\}$ & $\mathbf{1 1}\{108\}$ & $\mathbf{9}\{306,108\}$ & $\mathrm{Cc} 1 \mathrm{cscc} 1 \mathrm{Cn} 1 \mathrm{nnn} 1 \mathrm{~N} 1 \mathrm{CCC}(\mathrm{CC} 1) \mathrm{N} 1 \mathrm{CCCC} 1=\mathrm{O}$ & 0 \\
\hline 686 & $15\{306\}$ & $11\{177\}$ & $\mathbf{9}\{306,177\}$ & $\mathrm{CC}(\mathrm{C}) \mathrm{C}(=\mathrm{O}) \mathrm{N} 1 \mathrm{CCCN}(\mathrm{CC} 1) \mathrm{c} 1 \mathrm{nnnn} 1 \mathrm{Cc} 1 \mathrm{cscc} 1 \mathrm{C}$ & 0 \\
\hline 687 & $15\{306\}$ & $11\{261\}$ & $\mathbf{9}\{306,261\}$ & Cc1csec1Cn1nnnc1N1CCC(CC1)C1CCCO1 & 68 \\
\hline 688 & $15\{306\}$ & $11\{168\}$ & $\mathbf{9}\{306,168\}$ & Cc1cscc1Cn1nnnc1N1CCOC(C1)c1ccco1 & 31 \\
\hline 689 & $15\{306\}$ & $11\{497\}$ & $\mathbf{9}\{306,497\}$ & $\mathrm{Cc} 1 \operatorname{cscc} 1 \mathrm{Cn} 1 \mathrm{nnnn} 1 \mathrm{~N} 1 \mathrm{CCN} 2 \mathrm{C}(\mathrm{C} 1) \mathrm{C}(=\mathrm{O}) \mathrm{NC} 2=\mathrm{O}$ & 0 \\
\hline 690 & $15\{306\}$ & $11\{19\}$ & $\mathbf{9}\{306,19\}$ & $\mathrm{COC} 1(\mathrm{CCN}(\mathrm{C} 1) \mathrm{c} 1 \mathrm{nnnn} 1 \mathrm{Cc} 1 \mathrm{cscc} 1 \mathrm{C}) \mathrm{C}(\mathrm{F})(\mathrm{F}) \mathrm{F}$ & 0 \\
\hline 691 & $15\{306\}$ & $11\{375\}$ & $\mathbf{9}\{306,375\}$ & Cc1cscc1Cn1nnnc1N1CCN(CC1)c1nccen1 & 38 \\
\hline 692 & $\mathbf{1 5}\{307\}$ & $11\{288\}$ & $\mathbf{9}\{307,288\}$ & ${ }_{1}^{\mathrm{CCCC} 1}(\mathrm{Cn} 2 \mathrm{nnn} \mathrm{c} 2 \mathrm{~N} 2 \mathrm{CCCN}(\mathrm{CC} 2) \mathrm{C}(\mathrm{C})=\mathrm{O}) \mathrm{CCC}$ & 22 \\
\hline 693 & $\mathbf{1 5}\{308\}$ & $11\{424\}$ & $\mathbf{9}\{308,424\}$ & CCC $(\mathrm{OCCn} 1 \mathrm{nnnn} 1 \mathrm{~N} 1 \mathrm{CCCC}(\mathrm{O}) \mathrm{C} 1) \mathrm{c} 1 \mathrm{ccccc} 1$ & 0 \\
\hline 694 & $15\{308\}$ & $11\{423\}$ & $\mathbf{9}\{308,423\}$ & CCC $(\mathrm{OCCn} 1 \mathrm{nnnn} 1 \mathrm{~N} 1 \mathrm{CCNC}(=\mathrm{O}) \mathrm{C} 1) \mathrm{c} 1 \mathrm{ccccc} 1$ & 0 \\
\hline 695 & $\mathbf{1 5}\{308\}$ & $11\{321\}$ & $\mathbf{9}\{308,321\}$ & $\mathrm{CCC}(\mathrm{OCCn} 1 \mathrm{nnn} \mathrm{c} 1 \mathrm{~N} 1 \mathrm{CCC}(\mathrm{CO}) \mathrm{CC} 1) \mathrm{c} 1 \mathrm{ccccc} 1$ & 21 \\
\hline 696 & $\mathbf{1 5}\{308\}$ & $11\{328\}$ & $\mathbf{9}\{308,328\}$ & $\mathrm{CCC}(\mathrm{OCCn} 1 \mathrm{nnnn} 1 \mathrm{~N} 1 \mathrm{CCOCC} 1) \mathrm{c} 1 \mathrm{ccccc} 1$ & 28 \\
\hline 697 & $\mathbf{1 5}\{309\}$ & $11\{338\}$ & $\mathbf{9}\{309,338\}$ & $\mathrm{CCC} 1 \mathrm{CN}(\mathrm{CC}(\mathrm{CC}) \mathrm{O} 1) \mathrm{c} 1 \mathrm{nnnn} 1 \mathrm{CC} 1(\mathrm{CCOC}) \mathrm{CC} 1$ & 0 \\
\hline 698 & $15\{310\}$ & $11\{338\}$ & $\mathbf{9}\{310,338\}$ & ${ }_{1}^{\mathrm{CCOCCC} 1(\mathrm{Cn} 2 \mathrm{nnnc} 2 \mathrm{~N} 2 \mathrm{CC}(\mathrm{CC}) \mathrm{OC}(\mathrm{CC}) \mathrm{C} 2) \mathrm{CC}}$ & 45 \\
\hline 699 & $\mathbf{1 5}\{311\}$ & $\mathbf{1 1}\{355\}$ & $\mathbf{9}\{311,355\}$ & $\mathrm{COC}(\mathrm{Cn} 1 \mathrm{nnnn} 1 \mathrm{~N}(\mathrm{C}) \mathrm{Cc} 1 \mathrm{cnn}(\mathrm{C}) \mathrm{c} 1) \mathrm{C} 1 \mathrm{CCCCC} 1$ & 0 \\
\hline 700 & $15\{312\}$ & $11\{375\}$ & $\mathbf{9}\{312,375\}$ & $\mathrm{COC} 1(\mathrm{Cn} 2 \mathrm{nnnc} 2 \mathrm{~N} 2 \mathrm{CCN}(\mathrm{CC} 2) \mathrm{c} 2 \mathrm{ncccn} 2) \mathrm{CCC} 1$ & 25 \\
\hline 701 & $\mathbf{1 5}\{312\}$ & $11\{338\}$ & $\mathbf{9}\{312,338\}$ & $\mathrm{CCC} 1 \mathrm{CN}(\mathrm{CC}(\mathrm{CC}) \mathrm{O} 1) \mathrm{c} 1 \mathrm{nnnn} 1 \mathrm{CC} 1(\mathrm{CCC} 1) \mathrm{OC}$ & 27 \\
\hline 702 & $15\{312\}$ & $11\{287\}$ & $\mathbf{9}\{312,287\}$ & $\begin{array}{l}\mathrm{COC} 1(\mathrm{Cn} 2 \mathrm{nnn} \operatorname{c} 2 \mathrm{~N} 2 \mathrm{CCN}(\mathrm{CC} 2) \mathrm{C}(=\mathrm{O}) \mathrm{c} 2 \mathrm{ccco} 2) \mathrm{C} \\
\mathrm{CC} 1\end{array}$ & 0 \\
\hline 703 & $\mathbf{1 5}\{312\}$ & $11\{460\}$ & $\mathbf{9}\{312,460\}$ & ${ }_{1} \mathrm{COC} 1(\mathrm{Cn} 2 \mathrm{nnnn} 2 \mathrm{~N} 2 \mathrm{CCN}(\mathrm{CC} 2) \mathrm{c} 2 \mathrm{ccc}(\mathrm{F}) \mathrm{cc} 2) \mathrm{CCC}$ & 28 \\
\hline 704 & $\mathbf{1 5}\{312\}$ & $\mathbf{1 1}\{498\}$ & $\mathbf{9}\{312,498\}$ & $\mathrm{COC} 1(\mathrm{Cn} 2 \mathrm{nnn} \operatorname{coN} 2 \mathrm{CCN}(\mathrm{CC} 2) \mathrm{c} 2 \operatorname{ccccc} 2 \mathrm{~F}) \mathrm{CCC} 1$ & 0 \\
\hline
\end{tabular}




\begin{tabular}{|c|c|c|c|c|c|c|}
\hline 705 & $\mathbf{1 5}\{313\}$ & $\mathbf{1 1}\{338\}$ & $\mathbf{9}\{313,338\}$ & $\begin{array}{l}\mathrm{CCC} 1 \mathrm{CN}(\mathrm{CC}(\mathrm{CC}) \mathrm{O} 1) \mathrm{c} 1 \mathrm{nnnn} 1 \mathrm{CC}(\mathrm{C})(\mathrm{C}) \mathrm{C}(\mathrm{C})(\mathrm{C}) \\
\end{array}$ & 0 & 0 \\
\hline 706 & $\mathbf{1 5}\{314\}$ & $\mathbf{1 1}\{333\}$ & $\mathbf{9}\{314,333\}$ & $\mathrm{CCc} 1 \mathrm{nn}(\mathrm{C}) \mathrm{c}(\mathrm{CC}) \mathrm{c} 1 \mathrm{Cn} 1 \mathrm{nnn} \mathrm{c} 1 \mathrm{~N} 1 \mathrm{CCSC}(\mathrm{C})(\mathrm{C}) \mathrm{C} 1$ & 0 & 0 \\
\hline 707 & $\mathbf{1 5}\{315\}$ & $\mathbf{1 1}\{337\}$ & $\mathbf{9}\{315,337\}$ & CSc1 $\operatorname{ccc}(\mathrm{F}) \mathrm{c} 1 \mathrm{Cn} 1 \mathrm{nnn} \mathrm{c} 1 \mathrm{~N}(\mathrm{C}) \mathrm{CC} 1 \mathrm{CCOC} 1$ & 23 & 11 \\
\hline 708 & $\mathbf{1 5}\{316\}$ & $\mathbf{1 1}\{338\}$ & $\mathbf{9}\{316,338\}$ & $\begin{array}{l}\mathrm{CCC} 1 \mathrm{CN}(\mathrm{CC}(\mathrm{CC}) \mathrm{O} 1) \mathrm{c} 1 \mathrm{nnnn} 1 \mathrm{CCC} 1 \mathrm{CC} 2 \mathrm{CCC} 1 \\
\mathrm{O} 2\end{array}$ & 17 & 8 \\
\hline 709 & $\mathbf{1 5}\{317\}$ & $\mathbf{1 1}\{288\}$ & $\mathbf{9}\{317,288\}$ & $\mathrm{CCCC} 1(\mathrm{Cn} 2 \mathrm{nnn} \mathrm{n} 2 \mathrm{~N} 2 \mathrm{CCCN}(\mathrm{CC} 2) \mathrm{C}(\mathrm{C})=\mathrm{O}) \mathrm{CC} 1$ & 27 & 13 \\
\hline 710 & $\mathbf{1 5}\{317\}$ & $\mathbf{1 1}\{304\}$ & $\mathbf{9}\{317,304\}$ & $\begin{array}{l}\mathrm{CCCC} 1(\mathrm{Cn} 2 \mathrm{nnn} 2 \mathrm{~N} 2 \mathrm{CCCC}(\mathrm{CNC}(=\mathrm{O}) \mathrm{C}(\mathrm{C}) \mathrm{C}) \mathrm{C} 2 \\
) \mathrm{CC} 1\end{array}$ & 30 & 14 \\
\hline 711 & $\mathbf{1 5}\{318\}$ & $\mathbf{1 1}\{304\}$ & $\mathbf{9}\{318,304\}$ & $\begin{array}{l}\mathrm{CC}(\mathrm{C}) \mathrm{C}(=\mathrm{O}) \mathrm{NCC} 1 \mathrm{CCCN}(\mathrm{C} 1) \mathrm{c} 1 \mathrm{nnnn} 1 \mathrm{C} 1 \mathrm{CCC}(\mathrm{C} \\
\mathrm{C} 1\end{array}$ & 38 & 18 \\
\hline 712 & $\mathbf{1 5}\{319\}$ & $\mathbf{1 1}\{395\}$ & $\mathbf{9}\{319,395\}$ & $\begin{array}{l}\mathrm{CC}(\mathrm{C})(\mathrm{Cn} 1 \mathrm{nnn} n \mathrm{1N} 1 \mathrm{CCOC} 2(\mathrm{CCOC} 2) \mathrm{C} 1) \mathrm{C} 1 \mathrm{CC} \\
\mathrm{CC} 1\end{array}$ & 16 & 7 \\
\hline 713 & $\mathbf{1 5}\{319\}$ & $\mathbf{1 1}\{288\}$ & $\mathbf{9}\{319,288\}$ & $\begin{array}{l}\mathrm{CC}(=\mathrm{O}) \mathrm{N} 1 \mathrm{CCCN}(\mathrm{CC} 1) \mathrm{c} 1 \mathrm{nnnn} 1 \mathrm{CC}(\mathrm{C})(\mathrm{C}) \mathrm{C} 1 \mathrm{CC} \\
\mathrm{CC} 1\end{array}$ & 38 & 17 \\
\hline 714 & $\mathbf{1 5}\{320\}$ & $\mathbf{1 1}\{365\}$ & $\mathbf{9}\{320,365\}$ & $\begin{array}{l}\mathrm{CO}[\mathrm{C} @ \mathrm{H}] 1 \mathrm{C}[\mathrm{C} @ @ \mathrm{H}](\mathrm{C} 1) n 1 \mathrm{nnnc} 1 \mathrm{~N} 1 \mathrm{CC}(\mathrm{CC} 1 \mathrm{C} \\
) \mathrm{c} 1 \mathrm{ccc}(\mathrm{C}) \mathrm{cc} 1\end{array}$ & 60 & 28 \\
\hline 715 & $\mathbf{1 5}\{320\}$ & $\mathbf{1 1}\{327\}$ & $\mathbf{9}\{320,327\}$ & $\begin{array}{l}\mathrm{CO}[\mathrm{C} @ \mathrm{H}] 1 \mathrm{C}[\mathrm{C} @ @ \mathrm{H}](\mathrm{C} 1) n 1 n n n c 1 N 1 C C C C 1 \mathrm{c} 1 \\
\operatorname{ccsc} 1\end{array}$ & 189 & 89 \\
\hline 716 & $\mathbf{1 5}\{320\}$ & $\mathbf{1 1}\{302\}$ & $\mathbf{9}\{320,302\}$ & $\begin{array}{l}\text { CO[C@H]1C[C@@H](C1)n1nnnc1N(C)CCOc1 } \\
\operatorname{ccc}(\mathrm{F}) \mathrm{cc} 1\end{array}$ & 0 & 0 \\
\hline 717 & $\mathbf{1 5}\{321\}$ & $\mathbf{1 1}\{349\}$ & $\mathbf{9}\{321,349\}$ & $\mathrm{CCC} 1(\mathrm{Cn} 2 \mathrm{nnn} 22 \mathrm{~N} 2 \mathrm{CCCC}(\mathrm{CC} \# \mathrm{C}) \mathrm{C} 2) \mathrm{CCOCC} 1$ & 10 & 5 \\
\hline 718 & $\mathbf{1 5}\{321\}$ & $\mathbf{1 1}\{288\}$ & $\mathbf{9}\{321,288\}$ & $\begin{array}{l}\mathrm{CCC} 1(\mathrm{Cn} 2 \mathrm{nnn} \mathrm{n} 2 \mathrm{~N} 2 \mathrm{CCCN}(\mathrm{CC} 2) \mathrm{C}(\mathrm{C})=\mathrm{O}) \mathrm{CCOC} \\
\mathrm{C} 1\end{array}$ & 0 & 0 \\
\hline 719 & $\mathbf{1 5}\{322\}$ & $\mathbf{1 1}\{302\}$ & $\mathbf{9}\{322,302\}$ & $\mathrm{O}^{\mathrm{CN}(\mathrm{CCOc} 1 \mathrm{ccc}(\mathrm{F}) \mathrm{cc} 1) \mathrm{c} 1 \mathrm{nnnn} 1 \mathrm{C} 1 \mathrm{CC}(\mathrm{C} 1) \mathrm{C}(\mathrm{N})=}$ & 30 & 15 \\
\hline 720 & $\mathbf{1 5}\{323\}$ & $\mathbf{1 1}\{349\}$ & $\mathbf{9}\{323,349\}$ & COCC1CC(C1)n1nnnc1N1CCCC(CC\#C)C1 & 15 & 7 \\
\hline 721 & $\mathbf{1 5}\{323\}$ & $\mathbf{1 1}\{327\}$ & $\mathbf{9}\{323,327\}$ & COCC1CC(C1)n1nnnc1N1CCCC1c1ccsc1 & 68 & 34 \\
\hline 722 & $\mathbf{1 5}\{323\}$ & $\mathbf{1 1}\{302\}$ & $\mathbf{9}\{323,302\}$ & $\mathrm{COCC} 1 \mathrm{CC}(\mathrm{C} 1) \mathrm{n} 1 \mathrm{nnn} \operatorname{c} 1 \mathrm{~N}(\mathrm{C}) \mathrm{CCOc} 1 \mathrm{ccc}(\mathrm{F}) \mathrm{cc} 1$ & 16 & 8 \\
\hline 723 & $\mathbf{1 5}\{324\}$ & $\mathbf{1 1}\{349\}$ & $\mathbf{9}\{324,349\}$ & $\begin{array}{l}\mathrm{CS}(=\mathrm{O})(=\mathrm{O}) \mathrm{C} 1(\mathrm{Cn} 2 \mathrm{nnn} n \mathrm{2N} 2 \mathrm{CCCC}(\mathrm{CC} \# \mathrm{C}) \mathrm{C} 2) \mathrm{C} \\
\mathrm{C} 1\end{array}$ & 13 & 6 \\
\hline
\end{tabular}




\begin{tabular}{|c|c|c|c|c|c|}
\hline 724 & $\mathbf{1 5}\{324\}$ & $\mathbf{1 1}\{350\}$ & $\mathbf{9}\{324,350\}$ & $\mathrm{CN}(\mathrm{CCC}=\mathrm{C}) \mathrm{c} 1 \mathrm{nnnn} 1 \mathrm{CC} 1(\mathrm{CC} 1) \mathrm{S}(\mathrm{C})(=\mathrm{O})=\mathrm{O}$ & 22 \\
\hline 725 & $\mathbf{1 5}\{325\}$ & $\mathbf{1 1}\{338\}$ & $\mathbf{9}\{325,338\}$ & $\mathrm{CCC} 1 \mathrm{CN}(\mathrm{CC}(\mathrm{CC}) \mathrm{O} 1) \mathrm{c} 1 \mathrm{nnnn} 1 \mathrm{CC} 1 \mathrm{CCOC}(\mathrm{C}) \mathrm{C} 1$ & 18 \\
\hline 726 & $\mathbf{1 5}\{326\}$ & $\mathbf{1 1}\{179\}$ & $\mathbf{9}\{326,179\}$ & $\begin{array}{l}\mathrm{CC} 1(\mathrm{Cn} 2 \mathrm{nnn} n 2 \mathrm{~N} 2 \mathrm{C}[\mathrm{C} @ \mathrm{H}] 3[\mathrm{C} @ \mathrm{H}](\mathrm{CO})[\mathrm{C} @ \mathrm{H}] \\
\text { 3C2)CCCc2 } \operatorname{ccccc} 12\end{array}$ & 24 \\
\hline 727 & $\mathbf{1 5}\{327\}$ & $\mathbf{1 1}\{304\}$ & $\mathbf{9}\{327,304\}$ & $\begin{array}{l}\mathrm{CC}(\mathrm{C}) \mathrm{C}(=\mathrm{O}) \mathrm{NCC} 1 \mathrm{CCCN}(\mathrm{C} 1) \mathrm{c} 1 \mathrm{nnnn} 1 \mathrm{C} 1 \mathrm{CC} 2(\mathrm{C} \\
\mathrm{CC} 2) \mathrm{C} 1\end{array}$ & 30 \\
\hline 728 & $\mathbf{1 5}\{327\}$ & $\mathbf{1 1}\{472\}$ & $\mathbf{9}\{327,472\}$ & $\begin{array}{l}\mathrm{CC}(\mathrm{C})(\mathrm{C}) \mathrm{OC}(=\mathrm{O}) \mathrm{N}[\mathrm{C} @ @ \mathrm{H}] 1 \mathrm{CCN}(\mathrm{C} 1) \mathrm{c} 1 \mathrm{nnnn} 1 \\
\mathrm{C} 1 \mathrm{CC} 2(\mathrm{CCC} 2) \mathrm{C} 1\end{array}$ & 34 \\
\hline 729 & $\mathbf{1 5}\{328\}$ & $\mathbf{1 1}\{302\}$ & $\mathbf{9}\{328,302\}$ & $\begin{array}{l}\mathrm{CCO}[\mathrm{C} @ \mathrm{H}] 1 \mathrm{C}[\mathrm{C} @ \mathrm{H}](\mathrm{C} 1) \mathrm{n} 1 \mathrm{nnnn} 1 \mathrm{~N}(\mathrm{C}) \mathrm{CCOc} 1 \mathrm{c} \\
\mathrm{cc}(\mathrm{F}) \mathrm{cc} 1\end{array}$ & 0 \\
\hline 730 & $\mathbf{1 5}\{328\}$ & $\mathbf{1 1}\{327\}$ & $\mathbf{9}\{328,327\}$ & $\begin{array}{l}\mathrm{CCO}[\mathrm{C} @ \mathrm{H}] 1 \mathrm{C}[\mathrm{C} @ \mathrm{H}](\mathrm{C} 1) \mathrm{n} 1 \mathrm{nnn} n 1 \mathrm{~N} 1 \mathrm{CCCC} 1 \mathrm{c} 1 \\
\operatorname{ccsc} 1\end{array}$ & 34 \\
\hline 731 & $\mathbf{1 5}\{328\}$ & $\mathbf{1 1}\{349\}$ & $\mathbf{9}\{328,349\}$ & 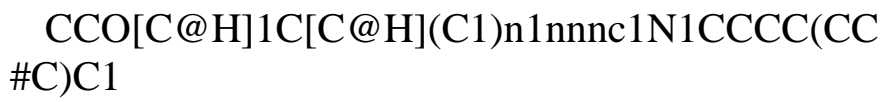 & 29 \\
\hline 732 & $\mathbf{1 5}\{329\}$ & $\mathbf{1 1}\{302\}$ & $\mathbf{9}\{329,302\}$ & $\begin{array}{l}\mathrm{CCO}[\mathrm{C} @ \mathrm{H}] 1 \mathrm{C}[\mathrm{C} @ @ \mathrm{H}](\mathrm{C} 1) n 1 \mathrm{nnnc} 1 \mathrm{~N}(\mathrm{C}) \mathrm{CCOc} \\
\text { 1ccc(F)cc1 }\end{array}$ & 18 \\
\hline 733 & $\mathbf{1 5}\{329\}$ & $\mathbf{1 1}\{327\}$ & $\mathbf{9}\{329,327\}$ & $\begin{array}{l}\text { CCO[C@H]1C[C@@H](C1)n1nnnc1N1CCCC1 } \\
\text { c1ccsc1 }\end{array}$ & 62 \\
\hline 734 & $\mathbf{1 5}\{329\}$ & $\mathbf{1 1}\{349\}$ & $\mathbf{9}\{329,349\}$ & $\begin{array}{l}\mathrm{CCO}[\mathrm{C} @ \mathrm{H}] 1 \mathrm{C}[\mathrm{C} @ @ \mathrm{H}](\mathrm{C} 1) n 1 \mathrm{nnn} 1 \mathrm{~N} 1 \mathrm{CCCC}( \\
\mathrm{CC \# C)C1}\end{array}$ & 33 \\
\hline 735 & $\mathbf{1 5}\{330\}$ & $\mathbf{1 1}\{499\}$ & $\mathbf{9}\{330,499\}$ & $\begin{array}{l}\mathrm{O}=\mathrm{S} 1(=\mathrm{O}) \mathrm{CCCCC} 1 \mathrm{Cn} 1 \mathrm{nnnn} 1 \mathrm{~N} 1 \mathrm{CCCC} 2(\mathrm{CC} 2) \mathrm{C} \\
\mathrm{C} 1\end{array}$ & 25 \\
\hline 736 & $\mathbf{1 5}\{330\}$ & $\mathbf{1 1}\{493\}$ & $\mathbf{9}\{330,493\}$ & $\begin{aligned} & \mathrm{CC} 1 \mathrm{CC} 2 \mathrm{CCC}(\mathrm{C} 1) \mathrm{N} 2 \mathrm{c} 1 \mathrm{nnnn} 1 \mathrm{CC} 1 \mathrm{CCCCS} 1(=\mathrm{O}) \\
= & \mathrm{O}\end{aligned}$ & 8 \\
\hline 737 & $\mathbf{1 5}\{331\}$ & $\mathbf{1 1}\{300\}$ & $\mathbf{9}\{331,300\}$ & $\mathrm{FC}(\mathrm{F})(\mathrm{F}) \mathrm{c} 1 \mathrm{cc}(\mathrm{Cn} 2 \mathrm{nnn} \mathrm{c} 2 \mathrm{~N} 2 \mathrm{CCCOCC} 2) \mathrm{cs} 1$ & 55 \\
\hline 738 & $\mathbf{1 5}\{331\}$ & $11\{337\}$ & $\mathbf{9}\{331,337\}$ & $\mathrm{CN}(\mathrm{CC} 1 \mathrm{CCOC} 1) \mathrm{c} 1 \mathrm{nnnn} 1 \mathrm{Cc} 1 \mathrm{csc}(\mathrm{c} 1) \mathrm{C}(\mathrm{F})(\mathrm{F}) \mathrm{F}$ & 25 \\
\hline 739 & $\mathbf{1 5}\{332\}$ & $\mathbf{1 1}\{500\}$ & $\mathbf{9}\{332,500\}$ & $\begin{array}{l}\text { CON1CCC }(\mathrm{CC} 1) \mathrm{n} 1 \mathrm{nnnn} 1 \mathrm{~N} 1 \mathrm{CCCC}(\mathrm{C} 1) \mathrm{c} 1 \mathrm{cc} 2 \mathrm{ccc} \\
\mathrm{cc} 2[\mathrm{nH}] 1\end{array}$ & 0 \\
\hline 740 & $\mathbf{1 5}\{333\}$ & $\mathbf{1 1}\{501\}$ & $\mathbf{9}\{333,501\}$ & $\mathrm{CCN}(\mathrm{CC}(\mathrm{C})=\mathrm{C}) \mathrm{c} 1 \mathrm{nnnn} 1 \mathrm{C} 1 \mathrm{COC} 2(\mathrm{CCC} 2) \mathrm{C} 1$ & 35 \\
\hline 741 & $\mathbf{1 5}\{333\}$ & $\mathbf{1 1}\{302\}$ & $\mathbf{9}\{333,302\}$ & ${ }_{1}^{\mathrm{CN}}(\mathrm{CCOc} 1 \mathrm{ccc}(\mathrm{F}) \mathrm{cc} 1) \mathrm{c} 1 \mathrm{nnnn} 1 \mathrm{C} 1 \mathrm{COC} 2(\mathrm{CCC} 2) \mathrm{C}$ & 47 \\
\hline
\end{tabular}




\begin{tabular}{|c|c|c|c|c|c|}
\hline 742 & $\mathbf{1 5}\{334\}$ & $\mathbf{1 1}\{391\}$ & $\mathbf{9}\{334,391\}$ & $\operatorname{CCS}(=\mathrm{O})(=\mathrm{O}) \mathrm{c} 1 \mathrm{ccc}(\mathrm{Cn} 2 \mathrm{nnn} \operatorname{coN}(\mathrm{C}) \mathrm{CC} 2 \mathrm{CC} 2) \mathrm{cc} 1$ & 6 \\
\hline 743 & $\mathbf{1 5}\{335\}$ & $\mathbf{1 1}\{430\}$ & $\mathbf{9}\{335,430\}$ & $\begin{array}{r}\mathrm{CCOC}(=\mathrm{O})[1 \mathrm{C} @ @ \mathrm{H}] 1[1 \mathrm{C} @ @ \mathrm{H}] 2 \mathrm{CCN}(\mathrm{C}[1 \mathrm{C} @ \\
\mathrm{H}] 12) \mathrm{c} 1 \mathrm{nnnn} 1[\mathrm{C} @ @ \mathrm{H}] 1 \mathrm{CCO}[\mathrm{C} @ @ \mathrm{H}] 1 \mathrm{C}\end{array}$ & 31 \\
\hline 744 & $\mathbf{1 5}\{335\}$ & $\mathbf{1 1}\{436\}$ & $\mathbf{9}\{335,436\}$ & $\begin{array}{l}\mathrm{COC}(=\mathrm{O})[1 \mathrm{C} @] 12 \mathrm{CCC}[1 \mathrm{C} @ \mathrm{H}] 1 \mathrm{~N}(\mathrm{CC} 2) \mathrm{c} 1 \mathrm{nnnn} \\
1[\mathrm{C} @ @ \mathrm{H}] 1 \mathrm{CCO}[\mathrm{C} @ @ \mathrm{H}] 1 \mathrm{C}\end{array}$ & 0 \\
\hline 745 & $\mathbf{1 5}\{335\}$ & $\mathbf{1 1}\{359\}$ & $\mathbf{9}\{335,359\}$ & $\begin{array}{l}\mathrm{C}[\mathrm{C} @ \mathrm{H}] 1 \mathrm{OCC}[\mathrm{C} @ \mathrm{H}] 1 \mathrm{n} 1 \mathrm{nnn} 1 \mathrm{~N} 1 \mathrm{C}[\mathrm{C} @ @ \mathrm{H}] 2 \mathrm{C} \\
{[\mathrm{C} @ \mathrm{H}](\mathrm{C} 1) \mathrm{c} 1 \mathrm{cccc}(=\mathrm{O}) \mathrm{n} 1 \mathrm{C} 2}\end{array}$ & 12 \\
\hline 746 & $\mathbf{1 5}\{335\}$ & $\mathbf{1 1}\{419\}$ & $\mathbf{9}\{335,419\}$ & $\begin{array}{l}\mathrm{C}[\mathrm{C} @ \mathrm{H}] 1 \mathrm{OCC}[\mathrm{C} @ \mathrm{H}] 1 \mathrm{n} 1 \mathrm{nnn} \mathrm{n} 1 \mathrm{~N} 1 \mathrm{C} 2 \mathrm{CCC} 1 \mathrm{CC} 1 \\
(\mathrm{CC} 1(\mathrm{~F}) \mathrm{F}) \mathrm{C} 2\end{array}$ & 0 \\
\hline 747 & $\mathbf{1 5}\{335\}$ & $\mathbf{1 1}\{502\}$ & $\mathbf{9}\{335,502\}$ & $\begin{array}{l}\mathrm{C}[\mathrm{C} @ \mathrm{H}] 1 \mathrm{OCC}[\mathrm{C} @ \mathrm{H}] 1 \mathrm{n} 1 \mathrm{nnn} n 1 \mathrm{~N} 1 \mathrm{CCC} 2(\mathrm{C} 1) \mathrm{Oc} \\
1 \mathrm{ccccc} 1 \mathrm{O} 2\end{array}$ & 0 \\
\hline 748 & $\mathbf{1 5}\{335\}$ & $\mathbf{1 1}\{371\}$ & $\mathbf{9}\{335,371\}$ & $\begin{array}{l}\mathrm{C}[\mathrm{C} @ \mathrm{H}] 1 \mathrm{OCC}[\mathrm{C} @ \mathrm{H}] 1 \mathrm{n} 1 \mathrm{nnn} n 1 \mathrm{~N} 1 \mathrm{CCOC} 2(\mathrm{CCc} \\
3 \mathrm{ccccc} 23) \mathrm{C} 1\end{array}$ & 0 \\
\hline 749 & $\mathbf{1 5}\{335\}$ & $\mathbf{1 1}\{408\}$ & $\mathbf{9}\{335,408\}$ & $\begin{array}{l}\text { C[C@H]1OCC[C@H]1n1nnnc1N1CCCC2(CCC } \\
\text { C2)C1 }\end{array}$ & 21 \\
\hline 750 & $\mathbf{1 5}\{335\}$ & $\mathbf{1 1}\{404\}$ & $\mathbf{9}\{335,404\}$ & $\begin{array}{l}\mathrm{COC}(=\mathrm{O})[1 \mathrm{C} @] 12 \mathrm{CN}(\mathrm{C}[1 \mathrm{C} @ \mathrm{H}] 1 \mathrm{CCCC} 2) \mathrm{c} 1 \mathrm{nnn} \\
\mathrm{n} 1[\mathrm{C} @ @ \mathrm{H}] 1 \mathrm{CCO}[\mathrm{C} @ @ \mathrm{H}] 1 \mathrm{C}\end{array}$ & 13 \\
\hline 751 & $\mathbf{1 5}\{335\}$ & $\mathbf{1 1}\{406\}$ & $\mathbf{9}\{335,406\}$ & $\begin{array}{l}\mathrm{C}[\mathrm{C} @ \mathrm{H}] 1 \mathrm{OCC}[\mathrm{C} @ \mathrm{H}] 1 \mathrm{n} 1 \mathrm{nnnc} 1 \mathrm{~N} 1 \mathrm{CCC}(\mathrm{C}) \mathrm{C} 2(\mathrm{C} \\
\mathrm{CC} 2) \mathrm{C} 1\end{array}$ & 18 \\
\hline 752 & $\mathbf{1 5}\{335\}$ & $\mathbf{1 1}\{413\}$ & $\mathbf{9}\{335,413\}$ & $\begin{array}{l}\text { C[C@H]1OCC }[\mathrm{C} @ \mathrm{H}] 1 \mathrm{n} 1 \mathrm{nnn} n 1 \mathrm{~N} 1 \mathrm{CC}(\mathrm{C}) \mathrm{OC} 2(\mathrm{C} \\
\mathrm{CCC} 2) \mathrm{C} 1\end{array}$ & 23 \\
\hline 753 & $\mathbf{1 5}\{335\}$ & $\mathbf{1 1}\{407\}$ & $\mathbf{9}\{335,407\}$ & $\begin{array}{l}\mathrm{C}[\mathrm{C} @ \mathrm{H}] 1 \mathrm{OCC}[\mathrm{C} @ \mathrm{H}] 1 \mathrm{n} 1 \mathrm{nnn} n 1 \mathrm{~N} 1 \mathrm{CCC} 2(\mathrm{C} 1) \mathrm{CC} \\
\mathrm{CCC} 2\end{array}$ & 40 \\
\hline 754 & $\mathbf{1 5}\{335\}$ & $\mathbf{1 1}\{457\}$ & $\mathbf{9}\{335,457\}$ & $\begin{array}{l}\text { CCc1ncc2C3CCC(Cc2n1)N3c1nnnn1[C@@H]1 } \\
\text { CCO[C@@H]1C }\end{array}$ & 0 \\
\hline 755 & $\mathbf{1 5}\{335\}$ & $\mathbf{1 1}\{503\}$ & $\mathbf{9}\{335,503\}$ & $\begin{array}{l}\mathrm{C}[\mathrm{C} @ \mathrm{H}] 1 \mathrm{OCC}[\mathrm{C} @ \mathrm{H}] 1 \mathrm{n} 1 \mathrm{nnn} n 1 \mathrm{~N} 1 \mathrm{CCCN} 2 \mathrm{C}(\mathrm{Cc} \\
3 \operatorname{ccccc} 23) \mathrm{C} 1\end{array}$ & 0 \\
\hline 756 & $\mathbf{1 5}\{335\}$ & $\mathbf{1 1}\{421\}$ & $\mathbf{9}\{335,421\}$ & $\begin{array}{l}\text { C }[\mathrm{C} @ \mathrm{H}] 1 \mathrm{OCC}[\mathrm{C} @ \mathrm{H}] 1 \mathrm{n} 1 \mathrm{nnnc1N} 1 \mathrm{CCC} 2(\mathrm{C} 1) \mathrm{OC} \\
\text { c1ccccc21 }\end{array}$ & 7 \\
\hline 757 & $\mathbf{1 5}\{335\}$ & $11\{467\}$ & $\mathbf{9}\{335,467\}$ & $\begin{array}{l}\mathrm{C}[\mathrm{C} @ \mathrm{H}] 1 \mathrm{OCC}[\mathrm{C} @ \mathrm{H}] 1 \mathrm{n} 1 \mathrm{nnn} n 1 \mathrm{~N} 1 \mathrm{C} 2 \mathrm{CCC} 1 \mathrm{CC}( \\
\mathrm{CC}(\mathrm{F})(\mathrm{F}) \mathrm{F}) \mathrm{C} 2\end{array}$ & 0 \\
\hline 758 & $\mathbf{1 5}\{335\}$ & $\mathbf{1 1}\{402\}$ & $\mathbf{9}\{335,402\}$ & $\begin{array}{l}\mathrm{C}[\mathrm{C} @ \mathrm{H}] 1 \mathrm{OCC}[\mathrm{C} @ \mathrm{H}] 1 \mathrm{n} 1 \mathrm{nnn} n 1 \mathrm{~N} 1 \mathrm{CC}(\mathrm{C}) \mathrm{OC} 2(\mathrm{C} \\
\mathrm{CCCC} 2) \mathrm{C} 1\end{array}$ & 0 \\
\hline 759 & $\mathbf{1 5}\{335\}$ & $\mathbf{1 1}\{104\}$ & $\mathbf{9}\{335,104\}$ & C[C@H]1OCC[C@H]1n1nnnc1N1CCOCC2(CC & 0 \\
\hline
\end{tabular}




\section{CC2)C1}

\begin{tabular}{|c|c|c|c|c|c|}
\hline 760 & $\mathbf{1 5}\{335\}$ & $\mathbf{1 1}\{409\}$ & $\mathbf{9}\{335,409\}$ & $\begin{array}{l}\mathrm{C}[\mathrm{C} @ \mathrm{H}] 1 \mathrm{OCC}[\mathrm{C} @ \mathrm{H}] 1 \mathrm{n} 1 \mathrm{nnn} n 1 \mathrm{~N} 1 \mathrm{CCSC} 2(\mathrm{CCC} \\
\mathrm{CC} 2) \mathrm{C} 1\end{array}$ & 0 \\
\hline 761 & $\mathbf{1 5}\{335\}$ & $\mathbf{1 1}\{412\}$ & $\mathbf{9}\{335,412\}$ & $\begin{array}{l}\mathrm{CON}(\mathrm{CC} 1 \mathrm{CC} 2 \mathrm{CCC} 1 \mathrm{C} 2) \mathrm{c} 1 \mathrm{nnnn} 1[\mathrm{C} @ @ \mathrm{H}] 1 \mathrm{CCO} \\
{[\mathrm{C} @ @ \mathrm{H}] 1 \mathrm{C}}\end{array}$ & 0 \\
\hline 762 & $\mathbf{1 5}\{335\}$ & $\mathbf{1 1}\{282\}$ & $\mathbf{9}\{335,282\}$ & $\begin{array}{l}\mathrm{C}[\mathrm{C} @ \mathrm{H}] 1 \mathrm{OCC}[\mathrm{C} @ \mathrm{H}] 1 \mathrm{n} 1 \mathrm{nnnn} 1 \mathrm{~N} 1 \mathrm{CCN}(\mathrm{C}(\mathrm{C})=\mathrm{O} \\
) \mathrm{C} 2(\mathrm{CCCCC} 2) \mathrm{C} 1\end{array}$ & 17 \\
\hline 763 & $\mathbf{1 5}\{336\}$ & $\mathbf{1 1}\{288\}$ & $\mathbf{9}\{336,288\}$ & $\begin{array}{l}\mathrm{CCC}[1 \mathrm{C} @ @ \mathrm{H}] 1 \mathrm{C}[1 \mathrm{C} @ \mathrm{H}] 1 \mathrm{n} 1 \mathrm{nnn} \mathrm{n} 1 \mathrm{~N} 1 \mathrm{CCCN}(\mathrm{C} \\
\mathrm{C} 1) \mathrm{C}(\mathrm{C})=\mathrm{O}\end{array}$ & 33 \\
\hline 764 & $\mathbf{1 5}\{336\}$ & $\mathbf{1 1}\{304\}$ & $\mathbf{9}\{336,304\}$ & $\begin{array}{l}\mathrm{CCC}[1 \mathrm{C} @ @ \mathrm{H}] 1 \mathrm{C}[1 \mathrm{C} @ \mathrm{H}] 1 \mathrm{n} 1 \mathrm{nnnn} 1 \mathrm{~N} 1 \mathrm{CCCC}(\mathrm{C} \\
\mathrm{NC}(=\mathrm{O}) \mathrm{C}(\mathrm{C}) \mathrm{C}) \mathrm{C} 1\end{array}$ & 17 \\
\hline 765 & $\mathbf{1 5}\{337\}$ & $\mathbf{1 1}\{322\}$ & $\mathbf{9}\{337,322\}$ & $\mathrm{CN}(\mathrm{C} 1 \mathrm{CC} 1) \mathrm{c} 1 \mathrm{nnnn} 1 \mathrm{C} 1 \mathrm{CCOC}(\mathrm{C} 1) \mathrm{c} 1 \mathrm{ccnn} 1 \mathrm{C}$ & 25 \\
\hline 766 & $\mathbf{1 5}\{337\}$ & $\mathbf{1 1}\{356\}$ & $\mathbf{9}\{337,356\}$ & $\mathrm{CN}(\mathrm{CC} 1 \mathrm{CCC} 1) \mathrm{c} 1 \mathrm{nnnn} 1 \mathrm{C} 1 \mathrm{CCOC}(\mathrm{C} 1) \mathrm{c} 1 \mathrm{ccnn} 1 \mathrm{C}$ & 30 \\
\hline 767 & $\mathbf{1 5}\{338\}$ & $\mathbf{1 1}\{322\}$ & $\mathbf{9}\{338,322\}$ & $\begin{array}{l}\mathrm{CN}(\mathrm{C} 1 \mathrm{CC} 1) \mathrm{c} 1 \mathrm{nnnn} 1 \mathrm{C}[\mathrm{C} @ @ \mathrm{H}] 1 \mathrm{CCCN}(\mathrm{C})[\mathrm{C} @ \\
\mathrm{H}] 1 \mathrm{c} 1 \mathrm{ccnn} 1 \mathrm{C}\end{array}$ & 0 \\
\hline 768 & $\mathbf{1 5}\{338\}$ & $\mathbf{1 1}\{391\}$ & $\mathbf{9}\{338,391\}$ & $\begin{array}{l}\text { CN(CC1CC1)c1nnnn1C[C@@H]1CCCN(C)[C } \\
@ \mathrm{H}] 1 \mathrm{c} 1 \mathrm{ccnn} 1 \mathrm{C}\end{array}$ & 33 \\
\hline 769 & $\mathbf{1 5}\{339\}$ & $\mathbf{1 1}\{288\}$ & $\mathbf{9}\{339,288\}$ & $\begin{array}{l}\mathrm{CC}(=\mathrm{O}) \mathrm{N} 1 \mathrm{CCCN}(\mathrm{CC} 1) \mathrm{c} 1 \mathrm{nnnn} 1 \mathrm{CC}(\mathrm{C} 1 \mathrm{CCC} 1) \mathrm{C} 1 \\
\mathrm{CCC} 1\end{array}$ & 21 \\
\hline 770 & $\mathbf{1 5}\{339\}$ & $\mathbf{1 1}\{395\}$ & $\mathbf{9}\{339,395\}$ & $\begin{array}{l}\mathrm{C}(\mathrm{C}(\mathrm{C} 1 \mathrm{CCC} 1) \mathrm{C} 1 \mathrm{CCC} 1) \mathrm{n} 1 \mathrm{nnn} \mathrm{n} 1 \mathrm{~N} 1 \mathrm{CCOC} 2(\mathrm{CC} \\
\mathrm{OC} 2) \mathrm{C} 1\end{array}$ & 32 \\
\hline 771 & $\mathbf{1 5}\{340\}$ & $\mathbf{1 1}\{504\}$ & $\mathbf{9}\{340,504\}$ & $\begin{array}{l}\mathrm{O}=\mathrm{C} 1 \mathrm{CN}(\mathrm{CCN} 1 \mathrm{C} 1 \mathrm{CC} 1) \mathrm{c} 1 \mathrm{nnnn} 1 \mathrm{CCC} 1 \mathrm{CCC} 2(\mathrm{C} \\
\mathrm{CC} 2) \mathrm{O} 1\end{array}$ & 11 \\
\hline 772 & $\mathbf{1 5}\{340\}$ & $\mathbf{1 1}\{319\}$ & $\mathbf{9}\{340,319\}$ & $\begin{array}{l}\mathrm{CN}(\mathrm{CC} 1 \mathrm{COCCO} 1) \mathrm{c} 1 \mathrm{nnnn} 1 \mathrm{CCC} 1 \mathrm{CCC} 2(\mathrm{CCC} 2) \\
\mathrm{O} 1\end{array}$ & 53 \\
\hline 773 & $\mathbf{1 5}\{341\}$ & $\mathbf{1 1}\{346\}$ & $\mathbf{9}\{341,346\}$ & $\mathrm{CCN}(\mathrm{C}) \mathrm{c} 1 \mathrm{nnnn} 1 \mathrm{Cc} 1 \mathrm{nc}(\mathrm{n} 2 \mathrm{CCCCc} 12) \mathrm{C}(\mathrm{F})(\mathrm{F}) \mathrm{F}$ & 70 \\
\hline 774 & $\mathbf{1 5}\{342\}$ & $\mathbf{1 1}\{288\}$ & $\mathbf{9}\{342,288\}$ & $\begin{array}{l}\mathrm{CC}(=\mathrm{O}) \mathrm{N} 1 \mathrm{CCCN}(\mathrm{CC} 1) \mathrm{c} 1 \mathrm{nnnn} 1 \mathrm{CC}(\mathrm{C})(\mathrm{C}) \mathrm{C} 1 \mathrm{CC} \\
\mathrm{C} 1\end{array}$ & 0 \\
\hline 775 & $\mathbf{1 5}\{343\}$ & $\mathbf{1 1}\{288\}$ & $\mathbf{9}\{343,288\}$ & $\begin{array}{l}\mathrm{CC}(=\mathrm{O}) \mathrm{N} 1 \mathrm{CCCN}(\mathrm{CC} 1) \mathrm{c} 1 \mathrm{nnnn} 1 \mathrm{CC} 1(\mathrm{C}) \mathrm{CC} 1(\mathrm{Cl}) \\
\mathrm{Cl}\end{array}$ & 0 \\
\hline 776 & $\mathbf{1 5}\{344\}$ & $\mathbf{1 1}\{304\}$ & $\mathbf{9}\{344,304\}$ & $\begin{array}{l}\mathrm{CC}(\mathrm{C})[1 \mathrm{C} @ @ \mathrm{H}] 1 \mathrm{C}[1 \mathrm{C} @ \mathrm{H}] 1 \mathrm{n} 1 \mathrm{nnn} n 1 \mathrm{~N} 1 \mathrm{CCCC}( \\
\mathrm{CNC}(=\mathrm{O}) \mathrm{C}(\mathrm{C}) \mathrm{C}) \mathrm{C} 1\end{array}$ & 36 \\
\hline
\end{tabular}




\begin{tabular}{|c|c|c|c|c|c|}
\hline 777 & $\mathbf{1 5}\{345\}$ & $\mathbf{1 1}\{304\}$ & $\mathbf{9}\{345,304\}$ & $\begin{array}{l}\mathrm{CC}(\mathrm{C}) \mathrm{C}[1 \mathrm{C} @ @ \mathrm{H}] 1 \mathrm{C}[1 \mathrm{C} @ \mathrm{H}] 1 \mathrm{n} 1 \mathrm{nnnc1N} 1 \mathrm{CCC} \\
\mathrm{C}(\mathrm{CNC}(=\mathrm{O}) \mathrm{C}(\mathrm{C}) \mathrm{C}) \mathrm{C} 1\end{array}$ & 34 \\
\hline 778 & $\mathbf{1 5}\{346\}$ & $\mathbf{1 1}\{391\}$ & $\mathbf{9}\{346,391\}$ & $\begin{array}{l}\mathrm{CN}(\mathrm{CC} 1 \mathrm{CC} 1) \mathrm{c} 1 \mathrm{nnnn} 1 \mathrm{C}[1 \mathrm{C} @ @ \mathrm{H}] 1 \mathrm{CCO}[1 \mathrm{C} @ \mathrm{H} \\
\text { ]1c1c(C)nn(C)c1C }\end{array}$ & 32 \\
\hline 779 & $\mathbf{1 5}\{347\}$ & $\mathbf{1 1}\{327\}$ & $\mathbf{9}\{347,327\}$ & $\begin{array}{l}\mathrm{CC}(\mathrm{C})(\mathrm{C}) \mathrm{O}[\mathrm{C} @ \mathrm{H}] 1 \mathrm{C}[\mathrm{C} @ \mathrm{H}](\mathrm{C} 1) n 1 \mathrm{nnnc} 1 \mathrm{~N} 1 \mathrm{CC} \\
\mathrm{CC} 1 \mathrm{c} 1 \mathrm{ccsc} 1\end{array}$ & 0 \\
\hline 780 & $\mathbf{1 5}\{347\}$ & $\mathbf{1 1}\{349\}$ & $\mathbf{9}\{347,349\}$ & $\begin{array}{l}\mathrm{CC}(\mathrm{C})(\mathrm{C}) \mathrm{O}[\mathrm{C} @ \mathrm{H}] 1 \mathrm{C}[\mathrm{C} @ \mathrm{H}](\mathrm{C} 1) n 1 \mathrm{nnnc} 1 \mathrm{~N} 1 \mathrm{CC} \\
\mathrm{CC}(\mathrm{CC} \# \mathrm{C}) \mathrm{C} 1\end{array}$ & 0 \\
\hline 781 & $\mathbf{1 5}\{348\}$ & $\mathbf{1 1}\{349\}$ & $\mathbf{9}\{348,349\}$ & $\begin{array}{l}\mathrm{COC}(=\mathrm{O}) \mathrm{CC} 1 \mathrm{CC}(\mathrm{C} 1) \mathrm{n} 1 \mathrm{nnnn} 1 \mathrm{~N} 1 \mathrm{CCCC}(\mathrm{CC} \# \mathrm{C}) \\
\mathrm{C} 1\end{array}$ & 14 \\
\hline 782 & $\mathbf{1 5}\{348\}$ & $\mathbf{1 1}\{327\}$ & $\mathbf{9}\{348,327\}$ & $\mathrm{COC}(=\mathrm{O}) \mathrm{CC} 1 \mathrm{CC}(\mathrm{C} 1) \mathrm{n} 1 \mathrm{nnn} \mathrm{n} 1 \mathrm{~N} 1 \mathrm{CCCC} 1 \mathrm{c} 1 \mathrm{ccsc} 1$ & 31 \\
\hline 783 & $\mathbf{1 5}\{349\}$ & $\mathbf{1 1}\{302\}$ & $\mathbf{9}\{349,302\}$ & ${ }_{1} \mathrm{CCN} 1 \mathrm{CCC}(\mathrm{C} 1=\mathrm{O}) \mathrm{n} 1 \mathrm{nnn} n \mathrm{1N}(\mathrm{C}) \mathrm{CCOc} 1 \mathrm{ccc}(\mathrm{F}) \mathrm{cc}$ & 0 \\
\hline 784 & $\mathbf{1 5}\{350\}$ & $\mathbf{1 1}\{302\}$ & $\mathbf{9}\{350,302\}$ & $\begin{array}{l}\mathrm{CN}(\mathrm{CCOc} 1 \mathrm{ccc}(\mathrm{F}) \mathrm{cc} 1) \mathrm{c} 1 \mathrm{nnnn} 1[\mathrm{C} @ \mathrm{H}] 1 \mathrm{C}[\mathrm{C} @ @ \\
\mathrm{H}](\mathrm{C} 1) \mathrm{C}(\mathrm{N})=\mathrm{O}\end{array}$ & 13 \\
\hline 785 & $\mathbf{1 5}\{351\}$ & $\mathbf{1 1}\{338\}$ & $\mathbf{9}\{351,338\}$ & CCC1CN(CC(CC)O1)c1nnnn1CCC1(COC)CC1 & 33 \\
\hline 786 & $\mathbf{1 5}\{352\}$ & $\mathbf{1 1}\{338\}$ & $\mathbf{9}\{352,338\}$ & $\mathrm{CCC} 1 \mathrm{CN}(\mathrm{CC}(\mathrm{CC}) \mathrm{O} 1) \mathrm{c} 1 \mathrm{nnnn} 1 \mathrm{CCCC} 1(\mathrm{O}) \mathrm{CCC} 1$ & 38 \\
\hline 787 & $\mathbf{1 5}\{353\}$ & $\mathbf{1 1}\{501\}$ & $\mathbf{9}\{353,501\}$ & $\mathrm{O}^{\mathrm{CCN}(\mathrm{CC}(\mathrm{C})=\mathrm{C}) \mathrm{c} 1 \mathrm{nnnn} 1 \mathrm{CCN} 1 \mathrm{CC} 2 \mathrm{CCCCN} 2 \mathrm{C} 1=}$ & 0 \\
\hline 788 & $\mathbf{1 5}\{354\}$ & $\mathbf{1 1}\{327\}$ & $\mathbf{9}\{354,327\}$ & C(C1SCCS1)n1nnnc1N1CCCC1c1ccsc1 & 8 \\
\hline 789 & $\mathbf{1 5}\{354\}$ & $\mathbf{1 1}\{349\}$ & $\mathbf{9}\{354,349\}$ & 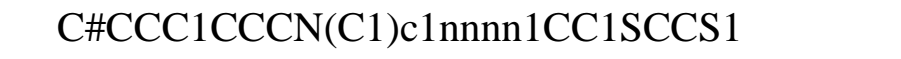 & 0 \\
\hline 790 & $\mathbf{1 5}\{355\}$ & $\mathbf{1 1}\{297\}$ & $\mathbf{9}\{355,297\}$ & $\begin{array}{l}\mathrm{CC} 1(\mathrm{OCCO} 1) \mathrm{C} 1 \mathrm{CCN}(\mathrm{CC} 1) \mathrm{c} 1 \mathrm{nnnn} 1 \mathrm{C} 1 \mathrm{COC}(\mathrm{C} 1) \\
\mathrm{C} 1 \mathrm{CC} 1\end{array}$ & 37 \\
\hline 791 & $\mathbf{1 5}\{355\}$ & $\mathbf{1 1}\{298\}$ & $\mathbf{9}\{355,298\}$ & $\begin{array}{l}\mathrm{CC} 1(\mathrm{OCCO} 1) \mathrm{C} 1 \mathrm{CCCN}(\mathrm{C} 1) \mathrm{c} 1 \mathrm{nnnn} 1 \mathrm{C} 1 \mathrm{COC}(\mathrm{C} 1) \\
\mathrm{C} 1 \mathrm{CC} 1\end{array}$ & 8 \\
\hline 792 & $\mathbf{1 5}\{356\}$ & $\mathbf{1 1}\{350\}$ & $\mathbf{9}\{356,350\}$ & $\mathrm{CN}(\mathrm{CCC}=\mathrm{C}) \mathrm{c} 1 \mathrm{nnnn} 1 \mathrm{C} 1 \mathrm{CCN}(\mathrm{C} 2 \mathrm{CCCCC} 2) \mathrm{C} 1=\mathrm{O}$ & 0 \\
\hline 793 & $\mathbf{1 5}\{357\}$ & $\mathbf{1 1}\{349\}$ & $\mathbf{9}\{357,349\}$ & $\begin{array}{l}\mathrm{CO}[\mathrm{C} @ @ \mathrm{H}] 1 \mathrm{C}[\mathrm{C} @ \mathrm{H}](\mathrm{Cn} 2 \mathrm{nnn} n 2 \mathrm{~N} 2 \mathrm{CCCC}(\mathrm{CC} \# \\
\mathrm{C}) \mathrm{C} 2) \mathrm{C} 1\end{array}$ & 60 \\
\hline 794 & $\mathbf{1 5}\{357\}$ & $\mathbf{1 1}\{327\}$ & $\mathbf{9}\{357,327\}$ & $\begin{array}{l}\mathrm{CO}[\mathrm{C} @ @ \mathrm{H}] 1 \mathrm{C}[\mathrm{C} @ \mathrm{H}](\mathrm{Cn} 2 \mathrm{nnn} n 2 \mathrm{~N} 2 \mathrm{CCCC} 2 \mathrm{c} 2 \mathrm{cc} \\
\mathrm{sc} 2) \mathrm{C} 1\end{array}$ & 44 \\
\hline 795 & $\mathbf{1 5}\{358\}$ & $\mathbf{1 1}\{349\}$ & $\mathbf{9}\{358,349\}$ & $\mathrm{O}=\mathrm{C} 1 \mathrm{C}(\mathrm{CCN} 1 \mathrm{C} 1 \mathrm{CCC} 1) \mathrm{n} 1 \mathrm{nnn} n 1 \mathrm{~N} 1 \mathrm{CCCC}(\mathrm{CC} \#$ & 0 \\
\hline
\end{tabular}


C) $\mathrm{C} 1$

\begin{tabular}{|c|c|c|c|c|c|}
\hline 796 & $\mathbf{1 5}\{358\}$ & $\mathbf{1 1}\{350\}$ & $\mathbf{9}\{358,350\}$ & $\mathrm{CN}(\mathrm{CCC}=\mathrm{C}) \mathrm{c} 1 \mathrm{nnnn} 1 \mathrm{C} 1 \mathrm{CCN}(\mathrm{C} 2 \mathrm{CCC} 2) \mathrm{C} 1=\mathrm{O}$ & 0 \\
\hline 797 & $\mathbf{1 5}\{359\}$ & $\mathbf{1 1}\{505\}$ & $\mathbf{9}\{359,505\}$ & $\begin{array}{l}\mathrm{CC} 1 \mathrm{CC} 2(\mathrm{CCC}(\mathrm{Cn} 3 n n n c 3 \mathrm{~N} 3 \mathrm{CC}(\mathrm{C}) \mathrm{CC}(\mathrm{C}) \mathrm{C} 3) \mathrm{O} 2) \\
\mathrm{CO} 1\end{array}$ & 34 \\
\hline 798 & $\mathbf{1 5}\{359\}$ & $\mathbf{1 1}\{465\}$ & $\mathbf{9}\{359,465\}$ & $\begin{array}{l}\mathrm{CC} 1 \mathrm{CC} 2(\mathrm{CCC}(\mathrm{Cn} 3 n n n c 3 \mathrm{~N} 3 \mathrm{CCC}(\mathrm{C}) \mathrm{C}(\mathrm{C}) \mathrm{C} 3) \mathrm{O} 2) \\
\mathrm{CO} 1\end{array}$ & 22 \\
\hline 799 & $\mathbf{1 5}\{359\}$ & $\mathbf{1 1}\{501\}$ & $\mathbf{9}\{359,501\}$ & $\begin{array}{l}\mathrm{CCN}(\mathrm{CC}(\mathrm{C})=\mathrm{C}) \mathrm{c} 1 \mathrm{nnnn} 1 \mathrm{CC} 1 \mathrm{CCC} 2(\mathrm{COC}(\mathrm{C}) \mathrm{C} 2) \\
\mathrm{O} 1\end{array}$ & 21 \\
\hline 800 & $\mathbf{1 5}\{359\}$ & $\mathbf{1 1}\{340\}$ & $\mathbf{9}\{359,340\}$ & CC1CC2(CCC(Cn3nnnc3N3CCCCCC3)O2)CO1 & 73 \\
\hline 801 & $\mathbf{1 5}\{359\}$ & $\mathbf{1 1}\{480\}$ & $\mathbf{9}\{359,480\}$ & $\begin{array}{l}\mathrm{CC} 1 \mathrm{CC} 2(\mathrm{CCC}(\mathrm{Cn} 3 \mathrm{nnn} \mathrm{n} 3 \mathrm{~N} 3 \mathrm{CCC}(\mathrm{C}) \mathrm{CC} 3) \mathrm{O} 2) \mathrm{C} \\
\mathrm{O} 1\end{array}$ & 17 \\
\hline 802 & $\mathbf{1 5}\{359\}$ & $\mathbf{1 1}\{479\}$ & $\mathbf{9}\{359,479\}$ & $\begin{array}{l}\mathrm{CC} 1 \mathrm{CC} 2(\mathrm{CCC}(\mathrm{Cn} 3 \mathrm{nnn} \mathrm{n} 3 \mathrm{~N} 3 \mathrm{CCCC}(\mathrm{C}) \mathrm{C} 3) \mathrm{O} 2) \mathrm{C} \\
\mathrm{O} 1\end{array}$ & 26 \\
\hline 803 & $\mathbf{1 5}\{360\}$ & $\mathbf{1 1}\{506\}$ & $\mathbf{9}\{360,506\}$ & $\mathrm{CN}(\mathrm{CC}=\mathrm{C}) \mathrm{c} 1 \mathrm{nnnn} 1 \mathrm{CC} 1(\mathrm{C}) \mathrm{CCOC} 2(\mathrm{CCC} 2) \mathrm{C} 1$ & 27 \\
\hline 804 & $\mathbf{1 5}\{360\}$ & $\mathbf{1 1}\{473\}$ & $\mathbf{9}\{360,473\}$ & $\begin{array}{l}\mathrm{CCO}[\mathrm{C} @ \mathrm{H}] 1 \mathrm{CCN}(\mathrm{C} 1) \mathrm{c} 1 \mathrm{nnnn} 1 \mathrm{CC} 1(\mathrm{C}) \mathrm{CCOC} 2( \\
\mathrm{CCC} 2) \mathrm{C} 1\end{array}$ & 56 \\
\hline 805 & $\mathbf{1 5}\{361\}$ & $\mathbf{1 1}\{350\}$ & $\mathbf{9}\{361,350\}$ & $\begin{array}{l}\mathrm{CN}(\mathrm{CCC}=\mathrm{C}) \mathrm{c} 1 \mathrm{nnnn} 1 \mathrm{C}[1 \mathrm{C} @ @ \mathrm{H}] 1 \mathrm{CCO}[1 \mathrm{C} @ \mathrm{H}] \\
1 \mathrm{c} 1 \mathrm{ccccc} 1\end{array}$ & 22 \\
\hline 806 & $\mathbf{1 5}\{362\}$ & $\mathbf{1 1}\{501\}$ & $\mathbf{9}\{362,501\}$ & $\begin{array}{l}\mathrm{CCN}(\mathrm{CC}(\mathrm{C})=\mathrm{C}) \mathrm{c} 1 \mathrm{nnnn} 1 \mathrm{CC} 12 \mathrm{COCCN} 1 \mathrm{C}(=\mathrm{O}) \mathrm{C} \\
\mathrm{OC} 2\end{array}$ & 9 \\
\hline 807 & $\mathbf{1 5}\{363\}$ & $\mathbf{1 1}\{501\}$ & $\mathbf{9}\{363,501\}$ & ${ }_{2} \mathrm{CCN}(\mathrm{CC}(\mathrm{C})=\mathrm{C}) \mathrm{c} 1 \mathrm{nnnnn} 1 \mathrm{CC} 1 \mathrm{CC} 2(\mathrm{CO} 1) \mathrm{CCOCC}$ & 16 \\
\hline 808 & $\mathbf{1 5}\{364\}$ & $\mathbf{1 1}\{501\}$ & $\mathbf{9}\{364,501\}$ & $\begin{array}{l}\mathrm{CCN}(\mathrm{CC}(\mathrm{C})=\mathrm{C}) \mathrm{c} 1 \mathrm{nnnn} 1 \mathrm{CC} 1 \mathrm{CC} 2 \mathrm{CCC}(\mathrm{C} 1) \mathrm{S} 2(= \\
\mathrm{O})=\mathrm{O}\end{array}$ & 43 \\
\hline 809 & $\mathbf{1 5}\{365\}$ & $\mathbf{1 1}\{501\}$ & $\mathbf{9}\{365,501\}$ & $\begin{array}{l}\mathrm{CCN}(\mathrm{CC}(\mathrm{C})=\mathrm{C}) \mathrm{c} 1 \mathrm{nnnnn} 1 \mathrm{CC} 1 \mathrm{CCC} 2(\mathrm{CCOCC} 2) \mathrm{C} \\
\mathrm{O} 1\end{array}$ & 24 \\
\hline 810 & $\mathbf{1 5}\{366\}$ & $\mathbf{1 1}\{501\}$ & $\mathbf{9}\{366,501\}$ & $\begin{array}{l}\mathrm{CCN}(\mathrm{CC}(\mathrm{C})=\mathrm{C}) \mathrm{c} 1 \mathrm{nnnn} 1 \mathrm{CCC} 1 \mathrm{CCC} 2(\mathrm{CCOCC} 2) \\
\mathrm{CO} 1\end{array}$ & 37 \\
\hline 811 & $\mathbf{1 5}\{367\}$ & $\mathbf{1 1}\{288\}$ & $\mathbf{9}\{367,288\}$ & $\begin{array}{l}\mathrm{CC}(=\mathrm{O}) \mathrm{N} 1 \mathrm{CCCN}(\mathrm{CC} 1) \mathrm{c} 1 \mathrm{nnnn} 1 \mathrm{CCC} 1 \mathrm{CCCC} 1(\mathrm{~F}) \\
\mathrm{F}\end{array}$ & 0 \\
\hline 812 & $\mathbf{1 5}\{368\}$ & $\mathbf{1 1}\{504\}$ & $\mathbf{9}\{368,504\}$ & $\begin{array}{l}\mathrm{O}=\mathrm{C} 1 \mathrm{CN}(\mathrm{CCN} 1 \mathrm{C} 1 \mathrm{CC} 1) \mathrm{c} 1 \mathrm{nnnn} 1 \mathrm{CC} 1 \mathrm{CCC} 2(\mathrm{CC} \\
\mathrm{C} 2) \mathrm{CO} 1\end{array}$ & 0 \\
\hline
\end{tabular}




\begin{tabular}{|c|c|c|c|c|c|c|}
\hline 813 & $15\{369\}$ & $11\{349\}$ & $\mathbf{9}\{369,349\}$ & $\mathrm{NC}(=\mathrm{O}) \mathrm{CC} 1 \mathrm{CC}(\mathrm{C} 1) \mathrm{n} 1 \mathrm{nnnc} 1 \mathrm{~N} 1 \mathrm{CCCC}(\mathrm{CC} \mathrm{C}) \mathrm{C} 1$ & 26 & 13 \\
\hline 814 & $15\{369\}$ & $11\{327\}$ & $\mathbf{9}\{369,327\}$ & $\mathrm{NC}(=\mathrm{O}) \mathrm{CC} 1 \mathrm{CC}(\mathrm{C} 1) \mathrm{n} 1 \mathrm{nnnn} 1 \mathrm{~N} 1 \mathrm{CCCC} 1 \mathrm{c} 1 \mathrm{ccsc} 1$ & 38 & 20 \\
\hline 815 & $15\{370\}$ & $11\{504\}$ & $\mathbf{9}\{370,504\}$ & $\begin{array}{l}\mathrm{O}=\mathrm{C} 1 \mathrm{CN}(\mathrm{CCN} 1 \mathrm{C} 1 \mathrm{CC} 1) \mathrm{c} 1 \mathrm{nnnn} 1 \mathrm{CC} 1 \mathrm{CC} 2 \mathrm{CCC}( \\
\mathrm{C} 1) \mathrm{S} 2\end{array}$ & 0 & 0 \\
\hline 816 & $15\{370\}$ & $11\{507\}$ & $\mathbf{9}\{370,507\}$ & ${ }_{) \mathrm{F}}^{\mathrm{OC} 1(\mathrm{CCN}(\mathrm{C} 1) \mathrm{c} 1 \mathrm{nnnn} 1 \mathrm{CC} 1 \mathrm{CC} 2 \mathrm{CCC}(\mathrm{C} 1) \mathrm{S} 2) \mathrm{C}(\mathrm{F}}$ & 24 & 11 \\
\hline 817 & $15\{371\}$ & $11\{350\}$ & $\mathbf{9}\{371,350\}$ & $\operatorname{COC} 1(\mathrm{Cn} 2 \mathrm{nnnn} 2 \mathrm{~N}(\mathrm{C}) \mathrm{CCC}=\mathrm{C}) \mathrm{CCS}(=\mathrm{O})(=\mathrm{O}) \mathrm{C} 1$ & 9 & 4 \\
\hline 818 & $15\{372\}$ & $11\{325\}$ & $\mathbf{9}\{372,325\}$ & $\begin{array}{l}\mathrm{O}=\mathrm{c} 1[\mathrm{nH}] \mathrm{ncn} 1 \mathrm{C} 1 \mathrm{CCN}(\mathrm{CC} 1) \mathrm{c} 1 \mathrm{nnnn} 1 \mathrm{CC} 12 \mathrm{CC} 1 \mathrm{C} \\
\mathrm{CCC} 2\end{array}$ & 32 & 15 \\
\hline 819 & $\mathbf{1 5}\{373\}$ & $11\{349\}$ & $\mathbf{9}\{373,349\}$ & $\begin{array}{l}\mathrm{FC}(\mathrm{F})(\mathrm{F}) \mathrm{C} 1 \mathrm{CCC}(\mathrm{Cn} 2 \mathrm{nnnc} 2 \mathrm{~N} 2 \mathrm{CCCC}(\mathrm{CC} \# \mathrm{C}) \mathrm{C} 2) \\
\mathrm{O} 2\end{array}$ & 5 & 2 \\
\hline 820 & $15\{373\}$ & $11\{350\}$ & $\mathbf{9}\{373,350\}$ & $\mathrm{CN}(\mathrm{CCC}=\mathrm{C}) \mathrm{c} 1 \mathrm{nnnn} 1 \mathrm{CC} 1 \mathrm{CCC}(\mathrm{O} 1) \mathrm{C}(\mathrm{F})(\mathrm{F}) \mathrm{F}$ & 15 & 7 \\
\hline 821 & $15\{374\}$ & $11\{508\}$ & $\mathbf{9}\{374,508\}$ & $\begin{array}{l}\mathrm{FC1}(\mathrm{F})[1 \mathrm{C} @ @ \mathrm{H}](\mathrm{Cn} 2 \mathrm{nnnc} 2 \mathrm{~N} 2 \mathrm{CCC} 3(\mathrm{COC} 3) \mathrm{C} 2 \\
)[1 \mathrm{C} @ @ \mathrm{H}] 1 \mathrm{c} 1 \mathrm{ccccc} 1\end{array}$ & 0 & 0 \\
\hline 822 & $15\{374\}$ & $11\{509\}$ & $\mathbf{9}\{374,509\}$ & $\begin{array}{l}\mathrm{FC1}(\mathrm{F})[1 \mathrm{C} @ @ \mathrm{H}](\mathrm{Cn} 2 \mathrm{nnnc} 2 \mathrm{~N} 2 \mathrm{C} 3 \mathrm{CCC} 2 \mathrm{CC} 3)[1 \\
\mathrm{C} @ @ \mathrm{H}] 1 \mathrm{c} 1 \mathrm{ccccc} 1\end{array}$ & 0 & 0 \\
\hline 823 & $15\{374\}$ & $11\{179\}$ & $\mathbf{9}\{374,179\}$ & $\begin{array}{l}\mathrm{OC}[\mathrm{C} @ \mathrm{H}] 1[\mathrm{C} @ @ \mathrm{H}] 2 \mathrm{CN}(\mathrm{C}[\mathrm{C} @ \mathrm{H}] 12) \mathrm{c} 1 \mathrm{nnnn} 1 \mathrm{C} \\
{[1 \mathrm{C} @ @ \mathrm{H}] 1[1 \mathrm{C} @ @ \mathrm{H}](\mathrm{c} 2 \mathrm{ccccc} 2) \mathrm{C} 1(\mathrm{~F}) \mathrm{F}}\end{array}$ & 0 & 0 \\
\hline 824 & $15\{374\}$ & $11\{510\}$ & $\mathbf{9}\{374,510\}$ & $\begin{array}{l}\mathrm{FC} 1(\mathrm{~F})[1 \mathrm{C} @ @ \mathrm{H}](\mathrm{Cn} 2 \mathrm{nnn} n 2 \mathrm{~N} 2 \mathrm{CC} 3 \mathrm{CC} 3 \mathrm{C} 2)[1 \mathrm{C} \\
@ \mathrm{H}] 1 \mathrm{c} 1 \mathrm{cccc} 1\end{array}$ & 0 & 0 \\
\hline 825 & $\mathbf{1 5}\{375\}$ & $\mathbf{1 1}\{511\}$ & $\mathbf{9}\{375,511\}$ & $\begin{array}{l}\mathrm{CN}(\mathrm{C}) \mathrm{C}(=\mathrm{O}) \mathrm{C} 1 \mathrm{CCN}(\mathrm{C} 1) \mathrm{c} 1 \mathrm{nnnnn} 1[1 \mathrm{C} @ @ \mathrm{H}] 1[1 \\
\mathrm{C} @ \mathrm{H}] 2 \mathrm{Cc} 3 \operatorname{ccccc} 3[1 \mathrm{C} @ @ \mathrm{H}] 12\end{array}$ & 71 & 36 \\
\hline 826 & $\mathbf{1 5}\{375\}$ & $\mathbf{1 1}\{504\}$ & $\mathbf{9}\{375,504\}$ & $\begin{array}{l}\mathrm{O}=\mathrm{C} 1 \mathrm{CN}(\mathrm{CCN} 1 \mathrm{C} 1 \mathrm{CC} 1) \mathrm{c} 1 \mathrm{nnnn} 1[1 \mathrm{C} @ @ \mathrm{H}] 1[1 \mathrm{C} \\
@ \mathrm{H}] 2 \mathrm{Cc} 3 \operatorname{ccccc} 3[1 \mathrm{C} @ @ \mathrm{H}] 12\end{array}$ & 0 & 0 \\
\hline 827 & $15\{376\}$ & $\mathbf{1 1}\{504\}$ & $\mathbf{9}\{376,504\}$ & $\begin{array}{l}\mathrm{O}=\mathrm{C} 1 \mathrm{CN}(\mathrm{CCN} 1 \mathrm{C} 1 \mathrm{CC} 1) \mathrm{c} 1 \mathrm{nnnn} 1 \mathrm{CC} 1 \mathrm{CCCC} 2(\mathrm{C} \\
\mathrm{CC} 2) \mathrm{O} 1\end{array}$ & 0 & 0 \\
\hline 828 & $\mathbf{1 5}\{377\}$ & $\mathbf{1 1}\{501\}$ & $\mathbf{9}\{377,501\}$ & ${ }_{1} \mathrm{CCN}(\mathrm{CC}(\mathrm{C})=\mathrm{C}) \mathrm{c} 1 \mathrm{nnnn} 1 \mathrm{CC} 1 \mathrm{CCC} 2(\mathrm{CCOCC} 2) \mathrm{O}$ & 17 & 8 \\
\hline 829 & $\mathbf{1 5}\{378\}$ & $\mathbf{1 1}\{511\}$ & $\mathbf{9}\{378,511\}$ & $\begin{array}{l}\mathrm{CN}(\mathrm{C}) \mathrm{C}(=\mathrm{O}) \mathrm{C} 1 \mathrm{CCN}(\mathrm{C} 1) \mathrm{c} 1 \mathrm{nnnn} 1 \mathrm{C}[1 \mathrm{C} @] 12 \mathrm{C}[1 \\
\mathrm{C} @ \mathrm{H}] 1 \mathrm{CCC} 2\end{array}$ & 26 & 13 \\
\hline 830 & $\mathbf{1 5}\{378\}$ & $11\{472\}$ & $\mathbf{9}\{378,472\}$ & $\begin{array}{l}\mathrm{CC}(\mathrm{C})(\mathrm{C}) \mathrm{OC}(=\mathrm{O}) \mathrm{N}[\mathrm{C} @ @ \mathrm{H}] 1 \mathrm{CCN}(\mathrm{C} 1) \mathrm{c} 1 \mathrm{nnnn} 1 \\
\mathrm{C}[1 \mathrm{C} @] 12 \mathrm{C}[1 \mathrm{C} @ \mathrm{H}] 1 \mathrm{CCC} 2\end{array}$ & 21 & 11 \\
\hline
\end{tabular}


${ }^{1} \mathrm{H}$ NMR spectrum of the compound $\mathbf{8}\{2,8\}$.
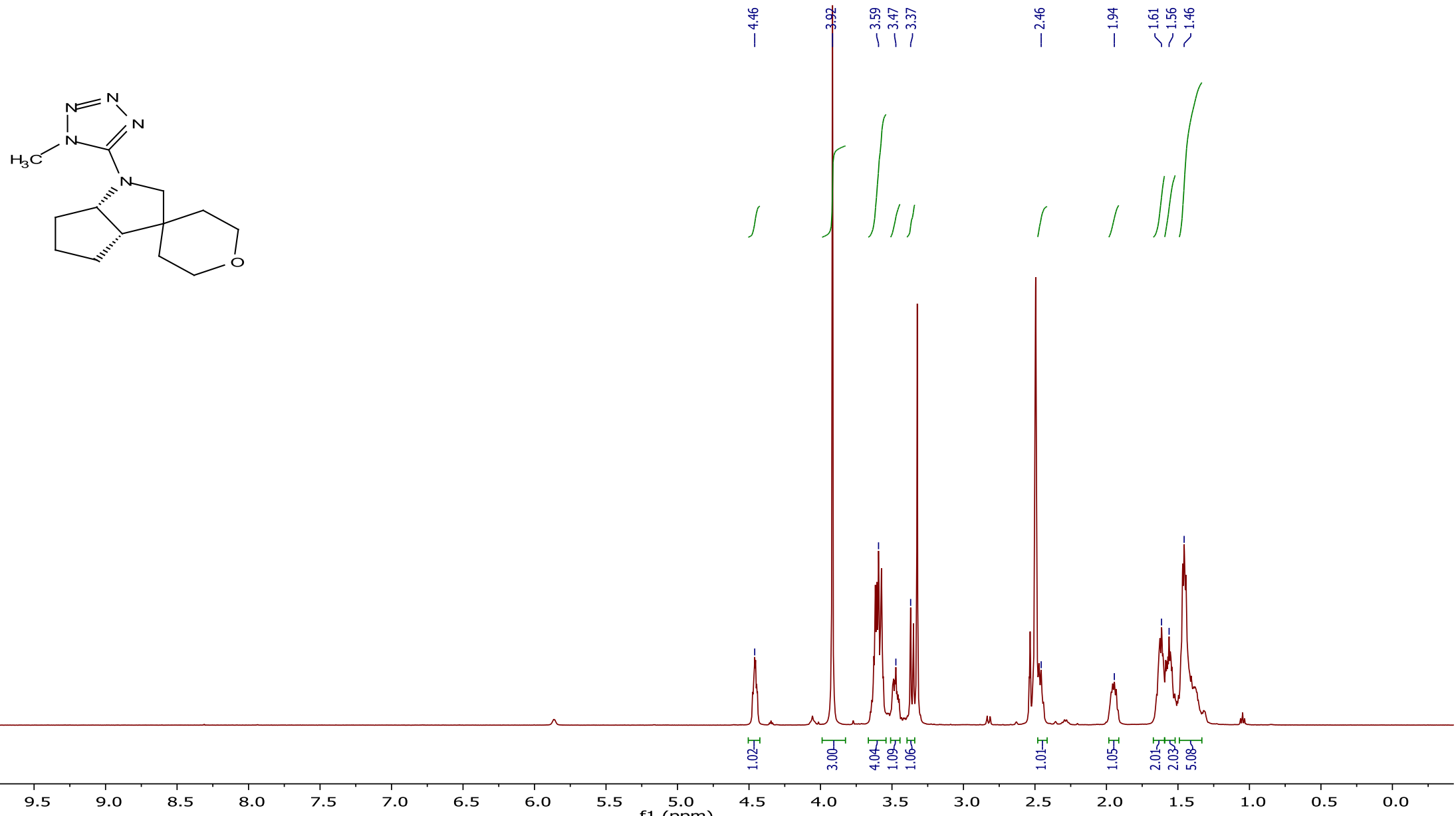
${ }^{13} \mathrm{C}$ NMR spectrum of the compound $\mathbf{8}\{2,8\}$.
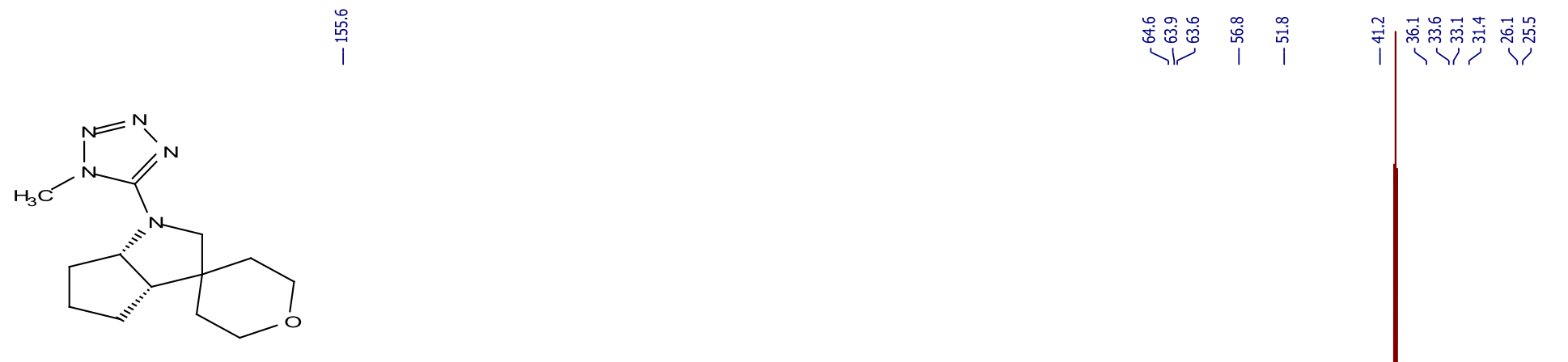
${ }^{1} \mathrm{H}$ NMR spectrum of the compound $\mathbf{8}\{10,50\}$.

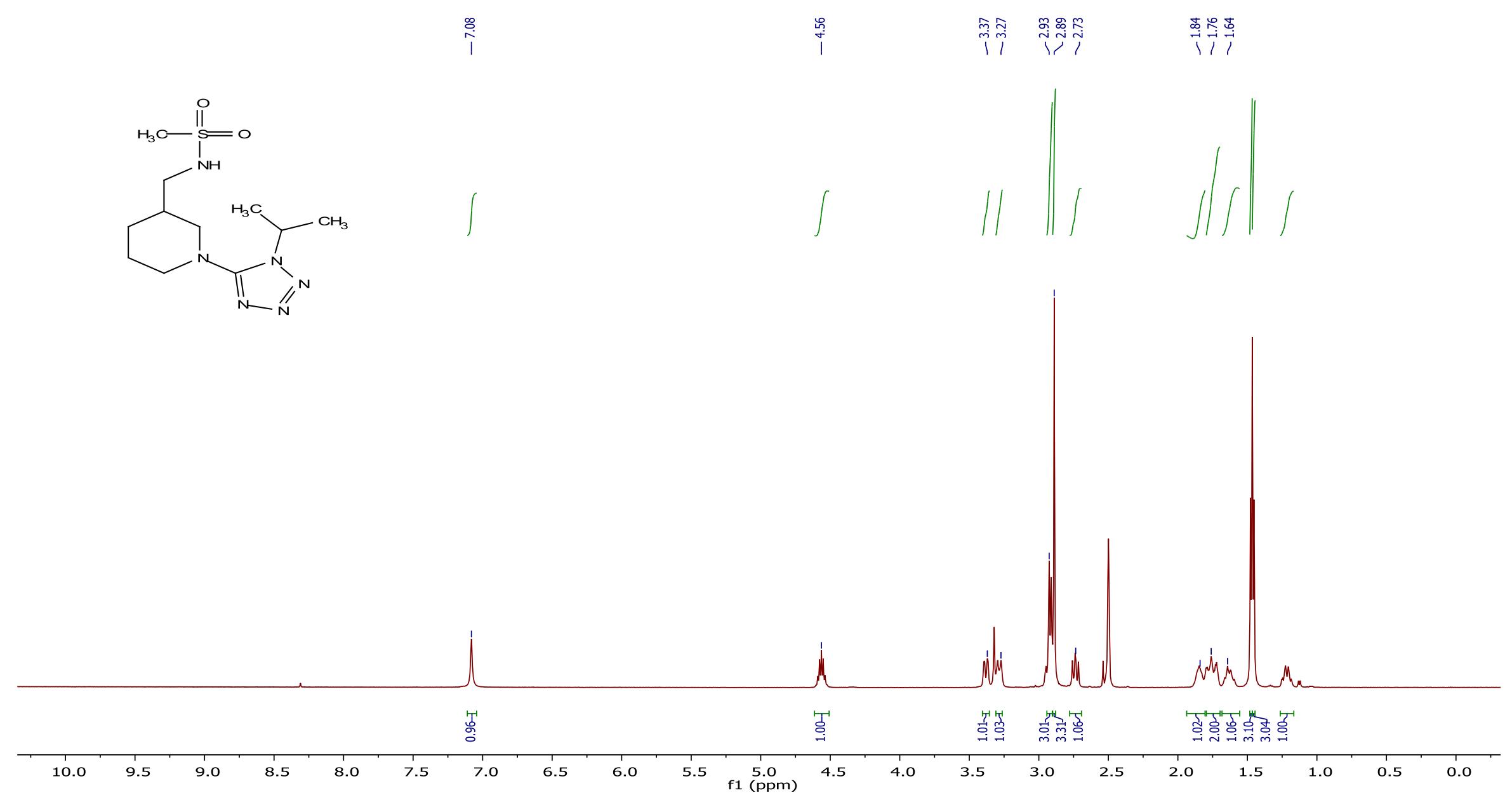


${ }^{13} \mathrm{C}$ NMR spectrum of the compound $\mathbf{8}\{10,50\}$.

$\stackrel{\text { 驾 }}{\stackrel{2}{1}}$
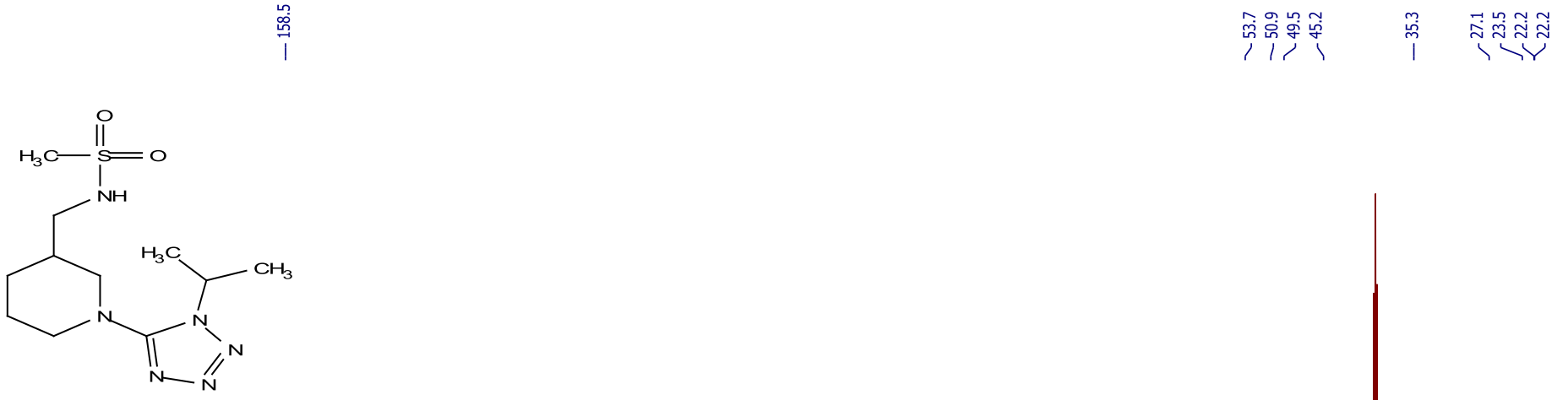

|||||||||| $\mid$ 
${ }^{1} \mathrm{H}$ NMR spectrum of the compound $\left.\mathbf{8}\{15,8)\right\}$.

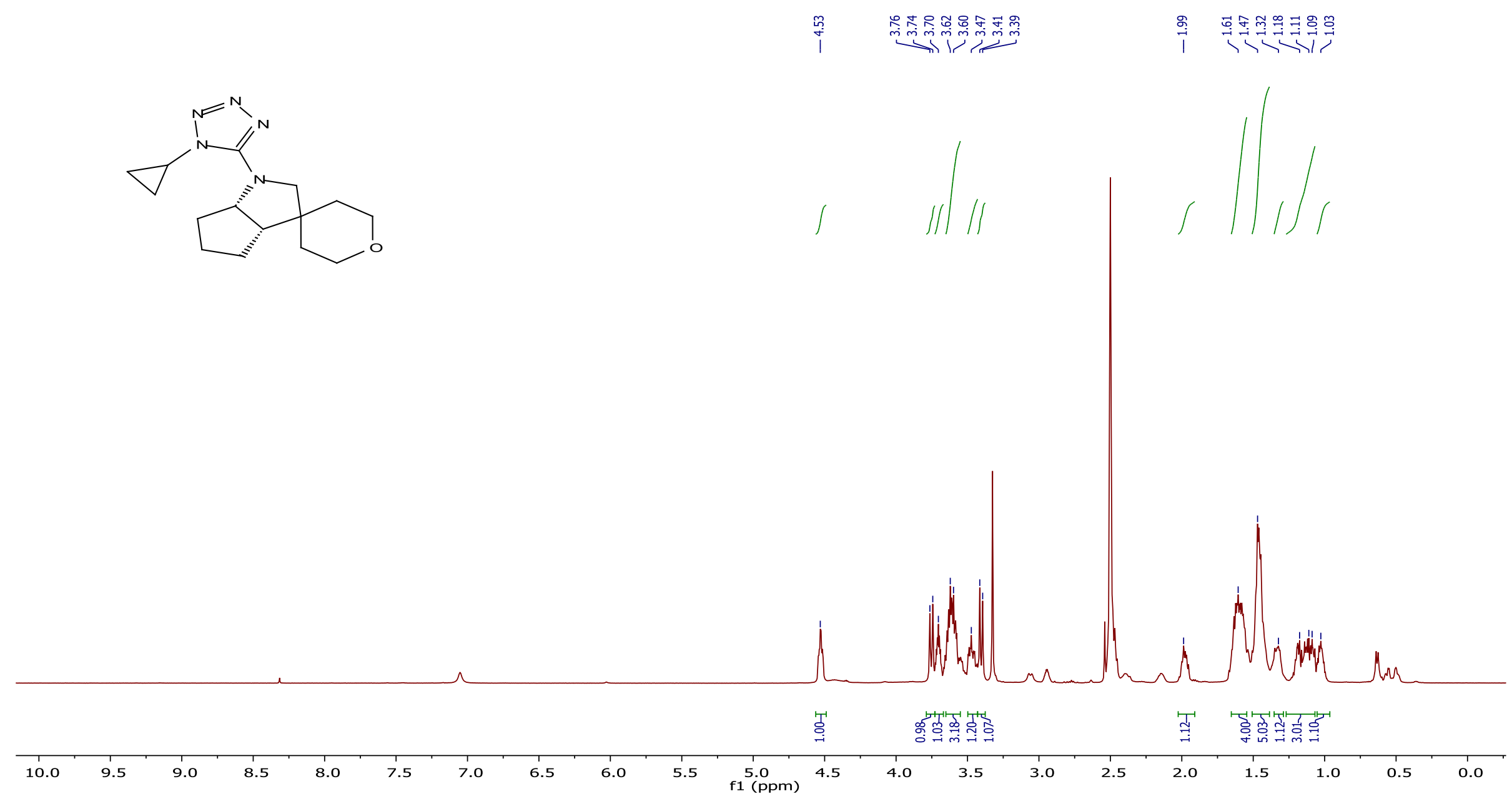


${ }^{13} \mathrm{C}$ NMR spectrum of the compound $\left.\mathbf{8}\{15,8)\right\}$.

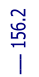
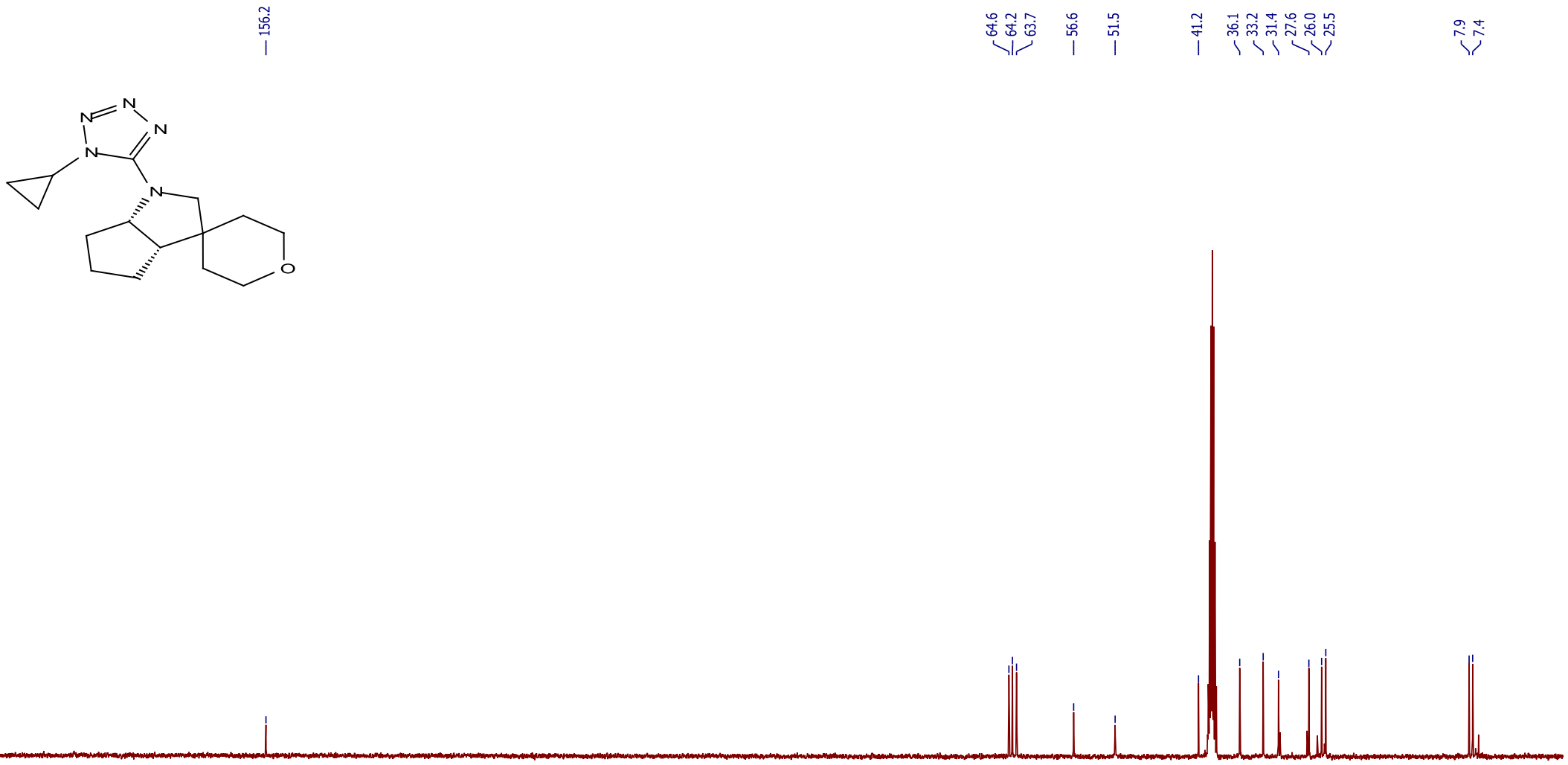

$200-190-180-170$

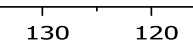

$110 \stackrel{100}{100}$

$90 \quad 80$

$70 \quad 60$

$50 \quad 40$

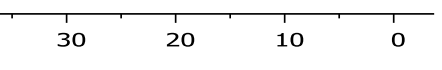


${ }^{1} \mathrm{H}$ NMR spectrum of the compound $\mathbf{8}\{15,71\}$.

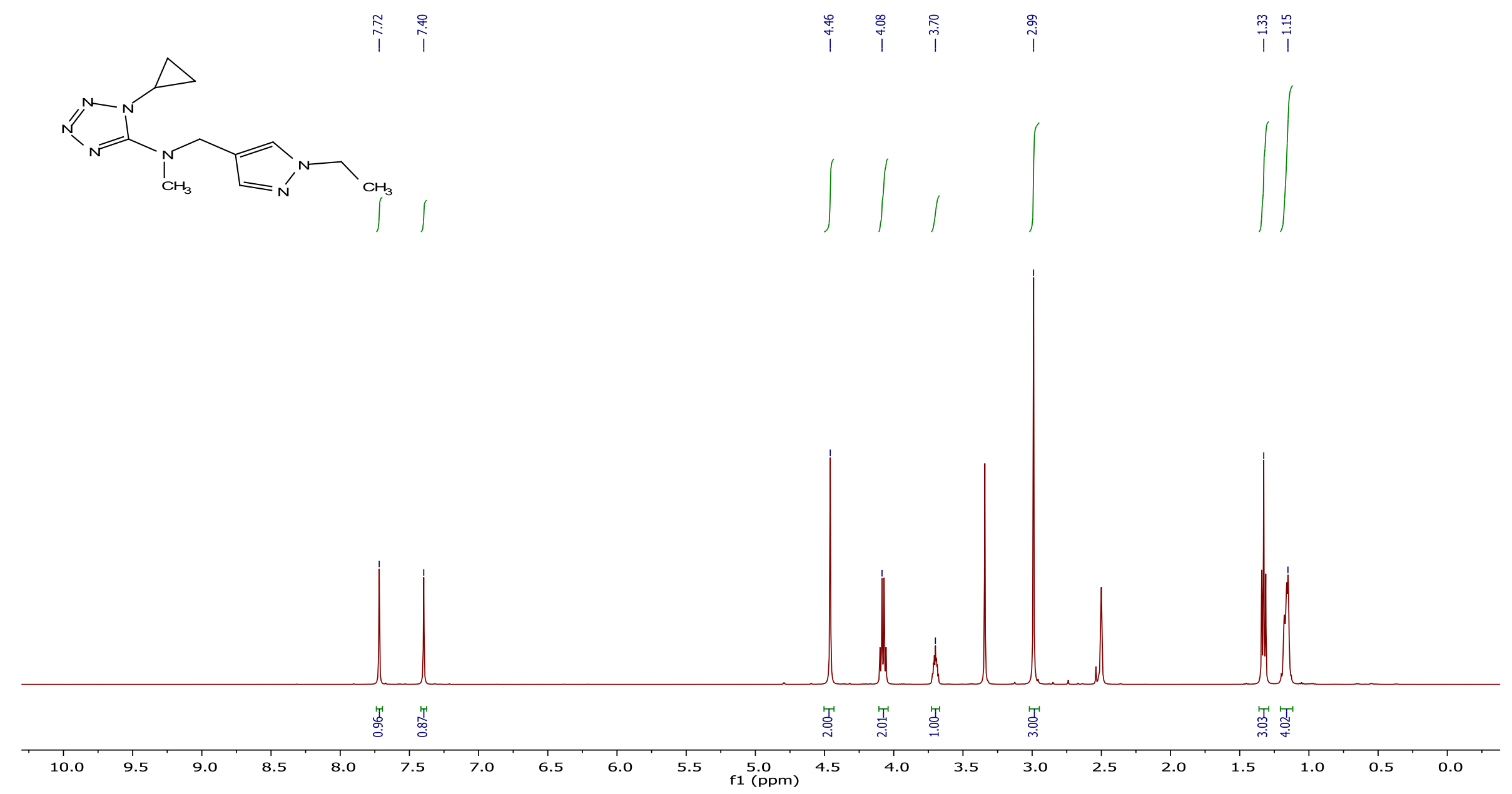


${ }^{13} \mathrm{C}$ NMR spectrum of the compound $8\{15,71\}$.
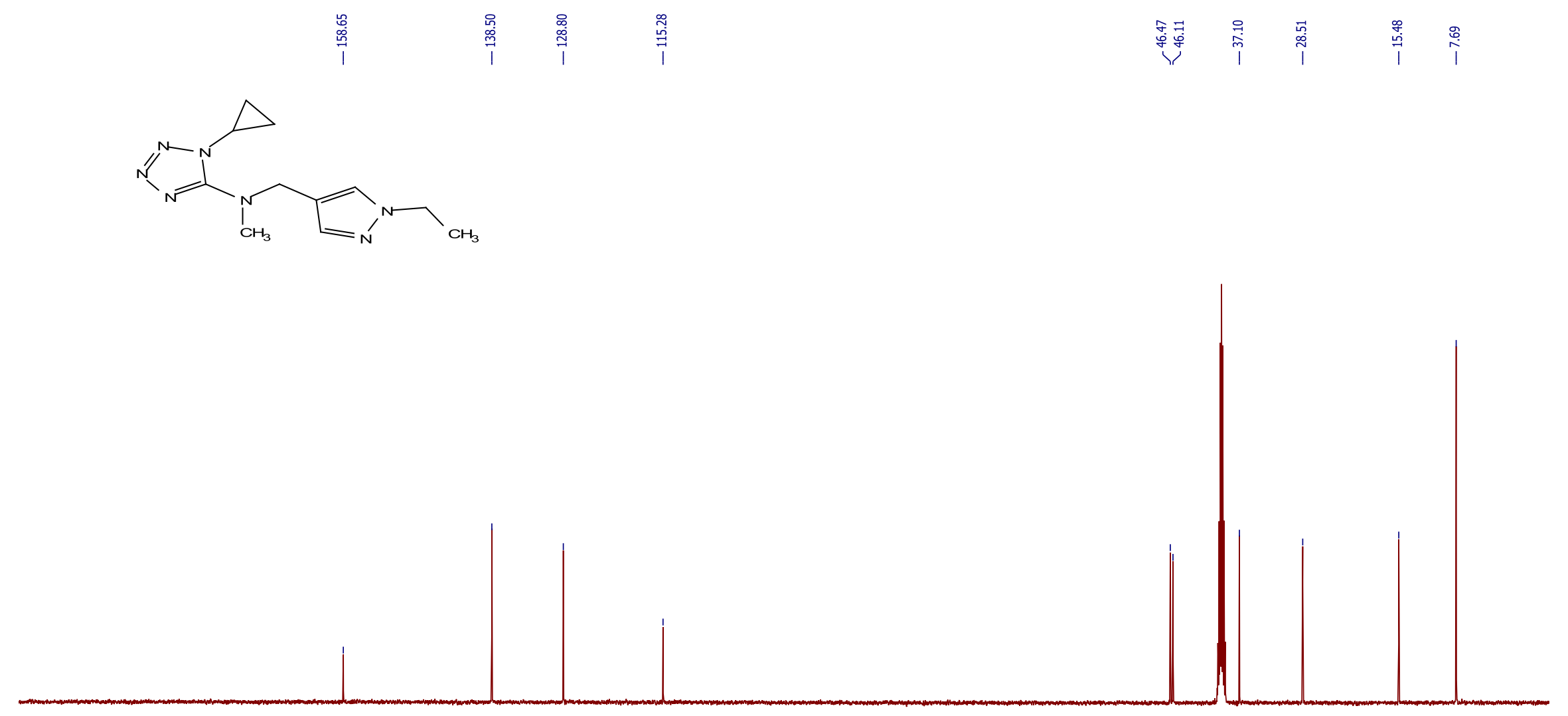

$200 \quad 190 \quad 180,170$

150

$130 \quad 120$

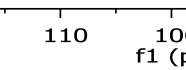

100
$f 1(\mathrm{ppm})$

$90 \quad 80$

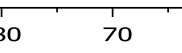

$60 \quad 50$

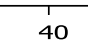

30

20

10 o 
${ }^{1} \mathrm{H}$ NMR spectrum of the compound $\left.\mathbf{8}\{15,72)\right\}$.

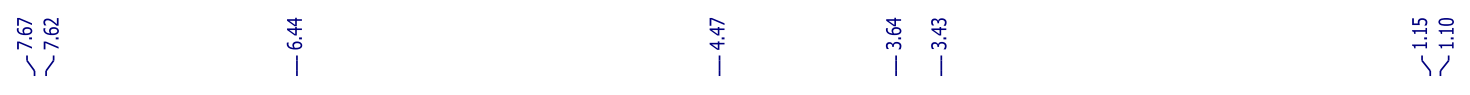
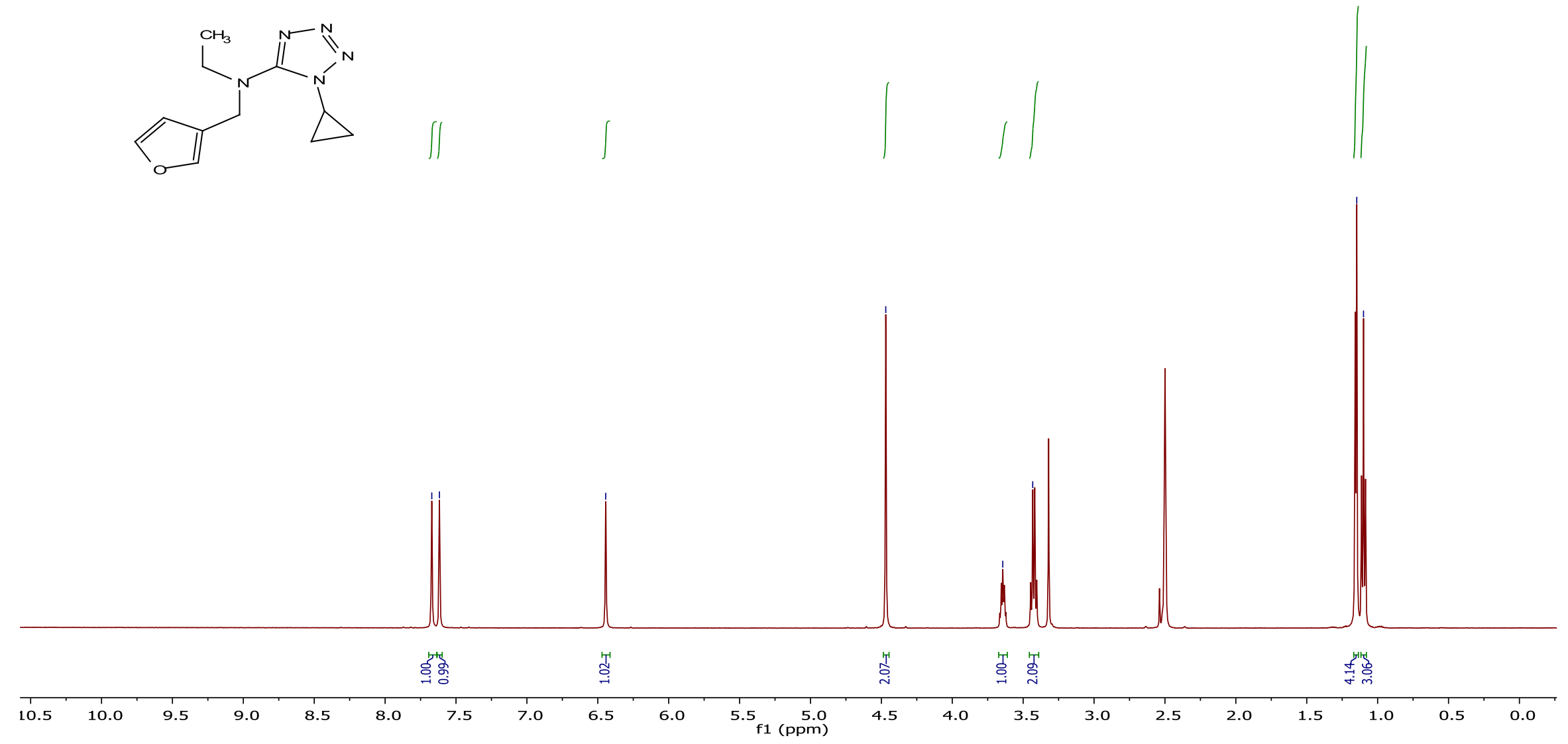
${ }^{13} \mathrm{C}$ NMR spectrum of the compound $\left.\mathbf{8}\{15,72)\right\}$.

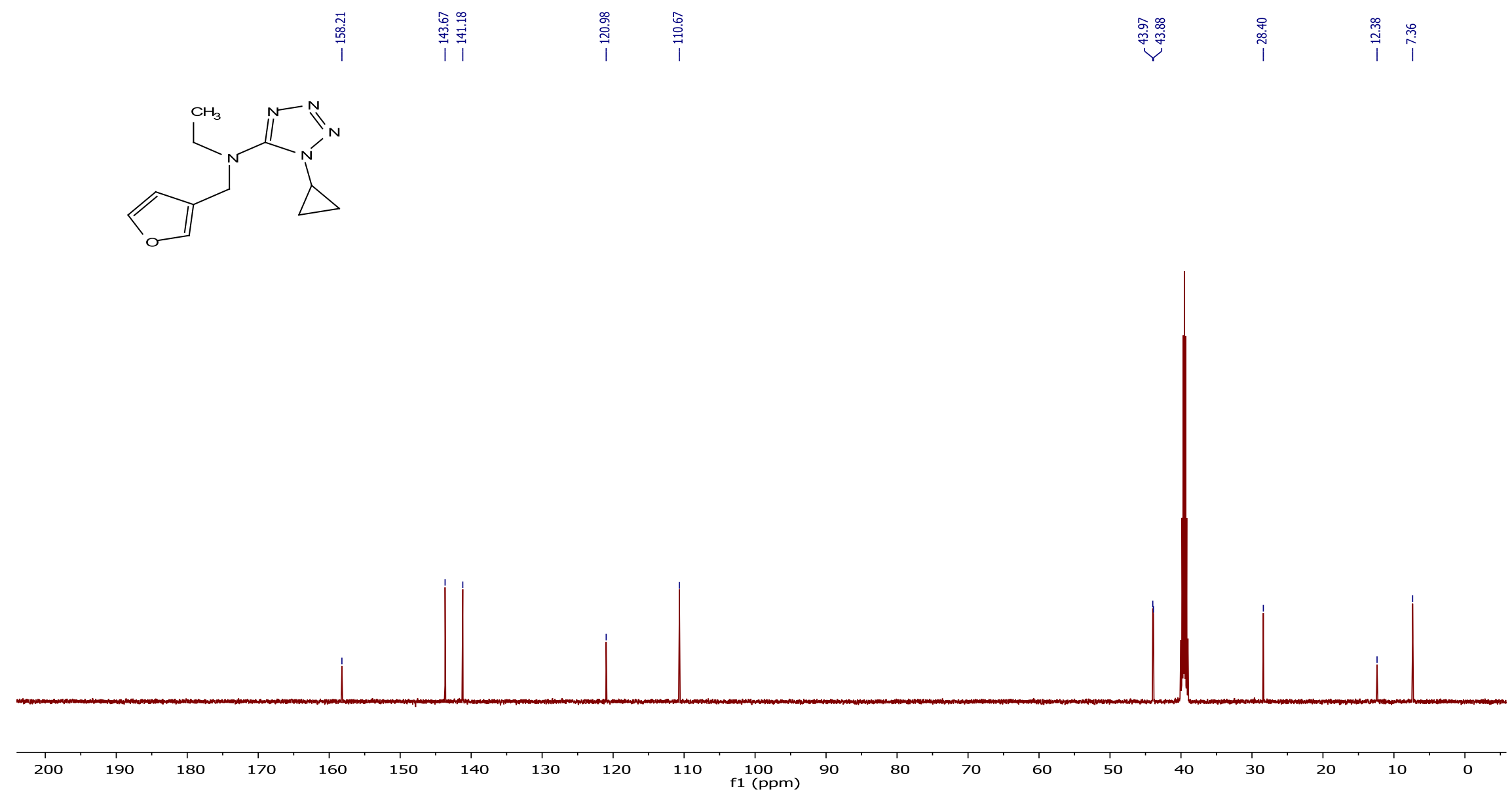


${ }^{1} \mathrm{H}$ NMR spectrum of the compound $\mathbf{8}\{36,164\}$.

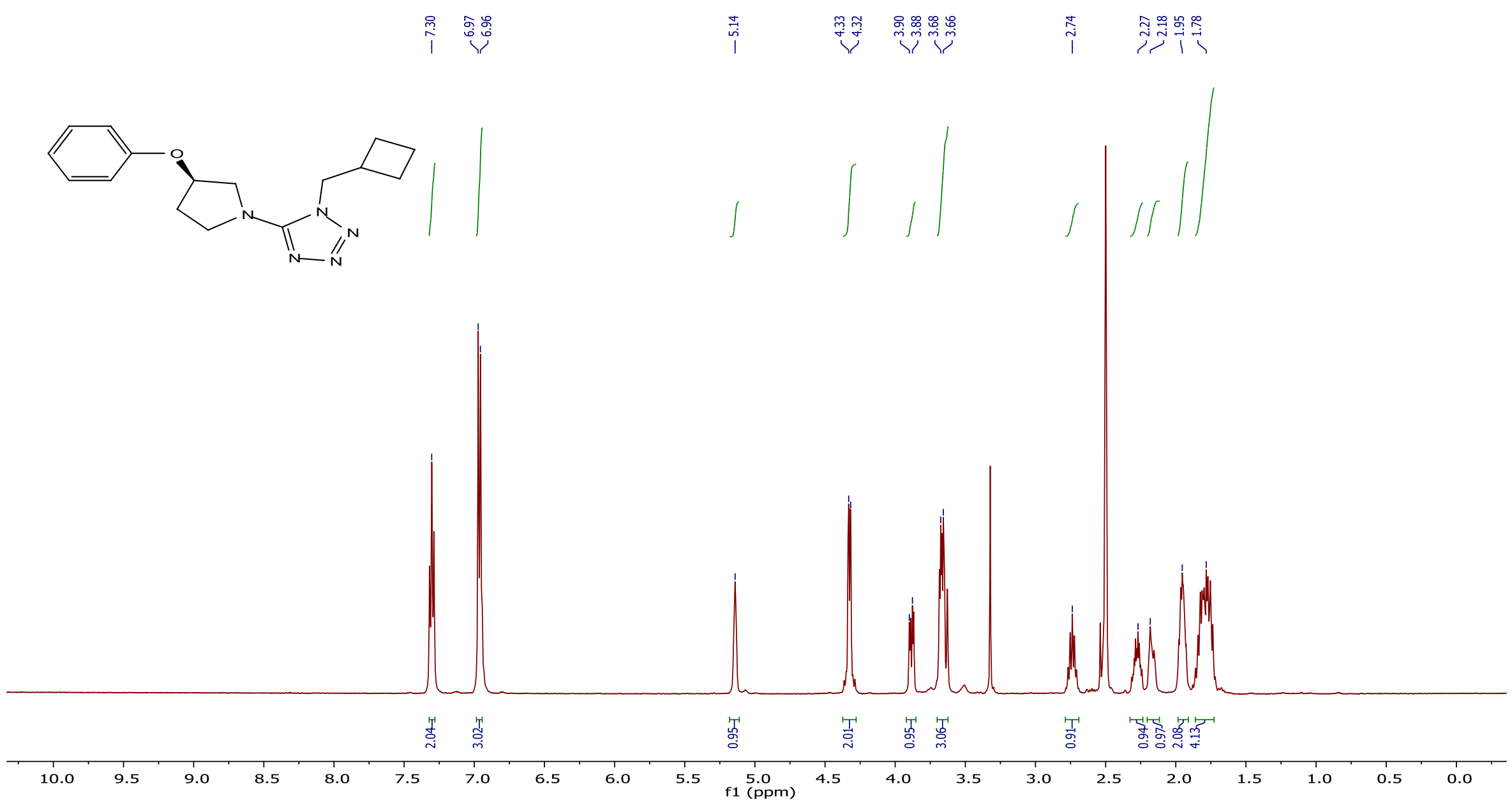


${ }^{13} \mathrm{C}$ NMR spectrum of the compound $\mathbf{8}\{36,164\}$.
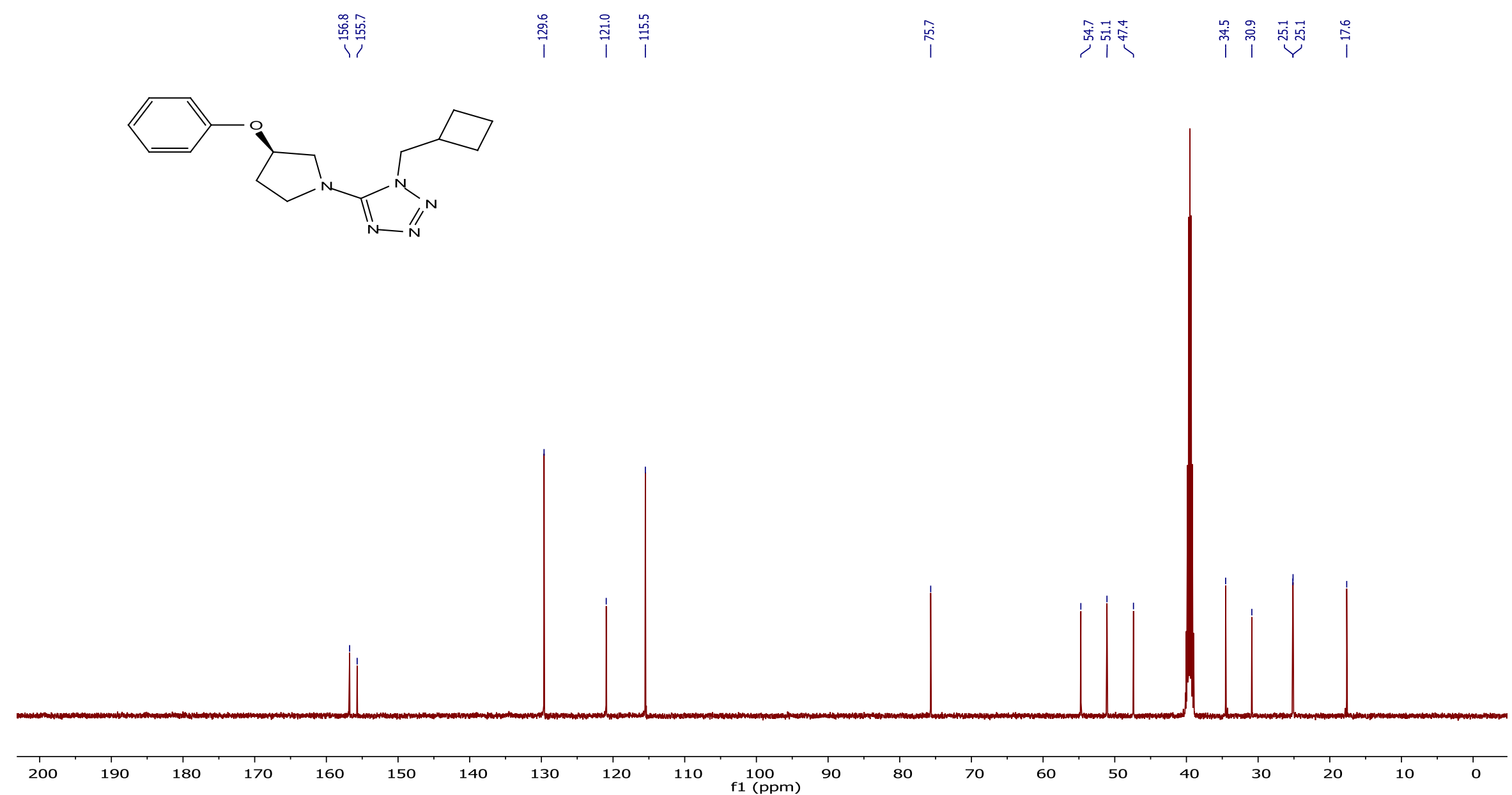
${ }^{1} \mathrm{H}$ NMR spectrum of the compound $\mathbf{8}\{40,188\}$.

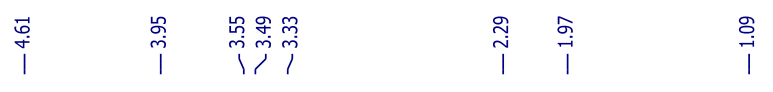
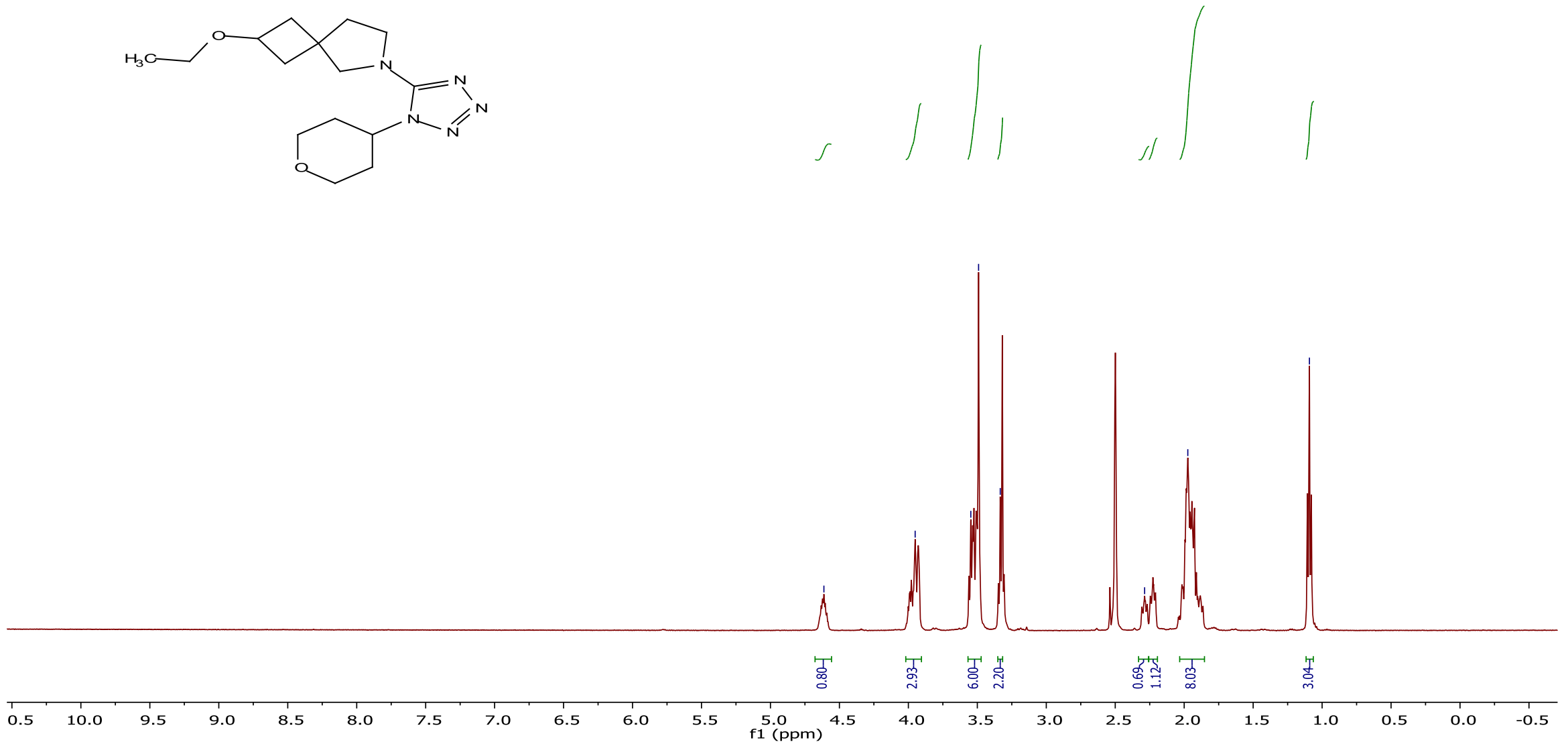
${ }^{13} \mathrm{C}$ NMR spectrum of the compound $\mathbf{8}\{40,188\}$.

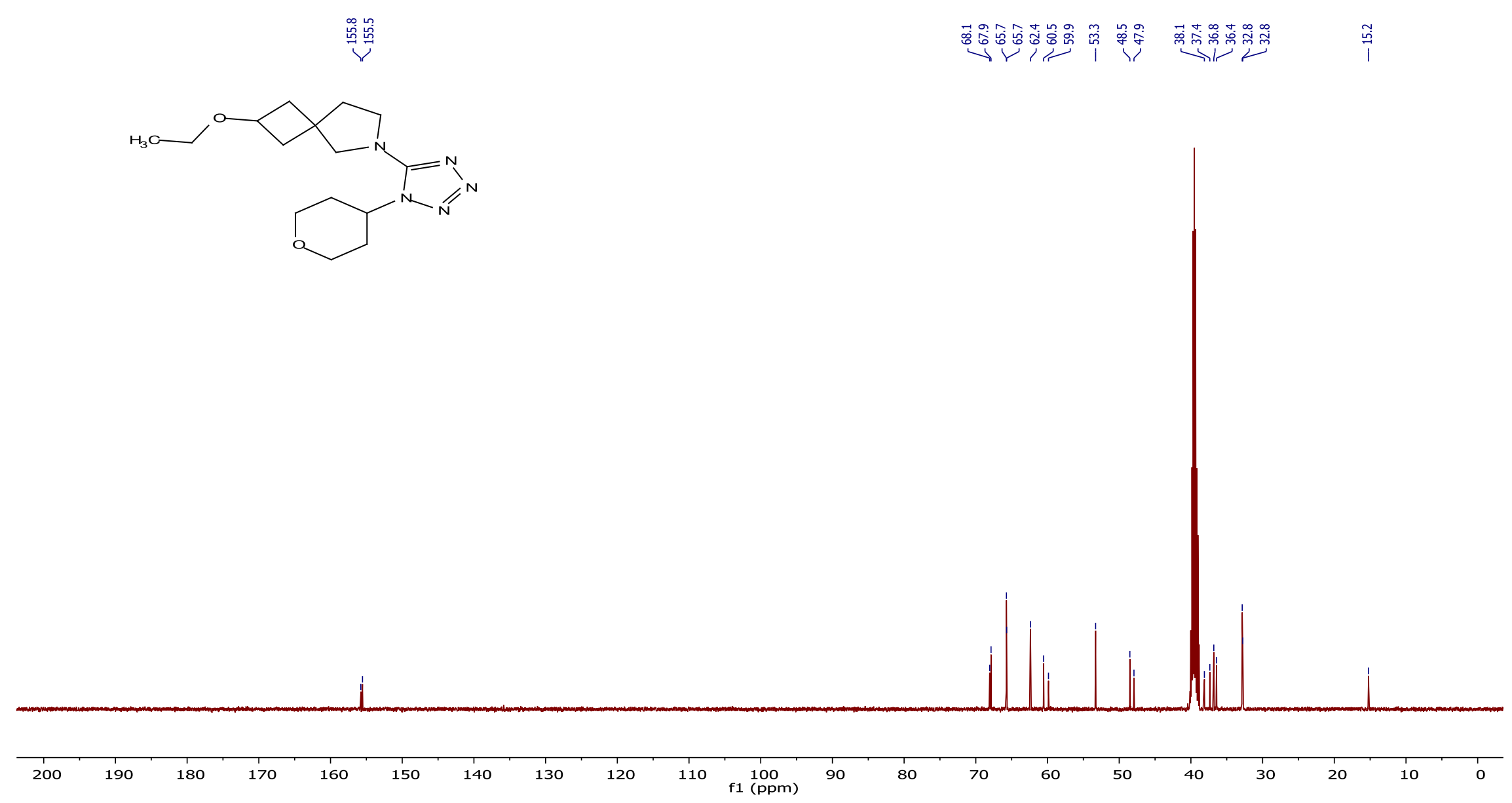


${ }^{1} \mathrm{H}$ NMR spectrum of the compound $\mathbf{8}\{40,192\}$.

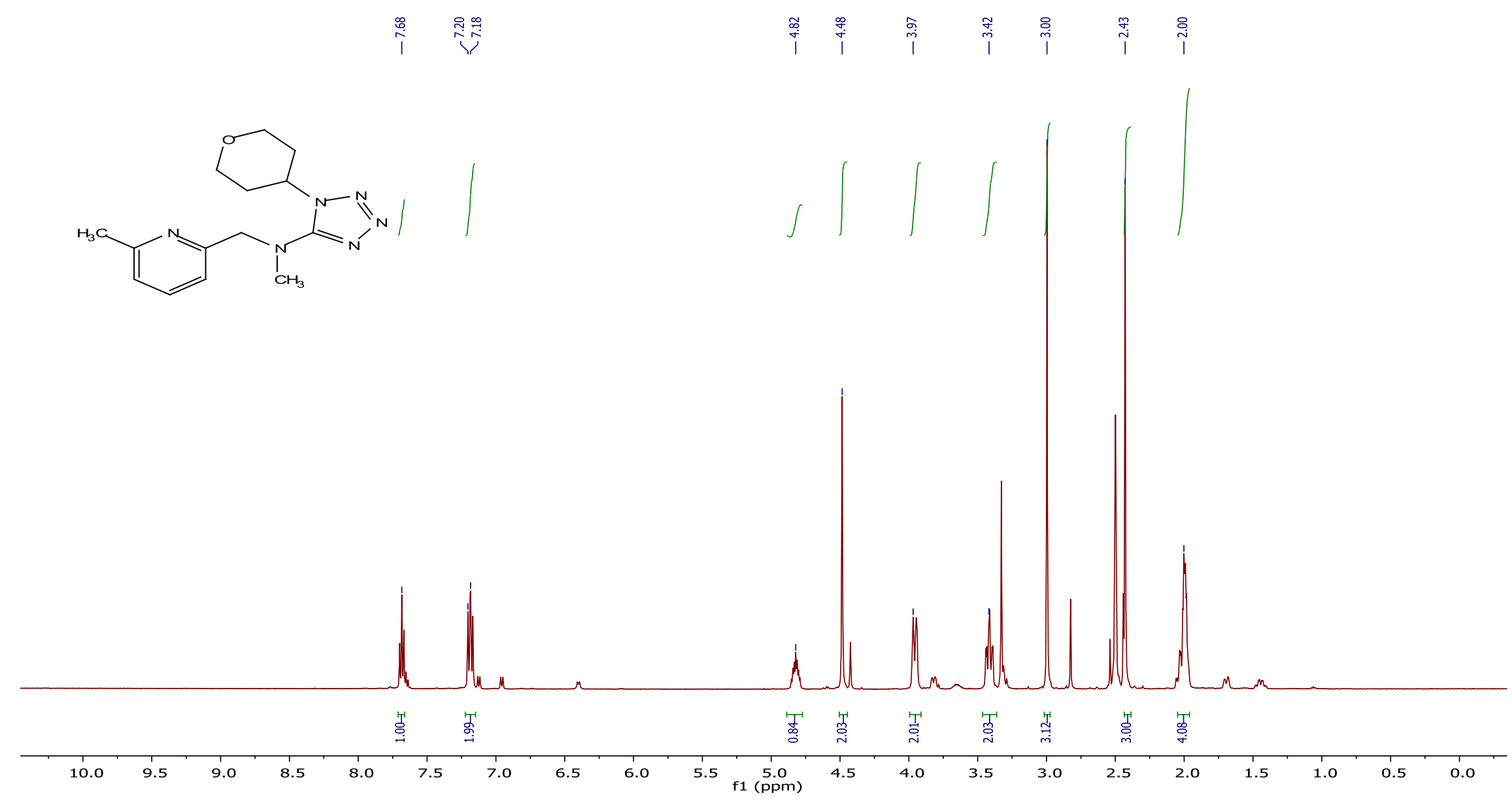


${ }^{13} \mathrm{C}$ NMR spectrum of the compound $\mathbf{8}\{40,192\}$.
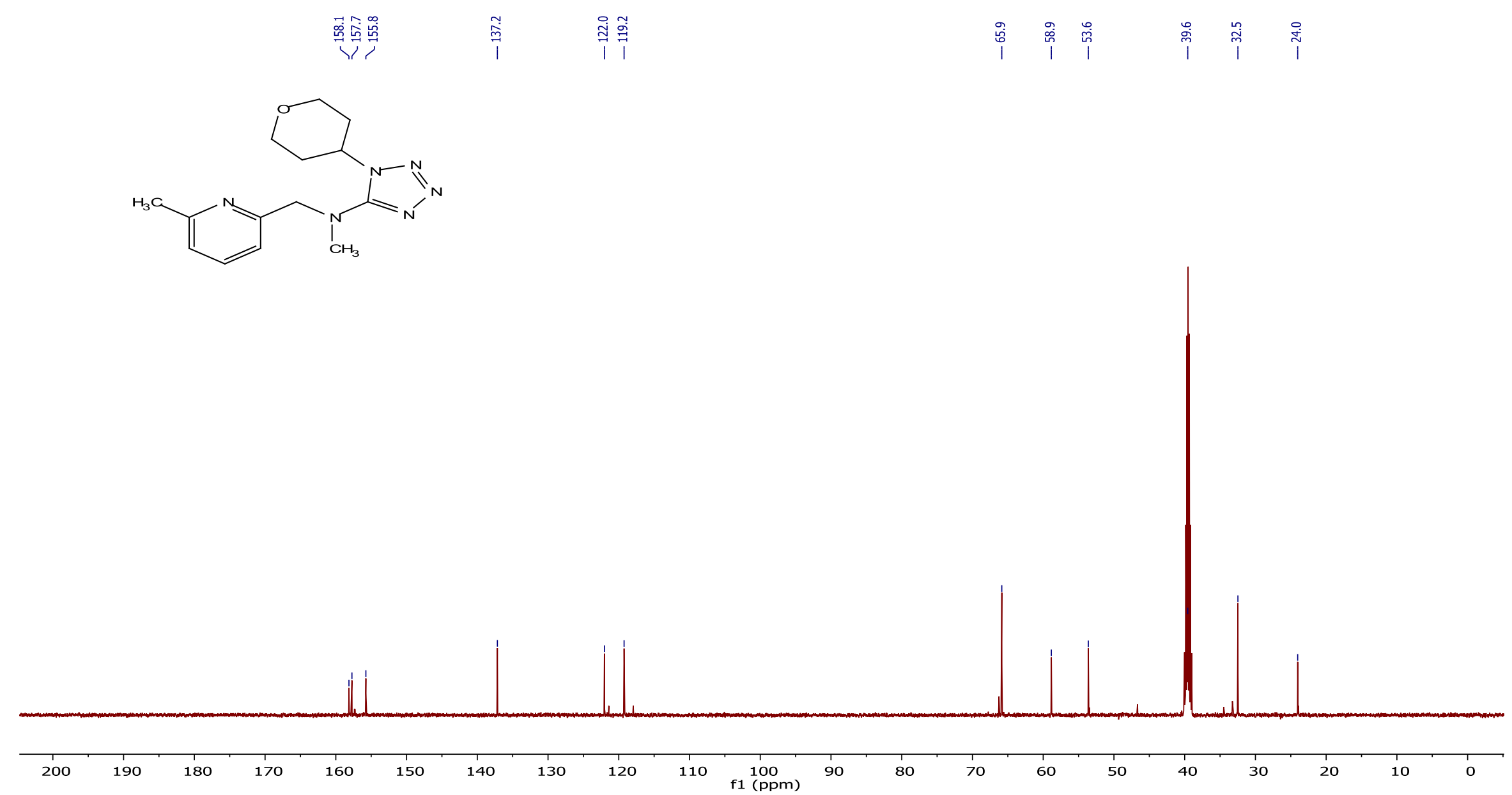
${ }^{1} \mathrm{H}$ NMR spectrum of the compound $\mathbf{8}\{41,147\}$.

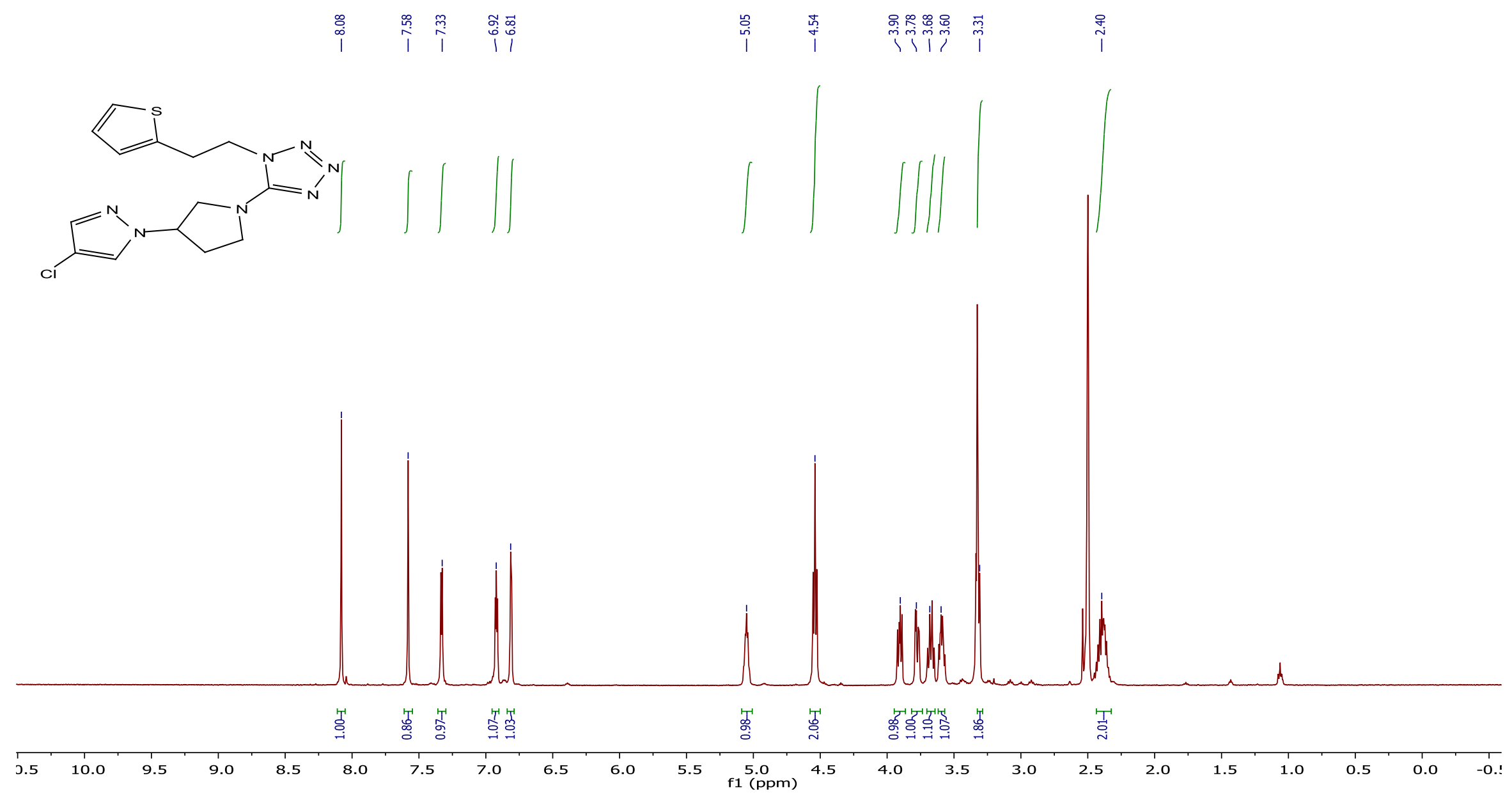


${ }^{13} \mathrm{C}$ NMR spectrum of the compound $\mathbf{8}\{41,147\}$.

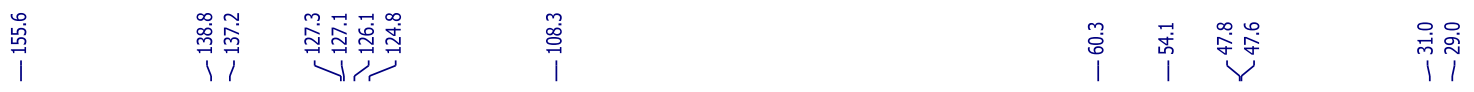

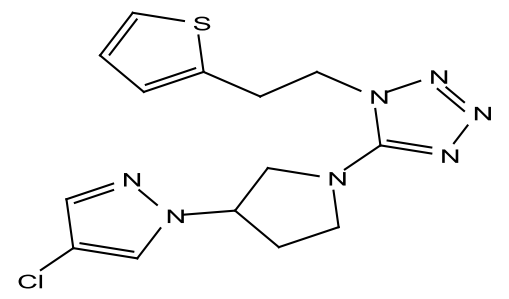


${ }^{1} \mathrm{H}$ NMR spectrum of the compound $\mathbf{8}\{41,148\}$.

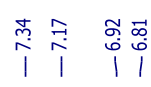

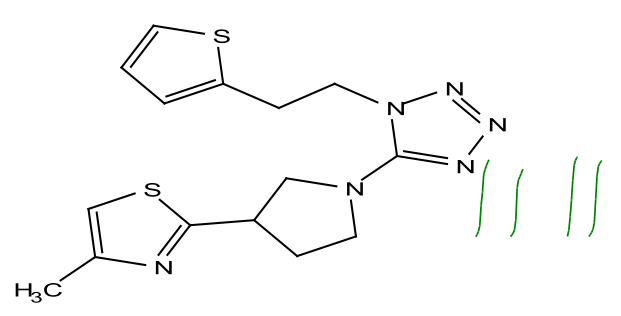

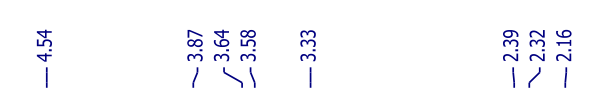

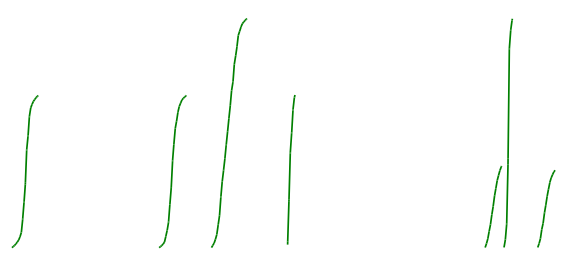

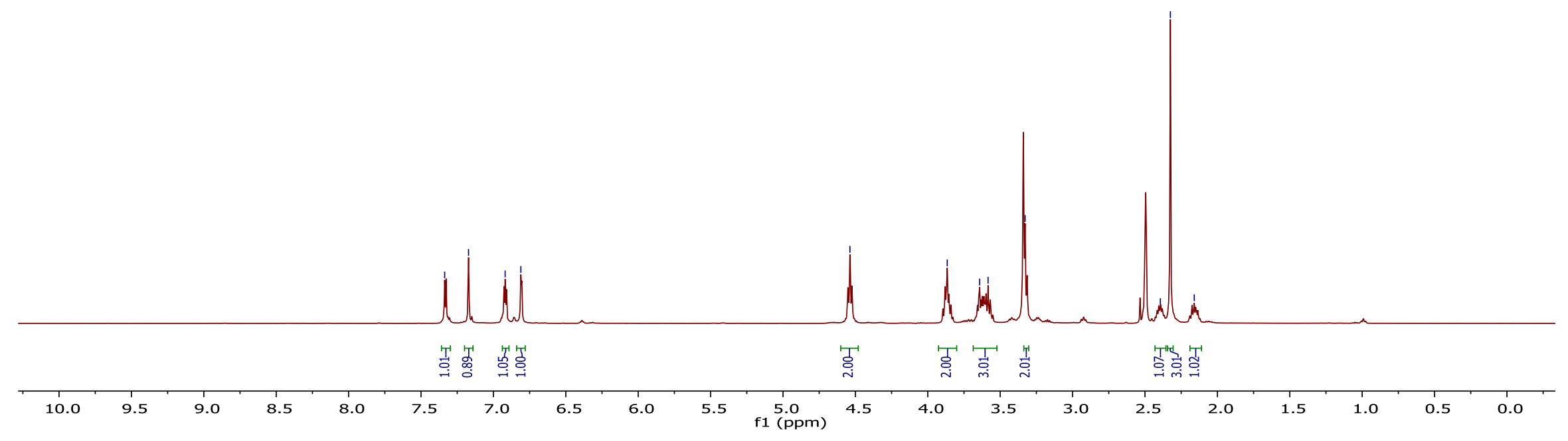


${ }^{13} \mathrm{C}$ NMR spectrum of the compound $\mathbf{8}\{41,148\}$.

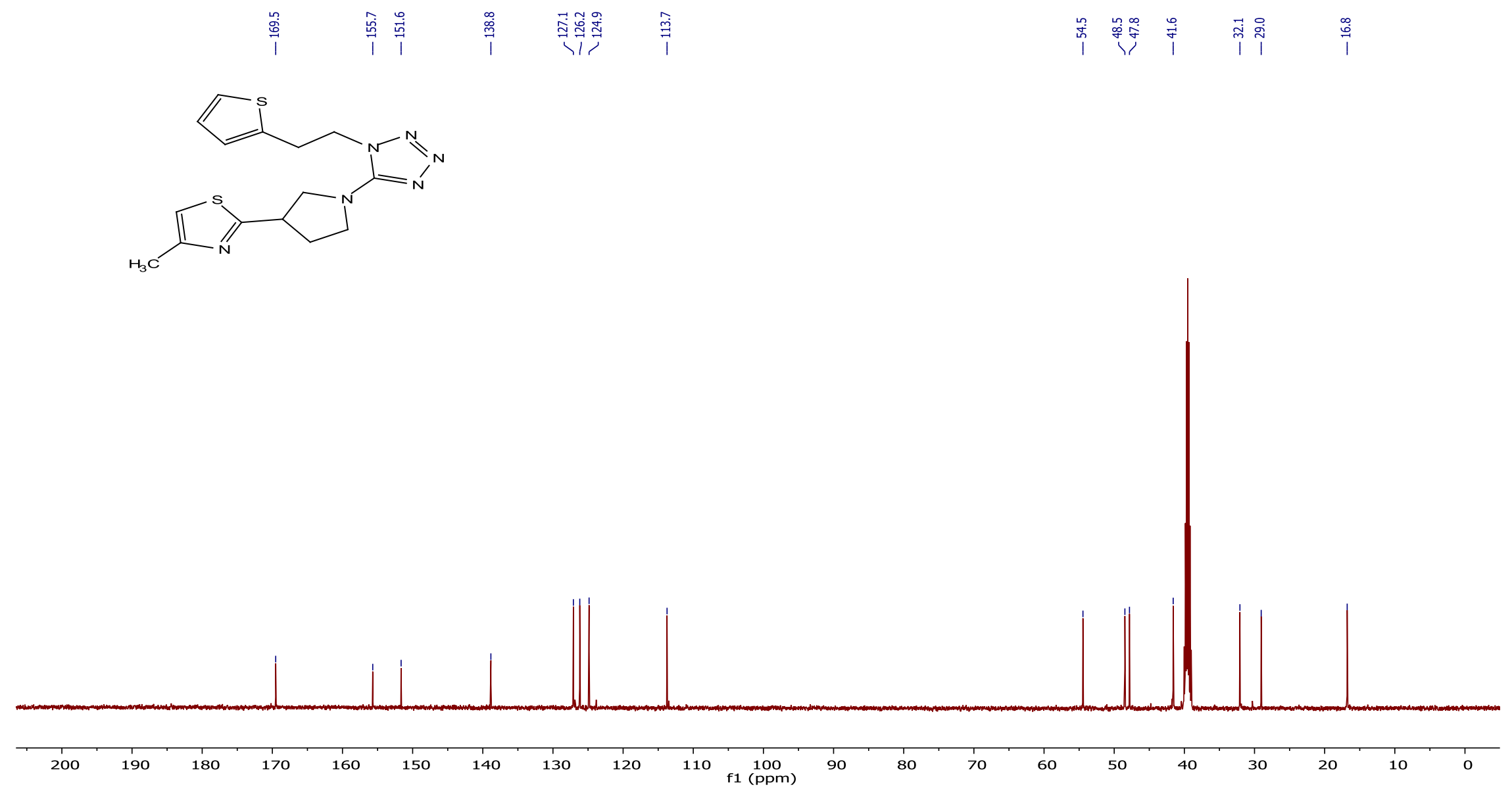


${ }^{1} \mathrm{H}$ NMR spectrum of the compound $\mathbf{8}\{41,210\}$.
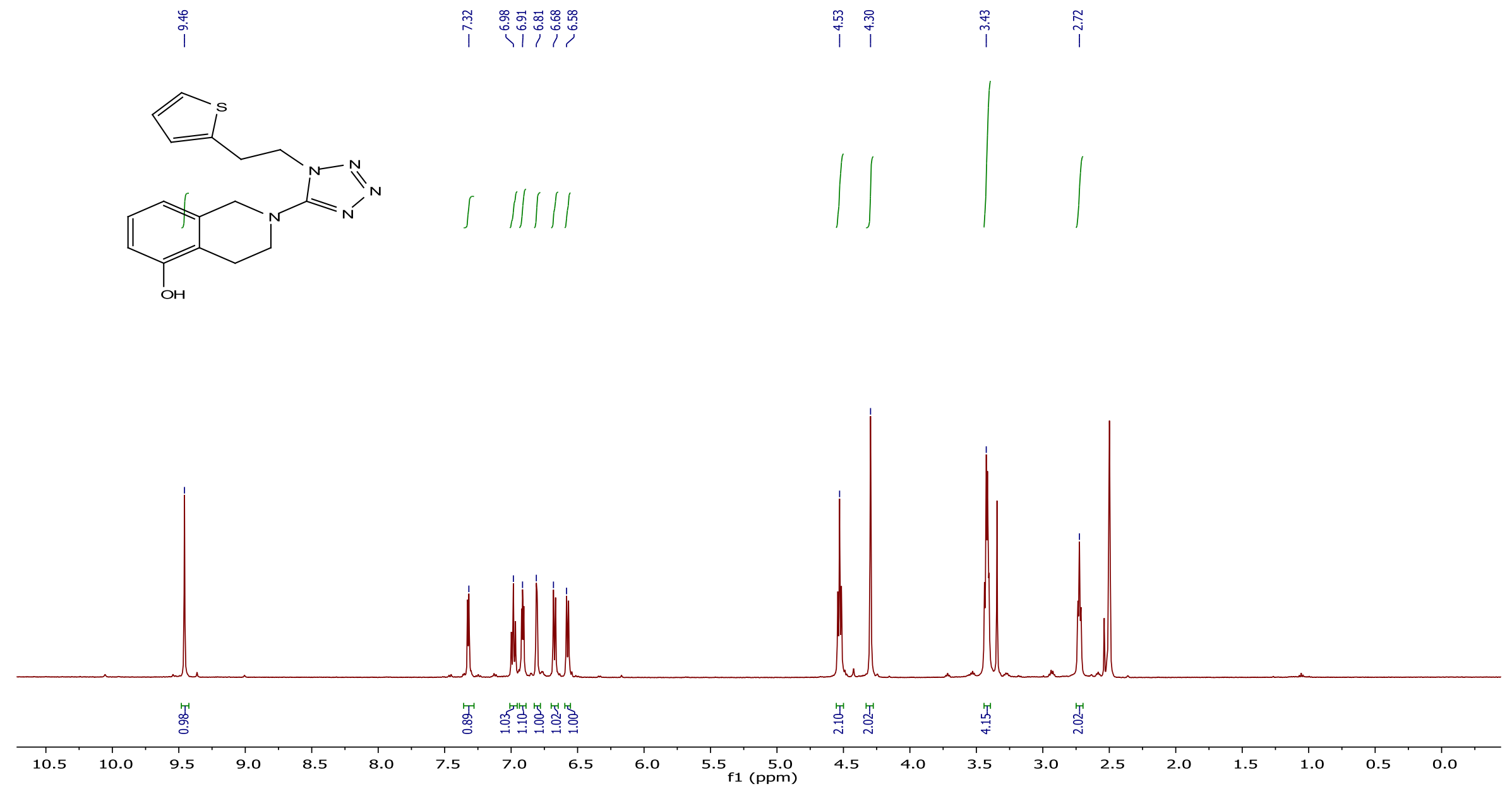
${ }^{13} \mathrm{C}$ NMR spectrum of the compound $\mathbf{8}\{41,210\}$.

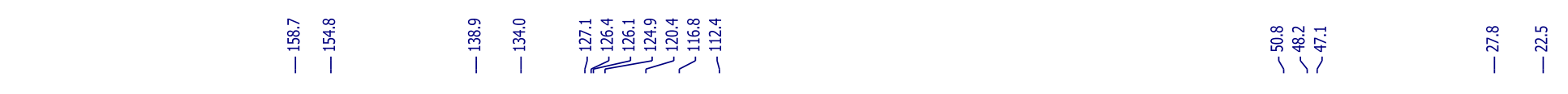
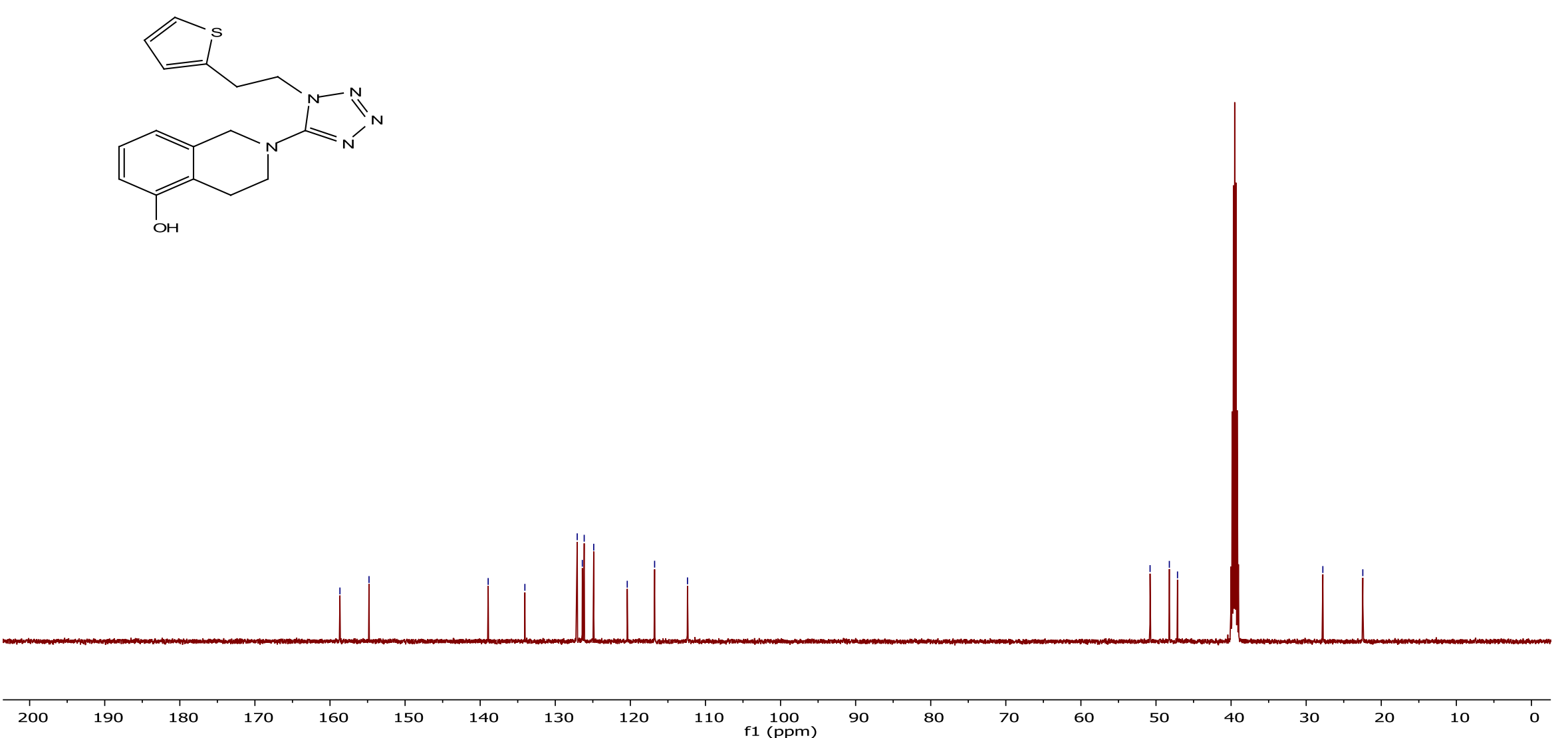
${ }^{1} \mathrm{H}$ NMR spectrum of the compound $\mathbf{8}\{41,211\}$.
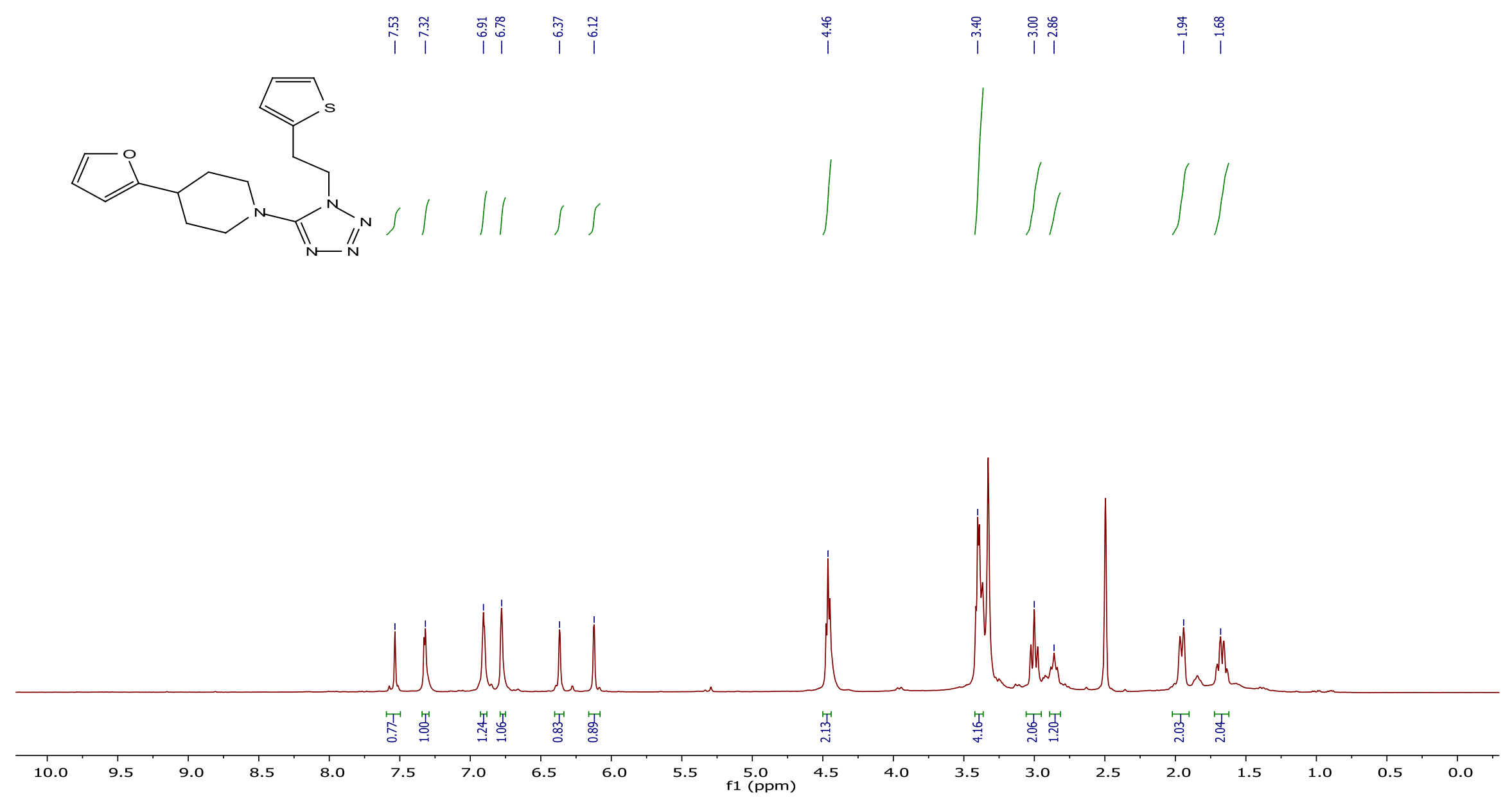
${ }^{13} \mathrm{C}$ NMR spectrum of the compound $\mathbf{8}\{41,211\}$.
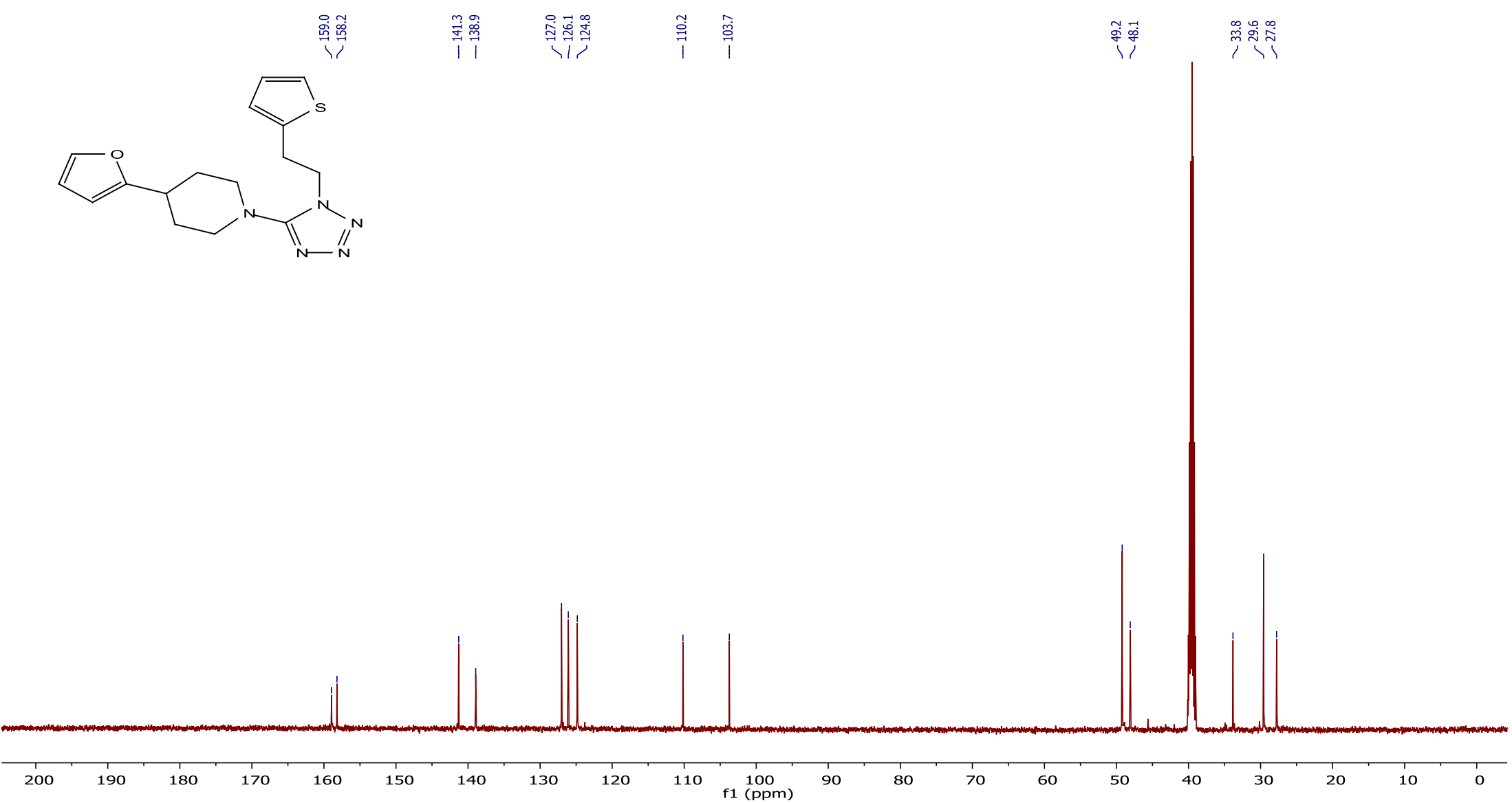
${ }^{1} \mathrm{H}$ NMR spectrum of the compound $\mathbf{8}\{41,212\}$.

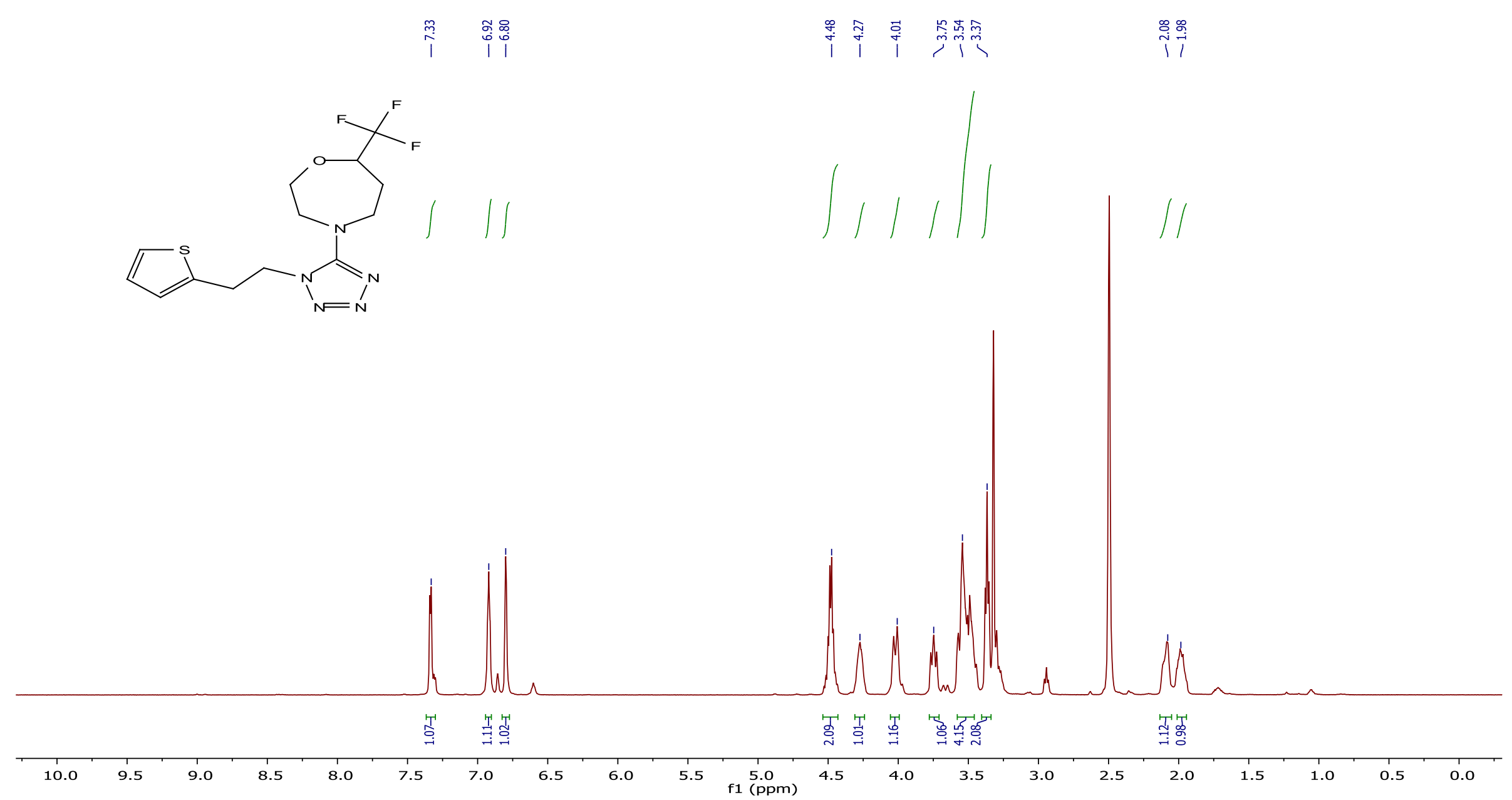


${ }^{13} \mathrm{C}$ NMR spectrum of the compound $\mathbf{8}\{41,212\}$.

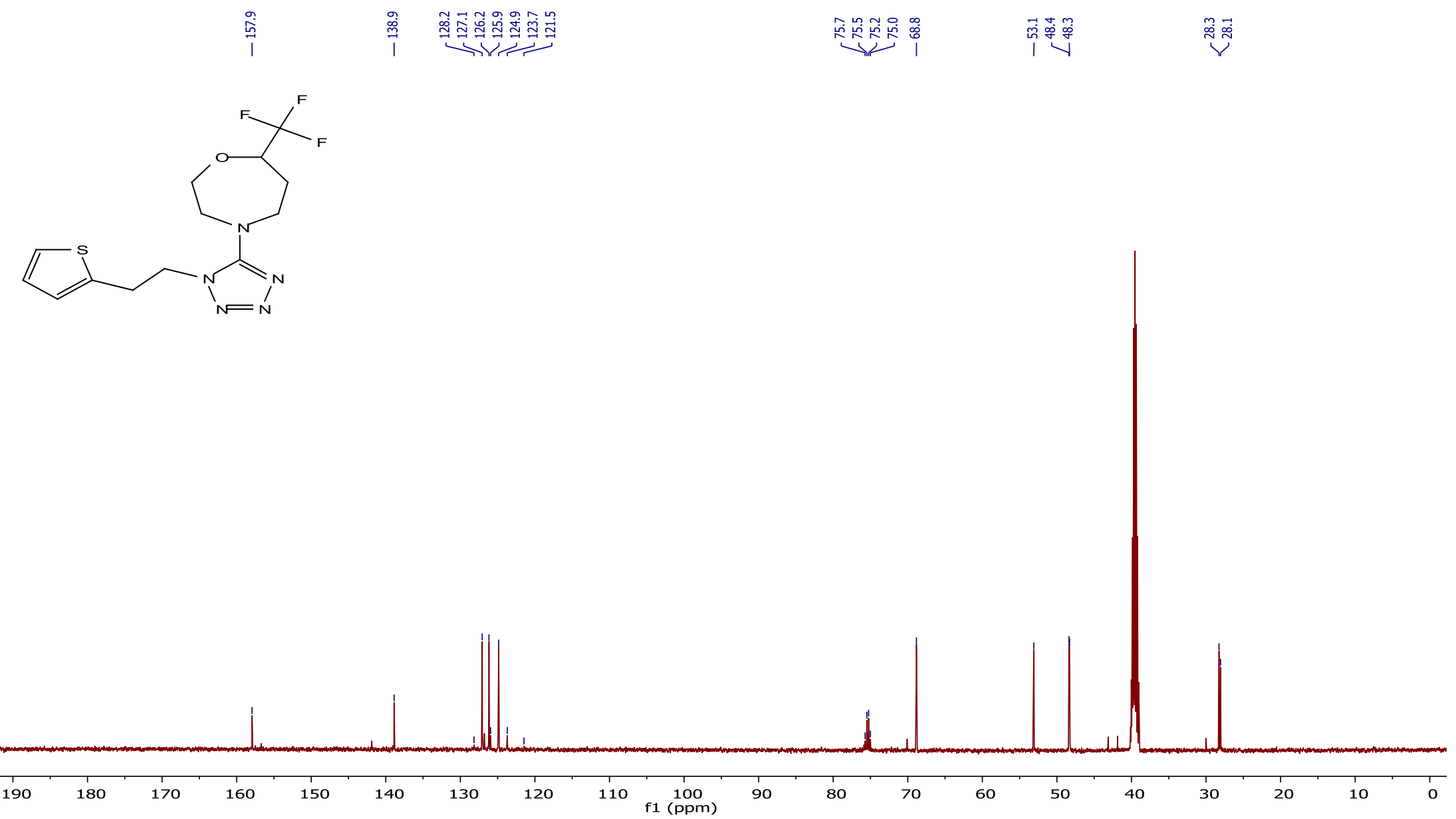


${ }^{19}$ F NMR spectrum of the compound $8\{41,212\}$.

$\stackrel{\circ}{i}$

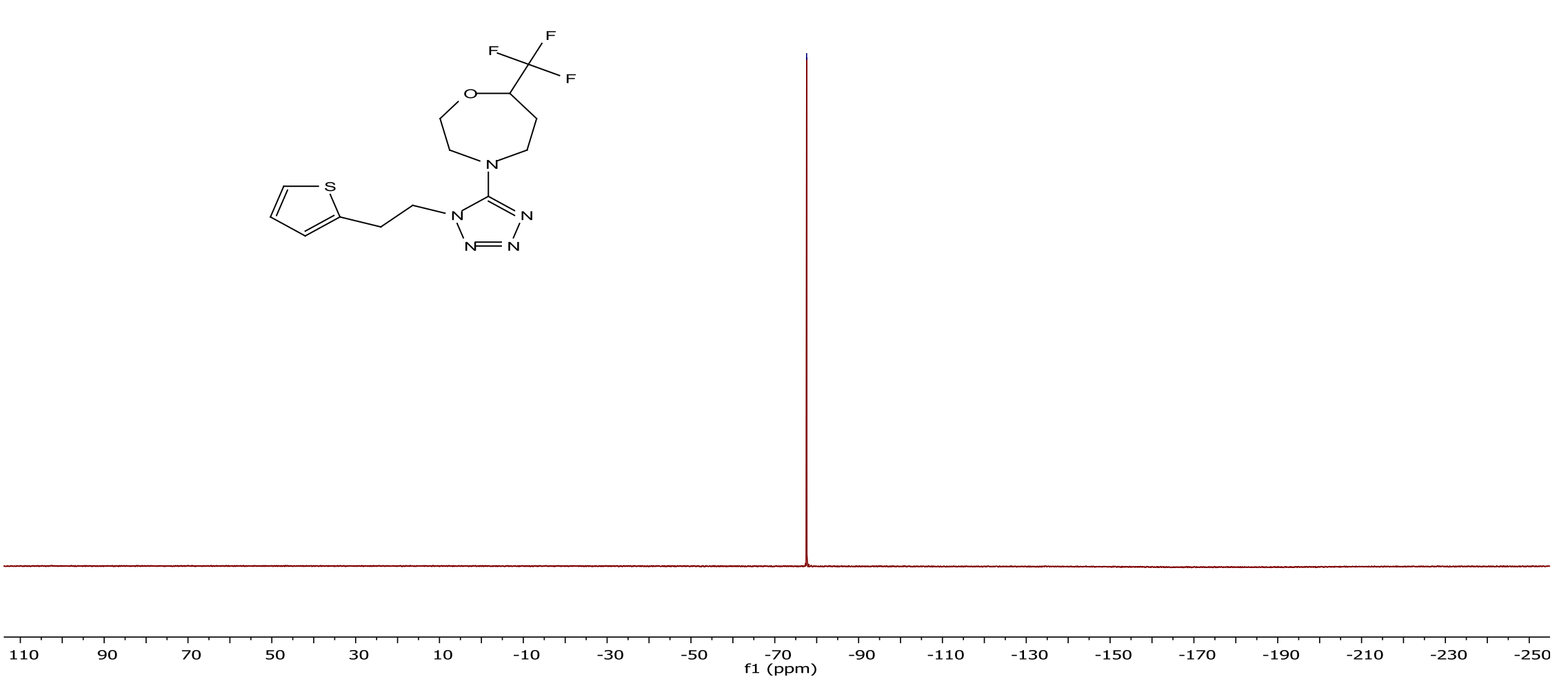

(n) 
${ }^{1} \mathrm{H}$ NMR spectrum of the compound $\mathbf{8}\{42,193\}$.

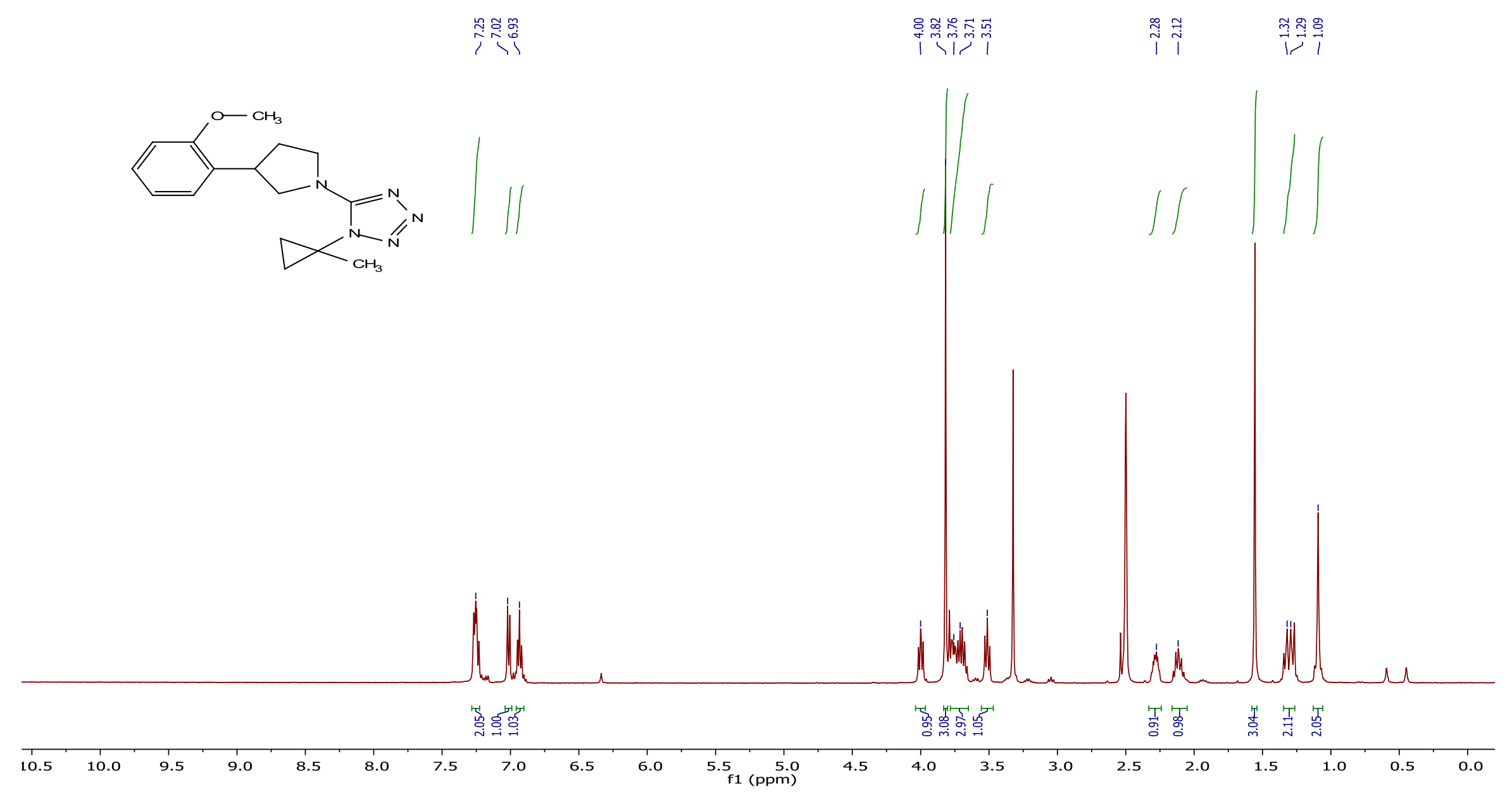


${ }^{13} \mathrm{C}$ NMR spectrum of the compound $\mathbf{8}\{42,193\}$.
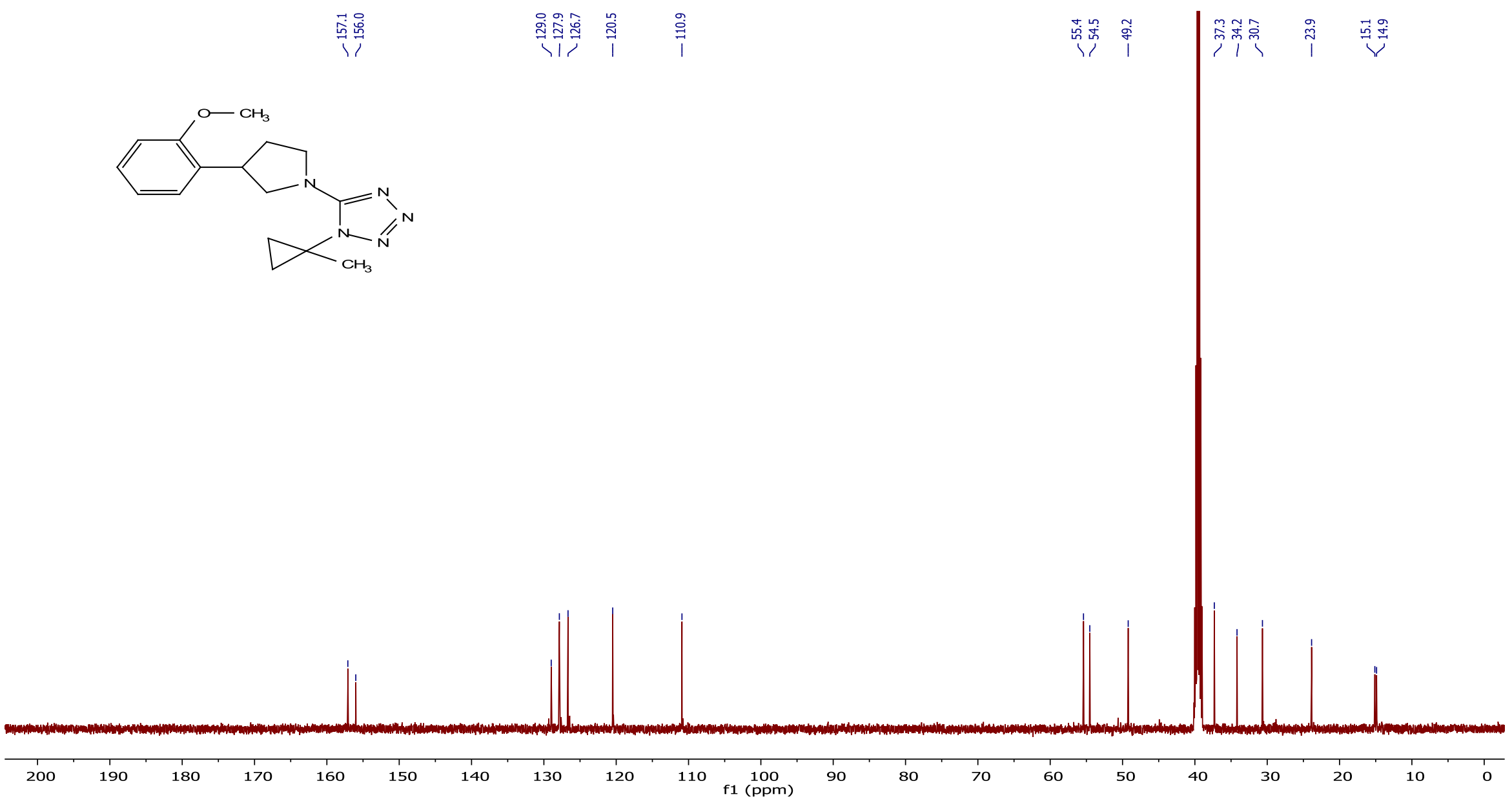
${ }^{1} \mathrm{H}$ NMR spectrum of the compound $\mathbf{8}\{42,227\}$.
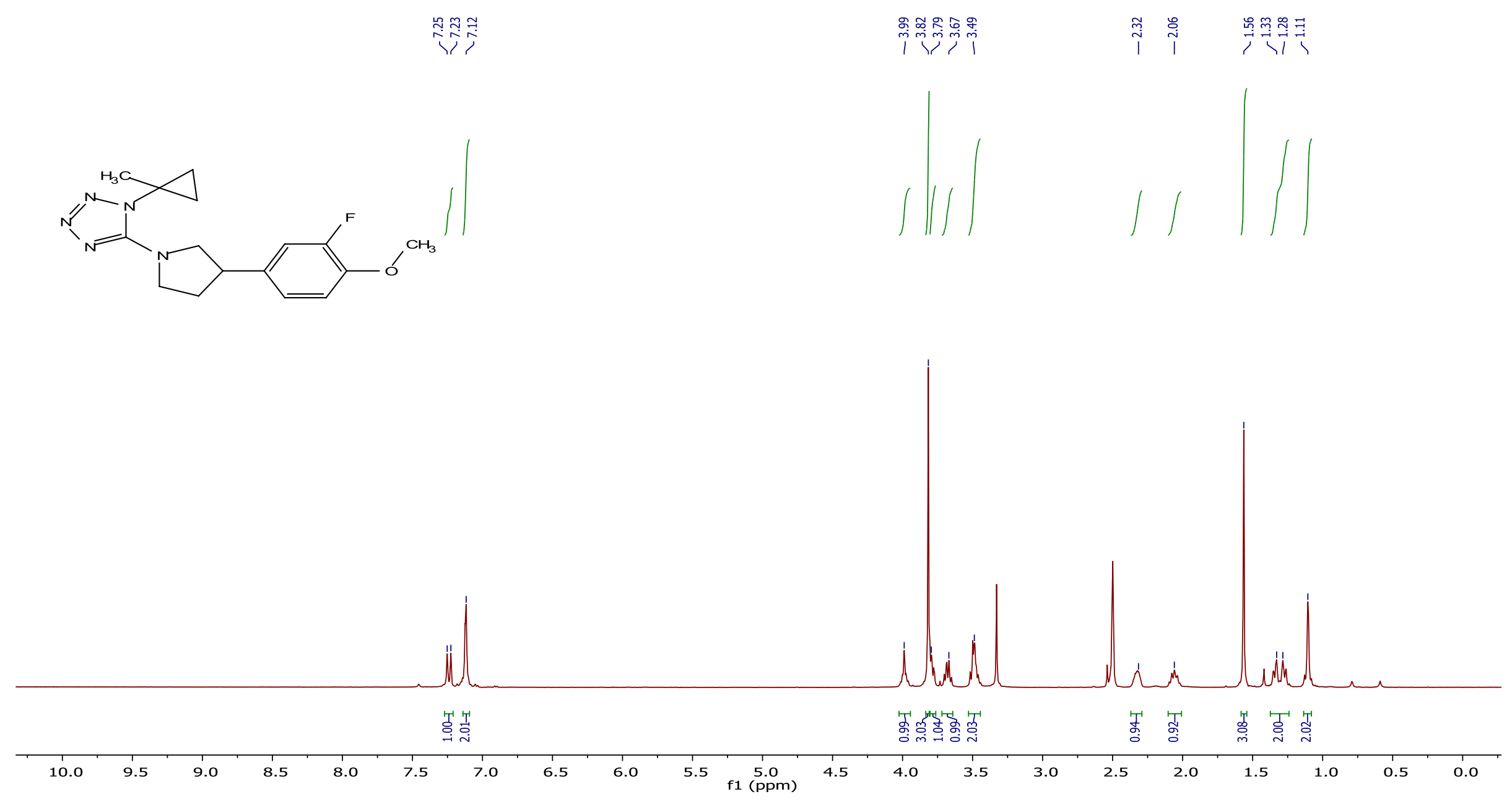
${ }^{13} \mathrm{C}$ NMR spectrum of the compound $\mathbf{8}\{42,227\}$.
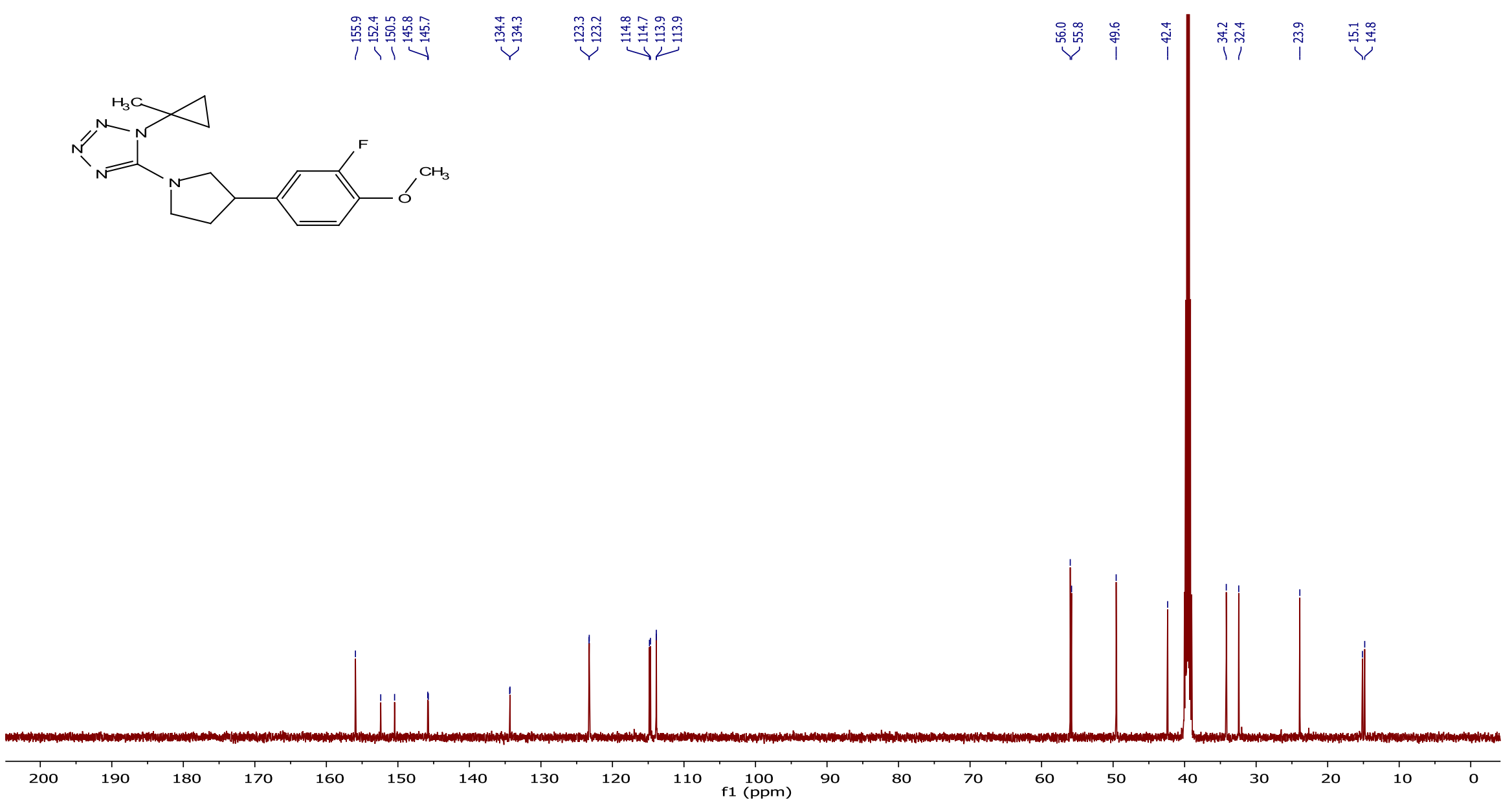
${ }^{19}$ F NMR spectrum of the compound $8\{42,227\}$.
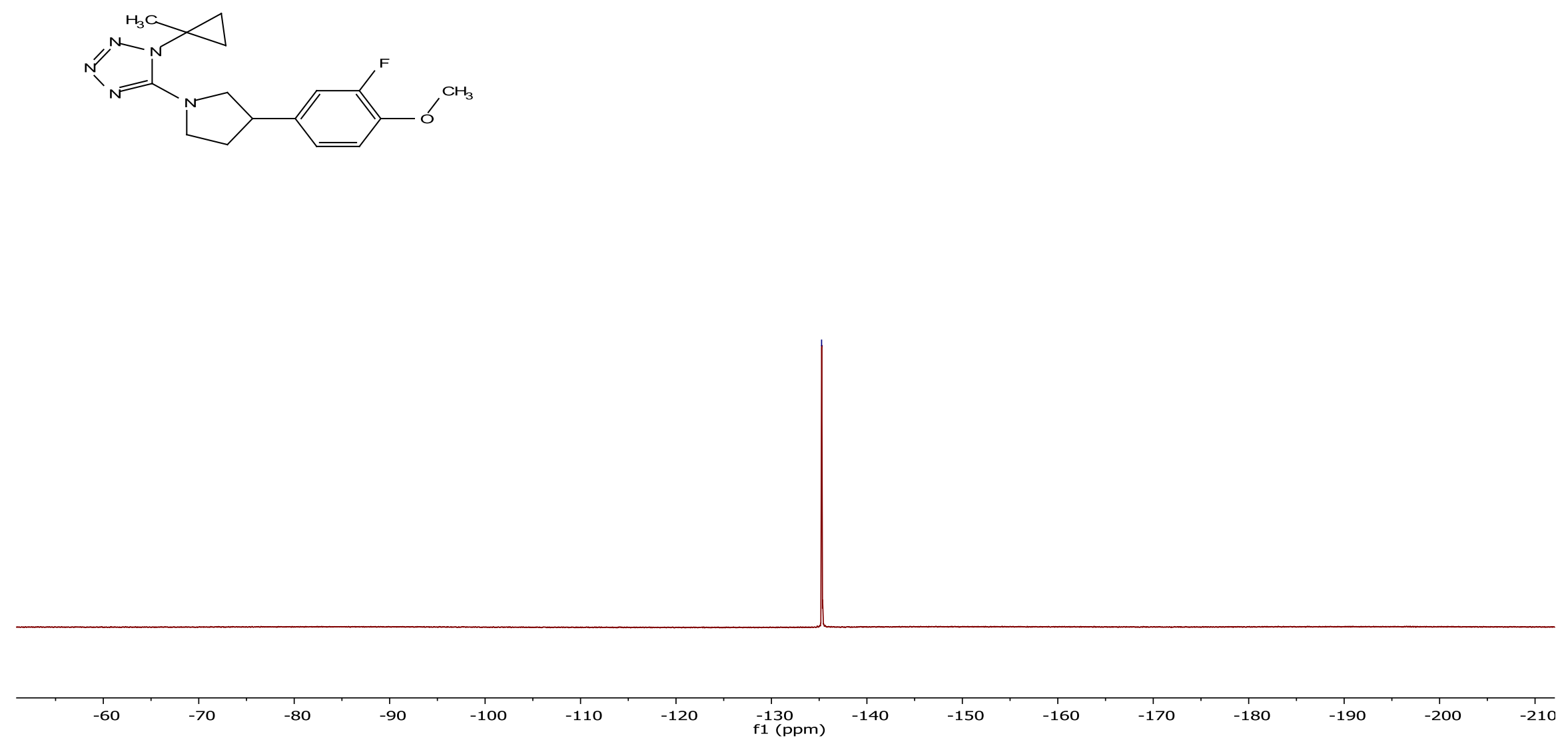
${ }^{1} \mathrm{H}$ NMR spectrum of the compound $\mathbf{8}\{51,251\}$.

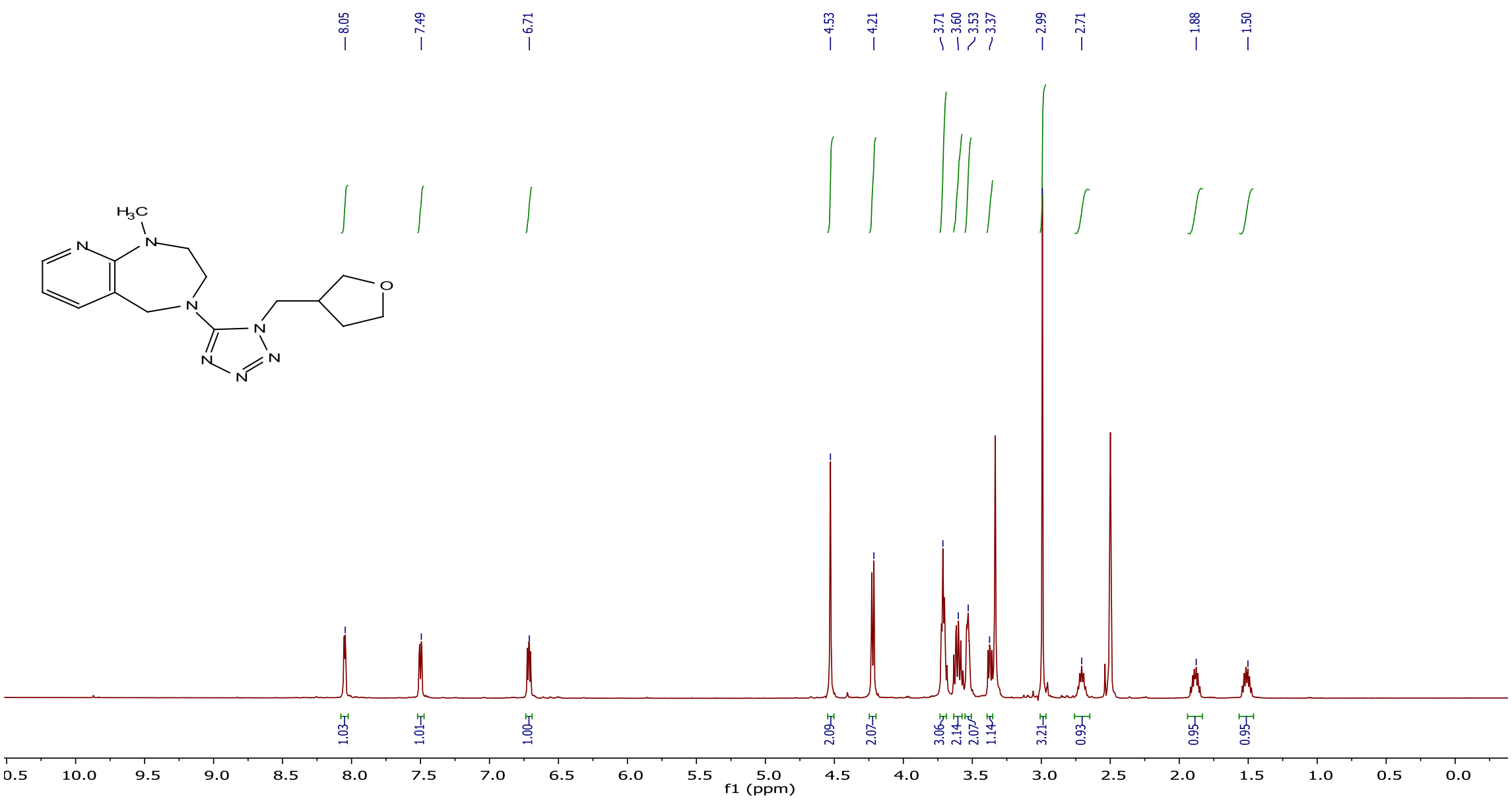


${ }^{13} \mathrm{C}$ NMR spectrum of the compound $\mathbf{8}\{51,251\}$.

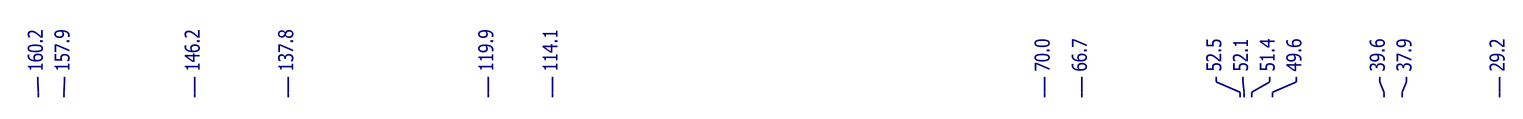
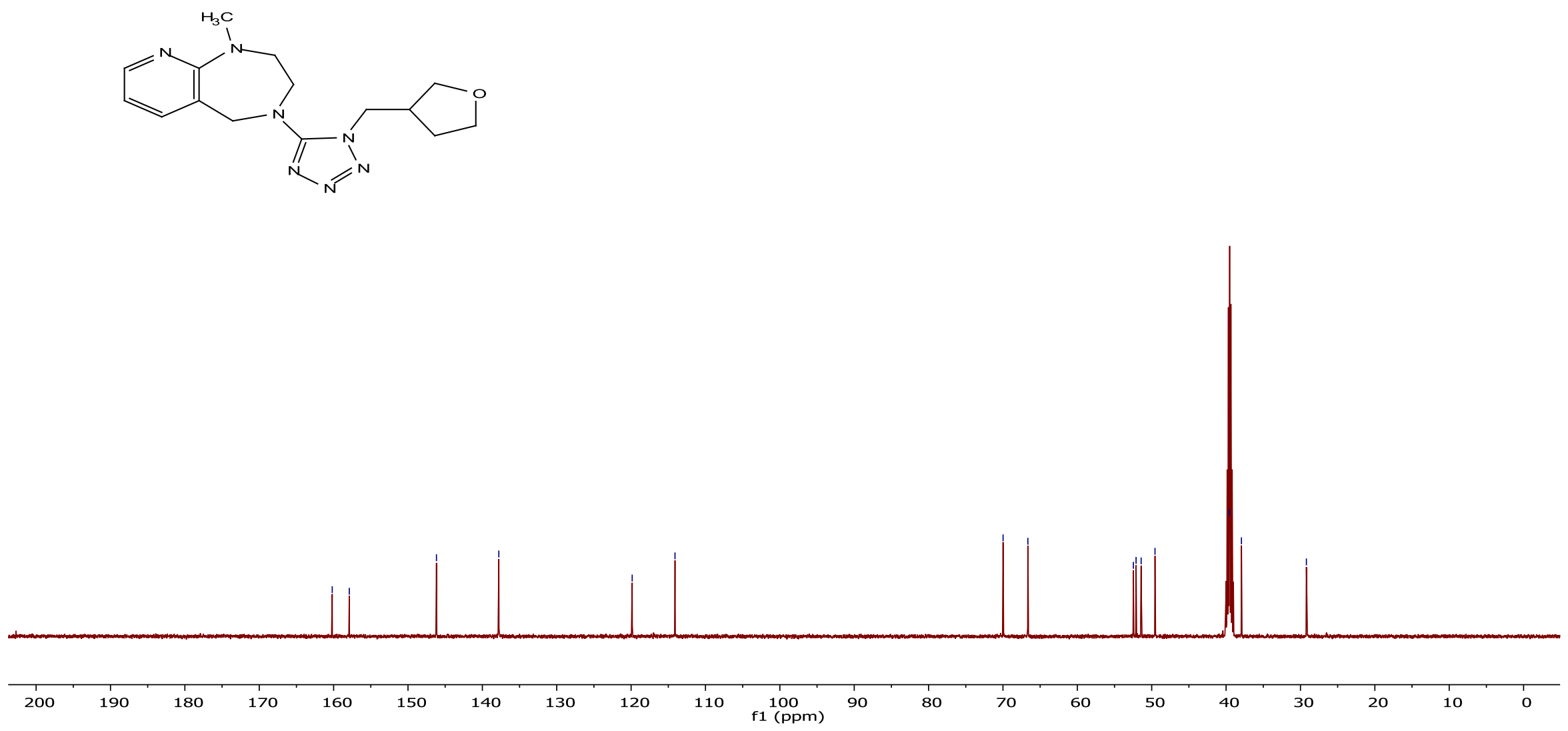
${ }^{1} \mathrm{H}$ NMR spectrum of the compound $\mathbf{8}\{53,236\}$.
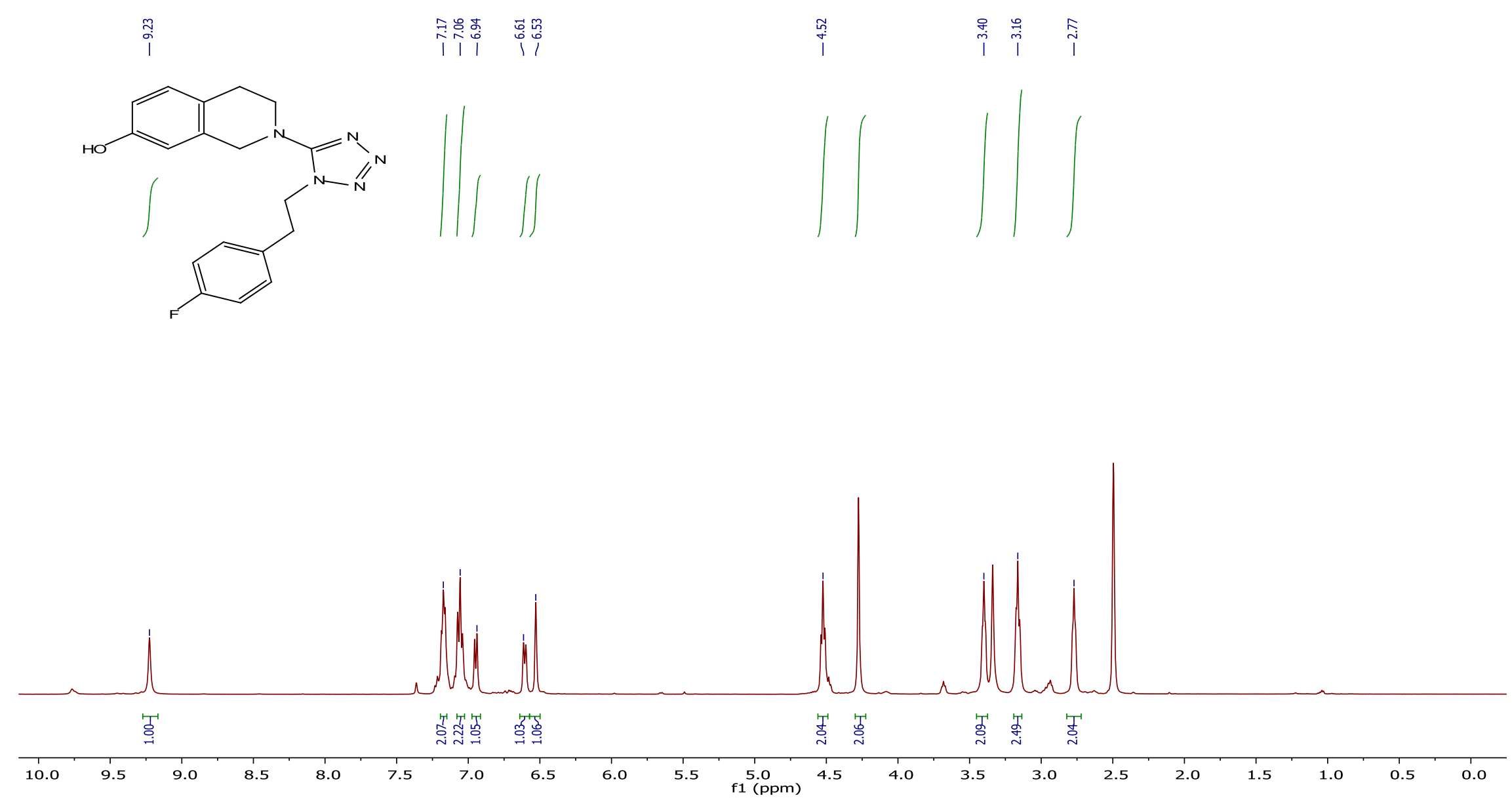
${ }^{13} \mathrm{C}$ NMR spectrum of the compound $\mathbf{8}\{53,236\}$.

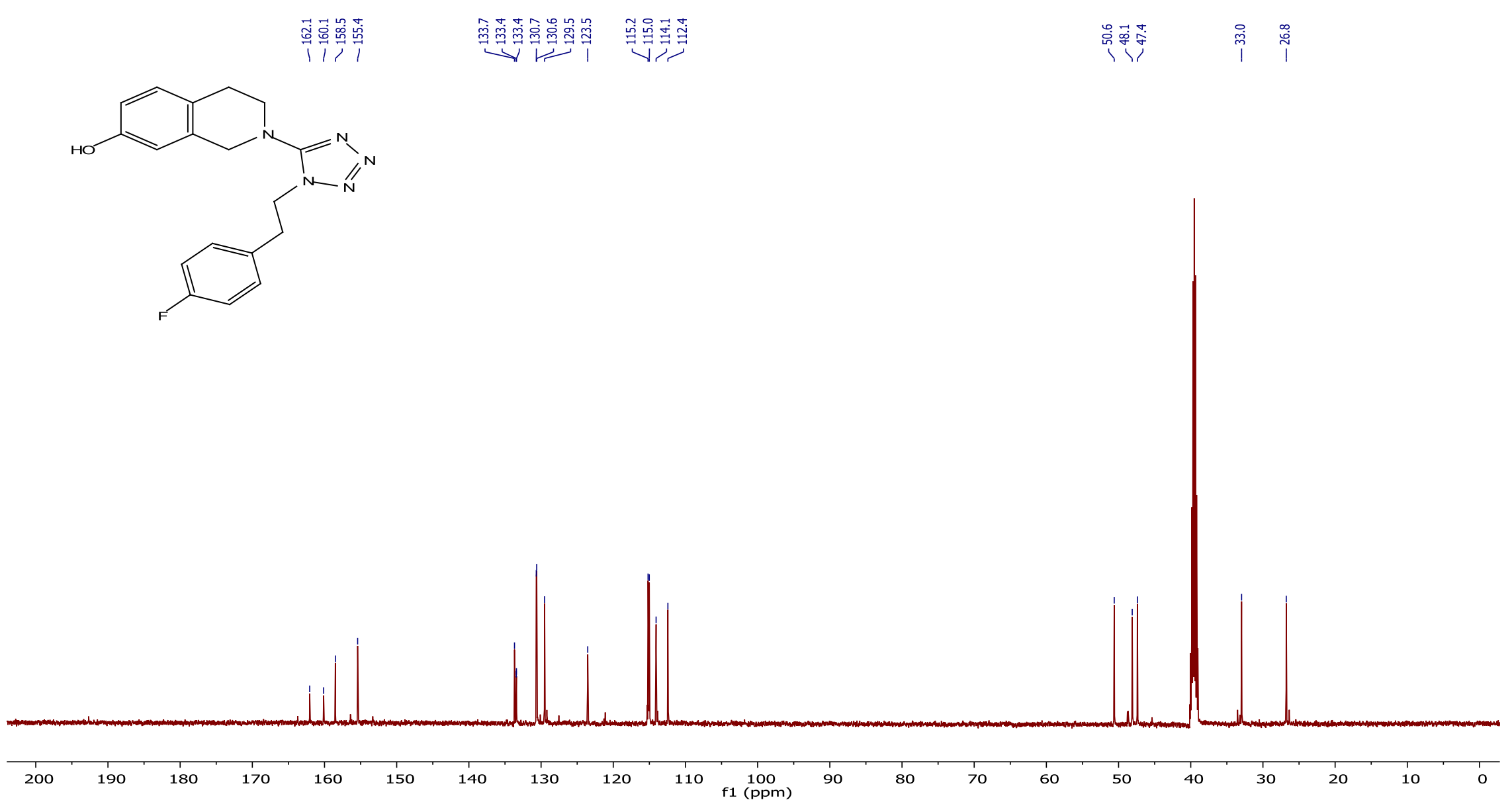


${ }^{19}$ F NMR spectrum of the compound $8\{53,236\}$.

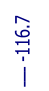
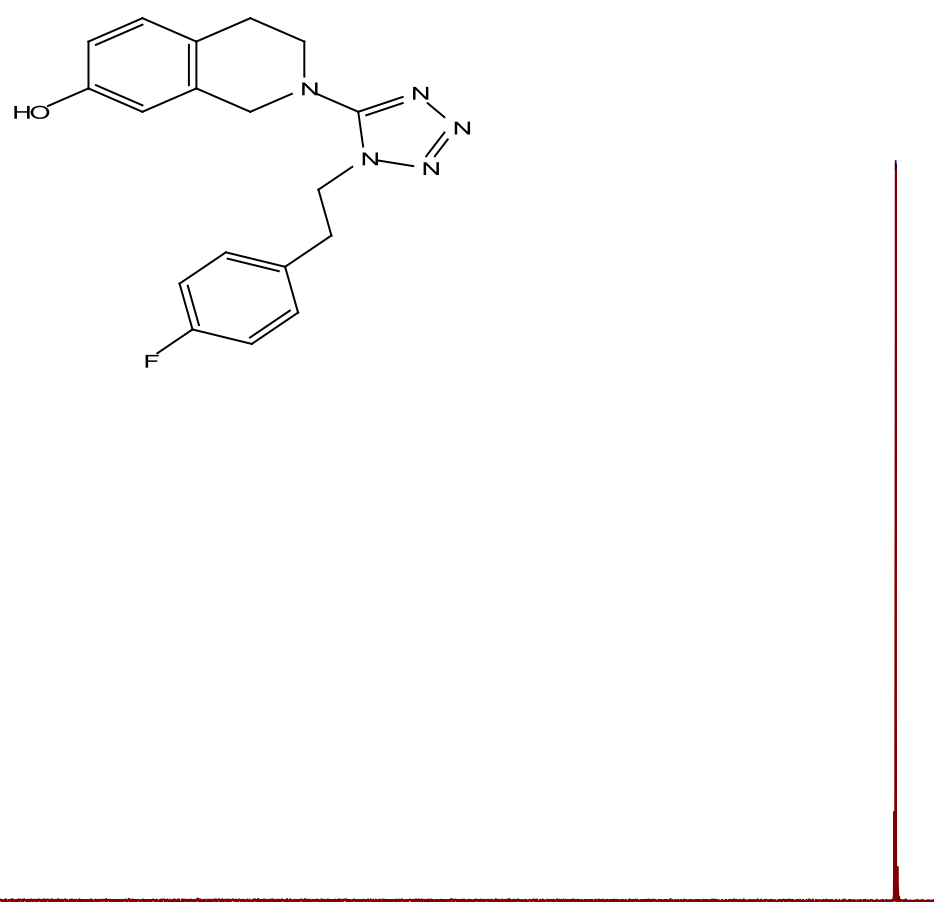

\begin{tabular}{lllllllllllllllllllllllllllllllllllll}
\hline 0 & -10 & -20 & -30 & -40 & -50 & -60 & -70 & -80 & -90 & -100 & -110 & -120 & -130 & -140 & -150 & -160 & -170 & -180 & -190 & -200 & -210 & -220 & -230 & -240 & -250
\end{tabular} 
${ }^{1} \mathrm{H}$ NMR spectrum of the compound $\mathbf{8}\{56,108\}$.

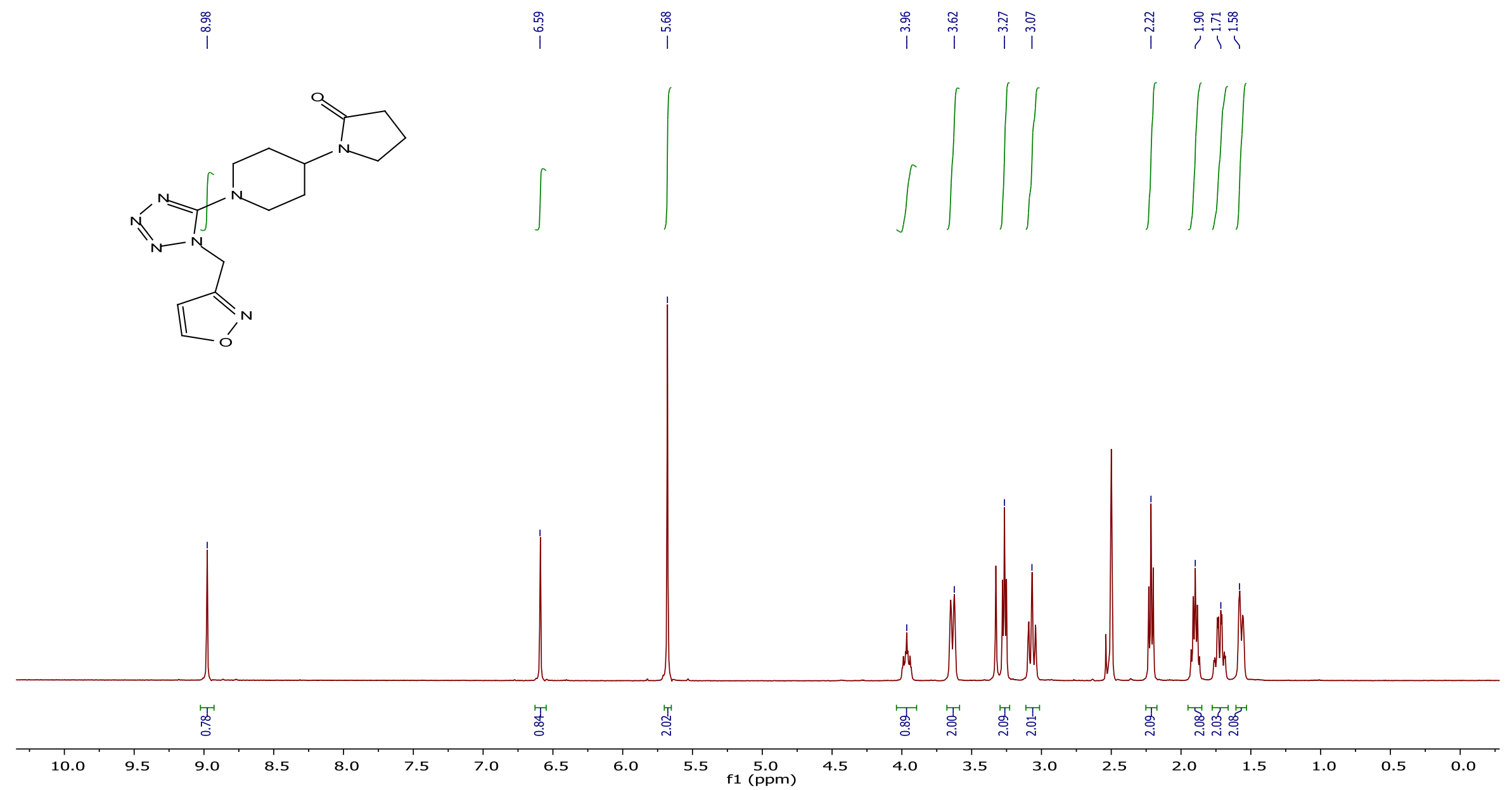


${ }^{13} \mathrm{C}$ NMR spectrum of the compound $\mathbf{8}\{56,108\}$.

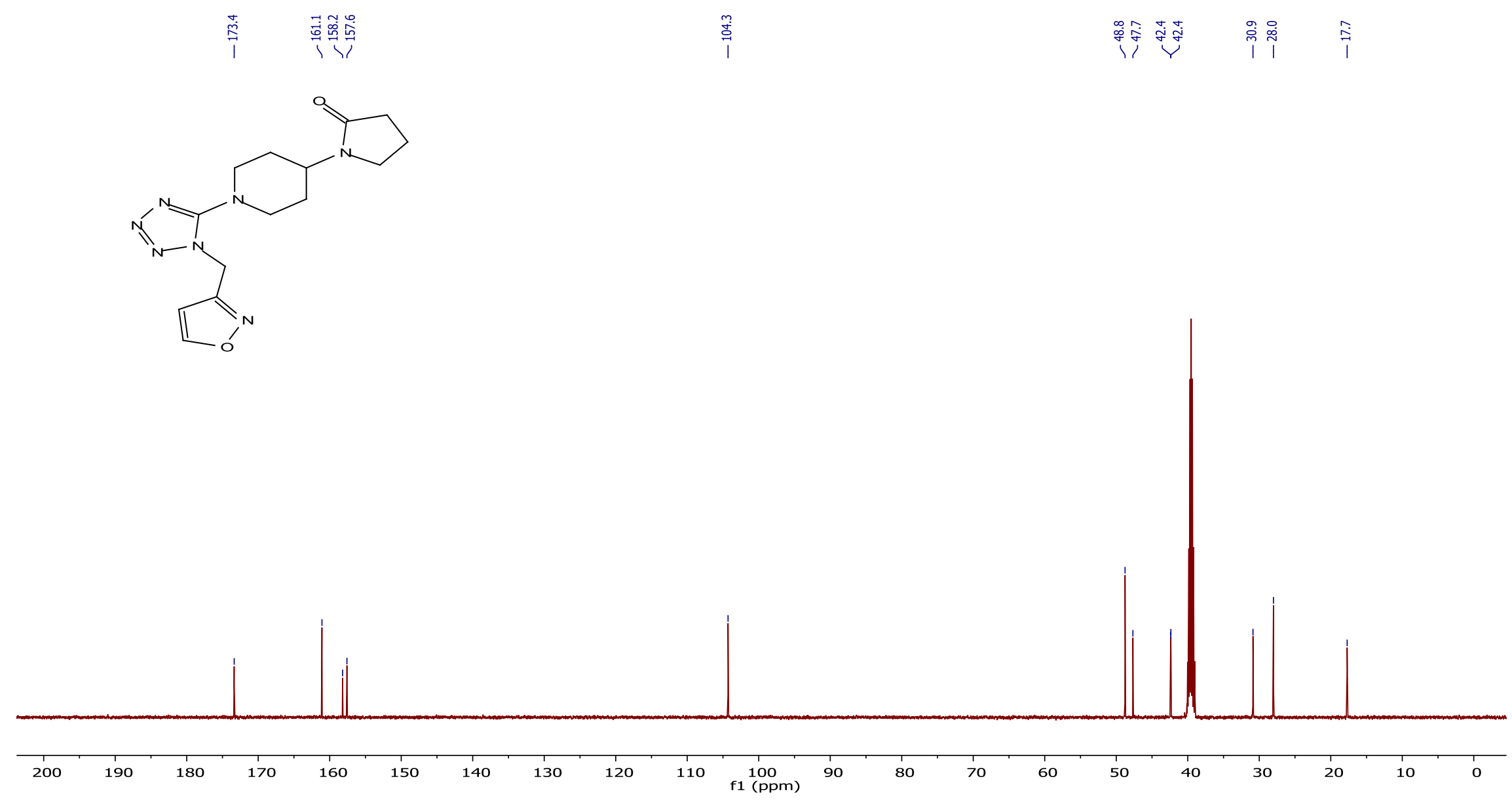


${ }^{1} \mathrm{H}$ NMR spectrum of the compound $\mathbf{8}\{56,271\}$.

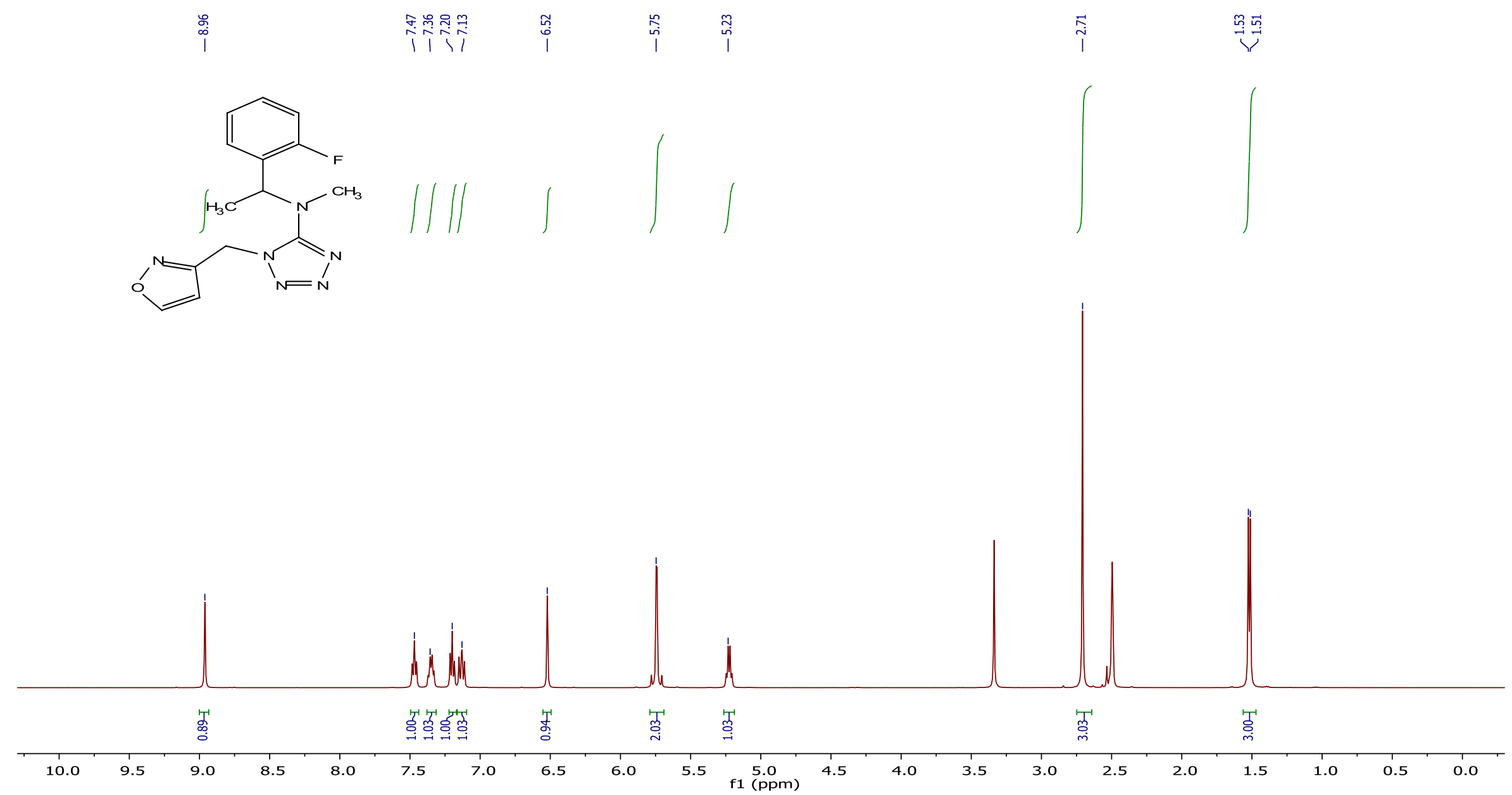


${ }^{13} \mathrm{C}$ NMR spectrum of the compound $\mathbf{8}\{56,271\}$.
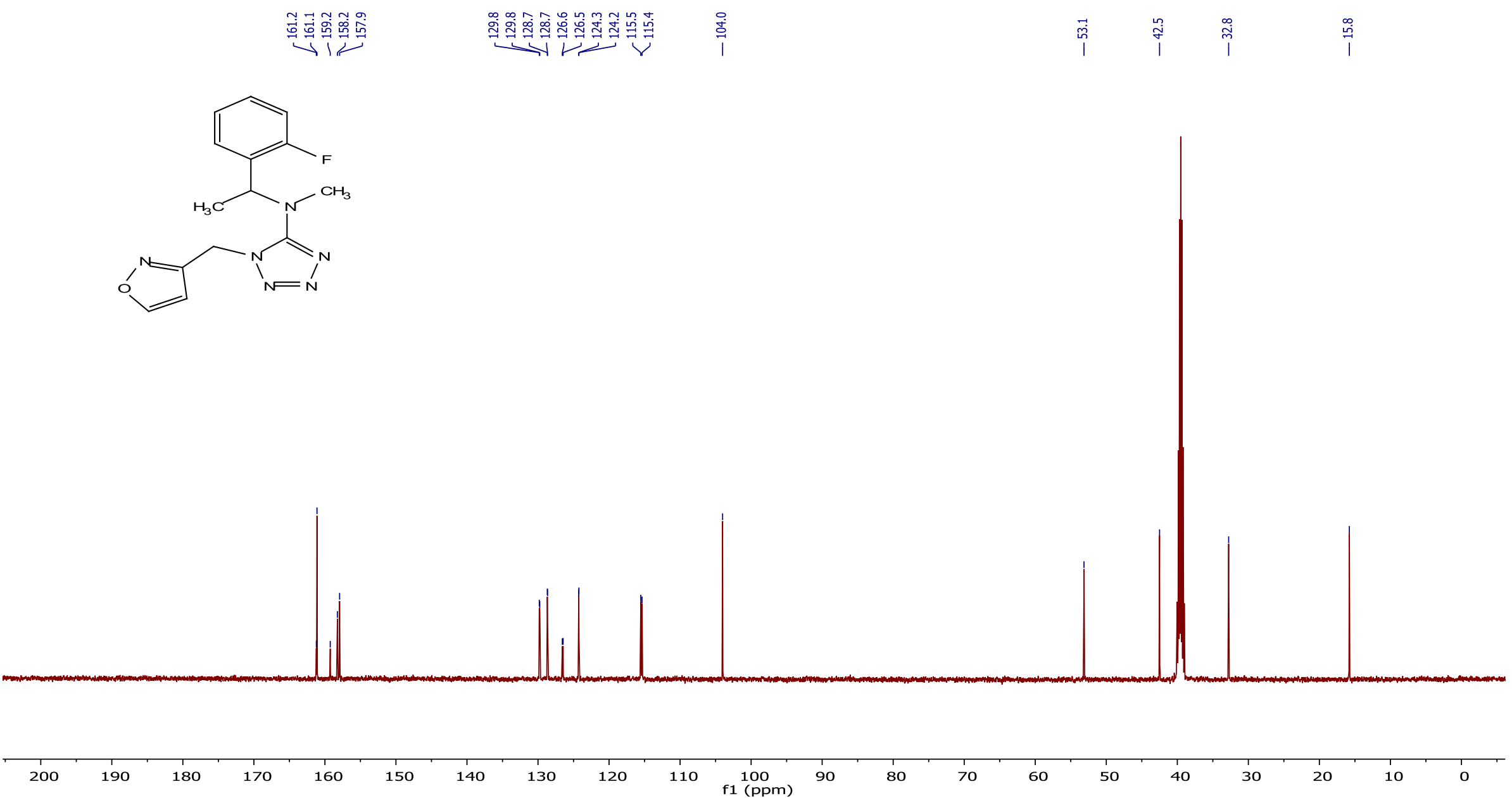
${ }^{19}$ F NMR spectrum of the compound $\mathbf{8}\{56,271\}$.

$\stackrel{8}{\stackrel{9}{i}}$

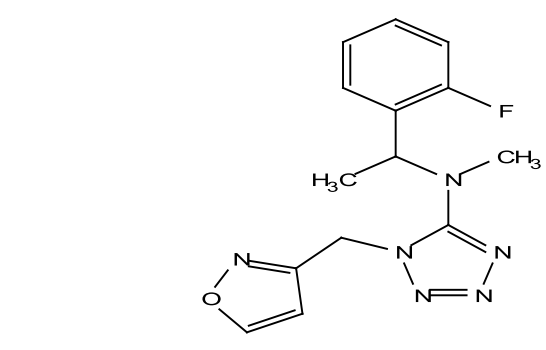


${ }^{1} \mathrm{H}$ NMR spectrum of the compound $\mathbf{8}\{58,280\}$.

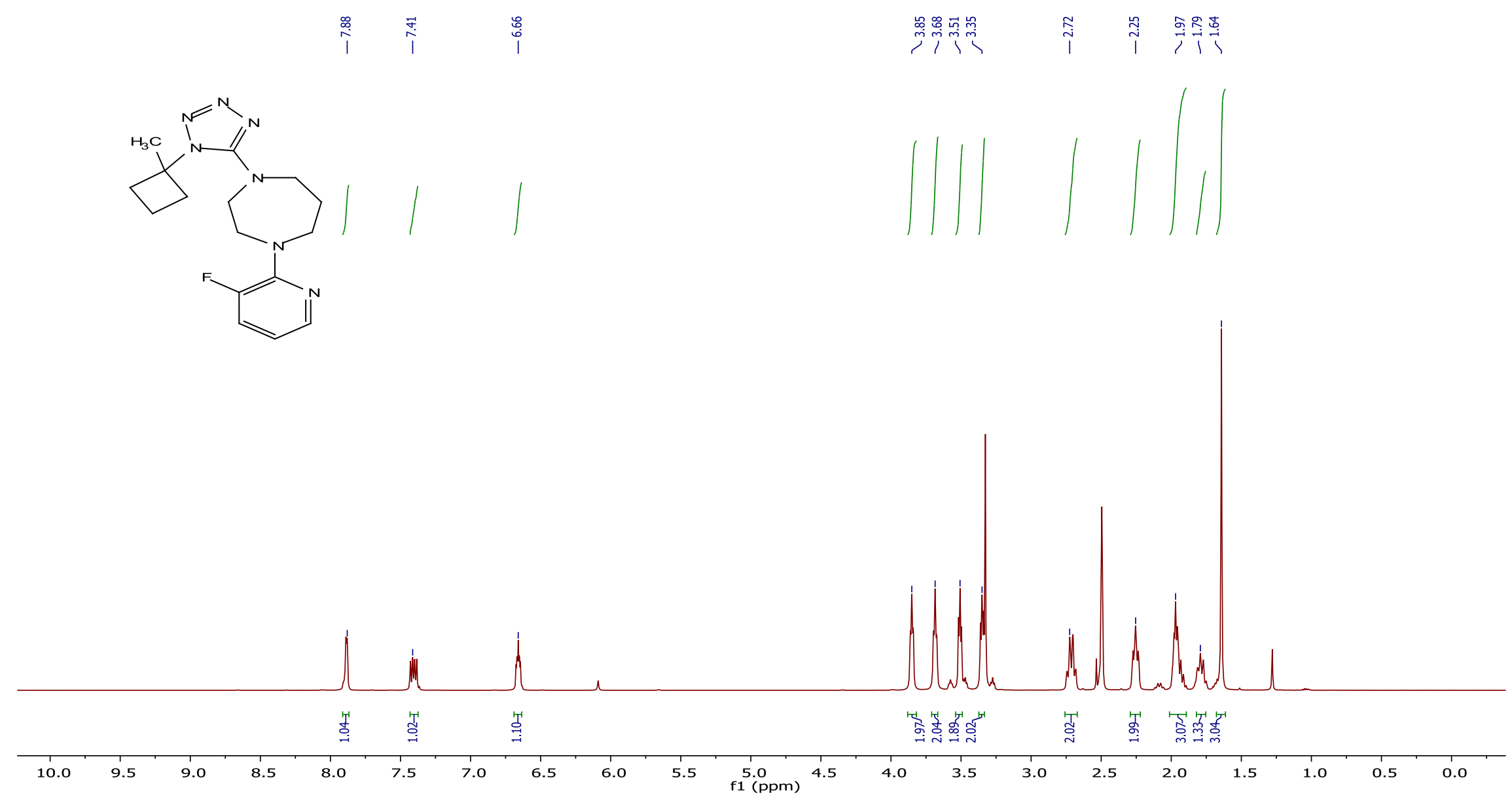


${ }^{13} \mathrm{C}$ NMR spectrum of the compound $\mathbf{8}\{58,280\}$.
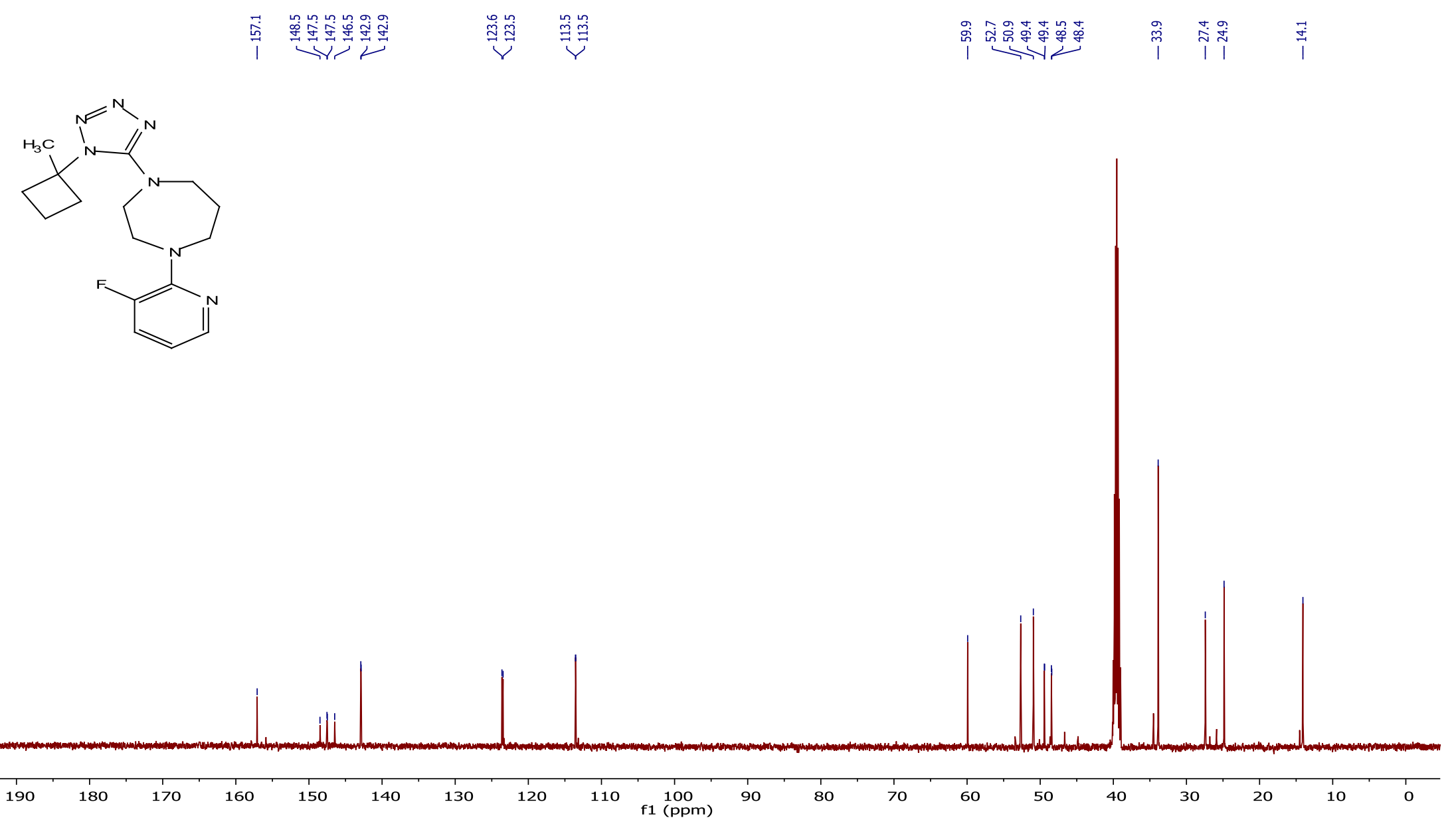
${ }^{19} \mathrm{~F}$ NMR spectrum of the compound $\mathbf{8}\{58,280\}$.

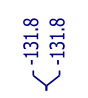
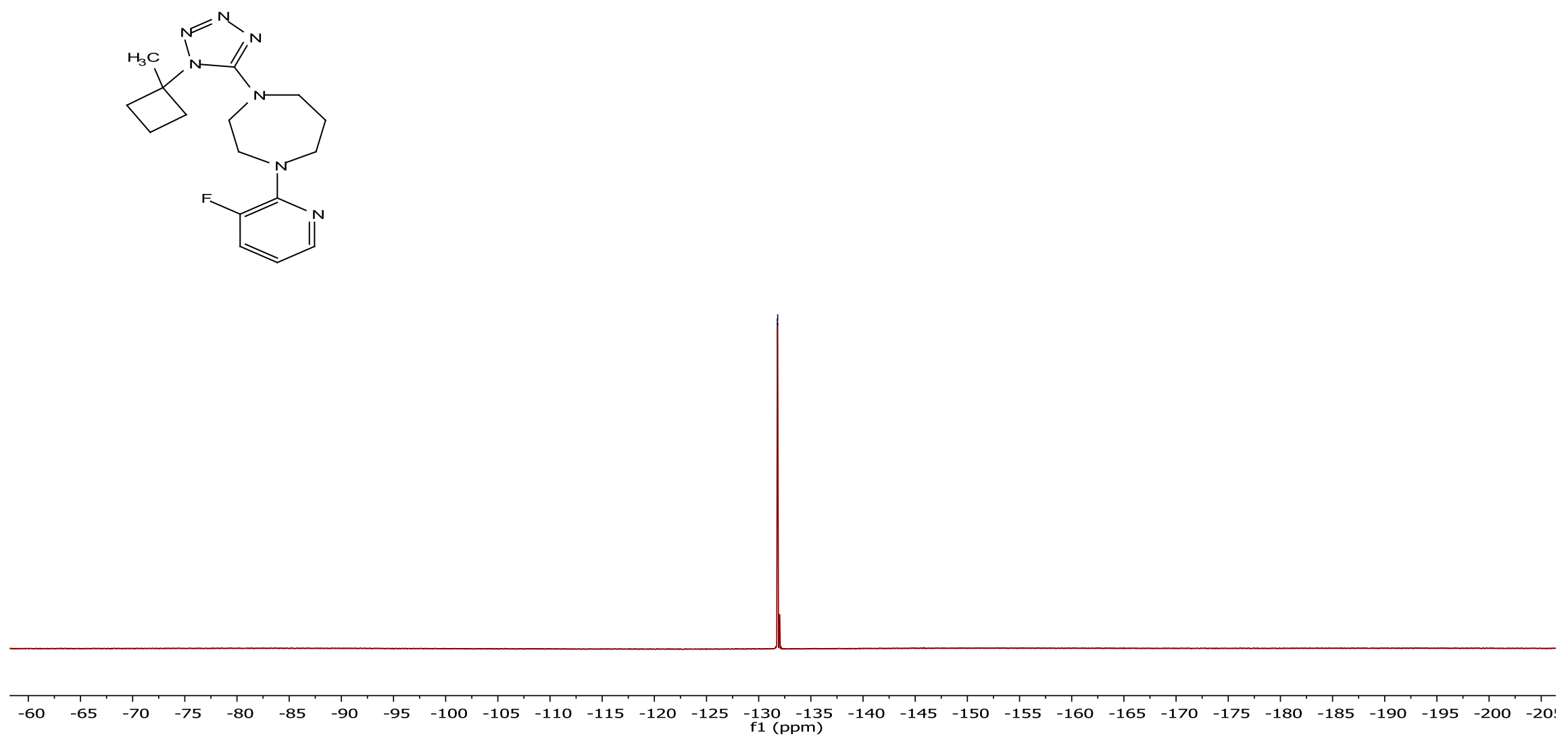
${ }^{1} \mathrm{H}$ NMR spectrum of the compound $\mathbf{9}\{4,289\}$.

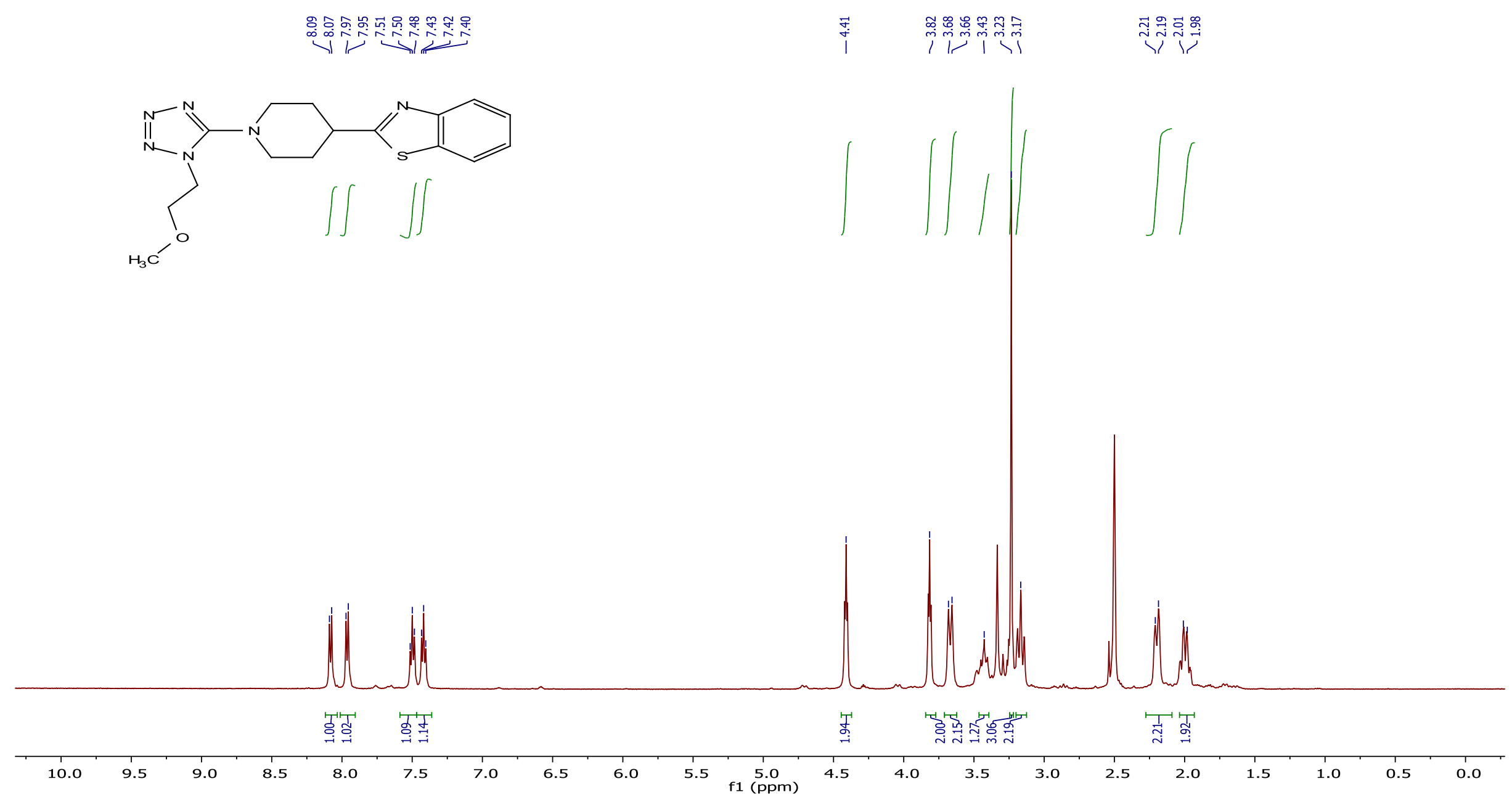


${ }^{13} \mathrm{C}$ NMR spectrum of the compound $\mathbf{9}\{4,289\}$.
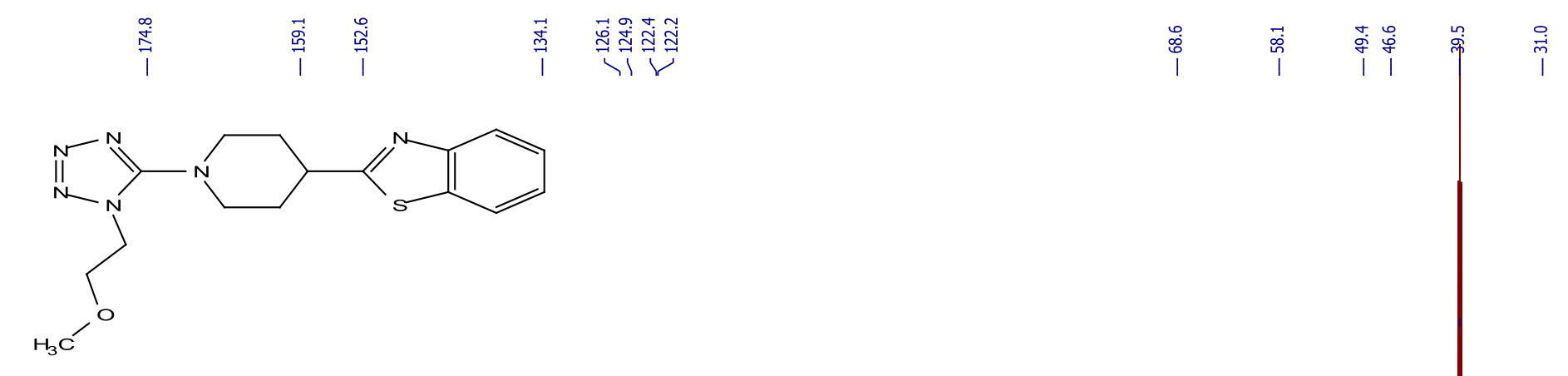
${ }^{1}$ H NMR spectrum of the compound $\mathbf{9}\{4,291\}$.

î
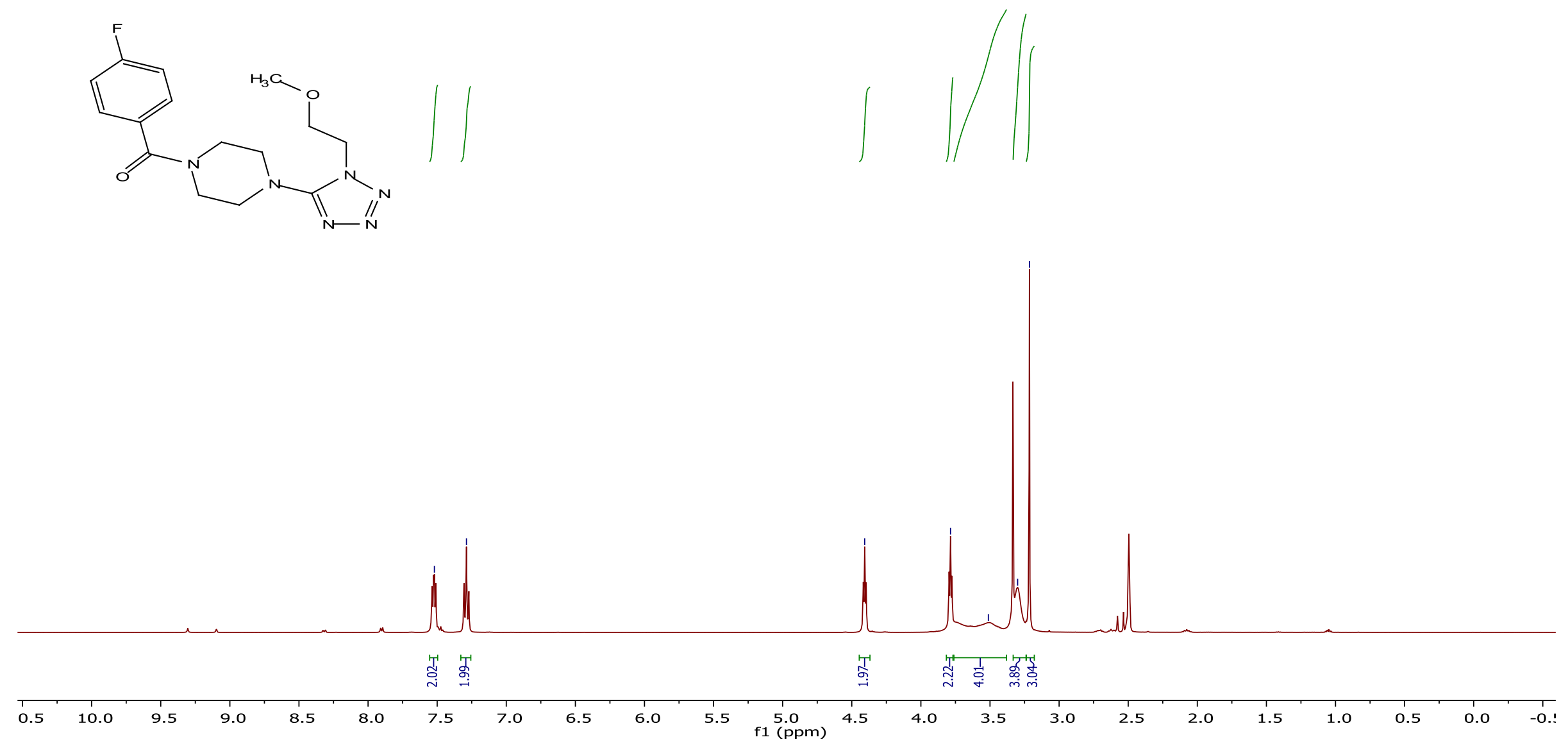
${ }^{13} \mathrm{C}$ NMR spectrum of the compound $\mathbf{9}\{4,291\}$.
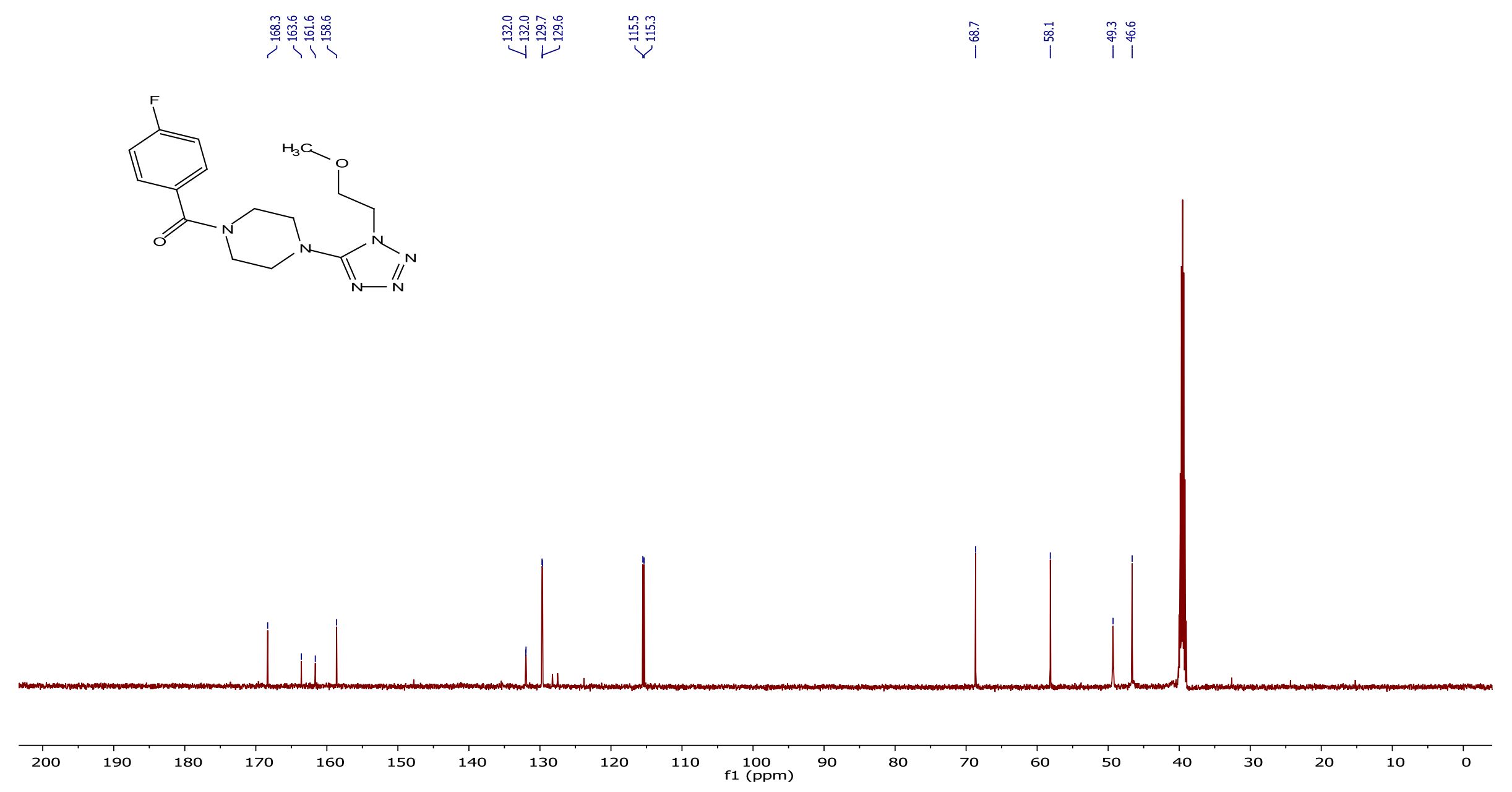
${ }^{19}$ F NMR spectrum of the compound $\mathbf{9}\{4,291\}$.

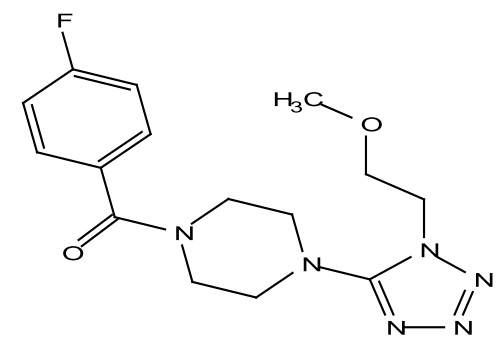

i

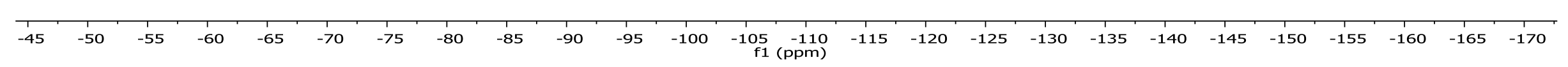

S229 
${ }^{1} \mathrm{H}$ NMR spectrum of the compound $\mathbf{9}\{18,300\}$.

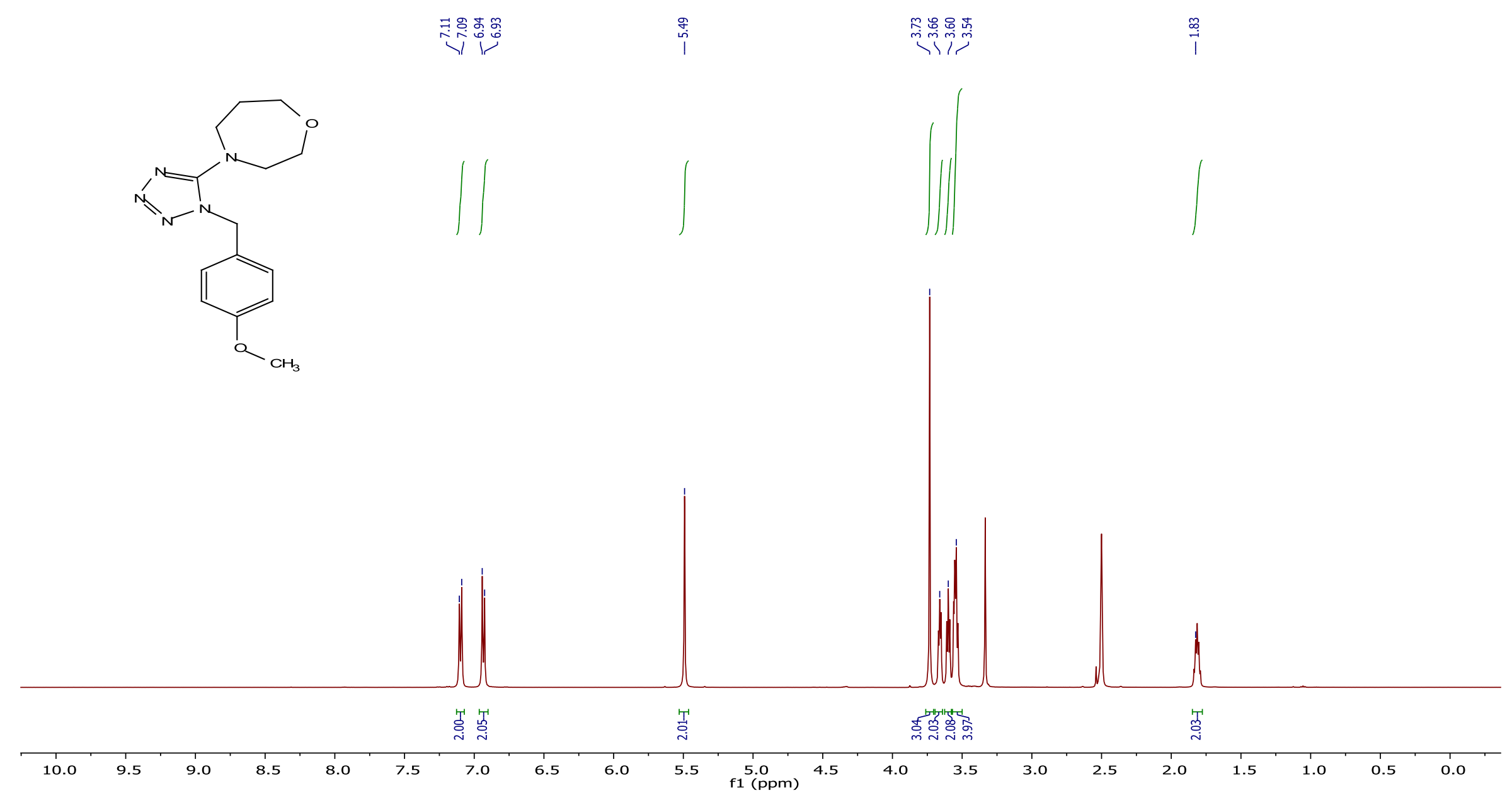


${ }^{13} \mathrm{C}$ NMR spectrum of the compound $\mathbf{9}\{18,300\}$.

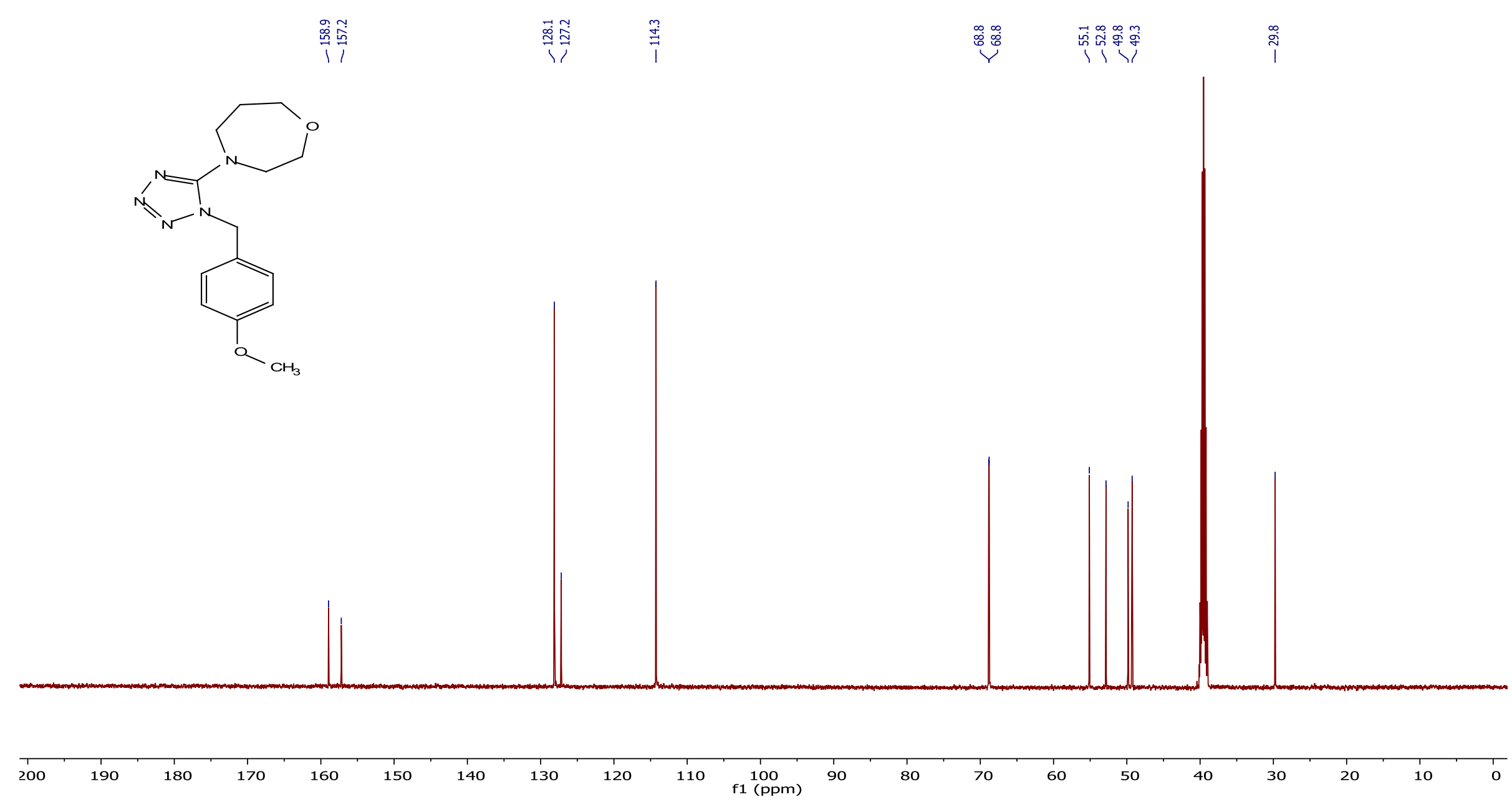


${ }^{1} \mathrm{H}$ NMR spectrum of the compound $\mathbf{9}\{25,147\}$.

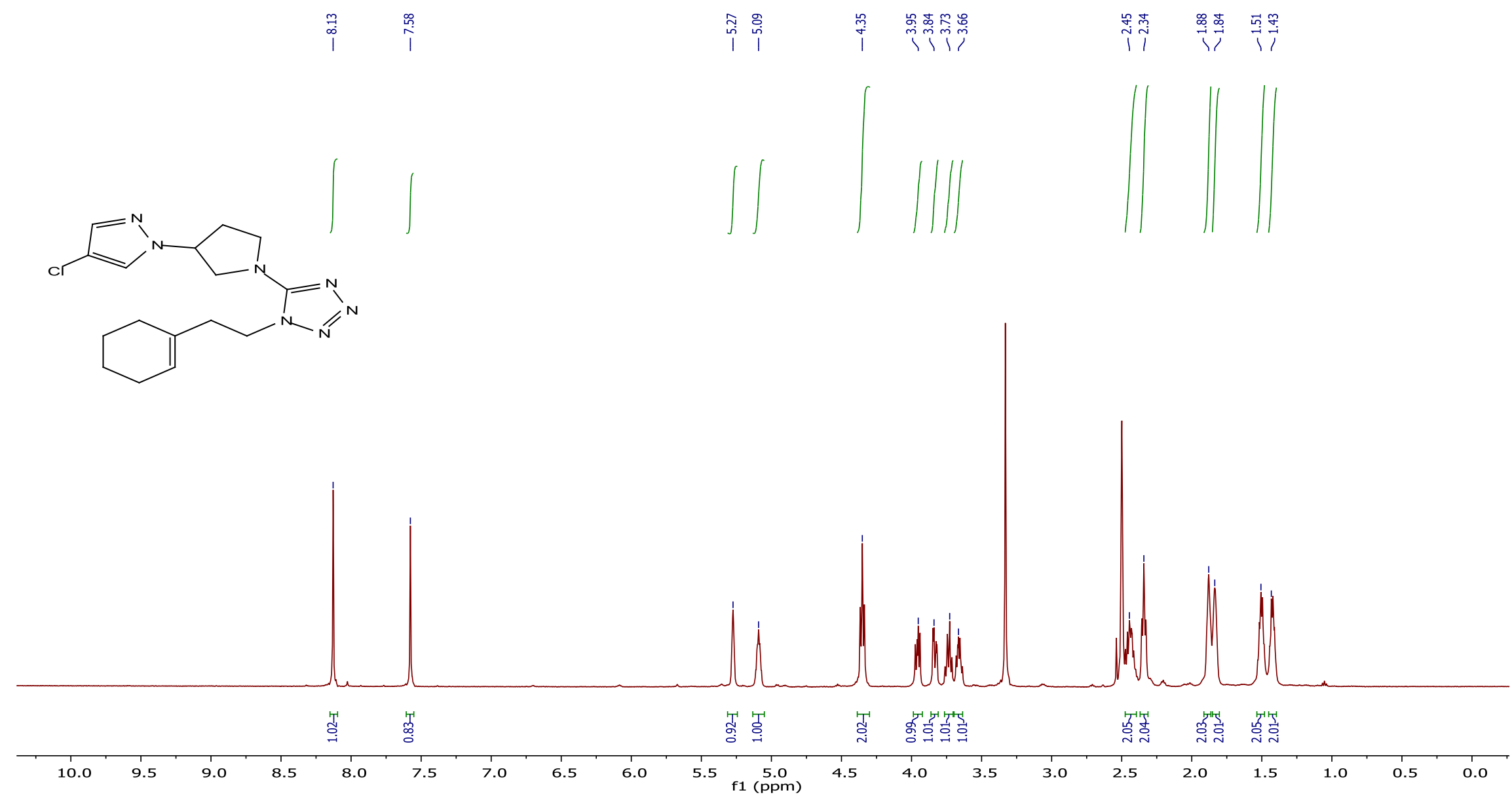


${ }^{13} \mathrm{C}$ NMR spectrum of the compound $\mathbf{9}\{25,147\}$.

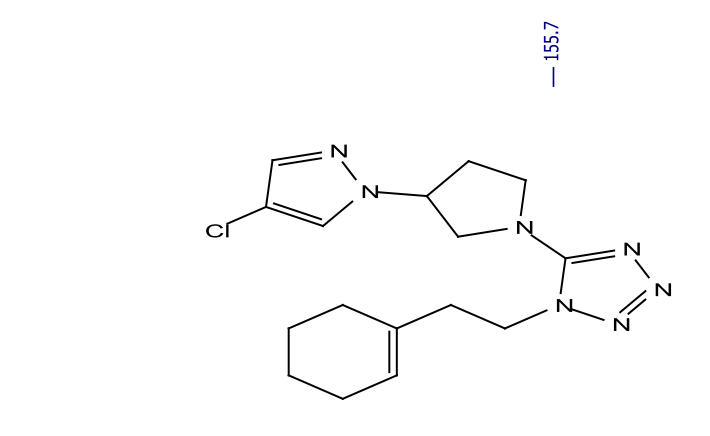

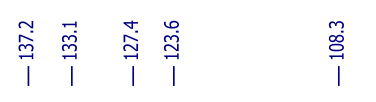

商

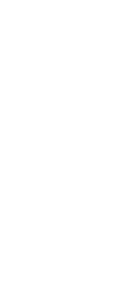

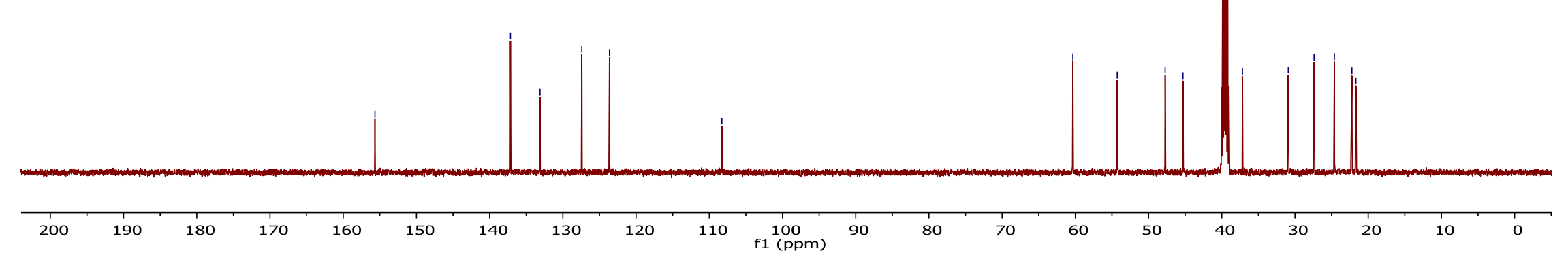


${ }^{1} \mathrm{H}$ NMR spectrum of the compound $\mathbf{9}\{52,319\}$.

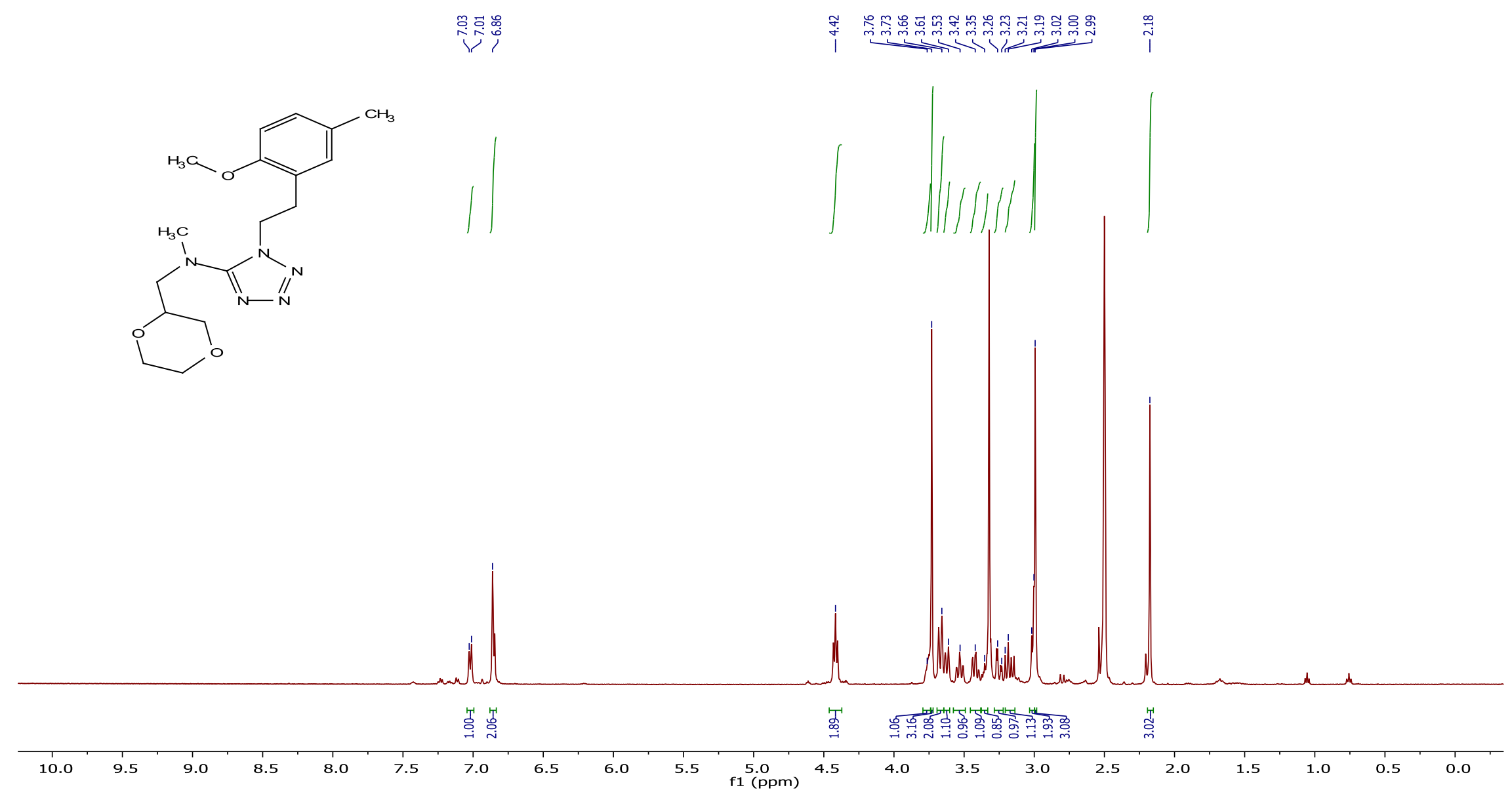


${ }^{13} \mathrm{C}$ NMR spectrum of the compound $\mathbf{9}\{52,319\}$.

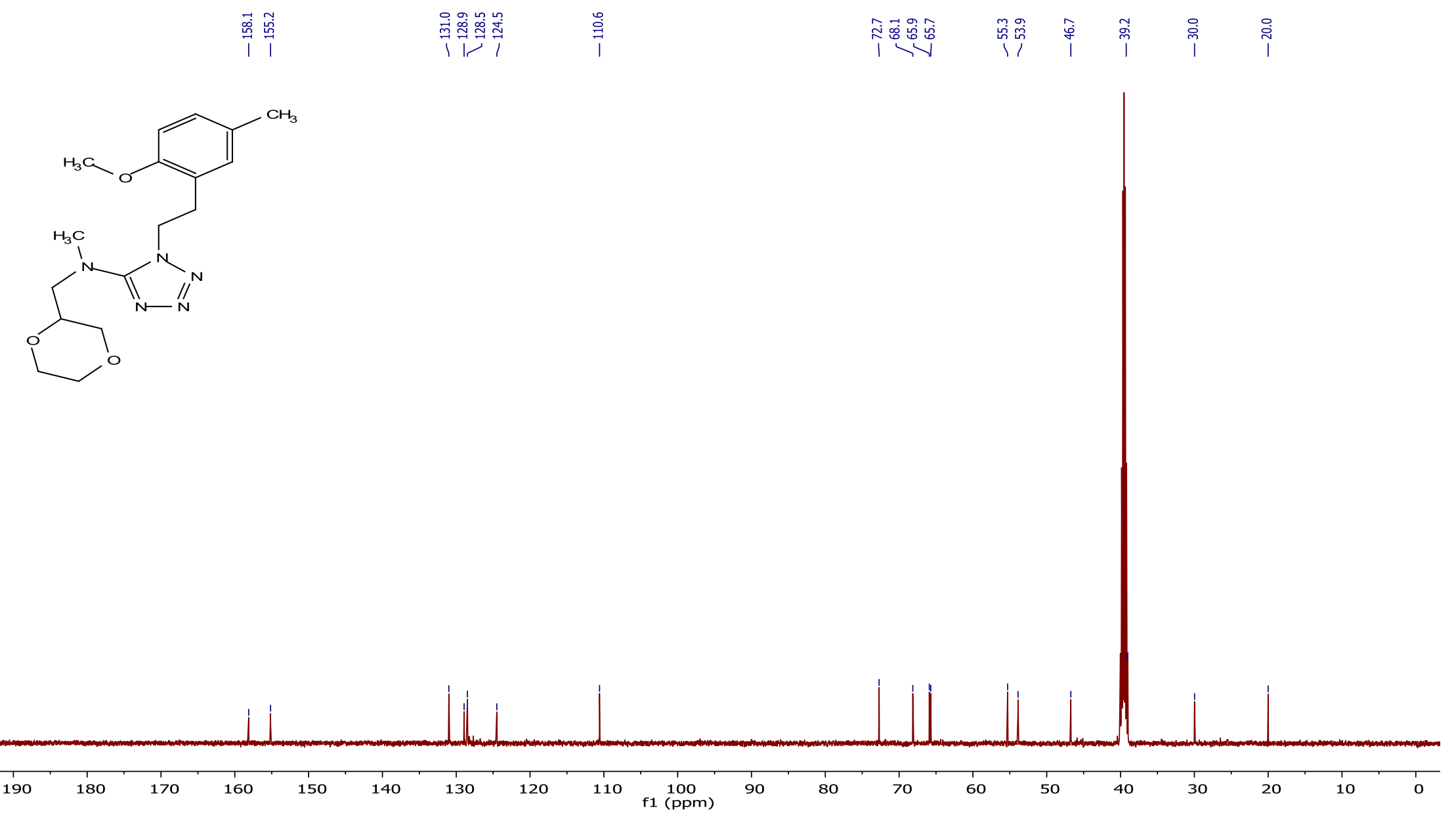


${ }^{1} \mathrm{H}$ NMR spectrum of the compound $\mathbf{9}\{55,337\}$.

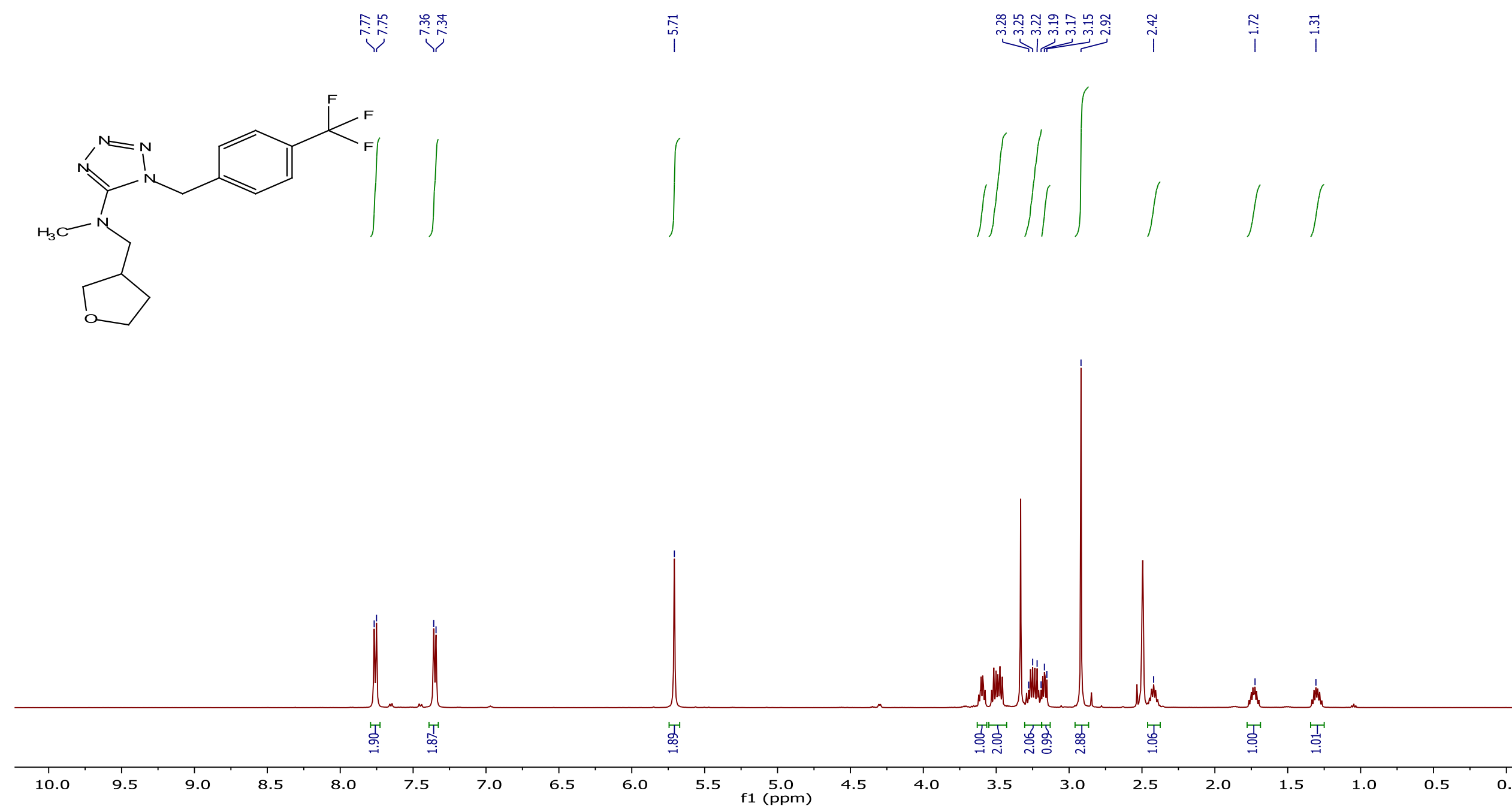


${ }^{13} \mathrm{C}$ NMR spectrum of the compound $\mathbf{9}\{55,337\}$.

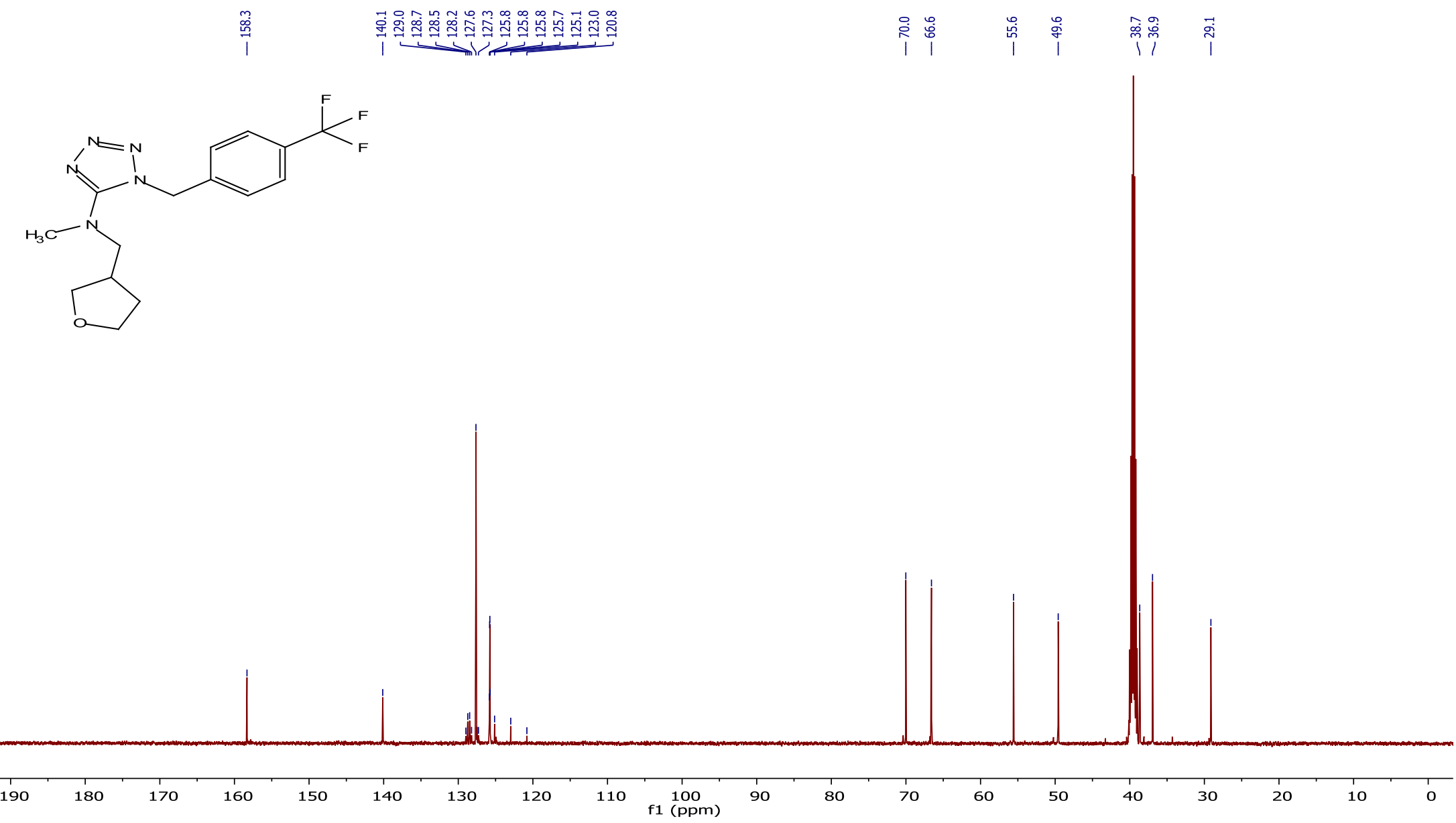


${ }^{19} \mathrm{~F}$ NMR spectrum of the compound $\mathbf{9}\{55,337\}$.

$\overrightarrow{\underline{i}}$
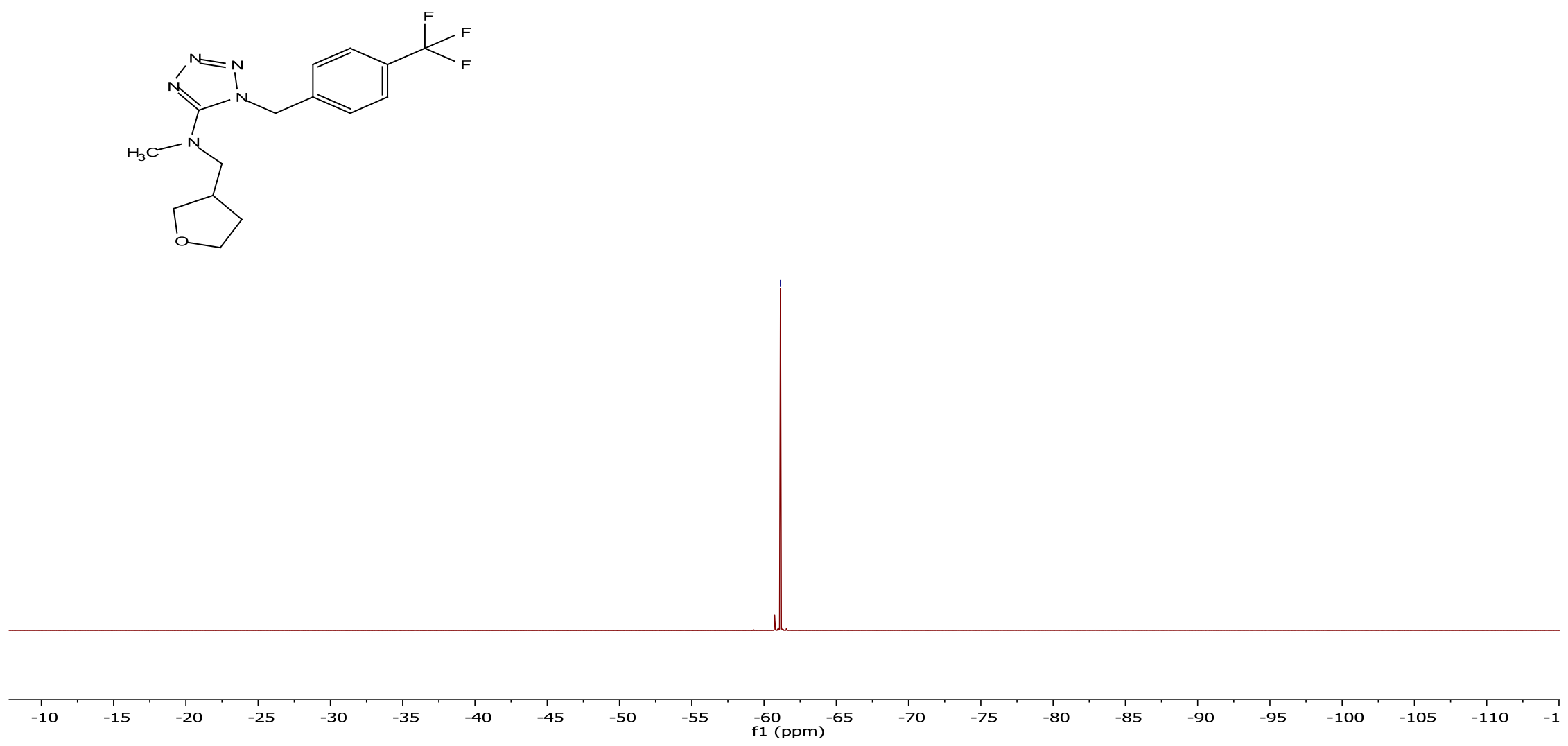
${ }^{1} \mathrm{H}$ NMR spectrum of the compound $\mathbf{9}\{57,261\}$.
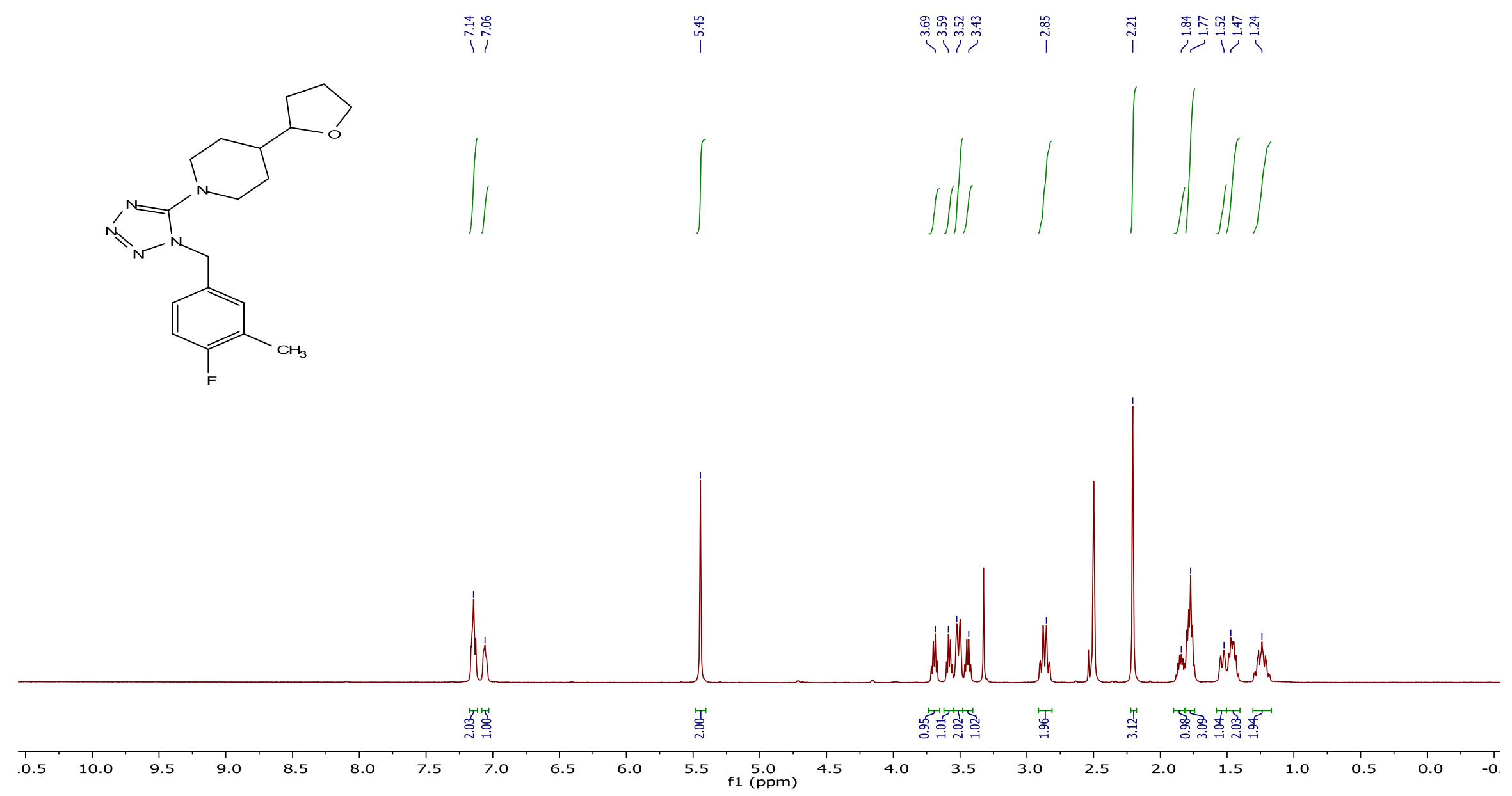
${ }^{13} \mathrm{C}$ NMR spectrum of the compound $\mathbf{9}\{57,261\}$.
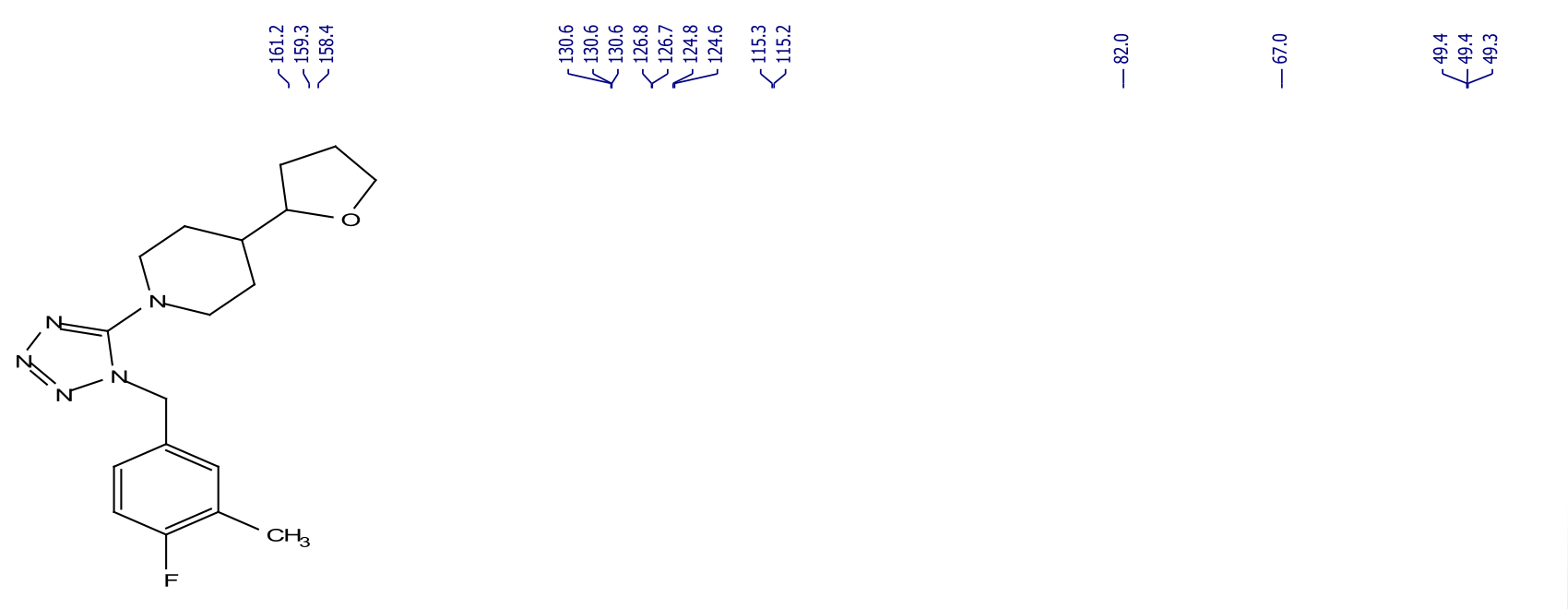

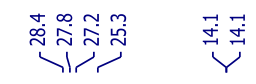

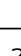


${ }^{19}$ F NMR spectrum of the compound $\mathbf{9}\{57,261\}$.

$\stackrel{\infty}{\stackrel{\infty}{\longrightarrow}}$
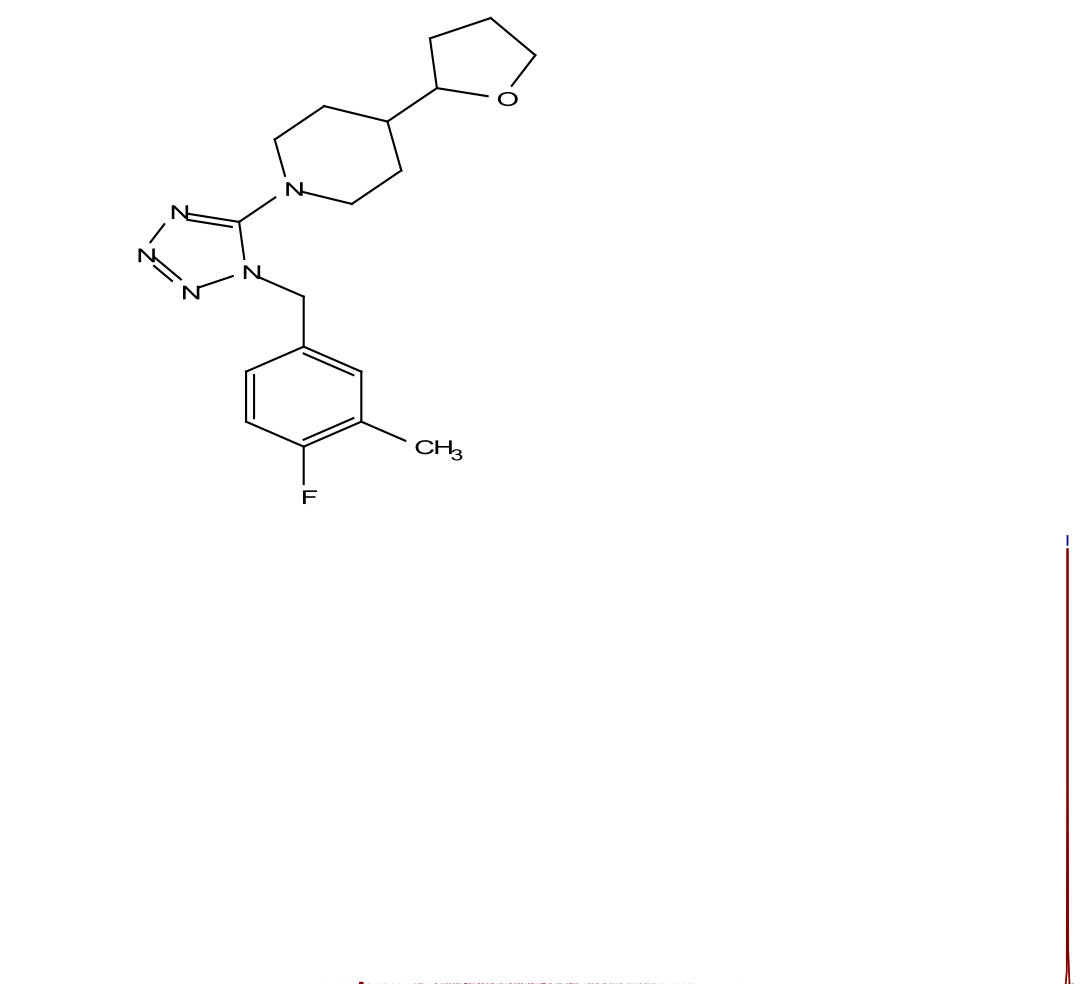
${ }^{1} \mathrm{H}$ NMR spectrum of the compound $\mathbf{9}\{57,300\}$.
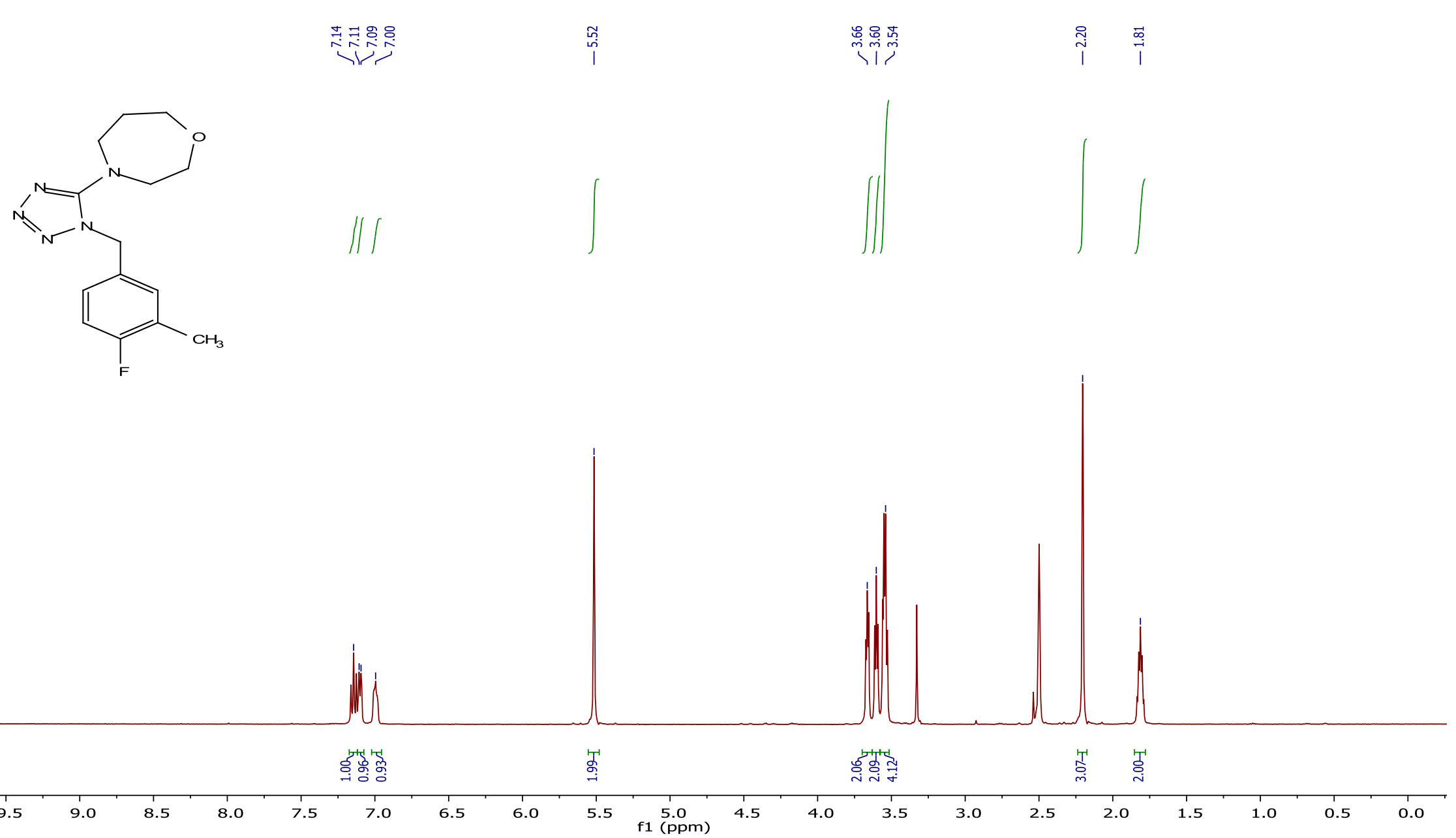
${ }^{13} \mathrm{C}$ NMR spectrum of the compound $\mathbf{9}\{57,300\}$.

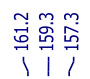

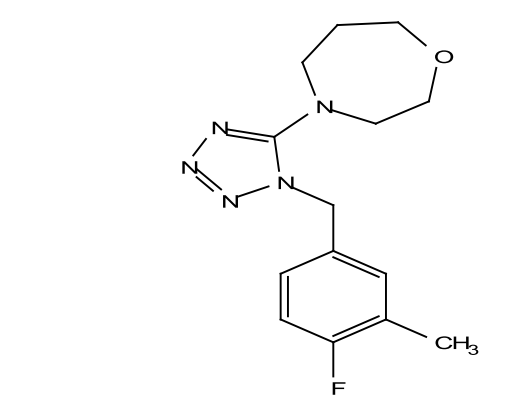

$\underbrace{\infty}_{\substack{\infty \\ \infty}}$

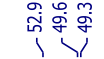

$\stackrel{\infty}{\substack{i \\ i}}$

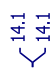

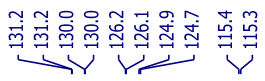
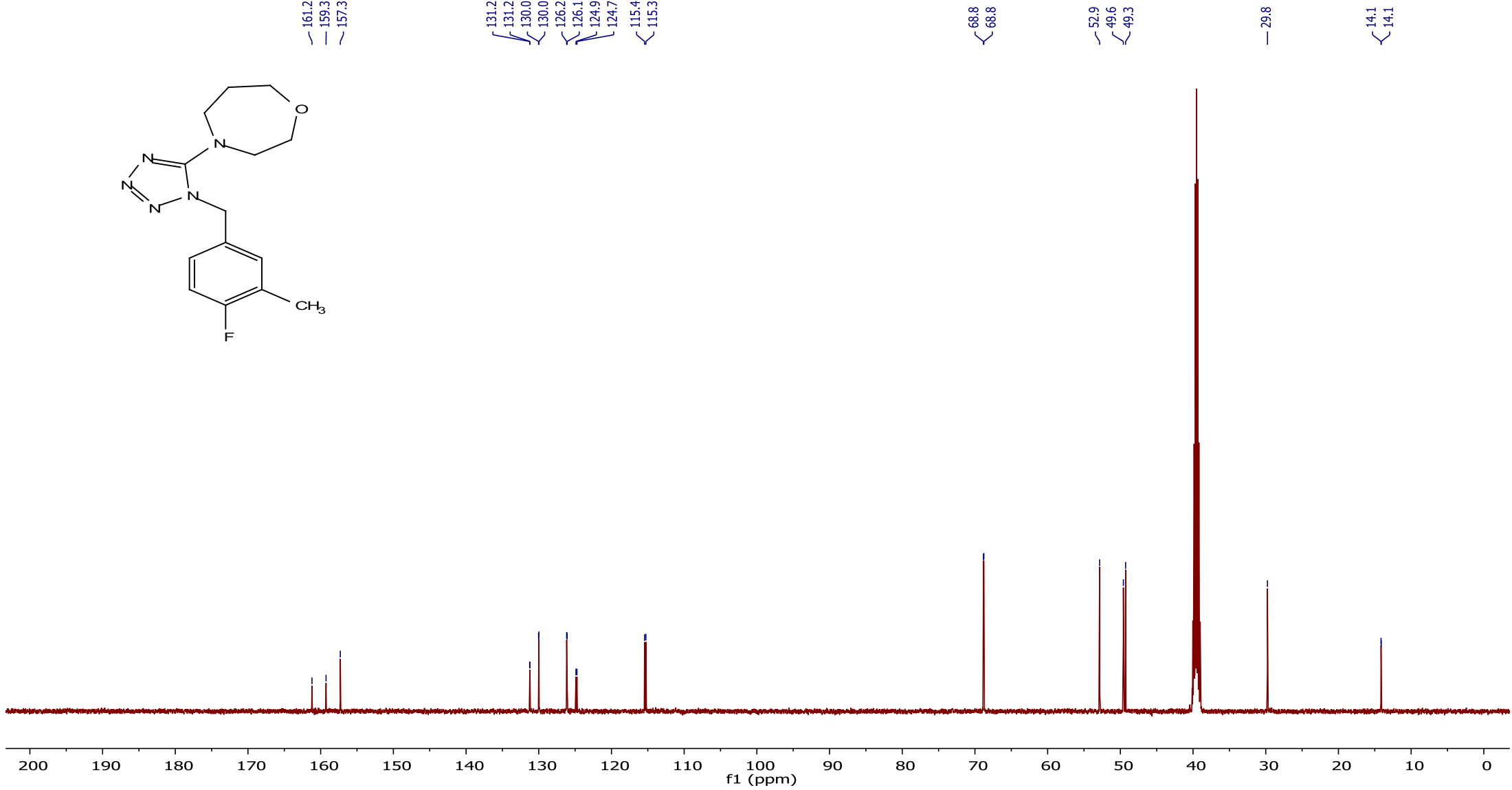

S243 
${ }^{19}$ F NMR spectrum of the compound $\mathbf{9}\{57,300\}$.

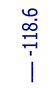
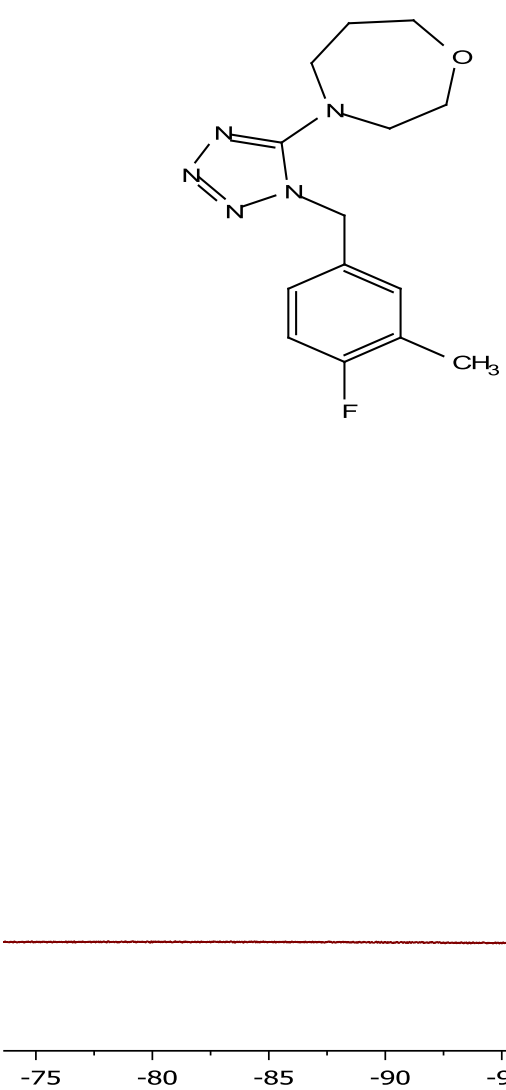

$-85$

$-90$

$-95$

$-100$

$-105$
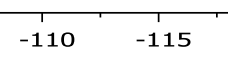

$-120 \quad-125$

$-130$

$-135$

$-140$

$-145$

$-150$

$-155$ 
${ }^{1} \mathrm{H}$ NMR spectrum of the compound $\mathbf{9}\{63,327\}$.
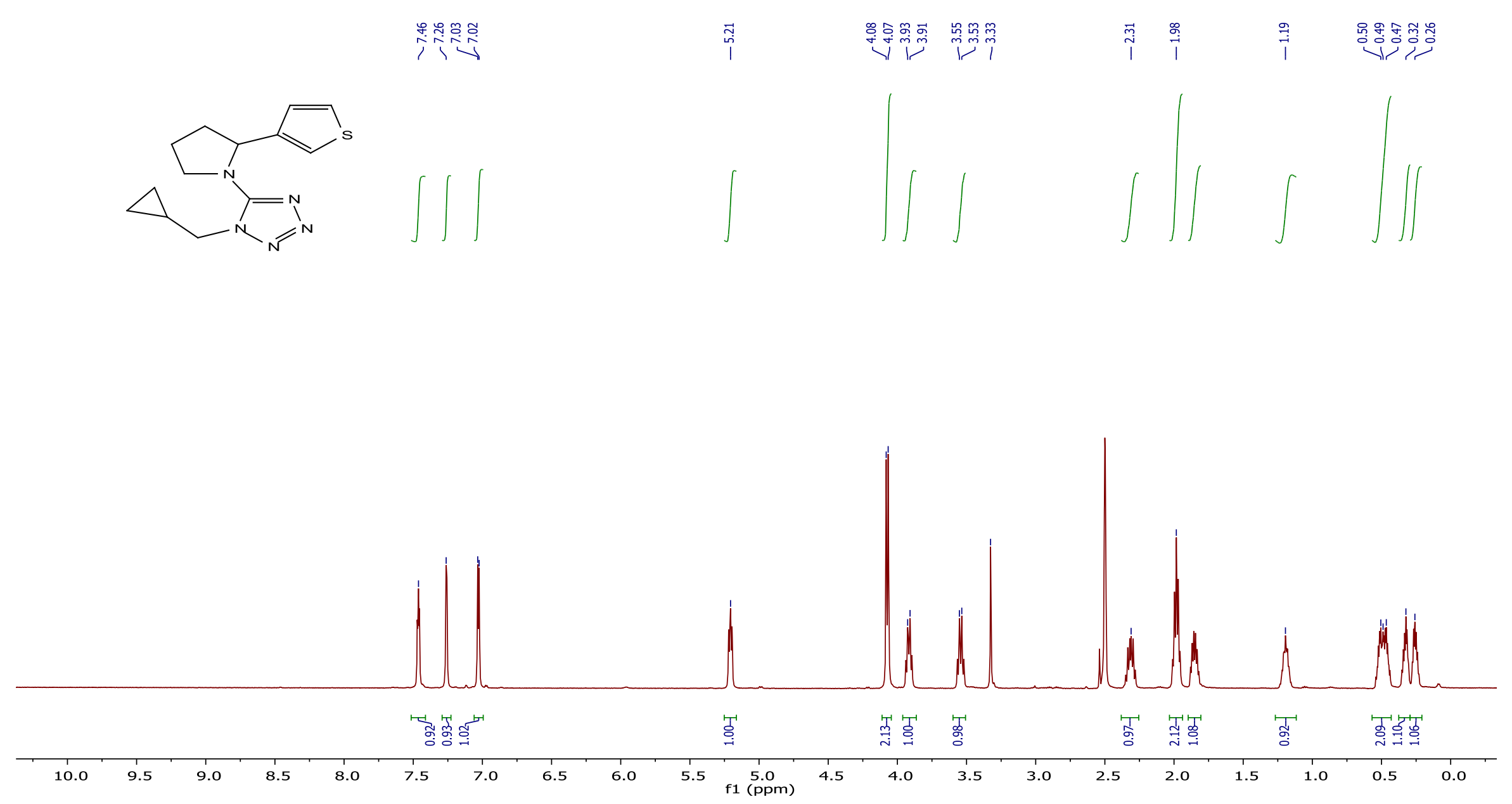
${ }^{13} \mathrm{C}$ NMR spectrum of the compound $\mathbf{9}\{63,327\}$.

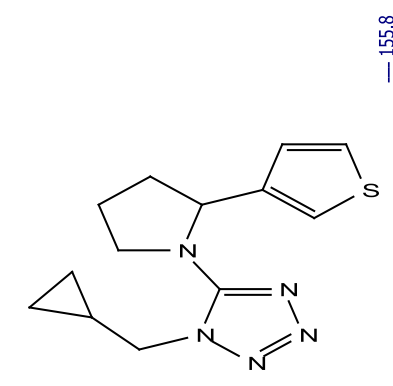

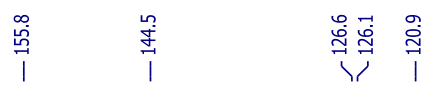

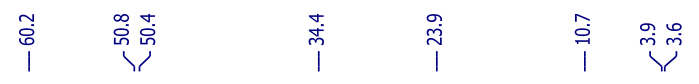

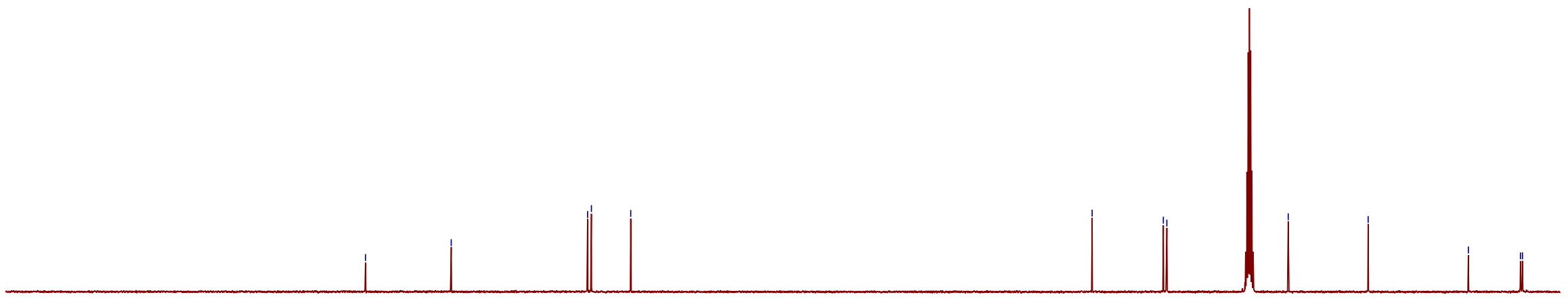

200

190

180

$170 \quad 160$

150

$140 \quad 130$

120

$110 \quad 100$

90

80

70

60

50

40

30

20

$10 \quad 0$ 
${ }^{1} \mathrm{H}$ NMR spectrum of the compound $\mathbf{9}\{68,300\}$.
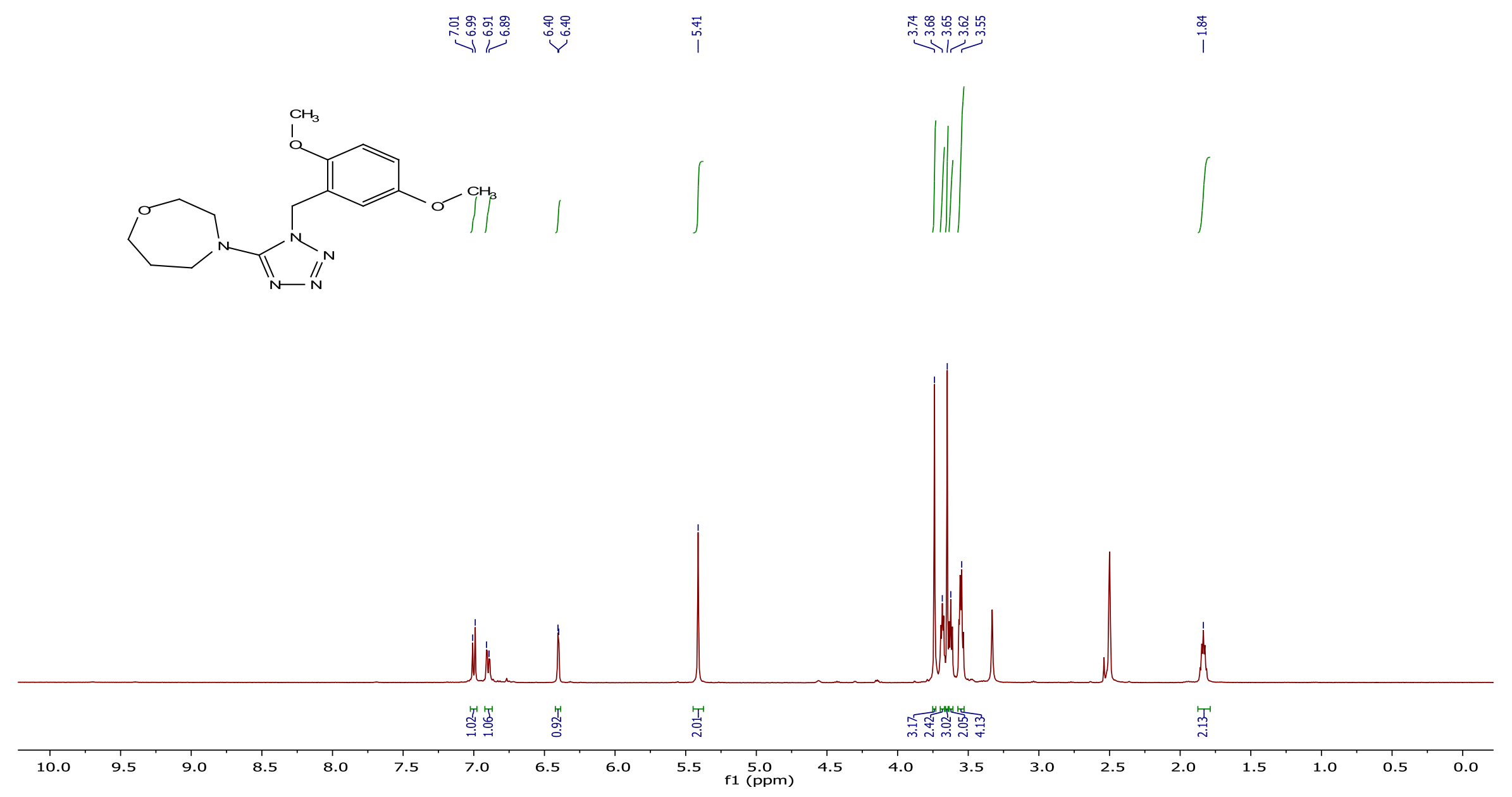
${ }^{13} \mathrm{C}$ NMR spectrum of the compound $\mathbf{9}\{68,300\}$.
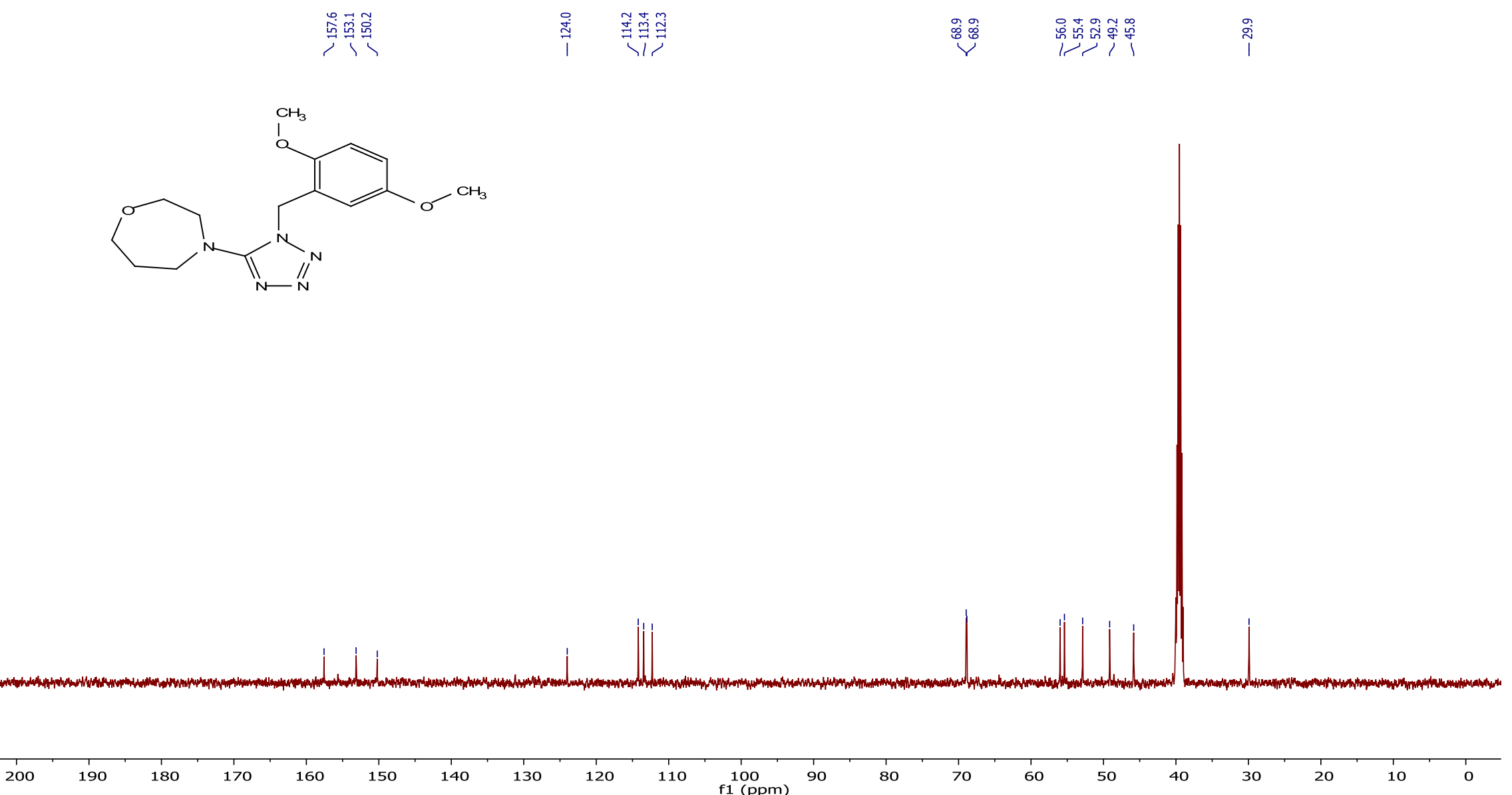
${ }^{1} \mathrm{H}$ NMR spectrum of the compound $\mathbf{9}\{77,363\}$.

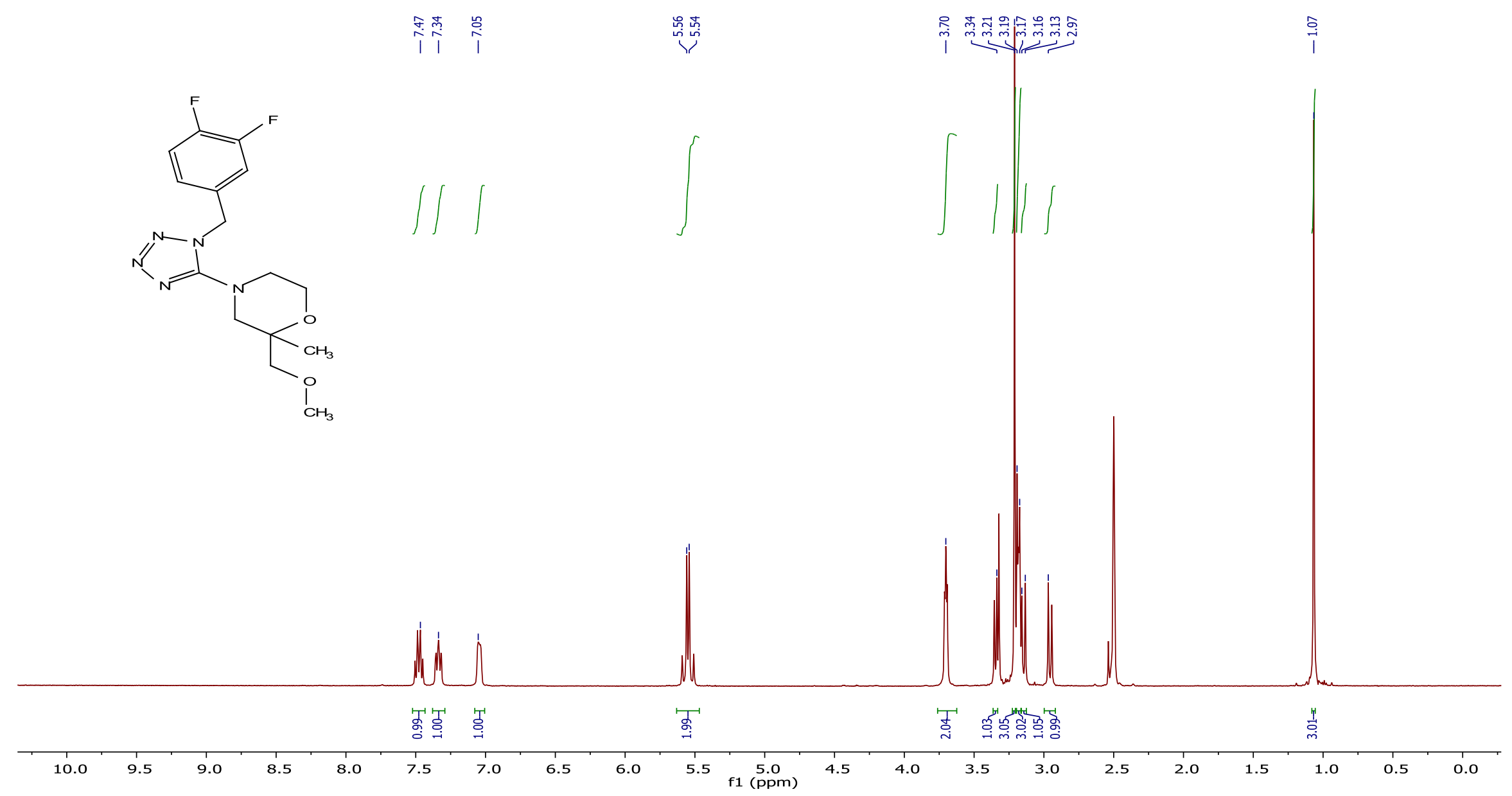


${ }^{13} \mathrm{C}$ NMR spectrum of the compound $\mathbf{9}\{77,363\}$.

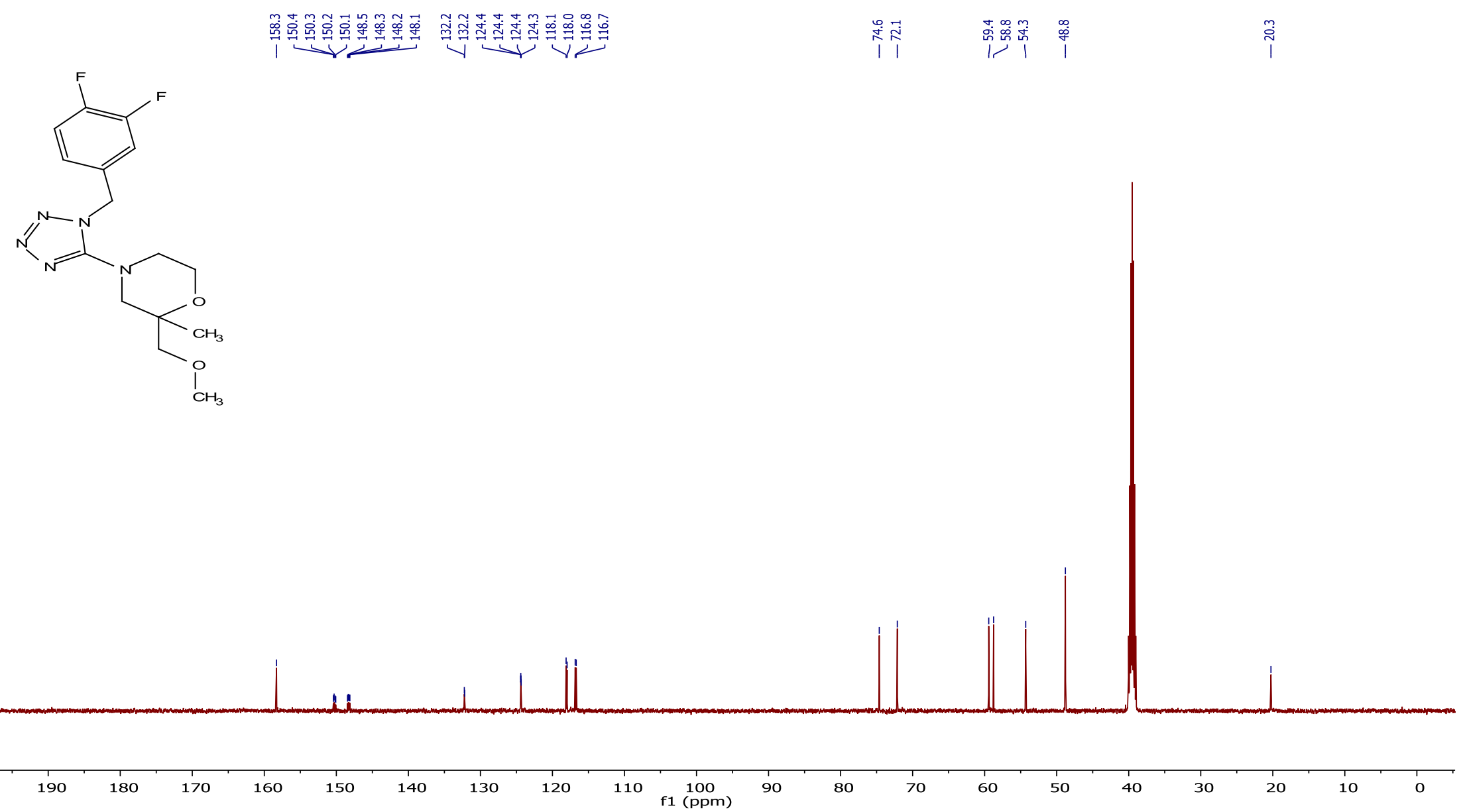


${ }^{19}$ F NMR spectrum of the compound $\mathbf{9}\{77,363\}$.

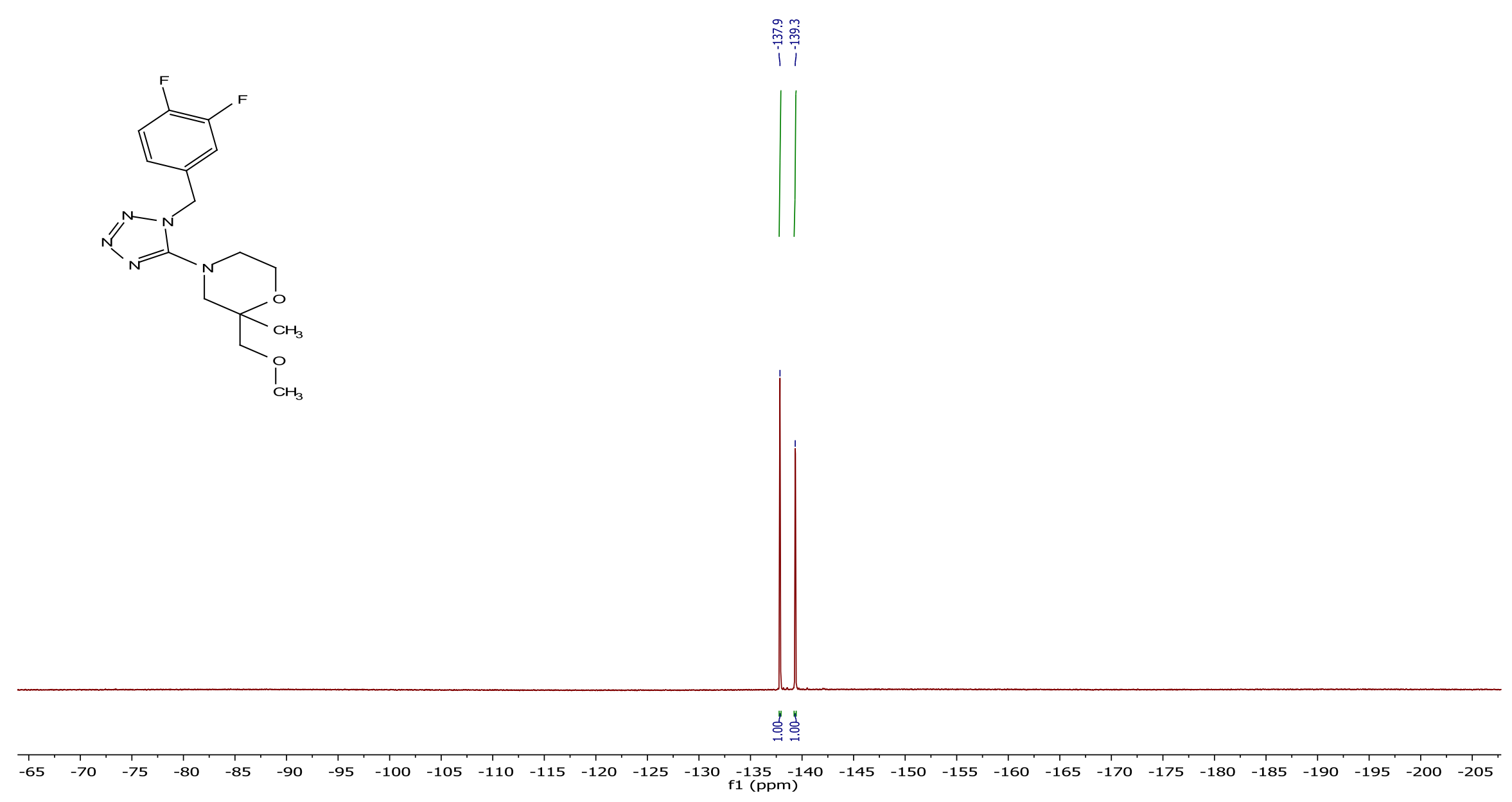


${ }^{1} \mathrm{H}$ NMR spectrum of the compound $\mathbf{9}\{84,319\}$.

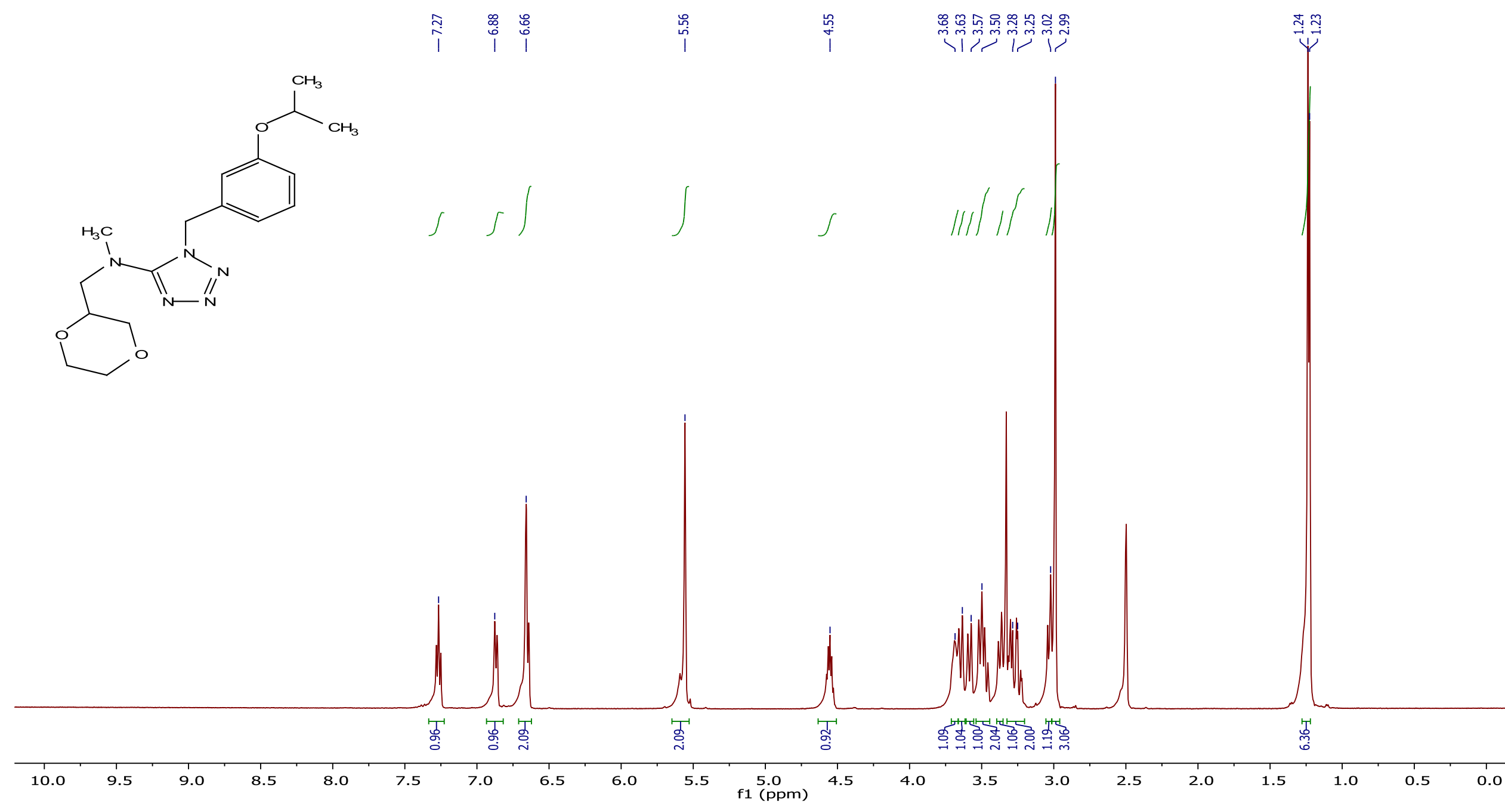


${ }^{13} \mathrm{C}$ NMR spectrum of the compound $\mathbf{9}\{84,319\}$.

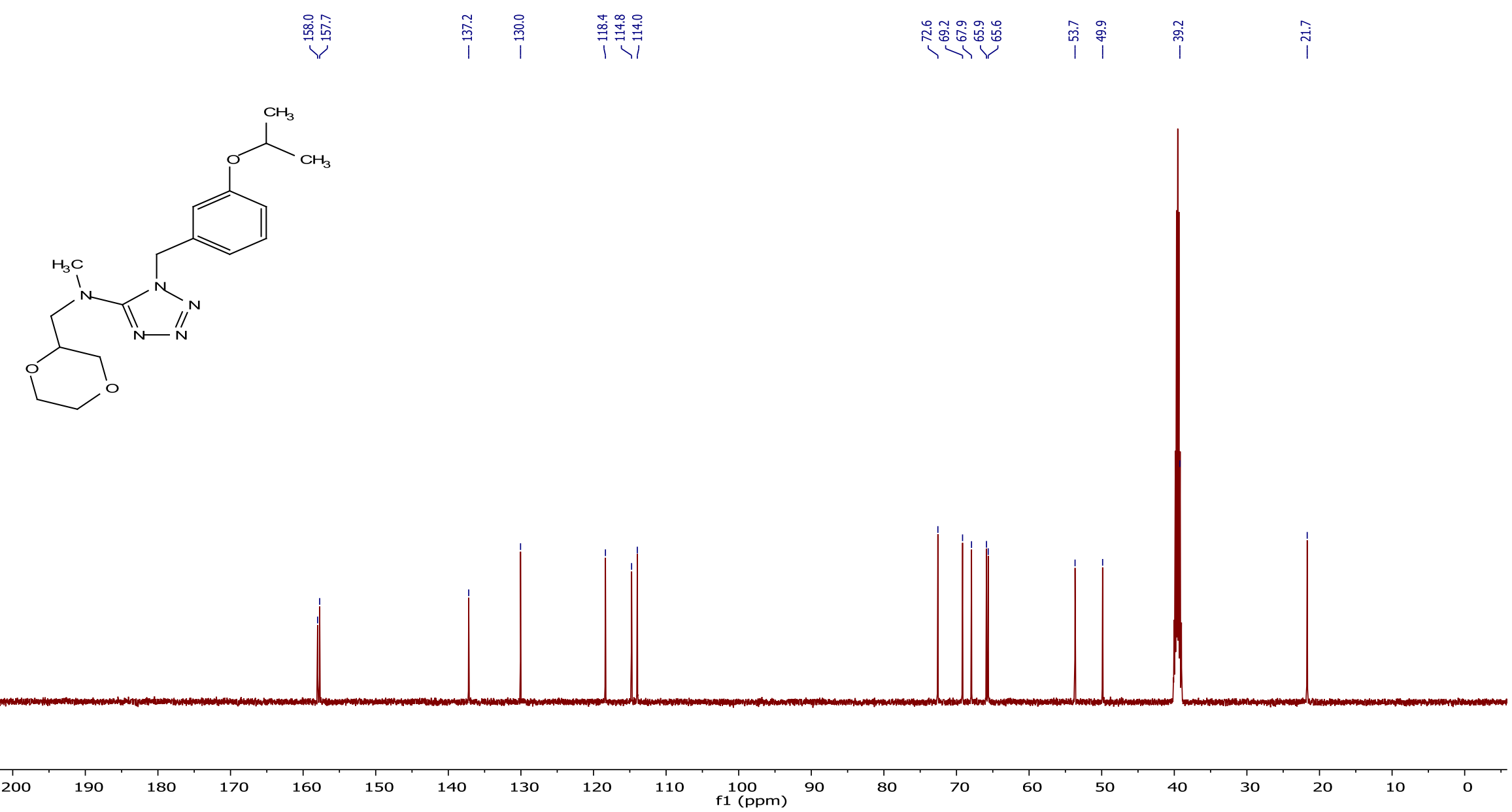


${ }^{1} \mathrm{H}$ NMR spectrum of the compound $\mathbf{9}\{90,371\}$.

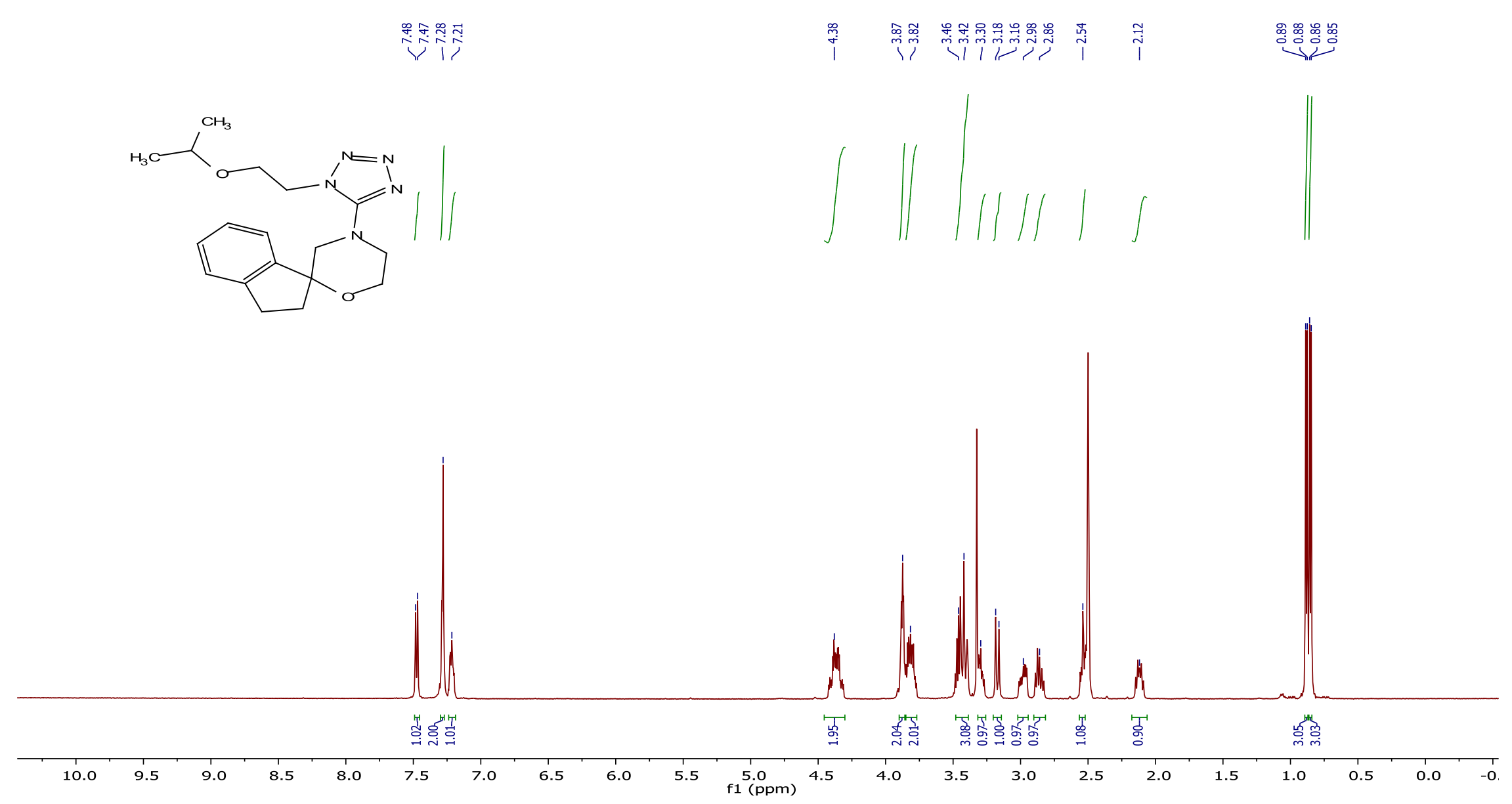


${ }^{13} \mathrm{C}$ NMR spectrum of the compound $\mathbf{9}\{90,371\}$.

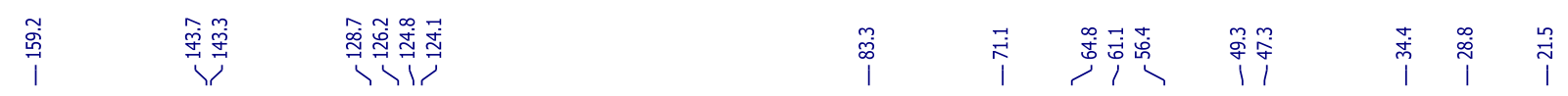
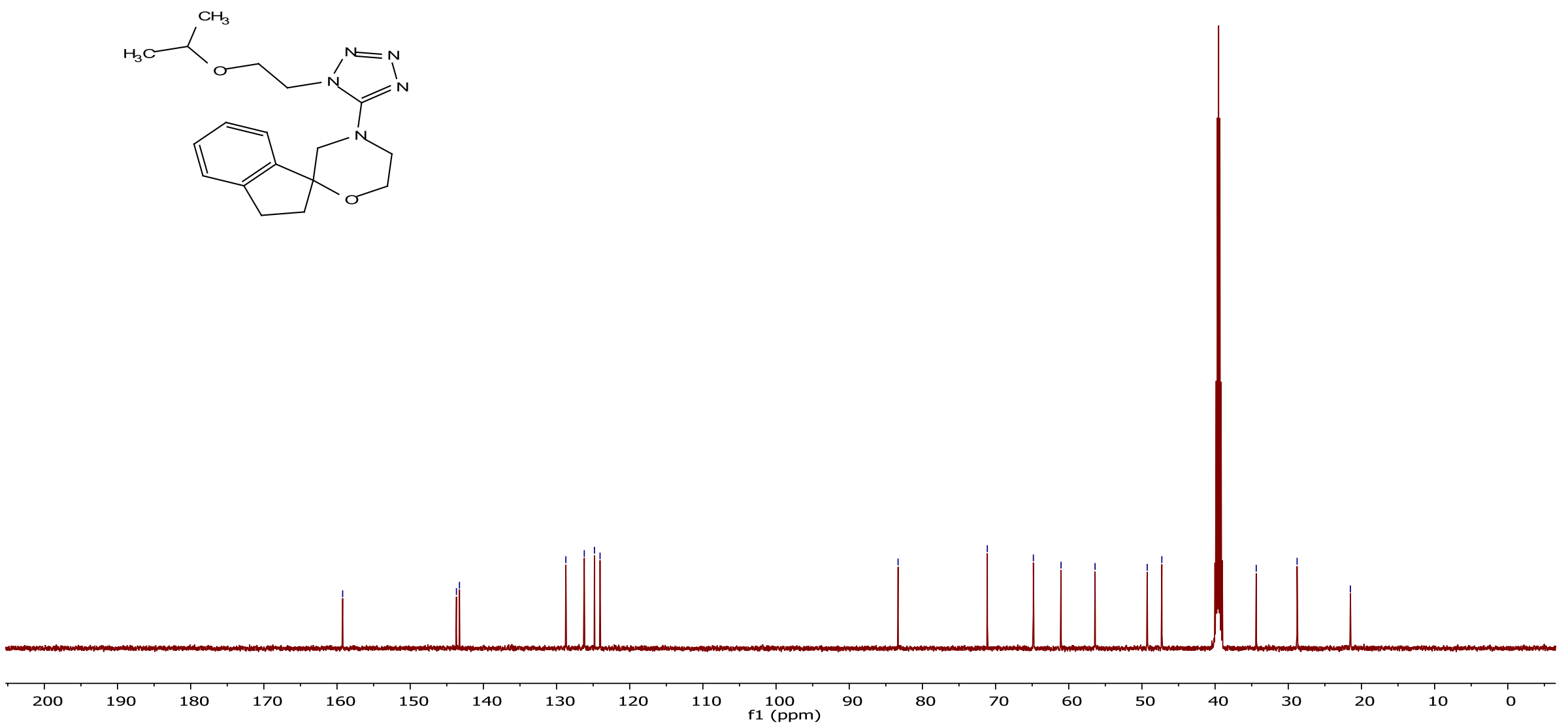
${ }^{1} \mathrm{H}$ NMR spectrum of the compound $\mathbf{9}\{102,356\}$.

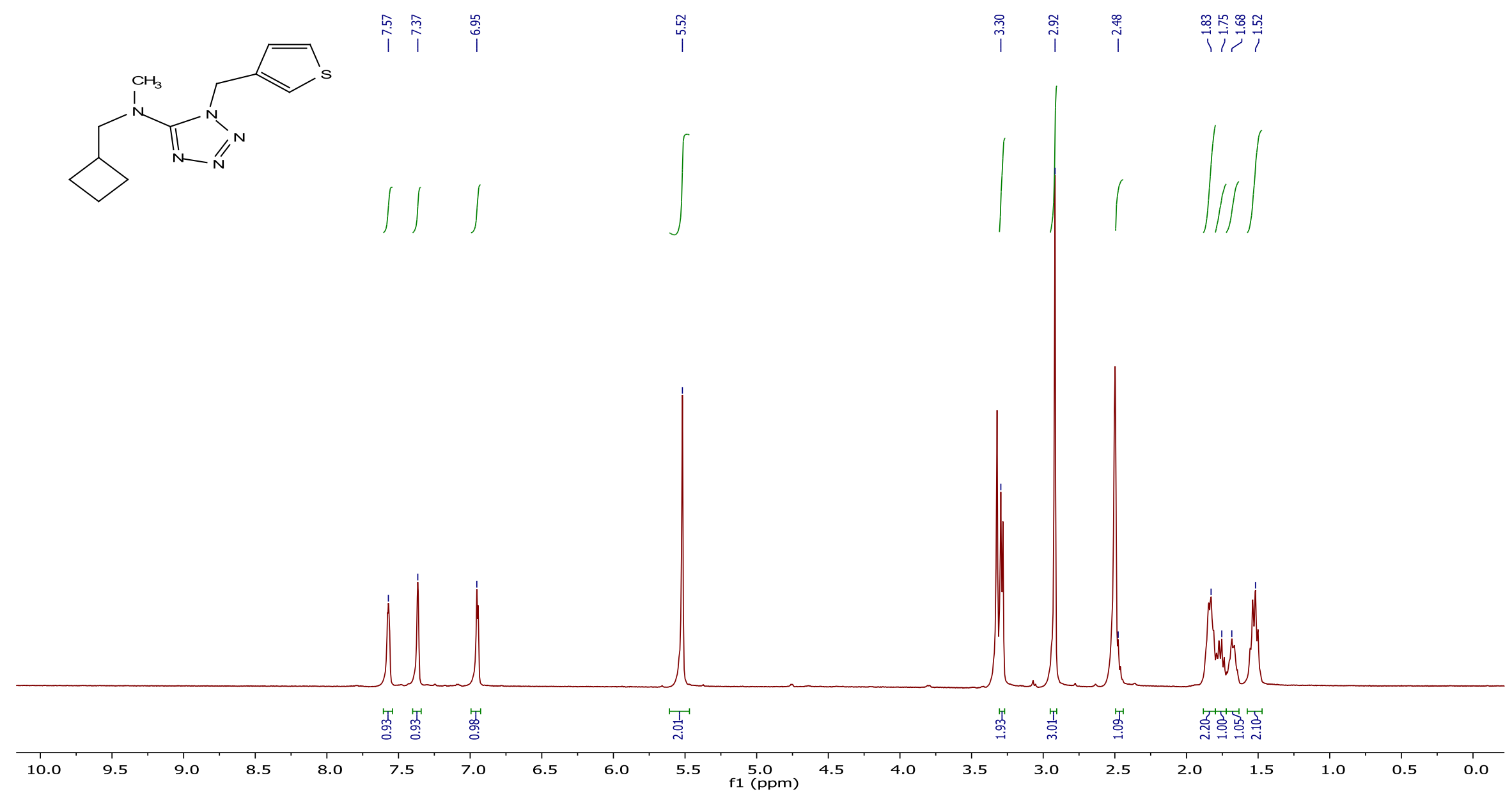


${ }^{13} \mathrm{C}$ NMR spectrum of the compound $\mathbf{9}\{102,356\}$.

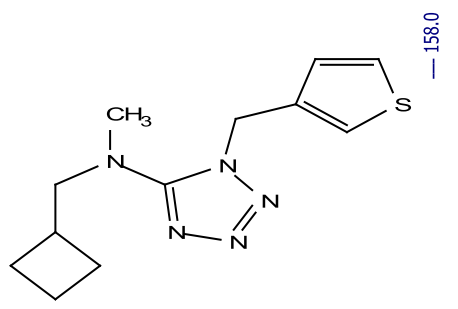

商

$\stackrel{\vec{\infty}}{\text { i }}$

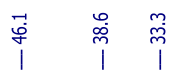

$\stackrel{\infty}{\stackrel{\infty}{i}}$

$\stackrel{\overrightarrow{0}}{\overrightarrow{1}}$

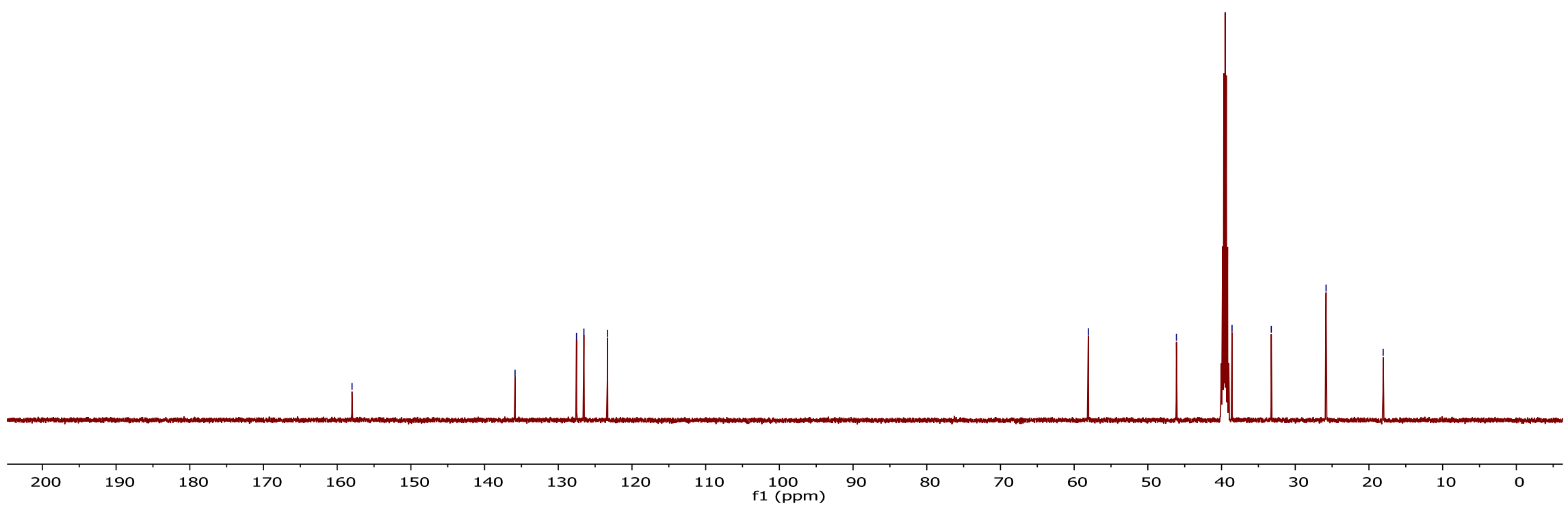

S257 
${ }^{1} \mathrm{H}$ NMR spectrum of the compound $\mathbf{9}\{103,386\}$.

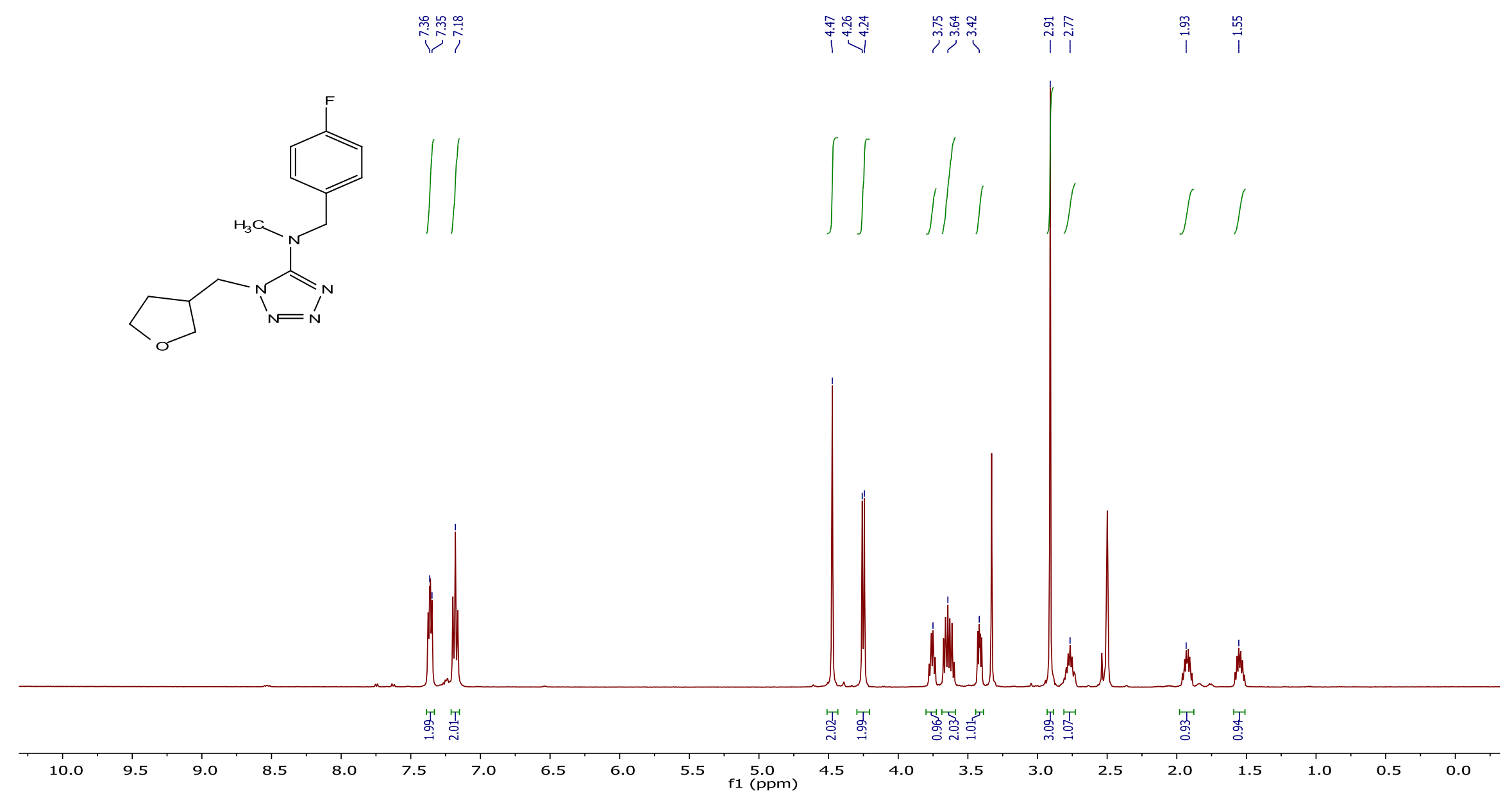


${ }^{13} \mathrm{C}$ NMR spectrum of the compound $\mathbf{9}\{103,386\}$.
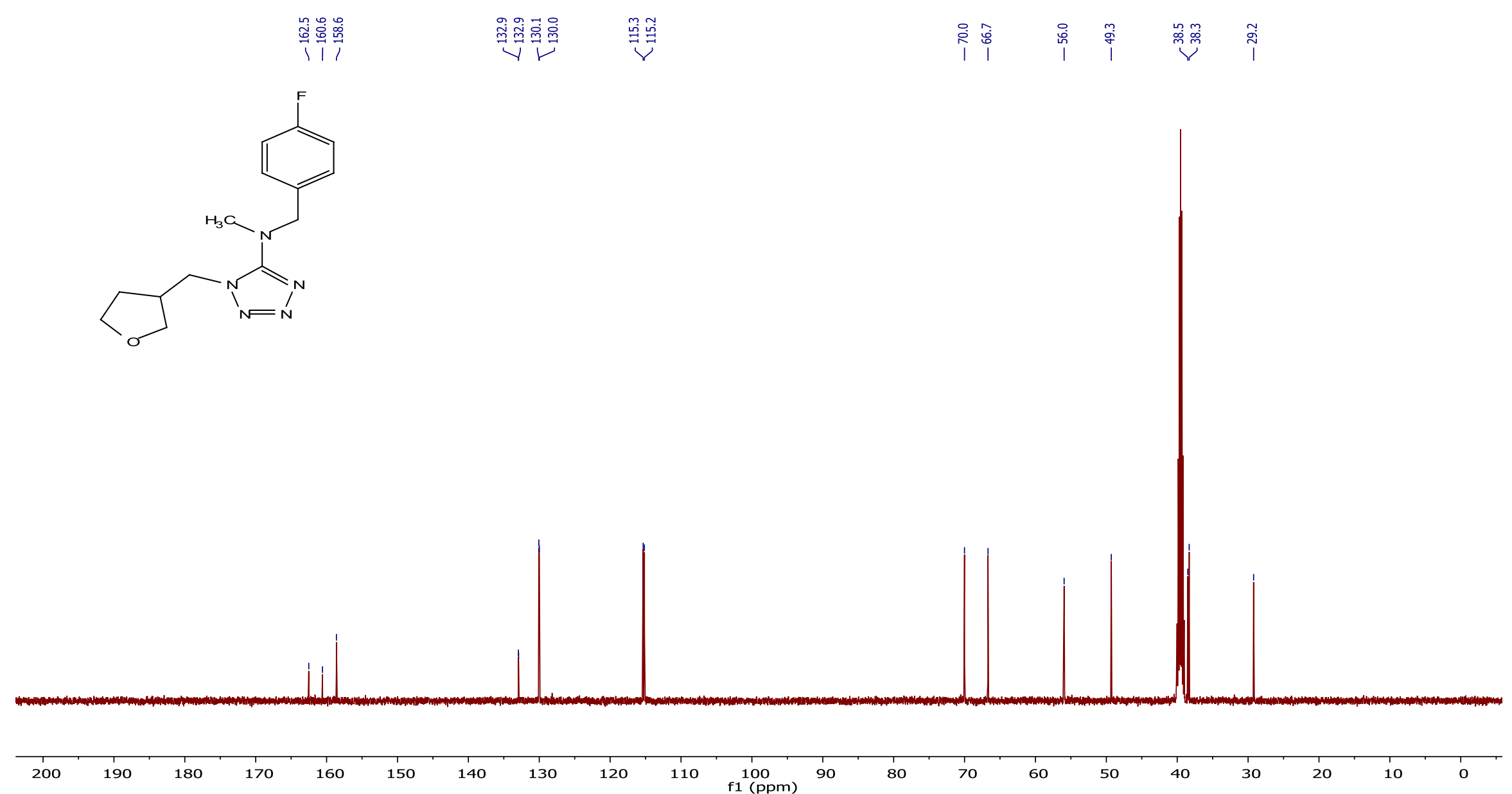
${ }^{19}$ F NMR spectrum of the compound $\mathbf{9}\{103,386\}$.

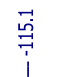
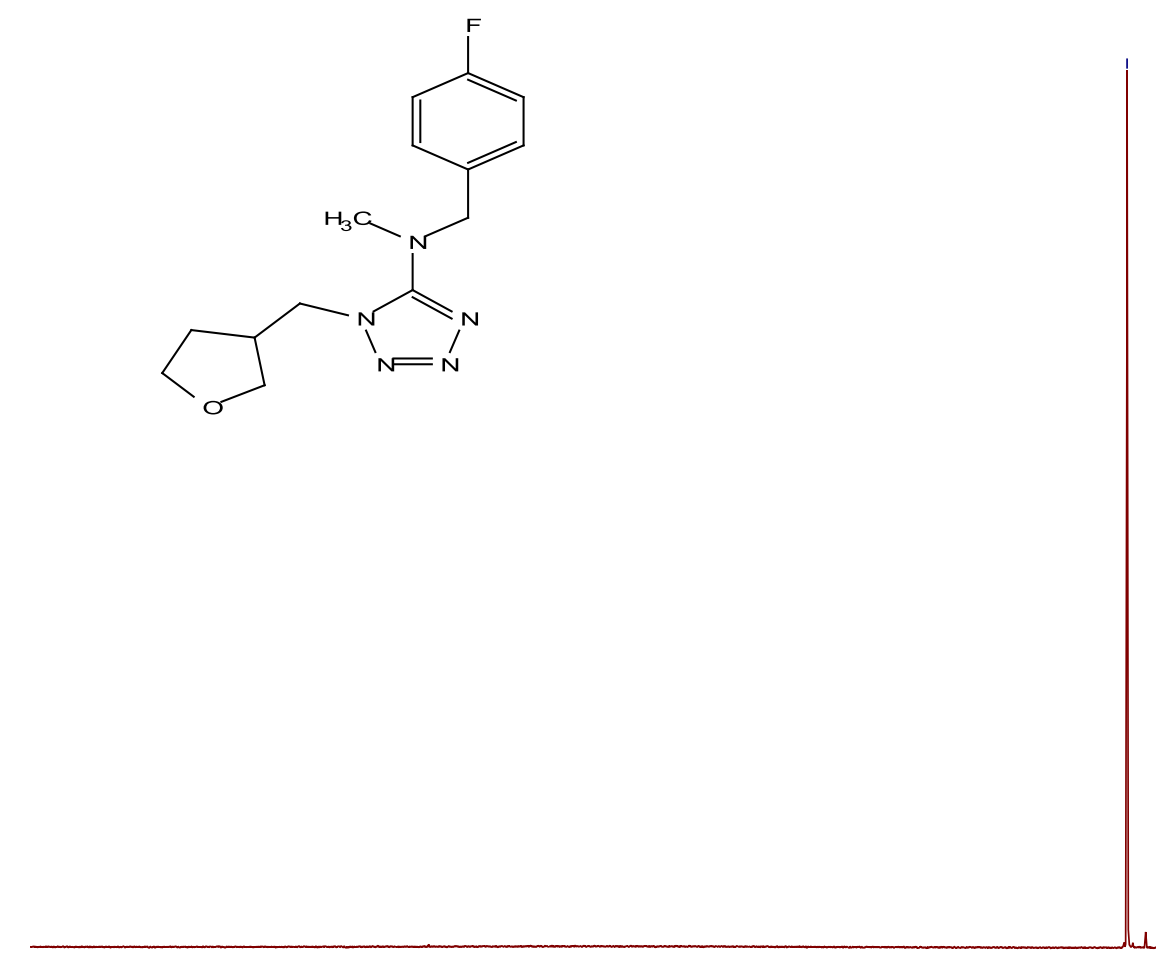

$\begin{array}{lllllll}50 & -55 & -60 & -65 & -70 & -75 & -80\end{array}$ 
${ }^{1} \mathrm{H}$ NMR spectrum of the compound $\mathbf{9}\{110,333\}$.

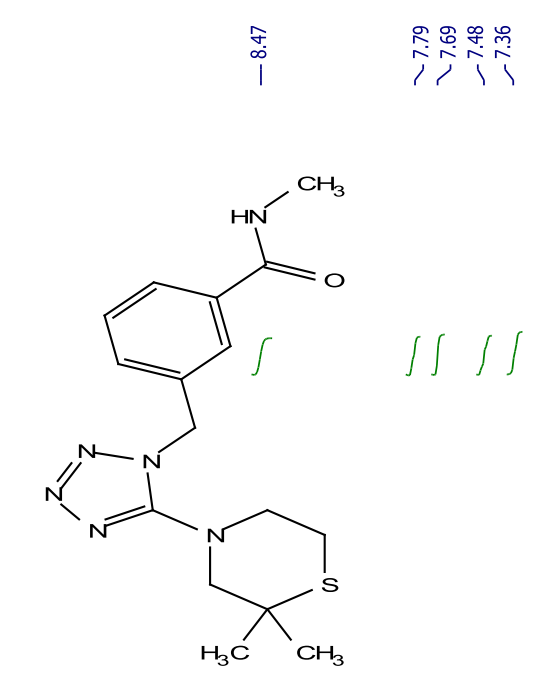

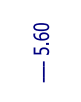

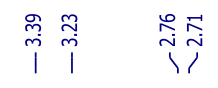

$\stackrel{7}{1}$
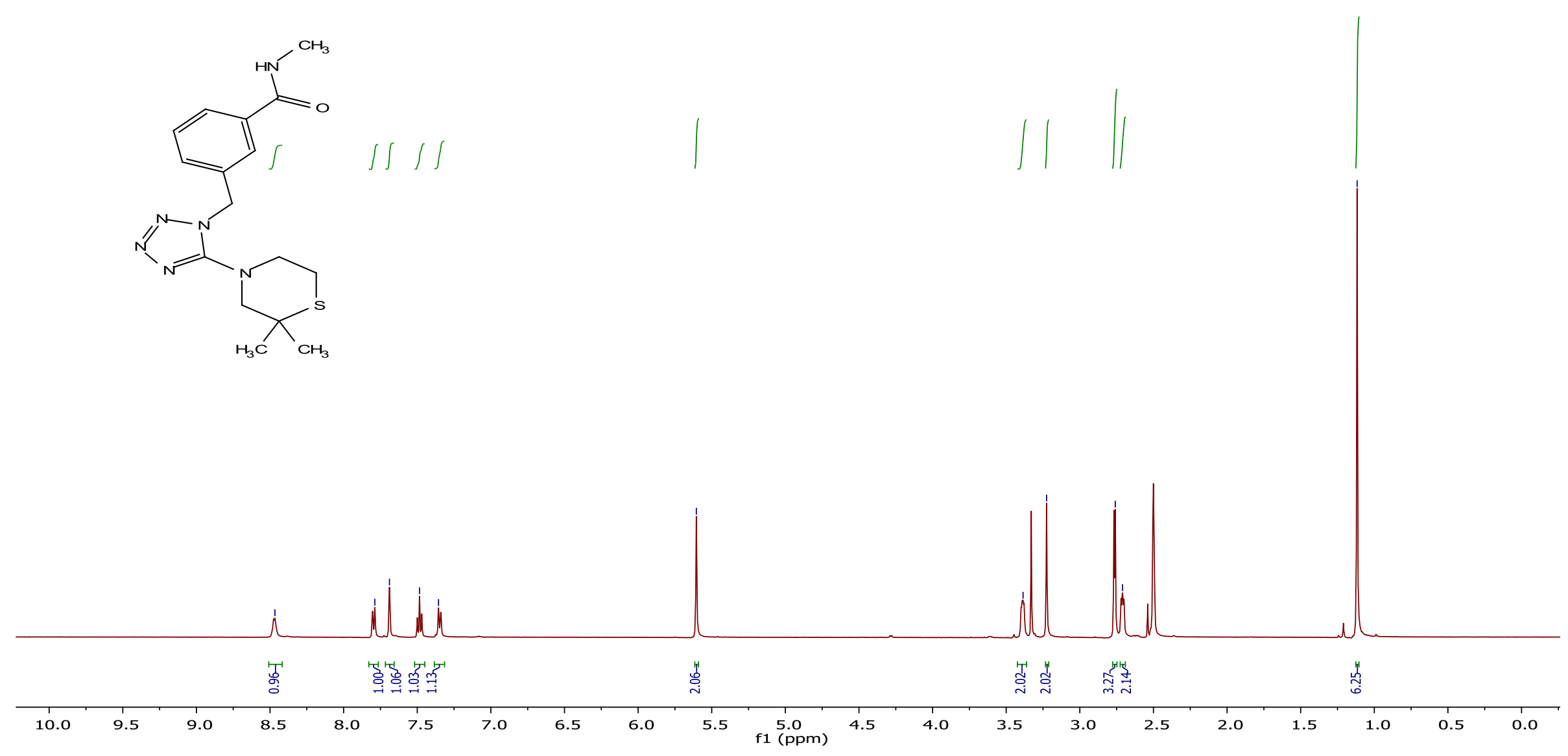
${ }^{13} \mathrm{C}$ NMR spectrum of the compound $\mathbf{9}\{110,333\}$.
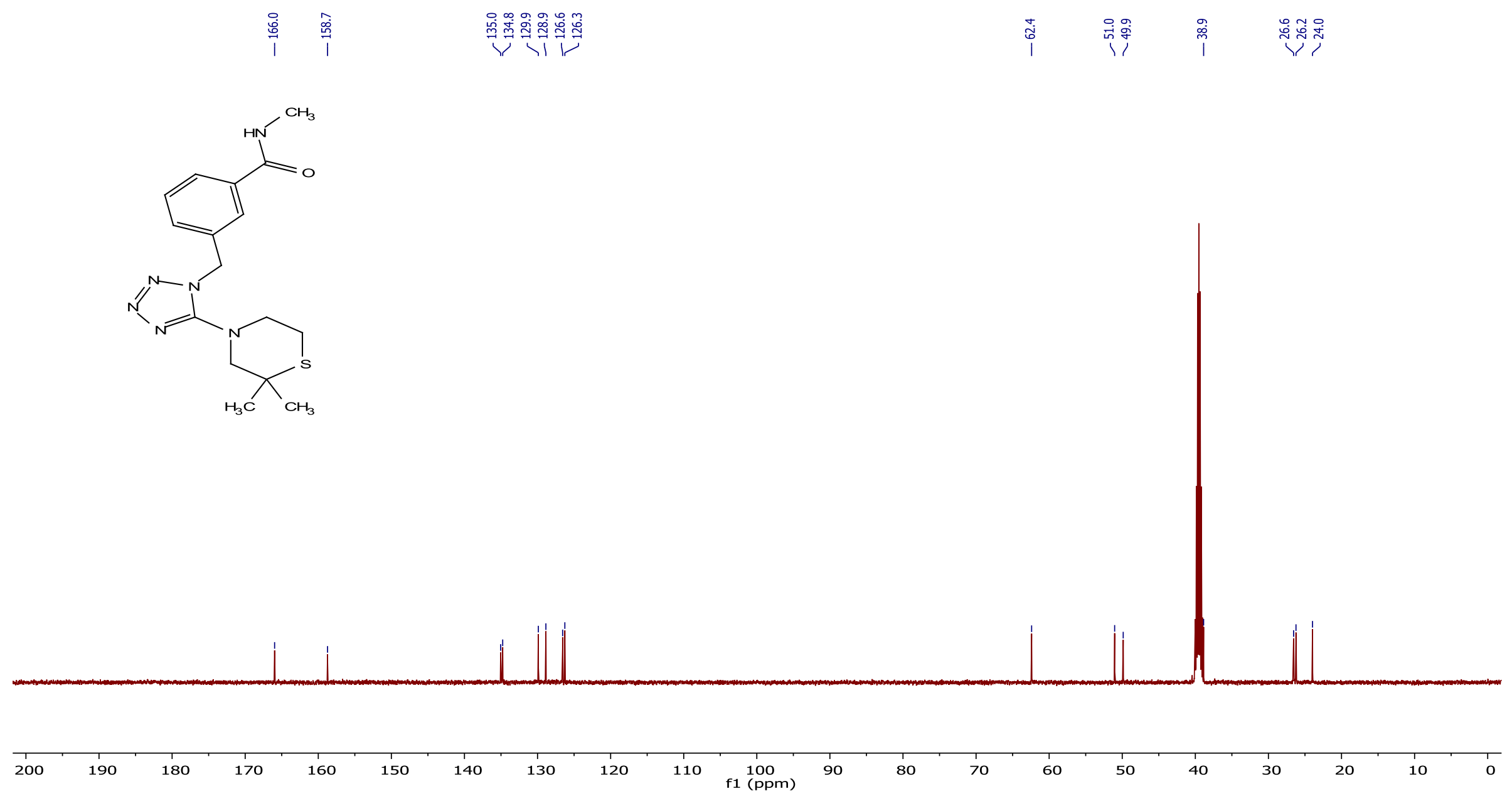
${ }^{1} \mathrm{H}$ NMR spectrum of the compound $\mathbf{9}\{121,302\}$.

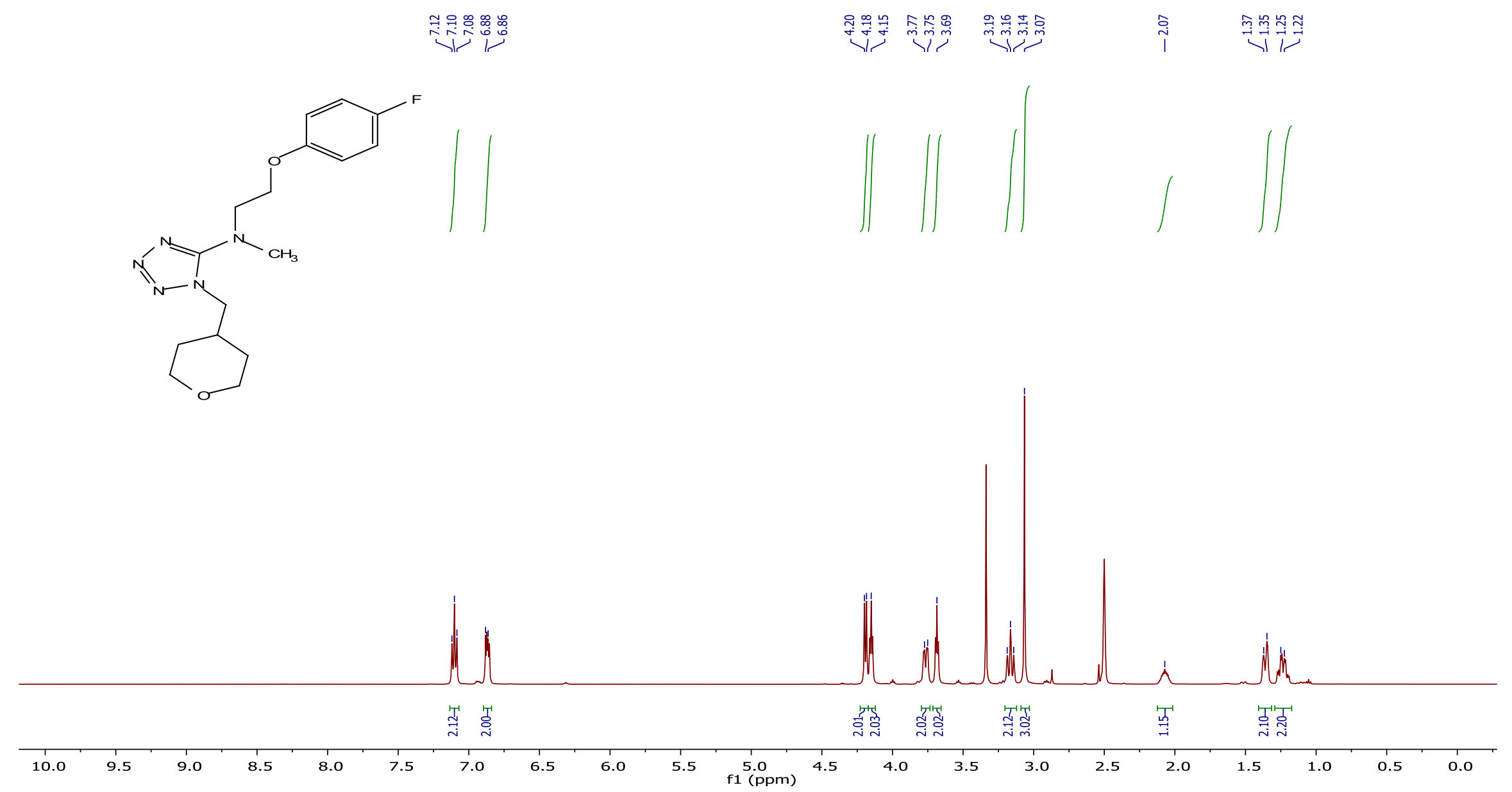


${ }^{13} \mathrm{C}$ NMR spectrum of the compound $\mathbf{9}\{121,302\}$.
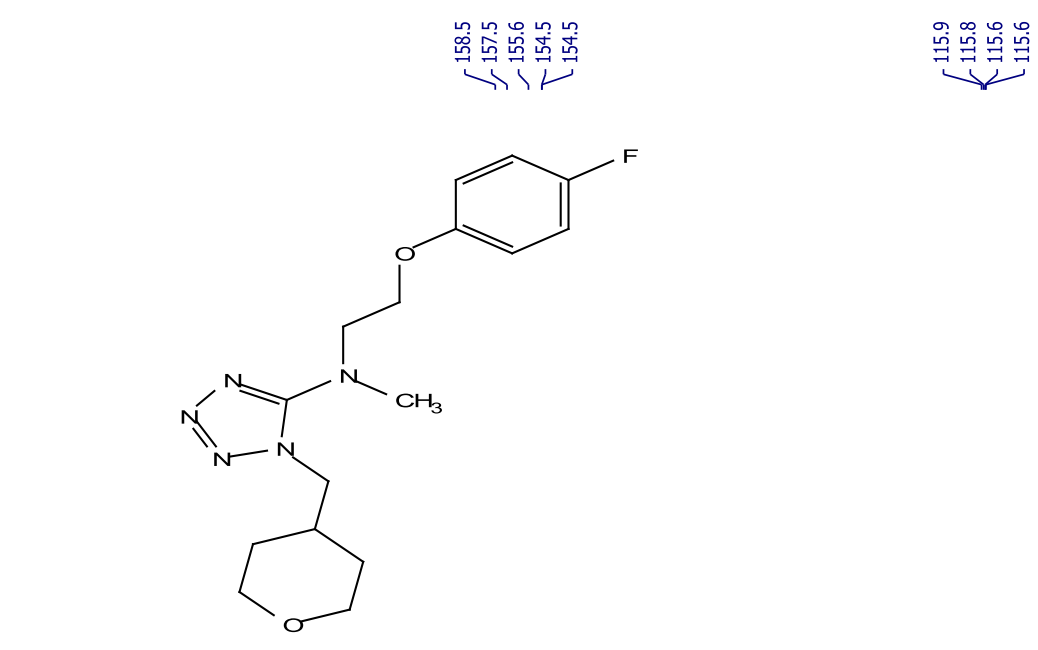

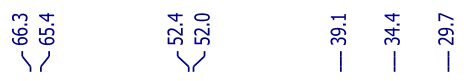

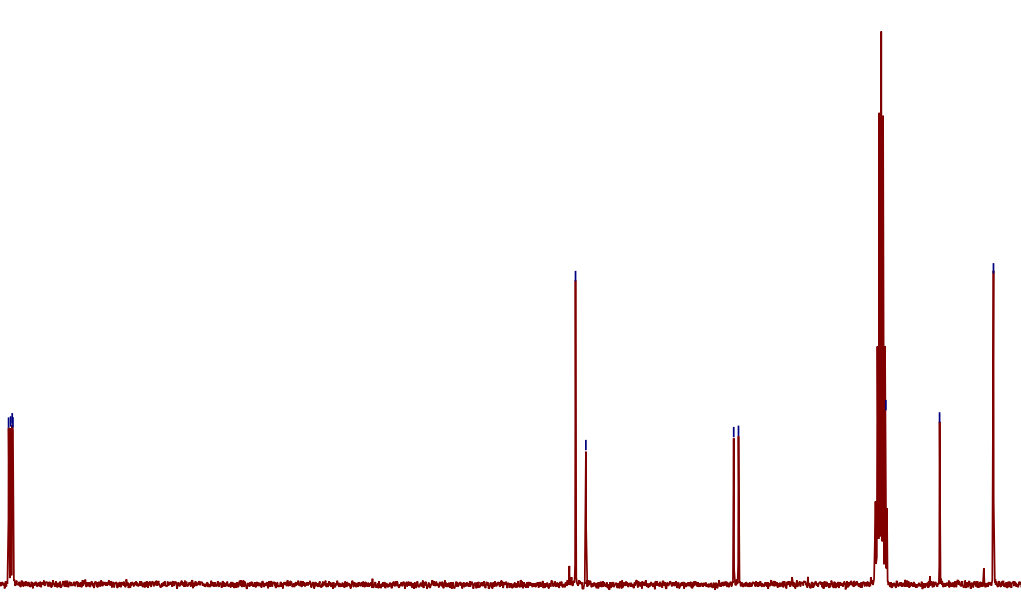

$\frac{1}{200}$ 
${ }^{19}$ F NMR spectrum of the compound $\mathbf{9}\{121,302\}$.
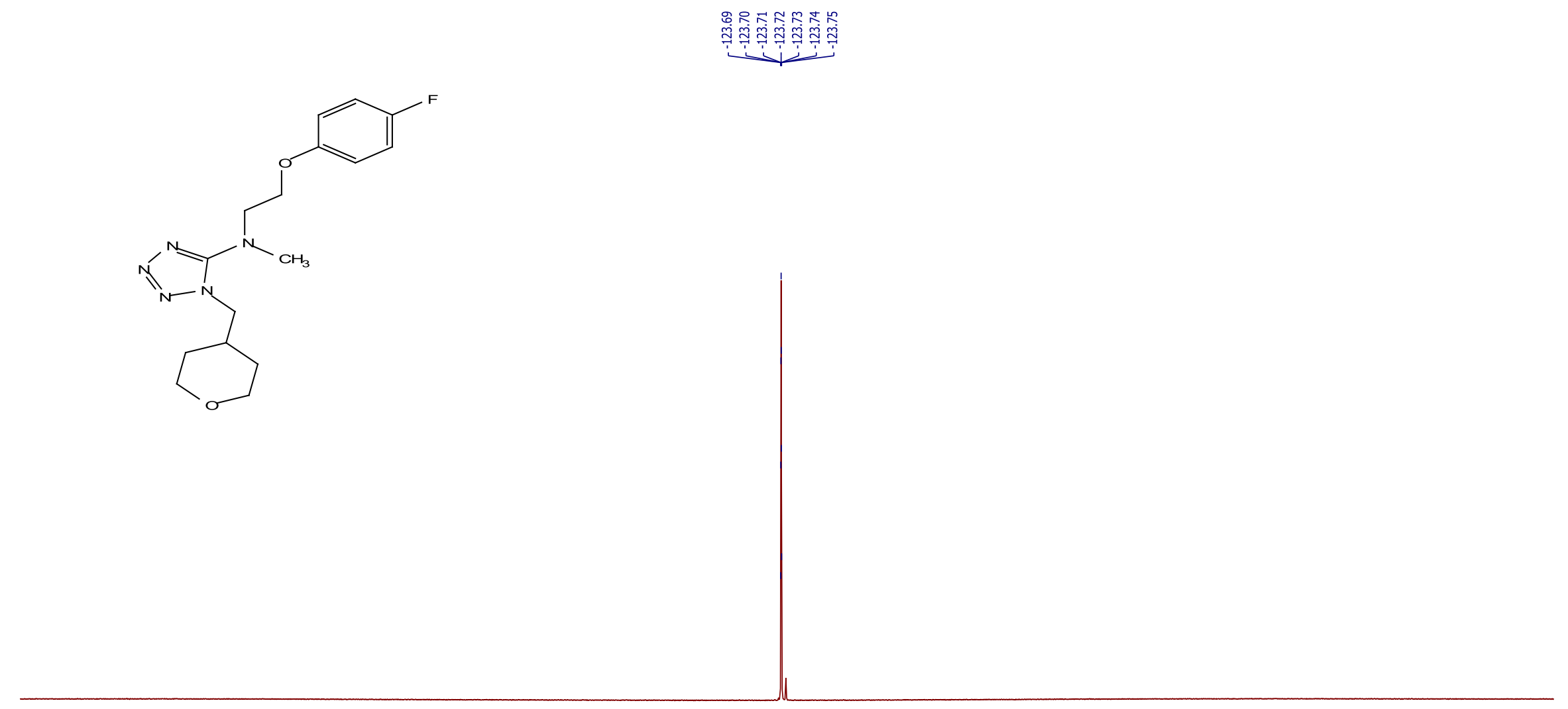
${ }^{1} \mathrm{H}$ NMR spectrum of the compound $\mathbf{9}\{(146,322\}$.
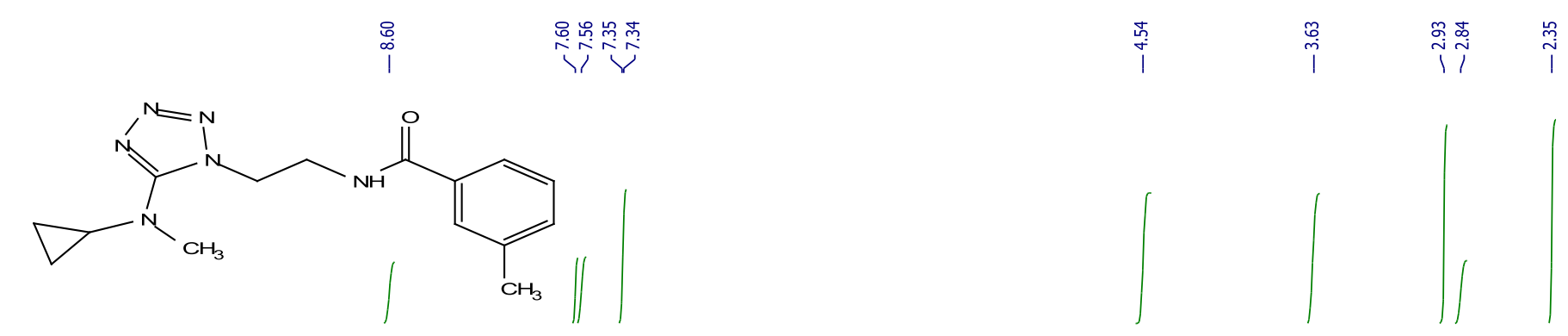

톤준 준
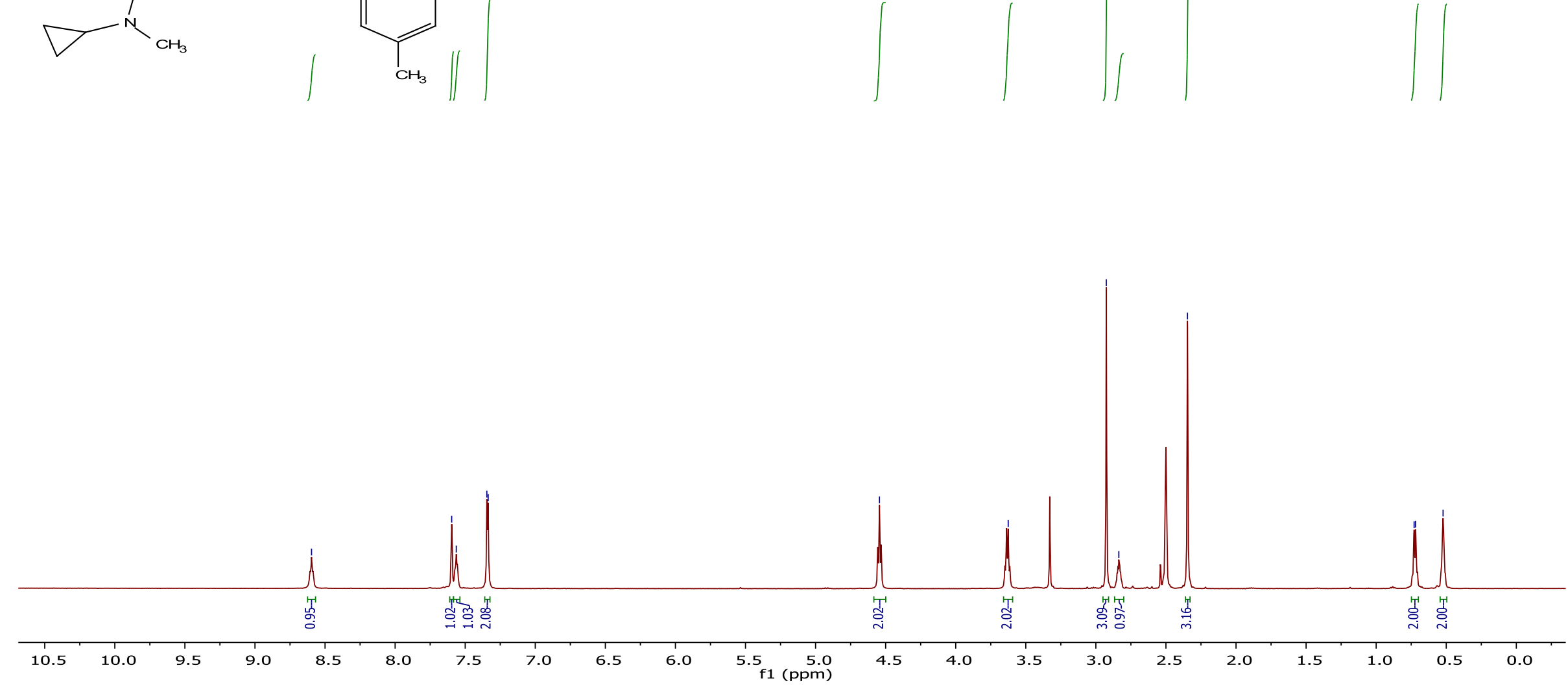
${ }^{13} \mathrm{C}$ NMR spectrum of the compound $\mathbf{9}\{(146,322\}$.

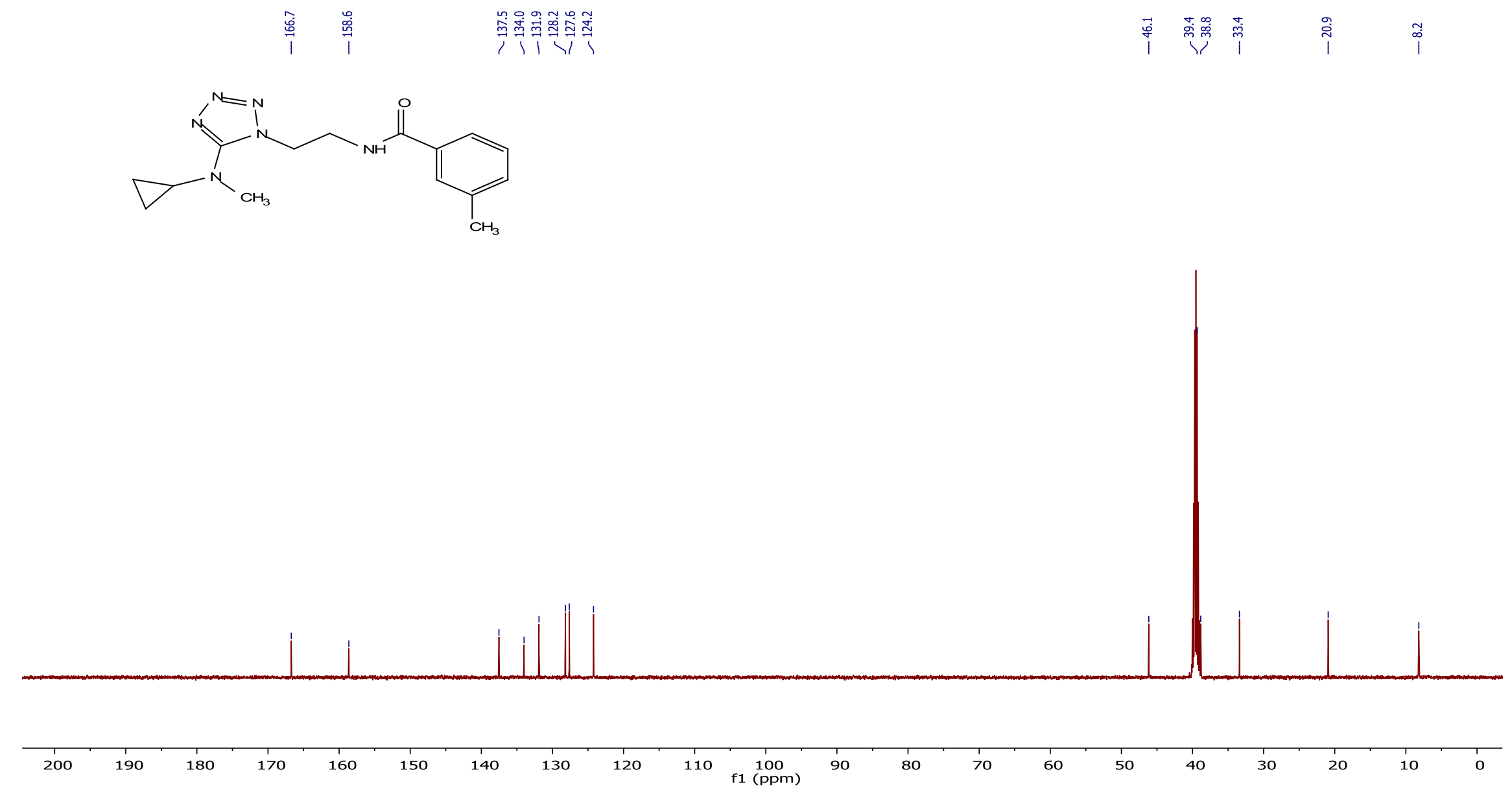

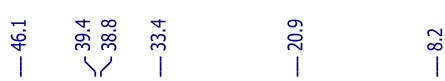


${ }^{1} \mathrm{H}$ NMR spectrum of the compound $\mathbf{9}\{175,299\}$.

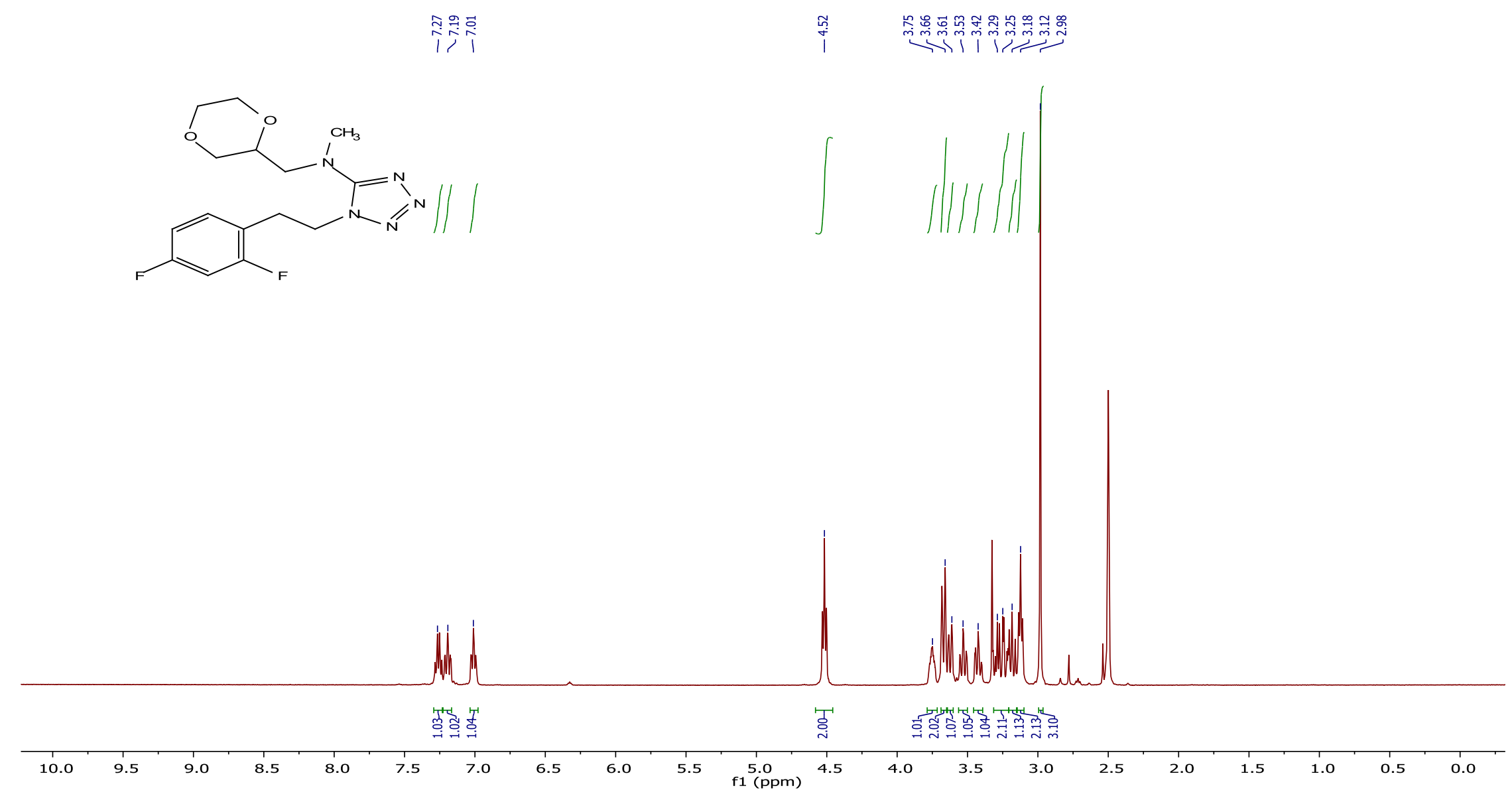


${ }^{13} \mathrm{C}$ NMR spectrum of the compound $\mathbf{9}\{175,299\}$.

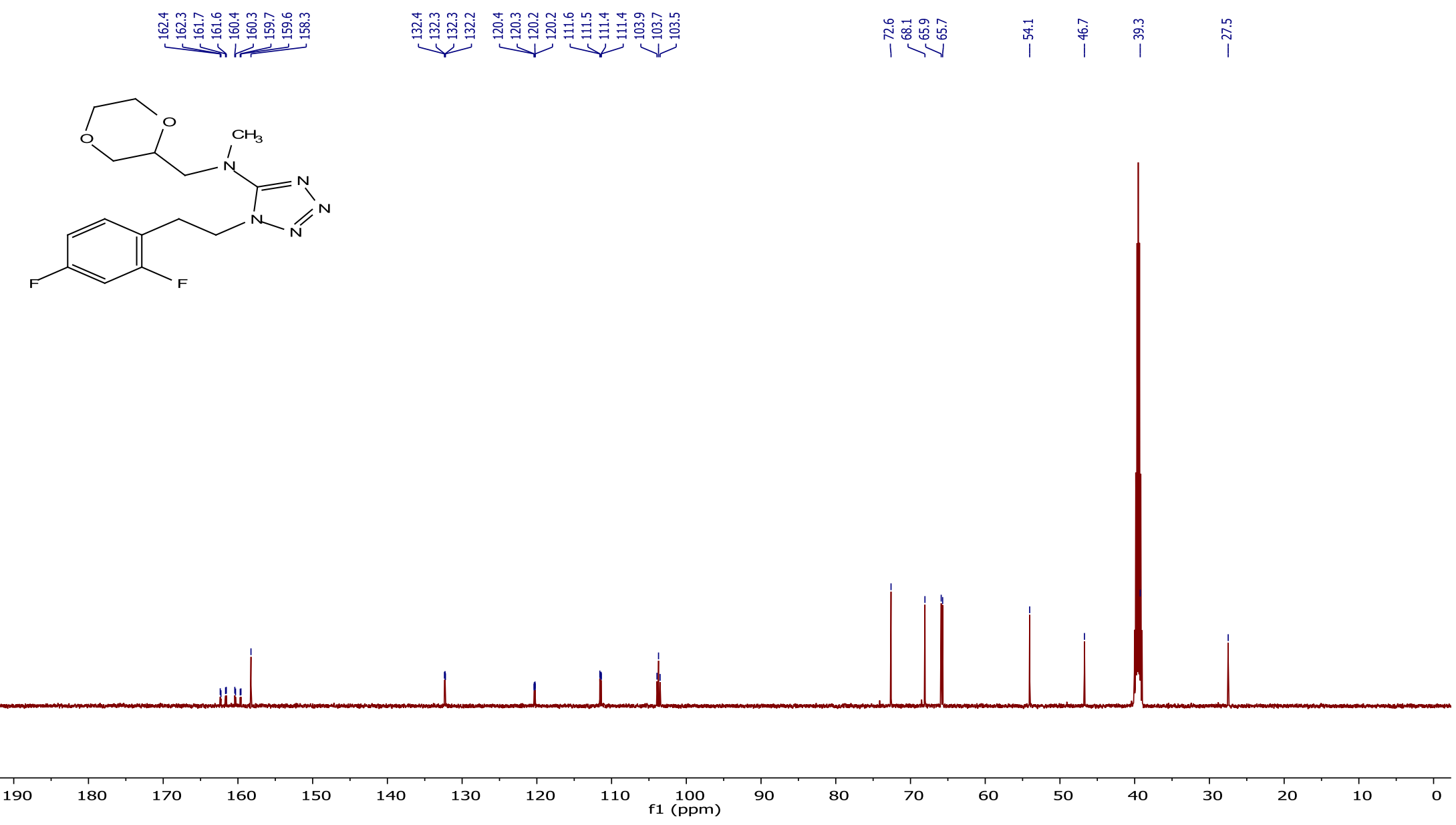


${ }^{19} \mathrm{~F}$ NMR spectrum of the compound $\mathbf{9}\{175,299\}$.

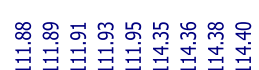
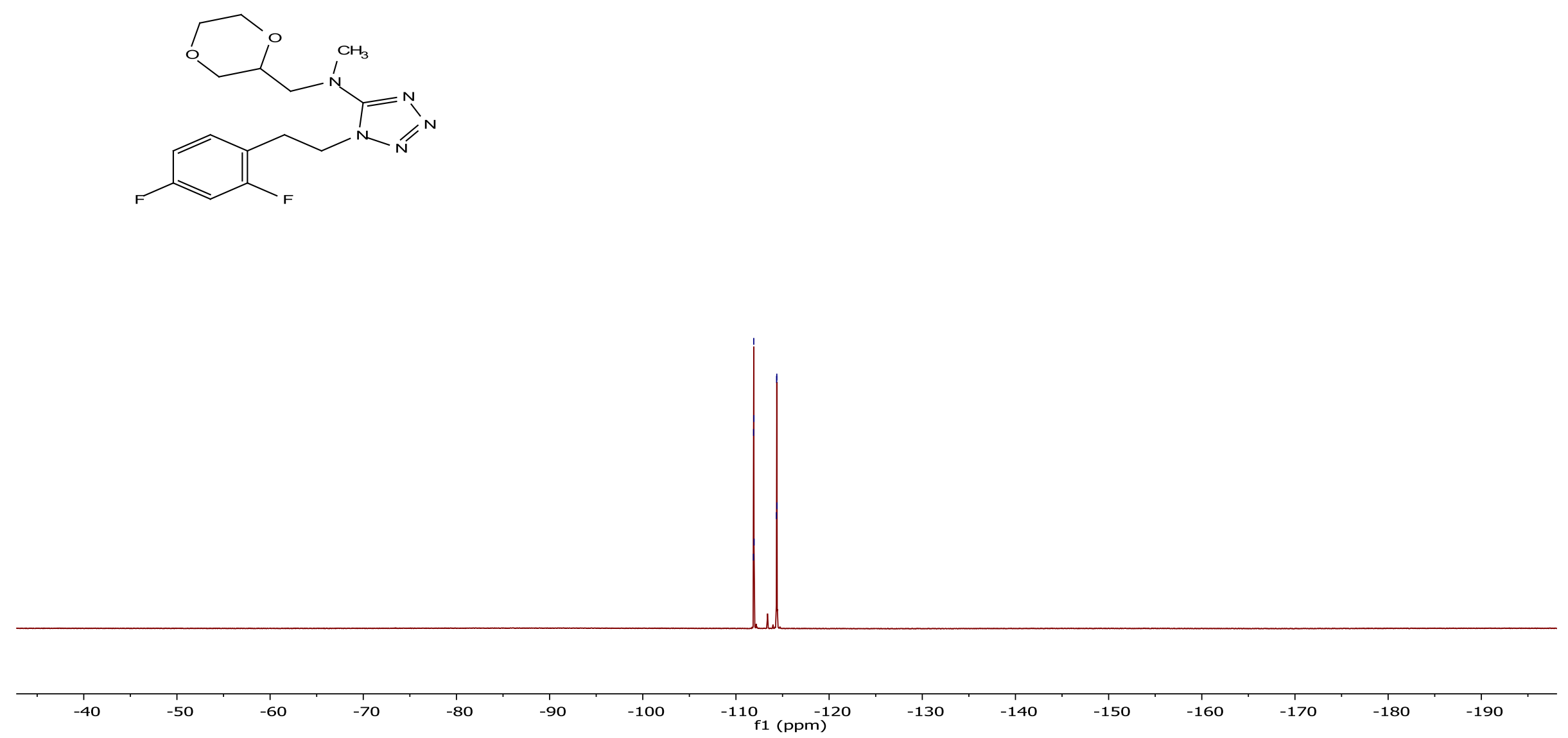
${ }^{1} \mathrm{H}$ NMR spectrum of the compound $\mathbf{9}\{151,319\}$.

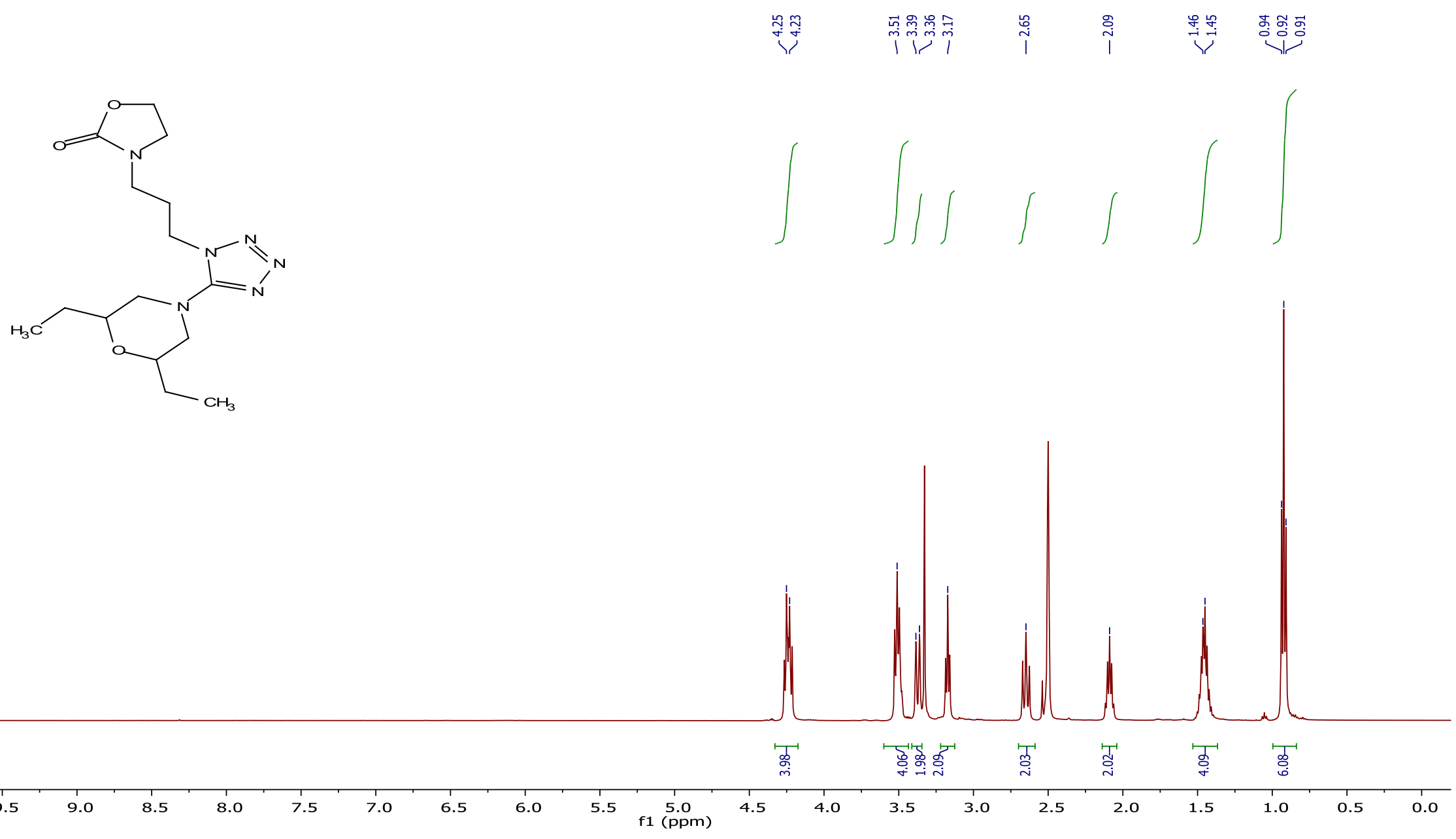


${ }^{13} \mathrm{C}$ NMR spectrum of the compound $\mathbf{9}\{151,319\}$.

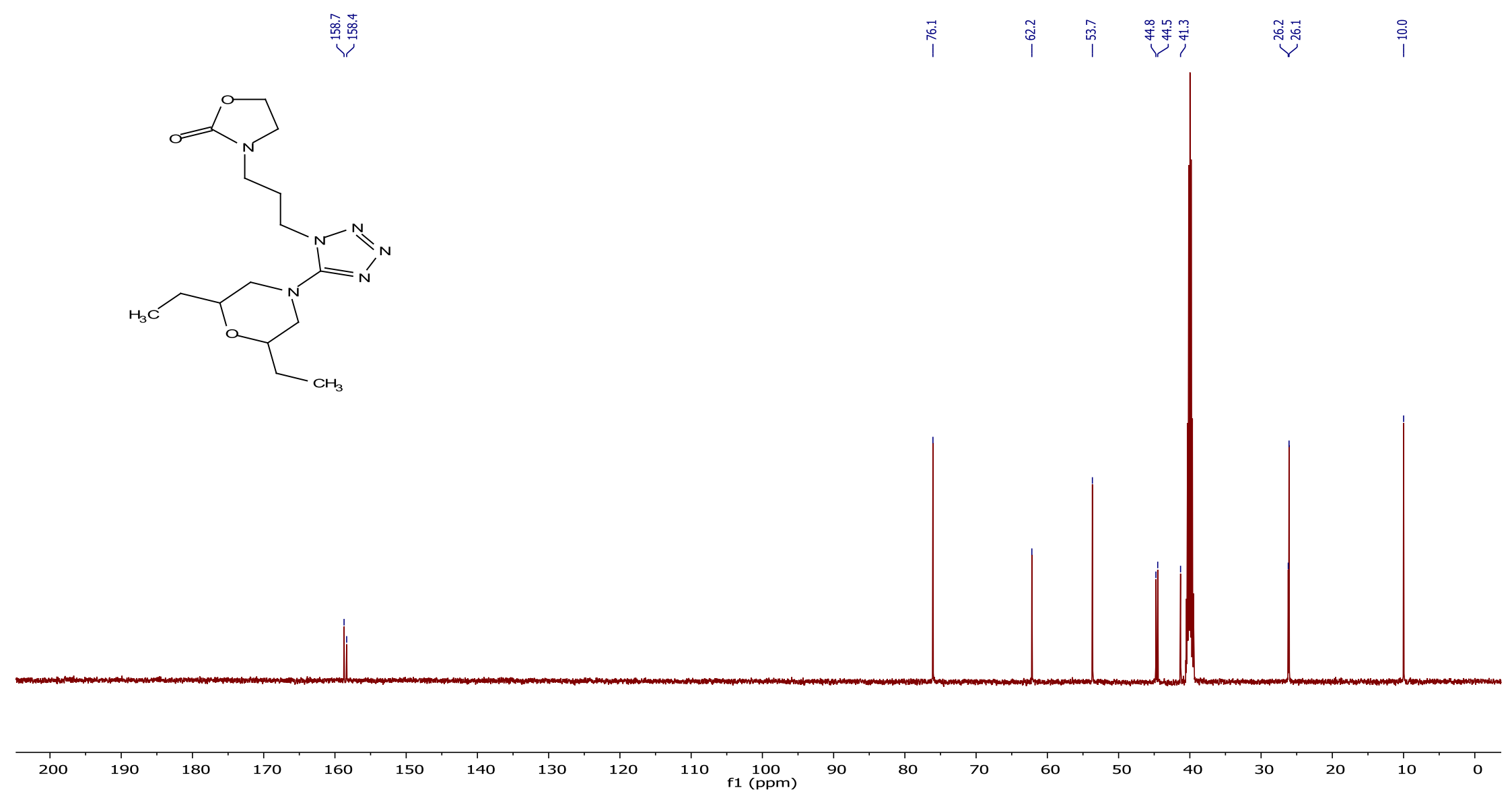


${ }^{1} \mathrm{H}$ NMR spectrum of the compound $\mathbf{9}\{165,299\}$.
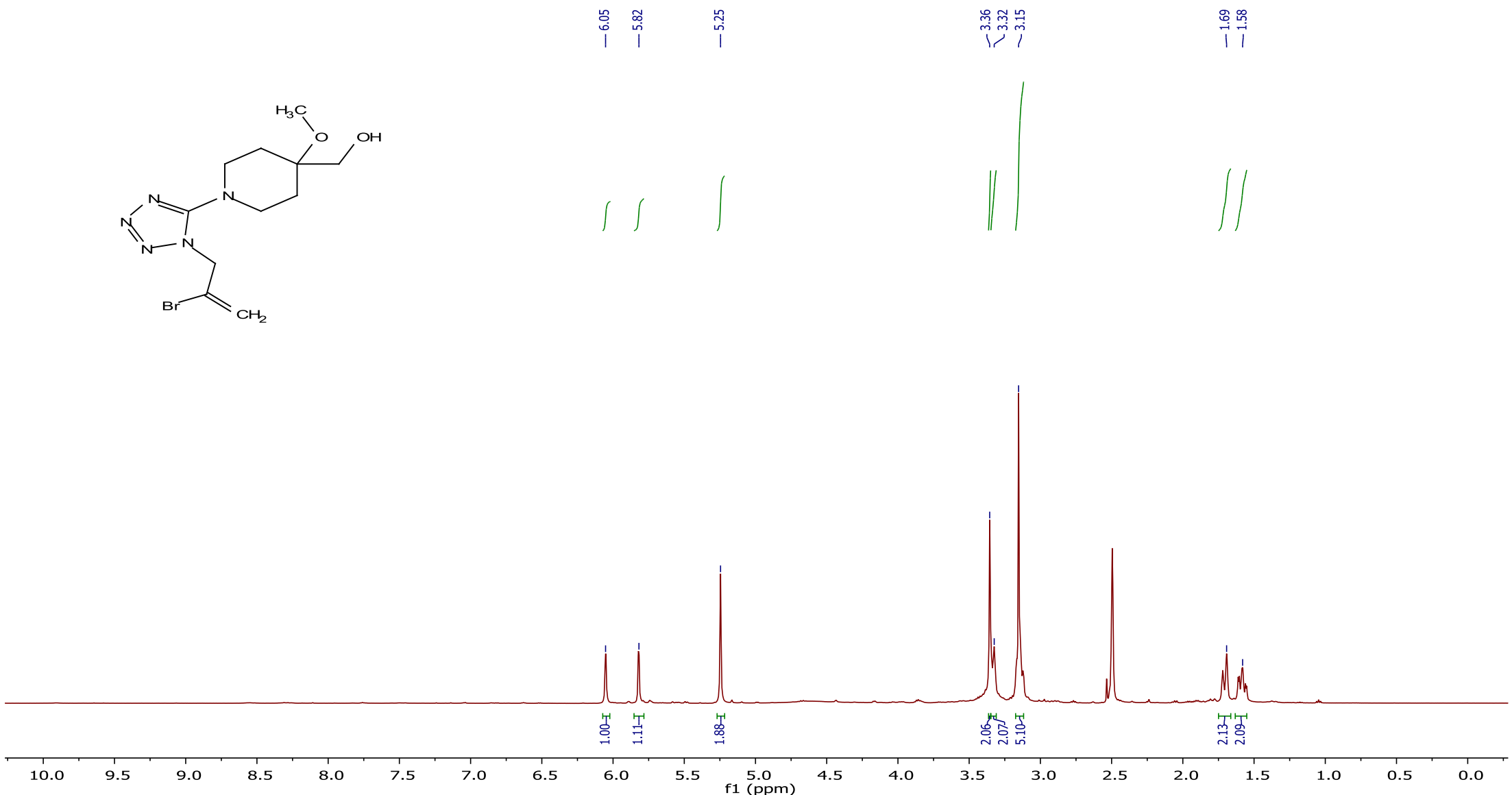
${ }^{13} \mathrm{C}$ NMR spectrum of the compound $\mathbf{9}\{165,299\}$.

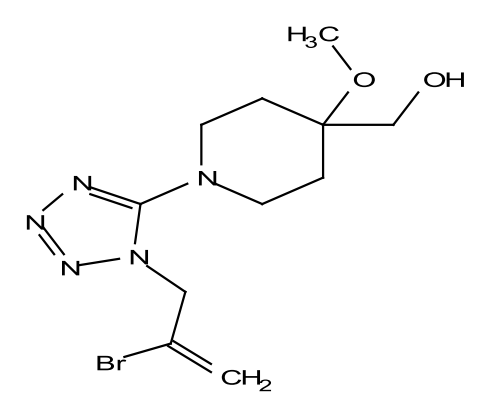


${ }^{1} \mathrm{H}$ NMR spectrum of the compound $\mathbf{9}\{175,299\}$.

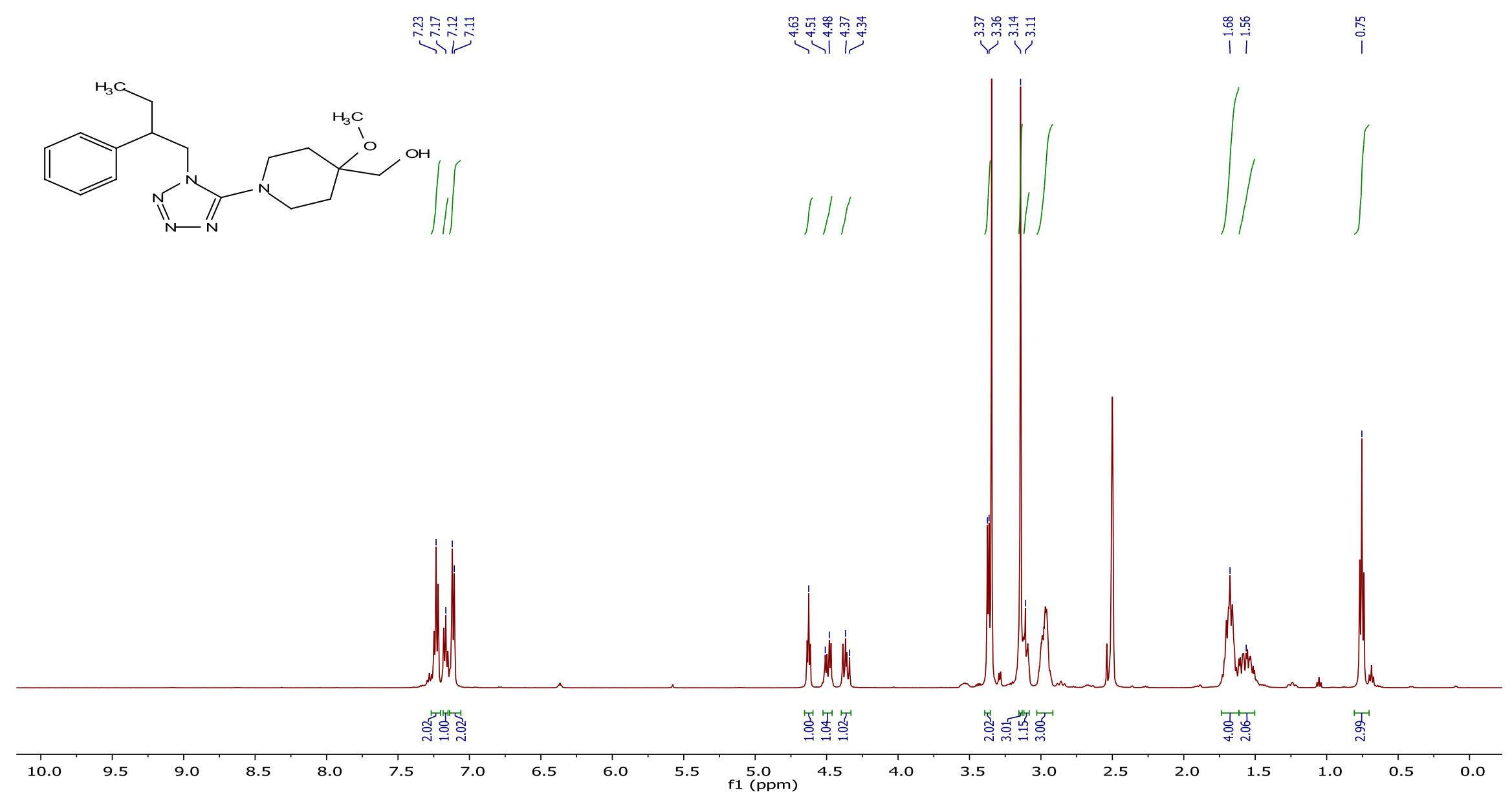


${ }^{13} \mathrm{C}$ NMR spectrum of the compound $\mathbf{9}\{175,299\}$.
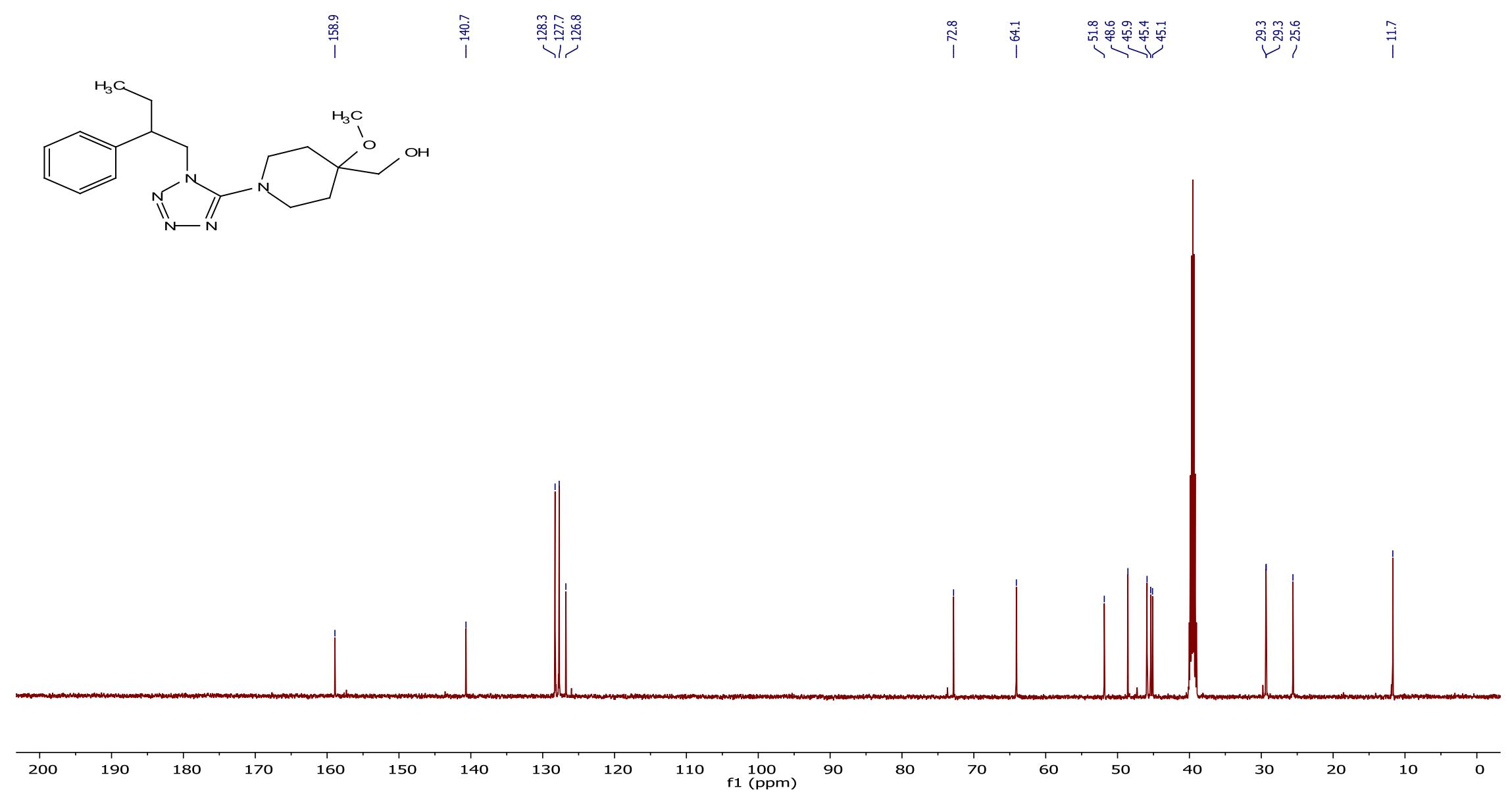
${ }^{1} \mathrm{H}$ NMR spectrum of the compound $\mathbf{9}\{188,338\}$.

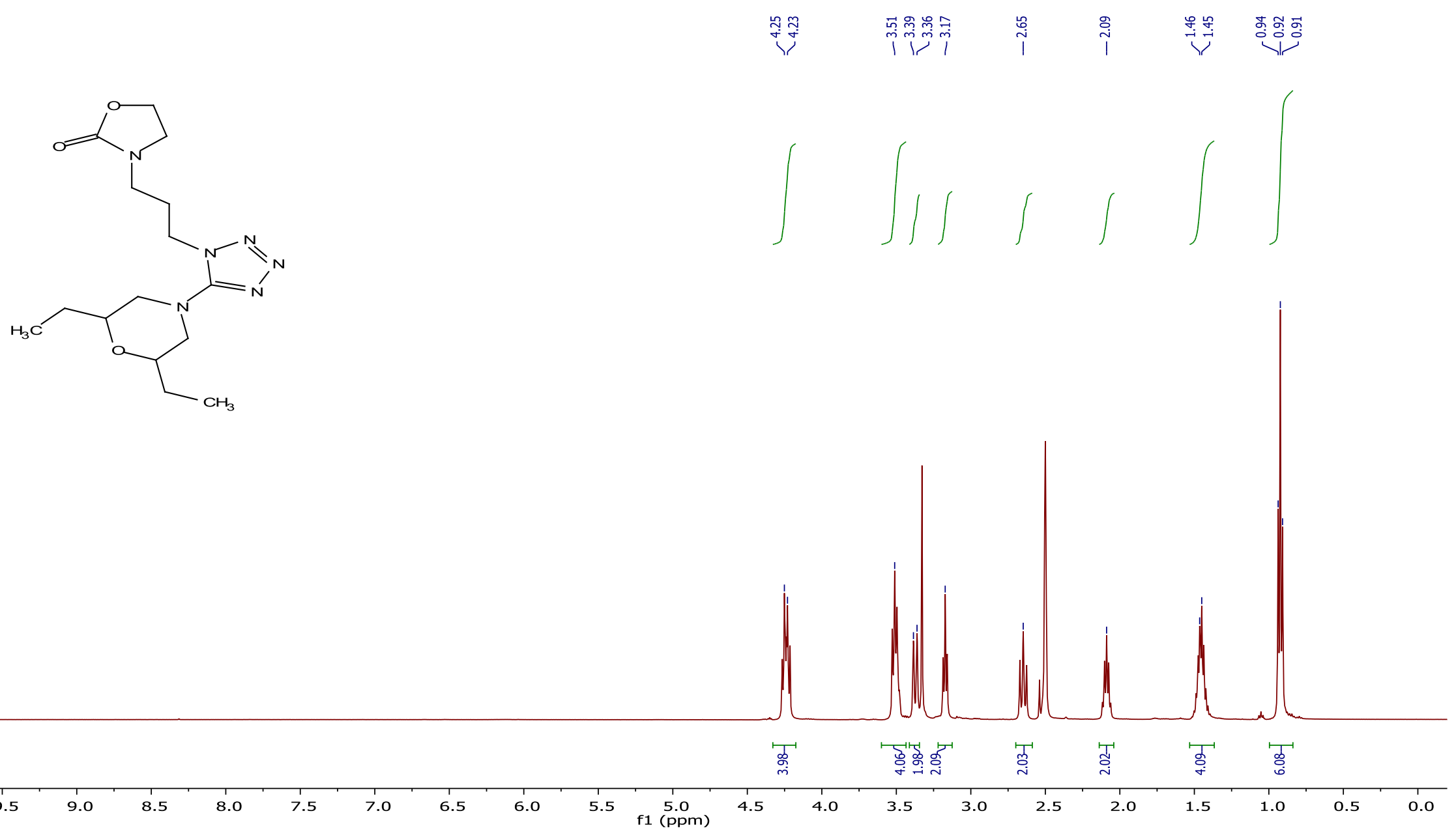


${ }^{13} \mathrm{C}$ NMR spectrum of the compound $\mathbf{9}\{188,338\}$.
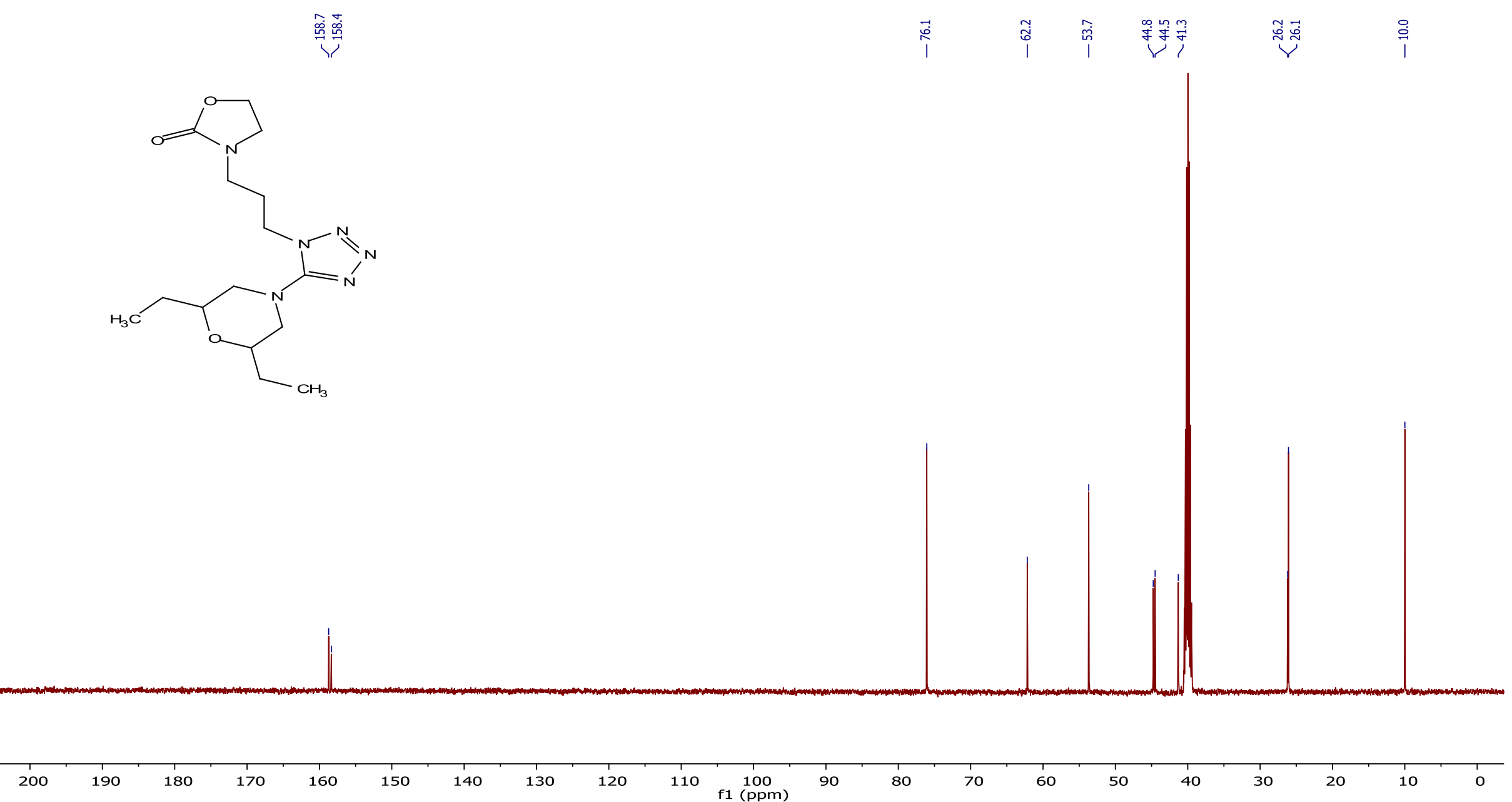
${ }^{1} \mathrm{H}$ NMR spectrum of the compound $\mathbf{9}\{190,341\}$.

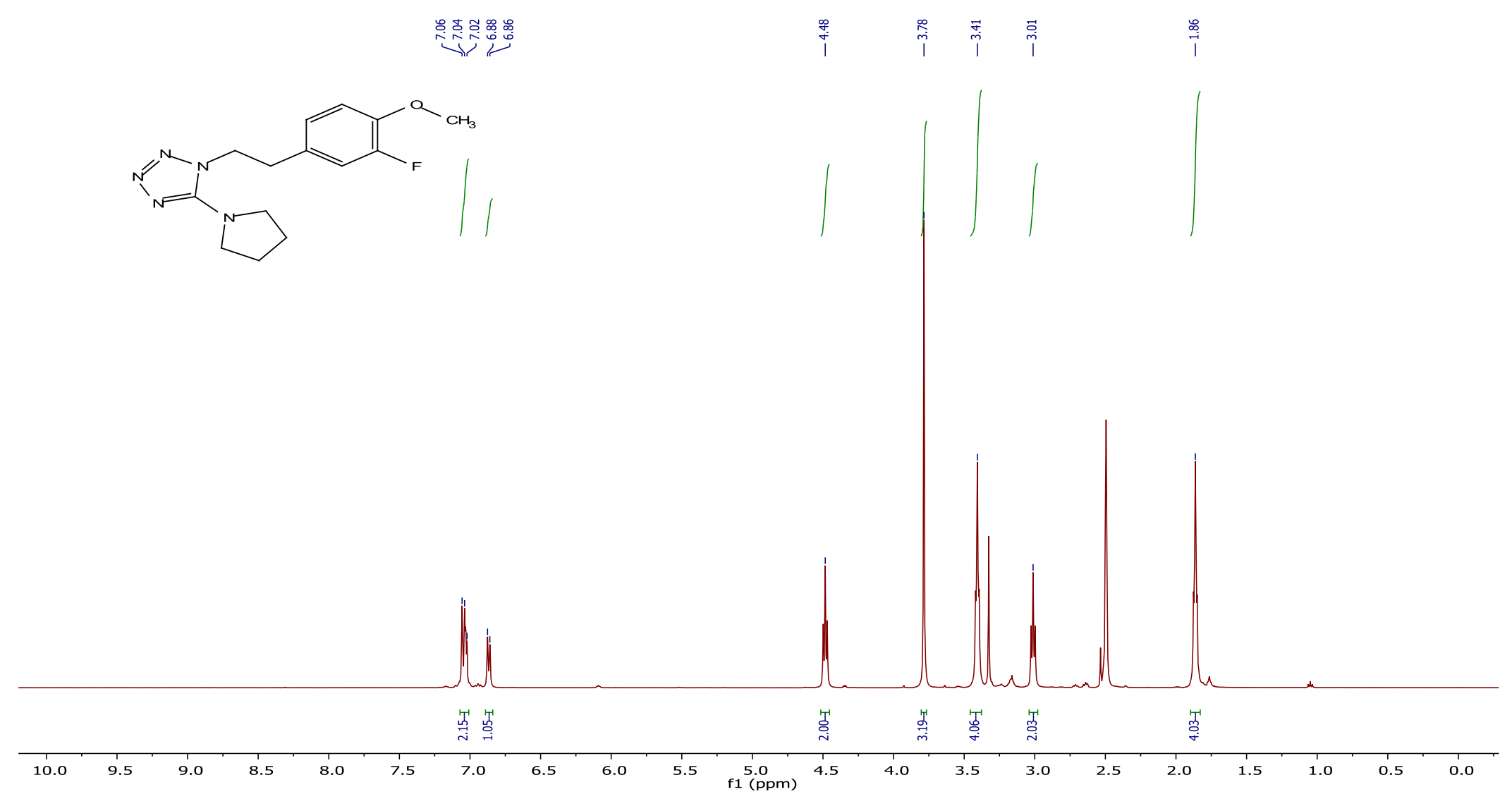


${ }^{13} \mathrm{C}$ NMR spectrum of the compound $\mathbf{9}\{190,341\}$.

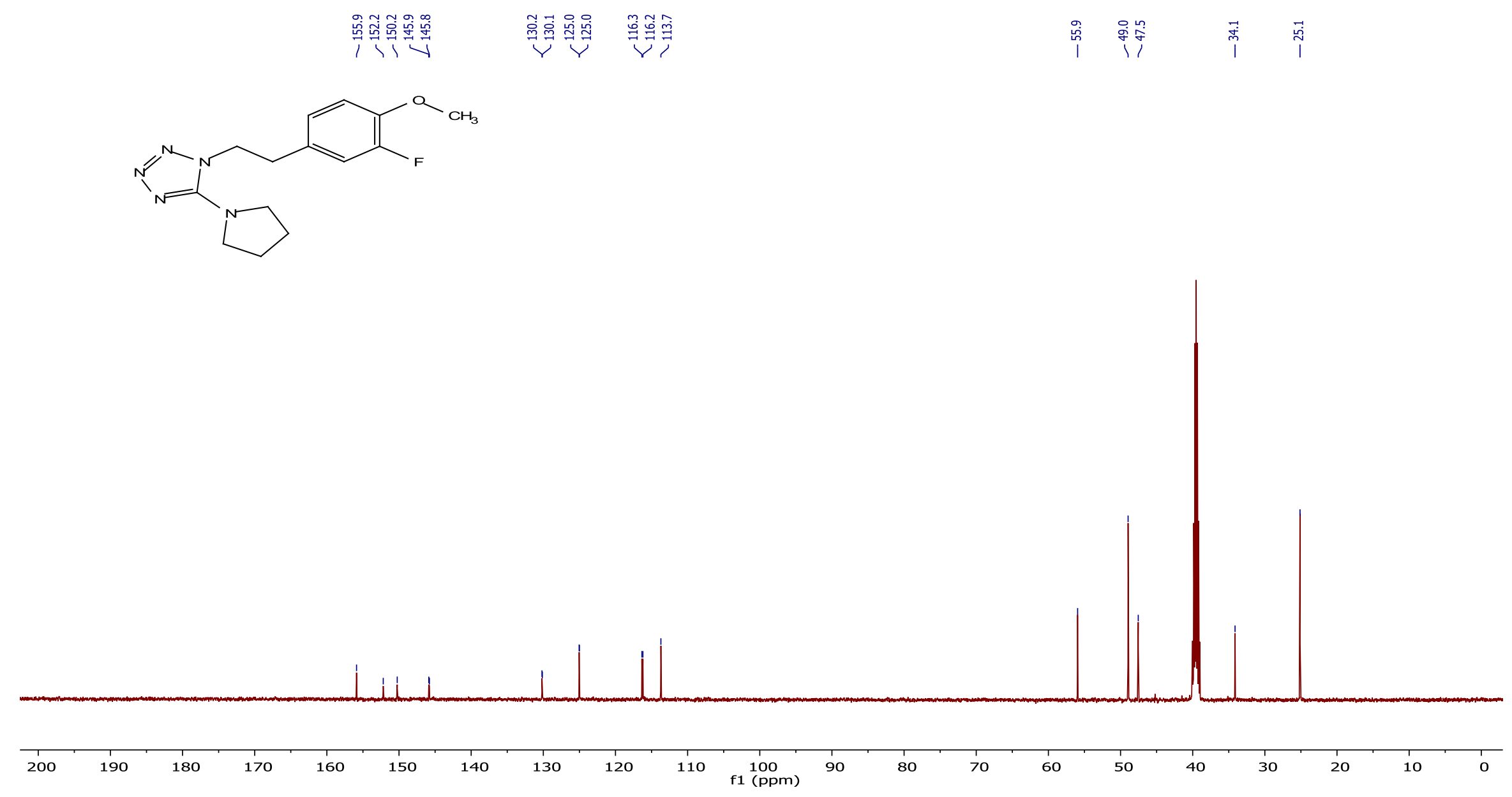


${ }^{19} \mathrm{~F}$ NMR spectrum of the compound $\mathbf{9}\{190,341\}$.

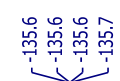
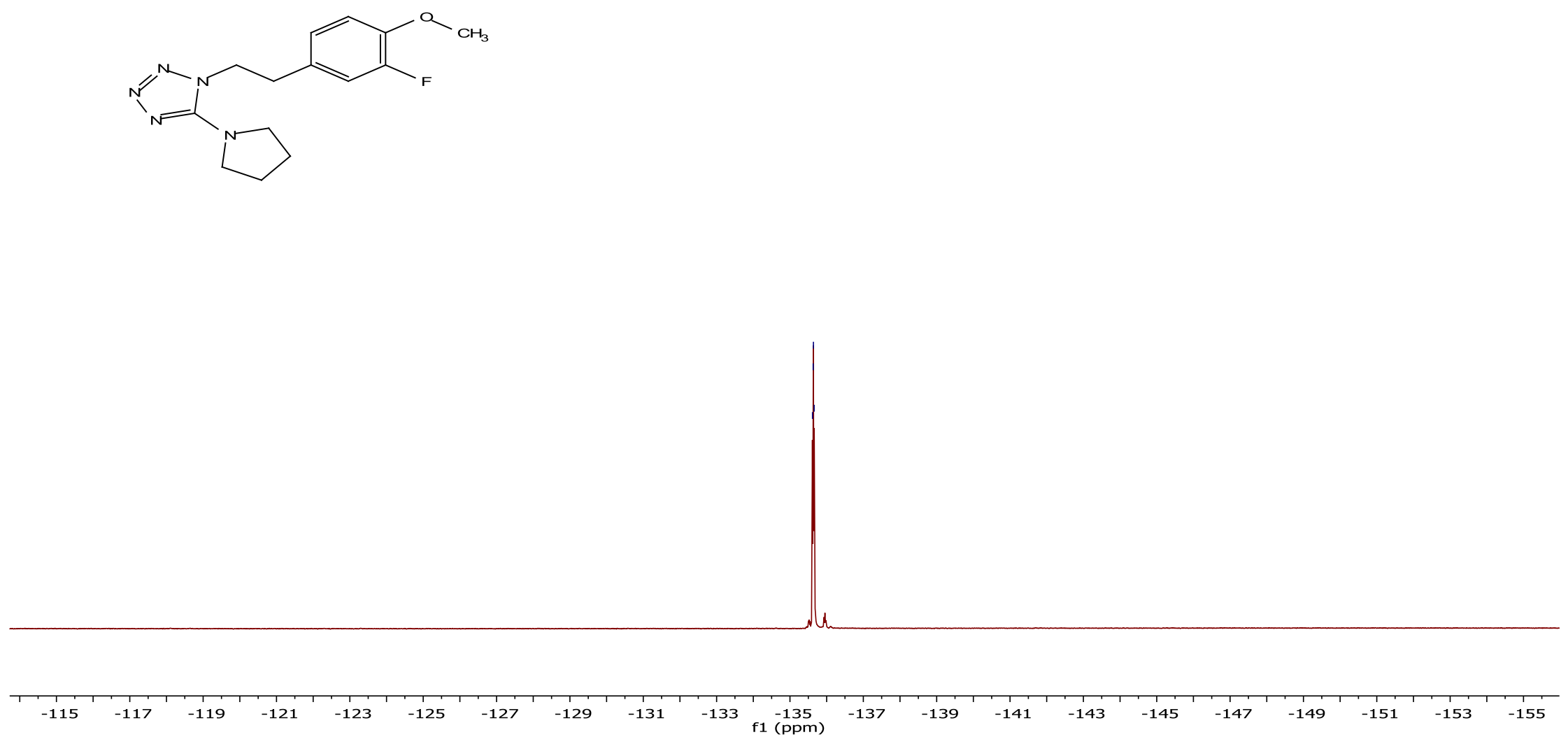
${ }^{1} \mathrm{H}$ NMR spectrum of the compound $\mathbf{9}\{262,319\}$.

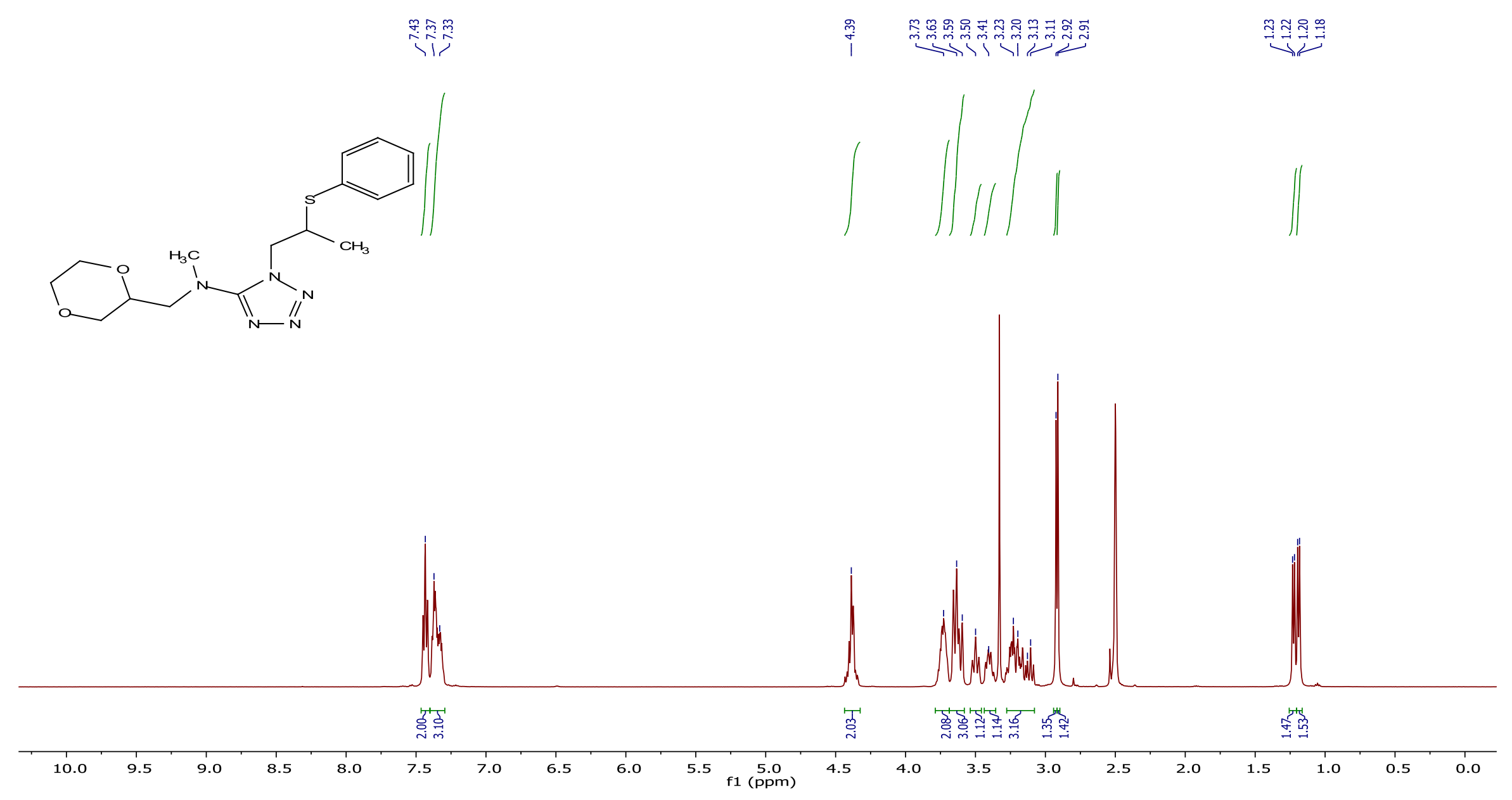


${ }^{13} \mathrm{C}$ NMR spectrum of the compound $\mathbf{9}\{262,319\}$.
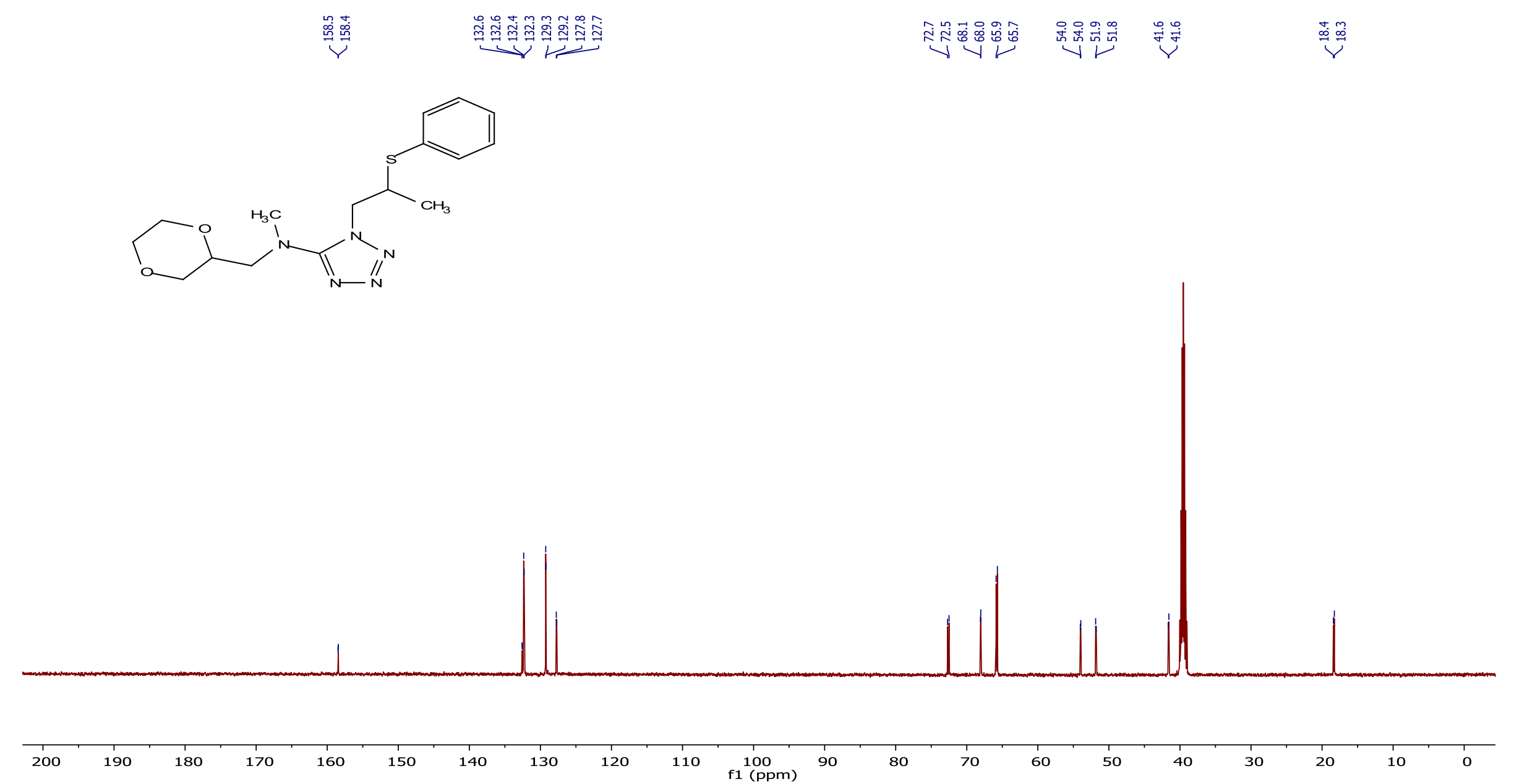
${ }^{1} \mathrm{H}$ NMR spectrum of the compound $\mathbf{9}\{263,341\}$.
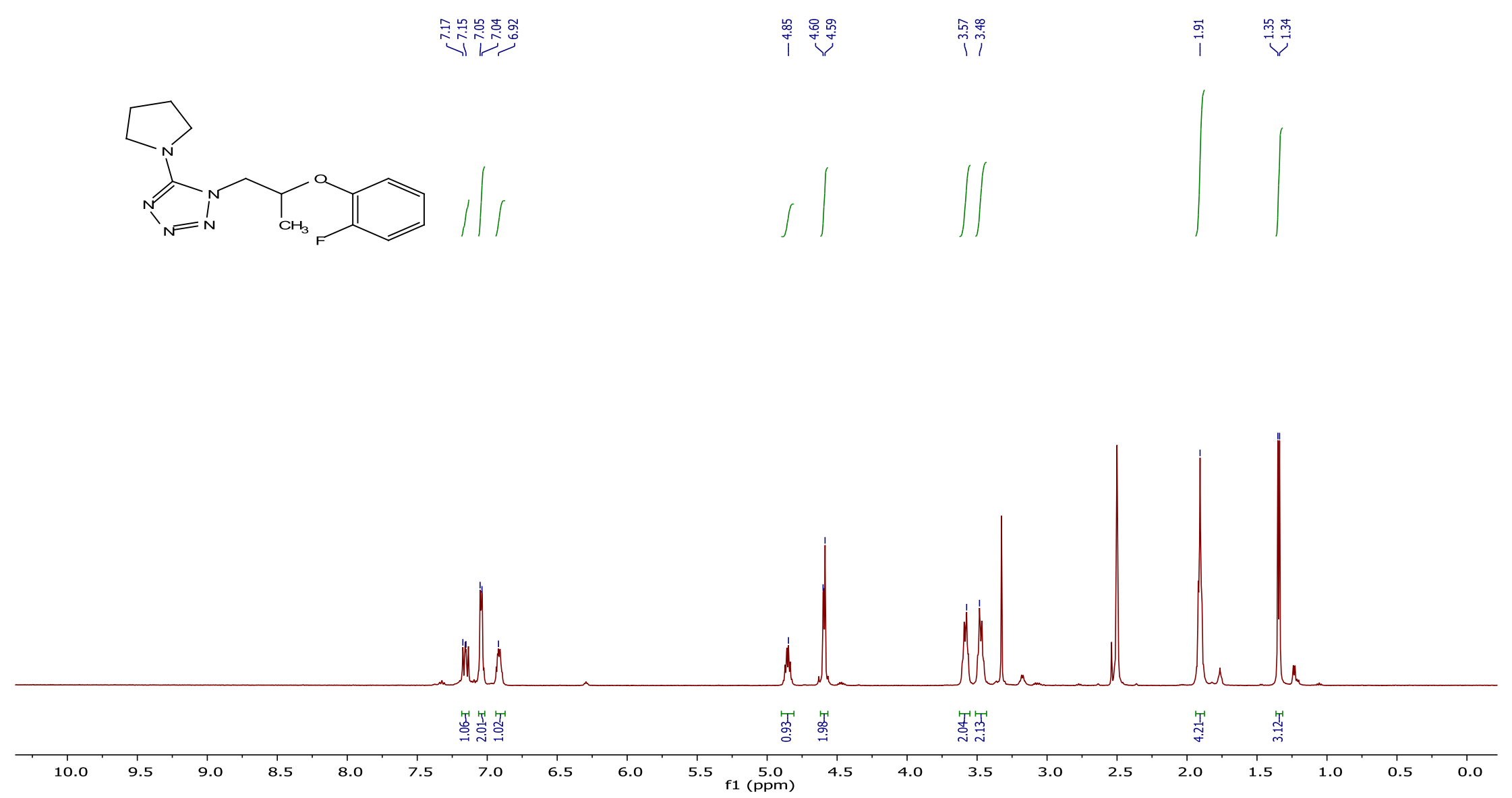
${ }^{13} \mathrm{C}$ NMR spectrum of the compound $\mathbf{9}\{263,341\}$.

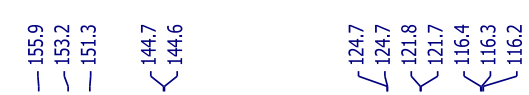
$\stackrel{\infty}{\prod_{1}^{\infty}}$

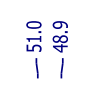

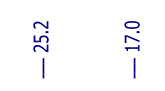

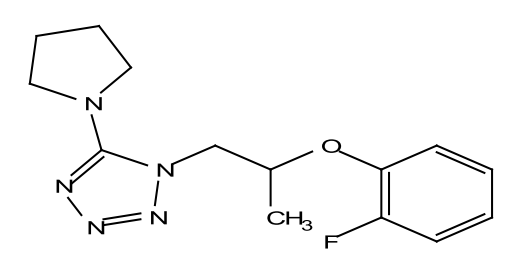

(ros

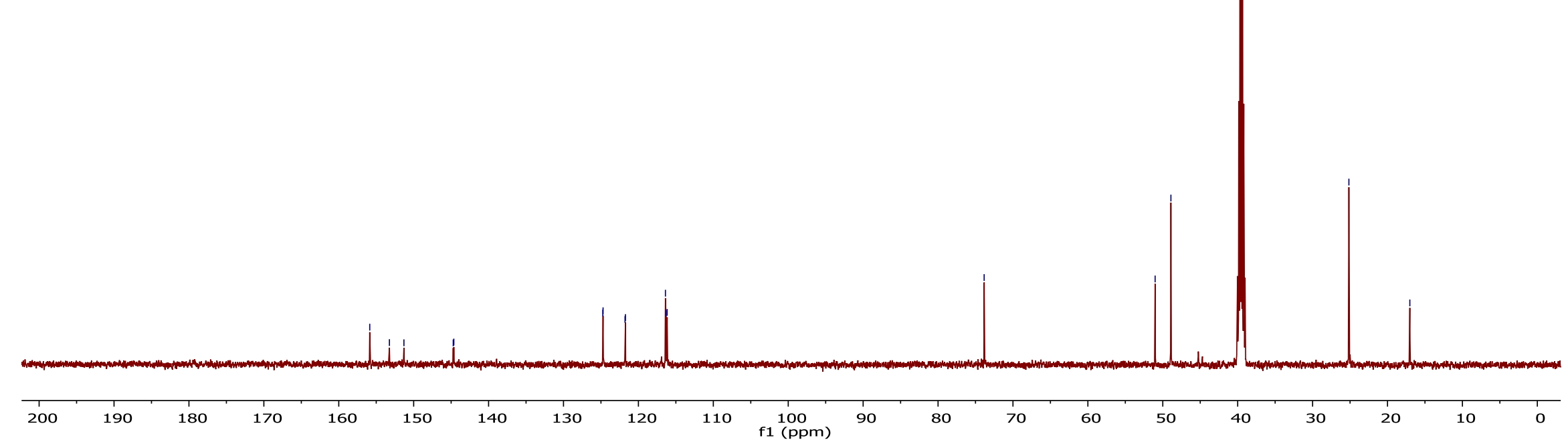


${ }^{19}$ F NMR spectrum of the compound $\mathbf{9}\{263,341\}$.

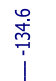
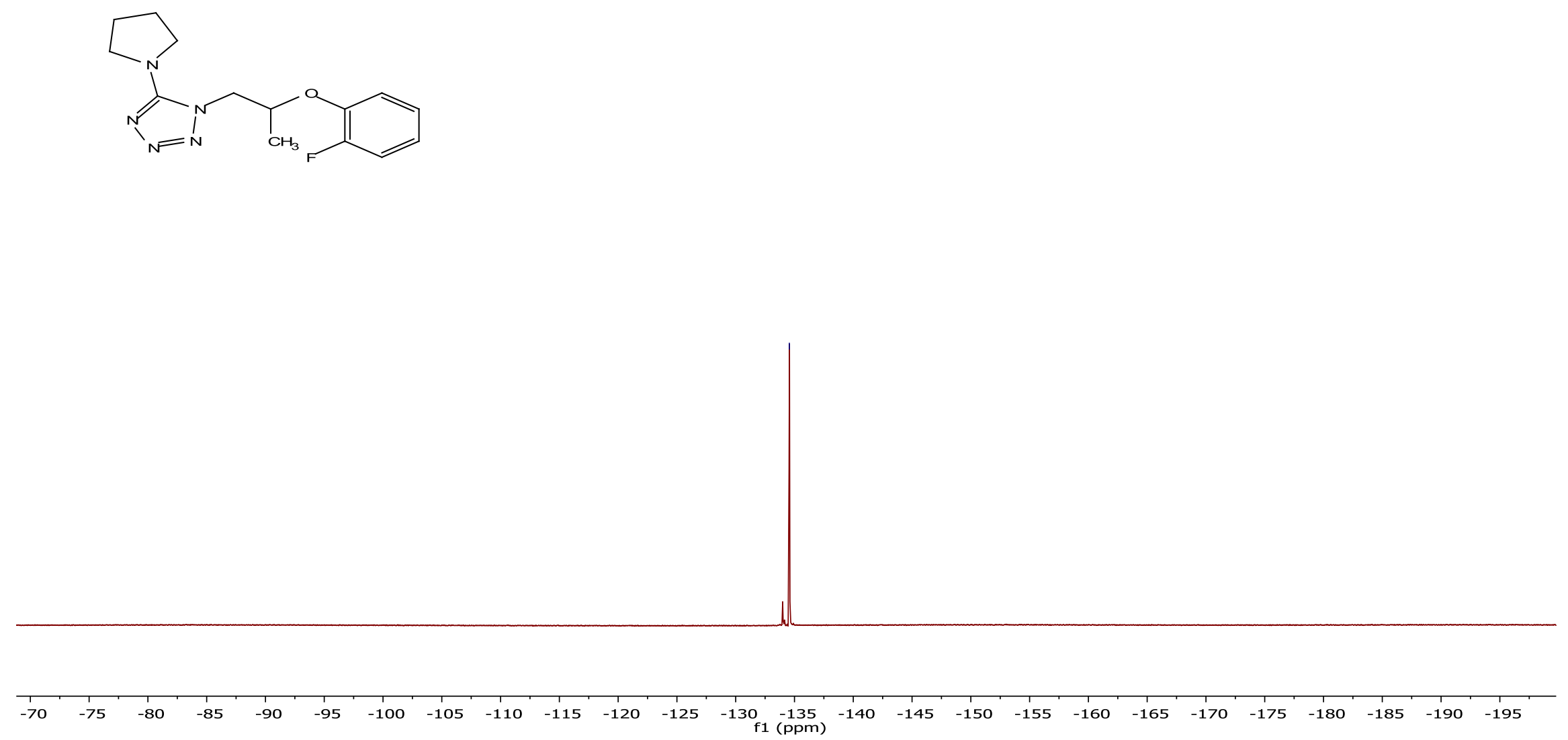
${ }^{1} \mathrm{H}$ NMR spectrum of the compound $\mathbf{9}\{272,319\}$.

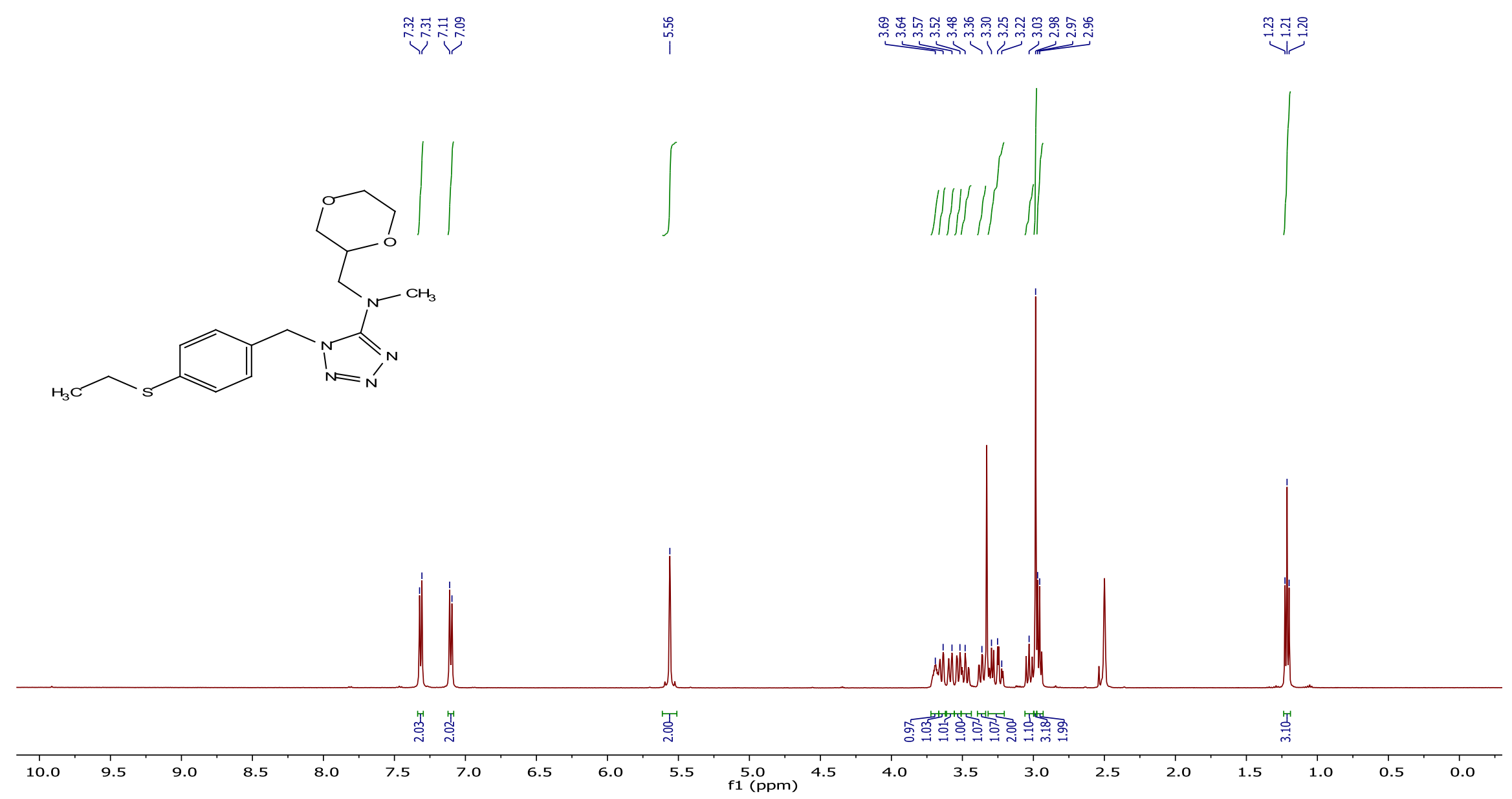


${ }^{13} \mathrm{C}$ NMR spectrum of the compound $\mathbf{9}\{272,319\}$.

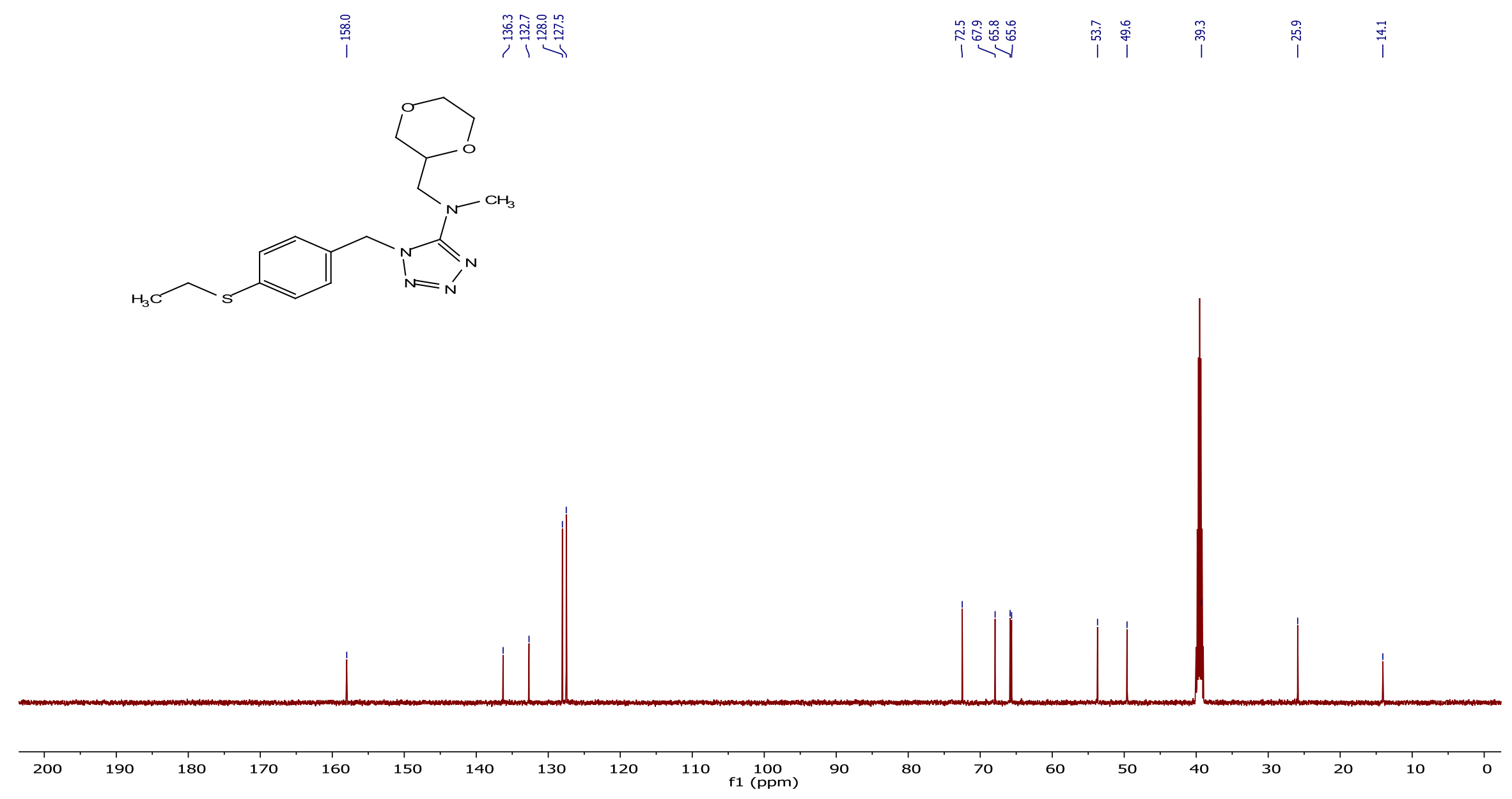


${ }^{1} \mathrm{H}$ NMR spectrum of the compound $\mathbf{9}\{280,391\}$.
พ
$\stackrel{\infty}{\stackrel{\infty}{*}}$

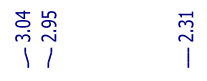

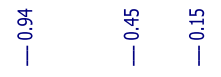
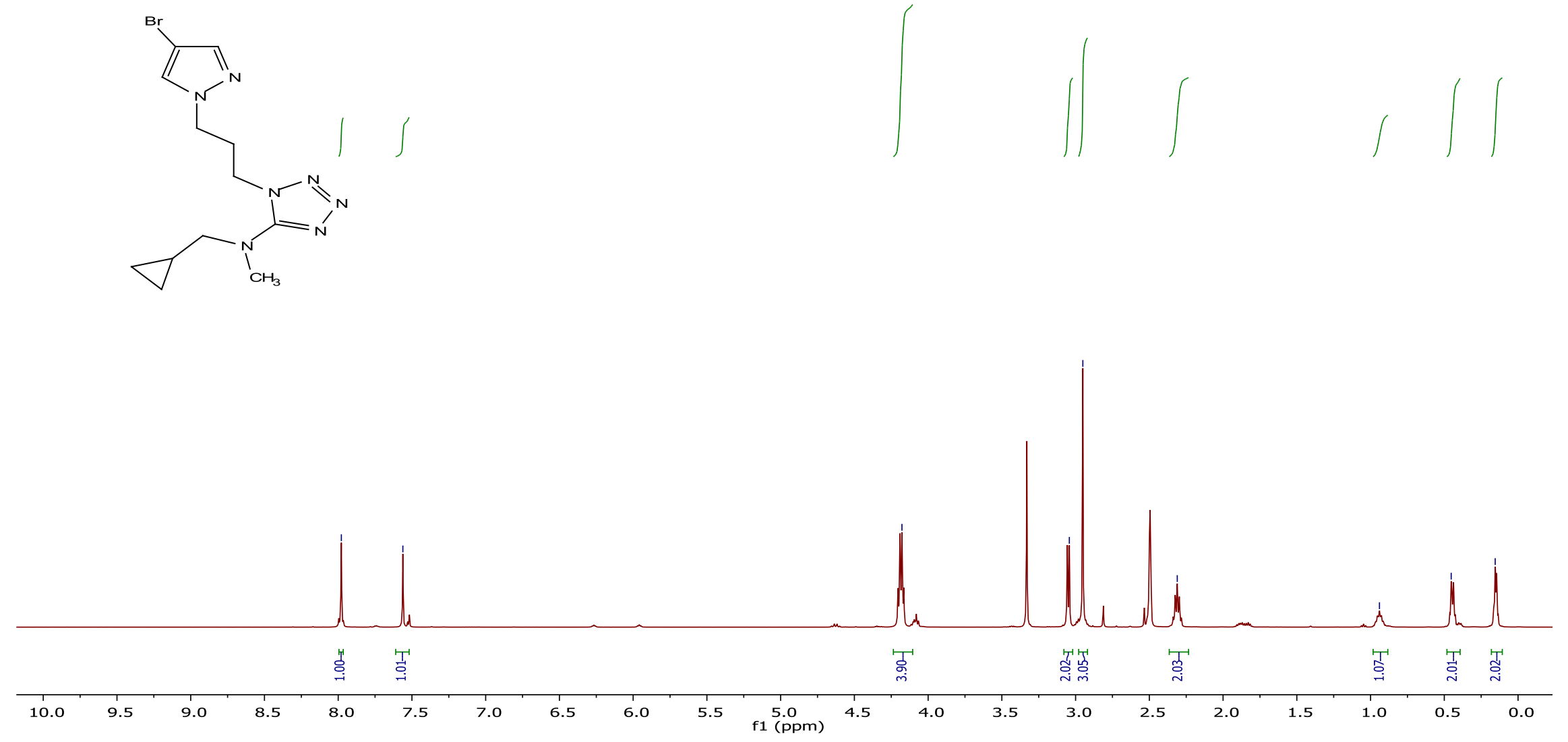
${ }^{13} \mathrm{C}$ NMR spectrum of the compound $\mathbf{9}\{280,391\}$.

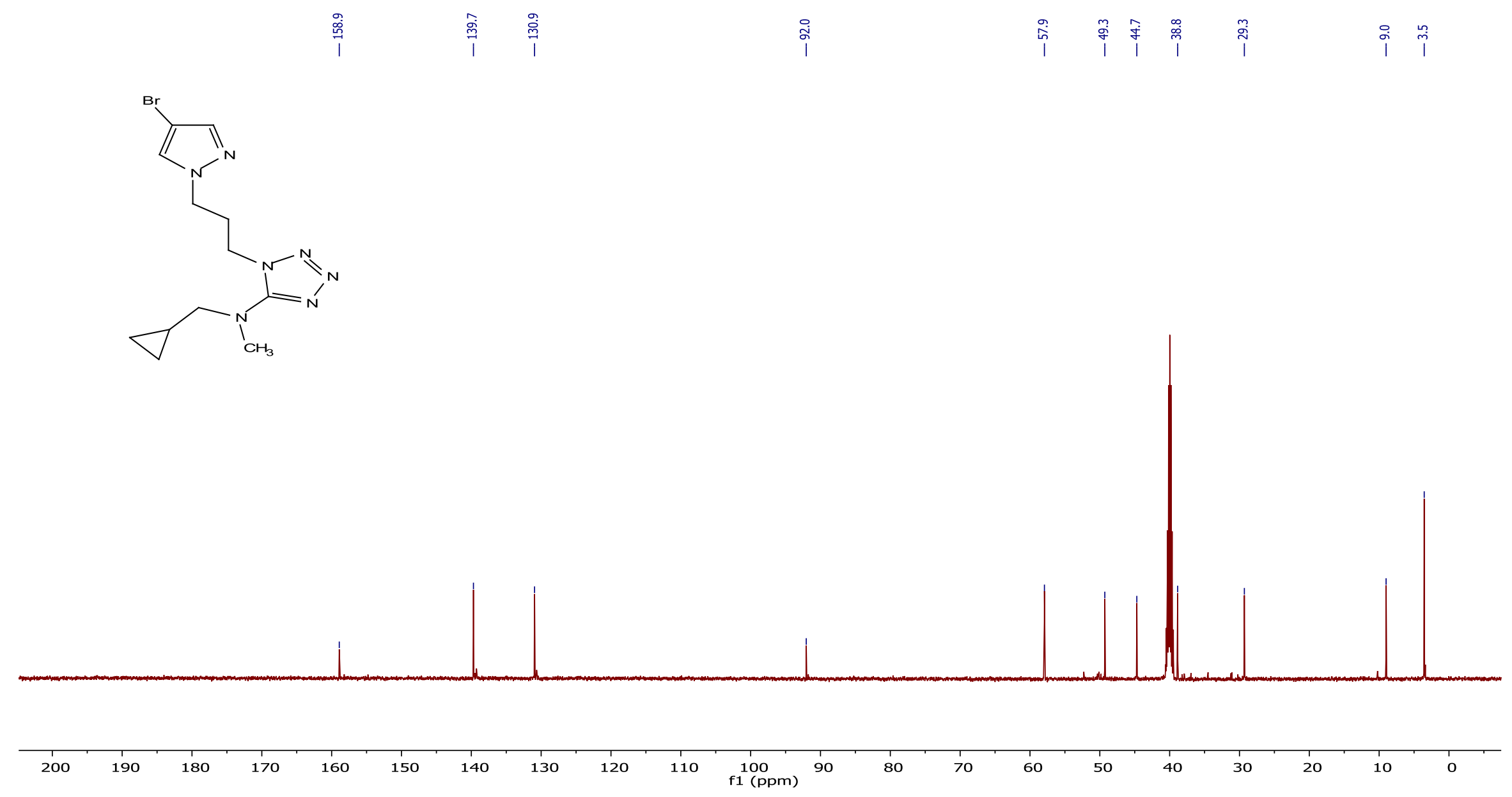


${ }^{1} \mathrm{H}$ NMR spectrum of the compound $\mathbf{9}\{306,261\}$.

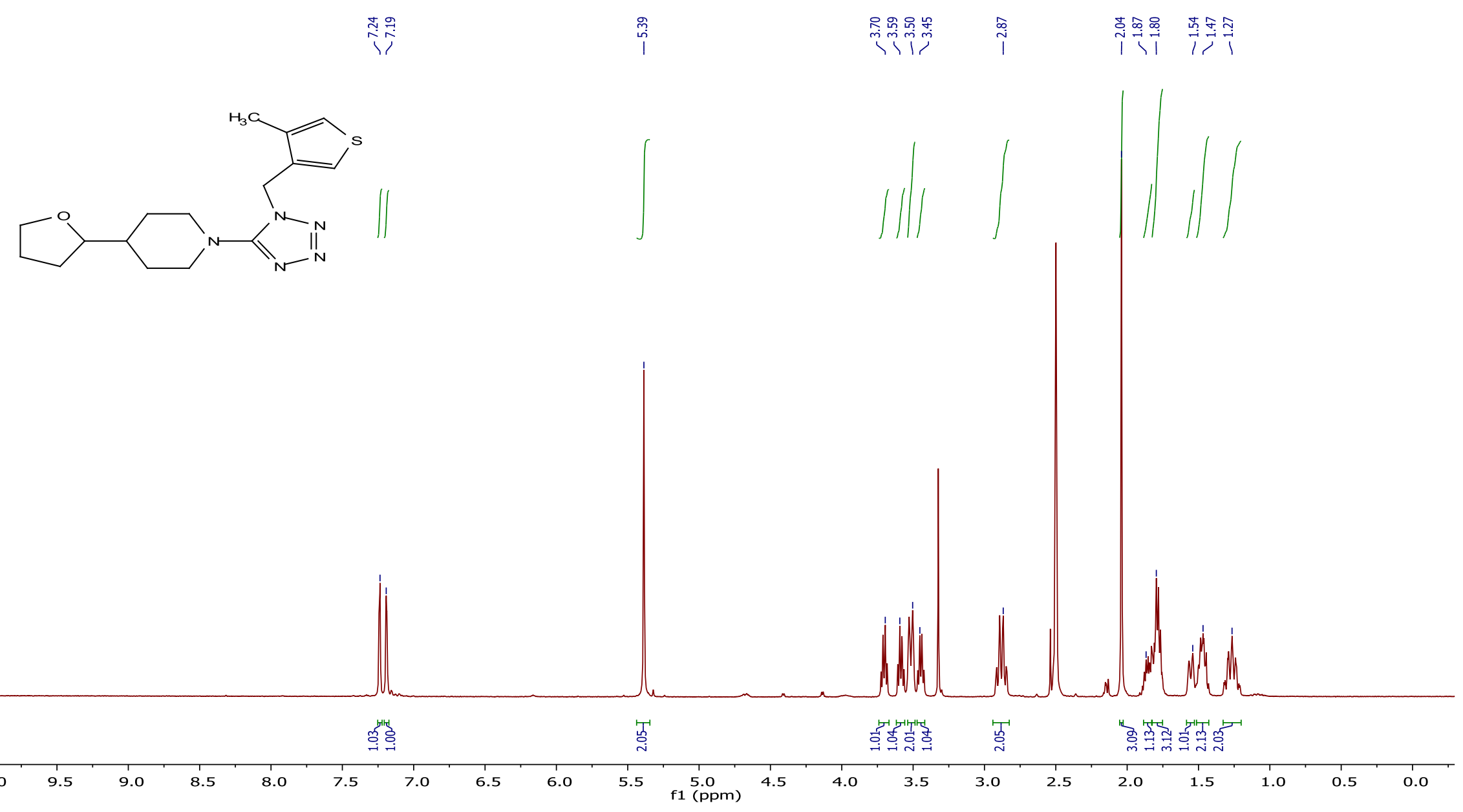


${ }^{13} \mathrm{C}$ NMR spectrum of the compound $\mathbf{9}\{306,261\}$.

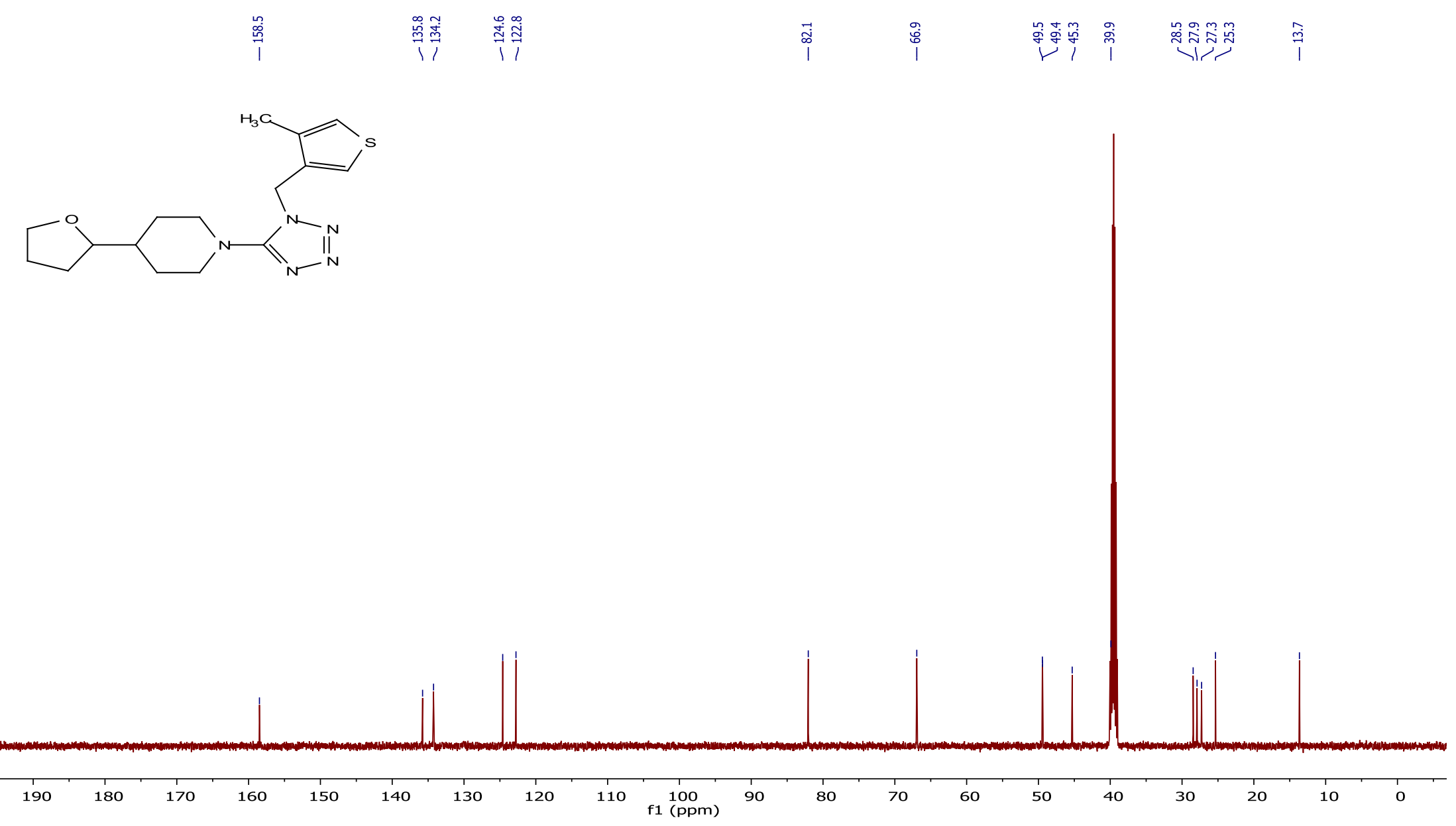


${ }^{1} \mathrm{H}$ NMR spectrum of the compound $\mathbf{9}\{341,346\}$.

总
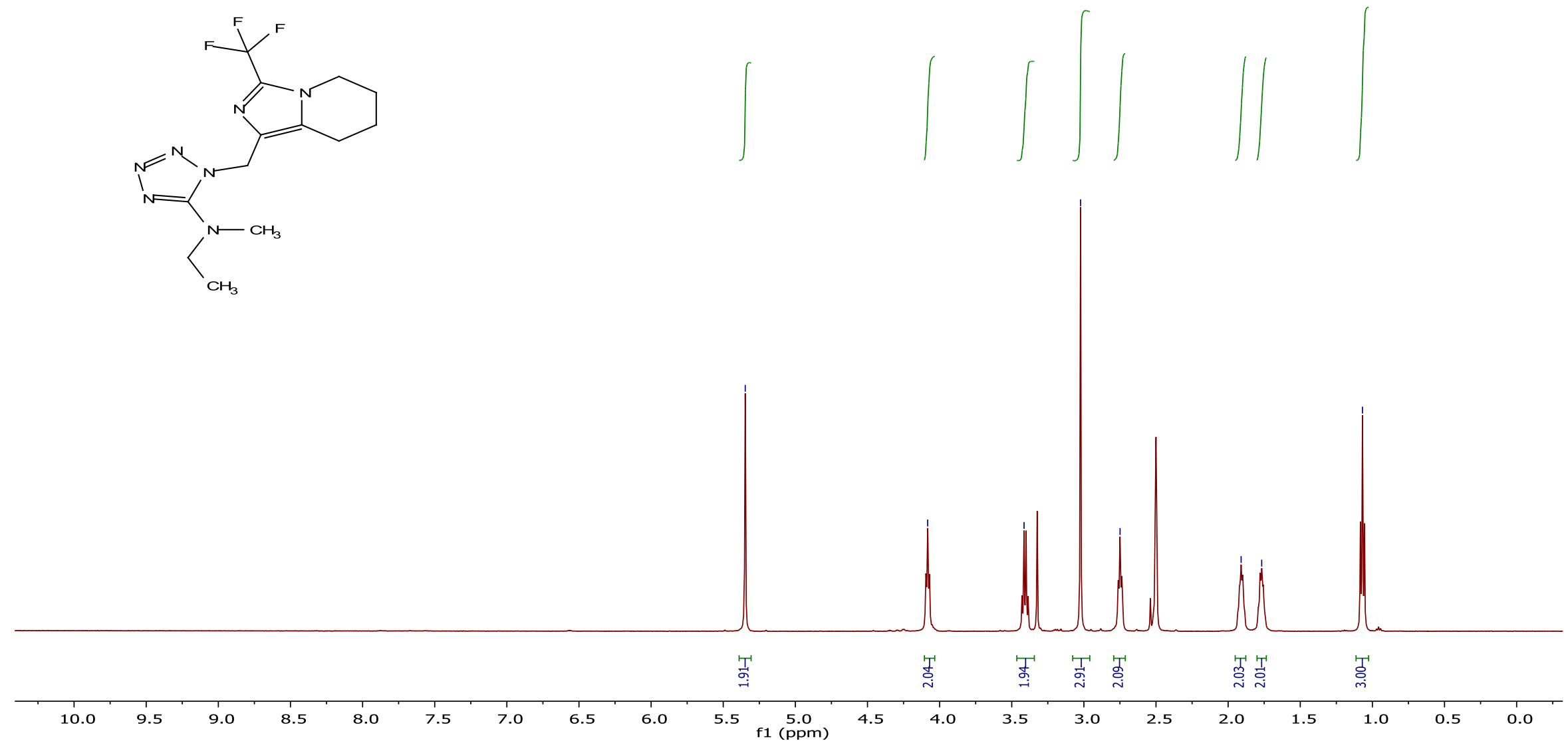
${ }^{13} \mathrm{C}$ NMR spectrum of the compound $\mathbf{9}\{341,346\}$.

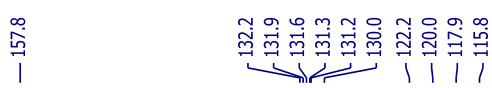

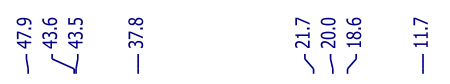
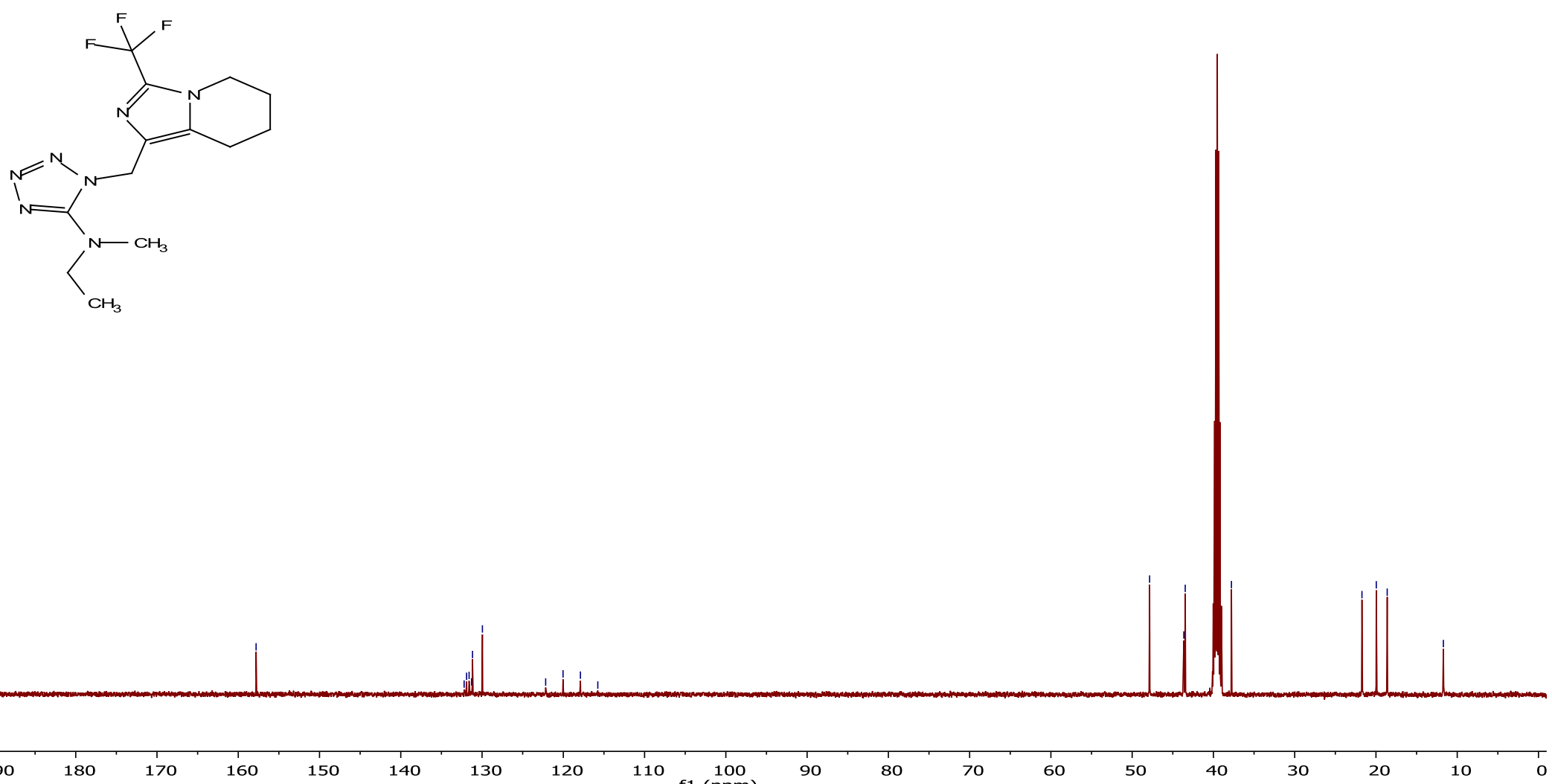
${ }^{19} \mathrm{~F}$ NMR spectrum of the compound $\mathbf{9}\{341,346\}$.

$\stackrel{9}{\grave{i}}$
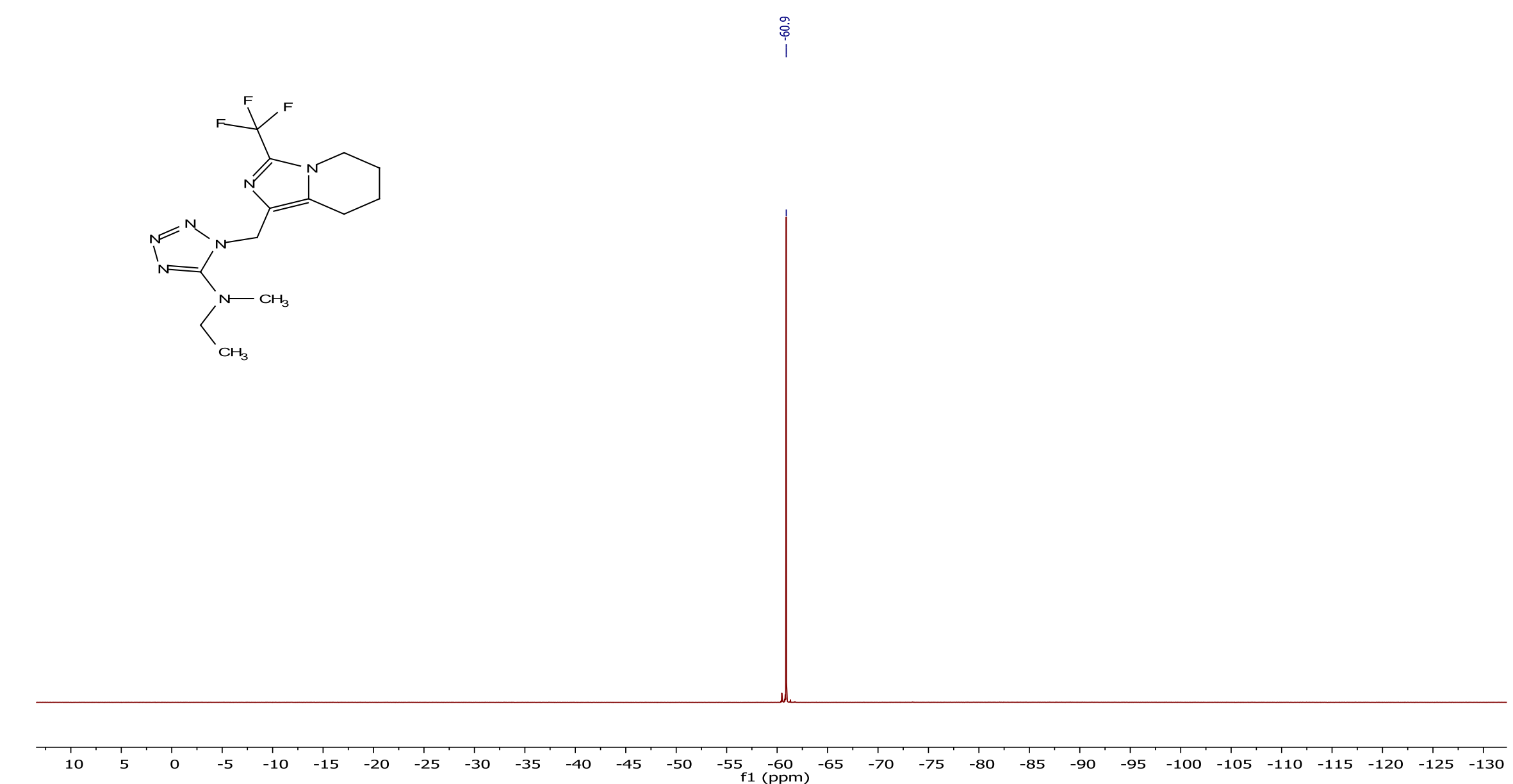
${ }^{1} \mathrm{H}$ NMR spectrum of the compound $\mathbf{9}\{375,511\}$.

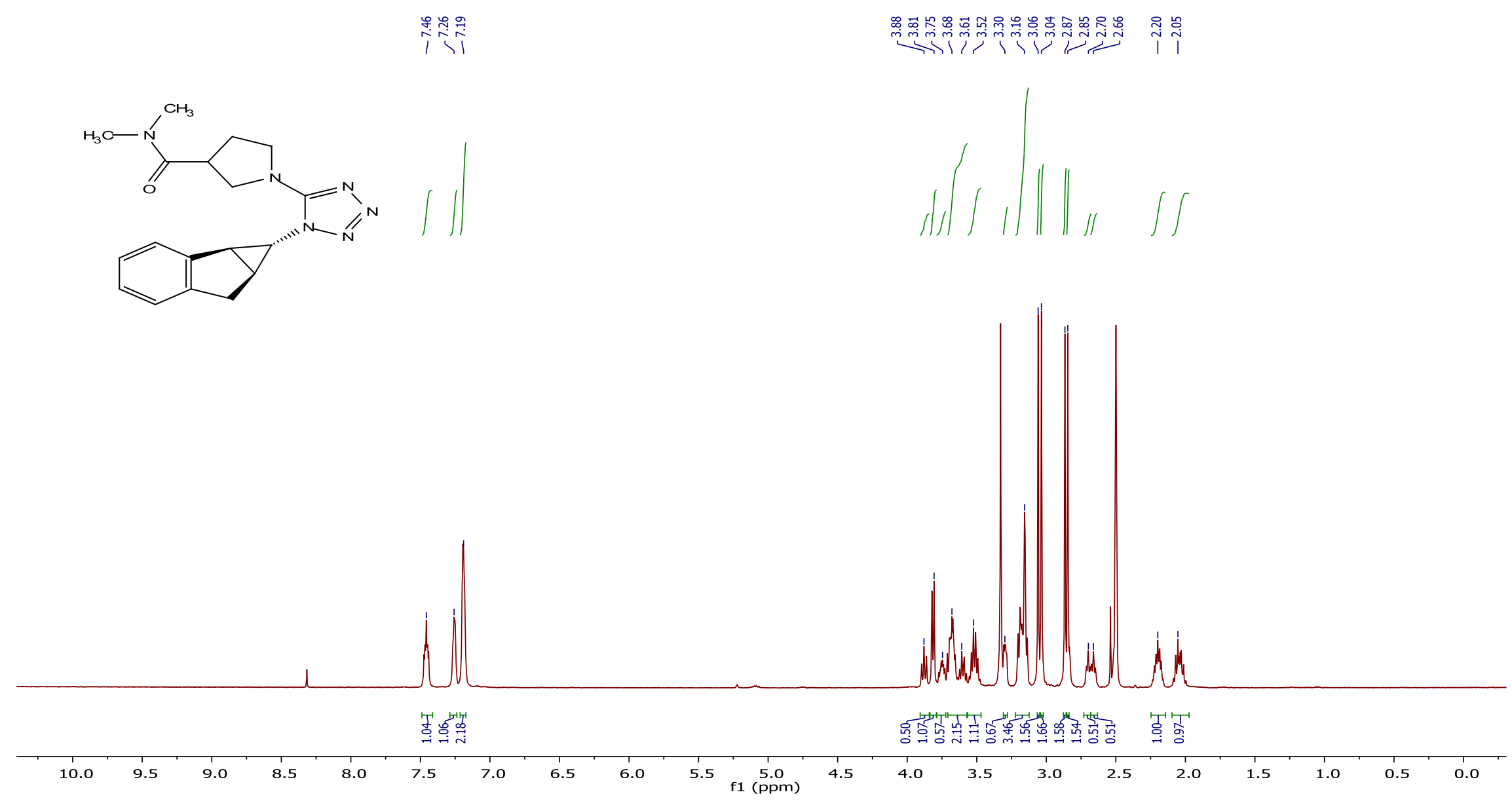


${ }^{13} \mathrm{C}$ NMR spectrum of the compound $\mathbf{9}\{375,511\}$.
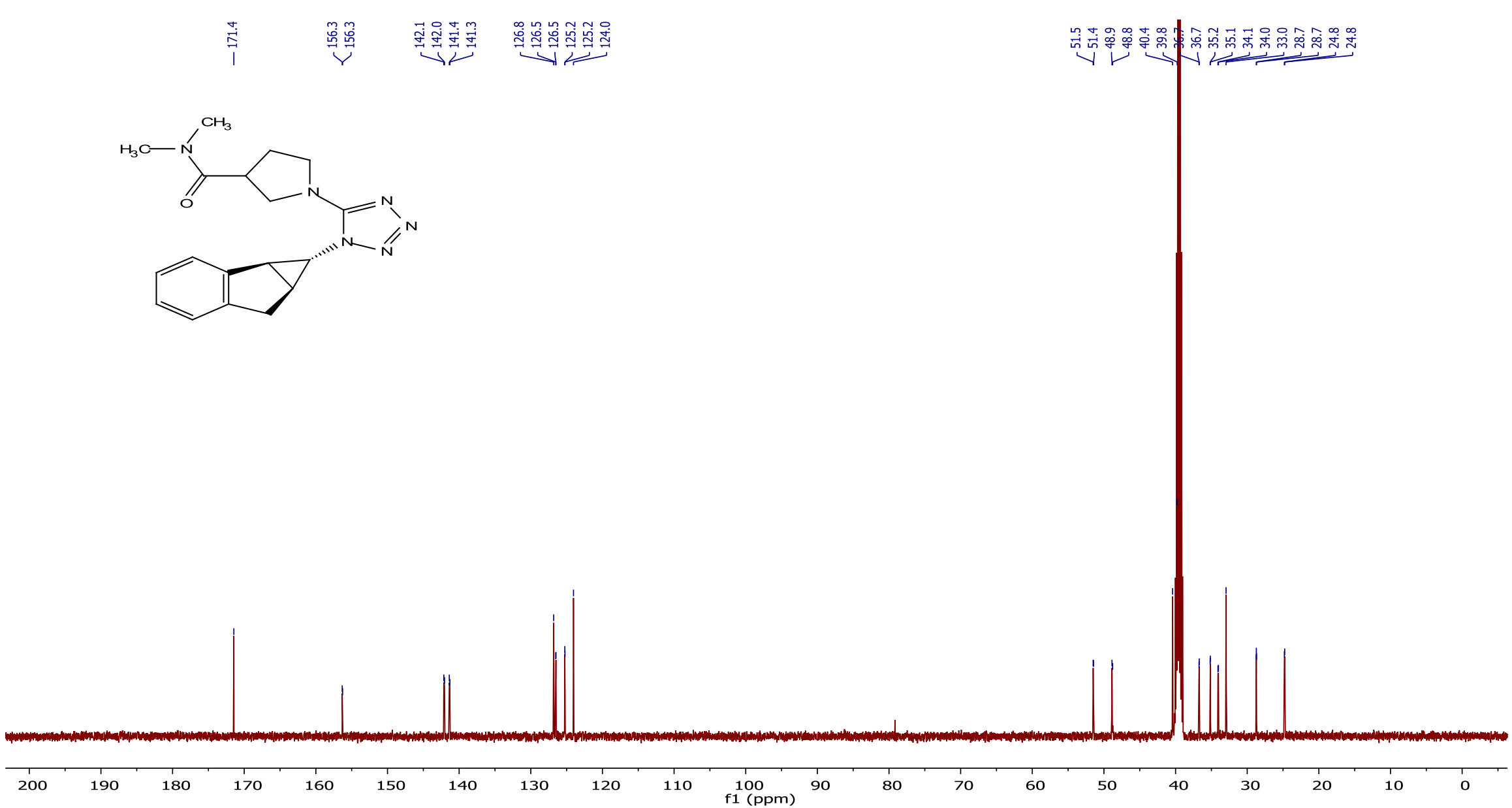